Jan Honsel

Das Kaufverhalten im Antiquitätenmarkt 


\section{Jan Honsel}

\section{Das Kaufverhalten im Antiquitätenmarkt}

Antiquitäten sprechen als einzigartige Individualgüter ein breites Spektrum sozialer, psychischer, ästhetischer und funktionaler Bedürfnisse an. Die Inhaltsanalyse der Käufermotivation, d.h. die Frage nach dem Warum von Antiquitätenkäufen, steht im Mittelpunkt der vorliegenden Arbeit. Zugleich wird nach den personalen, situativen und warenbezogenen Bestimmungsgrößen sowie den empirisch erfaßbaren Verhaltenswirkungen der Motive gefragt. Eine an den Motivationsstrukturen orientierte Käufertypologie bildet die Grundlage der Diskussion eines zielgruppenspezifischen Antiquitätenmarketing.

Jan Honsel, geboren 1953, studierte Betriebswirtschaftslehre an der Universität Münster von 1972 - 1977; Examen als Diplom-Kaufmann 1977 in Münster. 
Das Kaufverhalten im Antiquitätenmarkt

Jan Honsel - 978-3-631-75024-7

Downloaded from PubFactory at 01/11/2019 09:09:25AM

via free access 


\section{Schriften zum Marketine}

Herausgegeben von Prof. Dr. Heribert Meffert

\section{Band 9}

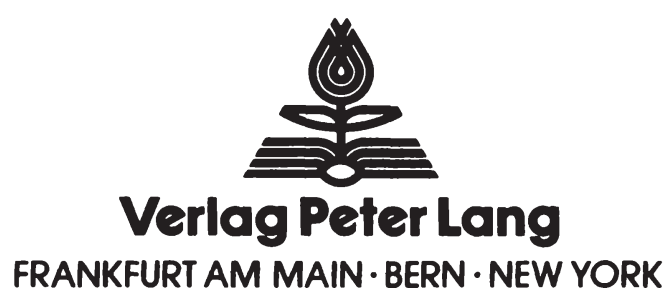




\section{Jan Honsel}

\section{Das Kaufverhalten im Antiquitätenmarkt \\ Eine empirische Analyse der Kaufmotive, ihrer \\ Bestimmungsfaktoren und \\ Verhaltenswirkungen}

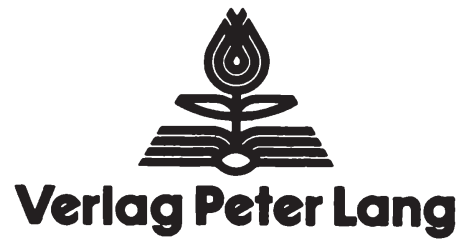

FRANKFURT AM MAIN · BERN - NEW YORK 
CIP-Kurztitelaufnahme der Deutschen Bibliothek

\title{
Honsel, Jan:
}

Das Kaufverhalten im Antiquitätenmarkt : e. empir. Analyse d. Kaufmotive, ihrer Bestimmungsfaktoren u. Verhaltenswirkungen / Jan Honsel. - Frankfurt am Main ; Bern ; New York : Lang, 1984.

(Schriften zum Marketing ; Bd. 9)

ISBN 3-8204-762.6-1

NE: GT

Open Access: The online version of this publication is published on www.peterlang.com and www.econstor.eu under the international Creative Commons License CC-BY 4.0. Learn more on how you can use and share this work: http:// creativecommons.org/licenses/by/4.0.

\section{(c) (i) \\ BY}

This book is available Open Access thanks to the kind support of ZBW - Leibniz-Informationszentrum Wirtschaft.

\author{
D 6 \\ ISSN $0721-5819$ \\ ISBN 3-8204-7626-1
}

ISBN 978-3-631-75024-7 (eBook)

(C) Verlag Peter Lang GmbH, Frankfurt am Main 1984

Alle Rechte vorbehalten.

Nachdruck oder Vervielfältigung, auch auszugsweise in allen Formen wie Mikrofilm, Xerographie, Mikrofiche, Mikrocard, Offset verboten.

Druck und Bindung: Weihert-Druck GmbH, Darmstadt 


\section{Vorwort des Herausgebers}

Die heutige Vielfalt von Antiquitätenhandlungen, Auktionsund Messeveranstaltungen sowie eine anhaltend hohe Medienpublizität spiegeln anschaulich das seit Jahren breite Interesse der Konsumenten an Antiquitäten wider. Weder diese Tatsache noch ein inzwischen beachtenswertes Marktvolumen, welches Antiquitäten $z u$ einem "aggressiven Konkurrenzprodukt" im Wettbewerb um das verfügbare Verbraucherbudget werden läßt, konnte bislang die Aufmerksamkeit der Marketingforschung auf diesen interessanten und durch eine Vielzahl von Besonderheiten einzigartigen Teilmarkt lenken.

Mit dem Anspruch einer explorativen Pilot-Studie wird in der vorliegenden Arbeit auf der Basis eines empirisch erhobenen Datensatzes das Kaufverhalten im Markt für Antiquitäten untersucht. Schwerpunkt bildet die Analyse der Motivationen von Antiquitätenkäufern sowohl im Hinblick auf ihre personalen, situativen und warenbezogenen Bestimmungsgrößen als auch im Hinblick auf ihre Erklärungsbeiträge zu konativen und kognitiven Verhaltensweisen der Käufer. Aus der Sicht der Käuferverhaltensforschung wird damit ein Problemkreis aufgegriffen, der unter Berücksichtigung des spezifischen Marktumfeldes bislang weder in empirisch-praktischer noch theoretischer sicht Beachtung gefunden hat.

Ausgehend von einem Verständnis der Käufermotivation als psychisch-kognitives aber zugleich situativ-warenspezifisches Verhaltenskonstrukt wird ein verhaltenswissenschaftliches S-O-R-Modell als theoriegeleiteter Bezugsrahmen gewählt. Zum besseren Verständnis wird der detaillierten Analyse der Käufermotivation eine systematische Bestandsaufnahme des motivational relevanten "Variablenumfeldes" vorangestellt, welche neben einem interessanten Definitionsversuch des Antiquitätenbegriffes aus der sicht von Antiquitätenkäufern eine strukturierende Marktübersicht gibt. 
Neben der umfassenden Analyse einzelner Motivationsinhalte und der empirischen Prüfung ihrer Beziehungen zu Motivationsdeterminanten und motivationsgeleitetem Verhalten verdient das anschließend vorgestellte Motivationstypen-Konzept nicht zuletzt auch unter statistisch-methodischen Gesichtspunkten besondere Beachtung. Die Vorteile dieses Konzeptes liegen nicht nur in einer erhöhten Transparenz und Uberschaubarkeit, sondern zugleich auch in einer größeren Realitätsnähe der implizierten motivationalen Erklärungsansätze des Käuferverhaltens. Dies wird insbesondere dadurch deutlich, daB Antiquitätenkäufer nur im Ausnahmefall monothematisch motiviert sind und die gefundenen neun Motivationstypen als Repräsentanten weitgehend homogener, aber zugleich multithematischer Motivationsstrukturen zu interpretieren sind. Im Zusammenhang mit der vom Autor vorgenommenen sozio-demographischen Typenbeschreibung ist in der Typenkonzeption ein praxisnaher Ansatz einer motivationsorientierten Marktsegmentierung der Antiquitätenkäufer als Grundlage eines Antiquitätenmarketing zu sehen.

Insgesamt gesehen leistet der Verfasser einen beachtenswerten Beitrag zur wissenschaftlichen Analyse des Käuferverhaltens auf einem bislang wenig bearbeiteten Gebiet. Die sorgfältig interpretierten empirischen Ergebnisse liefern vielfältige Ansatzpunkte für ein Antiquitätenmarketing. Es ist deshalb zu wünschen, daß die innovative Arbeit über den engeren Kreis der Fachwissenschaften hinaus auch in der Praxis Interesse findet. 
Ein stetig wachsender Kreis privater Antiquitätenkäufer hat in der Vergangenheit die Marktperspektive des Antiquitätenhandels vorwiegend auf das Problem der Warenakquisition verengt. Erste Anzeichen einer fortschreitenden Marktsättigung mit der Folge eines zunehmenden Wettbewerbsdrucks sowie eine gerade in jüngerer zeit spürbare Konjunktur- und insbesondere Rezessionsempfindlichkeit des Marktes fordern ein Umdenken in Richtung einer absatzpolitisch fundierten Kundenakquisition. Unabdingbare Voraussetzung eines in diesem Sinne erfolgversprechenden "Antiquitätenmarketing" ist ein gesichertes Grundlagenwissen über das Kaufverhalten im Antiquitätenmarkt.

Ziel der vorliegenden Arbeit ist es, im Rahmen einer explorativen Pilot-Studie theoriegeleitete Aussagen über das Verhalten von Antiquitätenkäufern durch eine empirische Untersuchung abzusichern und damit Entscheidungshilfen für ein kundengerechtes Antiquitätenmarketing bereitzustellen. Ausgehend von dem Grundgedanken, daB Antiquitäten als heterogene Individualgüter mit Unikatcharakter die unterschiedlichsten sozialen, psychischen, ästhetischen und funktionalen Nutzen repräsentieren können, wird der Käufermotivation eine zentrale Bedeutung zur Verhaltensexplikation beigemessen. Die Inhalte einzelner Kaufmotive und die Identifizierung übergreifender Motivkonfigurationen (Motivations-Typen) stehen dabei zusammen mit ihren situativen, personalen und warenspezifischen Bestimmungsfaktoren sowie ihren möglichen Verhaltenswirkungen im Mittelpunkt des Forschungsinteresses.

Die Entstehung dieser in erster Linie empirisch angelegten Arbeit war auf eine tatkräftige Unterstützung verschiedener Personen angewiesen. Hervorzuheben ist an dieser Stelle die umfassende Förderung durch meinen akademischen Lehrer, Herrn Professor Dr. Heribert Meffert. Ihm verdanke ich nicht nur die Anregung der Themenstellung, sondern ebenso eine Vielzahl richtungsweisender Impulse bei der Themenbearbeitung. 
Im Zusammenhang mit der notwendigen Datenerhebung danke ich den Trägern und Organisatoren der Münchener Kunst- und Antiquitäten-Messe, der Internationalen Antiquitätenausstellung in Essen und des Antiquitätenmarktes in Münster für die Erlaubnis, eine schriftliche Befragung durchführen zu können. Bei der z. T. langwierigen und programmtechnisch komplexen EDV-Datenaufbereitung erwies sich Herr V. Lückemeier als kompetente Hilfe.

Nicht zuletzt bedanke ich mich herzlich bei allen Kolleginnen und Kollegen des Instituts für Marketing der Universität Münster für ihre freundliche Unterstützung und ständige Diskussionsbereitschaft. In der konzeptionellen Anfangsphase gilt dies insbesondere für Herrn Dr. M. Bruhn, Herrn Dr. W. Kook und Herrn Dr. G. Kimmeskamp. Zu ganz besonderem Dank bin ich meinem Freund und Kollegen, Herrn Dr. J. Althans, verpflichtet, dessen fachlicher Rat und umfassende Hilfe die Entstehung dieser Arbeit begleiteten.

Abschließend danke ich Frau M. Bürger und Frau E. Thomas für die problemlose Abwicklung der zumal für die zahlreichen Tabellen und Abbildungen umfangreichen Schreibarbeiten.

Bielefeld, im Januar 1983 Jan Honsel 


\section{INHALTSVERZEICHNIS}

Seite

A. Die Motivation von Antiquitätenkäufern als Problem der Käuferverhaltensforschung

1. Motivationsanalyse als Grundlage des Antiquitätenmarketing

2. Motivation als psychische Determinante des Verhaltens von Antiquitätenkäufern

2.1 Motivation als hypothetische Erklärungsvariable des Käuferverhaltens

2.2 Bestimmungsfaktoren der Motivation

3. Ziel und Gang der Untersuchung

B. Antiquitäten, ihr Markt und ihre Käufer - Ergebnisse einer empirischen Exploration

1. Analyse des Antiquitätenbegriffes

1.1 Strukturelle Merkmale von Antiquitäten

1.2 Käuferbezogene Begriffsauffassung von Antiquitäten

1.21 Operationalisierung des Begriffsraumes

1.22 Dimensionen der Begriffsauffassung

1.23 Vorstellungsbild privater Käufer

2. Analyse der Strukturen und Prozesse im Antiquitätenmarkt

2.1 Käuferbezogenes Modell des Antiquitätenmarktes als Orientierungsrahmen

2.2 Kennzeichnung der Marktpartner von privaten Antiquitätenkäufern

2.21 Antiquitätenhandel

2.22 Auktionswesen

2.23 Serviceanbieter

2.3 Antiquitätenkreislauf und Marktregeneration

2.4 Rahmenbedingungen des Antiquitätenmarktes 
Seite

3. Analyse des Käuferverhaltens privater Antiquitätenkäufer

3.1 Personale Dispositionen

3.11 Soziodemographische Merkmale

3.12 Psychographische Merkmale

3.121 Persönlichkeitsmerkmale

3.122 Lebensstil

3.1221 Aktivitäten

70

3.1222 Allgemeine Wertorientierungen

3.1223 Wohnstil

3.123 Wahrgenommene Instrumentalität und Leitbild

3.124 Interessenentstehung

3.2 Verhaltensmerkmale

3.21 Entscheidungsverhalten

3.211 Typen und Träger der Kaufentscheidung

3.212 Kaufbegleitende Kognitionen

\subsection{Involvement \\ 3.2122 Kognitive Konflikte}

3.2123 Preisbereitschaft

3.22 Einkaufsstättenwahl

3.221 Anforderungen an Einkaufsstätten

3.222 Nutzung ausgewählter Einkaufsstätten

3.23 Informationsverhalten

3.231 Merkmale des Informationsstils

3.232 Nutzungsintensitäten von Informationsquellen

3.233 Thematische Ausrichtung des Informationsinteresses

C. Die Motivation von Antiquitätenkäufern - Erklärungsansätze und ihre empirische Prüfung

1. Bezugsrahmen der Erklärungsansätze

2. Motivationen und ihre Bestimmungsfaktoren 
2.1 Operationalisierung

Seite

2.11 Bedeutung einzelner Motivationsinhalte

2.12 Dimensionen der Motivation

2.2 Analyse der Käufermotivation

2.21 Streben nach Selbstverwirklichung und Selbstdarstellung

2.22 Streben nach Konsumerlebnis

2.23 Antiquitätenkauf als ökonomisches Kalkül

2.24 Sammelleidenschaft

2.25 Liebhaberei

2.26 Familiär bedingte Motivationen

2.27 Repräsentationsstreben

2.28 Endogener Geschmackswandel

2.29 Streben nach Ästhetik

3. Motivationstypen (Motivationskonfigurationen) und ihre Bestimmungsfaktoren

3.1 Operationalisierung

3. 11 Ansatzpunkte und Vorgehensweise bei der Typenbildung

3.12 Dimensionalität der Typenlösung 184

3.2 Analyse der Motivationstypen 192

3.21 Stark engagierte Typen 193

3.211 Nostalgiker

3. 212 Materialist

3.213 Individualist 199

3.214 Liebhaber 205

3.22 Schwach engagierte Typen $\quad 211$

3.221 Erlebniskäufer 217

3.222 Rationalist 217

3.223 Traditionalist 223

$\begin{array}{ll}3.224 \text { Sammler } & 228\end{array}$

3.3 Analyse der Typenzugehörigkeiten 234

3. 31 Wahrscheinlichkeit der Typenzugehörigkeit 240

3. 32 Prognose der Typenzugehörigkeit 246 
4. Vergleichende Analyse der Erklärungsbeiträge von Motivationen und Motivationskonfigurationen zum Verhalten

Seite

4. 1 Erklärungsbeiträge $\mathrm{zu}$ kognitiven Verhaltensmerkmalen

4.11 Kaufrelevanz von Antiquitäten-Merkmalen

4.12 Wahrgenommenes Risiko und kognitive Dissonanz

4.13 Involvement

4.14 Preisbereitschaft

4.15 Interessenausrichtung

4.16 Anforderungen an Einkaufsstätten

4.2 Erklärungsbeiträge zu konativen Verhaltensmerkmalen

274

4.21 Typen und Träger der Kaufentscheidung

274

4.22 Informationsverhalten

275

4.221 Merkmale des Informationsstils

4.222 Thematische Ausrichtung des Infor-

4.223 Nutzung von Informationsquellen

D. Implikationen der Motivationsanalyse für ein Antiquitäten-Marketing

1. Implikationen für die weltere Forschung

2. Implikationen für ein Antiquitätenmarketing

Anhang I : Untersuchungsdesign und Stichprobenstruktur

Anhang II : Abbildungen und Tabellen

Anhang III: Fragebogen 
Abb. 1: Die Stellung der Motivation im Rahmen eines S-O-R-Paradigma zur Verhaltensexplikation von Antiquitätenkäufern

Abb. 2: Strukturelle Merkmale und käuferbezogenes Vorstellungsbild von Antiquitäten

Abb. 3: Die Bedeutung begriffsbildender Beschreibungsmerkmale von Antiquitäten aus der Sicht von Antiquitätenkäufern

Abb. 4: "Reduzierter" Begriffsraum zur Kennzeichnung der Käufervorstellung von Antiquitäten

Abb. 5: Spannweiten der allgemeinen Käufervorstellung und durchschnittliche Vorstellungsbilder in Teilstichproben (Käufersegmenten)

Abb. 6: Modellhafte Darstellung der Grundstrukturen des Antiquitätenmarktes aus der Sicht privater Käufer

Abb. 7: Typisierung von Fachhandelsformen auf der Grundlage ihrer Sortimentsbreite und ihres Qualitätsgenres

Abb. 8: Waren- und Regenerationskreislauf als Determinanten des Antiquitätenangebotes

Abb. 9: Ausgewählte Rahmenbedingungen des Antiquitätenmarktes

Abb. 10: Semantisches Differential zur Kennzeichnung der psychographischen Selbsteinschätzung

Abb. 11: Aktivitätsniveaus in ausgewählten Lebensbereichen 
Abb. 12: Allgemeine Wertorientierungen von Antiquitätenkäufern

Abb. 13: Merkmale der Wohnumgebung von Antiquitätenkäufern

$\mathrm{Abb}$. 14: Wahrgenommene Instrumentalitäten und Leitbildmerkmale - Vorstellungsbilder privater Käufer 80

Abb. 15: Ausgangspunkte der Interessenentstehung für Antiquitäten

Abb. 16: Entscheidungstypen beim Antiquitätenkauf

Abb. 17: Indikatoren für das Involvement gegenüber Antiquitäten

Abb. 18: Preisbereitschaft von Antiquitätenkäufern in abgegrenzten Preisklassen

Abb. 19: Häufigkeitsverteilung individueller Preisgrenzen beim Kauf von Antiquitäten

Abb. 20: Anforderungen an Einkaufsstätten

Abb. 21: Merkmale des Informationsstils

Abb. 22: Nutzung von Informationsquelien

$\mathrm{Abb}$. 23: Interesse an antiquitätenspezifischen Informationsinhalten

$\mathrm{Abb}$. 24: Interesse an Sach- bzw. Sammelgebieten 
Abb. 25: Bezugsrahmen motivationaler Erklärungsansätze

Abb. 26: Bedeutung einzelner Motivationsinhalte als Beweggrund eines Antiquitätenkaufes

Abb. 27: Bestimmungs faktoren der Motivationsdimension "Streben nach Selbstverwirklichung" und Selbstdarstellung

Abb. 28: Bestimmungsfaktoren der Motivationsdimension "Streben nach Konsumerlebnis"

Abb. 29: Bestimmungsfaktoren der Motivationsdimension "Antiquitätenkauf als ökonomisches Kalkül"

Abb. 30: Bestimmungs faktoren der Motivationsdimension "Sammeln von Antiquitäten als freizeitgestaltendes Hobby"

Abb. 31: Bestimmungsfaktoren der Motivationsdimension "Liebhaberei"

Abb. 32: Bestimmungsfaktoren familiär bedingter Motivationen

Abb. 33: Bestimmungsfaktoren der Motivation "Repräsentation"

Abb. 34: Bestimmungs faktoren der Motivationsdimension "Endogener Geschmackswandel"

Abb. 35: Das "Ästhetik-Funktion-Psyche-Dreieck" zur Kennzeichnung struktureller Nutzenwerte von Antiquitäten 
Abb. 36: Bestimmungsfaktoren der Motivation "Streben

Seite nach Ästhetik"

Abb. 37: Bestimmungsfaktoren der Motivation

"Freude am Schenken"

$\mathrm{Abb}$. 38: Dendogramm für das voroptimierende, agglomerative "Ward-Verfahren" zur graphischen Darstellung der Clusterbildung und "Startpartitionierung"

Abb. 39: Motivationstypen und -konfigurationen im 2-dimensionalen ("reduzierten") Diskriminanzraum

$\mathrm{Abb}$. 40: Motivationskonfiguration "Nostalgiker"

Abb. 41: Ausprägungen motivationaler Bestimmungsfaktoren im Motivationstyp "Nostalgiker"

Abb. 42: Soziodemographie der "Nostalgiker

Abb. 43: Motivationskonfiguration "Materialist"

Abb. 44: Ausprägungen motivationaler Bestimmungsfaktoren im Motivationstyp "Materialist"

Abb. 45: Soziodemographie der "Materialisten"

Abb. 46: Motivationskonfiguration "Individualist"

Abb. 47: Ausprägungen motivationaler Bestimmungsfaktoren im Motivationstyp "Individualist"

Abb. 48: Soziodemographie der "Individualisten"

Abb. 49: Motivationskonfiguration "Liebhaber" 
Seite

Abb. 50: Ausprägungen motivationaler Bestimmungsfaktoren im Motivationstyp "Liebhaber"

Abb. 51: Soziodemographie der "Liebhaber"

Abb. 52: Motivationskonfiguration "Erlebniskäufer"

Abb. 53: Ausprägungen motivationaler Bestimmungsfaktoren im Motivationstyp "Erlebniskäufer"

Abb. 54: Soziodemographie der "Erlebniskäufer"

Abb. 55: Motivationskonfiguration "Rationalist"

Abb. 56: Ausprägungen motivationaler Bestimmungsfaktoren im Motivationstyp "Rationalist"

Abb. 57: Soziodemographie der "Rationalisten"

Abb. 58: Motivationskonfiguration "Traditionalist"

Abb. 59: Ausprägungen motivationaler Bestimmungsfaktoren im Motivationstyp "Traditionalist"

Abb. 60: Soziodemographie der "Traditionalisten"

Abb. 61: Motivationskonfiguration "Sammler"

Abb. 62: Ausprägungen motivationaler Bestimmungsfaktoren im Motivationstyp "Sammler"

Abb. 63: Soziodemographie der "Sammler"

Abb. 64: Motivationskonfiguration "Einrichter" 
Seite

Abb. 65: Ausprägungen motivationaler Bestimmungsfaktoren im Motivationstyp "Einrichter"

Abb. 66: Soziodemographie der "Einrichter"

Abb. 67: Demographische Struktur der befragten Antiquitätenkäufer

Abb. 68: Sozioökonomische Struktur der befragten Antiquitätenkäufer 
Tab. 1: Dimensionen des Antiquitätenbegriffs - Ergebnisüberblick einer Faktorenanalyse

Tab. 2: Persönlichkeitsdimensionen von Antiquitätenkäufern - Ergebnisüberblick einer Faktorenanlyse

Tab. 3: Wohnstile von Antiquitätenkäufern - Ergebnisüberblick einer Faktorenanalyse

Tab. 4: Ausgewählte Aspekte des wahrgenommenen Kaufrisikos - relative Häufigkeitsverteilungen

Tab. 5: Ausgewählte Aspekte der kognitiven Dissonanz relative Häufigkeitsverteilungen

Tab. 6: Nutzung von Einkaufsstätten - Häufigkeiten bei Mehrfachnennungen

Tab. 7: Inhaltsdimensionen der Motivation von Antiquitätenkäufern - Ergebnisüberblick einer Faktorenanalyse

Tab. 8: Erklärungsbeiträge motivationaler Bestimmungsfaktoren für die Kaufbedeutung einzelner Motivationsinhalte - Ergebnisüberblick multipler stufenweiser Regressionsanalysen

Tab. 9: Motivationstypen im Uberblick

Tab. 10: Die Bedeutung von Einzelmotivationen zur Diskrimination von Motivationstypen - Ergebnisüberblick einer multiplen schrittweisen Diskriminanzanalyse

Tab. 11: Zugehörigkeitswahrscheinlichkeiten zu Motivationstypen 
Seite

Tab. 12: Diskriminanzanalytische Klassifikationsergebnisse für die Typenzugehörigkeit auf der Basis motivationaler Bestimmungs faktoren

Tab. 13: Motivationale Erklärungsbeiträge zur Kaufrelevanz von Antiquitäten-Merkmalen - Ergebnistableaus multipler schrittweiser Regressionsanalysen

Tab. 14: Der Einfluß von Motivationskonfigurationen (Typenzugehörigkeiten) auf die Kaufrelevanz von Antiquitätenmerkmalen - Ergebnisüberblick einfacher Varianzanalysen

Tab. 15: Motivationale Erklärungsbeiträge zum Involvement gegenüber Antiquitäten - Ergebnisüberblick multipler schrittweiser Regressionsanalysen

Tab. 16: Der Einfluß von Motivationskonfigurationen (Typenzugehörigkeiten) auf das Involvement gegenüber Antiquitäten - Ergebnisüberblick einfacher Varianzanalysen

Tab. 17: Motivationale Erklärungsbeiträge zur Interessensausrichtung von Antiquitätenkäufern Ergebnistableaus multipler schrittweiser Regressionsanalysen

Tab. 18: Der Einfluß von Motivationskonfigurationen (Typenzugehörigkeiten) auf die Interessensausrichtung von Antiquitätenkäufern Ergebnisüberblick einfacher Varianzanalysen

Tab. 19: Motivationale Erklärungsbeiträge für Anforderungen von Antiquitätenkäufern an Einkaufsstätten - Ergebnistableaus multipler schrittweiser Regressionsanalysen

Tab. 20: Der Einfluß von Motivationskonfigurationen (Typenzugehörigkeiten) auf Anforderungen an Einkaufsstätten - Ergebnisüberblick einfacher Varianzanalysen 
Seite

Tab. 21: Motivationale Erklärungsbeiträge zu Stil und Kollektivität der Kaufentscheidung bei Antiquitäten - Ergebnisüberblick multipler schrittweiser Regressionsanalysen

Tab. 22: Motivationale Erklärungsbeiträge zum Informationsstil - Ergebnistableaus multipler schrittweiser Regressionsanalysen

Tab. 23: Der Einfluß von Motivationskonfigurationen (Typenzugehörigkeiten) auf den Informationsstil - Ergebnisüberblick einfacher Varianzanalysen

Tab. 24: Motivationale Erklärungsbeiträge zum thematischen Informationsinteresse - Ergebnistableaus multipler schrittweiser Regressionsanalysen

Tab. 25: Der EinfluB von Motivationskonfigurationen (Typenzugehörigkeiten) auf die Interessenausrichtung - Ergebnisüberblick einfacher Varianzanalysen

Tab. 26: Motivationaler Erklärungsbeitrag zur Nutzung von Fachbüchern als Informationsquelle Ergebnistableau einer multiplen schrittweisen Regressionsanalyse

Tab. 27: Der Einfluß von Motivationskonfigurationen (Typenzugehörigkeiten) auf die Nutzung von Informationsquellen - Ergebnisüberblick einfacher Varianzanalysen

Tab. 28: Motivationen und Nutzung von Einkaufsstätten Ergebnisüberblick von Kreuztabulierungen

Tab. 29: Motivationskonfigurationen (Typenzugehörigkeiten) und Nutzung von Einkaufsstätten Kreuztabulierungen

Tab. 30: Kennzeichnung der Stichprobe 


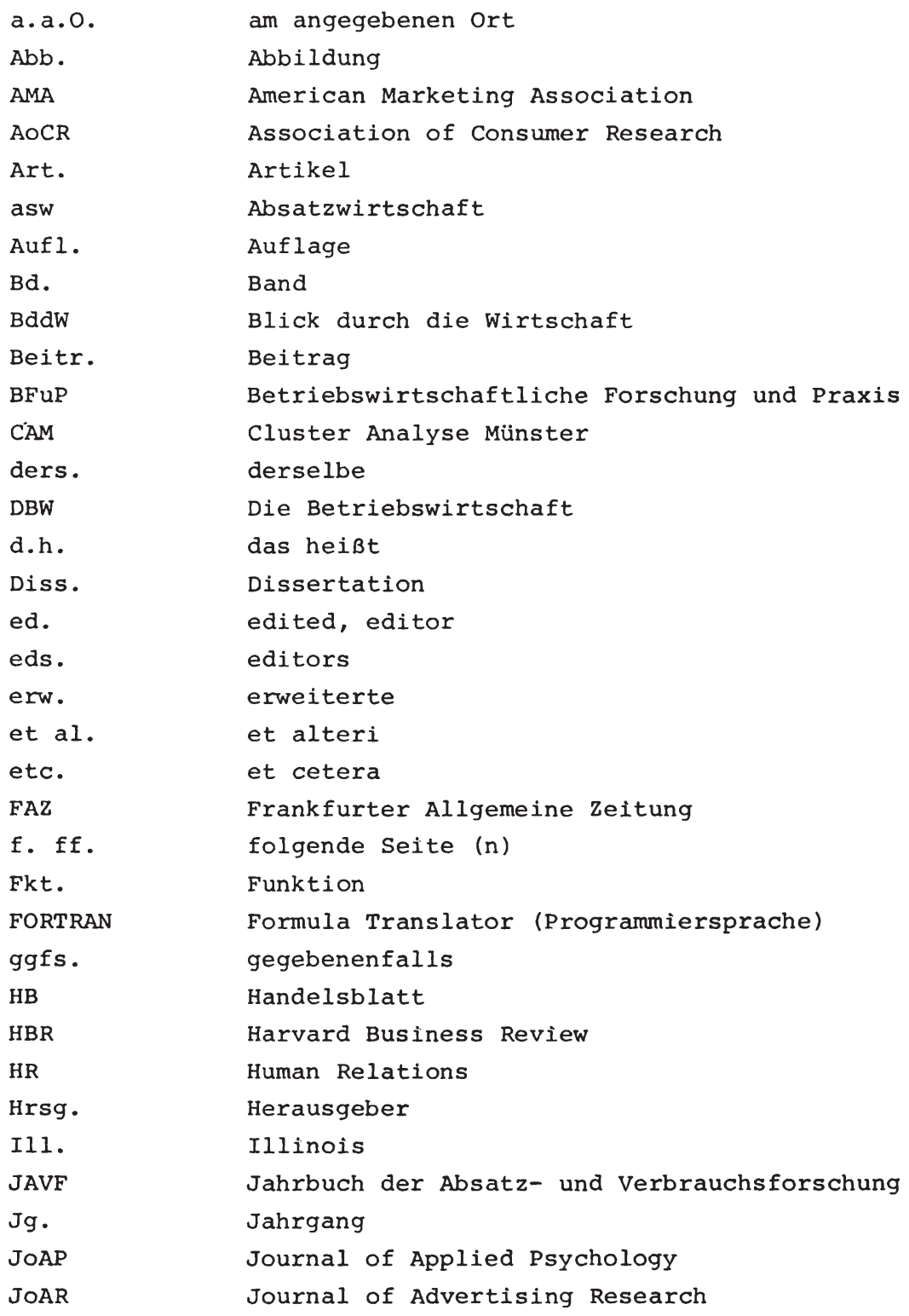




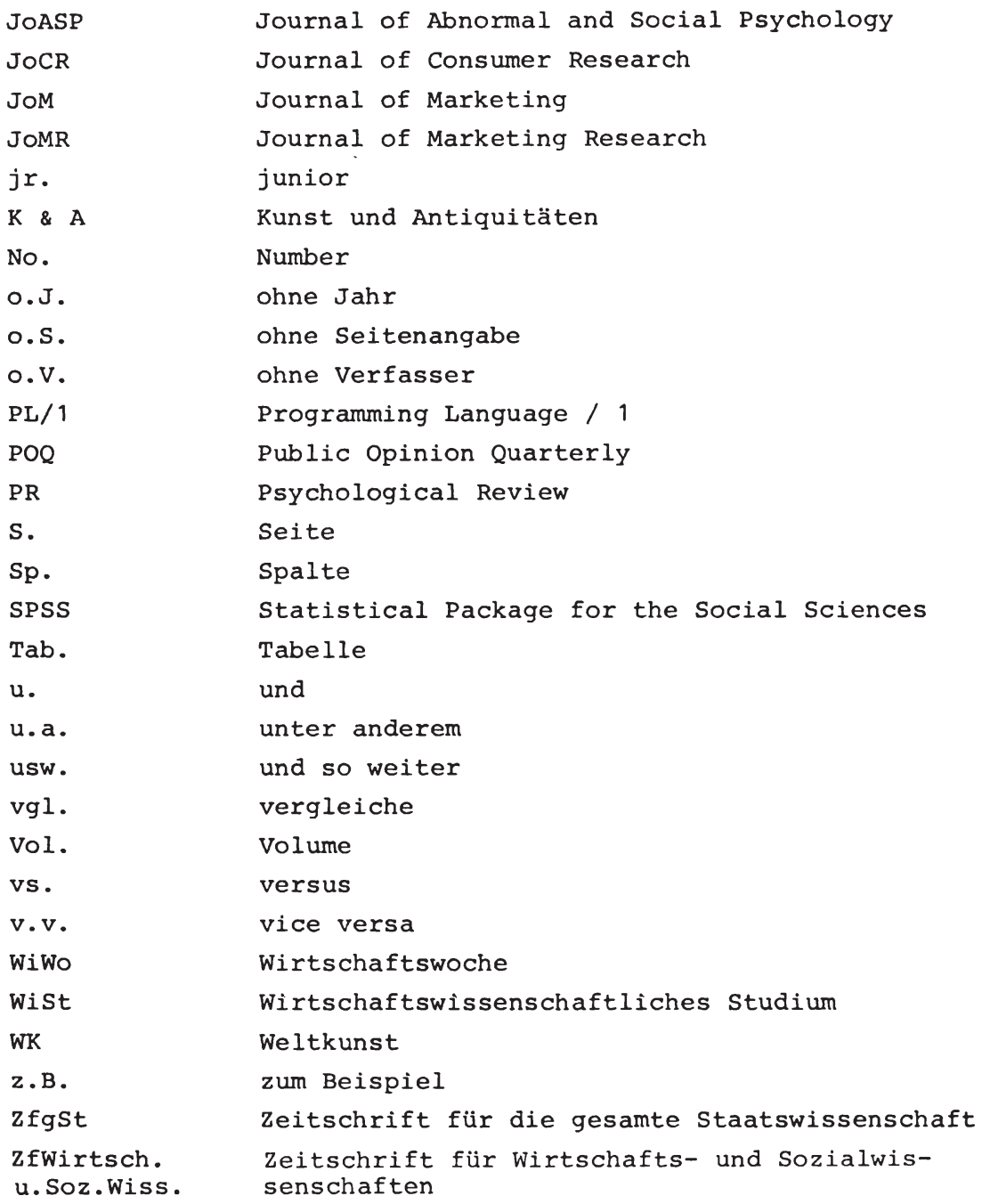


Jan Honsel - 978-3-631-75024-7 Downloaded from PubFactory at 01/11/2019 09:09:25AM via free access 


\section{A. Die Motivation von Antiquitätenkäufern als Problem der Käuferverhaltensforschung}

\section{Motivationsanalyse als Grundlage des Antiquitäten- Marketing}

Der Begriff "Antiquität" besitzt sowohl bei Laien und Käufern als auch bei Fachleuten ein weites und uneinheitliches Interpretationsspektrum. Gemeinsamkeiten in der Begriffsauffassung finden sich nur auf hohem Abstraktionsniveau, so daß Antiquitäten zunächst allgemein als Sammelbegriff für Gegenstände des Kunsthandwerks vergangener und in sich abgeschlossener Stil-, Zeit- und Geschichtsepochen stehen ${ }^{1}$.

Antiquitäten werden üblicherweise nach Gesichtspunkten des eingesetzten Materials oder ihrer ursprünglichen Gebrauchsfunktion in nicht immer überschneidungsfreie Sachund Sammelgebiete eingeteilt und als "antike (s)" Möbel, Uhren, Teppiche, Silber, Glas, Porzellan etc. auf einem weitgehend eigenständigen, durch vielfältige Besonderheiten

1 Kunstwissenschaftlich gesehen ist das "Kunsthandwerk" seinerseits Teil der sog. "angewandten Kunst", die neben der bildenden Kunst, Musik, Poesie, Epik und Bühnenkunst eine Grunddisziplin künstlerischer Ausdrucks- und Mitteilungsformen darstellt.

Zum Marketing von Organisationen, die in den genannten Kunstgattungen überwiegend kulturelle und gesellschaftsbezogene Aufgaben übernehmen (Museen, Theater, Ballet, Konzert etc.) vgl. Mokwa, M.P., Dawson, W.M., Prieve, E.A., Marketing the Arts, in: Permut, S.E. (Hrsg.), Series in Public and Nonprofit Sector Marketing, No. 1, New York 1980; Andreasen, A.R., Beek, R.W., Predictors of Attendance at the Performing Arts, in: JoCR, Vol. 7 (September 1980), S. 112-120: Nielsen, R.P., McQueen, Ch., Performing Arts Consumer Behavior: An Exploratory Study, in: AMA Combined Proceedings 1974, Chicago 1975 , s. 392 ff.. 
gekennzeichneten Antiquitätenmarkt gehandelt ${ }^{1}$.

An Antiquitäten werden in Abgrenzung $z u$ "zeitgenössischem Kunsthandwerk" sowohl altersmäßige als auch qualitative Mindestanforderungen gestellt, wodurch sich das Marktangebot insgesamt (Marktpotential) im Zeitablauf nur langsam und sachlich begrenzt erweitert ${ }^{2}$. Der aktuelle Marktkreislauf für Antiquitäten als Handelswaren erhält und regeneriert sich dagegen durch wechselseitige An- und Verkaufsbeziehungen zwischen den unterschiedlichsten Marktpartnern.

Private Käufer, kommerzieller Handel (Fachhandel, Auktion etc.) und öffentlich-rechtliche Institutionen (Museen, Verwaltungen etc.) stehen sowohl als Anbieter als auch als Nachfrager in einem intensiven Wettbewerb um das verfügbare Antiquitätenangebot, welches u.a. durch "Heterogenität", "Individualität" und"Seltenheit" jedes Einzelstücks charakterisiert ist.

1 Der Antiquitätenmarkt ist in der Handelspraxis sehr eng mit dem Markt für bildende Kunst verknüpft. Der Kunstund Antiquitätenhandel trifft dabei eine exakte sachliche Unterscheidung in Bezug auf das Warenangebot, die auch zu einem begrifflichen Nebeneinander von "Kunst" und "Antiquitäten" führt.

Unter "Kunst" werden aus handelsbezogener Sicht (aufgrund einer sprachlichen Gleichsetzung) traditionsgemäß ausschließlich Objekte der "bildenden Kunst" wie z.B. Gemälde, Aquarelle, Zeichnungen, serielle Graphiken sowie Plastiken verstanden.

Für Antiquitäten variiert Inhalt und Umfang vom Handel angebotener Sachgebiete in Abhängigkeit von Art und Zahl der berücksichtigten Abgrenzungskriterien (z.B. Alter, geographische Herkunft etc.). Als Beispiel führen die Auktionshäuser "Christie's" und "Sotheby's" für über 7o unterschiedliche Sach- bzw. Sammelgebiete eigenständige Auktionen durch.

2 Ein Beispiel ist die in den letzten Jahren zunehmende Akzeptanz und Bedeutung von kunsthandwerklichen Arbeiten des "Jugendstils". Vgl. z.B. Herchenröder, Chr., Nach kalter Polarluft eine gewisse Belebung - Freundlichere Tendênzen auf dem Kunstmarkt zum Jahresende, in: Kunst und Antiquitäten, Heft 6 (November/Dezember) 1981, S. 6 f.. 
Eine bedarfsgerechte Warenakquisition (Sortimentsbildung) bereitet dem Antiquitätenhandel aufgrund einer zumeist ausgeprägten privaten Besitzbindung einerseits und der weiten geographischen Besitzstreuung andererseits traditionell besondere Probleme ${ }^{1}$. Vermutlich liegt speziell hierin und weniger in der oft gefühlsmäßig empfundenen Unvereinbarkeit von Antiquitäten (als Kunst) und ihrer bewußten Kommerzialisierung ${ }^{2}$ die bis heute untergeordnete Bedeutung eines verkaufs- und marketingorientierten Denkens im Antiquitätenhandel begründet.

Grundgedanke eines Antiguitäten-Marketing im Sinne einer Management-Konzeption des professionellen Antiquitätenhandels ist, die Wünsche, Strebungen und Bedürfnisse aktueller und potentieller Kunden (Marktpartner) zum Ausgangspunkt einer gezielten Gestaltung angestrebter Austauschprozesse zu machen. Die damit verbundene Gestaltungsauf-

1 Die Dominanz des "gewußt wo" des Wareneinkaufs erinnert zusammen mit dem für den Antiquitäterımarkt in den letzten Jahrzehnten typischen Nachfrageüberhang an "produktionsorientierte" Entwicklungsphasen von Konsumgütermärkten. $\mathrm{Zu}$ den Entwicklungsphasen des Absatzbereiches in Konsumgütermärkten vgl. Meffert, H., Marketing. Einführung in die Absatzpolitik, 5. Aufl., Wiesbaden 1980, S. 33 f.; Kotler, $\mathrm{Ph} .$, Marketing-Management. Analyse, Planung und Kontrolle, Stuttgart 1974, S. 15 f..

2 Zur kritischen Diskussion des Verhältnisses von "Kunst und Kommerz" vgl. u.a. Bongard, W., Kunst \& Kommerz, Zwischen Passion und Spekulation, Oldenburg-Hamburg 1967; Reitlinger, G., The Economics of Taste, Bd. I-III, London, New York 1961-1970; v.Rath, K., Simmat, E.W., Schultze, B., Schreib, W., Thwaites, A.I., Ein provokatorischer Dialog, in: Neven DuMont, A. (Hrsg.), Der Kunstmarkt, dumont magnum Heft 50 (Oktober 1968), S. 72-76; Schloz, G., Kunst und Kommerz - Frivolés Verhältnis, in: Wirtschaftswoche Heft 10 (vom 7. März 1980), S. 50-51; Dawson, M.W., The Arts and Marketing, in: Mokwa,P. et al., Marketing The Arts, a.a.o., S. 7 ff.. 
gabe eines Antiquitäten-Marketing umfaßt Planung, Koordination und Kontrolle aller Aktivitäten des Antiquitätenhandels, die mit Ankauf, Verkauf, Tausch sowie Vermittlung von Antiquitäten und den sie begleitenden Kommunikationsprozessen in Zusammenhang stehen ${ }^{1}$.

Die Tatsache, daß eine umfassende markt- und insbesondere kundenorientierte Ausrichtung des Antiquitätenhandels nur gering ausgeprägt ist, erstaunt um so mehr, da sich die situativen Rahmenbedingungen des Antiquitätenmarktes entscheidend geändert haben ${ }^{2}$ :

(1) In Verbindung mit langfristig gestiegenem Realeinkommen sind Antiquitäten ins Bewußtsein einer breiten privaten Käuferschicht gerückt, wodurch sie ihre Bedeutung als ausschließliche "Expertenware" z.B. für Museen, Sammler und Kunstkenner verloren und sich $\mathrm{zu}$ einer Handelsware mit "Konsumcharakter" entwickelt haben.

(2) Der Verkauf von Antiquitäten ist durch einen zunehmenden Wettbewerbsdruck gekennzeichnet, wie er sich in einer zum Teil explosionsartigen Zunahme von Messen, Märkten, Verkaufsausstellungen, Auktionen und Neueröffnungen von Handelsgeschäften widerspiegelt.

$1 \mathrm{Zu}$ Begriff und grundsätzlichen Merkmalen des Marketing vgl. Meffert, H., Beitr. Marketing, in: Management-Enzyklopädie, Bd. 4, München 1971, S. 383 ff.; Meffèrt, H., Marketing, a.a.O., S. 34 f.; Kotler, Ph., Marketing-Management, a.a.0., S. 11 ff.; Hill, W., Marketing, Bd. 1, 3. Aufl., Bern 1973, S. $33 \mathrm{ff.;}$ Ahlert, D., Grundzüge des Marketing, Düsseldorf $1980, S .2$ f..

2 Vgl. beispielhaft Müller-Mehlis, R., Stärkung der mittleren und unteren Ebene. Auktionen 1980 im Rückblick, in: Weltkunst (WK), 51. Jg. (1981), Heft 3, S. 203 ff.; derselbe, Nachbericht zur 25. Deutschen Kunst- und Antiquitäten-Messe in München, in: WK, 50. Jg. (1980), Heft 23, S. $3541 \mathrm{ff}$.; Herchenröder, Chr., Der Kunstmarkt 1981 - besser als sein Ruf, in: WK, 51. Jg. (1981), Heft 20, S. 2964 f.; derselbe, Die Kunstmärkte, DüsseldorfWien 1978, S. $15 \mathrm{ff.;}$ Paschke, M., Zur Marktsituation des Kunst- und Antiquitätenhandels im Frühjahr 1981, in: WK, 51. Jg. (1981), Heft 10, S. 1531.0.V., Zwischen Expansion und Rezession, in: FAZ Nr. 197 vom 27.8.1981, S. 23. O.V. Amerikas Auktionshäuser müssen den Gürtel enger schnallen - Die Flucht in die Sachwerte ebbt ab, in: FAz, $\mathrm{Nr} .56$ vom 8.3.1982, S. 23 . 
(3) Die zunehmende Kommerzialisierung einerseits und Konsumorientierung andererseits hat den Antiquitätenmarkt gegenüber gesamtwirtschaftlichen Entwicklungen (Inflation, Kapitalzins etc.) kurzfristig reagibler gemacht, wodurch "Rezessionsphasen" auch für Antiquitäten typisch geworden sind.

(4) Der langfristige und kontinuierliche Preisaufschwung führt für fast alle Marktbereiche, insbesondere aber die gehobenen Qualitätsgenres, zu einer "künstlichen Marktverengung", die zusammen mit erkennbaren Marktsättigungstendenzen ein inzwischen überwiegend selektives Käuferverhalten in bezug auf Preis und Qualität gefördert hat.

Angesichts der aufgezeigten Markttendenzen ist eine Neuorientierung der Aktivitäten des Antiquitätenhandels von der Warenakquisition $z u$ einer absatzpolitisch fundierten Kundenakquisition notwendig geworden.

Für die Anbieter von Antiquitäten gewinnt damit die systematische Sammlung von Informationen über Ziele, Bedürfnisse und Verhaltensweisen ihrer aktuellen und potentiellen Kunden als Grundlage einer zielgerichteten Marktansprache besondere Bedeutung.

"Therefore, from the point of view of marketing, all marketing activities must begin and end with some statement or understanding, either implicit or explicit, of consumer behavior" 1 .

Aufgrund der einzigartigen Warencharakteristika (Produktmerkmale) kann der Käufer mit dem Erwerb von Antiquitäten die unterschiedlichsten instrumentellen und imaginären Nutzenerwartungen verbinden. Die Exploration und Deskription individueller "Bedürfnisse" von Antiquitätenkäufern, zu

1 Markin, R.J. (jr.), Consumer Behavior. A Cognitive Orientation, New York 1974, S. 5. 
denen beispielsweise das Streben nach "individueller Selbstverwirklichung", "sozialer Selbstdarstellung", "Vermögenssicherung", "Dekoration der Wohnung" oder auch die "Freude am Einkaufserlebnis" zählen, ist daher zentraler Ausgangspunkt des Verständnisses ihres Verhaltens.

Der zunächst definitorisch nicht näher bestimmte "Bedürfnis-Begriff" erfährt in verhaltenswissenschaftlich und insbesondere psychologisch orientierten Erklärungsansätzen des Käuferverhaltens ${ }^{1}$ eine inhaltliche Konkretisierung im Motiv- bzw. Motivationsbegriff ${ }^{2}$.

\section{Motivation als psychische Determinante des Verhaltens von Antiquitätenkäufern}

Obwohl von motivationalen Erklärungsansätzen entscheidende Beiträge zum Verständnis des psychologischen Hintergrunds allgemeiner und situationsspezifischer Verhaltensweisen

1 Eine Übersicht über Erklärungsansätze, geordnet nach ihrer disziplinären Herkunft, gibt u.a. Meffert. Vgl. Meffert, H., Modelle des Käuferverhaltens und ihr Aussagewert für das Marketing, in: ZfgSt, Nr. 127 (1971), S. 329 ff.. Ubbersichten theoretischer Ansätze zur modellmäßigen Erfassung komplexer Kaufentscheidungsprozesse geben u.a. auch Schulz, Markin und Schnabel. Vgl. Schulz, R., Kaufentscheidungsprozesse des Konsumenten, Schriftenreihe Unternehmensführung und Marketing, Hrsg. Meffert, H., Bd. 2, Wiesbaden 1972, S. 41 ff.; Schnabel, H., Verhaltenswissenschaftliche Konsumtheorie, Stuttgart etc. 1979, S. $17 \mathrm{ff}$. Uberblicke über psychologische Ansätze finden sich bei Schewe, C.D., Selected Social Psychological Models for Analyzing Buyers, in: JoM, Vol. 37 (July 1973), S. 31-39; Hansen, F., Psychological Theories of Consumer Choice, in: JoCR, Vol. 3 (December 1976), S. 117-142.

2 Vgl. Kroeber-Riel, W., Konsumentenverhalten, 2. Aufl., München 1980, S. 146; Wiswede, G., Motivforschung. Eine Analyse ihrer Erkenntnisgrenzen, Schriftenreihe Marktwirtschaft und Verbrauch, GfK (Hrsg.), Bd. 18, Nürnberg 1962, S. 15 ff.; Rüttinger, B., v. Rosenstiel, L., Molt, W., Motivation wirtschaftlichen Verhaltens, Stuttgart usw. 1974 , S. $17 \mathrm{ff}$. . 
erwartet werden können, haben sie in den letzten Jahren wenig Eingang in die Käuferverhaltensforschung gefunden ${ }^{1}$.

Im gegenwärtigen Forschungs-Stadium besteht zudem weder ein einheitliches Begriffsverständnis des Motiv- bzw. Motivationskonstrukts ${ }^{2}$ noch ein allgemein akzeptierter motivationstheoretischer Erklärungsansatz mit übergreifender Gültigkeit ${ }^{3}$.

1 Vgl. Raaij, W.F. van, Wandwossen, K., Motivation - Need Theories and Consumer Behavior, in: Faculty Working Paper No. 432, Hrsg. College of Commerce and Business Administration, University of Illinois, Urbana-Champaign 1977, S. 1; Kroeber-Riel, W., Konsumentenverhalten, a.a.O.. S. 10; Fennell, G., Motivation Research Revisited, in: JoAR, Vol. 15, No. 2 (June 1975), S. 23-27.

2 "Motivation is one of the most difficult topics in the literature of psychology. There appears to be a little commonality in terms and definitions, and the reader faces real difficulties in arriving at a meaningful conceptual perspective". Kollat, D.T., Blackwell, R.D., Engel, F.E., in: dieselben (Hrsg.), Research in Consumer Behavior, New York, Chicago etc. 1970, S. 23. Inhaltliche Unterschiede der Begriffsauffassungen einzelner motivationstheoretischer schulen spiegeln sich repräsentativ in einer Sammlung von Originalaufsätzen u.a. von Levine, Freud, McClelland, Atkinson, Hebb, Hull, Toleman, die von T':omae zusammengestellt wurde, wider. Vgl. Thomae, H. (Hrsg.), Die Motivation menschlichen Handelns, Köln-Berlin 1965.

3 Vgl. Markin,R.J. (jr.), Consumer Behavior, a.a.O., S. 167 f. und S. $171 \mathrm{ff.;}$ Schnabel, H., Verhaltenswissenschaftliche Konsumtheorie, a.a.O., S. 39 ff.; Wiswede, G., Motivation und Verbraucherverhalten, a.a.0., S. $78 \mathrm{ff} .$.

Als kritische Aufsätze, insbesondere gegenüber einem Transfer von Aussagen motivationaler Erklärungsansätze der klinischen Psychologie und der Psychoanalyse auf Problemstellungen der Käuferverhaltensforschung sind zu nennen: z.B. Polnitz, A., Motivation Research from a Research Viewpoint, in: POQ, Vol. 20 (Winter 1956/57), S. $663 \mathrm{ff.;}$ Rothwell, N.D., Motivation Research Revised, in: IoM, Vol. 19 (1955), S. $150 \mathrm{ff.;}$ Sheth, J.N., Role of Motivation Research in Consumer Psychology, in: Faculty Working Paper No. 153, Hrsg. College of Commerce and Business Administration, University of Illinois, Urbana-Champaign 1974, O.S. . 
Eine theoriegeleitete empirische Analyse der Motivation von Antiquitätenkäufern hat demnach bei einer standortbestimmung des Motivationsbegriffes und seines Aussagegehalts für die Verhaltenserklärung in diesem spezifischen Markt anzusetzen.

\subsection{Motivation als hypothetische Erklärungsvariable des Käuferverhaltens}

Motive und Motivationen lassen sich als psychische Vorgänge des Antiquitätenkäufers kennzeichnen, die seinem allgemeinen wie auch seinem speziell auf Antiquitäten bezogenen Verhalten sowohl energetischen Antrieb als auch Zielgerichtetheit verleihen ${ }^{1,2}$.

"An organism is said to be motivated only when it is characterized both by a state of energy mobilization and by the direction of behavior toward some goal that is selected in preference to all other possible goals. Motive, then, is a concept that joins together a state of energy mobilization and a goal" 3 .

1 Vgl. beispielhaft Markin, R.J. (jr.), Consumer Behavior, a.a.O., S. $166 \mathrm{f}$. und S. $174 \mathrm{f}$.; Schnabel, H., Verhaltenswissenschaftliche Konsumtheorie, a.a.O., S. 31; KroeberRiel, W., Konsumentenverhalten, a.a.O., S. $146 \mathrm{ff}$..

2 Oft wird eine begriffliche Differenzierung von "Motiv" und "Motivation" vorgenommen: Motive beschreiben dabei latente Persönlichkeitsdispositionen des Verhaltens, die erst durch situationsspezifische Reize zur aktuellen Motivation werden. Vgl. z.B. Heckhausen, H., Motiv und Motivation, in: Herrmann, T., Hofstätter, P.R., Huber, H.P., Weinert, F.E. (Hrsg.), Handbuch psychologischer Grundbegriffe, München 1972, S. 298 f.. Kroeber-Riel, dessen Auffassung in dieser Untersuchung gefolgt werden soll, geht sinnvoll davon aus, daß latente Motive bei der Analyse aktueller motivationaler Prozesse implizit miterfaßt werden. Der Motiv- und Motivationsbegriff wird daher im weiteren synonym verwandt. Vgl. Kroeber-Riel, W., Konsumentenverhalten, a.a.0., S. 64 und S. 147 .

3 Newcomb, T.M., Turner, R.H., Converse, Ph.E., The Nature of Motivation, Beitr. in: Research in Consumer Behavior, Hrsg. Kollat, D.T.,Blackwell,R.D.,Engel,J.F., New York,Chicago etc. 1970, S. 25. 
Die Frage nach dem inhaltlich-interpretatorischen Schwerpunkt von Motivationen als mehr oder ausschließlich aktivierend-energetische prozesse oder als mehr oder ausschlieBlich zielorientierend-lenkende Prozesse ist dabei wesentlich vom motivationstheoretischen standpunkt abhängig ${ }^{1,2}$.

Als verhaltensrelevante Motivationen von Antiquitätenkäufern werden im Rahmen der vorliegenden Untersuchung tendenziell die situationsspezifisch aktualisierten und durch kognitive Prozesse begleiteten Beweggründe des Verhaltens als Motivationen interpretiert, wodurch die zielorientierendlenkende Motivationskomponente in den Mittelpunkt der Betrachtung rückt ${ }^{3}$.

Die Untersuchung von Motivation und motivationsgeleitetem Verhalten gründet sich daher nicht auf eine isolierte Betrachtung und Operationalisierung einzelner Motive im Sinne angeborener oder erlernter Persönlichkeitsdispositionen, sondern fabt im Gegenteil Verhalten als das Resultat eines Wechselspiels zwischen der wahrgenommenen motivationalen Situation und dem (motivierten) Käufer auf.

1 Vgl. Wiswede, G., Motivation und Verbraucherverhalten, a.a.O., S. 66; Madsen, K.B., Theories of Motivation. A comparative study of modern theories of motivation, 4 . Aufl., Kent (Ohio)-Munksgaard 1968, s. 45 ff..

2 Von einer Exploration elementarer, situationsungebundener und allen Menschen ähnlicher Grundmotive (Triebe, Instinkte etc.) des Verhaltens, werden nur geringe Erklärungsbeiträge für das Verhalten von Antiquitätenkäufern erwartet. Zum Hintergrund der psychoanalytischen Motivforschung vgl. Thomae, H., Feger, H., Hauptströmungen der neueren Psychologie, in der Reihe: Graumann, C.F. (Hrsg.). Einfürung in die Psychologie, Bd. 7, 2. Aufl., Frankfurt 1972 , S. 122-136. Zu einem kurzen Uberblick persönlichkeitstheoretischer Ansätze vgl. u.a. Krohne, H.W., Persönlichkeitstheorie, in: Herrmann, T. et al., Handbuch psychologischer Grundbegriffe, a.a.O., S. 352 ff.;

Fennell, G., a.a.O., S. 25.

3 Vgl. Markin, J.R. (jr.), Consumer Behavior, a.a.o., S. $174 \mathrm{f}$. und S. $188 \mathrm{ff}$.; Hoepfner, F.G., Verbraucherverhalten, Stuttgart usw. 1976, S. 58 ff.. 
Ein derartiger motivationaler Erklärungsansatz hat die Grundstruktur eines Verhaltensmodells, welches einem StimulusOrganismus-Reaktions-Paradigma folgt ${ }^{1}$, in dem die Motivation als "hypothetisches Konstrukt" zwischen motivationalen Bestimmungsfaktoren und motivationsgeleitetem Verhalten interveniert (vgl. Abbildung 1$)^{2}$.

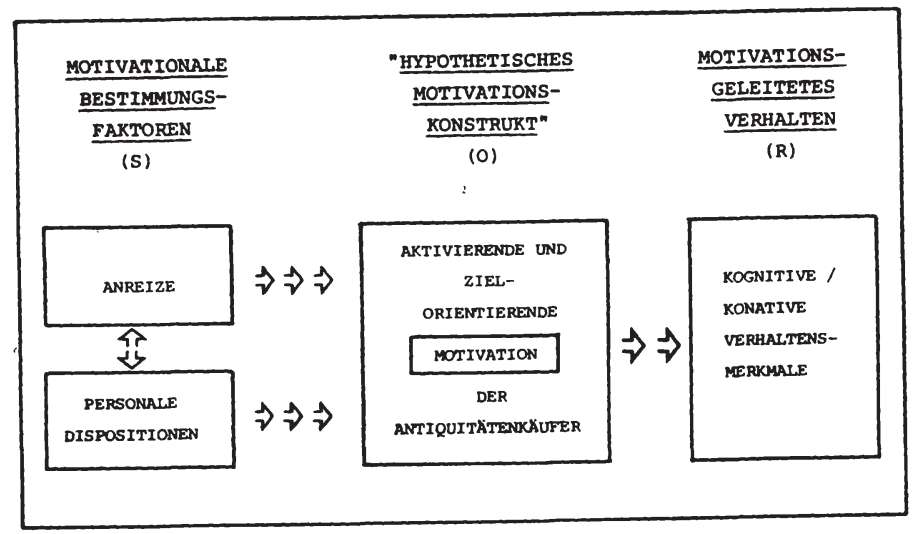

Abb. 1: Die Stellung der Motivation im Rahmen eines S-O-RParadigma zur Verhaltensexplikation von Antiquitätenkäufern

1 Vgl. Howard, I.A., Sheth, I.N., The Theory of Buyer Behavior, New York usw. 1969, S. 53 ff.; Schulz, R., Kaufentscheidungsprozesse ..., a.a.O., S. 35 f.; Jones, E.E., Gerard, H.B., Foundations of Social Psychology, New York usw. 1967, S. 57 f.; Freter, H., Mehrdimensionale Einstellungsmodelle im Marketing. Interpretation, Vergleich und Aussagewert. Arbeitspapier $\mathrm{Nr}, 12$ des Instituts für Marketing der Universität Münster, Meffert, H. (Hrsg.), Münster 1976, S. 48; Andritzky, K., Die Operationalisierbarkeit von Theorien zum Konsumentenverhalten, Berlin 1976, S. $9 \mathrm{f..}$

2 Vgl. u.a. Meffert, H., Modelle des Käuferverhaltens und ihr Aussagewert für das Marketing, in: ZfgSt, Nr. 127 (1971), S. 333 ff.; Thomae, H. , Feger, H., Hauptströmungen, a.a.O., S. 49 und S. 149 ff.; Madson, K.B., Motivation, a.a.O., S. 42 und S. 80 ff.; Rothwell, N.D., Motivation, a.a.o., s. $150 \mathrm{ff}$. und die dort angegebene Literatur. 
Entwicklung und Bedeutung des "Motivations-Konstrukts" zur Verhaltensexplikation von Antiquitätenkäufern begründet sich in den beobachtbaren inter- und intrapersonalen Verhaltensvariationen bei Antiquitätenkäufern ${ }^{1}$. Die Auswahl von "Motivation" als Erklärungsvariable des individuellen Kaufverhaltens basiert auf der zusätzlichen Hypothese, daß Verhaltensvariationen besonders hoch mit einzelnen Motivationsinhalten bzw. gesamtheitlichen Motivationskonfigurationen und den jeweiligen Motivationsintensitäten (-ausprägungen) korrelieren.

Die Korrelationsbeziehung zwischen Motivation und Verhalten, d.h. ihr Erklärungsbeitrag, ist nicht auf einen speziellen Verhaltensaspekt beschränkt. Zum motivationsgeleiteten Verhalten von Antiquitätenkäufern zählen daher hypothetisch eine Reihe sowohl kognitiver/affektiver Merkmalsklassen des Verhaltens, wie: Interesse, Anforderungen, Risikoempfinden und Involvement als auch beobachtbare konative Verhaltensmerkmale, wie: Informationsverhalten, Einkaufsstättenwahl und Merkmale des Kaufentscheidungsprozesses.

Werden Verhaltensunterschiede und -änderungen modellhaft als ausschließliches Resultat individueller Motivationsinhalte

1 "Motivation" ist nicht die einzige anzutreffende und diskutierte "gedankliche Konstruktion" zur Verhaltenserklärung. Besondere Aufmerksamkeit wurde im Rahmen der Verhaltensforschung in den letzten Jahren den "Einstellungen" geschenkt. Von "Einstellungen" wird dabei aufgrund ihrer "Verhaltensnähe" eine hohe prognistische Relevanz für spezifische Verhaltensweisen (i.e.s. Kauf vs. Nicht-Kauf) erwartet. "Nun ist der Prognosewert und Erklärungswert eines Konstrukts nicht identisch mit seiner operationalen Griffigkeit. So führen Einstellungsvariablen oftmals zu nichtssagenden oder beinahe tautologischen Aussagen ... Einstellungen sollten also "hinterfragt" werden, d.h. motivational begründet werden, um echte Erklärungen für faktisches Verhalten zu bieten". Wiswede, G., Motivation und Verbraucherverhalten, a.a.0., S. 12 und S. 13. Daneben werden auch "Emotionen", "Involvement", "Wahrnehmung" u.a. Konstrukte auf ihre Verhaltensrelevanz untersucht. 
und Motivationsausprägungen angesehen ${ }^{1}$, wird erwartet, daß die Höhe motivationaler Erklärungsbeiträge aufgrund der unterschiedlichen "relativen Motivationsnähe" einzelner Verhaltensaspekte verschieden stark ausgeprägt sind ${ }^{2}$.

Zudem variiert auch die "Art" der motivationalen Erklärungsbeiträge in Abhängigkeit von den Inhaltsdimensionen einzelner Verhaltensaspekte. Als Beispiel sind beim Informationsverhalten von Antiquitätenkäufern sowohl Einflüsse der Motivation auf die Wahl von Informationsquellen als auch der Intensität ihrer Nutzung oder den jeweils interessierenden Themen $\mathrm{zu}$ erwarten ${ }^{3}$.

Die Annahme, individuelle Verhaltensunterschiede auf den motivational-psychologischen Hintergrund einzelner Antiquitätenkäufer zurückführen zu können, impliziert zugleich die Frage nach den empirisch erfaßbaren Bestimmungsfaktoren einzelner Motivationsarten (Inhaltsklassen), ihren Erscheinungsstrukturen (Konfigurationen) wie auch ihrer isolierten und kombinierten Ausprägungen (Intensitäten / Dominanzen).

1 Dies entspricht einer "ceteris-paribus-Bedingung" des motivationalen Erklärungsansatzes.

2 Die generelle und auch theoretische Bedeutung des hypothetischen Motivationskonstrukts wird um so größer zu beurteilen sein, um so thematisch verschiedenartiger und enger die erwarteten Korrelationszusammenhänge empirisch nachvollzogen werden können. In Bezug auf die zeitliche Stabilität des Konstrukts wird unterstellt, daß Motivationen relativ zeitüberdauernd Handlungstendenzen auslösen bzw. steuern und sich tendenziell nur langfristig durch Lern- und Erfahrungsprozesse verändern.

3 Vgl. Burnkrant, R.E., A Motivational Model of Information Processing Intensity, in: JoCR, Vol. 3 (June 1976), S. 21-30. Rüttinger, B., v. Rosenstiel, L., Molt, W., a.a.O., S. $159 \mathrm{ff.;}$ Wiswede, G., Motivation zur Information - Determinanten konsumrelevanter Informationssuche, in: Jahrbuch der Absatz- und Verbrauchsforschung 1975, S. $221 \mathrm{ff}$.. 


\subsection{Bestimmungsfaktoren der Motivation}

Das in der vorliegenden Untersuchung vertretene kognitive Begriffsverständnis von Motivation beinhaltet, daß motivational bedingte $\mathrm{Zielorientierungen} \mathrm{des} \mathrm{ausgeführten}$ Verhaltens bzw. der Verhaltenstendenzen von Antiquitätenkäufern auf eher mentalen steuerungs-, Vermittlungs- und Wahrnehmungsprozessen beruhen.

Die individuelle Aktualisierung antiquitätenspezifischer Motivationsinhalte als Ausgangspunkt des Motivationsprozesses werden durch motivationale Anreize oder personale Dispositionen initiiert ${ }^{1}$.

Anreizfunktionen beim Kauf von Antiquitäten besitzen dabei zwei wichtige Gruppen von Bestimmungsfaktoren in ihren individuellen Ausprägungen: die warenbezogenen und die situationalen ${ }^{2}$ Motivationsdeterminanten. Die warenbezogenen Determinanten umfassen alle Eigenschaften und Merkmale von Antiquitäten als Kaufgegenstände. $\mathrm{Zu}$ den situationalen Einflußgrößen zählen dagegen alle motivationsrelevanten Merkmale und Entwicklungstendenzen des Antiquitätenmarktes und der spezifischen (engeren) Kaufsituation. Gemeinsam setzen und begrenzen beide Determinantengruppen die äußeren Rahmenbedingungen des motivationalen Geschehens.

Die kognitiv orientierte Interpretation des Motivationsprozesses legt nahe, die Wirkung motivationaler Reize nicht

1 Vgl. Hansen, F., Psychological Theories ...., a.a.0., s. $120 \mathrm{ff} . ;$ Rüttinger, V., v. Rosenstiel, L., Molt, W., a.a.O., S. $20 \mathrm{ff.;}$ Thomae, H., Kognition und Motivation Moderne Aspekte eines alten Problems, Beitrag in: Hartmann, K.D., Koeppler, K. (Hrsg.), Fortschritte der Marktpsychologie, Bd. 1, Frankfurt 1977, S. $10 \mathrm{ff.}$; Madson, K.B., a.a.O., S. 324 ; Sanchez, H.T., O'Brien, T.V., Summers, G.W., Self Concept and Consumer Motivation, in: AMA Combined Proceedings 1975, Mazze, E.M. (ed.), S. 224 ff..

2 Vgl. u. a. Belk, R.W., Situational Variables and Consumer Behavior, in: JoCR, Vol. 2 (1975), S. $157 \mathrm{ff.;}$ Rokeach, M., Beliefs, Attitudes, and Values, San Francisco 1968, S. $133 \mathrm{ff.}$. 
auf ihre "objektiv-reale" Erscheinung, sondern auf ihre subjektiv vom Käufer empfundenen Bedeutungsinhalte und den damit verbundenen motivationalen Aufforderungsgehalten zurückzuführen ${ }^{1}$. In diesem Sinne wird davon auszugehen sein, daß erst die individuellen Vorstellungsbilder der Käufer von Antiquitäten und ihre individuellen Einschätzungen von Markt- und Kaufsituation motivationale Reize darstellen. Kaufobjekte und Kaufsituationen sind Grundlage einer gedanklichen Antizipation möglicher Zielbeiträge des (geplanten oder realisierten) Kaufverhaltens (Besitz, Information etc.) und der Verhaltensfolgen (soziale Anerkennung, Selbstwert etc.) als Bestandteil der erwarteten Motivationsbefriedigung.

Erst die Berücksichtigung personaler Determinanten der Motivation und die daraus resultierenden unterschiedlichen Bewertungen und Akzentuierungen der motivationalen Reize, erlaubt eine umfassende Erklärung individueller Motivationen bei ansonsten gleichen situationalen und warenbezogenen Rahmenbedingungen.

Personale Motivationsdeterminanten ${ }^{2}$ bestimmen sowohl die selektive Wahrnehmung als auch die Reizbewertung (Zielbeiträge) in Bezug auf die angestrebten Verhaltensfolgen (Verhaltensziele). Aus der vielzahl möglicher personengebundener Dispositionen von Antiquitätenkäufern werden insbesondere von einer psychographischen selbsteinschätzung anhand vorgegebener Persönlichkeits- und Lebensstilmerkmale sowie in geringem Umfang auch von soziodemographischen Ausgangs-

1 Vgl. Mischel, W., Toward a cognitive social learning reconceptualization of personality, in: Psychological Review 80 (1973), S. 252-283.

2 Vgl. Hansen, F., a.a.O., S. 126; Kassarjian, H.H., Personality and Consumer Behavior: A Review, in: JoMR, Vol. 8 (November 1971), S. 409-418. Sanchez, H.T., O'Brien, T.V., Summers, G.W., a.a.O., S. 225 ff.. 
daten wichtige Erklärungsbeiträge zu Motivationsinhalten und-ausprägungen erwartet.

Abschließend ist festzuhalten, daß die Motivation von Antiquitätenkäufern weder in ihren inhaltlichen Erscheinungsformen (Merkmalsklassen) noch in ihrer Bedeutung für die Verhaltensexplikation und ggf. Prognose (Merkmalsausprägungen) durch nur eine einzige Determinante erklärbar werden. Warenbezogene, situative und personale Bestimmungsfaktoren üben in einem interdependenten Beziehungsgefüge gemeinschaftlich Einfluß auf die Motivation von Antiquitätenkäufern aus ${ }^{1}$.

\section{Ziel und Gang der Untersuchung}

Angesichts des offenkundigen Forschungsdefizits im Rahmen motivationaler Erklärungsansätze des Käuferverhaltens, soll die vorliegende Arbeit am Beispiel von Antiquitätenkäufern die Bedeutung des Motiv- bzw. Motivationskonstrukts zur Verhaltenserklärung in einem spezifischen Markt untersuchen. Zentrale inhaltliche ziele sind entsprechend eine möglichst umfassende Analyse von Motivationen des Antiquitätenkaufs, ihrer Bestimmungsfaktoren sowie ihrer Beziehung zu kognitiven und konativen Verhaltensmerkmalen des Antiquitätenkäufers.

Das grundlegende Verständnis von Motivation als psychischkognitives aber insbesondere auch situativ-warenspezifisches Konstrukt, setzt ein Mindestmaß an Kenntnissen über Antiquitäten, ihren Markt und ihre Käufer voraus.

1 In Abgrenzung $\mathrm{zu}$ rein situativen oder rein dispositionsorientierten Ansätzen, geht der hier vertretene Erklärungsversuch von Motivationen von einer Interaktion aller Bestimmungsfaktoren aus. Vgl. Hansen, F., a.a.O., S. 118; Dolich, J.I., Congruence Relationships Between Self Images and Product Brands, in: JoMR, Vol. 6 (February 1969), S. $80-84$. 
So fordert der Warenbezug motivationaler Prozesse zunächst den Versuch einer motivationsrelevanten, d.h. käuferbezogenen Definition des Antiquitätenbegriffs. Literaturmeinungen existieren zu diesem Problemkreis nicht. Auch Versuche einer strukturierenden Durchdringung des Antiquitätenbegriffs sind, abgesehen von summarischen Aufzählungen einzelner Sammel- bzw. Sachgebiete, bislang unvollständig und unzulänglich. Ausgehend von einer umfassenden strukturell-inhaltlichen Begriffsbestimmung von Antiquitäten soll daher auf empirischer Basis versucht werden, die wichtigsten Merkmale und Dimensionen des Antiquitätenbegriffs aus Käufersicht zu ermitteln (Kapitel B.1.).

Die Ermittlung des Vorstellungsbildes von Antiquitäten aus der Sicht privater Antiquitätenkäufer bietet die Möglichkeit einer Abgrenzung des "relevanten Antiquitätenmarktes". Die Kenntnis der grundsätzlichen marktlichen Gegebenheiten und wichtiger noch seiner charakteristischen Eigenarten, die ihn gegenüber "normalen" Konsumgütermärkten zu einem atypischen Sondermarkt werden lassen, sind ihrerseits Vorbedingung eines tiefergehenden Verständnisses motivationaler Prozesse des Antiquitätenkäufers. Es besteht daher die Notwendigkeit einer kurzen systematischen Marktübersicht unter besonderer Berücksichtigung wichtiger Bezüge zur Motivation (Kapitel B.2.).

Bildet das Marktgeschehen den übergeordneten Bezugsrahmen der Motivationsanalyse, so dient die Beschreibung wichtiger demographischer und psychoqraphischer Verhaltensmerkmale des Antiquitätenkäufers in ihrer typischen Ausprägung dem Verständnis des engeren motivationalen Hintergrunds (Kapitel B.3.).

Ziele und Inhalte der sich anschließenden Motivationsanalyse verdeutlicht der folgende Aufgabenkatalog (Kapitel C): 
(1) Exploration und Beschreibung antiquitätenspezifischer Einzelmotivationen und Motivationsdimensionen (Motivationsfaktoren) durch Bestimmung ihrer personalen, situationalen und warenbezogenen Determinanten sowie die Analyse ihrer Einflußrichtung und -stärke.

(2) Untersuchung des Erklärungsbeitrags von Motivationen des Antiquitätenkäufers $\mathrm{zu}$ ausgewählten kognitiven und konativen Verhaltensaspekten zur Uberprüfung von Tragfähigkeit und Anwendungsbereich des Motivationskonstrukts in einem spezifischen Markt.

(3) Ermittlung verhaltenswirksamer Motivationskonfigurationen durch Aggregation von motivationsähnlichen Antiquitätenkäufern zu abgrenzbaren waren- und marktspezifischen Motivationstypen bzw. -clustern.

(4) Beschreibung der Typenlösung sowie diskriminatorische Analyse der Bestimmungsfaktoren und Dimensionalität der Typenzugehörigkeit.

(5) Untersuchung des Erklärungsbeitrags von Motivationskonfigurationen (Typenzugehörigkeit) für ausgewählte kognitive und konative Verhaltensweisen von Antiquitätenkäufern.

Da die vorliegende Untersuchung sich zur Zeit nicht auf ein geschlossenes motivationstheoretisches Konzept als Erklärungsansatz des Käuferverhaltens beziehen kann, wurde ein durch die allgemeine Käuferverhaltensforschung theoriegeleitetes aber zugleich auf die spezifischen Bedingungen des Antiquitätenmarktes zugeschnittenes explorativ-empirisches Vorgehen gewählt.

Ausgehend von dem Grundgedanken, in ein empirisch fundiertes Antiquitäten-Marketing die Motivationen der Antiquitätenkäufer einzubeziehen, wurde im Rahmen des beschriebenen Forschungsprojektes ein umfassender Fragebogen zur Einschätzung relevanter Motivationen, personen-, waren- und situationsbezogener motivationaler Bestimmungsfaktoren sowie motivationsgeleiteter Verhaltensweisen zusammengestellt. Grundlage bildeten dazu die Ergebnisse einer Vorstudie, 
welche anläßlich der Westdeutschen Kunst- und Antiquitätenmesse in Düsseldorf durchgeführt wurde.

In seiner Endfassung enthielt der schriftlich auszufüllende Fragebogen der Hauptstudie 32 übergreifende Fragestellungen mit ca. 230 Einzelitems. Aus insgesamt 600 auf der Münchener Kunst- und Antiquitätenmesse, der Internationalen Antiquitätenausstellung in Essen und dem Antiquitätenmarkt in Münster/Westf. im Rahmen eines kurzen persönlichen Gesprächs verteilten Fragebögen resultierte ein auswertbarer Netto-Rücklauf von 151 stück.

Das Design der empirisch durchgeführten studie bewegt sich damit sowohl hinsichtlich der Repräsentanz (Stichprobenauswahl und-größe) als auch des methodischen Vorgehens (Vorstudie, Befragungstechnik und -umfang etc.) auf dem Niveau einer explorativen Pilot-Studie ${ }^{1}$. Die gewonnenen Ergebnisse sind als Tendenzaussagen und Denkansätze zu werten, von denen Impulse für die motivationsorientierte Käuferverhaltensforschung erwartet werden.

Nicht zuletzt bieten die Ergebnisse der empirischen Studie die Chance, praktisch normative Aussagen in Form von Handlungsvorschlägen für konkrete Entscheidungssituationen des Antiquitäten-Marketings $\mathrm{zu}$ entwickeln und $\mathrm{zu}$ diskutieren (Kapitel D.). Implikationen dieser Art besitzen trotz ihrer eingeschränkten generellen Gültigkeit einen nicht unerheblichen pragmatischen wert.

1 Zur ausführlichen Beschreibung des Untersuchungsdesigns und der Stichprobenstruktur vgl. die Präzisierungen im Anhang I (S. $272 \mathrm{ff.}$ ) dieser Arbeit. 
B. Antiguitäten, ihr Markt und ihre Käufer - Ergebnisse einer empirischen Exploration

1. Analyse des Antiguitätenbegriffes

Eine inhaltlich-aufzählende oder klassifikatorische Beschreibung des Antiquitätenbegriffs muB aufgrund der Vielzahl realer Erscheinungsformen von Antiquitäten als Handelsware zwangsläufig unvollständig bleiben. Beispielhaft sind Möbel, Uhren, Porzellan, Silber, Ostasiatika, Schmuck, Glas und Teppiche als marktlich bedeutende Sachgebiete $z$ u nennen, die zudem beim Käufer einen engen assoziativen Bezug zum Antiquitätenbegriff besitzen ${ }^{1}$. Diskriptive Aufzählungen von Sach- bzw. Sammelgebieten können nur unzulänglich strukturierende und inhaltlicherklärende Funktionen erfüllen.

Grundlage des für ein gezieltes Antiquitäten-Marketing notwendigen Verständnisses motivationsgeleiteten Verhaltens von Antiquitätenkäufern ist daher eine zusätzliche Begriffsbestimmung aus struktureller, d.h. eher inhaltlich objektivierender sicht einerseits und käuferbezogener, d.h. eher subjektiv wertender sicht andererseits.

Bindeglied struktureller und käuferbezogener Betrachtungsweise sind die interpretierenden Wahrnehmungsprozesse des Käufers: Strukturmerkmale von Antiquitäten sind ihr Gegenstand, das allgemeine Vorstellungsbild des Käufers von Antiquitäten ihr Resultat. Abbildung 2 zeigt den Zusammenhang im Uberblick.

1 In gleicher Reihenfolge wurden (geordnet nach der Anzahl von Nennungen) Sammel- bzw. Sachgebiete von Antiquitätenkäufern in der empirischen Studie begrifflich assoziiert. Vgl. auch Frage 2 des Originalfragebogens im Anhang. 


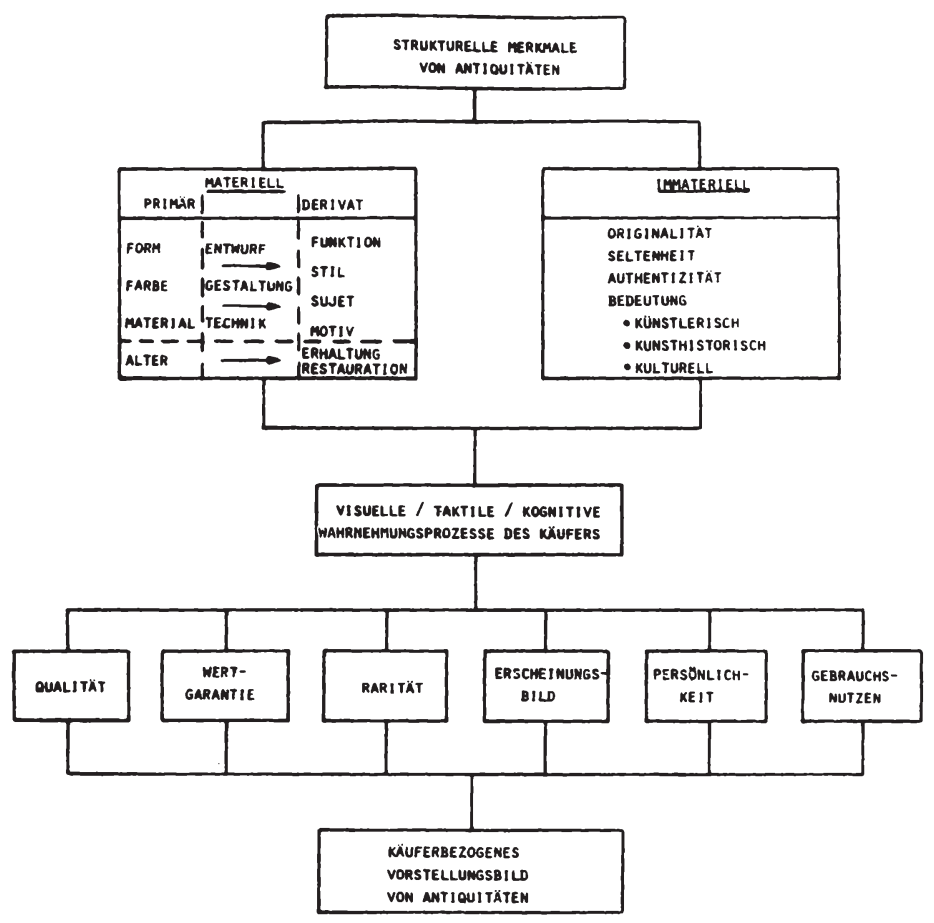

Abb. 2: Strukturelle Merkmale und käuferbezogenes Vorstellungsbild von Antiquitäten

\subsection{Strukturelle Merkmale von Antiguitäten}

Strukturelle Merkmale von Antiquitäten treten in zwei möglichen Erscheinungsformen auf:

Materielle Strukturelemente besitzen physische Korrelate mit spezifischen materiellen, optischen und haptischen Eigenschaften, die vom Käufer tendenziell wahrnehmbar sind. Immaterielle itrukturelemente dagegen sind Abstraktionen bzw. Bedeutungsinhalte, die sprachlicher, schriftlicher oder gedanklicher Konkretisierung bedürfen, um dem Käufer durch eher mentale Wahrnehmungsprozesse erschließbar zu werden. 
Die materiellen Strukturelemente lassen sich ihrerseits in primäre und derivate Bestandteile von Antiquitäten gliedern.

Zur primären Grundsubstanz jeder Antiquität gehören dabei zunächst Material, Form und Farbe, die in der summe realer Ausprägungs- und Kombinationsmöglichkeiten das theoretische Gestaltungspotential bei der kunsthandwerklichen Anfertigung von Antiquitäten bilden ${ }^{1}$. Gedanklicher Entwurf und konkreter GestaltungsprozeB beinhalten die intuitive und/oder willentlich selektive Ausschöpfung der durch Farbe, Form und Material gegebenen Gestaltungsmöglichkeiten durch einen Kunsthandwerker $^{2}$. Der Einsatz spezieller kunsthandwerklicher Techniken $^{3}$ orientiert sich an den beim Entwurf antizipierten und festgelegten derivaten Merkmalen (Gestaltungszielen) Funktion, stil und Sujet/Motiv.

Als Gebrauchsgegenstand besitzt jede Antiquität neben ästhetisch-dekorativen Eigenschaften stets eine Zweckbestimmtheit im Sinne einer Gebrauchsfunktion. Die ursprüngliche Funktion einer Antiquität ist schon aus historischer Sicht und insbesondere - soweit erhalten - aus heutiger sicht nicht mit ihrem Grundnutzen gleichzusetzen ${ }^{4}$.

1 Wichtige abstrahierte Merkmalskategorien sind:

- Materialarten (Holzarten, Edelmetalle, Glassorten, textile Stoffe etc.)

- Materialeigenschaften (Gewicht, Reinheit, Festigkeit, Struktur etc.)

- Farbeigenschaften (Ton, Intensität, Kontrast, Komplementarität etc.)

- Formeigenschaften (Geometrie, Dynamik, Volumen,Format etc.).

2 Der Gestaltungsproze $\beta$ wird daher auch insbesondere durch seine Personengebundenheit, d.h. durch individuelle Kreativität, Zielsetzung und handwerkliches Können des Kunsthandwerkers beeinflußt und begrenzt.

3 Eine umfassende Beschreibung kunsthandwerklicher Techniken muB der Kunstwissenschaft vorbehalten bleiben. Zur Begriffserläuterung werden exemplarisch einige wichtige Techniken von Ebenisten (Kunstschreinern) genannt: Furnier, Politur, Intarsie, Marqueterie, Boulle-Technik, Firnis etc..

4 Vielmehr ist es ein typisches Kennzeichen für Antiquitäten, daß sich ihre primäre Nutzenstiftung für den heutigen Käufer vom instrumentellen Gebrauchswert $\mathrm{zu}$ mehr individuellen und imaginären (Zusatz-) Nutzenerwartungen verschoben haben. 
Dies impliziert zugleich eine Aufwertung stilistischer Merkmale und Aussagen. Der Stilbegriff knüpft an die Vorstellung an, daß sich kulturelle, soziale, politische und religiöse Einflüsse einer Epoche in Gestaltungsprinzipien niederschlagen. Der Stil einer Antiquität ist damit Ausdruck einer zeittypischen Formvorstellung und-interpretation ${ }^{1}$. Die Entwicklung von Antiquitätenstilen ist wie die ihrer Einflußgrößen dynamisch ${ }^{2}$.

Als letztes durch den Gestaltungsprozeß geprägtes derivates Strukturmerkmal ist das sujet oder Motiv zu nennen. Antiquitäten dienen in ihrer gegenständlichen Form (Schrank, Schreibtisch, Uhr usw.) zugleich als Träger dekorativer und symbolischer Elemente, denen eine bewußte Gestaltungsidee, eine Thematik zugrundeliegt. Die möglichen Ausprägungen reichen von einfachen ornamenten bis zu bildlichen Darstellungen.

1 Die praktische Bedeutung einer Stilanalyse von Antiquitäten liegt darin, daß sie Anhaltspunkte für die zeitliche Zuschreibung eines Stückes unbekannter Herkunft geben kann. Wichtig in diesem Zusammenhang ist der Begriff der Stilkopie. Mit ihr sind Antiquitäten zu bezeichnen, die typische stilelemente bereits abgeschlossener Stilepochen wieder aufgreifen. Extremes Beispiel dieses Prinzips sind heutige Stilmöbel.

2 Gleiche Stilrichtungen entwickeln sich geographisch gesehen i.d.R. zu unterschiedlichen Zeitpunkten. Dabei werden zeitliche Entwicklungsphasen durchlaufen, wie: Stilentstehung, Stilblüte (Hochform) und Stilniedergang. Ubberlagerungen von Stilphasen bezogen auf unterschiedliche Stilrichtungen sind üblich. Ihre zeitliche Abfolge ist bis zum Anfang des 19. Jahrhunderts in Europa indes im wesentlichen gleichartig: Gotik, Renaissance, Barock, Rokoko und Klassizismus. Spätere Stile (Biedermeier, Jugendstil, Art Nouveau etc.) sind von eher regionaler Prägung und geringerer kunsthistorischer Reichweite. Die Stilbezeichnung für das Kunsthandwerk sind ebenso wie diejenigen für andere Kunstgattungen willkürlich gewählte klassifikatorische Begriffe. Als Standardwerk zur Stilgeschichte vgl. beispielhaft Rettelbusch, E., Stilhandbuch, Ornamentik, Möbel, Innenausbau von den ältesten Zeiten bis zum Biedermeier, 10. erw. Aufl., Stuttgart 1979 . 
Formal gesehen steht am Ende des Gestaltungsprozesses - als Gesamtheit aller derivaten Strukturelemente - ein mit eigenständiger "Persönlichkeit" versehenes kunsthandwerkliches Objekt. Erst die Berücksichtigung des Alters als zusätzliches primäres Strukturmerkmal, d.h. die Berücksichtigung des Entstehungszeitpunktes, trennt definitorisch "zeitgenössisches" Kunsthandwerk von Antiquitäten. Antiquitäten "entstehen" demnach nicht allein durch ihre materielle Ausgestaltung, sondern insbesondere auch durch einen Alterungsund AusleseprozeB.

Genaue Altersbestimmungen sind unter einschränkenden Voraussetzungen möglich ${ }^{1}$. Sie lösen jedoch keinesfalls das Problem der Formulierung eines Anspruchsniveaus im Sinne eines Mindestalters, das ein kunstgewerblicher Gegenstand besitzen muB, um aus struktureller Sicht als Antiquität zu gelten. Resultierende subjektive Auffassungs- und Interpretationsspielräume sind daher begriffsimmanent ${ }^{2}$.

Der AlterungsprozeB ist zugleich mitverantwortlich für den Erhaltungszustand einer Antiquität. Antiquitäten unterliegen im Laufe der zeit einem natürlichen und durch Gebrauch oder Beschädigung verursachten Substanzverlust. Die Erhaltung ist daher ihrerseits derivates strukturmerkmal (vgl. Abbildung 2). Ihre Beurteilung umfaBt neben vielen anderen Gesichtspunkten die des Farb- und Materialzustands ebenso wie die der Gebrauchstüchtigkeit und Vollständigkeit. Der Versuch, durch Aufarbeitung und Ersatz schadhafter Bestand-

1 Naturwissenschaftliche Methoden oder auch Beschauzeichen (Marken, Signaturen, Punzen, Stempel etc.) können relativ exakte Anhaltspunkte geben.

2 Ihnen könnte nur durch eine axiomatisch festageleơte Altersgrenze begegnet werden, wie es z.B. im deutschen Zoiltarif (Nr. 99.06) mit einem geforderten Mindestalter von $100 \mathrm{Jah}-$ ren geschieht. Aus kunstwissenschaftlicher Sicht muB eine derartige Grenzziehung unbefriedigend bleiben. Auch vom Standpunkt der Käuferverhaltensforschung ist sie abzulehnen, da sie einer überwiegend individuell geprägten Begriffsauffassung des Antiquitätenkäufers nur ungenügend Rechnung trägt. 
teile den historischen Zustand einer Antiquität möglichst exakt wiederherzustellen, wird als Restauration bezeichnet. Anteil und Güte ersetzter und aufgearbeiteter Bestandteile bestimmt den sogenannten Restaurationsgrad als ein zusätzliches Kriterium des Erhaltungszustands.

Oft werden unrestaurierte Antiquitäten als originale bezeichnet. Die originalität als wichtiges immaterielles Strukturmerkmal meint jedoch mehr als nur den originalzustand von Antiquitäten. Originalität ist Ausdruck der absoluten oder zumindest tendenziellen Einmaligkeit jedes Einzelstücks, die in originärem Entwurf und handwerklicher Ausführung bedingt ist. Antiquitäten werden daher auch oft als Unikate bezeichnet ${ }^{1}$. Die Originalität begründet schließlich zusammen mit der regionalen Verteilung von Antiquitätenbesitz und-angebot die auf Einzelstücke bezogene absolute und auf manufakturielle serien bezogene relative seltenheit.

Zusätzlich gibt sie dem Einzelstück, dem Original, eine über die materielle Erscheinung hinausgehende Identität, indem sie es mit seiner geographischen, zeitlich-kulturellen und personalen Herkunft verknüpft: Entstehungsort und -epoche sowie der prägende Einfluß des Kunsthandwerkers sind im Sinne des Originals immaterielle Bestandteile einer Antiquität ${ }^{2}$.

Die Utbereinstimmung von tatsächlicher und zugesicherter Originalität, d.h. die Frage nach der Echtheit oder Authentizität stellt sich überwiegend als eine Dokumentationsaufgabe. Vollständigkeit, Genauigkeit und Sicherheitsgrad retrograd vorgenommener zuschreibung sowie die Art ihrer

1 Schütte vergleicht diese Tatsache mit dem Zustand einer extremen Produktdifferenzierung und zieht den SchluB, daB es auf dem Kunstmarkt nur Surrogatkonkurrenz geben kann. Vgl. Schütte, R., Kunst- und Antiquitätenhandel, in: Tietz, B. (Hrsg.), Handwörterbuch der Absatzwirtschaft, stuttgart 1974, Sp. 1167.

2 Vgl. Straub, E., Das Original in der Kunst - ein Fetisch?, in: FAZ, Nr. 100 vom 30.4.1979, S. 21. 
Dokumentation (Gutachten, Expertise, Zertifikate u.ä.) sind praktische Bestimmungsgrößen der Authentizität.

Letztlich ist die "Bedeutung" von Antiquitäten als immaterielles Strukturmerkmal zu nennen. Gerade hier stößt eine intersubjektiv nachprüfbare Beurteilung auf Schwierigkeiten, da die künstlerische, kunsthistorische, kulturelle und individuelle Bedeutung einer Antiquität auch bei gröBtem Bestreben nach sachlicher Objektivität stets Ausdruck einer zeitbedingten und subjektivistisch verengten Interpretation und Wertbeimessung ist.

Aus struktureller Sicht ist daher eine abschlieBende Begriffsfindung im Sinne einer allgemeingültigen Definition nicht möglich. Eine Antiquität in den Augen eines Museumsdirektors oder eines Händlers ist aufgrund unterschiedlicher Anspruchsniveaus und Sichtweisen nicht notwendigerweise identisch mit derjenigen des antiquitäteninteressierten oder antiquitätenkaufenden Konsumenten. Die individuelle Wertschätzung des Antiquitätenkäufers ist Resultat seiner selektiv interpretierenden Wahrnehmung mehrerer oder auch aller strukturellen Merkmale. Sie legt damit zugleich die Grundlage zur käuferbezogenen Definition von Antiquitäten.

\subsection{Käuferbezogene Begriffsauffassung von Antiquitäten}

In Theorie und Praxis hat sich eine käuferbezogene Definition des Antiquitätenbegriffs bislang nicht herausgebildet. Es erscheint jedoch notwendig, gerade diesen Aspekt des Antiquitätenbegriffs sowohl theoretisch als auch empirisch zu durchdringen, da

(1) starre klassifikatorische Definitionen durch axiomatische Setzung von Anspruchsniveaus an strukturelle Merkmale der Tatsache empirisch individueller Vorstellungsbilder und Begriffsauffassungen nicht gerecht werden, 
(2) die Abgrenzung eines aus Käufersicht relevanten Marktumfeldes nur auf der Grundlage der käuferbezogenen Begriffsauffassung möglich ist, und

(3) die angestrebte Analyse der Beziehung zwischen Antiquitäten als Handelsware und Motiven von Antiquitätenkäufern nur sinnvoll auf der Basis eines Verständnisses der käuferbezogenen Sichtweise ist, 1 da Antiquitätenkäufer nur dann ihre "Bedürfnisse" befriedigen können, wenn ihr Vorstellungsbild von Antiquitäten mit ihrer Motivstruktur kongruent ist.

\subsection{Operationalisierung des Begriffsraumes}

Ausgangspunkt der empirischen Fragestellung war die Sammlung semantischer Begriffsbestandteile, welche assoziierte Eigenschaften, Vorstellungen, Meinungen und Gefühle von Antiquitätenkäufern bezogen auf Antiquitäten weitgehend (inhaltlich) repräsentativ widerspiegeln sollten ${ }^{2}$.

In ihrer Gesamtheit bilden und begrenzen diese Begriffsbestandteile den vom Käufer assozjierten Begriffsraum für Antiquitäten. Sie umschreiben damit die semantische Begriffsextension und charakterisieren mit ihren Mittelwerten die typischen Schwerpunkte einzelner Begriffsinhalte des Antiquitätenbegriffs.

Die standardisierte Abfrage der Beschreibungsmerkmale führte $z u$ dem in Abbildung 3 wiedergegebenen Mittelwertprofil .

1 Vgl. dazu die Darstellung bei Wiswede, G., Motivation ..., a.a.O., S. 95 und $z u$ analogen tberlegungen im Rahmen der Einstellungsforschung Böhler, H., Beachtete Produktalternativen und ihre relevanten Eigenschaften im Kaufentscheidungsproze $B$ von Konsumenten, Beitr. in: Meffert, H., Steffenhangen, H., Freter, H. (Hrsg.), Konsumentenverhalten und Information, Wiesbaden 1979, S. 261 f. und S. 265 ff..

2 Durch Auswertung vorhandener Literatur und freier Interviews mit Experten und Antiquitätenkäufern wurde ein umfangreicher Katalog beschreibender Merkmale und Begriffsassoziationen gesammelt. Unter Berücksichtigung von Nennungshäufigkeiten und unter Beachtung eines mittleren $\mathrm{Ab}-$ straktionsniveaus wurde im folgenden schritt eine heuristische Auswahl getroffen, die zu einem Set von 22 als relevant erachteten Beschreibungsmerkmalen (Items) führte. 
Das dargestellte Profil macht deutlich, daB der Begriffsraum in den Augen der Käufer inshesondere durch die subjektiv empfundenen Begriffsinhalte: Schönheit, Dekorativität, Qualität und Originalität geprägt wird. Aus entgegengesetzter Blickrichtung sind Mobilität, Funktionalität, Ausgefallenheit, künstlerische Bedeutung, Repräsentativität und Exklusivität weniger zutreffende Einzelvorstellungen von Antiquitä$\operatorname{ten}^{1}$.

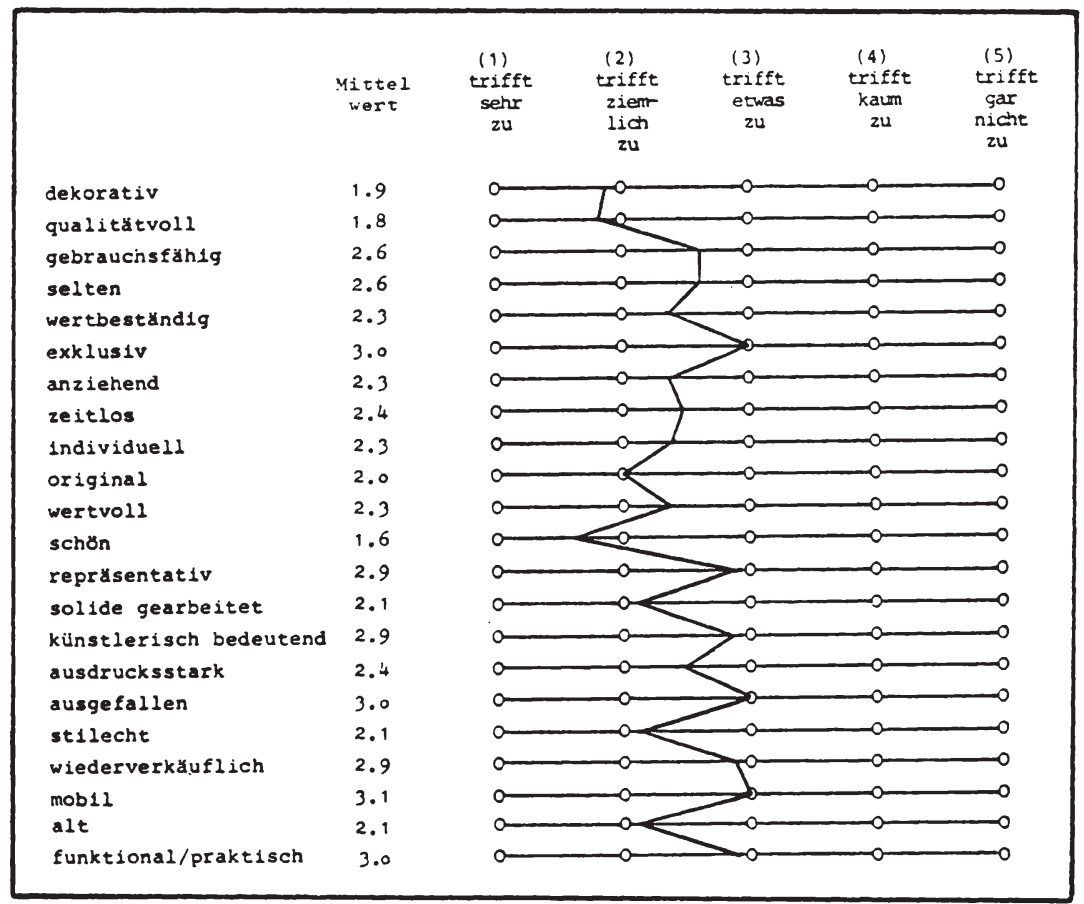

Abb. 3: Die Bedeutung begriffsbildender Beschreibungsmerkmale von Antiquitäten aus der Sicht von Antiquitätenkäufern

1 Da sich alle Item-Mittelwerte tendenziell im "positiven" $\mathrm{Zu}-$ stimmungsbereich von "trifft sehr zu" bis "trifft etwas zu" bewegen, zeigt sich, daB das heuristische Auswahlverfahren insgesamt bedeutsame, begriffsbildende Merkmale generiert hat.

Bei Mittelwerten von 2,9 (repräsentativ) und 3,0 (exklusiv), die auf einer Zustimmung von jeweils ca. 40 \& der Befragten zu den Skalenstufen (1) und (2) basieren, wird zudem eindrucksvoll bestätigt, daB sich Antiquitäten aus Käufersicht ganz allgemein zur Repräsentation und Dokumentation von Exklusivität eignen. 
Bedenkt man, wie stark die genannten Begriffsassoziationen in individuell unterschiedlich ausgeprägten Wertkategorien (z.B. "Ästhetik", "Status" und "Prestige") verwurzelt sind, wird das weite spektrum konkreter Interpretationsmöglichkeiten', aber auch ihr enger Bezug zur individuellen Kaufmotivation deutlich.

Insgesamt läßt die isolierte Betrachtung einzelner Begriffsbestandteile (Assoziationen) die Möglichkeit unterschiedlicher Abstraktionsniveaus und inhaltlich-semantischer Uberschneidungen außer acht. Fast zwangsläufig stellt sich damit die Frage nach den grundsätzlichen und unabhängigen Dimensionen einer käuferbezogenen Interpretation des Antiquitätenbegriffs bzw. der dimensionalen Struktur des Begriffsraumes ${ }^{2}$.

\subsection{Dimensionen der Begriffsauffassung}

Einen tuberblick über die empirisch ermittelten Dimensionen

1 So sind z.B. keine Aussagen möglich, ob die käuferbezogene Sicht von "Originalität" mit der bereits diskutierten strukturellen Sicht inhaltlich übereinstimmt.

2 Die Statistik stellt mit der multivariaten Faktorenanalyse ein problemadäquates Rechen- und Auswertungsverfahren zur Verfügung. Ziel der Faktorenanalyse ist die Extraktion möglichst unabhängiger Faktoren (Begriffsdimensionen) aus einem set vorgegebener Variablen (begriffsbeschreibende Items) und die damit verbundene Datenreduktion. Zur Anwendung vgl. Green, P.E., Analyzing Multivariate Data, Hinsdale 1978, S. 342 ff.; Uberla, K., Faktorenanalyse, 2. Aufl., Berlin 1971; Schuchard-Ficher, Chr. et al., Multivariate Analysemethode - Eine anwendungsorientierte Einführung, Berlin-Heidelberg-New York 1980, S. $213 \mathrm{ff}$.. Soweit nicht gesondert erwähnt, wurden alle statistischen Auswertungen mit dem SPSS-Programmpaket (in der Version 8) des Rechenzentrums der Westf. Wilhelms-Universität Münster durchgeführt. Zu Aufbau und Anwendung des SPSS vgl. Nie, N.H. et al., SPSS - Statistical Package for the Social Sciences, 2. Auf1., New York etc. 1975; Hull, C.H., Nie, N.H., SPSS Up-date, New York etc. 1979; Beutel, P. et al., SPSS - Statistik Programmsystem für die Sozialwissenschaften, 2. Aufl., Stuttgart 1978. Böhler, H., a.a.0., S. $280 \mathrm{ff.}$. 
(Faktoren), ihre Bedeutung (Varianzerklärungsanteile) sowie ihre Beziehung (Faktorladungen) zu den wichtigsten ihrer Interpretation zugrundeliegenden Begriffsmerkmalen von Antiquitäten gibt die Tabelle 1 .

Der käuferbezogene Antiquitätenbegriff wird durch die sechs Dimensionen: Qualität, Wertgarantie, Rarität, Erscheinungsbild, Persönlichkeit und Gebrauchsnutzen beschrieben. Ihr gemeinsamer Informationsgehalt, bezogen auf die Ausgangsinformation aller 22 Einzel-Items, ist mit einem absoluten Varianzerklärungsanteil von $53 \%$ eindrucksvoll. Die einzelnen Dimensionen tragen entsprechend ihrer individuellen Bedeutung zu diesem Ergebnis in unterschiedlichem Maße bei (vgl. relative Varianzanteile).

Der empirische Befund zeigt die Dominanz einer subjektiv empfundenen "Qualität" als Dimension des käuferbezogenen Antiquitätenbegriffs. Das direkte Qualitätsattribut "qualitätvoll" zusammen mit den zusätzlichen Qualitätskomponenten "schön", "solide gearbeitet", "stilecht" und "alt" prägen inhaltlich die allgemeine Qualitätsvorstellung des Käufers bei Antiquitäten. Dabei besteht jeweils eine etwa gleich enge Korrelation (vgl. Faktorladungen) mit der übergreifenden Begriffsdimension "Qualität" ${ }^{2}$. Obwohl das indivi-

1 Die Varianzerklärungsanteile beziehen sich auf die vollständige, rotierte Faktorladungsmatrix. Die Tabelle 1 gibt dagegen aus Gründen der Verständlichkeit und Úbersicht die rotierte Faktorladungsmatrix nur im Ausschnitt, d.h. unter Berücksichtigung der für die einzelnen Dimensionen aufgrund hoher Faktorladungen dominanten Beschreibungsmerkmale wieder.

2 Händleraussagen stützen die angestellten Überlegungen: Fragen nach allgemeiner Qualität, Alter und Echtheit stehen im Mittelpunkt fast jeden Verkaufsgesprächs und ersetzen ebenso wie Schönheit und Solidität als orientierungsgrößen die-aufgrund fehlender sachlicher Kompetenz erschwerte-"objektive" bzw. kunstwissenschaftliche Qualitätsbeurteilung. 


\begin{tabular}{|c|c|c|c|}
\hline \multicolumn{2}{|c|}{$\begin{array}{l}\text { DIMENSIONEN DES ANTIQUITÄTEN-BEGRIFFS } \\
\text { AUS DER SICHT VON ANTIQUITÄTENKÄUFERN }\end{array}$} & $\begin{array}{l}\text { relativer } \\
\text { Varianz- } \\
\text { erklarungs- } \\
\text { anteil }\end{array}$ & $\begin{array}{l}\text { absoluter } \\
\text { Varianz- } \\
\text { erklarungs- } \\
\text { anteil }\end{array}$ \\
\hline \multicolumn{2}{|l|}{ QUALITÄT } & \multirow[b]{2}{*}{$47,8 \%$} & \multirow[b]{2}{*}{25,58} \\
\hline $\begin{array}{l}\text { qualitatvoll } \\
\text { schon } \\
\text { solide gearbeitet } \\
\text { stilecht } \\
\text { alt }\end{array}$ & $\begin{array}{l}0,61 \\
0,59 \\
0,53 \\
0,50 \\
0,48\end{array}$ & & \\
\hline \multirow{2}{*}{\multicolumn{2}{|c|}{$\begin{array}{l}\text { wiederverkaufich } \\
\text { wertbestzndig } \\
\text { mobil } \\
\text { wertvoll }\end{array}$}} & \multirow[b]{2}{*}{$18,6 \%$} & \multirow[b]{2}{*}{35,48} \\
\hline & & & \\
\hline \multirow{2}{*}{\multicolumn{2}{|c|}{$\begin{array}{l}\text { selten } \\
\text { künstlerisch bedeutend } \\
\text { ausgefallen }\end{array}$}} & \multirow[b]{2}{*}{11,88} & \multirow[b]{2}{*}{41,78} \\
\hline & & & \\
\hline \multicolumn{2}{|l|}{ ERSCHEINUNGSBILD } & \multirow[b]{2}{*}{$8,1 \%$} & \multirow[b]{2}{*}{46,08} \\
\hline $\begin{array}{l}\text { reprasentativ } \\
\text { dekorativ } \\
\text { exklusiv } \\
\text { wertvoli } \\
\text { anziehend } \\
\text { zedtlos }\end{array}$ & $\begin{array}{c}\text { Faktor ladungen } \\
0,58 \\
0,55 \\
0,48 \\
0,47 \\
0,40 \\
0,38 \\
\end{array}$ & & \\
\hline \multicolumn{2}{|l|}{ PERSÖNLICHKEIT } & \multirow[b]{2}{*}{7,68} & \multirow[b]{2}{*}{50,18} \\
\hline $\begin{array}{l}\text { original } \\
\text { indigiduell } \\
\text { ausdrucksstark } \\
\text { stilecht }\end{array}$ & \begin{tabular}{|c|} 
Faktorladungen \\
0,73 \\
0,61 \\
0,50 \\
0,45 \\
\end{tabular} & & \\
\hline \multicolumn{2}{|l|}{ GEBRAUCHSNUTZEN } & \multirow[b]{2}{*}{6,18} & \multirow[b]{2}{*}{53,38} \\
\hline $\begin{array}{l}\text { funktional/praktisch } \\
\text { gebrauchsfahig }\end{array}$ & $\begin{array}{c}\text { raiktor? adungen } \\
0,85 \\
0.81 \\
\end{array}$ & & \\
\hline & & $100,00 x$ & $53,33 \%$ \\
\hline
\end{tabular}

Tab. 1: Dimensionen des Antiquitätenbegriffs - Ergebnisüberblick einer Faktorenanalyse 
duelle Qualitätsempfinden und der Qualitätsanspruch gegenüber einem Einzelstück variieren kann, ist "Qualität" aus Käufersicht fester Begriffsbestandteil, d.h. ein kunsthandwerklicher Gegenstand kann erst Antiquität sein, wenn er subjektiv das Merkmal "Qualität" besitzt".

Eine zweite empirisch ermittelte Begriffsdimension, die als "Wertgarantie" interpretiert wird, liefert immerhin fast $20 \%$ des Erklärungsanteils aller Dimensionen. Zum Begriffsbestandteil "Wertgarantie" fügt sich ein set von Einzelmerkmalen, in denen die wertbewahrende Eigenschaft von Antiquitäten zum Ausdruck kommt. Als Sachwerte besitzen Antiquitäten materiellen Wert (sind wertvoll), konservieren ihn im Zeitablauf (sind wertbeständig), sichern ihn in möglichen Krisenfällen durch Transportfähigkeit (sind mobil) und gewährleisten in den Augen ihrer Käufer eine Rekonvertibilität in andere Real- oder Nominalwerte (sind wiederverkäuflich). Insbesondere die Vorstellung der Wiederverkäuflichkeit besitzt einen sehr engen korrelativen zusammenhang mit der übergeordneten Begriffsdimension "Wertgarantie".

1 Welche Qualitätskomponenten der Käufer zum Maßstab seiner individuellen Beurteilung eines Einzelstücks macht, in welcher Beziehung die Qualitätskomponenten zueinander und zum gesamtheitlichen Qualitätsurteil stehen (substitutiv, komplementär, interdependent) oder wie hoch die jeweiligen Mindestanforderungen sind, kann nicht gesagt werden. Auch die Frage, welche Eigenschaften von Antiquitäten den individuellen Qualitätsansprüchen gerecht werden und welche Hilfsmaßstäbe der Käufer als Qualitätsindikatoren (Preis, Expertisen, Sachverständigenurteile etc.) akzeptiert und zur Beurteilung von "Qualität" heranzieht, ist nur im Einzelfall beantwortbar. Analoge uberlegungen können für alle nachfolgend diskutierten Begriffsdimensionen angestellt werden. 
Einen nicht unerheblichen Erklärungsbeitrag von ca. 12 \& zur Begriffsauffassung von Käufern leistet die empfundene "Rarität" von Antiquitäten. "Rarität" ist ein häufig von Fachleuten genanntes Begriffsmerkmal, das dabei in engem Zusammenhang mit dem Unikatcharakter und der Seltenheit von Antiquitäten gesehen wird. Auch das käuferbezogene Verständnis von "Rarität" umschließt die "Seltenheit". Dazu treten gleichrangig und inhaltlich ergänzend die Assoziationen einer "künstlerischen Bedeutung" und "Ausgefallenheit" als Komponenten von "Rarität". Die Beziehung zwischen den drei Beschreibungsmerkmalen und der extrahierten Begriffsdimension sind von etwa gleich starker Intensität.

Ein inhaltlich interessantes Gegensatzpaar von Dimensionen sind "Erscheinungsbild" und "Persönlichkeit" als unabhängige Teilaspekte des käuferbezogenen Antiquitätenbegriffs.

"Erscheinungsbild" umschließt ein Set von Merkmalen, die sich auf subjektiv wahrgenommene Eindrücke des Äußeren von Antiquitäten beziehen. Gemeint sind Anmutungen und Ausstrahlungen wie "repräsentativ", "dekorativ", "exklusiv" oder "wertvoll". Als definitorische Begriffsbestandteile sind die genannten Beschreibungsmerkmale zunächst wertneutral. Erst die bewußte oder unbewußte Ausnutzung ihres Dokumentationswertes durch den Käufer unterstellt eine antizipierte Wertung der Umgebung : das "Erscheinungsbild" wird instrumentell im Sinne einer erwünschten Wirkung bei anderen eingesetzt.

Anders die "Persönlichkeit" als Begriffsdimension, die durch die Attribute "original", "individuell", "ausdrucksstark" und "stilecht" gekennzeichnet ist. Sie bezieht sich auf innere Eigenschaften oder auch Wesensmerkmale von Antiquitäten, die Ursache oder Ausgangspunkt einer intensi- 
ven individuellen Beziehung des Käufers zu seinem Antiquitätenbesitz darstellen können ${ }^{1,2}$. Die Wirkungsrichtung der genannten "Persönlichkeitsmerkmale" von Antiquitäten ist im Gegensatz zum "Erscheinungsbild" nicht auf andere (außenstehende) Personen, sondern auf die Käuferpersönlichkeit selbst (nach innen) gerichtet.

Der gemeinsame, relative Erklärungsbeitrag des Dimensionspaares zur faktoranalytischen Begriffsexplikation liegt bei ca. 158 .

Erheblich geringer ist er schließlich beim "Gebrauchsnutzen" mit ca. 6 \% . Kommt darin auch eine insgesamt untergeordnete begriffsbildende Rolle von "Gebrauchsnutzen" zum Ausdruck, ist andererseits diese Dimension inhaltlich, d.h. in ihren Korrelationen $z u$ den durch sie repräsentierten

1 Ein methodisch und semantisch aufschlußreicher Aspekt ergibt sich für das Beschreibungsmerkmal "wertvoll". Es besitzt einen hohen korrelativen Zusammenhang (vgl. Faktorladungen Abbildung 4) mit der "Wertgarantie" und dem "Erscheinungsbild" als unabhängige Dimension des Antiquitätenbegriffs. Hierin spiegelt sich eindrucksvoll eine mögliche Doppeldeutigkeit. Im Zusammenhang mit "Wertgarantie" ist wertvoll eher im Sinne "besitzt Wert" oder "ist voll Wert" zu verstehen - in Verbindung mit "Erscheinungsbild" zählt allein die Äußerlichkeit: Antiquitäten "wirken wertvoll", unabhängig davon, ob sie auch tatsächlich wertvoll im materiellen oder kunstwissenschaftlichen Sinn sind. Methodisch ist bemerkenswert, da $B$ die vorgenommene Faktorenanalyse eine sinngerechte Differenzierung und Zuordnung nachvollzieht.

2 Empirisch bestätigt sich die Existenz einer intensiven Beziehung zum Antiquitätenbesitz in einer außerordentlich hohen Zustimmung der Antiquitätenkäufer zu dem Statement, daß "man sich von einer einmal erworbenen Antiquität nur sehr schwer wieder trennen kann". Bezogen auf eine 5-stufige Skala von "trifft sehr $z u$ " (1) bis "trifft gar nicht $z u "$ (5) erreichte die Frage einen Mittelwert von 1,6. Der gesamte Mittelwert entspricht einer Zustimmung von fast 60 o (90\%) der Befragten zu der (den) Skalenstufe(n) 1 ( 1 und 2). 
Beschreibungsmerkmalen, sehr kompakt. Hohe Faktorladungen $(-0,8)$ für "Funktionalität" und "Gebrauchsfähigkeit" zeigen, daß die ursprüngliche Funktion von Antiquitäten auch heute noch ein sehr eigenständiger Begriffsbestandteil in den Augen der Käufer ist.

Der motivationale Erklärungswert der extrahierten Begriffsdimensionen liegt in den subjektiv interpretierenden Wahrnehmungen von Antiquitäten begründet. In den individuellen Vorstellungsbildern kommen bewußte oder unbewußte Erwartungshaltungen bezogen auf Eigenschaften und Merkmale von Antiquitäten zum Ausdruck, die aufgrund ihres Aufforderungsgehalts (antizipierte Motivbefriedigung) als motivationale Anreize interpretiert werden können.

Zur Eingrenzung der "Käuferdefinition" von Antiquitäten interessiert allerdings nicht nur, ob und wieweit die vielfältigen (Einzel-) Merkmale und Eigenschaften auf eine geringe Anzahl von Begriffs-bzw. Vorstellungsdimensionen reduziert werden können, sondern auch die Ausprägung der Käufervorstellung auf diesen Dimensionen.

\subsection{Vorstellungsbild privater Käufer}

Der Übergang von der Diskussion struktureller Dimensionen des käuferbezogenen Antiquitätenbegriffs zur Charakterisierung des käuferbezogenen Vorstellungsbildes beinhaltet die Frage nach der Beurteilung von "Antiquitäten" anhand der extrahierten Begriffsdimensionen (Ermittlung der Faktorenwerte 1,2 ).

1 Da das SPSS-Programmpaket keine Option zum Ausdruck von Faktorenwerten besitzt, mußte ein (im Anhang wiedergegebenes) PL 1 Programm aufgebaut werden, mit dem gleichzeitig Faktorenwert-Mittelwerte für unterschiedliche Käufergruppen ermittelt werden können.

2 Die im SPSS implementierte regressionsanalytische Schätzung der Faktorwerte ist eine mögliche Schätzmethode, wodurch die Anwendung anderer Verfahren $\mathrm{zu}$ abweichenden Ergebnissen führen kann. Vgl. Schuchard-Ficher, Chr., et al., Multivariate Analysemethoden,a.a.0., S. $255 \mathrm{ff}$. 
Jedes individuelle Vorstellungsbild eines Käufers wird im gegebenen Fall durch 8 (Faktoren-) Werte auf den extrahierten Begriffsdimensionen "Qualität", "Wertgarantie", "Rarität", "Erscheinungsbild", "Persönlichkeit" und "Gebrauchsnutzen" repräsentiert und damit (theoretisch) in einem 6-dimensionalen Raum positionierbar.

Die vorausgegangene Analyse hat gezeigt, daB die drei erstgenannten Begriffsđimensionen fast $80 \%$ des gemeinsamen (relativen) Erklärungsanteils tragen. Abbildung 4 zeigt den auf diese drei Dimensionen "reduzierten" Begriffsraum, ohne daB durch dieses Vorgehen ein wesentlicher Informationsverlust $\mathrm{zu}$ erwarten ist.

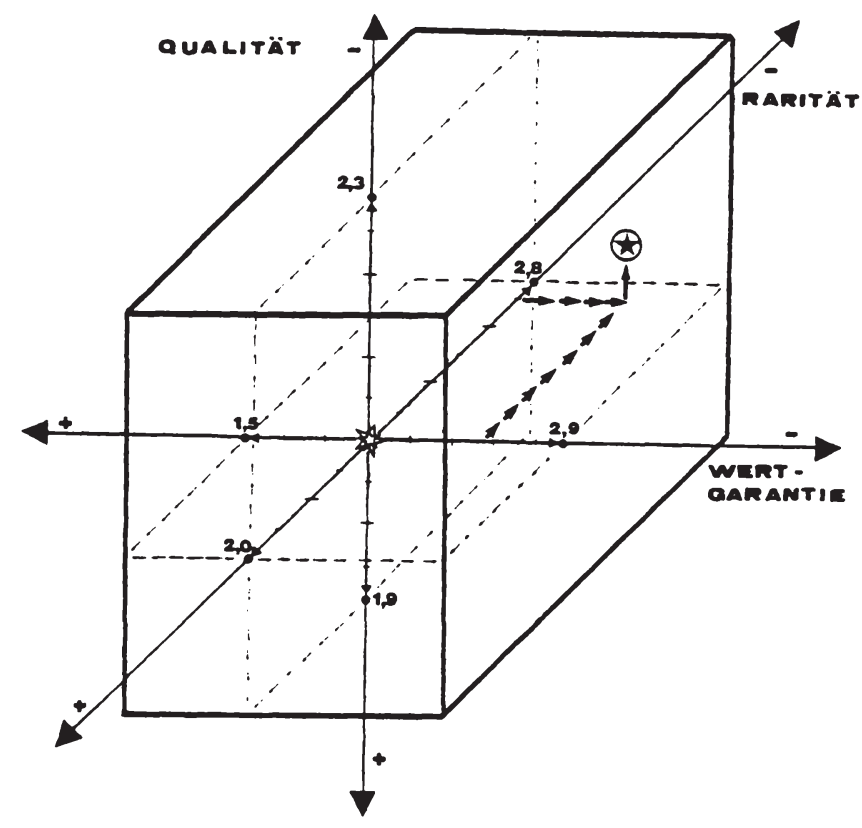

故

Mitelpunkt des Begriffsraumes

$\theta$

position eines individuellen vorstellungsblides

(1)

zahlenangaben - Faktorenwerte (Factor-scores)

Abb. 4: "Reduzierter" Begriffsraum zur Darstellung der Käufervorstellung von Antiquitäten 
Im dreidimensionalen Raum wird die individuelle Käufervorstellung durch seine Teilvorstellungen über "Qualität", "Wertgarantie" und "Rarität" beschrieben. Dabei kann als kognitiver Bezugspunkt der Käufervorstellung sein "evoked Set" bezogen auf die (sachlich überaus heterogene) Warengruppe "Antiquitäten" angesehen werden; d.h. sie bezieht sich auf die individuell beachteten Kaufalternativen aus der gesamten Warengruppe ${ }^{1}$.

Das beispielhaft gezeigte Vorstellungsbild eines einzelnen Käufers spiegelt eine ausgeprägte Bedeutung des "Raritätsaspektes", eine weniger starke des "Wertgarantieasnektes" und schließlich eine nochmals geringere des "Qualitätsaspektes" im Vorstellungsbild des Befragten wieder. Da die Beurteilungswerte (Faktorenwerte) hinsichtlich der Begriffsdimensionen bzw. Warenmerkmale standardisiert sind ${ }^{2}$, stehen die Dimensionen untereinander sowie auch die Ausprägungen der Käufervorstellungen auf ihnen in einer vergleichbaren Relation. Dies führt u.a. dazu, daß die "Abstände" zwischen den Positionen unterschiedlicher Vorstellungsbilder als "Maßgrößen" ${ }^{3}$ für ihre Ähnlichkeit bzw. Unähnlichkeit angesehen werden können. Zudem läßt sich jede individuelle Vorstellung als "nah" oder "weit" von der durchschnittlichen Käufervorstellung (Mittelpunkt des Begriffsraumes ${ }^{4}$ ) charakterisieren.

1 Vgl. Howard, I.A., Sheth, J.N., The Theory, a.a.O., S.98 ff.; Böhler, H., a.a.O., S. $262 \mathrm{ff.}$.

2 Vgl. Kim, I.-O., a.a.O., S. 489.

3 Die Metrik ist dabei vom gewählten Distanzmaß (z.B. quadrierte euklidsche Distanz, "City-Block-Verfahren" abhängig.

4 Aufgrund der Standardisierung ist der Faktorenwert-Mittelwert über die gesamte Stichprobe gesehen, für jeden Faktor (Dimension) O. Vgl. Kim, I.-O., a.a.O., S. 481, Fußnote 1 . 
Zur Kennzeichnung des allgemeinen Käuferbildes ist zu untersuchen, wie sich Antiquitäten aus Käufersicht im aufgespannten Begriffsraum insgesamt einordnen, abgrenzen bzw. "definieren" lassen. Für die Eingrenzung einer solchen "Käuferdefinition" geben die jeweils geringsten und höchsten Beurteilungen (Faktorenwerte) auf den Dimensionen Anhaltspunkte. Der in der Abbildung gezeigte Kubus begrenzt in diesem Sinne den durch die Teil-Vorstellungsbilder "Qualität", "Wertgarantie" und "Rarität" strukturierten, käuferbezogenen "Definitionsraum". Alle Vorstellungsbilder, die in einem oder mehreren Teilaspekten außerhalb der gesteckten "Definitionsgrenzen" liegen, beschreiben aus der Sicht der befragten Käufer - und damit der vorliegenden Untersuchung - keine Antiquitäten.

Die relative Lage des 3-faktoriellen Vorstellungsraumes zeigt für alle Dimensionen eine stärkere Ausdehnung in Richtung auf negative (Faktoren-) Werte. Dahinter ist eine größere Meinungsvielfalt und -breite bezogen auf das "Vorhandensein" von "Qualität", "Wertgarantie" und "Rarität" als Eigenschaften von Antiquitäten $z u$ verstehen, als sie in entgegengesetzter Richtung bezogen auf das "Nicht-Vorhandensein" dieser Merkmale festgestellt werden kann. Es scheint daher die Annahme plausibel, daß die Käuferdefinition insgesamt nach "oben" relativ cffen und weitgefaßt ist, oder m.a.W. die "Minimalvorstellungen" von den durch die Dimension repräsentierten Antgiuitätenmerkmalen relativ "hoch" ausfallen.

Wie aus Abbildung 5 ersichtlich, gilt das Gesagte für alle extrahierten Dimensionen der Käufervorstellung von Antiquitäten $^{1}$.

1 Die Abbildung 5 kann dabei graphisch nicht mehr der Unabhängigkeit der Dimensionen gerecht werden und ist daher auch nicht mehr exakt "räumlich" interpretierbar. 


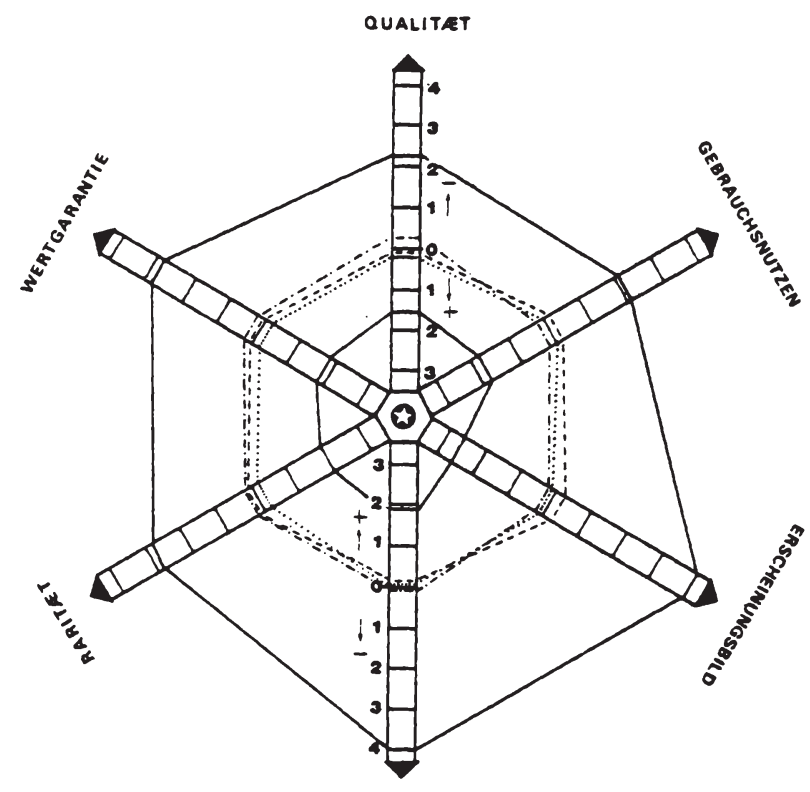

PERSCNLICHKEIT

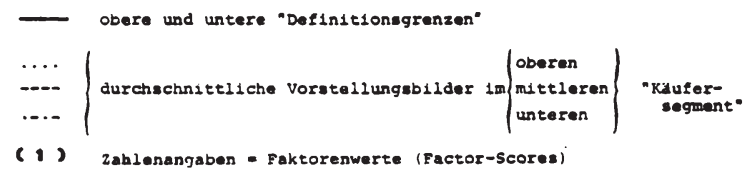

Abb. 5: Spannweiten der allgemeinen Käufervorstellung und durchschnittliche Vorstellungsbilder in Teilstrichproben ("Käufersegmenten")

Es zeigt sich, daB die niedrigsten Einstufungen der Käufer bei den Dimensionen "Persönlichkeit" und "Erscheinungsbild" bestehen. Auch die absolute Spannbreite der individuellen Käufervorstellungen ist hier am größten - ein Ergebnis, das aufgrund der hohen "Subjektivität" und "Individualität" dieser Dimensionen folgerichtig erscheint. Andererseits sind die Dimensionen "Qualität" und "Gebrauchsnutzen" vermutlich, da der Käufer hier eher auf "kognitive Bezugspunkte" (Erfah- 
rungen, wissen etc.) und "objektivierbare Beurteilungsindikatoren" (Gutachten, Preise etc.) zurückgreifen kann, am kompaktesten ${ }^{1}$.

Die Diskussion um den käuferbezogenen Antiquitätenbegriff läßt sich abschließend folgendermaßen zusammenfassen:

1. Die Käufervorstellung von Eigenschaften und Merkmalen von Antiquitäten ist multidimensional.

2. Sie kann durch 6 Dimensionen strukturiert und beschrieben werden, welche sowohl statistisch wie interpretatorisch die abgefragten Teilaspekte des Antiquitätenbegriffs gut repräsentieren.

3. Unter den Dimensionen dominieren inhaltlich die "Qualitäts-", "Wertgarantie-" und "Raritätsvorstellung".

4. Durch die Vorstellungsdimensionen läßt sich ein "Vorstellungs- bzw. Definitionsraum" abgrenzen, in dem alle erhobenen individuellen Vorstellungsbilder positionierbar sind.

5. Die Ausdehnung dieses Raumes erscheint relativ groB und die Vorstellungsbilder der Käufer breit gestreut und differenziert, d.h. in jedem Fall individuell ("evoked set").

6. Die durchschnittlichen Vorstellungsbilder, bezogen auf einzelne Stichprobensegmente (Käufergruppen) sind relativ invariant.

1 Interessant ist in diesem Zusammenhang die Frage, ob und in welchem ¿usma $B$ sich unterschiedliche Käufergruppen hinsichtlich ihres (durchschnittlichen) Vorstellungsbildes von Antiquitäten unterscheiden.

Ein mögliches Abgrenzungskriterium bilden die Teilstichproben der empirischen Erhebung und damit entsprechend der Besuch auf Antiquitätenmessen und -märkten unterschiedlicher Qualitätssegmente. Stellvertretend für diese segmente stehen die Angebote der Kunst- und Antiquitätenmesse ünchen, der Internationalen Antiquitätenausstellung Essen und des Antiquitätenmarktes Münster.

Die erhobenen durchschnittlichen vorstellungsbilder im oberen, mittleren und unteren segment sind ebenfalls der Abbildung $5 \mathrm{zu}$ entnehmen. Als Ergebnis ist festzustellen, da $B$ nur unwesentliche Differenzen in den Vorstellungsbildern der Kä̈ufergruppen (Teilstichproben) bestehen. Ein in der Tendenz unerwartetes Ergebnis, welches eindrucksvoll die "Unabhängigkei+" der durchschnittlichen Käufervorstellung von der Stichprobenzusammensetzung dokumentiert und zugleich ein Hinweis darauf, daB Käufer mit einem ausgeprägten Interesse an "hochklassigen Antiquitäten" und Käufer mit einem ausgeprägten Interesse an "Trödel" durchaus ein ähnliches (durchschnittliches) Vorstellungsbild von Antiquitäten besitren. 
2. Analyse der Strukturen und Prozesse im Antiquitätenmarkt

Eine Untersuchung der Motivation von Antiquitätenkäufern setzt ein Mindestmaß an Kenntnissen über Elemente und Strukturen des Marktes für Antiquitäten voraus.

Eigenschaften der Marktteilnehmer, ihre Beziehungen zueinander sowie allgemeine Marktentwicklungen und -prozesse sind in ihrer subjektiv vom Käufer wahrgenommenen Form motivations- und verhaltenswirksame Einflußgrößen. Das Marktgeschehen bildet daher einen Teil des situationalen Rahmens, in dem sich individuelle Motivationen und Verhaltensweisen äußern und prägen.

Die reale Vielfalt und Komplexität der Erscheinungsformen von Marktteilnehmern und Interaktionsbeziehungen verlangt eine modellhafte Vereinfachung. Erst dadurch wird die Grundstruktur des Antiquitätenmarktes transparent und die Isolation relevanter motivationaler Einflußgrößen möglich.

\subsection{Käuferbezogenes Modell des Antiquitätenmarktes als orientierungsrahmen}

Art und Zahl der in Abbildung 6 überblickhaft dargestellten Marktelemente und -beziehungen basieren - der käuferbezogenen Definition des Antiquitätenbegriffs folgend - auf einer Marktabgrenzung aus der Sicht des privaten Antiquitätenkäufers. Dieser nimmt entsprechend die zentrale Stellung im gezeigten Marktschema ein ${ }^{1}$.

1 Das gezeigte Marktmodell bezieht sich dabei auf einen "idealisierten" Käufer mit tendenziell vollständiger Marktinformation und -übersicht. Der im Einzelfall individuell wahrgenommene Marktausschnitt variiert in Abhängigkeit von Markttransparenz und Marktkenntnis. Im Gegensatz zur "käuferzentrierten" steht die in der Literatur überwiegend anzutreffende "sachgebietsbezogene" Marktdarstellung. Zu derartigen nach Teilmärkten gegliederten Marktbeobachtungen, deren Augenmerk zudem im wesentlichen auf Preisentwicklungen für (Fortsetzung siehe S. 42) 

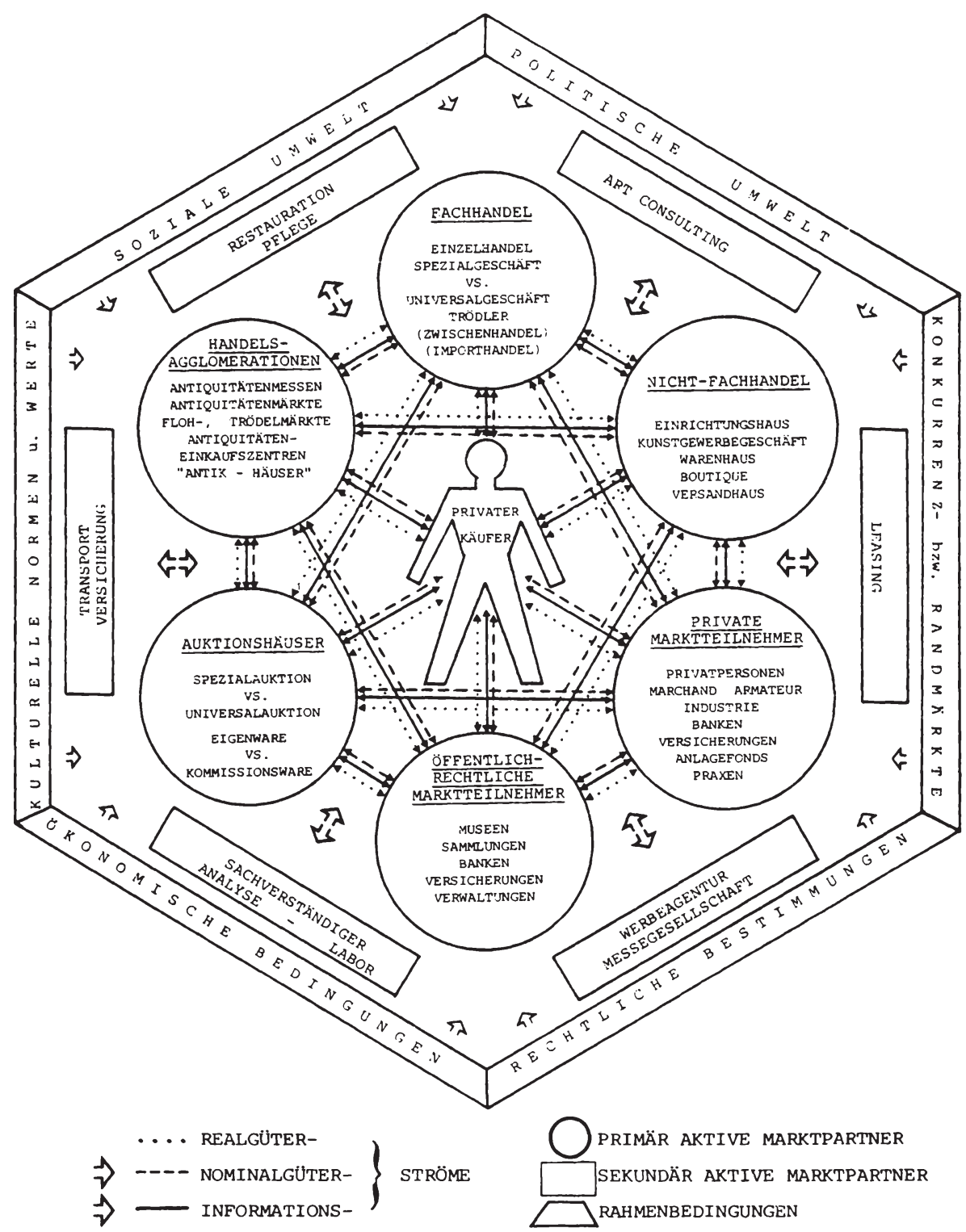

Abb. 6: Modellhafte Darstellung der Grundstrukturen des Antiquitätenmarktes aus der Sicht privater Käufer 
Im Rahmen motivationaler Erklärungsansätze des Verhaltens privater Antiquitätenkäufer sind als selektiver Marktausschnitt

(1) die Merkmale der vom privaten Käufer genutzten Einkaufsstätten und Serviceanbieter (Marktpartner) sowie

(2) die motivationsbeeinflussenden Variablen der wahrgenommenen Markt- und Kaufsituation (Marktprozesse und -entwicklungen)

für die Marktanalyse von zentraler Bedeutung.

\subsection{Kennzeichnung der harktpartner von privaten Antiquitä- tenkäufern}

Zum Kreis primär aktiver Marktpartner zählen alle Betriebsformen und Erscheinungen des professionellen Ilandels und als wichtige Besonderheit des Antiquitätenmarktes ein etabliertes und umfangreiches Auktionswesen. Als Einkaufsstätten stehen ilandel und Auktionshäuser durch die in den letzten Jahren zunehmende Beteiligung privater Antiquitätenkäufer an Auktionen in wachsender Konkurrenz. Speziell in rezessiven Marktphasen nimmt diese den Charakter eines Verdrängungswettbewerbs $a^{1}$.

Daneben ist auch der direkte Kauf, Verkauf und Tausch von Antiquitäten zwischen den privaten Marktteilnehmern üblich. Dabei kann zum Teil ein durchaus "halbprofessioneller" Cha-

Einzelstücke oder Marktsparten gerichtet ist; vgl. beispielhaft Seling, H. (Hrsg.), Keysers Kunst- und Antiquitätenbuch, 8. Aufl., München 1974; Grasser, W. (Hrsg.), Antiqui.täten als Kapitalanlage, 2. Auf1.. München 1975; Miller, J. und M. (Hrsg.), Miller's Antiques Price Guide, Professional Handbook 1981, Pinguin's Hall etc. 1981; Kunstpreis-Jahrbuch, der Markt der Kunst international, Bd. XXXVA und XXXVB, Zg. v. Wellensiek, H., Keyszelitz, R., München 1980; Herchenröder, Chr., Die Kunstmärkte, a.a.o., s. 35 ff..

$1 \mathrm{Vgl}$. Müller-Mehlis, R., Stärkung der mittleren und unteren Ebene, in: WK, 51. Jg. (1981), Heft 3 (Februar), S. 203; . .V., Sind Antiquitätengeschäfte antiquiert?, in: BddW Nr. 188 vom 15.08.79, S. 6; Paschke, M., Zur Marktsituation .... a.a.0., S. 1531 . 
rakter solcher Transaktionen bei den sog. "marchards amateurs" ${ }^{1}$ unterstellt werden ${ }^{2}$.

Die strukturellen Dimensionen des Erscheinungsbildes von Handels- und Auktionshäusern sind das Ergebnis einer unbewuBten Festlegung oder bewuBten Gestaltung der absatzpolitischen Instrumente (Politiken) des Antiquitätenhandels. Dabei lassen sich folgende Bereiche abgrenzen ${ }^{3}$ :

(1) Die Gestaltung bzw. Wahrnehmung des Angebotssortiments und der verkaufsbegleitenden serviceleistungen ist "Kernstück" des Erscheinungsbildes. Die Aufmerksamkeit des Antiquitätenkäufers richtet sich dabei nicht nur auf allgemeine Sortimentsdimensionen, wie die Anzahl der geführten Sach-bzw. Sammelgebiete (Sortimentsbreite), die Auswahl innerhalb dieser Gebiete (Sortimentstiefe) und das allgemeine Qualitätsniveau (Angebotsgenre), sondern insbesondere auch auf Einzelstücke mit spezieller Attraktions-

1 Vgl. Schütte, R., Kunst und Antiquitätenhandel, a.a.0., Sp. 1165.

2 In der empirischen Studie geben 40,4 \& der Befragten an, Antiquitäten schon einmal von "anderen Privatleuten" gekauft $\mathrm{zu}$ haben. Dieser im Vergleich $\mathrm{zu}$ anderen Einkaufsstätten überraschend hohe Prozentsatz (vgl. Kapitel B. 4.2) deutet auf einen nicht unerheblichen privaten "Schwarzhandel" mit Antiquitäten hin.

3 Absatzpolitische Instrumente werden in der Literatur in unterschiedlichster iorm systematisiert. Einen Uberblick gibt Meffert, H., Instrumente, absatzpolitische, Beitrag in: Tietz, B. (Hrsg.), Handwörterbuch der Absatzwirtschaft, Stuttgart 1974, Sp. 888. Zu handelsspezifischen Gliederungen vgl. beispielhaft Nieschlag, R., Dichtl, E., Hörschgen, H., Marketing, a.a.O., S. $113 \mathrm{ff.;}$ Hansen, U., Absatz- und Beschaffungsmarketing des Einzelhandels, 1. Teilband, Göttingen 1976, S. $114 \mathrm{ff} . ;$ Meffert, H., Handelsmarketing und Handelsfunktionen, in: Würfel und Ecken, Heft 2, 1972, S. $4-6$. 
wirkung $^{1}$. Die Variationsbreite und zugleich hohe akquisitorische Wirkung von Serviceleistungen begründet sich in den besonderen Warenmerkmalen von Antiquitäten (Individualität, Beratungsbedürftigkeit, Wertunsicherheit etc.).

(2) Im Rahmen der Kommunikationspolitik dominieren Art und Atmosphäre der Warenpräsentation sowie die wahrgenommene Beratungs - und Fachkompetenz des Händlers, Auktionators oder seines Personals das Erscheinungsbild. Daneben sind Lager- und Auktionskataloge, "direct mail" Aktionen, Pressemitteilungen, Verkaufsausstellungen, Messen und Märkte von kommunikativer Bedeutung. Die klassische (Medien-) Werbung bleibt in der Regel auf Anzeigen in Fachzeitschriften beschränkt.

(3) Das wahrgenommene Preisniveau, die Preiswürdigkeit und "Preischance" (Möglichkeit von Trouvaillen, Preisnachlässe durch Verhandlung, günstiger $\mathrm{Zu}-$ schlag auf Auktionen) sind Ergebnisse der preispolitischen Entscheidungen von Handel und Auktion. Diese erfordern die Festlegung eines generellen Preisbildungsmechanismus (Preiskalkulation vs. Bietgefecht), sowie die Ermittlung von Einzelpreisen und Entscheidungen über Preiskonditionen (Nachlässe, Kredite und Zahlungsbedingungen).

(4) Der gewählte Standort wird vom Käufer in erster Linie durch die aufzuwendende Mühe zum Erreichen der Einkaufsstätte (Entfernung, Zeitinanspruchnahme, Parkplätze etc.) wahrgenommen. Ferner ist gerade

1 Typisches Beispiel ist die überdurchschnittliche Aufmerksamkeit von Käufern gegenüber Antiquitäten, die auf Messen oder Auktionen zu "Rekordpreisen" angeboten werden. Die bewußte Ausnutzung solcher Ausstrahlungseffekte auf das "Image" des übrigen Sortiments oder der Einkaufsstätte insgesamt wird oft versucht. Hohe Medienpublizität eines "spektakulären" Verkaufserfolges sorgt darüber hinaus für zusätzliche kommunikative Wirkungen. Vgl. beispielhaft Fischer, L., Weltrekordpreise nutzen dem Kunstmarkt, in: Die Welt, Nr. 192 vom 19. August 1978, S. 19; 0.V., Teutonischer Blitz, in: Der Spiegel Nr. 27 (Juli) 1978, S. 113-135; 0.V., Die Geheimnisse des großen Bietens, in: FAZ Nr. 133 vom 27. Juni 1978, S. 19; Herchenröder, Chr., Die Kunstmärkte, a.a.O., S. 16-21; v. Hassell, Chr., Ein Jahr der Rekorde, in: WK, 51. Jg. (1981), Heft 3 (Februar), S. $207 \mathrm{f} . ;$ Herchenröder, Chr., Kunstmarkt - Eine bessere Markttransparenz ist anzustreben, in: Deutsche Bank (Hrsg.), Börsenbild und Anlage-Spiegel, Nr. 1/82, Frankfurt 1982, S. 6 . 
für Antiquitäten die Standortumgebung (erhöhte Attraktionswirkung von Handelsagglomerationen, "Imagepartizipationseffekte" durch die Nähe zu anderen Händlern oder durch die Lage in einer Stadt, die den Ruf eines "Kunstzentrums" besitzt") wichtig.

(5) Im engen Zusammenhang mit der Stadtortwahl steht schließlich die Entscheidung für die Art der Verkaufsorganisation (Residenz-, Treff- und Distanzprinzip ${ }^{2}$. Für den Käufer sind Einkaufsstätten durch den Verkauf in Ladengeschäften (ständige Präsenz des Antiquitätenangebots, Möglichkeit der Warenbegutachtung, persönliche Beratung etc.) und/oder auf Messen, Märkten und Verkaufsausstellungen (breitere Auswahl, Preisvergleichsmöglichkeit, Einkaufserlebnis etc.) und/oder der Möglichkeit eines schriftlichen oder fernmündlichen Kaufs (Versandhandel, Auktion etc.) gekennzeichnet.

Die im folgenden vorgenommene Klassifikation von Marktpartnern versucht, in heuristischer Vorgehensweise die jeweils typische Ausprägungskombination der Einzelinstrumente als Ausdruck der gesamtheitlichen Absatzkonzeption in den Vordergrund zu stellen. Das Resultat sind "äuBerlich" differenzierbare Handelssegmente (Gruppen von Handelspartnern), welche sich "nach innen" durch ein weitgehend einheitliches Erscheinungsbild, d. h. durch ähnliche Eigenschaften, Ausstrahlungen und Einkaufsatmosphären für den Käufer auszeichnen.

\subsection{Antiquitätenhandel}

Die unterschiedlichen Erscheinungsformen des professionellen Antiquitätenhandels lassen sich typisierend in die drei seg-

$1 \mathrm{zu}$ Merkmalen von "Kunstzentren" vgl. Fischer, I., London bleibt die $\mathrm{Nr} .1$ am Kunstmarkt, in: Die Welt $\mathrm{Nr} .180$ vom 5.8.1978, S. 17; Wille, I,G., Zürich als internationaler Kunsthandelsplatz, in: SKA (Hrsg.), Bulletin Januar/Februar 1979 , S. 38 f..

2 Vgl. Hansen, U., Absatz- und Beschaffungsmarketinọ, a.a.o., S. 273 ff.. 
mente Fachhandel, Nichtfachhandel und Handelsagglomerationen gliedern ${ }^{1}$.

Der Antiquitätenfachhandel firmiert im Markt überwiegend unter der Bezeichnung "Kunst- und Antiquitätengeschäft" oder "Kunsthanālung". Reine "Antiquitätenhandlungen" sind in der Minder$z_{a h l^{2}}$. Der Fachhandelsbegriff beinhaltet eine allgemeine Sortimentsbegrenzung auf Antiquitäten und objekte der bildenden

Kunst. Diese zunächst undifferenzierte Sortimentsbeschreibung deutet auf zusätzliche Abgrenzungsmöglichkeiten innerhalb der Fachhandelssortimente hin. Als Klassifikationsmerkmale bieten sich unterschiedliche Ausgestaltungen einzelner Dimensionen der Sortimentsstruktur an.

Nur geringe Dynamik entwickelt das Gesamtangebot von Antiquitätenhändlern in der Regel bezogen auf die Dimensionen der Sortimentsbreite (Spezialisierungsgrad) und des allgemeinen Qualitätsniveaus (Preislage). Idealtypisch lassen sich darauf aufbauend die in Abbildung 7 gezeigten Fachhandelstypen abgrenzen.

1 Antiguitätengroßhandlungen lassen sich im Antiquitätenmarkt als eigenständige Unterformen nicht herausgrenzen. Nur im Einzelfall wird von spezialisierten Importfirmen oder von Fachhändlern ein ausschlieBlicher Zwischenhandel übernommen. Da Handel-an-Handel-Transaktionen (Ankäufe, Verkäufe und Kommissionen) für alle Handelsformen typisch sind, besitzt der spezialisierte $\mathrm{zwischenhandel}$ nur geringe Bedeutung.

2 Aufgrund der intensiven Verflechtungen zwischen Kunst("bildende Kunst") und Antiquitätenhandel und unterschiedlichster Begriffsauffassungen von Antiquitäten sind exakte Schätzungen quantitativer Marktgrößen nicht möglich. Einen Anhaltspunkt für die Anzahl von Fachhändlern mit "guten Qualitäten" bilden die ca. 550 Mitglieder des "Bundesverband des Deutschen Kunst- und Antiquitätenhandels e.v." . Für einen weitergefaßten Qualitätsstandard weist das "Kunstadressbuch für die Bundesrepublik Deutschland 1977/78" ca. 2000 Händler aus.vgl. Art-Address-Verlag (Hrsg.), Kunstadressbuch für die Bundesrepublik Deutschland $1977 / 78$, Frankfurt 1977 , S. 2-42. 


\begin{tabular}{|c|l|l|}
\hline $\begin{array}{c}\text { QUAITATIS- } \\
\text { GENRF } \\
\text { SORTIMENTS- } \\
\text { BREITE }\end{array}$ & hoch & niedrig \\
\hline breit & $\begin{array}{l}\text { universeller Anti- } \\
\text { quitatenfachhande) } \\
\text { (Universalgeschäfte) }\end{array}$ & Trödler \\
\hline schmal & $\begin{array}{l}\text { spezialisierter Anti- } \\
\text { quitätenfachhandel } \\
\text { (Spezialgeschäft) }\end{array}$ & \\
\hline
\end{tabular}

Abb. 7: Typisierung von Fachhandelsformen auf der Grundlage ihrer Sortimentsbreite und ihres Qualitätsgenres

Nichtfachhändler führen Antiquitäten im Sortimentsverbund mit anderen Warengruppen. Zum Nichtfachhandel gehören u.a. warenhäuser, Einrichtungshäuser, Versender, runstgewerbegeschäfte oder Boutiquen, deren Antiquitätenangebot entweder in Form eines eigenständigen Sortimentsbereichs (Abteilung) oder als reines Nebensortiment geführt wird.

Antiquitätenabteilungen in Warenhäusern und im Programm von Versendern sind neuere Markterscheinungen ${ }^{1}$. Verfolgt das zusätzliche Angebot von Antiquitäten die Ziele, an einem "Wachstumsmarkt" zu partizipieren oder eines bewuBten "trading-up" ${ }^{2}$, ist aus der Sicht des Käufers auf mögliche

1 Der Kaufhauskonzern Horten betreibt seit 1980 in mehreren Filialen (u.a. Düsseldorf mit einer Verkaufsfläche von über $2.000 \mathrm{gm}$ ) eine "Wohngalerie" mit Antiquitäten, Stilmöbeln und Wohnaccessoires. Das Angebot bei Uhren, Silber etc. reicht in mittlere bis hohe preislagen von ca. 20 30 TSD DM. Vgl. O.V., Antiquitäten im varenhaus, BddW, Nr. 129 vom 9.5.1980, S. 5.

$2 \mathrm{Vgl}$. Meffert, H., Perspektiven des Marketing in den 80er Jahren - Ein Uberblick des Herausgebers, in: derselbe

(Hrsg.), Marketing im Wandel, Wiesbaden 1980, S. 23 u. 30 . 
"Imageunverträglichkeiten" zwischen Angebotsform und Warencharakter hinzuweisen. Dies gilt insbesondere für wahrgenommene Diskrepanzen zwischen erforderlichen bzw. vom privaten Käufer gewünschten Service- und Beratungsleistungen und ihrem tatsächlichen Niveau. Einrichtungshäuser, Kunstgewerbegeschäfte und Boutiquen praktizieren bereits seit längerem ständige oder zeitlich begrenzte (ausstellungsartige) offerten von Antiquitäten zur Sortimentsabrundung und -aufwertung ${ }^{1}$.

Konstituierendes Merkmal von Handelsagglomerationen ist die örtliche Konzentration eines besonders umfangreichen Warenangebots, welchesdem Käufer eine vergleichsweise hohe preisliche und qualitative vergleichsmöglichkeit gibt ${ }^{2}$.

Aufgrund ihrer spezifischen Verkaufsorganisation lassen sich unter den Handelsagglomerationen Antiquitätenmessen, -märkte und-verkaufsausstellungen einerseits und bestimmte Typen von Antiquitäteneinkaufszentren andererseits unterscheiden.

Messen, Märkte und Verkaufsausstellungen sind örtlich und zeitlich begrenzte Marktveranstaltungen einer Vielzahl von Händlern, bei denen sich Käufer und Verkäufer am gewählten Veranstaltungsort treffen (Treffprinzip). Aus struktureller Sicht läBt sich eine idealtypische Klassifikation von Marktveranstaltungen - aufgrund durchgängig hoher sortimentsbreite - auf die unterschiedlichen Qualitätsniveaus des Warenangebots reduzieren. Der Qualitätswahrnehmung des Käufers ent-

1 Insbesondere einmalige Verkaufsauktionen bzw. -ausstellungen (beispielsweise antiker Taschenuhren bzw. antiker Schmuckstücke bei Juwelieren oder auch antiker Teppiche in Einrichtungsgeschäften) sind aufgrund vermuteter Imageund Kommunikationswirkungen als Verkaufsförderungsaktionen beliebt. Initiator ist oft ursächlich der Antiquitätenfachhandel, der seine Ware (z.B. als Kommissionsware) zur Verfügung stellt, um sie einem themenspezifisch interessierten Käuferkreis zugänglich zu machen, der im eigenen Geschäftslokal nicht erreicht wird.

2 Vgl. Schütte, R., Kunst- und Antiquitätenhandel, a.a.o., Sp. 1166 . 
spricht dabei am ehesten eine marktliche Dreiteilung, die sich auch sprachlich weitgehend etabliert hat: "Kunst- und Antiquitätenmessen" ${ }^{1}$ bilden das obere, "Antiquitätenmärkte und -verkaufsausstellungen" das mittlere und "Floh- und Trödelmärkte" das untere Qualitätssegment.

Antiquitäteneinkaufszentren wie zum Beispiel "Antik-Häuser" oder "Antik-Passagen" basieren in ihrer Verkaufsorganisation auf dem Domizil-Prinzip, bei dem das Warenangebot in ständiger Präsenz in (ortsgebundenen) Geschäftslokalen offeriert wird ${ }^{2}$.

Einkaufszentren können sowohl historisch gewachsen als auch kooperativ geplant sein. Der konkurrenzintensiveren Standortsituation des einzelnen Händlers steht als wichtigster Ausgleich die attraktionssteigernde wirkung eines reichhaltigen, integriert wahrgenommenen Antiquitätenangebots mit den sich ergebenden Auswahl- und Vergleichsmöglichkeiten für den Käufer gegenüber.

$1 \mathrm{zu}$ den qualitativ hochstehenden überregional und international bedeutenden deutschen Messen zählen: Deutsche Kunstund Antiquitätenmesse München (ca. 150 Aussteller), Hannover (ca. 75 Aussteller) und - im jährlichen Wechsel Köln/Düsseldorf (ca. 170 Händler). Die Besucherzahl hat sich in den letzten Jahren auf jeweils ca. 50.000 eingespielt. Zu Fakten und Konzeptionen der Messen vgl. beispielhaft Harenberg, H., Sammeln mit Gewinn, München 1976, S. $133 \mathrm{ff.;}$ Herchenröder, Chr., Die Kunstmärkte, a.a.o., S. $245 \mathrm{ff.;} \mathrm{Häußermann,} \mathrm{B.} \mathrm{,} \mathrm{Ein} \mathrm{breitgefächertes} \mathrm{Programm,}$ in: WK, 50. Jg. (1980), Heft 7 (April), S. $852 \mathrm{f}$.; o.V., Reger Konsum honoriert Messearbeit, in: HB Nr. 61 vom 27.3.1979, S. 19; Hegewisch, K., Die Flaute im Mittelfeld - Antiquitätenmesse in München, in: FAZ Nr. 250 vom 28.10.1981, S. 28; v. Helmolt, Chr., wo das Alter sehr hoch bewertet wird, in: FAz Nr. 55 vom 5.3.1980, S. 27; Paschke, M. , Marktsituation .... a.a.0.. S. 1531.

2 Extreme Gesichtspunkte, daß zum Beispiel ganze Städte "Kunstzentren" (München, Düsseldorf, Zürich, London, New York etc.) oder sogar Länder als Abstraktum für Einkaufsstätten stehen können, wurden nicht berücksichtigt, obwohl Generalisierungen wie "Ich kaufe meine Antiquitäten ausschließlich in ..." für Antiquitätenkäufer nicht untypisch sind. Hierin spiegelt sich wohl eher der Wunsch, die gekaufte Antiquität am "Image" oder "Prestige" der Einkaufsstätte partizipieren zu lassen. 


\subsection{Auktionswesen}

Antiquitätenauktionen sind sowohl für private Käufer als auch für Händler bedeutende Ein- und Verkaufsquellen. Als börsenähnliche "Marktplätze" bestimmen Auktionen Preisund Marktentwicklungen für Einzelstücke und Sammelgebiete maßgeblich. Von einer Veröffentlichung erzielter Auktionsergebnisse in Presse und "hauseigenen" Ergebnislisten, können stimulierende oder dämpfende Effekte insbesondere auf die private Nachfrage ausgehen ${ }^{1}$.

Auktionshäuser versteigern in der Regel Antiquitäten als Kommissionäre im eigenen Namen aber für Rechnung der zumeist ungenannt bleibenden Einlieferer (Kommittenten) ${ }^{2,3}$. Daneben kommt in geringerem Umfang sog. Eigenware zum Ausruf ${ }^{4}$. Schließlich betätigen sich Auktionshäuser auch als Facheinzelhändler und verkaufen Antiquitäten im Freiverkauf in eigenen Geschäftslokalen.

1 Vgl. Fischer, L., Weltrekordpreise ..., a.a.O., S. 19.

2 Nur in wenigen Ausnahmen versteigern Auktionshäuser im Namen und auf Rechnung des Einlieferers (z.B. vollständige Sammlungen oder Nachlässe).

3 Die Zahl im Bereich Antiquitäten tätiger Auktionshäuser in der Bundesrepublik ist nicht exakt bestimmbar. Für den gemeinsamen Bereich Kunst und Antiquitäten werden im "Kunstadressbuch für die Bundesrepublik Deutschland" ca. 110 Auktionshäuser genannt. In der Marktübersicht der "Keyser'schen Verlangsbuchhandlung" finden sich dagegen nur ca. 40 Auktionatoren. Verbandsmäßig organisiert im "Bundesverband Deutscher Kunstversteigerer e.v." sind ca. 25 Häuser. Vgl. Kunstadressbuch für die Bundesrepublik Deutschland, a.a.O., S. 95-102; Keyser'sche Verlangsbuchhandlung (Hrsg.), Der Kunst- und Antiquitäten-Markt $1979 / 80$, München 1978, S. 243.

4 Grundsätzlich ist einem Versteigerer der verkauf von Waren, die er in seinem Handelsgeschäft führt (Eigenware), untersagt. Der Gesetzgeber macht mit der Formulierung "soweit dies nicht üblich ist" jedoch eine Einschränkung, die für den Antiquitätenhandel Anwendung findet (vgl. 1 GewO $\S 34$ b Abs.6 Nr.4). Eigenware ist in Auktionen üblich. Andererseits ist sie für den Käufer nicht als solche ersichtlich, wodurch sich Auktionatoren den "atmosphärischen" Vorteil von Versteigerungen als Verkaufsform für die Eigenware sichern. Dabei gilt i.d.R. auch für die Eigenware der für die Kommissionsware von Auktionshäusern übliche Gewährleistungsausschluß. 
In ihrem Auktionsangebot unterscheiden sich Auktionshäuser untereinander ebenso wie der Fachhandel in thematischer (Sachgebiete bzw. Angebotsbreite), qualitativer (Genre) und quantitativer (Angebotstiefe) Hinsicht.

Analog zur Gliederung des Fachhandels lassen sich Auktionen in Spezial- und Universalauktionen klassifizieren. Die bedeutenden national und international tätigen Auktionshäuser führen i.d.R. beide Auktionstypen durch. Darüber hinaus sind auch Sonderauktionen z.B. für geschlossene Sammlungen/Nachlässe oder qualitativ ausgesonderte Sammelqebiete ("Collectors Items" wie Spielzeug, Puppen etc.) üblich". Auktionskäufe beinhalten für den Käufer hohes Eigenrisiko, da Kunstversteigerer für Mängel und Fehler auch bei anderslautender Katalogbeschreibung eine Gewährleistung in der kegel ausschlieBen ${ }^{2}$.

Der Versteigerungsvorgang vollzieht sich in einem offenen und direkten AufeinanderstoBen von Angebot und Nachfrage: Die Aukticnsteilnehmer leisten sich ausgehend von einem Ausrufpreis ein Bietgefecht. Neben überwiegend persönlich abgegebenen Geboten werden auch schriftliche und telefonische berücksichtigt. Den Zuschlag bekommt nach dreimaligem Ausruf der Käufer mit dem höchsten Gebot. Die bei einer Auktion aurch Konkurrenz, Direktheit, Schnelligkeit, Spontaneität, Chance, Risiko und Ausstrahlung des Auktionators entstehende Atmosphäre sind wichtiger Bestandteil der vom privaten Käufer erlebten engeren Kaufsituation auf Auktio-

1 Vgl. v. Gehren, G., Sorgfältige Sammleroflege, in: HB Nr. 155 vom 14.8.1979, S. 15.

2 Katalogbeschreibungen sind keine Zusicherungen von Eigenschaften im Sinne des Kaufrechts (vgl. §§ $459 \mathrm{ff}$. BGB). Sie werden ausdrücklich nach "bestem Wissen und Gewissen" erstellt und bieten daher für den Käufer keine Grundlage für Gewährleistungsansprüche gegenüber dem Auktionator. Zur Diskussion vgl. Löhr, H.D., Schütte, R., Kunstumsatz findet auf einem Sondermarkt statt - Prinzipielles und Aktuelles über Kunstversteigerungen, Beitrag in: Harten, I., de la Motte, M. et al. (Hrsg.), Das Kunstjahrbuch, Bamberg 1979, S. 63-69; Paschke, M., Rechtliche Aspekte des Kunsterwerbs und -sammelns, Beitrag in: FAZ (Hrso.), Kunst- und Antiquitätenkalender 1982, Frankfurt 1981, S. 148-153. 
nen. Einflüsse auf den momentanen KaufentscheidungsprozeB (z.B. in Form eines erlebten Zeitdrucks oder einer sich spontan aufbauenden Rivalität $z u$ einem Mitbieter) sowie eine "psychologische" Selbstselektion von Auktionskunden und Nicht-Kunden sind denkbar.

Bei Kauf und Verkauf auf Auktionen fallen feste Provisionssätze in Form von Aufgeldern und Abschlägen auf den erzielten Verkaufspreis für den Auktionator an: in der Bundesrepublik zwischen ca. 10 - 25 \& für den Einlieferer und zwischen ca. 10 und $20 \%$ für den Käufer ${ }^{1}$.

Insgesamt leisten Auktionshäuser durch die Akquisition von Nachlässen, Sammlungen und Einzelstücken für ihre Auktionen einen groBen Beitrag zur Aufrechterhaltung und Regeneration des Waren- und Marktkreislaufs. Die damit einhergehende Ubernahme von Suche und Vorselektion des Antiquitätenangebots ist ebenso wie die spezifische Auktionsatmosphäre Grundlage ihrer Bedeutung als Einkaufsstätte ${ }^{2}$.

1 Höhe und Verteilung der Provisionssätze auf Käufer und Einlieferer sind Kernstück der preispolitischen Entscheidung eines Auktionshauses. Ein Auktionator kann sich nicht nur Wettbewerbsvorteile gegenüber Konkurrenzhäusern verschaffen, sondern sich auch flexibel an die allgemeine Entwicklung von Einliefererangebot und Käufernachfrage in unterschiedlichen Konjunkturphasen des Antiquitätenmarktes anpassen. Christie's und Sotheby's haben erst Mitte der 70 J Jahre Käuferprovisionen (ca. $10 \%$ ) eingeführt und sich damit Verhandlungsspielraum bei den Verkaufs- bzw. Einliefererprovisionen (jetzt zwischen 2 und $10 \%$, zuvor zwischen 12 und 25 \%) geschaffen. Sie reagierten damit nicht zuletzt auf zunehmende Schwierigkeiten der Warenbeschaffung. Vgl. Hawkins, J., Londons Auktionshäuser auf der Suche nach Millionenobjekten, in: FAZ, Nr. 251 vom 28.10.1980, S. 18; v. Hassel, Chr., Auktionssaison, New York, August 1980, in: Weltkunst, Heft 17, 50. Jg., 1980, S. $2317 \mathrm{f}$..

2 Die steigende Zahl privater Auktionskunden läBt sich dabei nicht zuletzt auch auf die traditionelle Vorstellung von Auktionen als wichtigste Beschaffungsquelle des Handels zurückführen: durch die Umgehung von Handelsspannen können sich dem Käufer vermutete oder tatsächliche Preisvorteile bieten. 


\subsection{Serviceanbieter}

Serviceanbieter zählen $z u$ den sekundär aktiven Marktpartnern privater Antiquitätenkäufer bzw. -besitzer, da sie im Markt nicht unmittelbar als An- oder Verkäufer von Antiquitäten, sondern als Dienstleistungsunternehmen aufreten.

Serviceangebote können sowohl direkt als auch über die Vermittlung primär aktiver Marktpartner (Handel/Auktion etc.) in Anspruch genommen werden. Dies gilt insbesondere für grundsätzliche, objektbezogene Leistungen, die die allgemeine Wertschätzung des Käufers und damit zugleich die Verkäuflichkeit von Antiquitäten wesentlich beeinflussen. Hierzu zählen u.a. Restauration, Reparatur, Kauf- und Anlageberatung $^{1}$ sowie unterschiedliche Arten von Gutachten ${ }^{2}$.

Andere Serviceanbieter wie z. B. Messegesellschaften, Werbeagenturen, Spezialtransportunternehmen oder Spezialversicherungen arbeiten dagegen überwiegend für Handels- und Auktionsfirmen.

1 Die Kauf- und Anlageberatung für Unternehmen und Banken hat sich in den USA unter der Bezeichnung "Art Advice" bzw. "Art Consulting" als neue Dienstleistungsbranche etabliert. Vgl. o.V., Kunstgriff. Art Consulting für Unternehmen, in: Capital, 19. Jg. (1980), Heft 9, S. 158 ff.. In der Bundesrepublik bieten inzwischen "Antik-Investitionsgesellschaften" insbesondere den "Anlegern" unter den Käufern ähnliche Dienstleistungen.

2 "Expertisen" werden Gutachten von (unabhängigen) Sachverständigen (überwiegend selbst Händler) genannt, die von örtlichen Industrie- und Handelskammern bestellt und vereidigt sind. Zur Problematik und Aussagewert von Expertisen vgl. Herchenröder, Chr., Die Kunstmärkte, a.a.o., S. 287 ff.; "Schätzungen", "Zertifikate", "Atteste" o.ä. sind dagegen Objektbeschreibungen (im Sinne "zugesicherter Eigenschaften" vgl. Kapitel B./2.21) des verkaufenden Händlers. Daneben sind auch naturwissenschaftlich fundierte Gutachten von spezialisierten Analyse-Labors (insbesondere Altersbestimmungen) im Einzelfall möglich. Zur Verfahrenstechnik vgl. Harenberg, H., Sammeln mit Gewinn, a.a.o., S. 247 f.. 
Eine Sonderstellung unter den Serviceanbietern nehmen Antiquitäten-Leasinggesellschaften ein ${ }^{1}$. Grundgedanke des Leasing-Konzeptes ist die Vorfinanzierung und Übernahme der Eigentümerfunktion durch einen Leasinggeber bei gleichzeitiger Vermietung des Leasingobjekts (Antiquität) an einen Leasingnehmer (Käufer). Durch Kombination eines Mietpreises, der u.a. ein monetäres Äquivalent für die nutzungsbedingten "Wertverluste" beinhaltet, und einer Kaufmöglichkeit am Ende der vereinbarten Mietdauer zu einem nur noch geringen "Restwert" kann Miete (Besitz) und Kauf (Eigentum) steuerlich als Betriebsaufwand geltend gemacht werden ${ }^{2}$. Als private Kunden von Leasinggebern kommen daher selbständige Unternehmer und insbesondere Freiberufler (Ärzte, Anwälte etc.) in Betracht.

Die Erfahrung zeigt, daB Leasingnehmer während der Mietdauer eine "persönliche Bindung" zur geleasten Antiquität bekommen, so daB sie in der Regel von ihrer Kaufmöglichkeit Gebrauch machen ${ }^{3}$. Antiquitäten-Leasinggesellschaften wachsen damit über die Rolle eines ausschließlichen Serviceanbieters hinaus und werden zu einer weiteren Einkaufsstättenalternative für den privaten Käufer.

1 Vgl. als praktisches Beispiel das Portrait einer Antiquitäten-Leasinggesellschaft, o.V., Antiquitäten. Fast wie Eigentum, in: Der Spiegel, Nr. 46 (November) 1980, S. $254 \mathrm{f}$.. Zum Konzept des Leasing vgl. beispielhaft Fischer, $0 .$, Finanzwirtschaft der Unternehmung I. Tübingen, Düsseldorf 1977 , S. $122 \mathrm{ff}$; Holz, D., Die Optimierungsbestimmung bei Kauf-Leasing-Entscheidungen, Frankfurt, Zürich 1973; 0.V., Der Schwerpunkt: Leasing macht Karriere, in: WiWo $\mathrm{Nr} .47$ vom 24.11 .1972 , S. $84 \mathrm{ff}$.

2 Der Kauf von hochwertigen Antiquitäten zu "Neu- bzw. Marktpreisen" wird aufgrund einer preislichen "Unverhältnismäßigkeit" zu "normalen" betrieblich genutzten Einrichtungs- und Repräsentationsgegenständen i.d.R. nicht als Betriebsaufwand anerkannt.

3 Vgl. o.V., Antiquitäten. Fast wie Eigentum, a.a.O., S. 255.

4 In Abhängigkeit von der konkreten Ausgestaltung des Leasingvertrages können hierfür zusätzliche materielle Gründe sprechen: Hohe Mietpreise mit hohen anteiligen "Kaufpreisraten" führen im Zeitablauf zu einem niedrigen Restkaufpreis. Durch die Nichtwahrnehmung der Kaufmöglichkeit entstehen finanzielle Nachteile, die darüber hinaus i.d.R. nicht von einer Wertminderung der Antiquität durch Gebrauch, sondern im Gegenteil von einem steigenden Marktwert begleitet werden. 


\subsection{Antiquitätenkreislauf und Marktregeneration}

Kauf, Verkauf oder Tausch von Antiquitäten beinhalten als aktuelle Transaktionsprozesse neben Geld- und Informationstransfers einen realen Warenfluss von Antiquitäten zwischen den beteiligten Marktpartnern. Besondere Bedeutung besitzt dieser Warenfluss bei der Aufrechterhaltung des Marktkreislaufs: Er ist Grundlage der stetigen Erneuerung (Regeneration) eines lantent vorhandenen in ein aktuelles Antiquitätenangebot. Die Prozeßstruktur des Warenflusses ist als abstrahiertes Spiegelbild seiner realen Erscheinungsformen anzusehen (vgl. Abbildung 8).

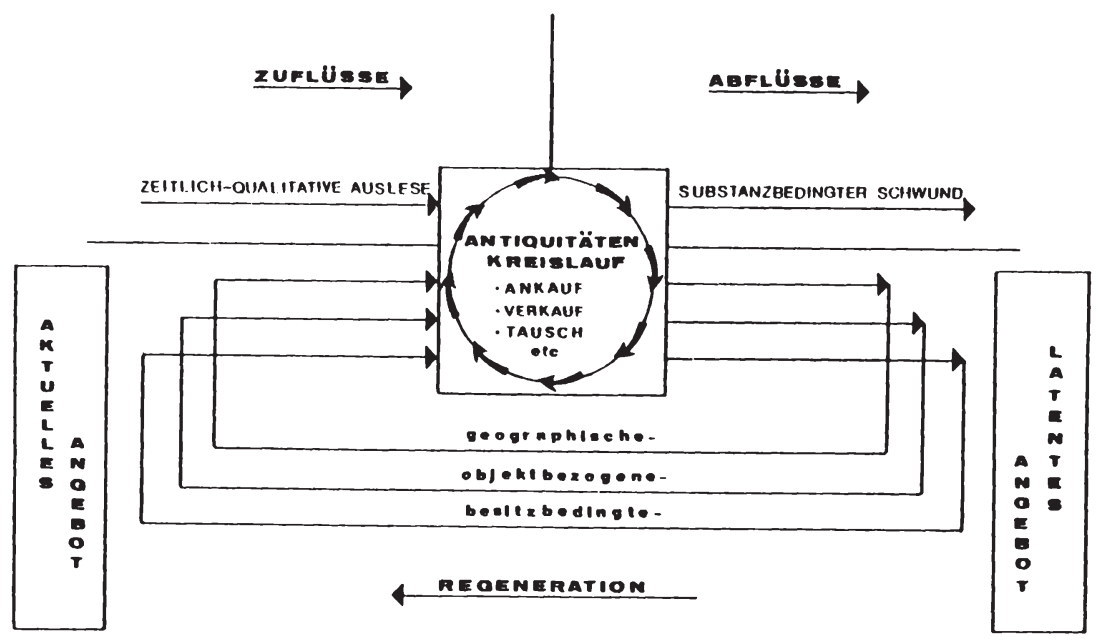

Abb. 8: Waren- und Regenerationskreislauf als Determinanten des Antiquitätenangebots 
Kennzeichnendes Merkmal und Besonderheit ${ }^{1}$ des Antiquitätenmarktes ist die Zirkulation von Antiquitäten in einem nahezu geschlossenen Warenkreislauf.

Ursache der zirkulären Struktur des Warenflusses ist die ex definitione ausgeschlossene Warenproduktion und die resultierende relative Konstanz des verfügbaren Antiquitätenpotentials. Nur langfristig und in geringem Umfang kommt es zu einer Erweiterung dieses Potentials durch ein "Hineinwachsen" jüngeren Kunstgewerbes in den Marktkreislauf als Einzelstücke, als neue Sammelgebiete oder als abgeschlossene stilrichtungen.

Derartige Zuflüsse sind stets das Ergebnis zeitlicher und qualitativer Ausleseprozesse, die u.a. durch kommerzielle Interessen von Handel und Auktionshäusern gefördert, sowie durch individuell-motivationsbedingte und kulturell bestimmte Wert- und Geschmacksvorstellungen der Käufer beeinflußt werden.

Den Erweiterungstendenzen des Gesamtpotentials stehen kompensierend substanzbedingte Abflüsse einzelner Antiquitäten durch Gebrauch, Zerstörung, Beschädigung, Verfall etc. gegenüber.

Marktkreislauf und aktuelles Antiquitätenangebot leben daher fast ausschließlich von einer Marktregeneration, d.h.

1 Der Antiquitätenmarkt unterscheidet sich hierin wesentlich von Produkt- bzw. Konsumgütermärkten mit einer stetigen Produktion, die überwiegend durch hierarchisch-vertikale Verkaufsbeziehungen bei der Güterdistribution gekennzeichnet sind. Vgl. die Darstellung solcher Distributionswege z.B. bei Kotler, Ph., Marketing Management ..., a.a.O., S. 546 ff.; Nieschlag, R., Dichtl, E., Hörschgen, H., Marketing, a.a.0., S. 129 ff.. 
der kurzfristigen und wiederholten Aktualisierung des bereits latent vorhandenen Antiquitätenangebots. Aus struktureller Sicht können dabei geographische, objektbezogene und besitzbedingte Regenerationskreisläufe (Latenz-Aktualisierungskreisläufe) unterschieden werden.

Der geographische Regenerationsproze $\beta$ basiert auf der regionalen Begrenztheit des tatsächlichen Antiquitätenangebots einerseits und der verengten individuellen Marktübersicht des privaten Käufers andererseits ${ }^{1}$. Der vom Käufer wahrgenommene Markt- bzw. Angebotsausschnitt ändert sich beispielsweise in Abhängigkeit von motivational geprägtem Engangement, Interesse und Informationsstand: ein engagierter, thematisch spezialisierter und kenntnisreicher "Sammler" , wird eine breitere und i.d.R. sogar internationale Marktübersicht besitzen. Für den "Einrichtungs- und Dekorationskäufer" ist c.p. vermutlich das Gegenteil zutreffend.

Die objektbezogene Regeneration des Marktkreislaufes basiert auf der besonderen Eigenart von Antiquitäten, wiederholt neue und in sich geschlossene "marktliche Lebenszyklen" durchlaufen zu können. Dabei ist nicht nur an zeitweiliọ verschollene Einzelstücke $(z$. B. auf Speichern oder in Nachlässen) zu denken, sondern wichtiger noch an motivational-

1 Oft anzutreffende willkürliche Abgrenzungen eines "relevanten " Marktgebietes beispielsweise durch politische Grenzen - die Regeneration würde dann durch einen ImportExport-Kreislauf vollzogen - wird weder der Handels- noch der Käuferauffassung gerecht.

Gerade für Antiquitäten geht mit zunehmenden Qualitätsansprüchen und thematischen Spezialisierungen eine "Internationalisierung" der Marktsicht einher. Vgl. v. Gehren, G., Tendenzen zur Internationalisierung, in: Wk, 51 . Jg. (1981), Heft 3 (Februar), S. 205; Herchenröder, Chr., Die Kunstmärkte, a.a.0., s. $31 \mathrm{ff.;}$ 0.V., Die Saisonbilanzen der Londoner Auktionshäuser, in: Wk, 50.Jg. (1980), Heft 17 (September), S. 2318; Sturm, A.G., Schlag auf Schlag, in: Plus Nr. 20 vom 13.5.1981, Düsseldorf, Hamburg, Zürich, S. 28; Giachi, A., Die Spezialisten geben den Ton an, in: FAZ Nr. 81 vom 21.4.1978, S. 27. 
ästhetisch beeinfluBte und geschmacksbedingte "Modezyklen" für ganze Sammelgebiete und Stilrichtungen ${ }^{1}$. Die Erneuerung des Marktangebots vollzieht sich dabei für den Käufer im Wechsel eines "aus der Mode- bzw. in Vergessenheitgeratens" einerseits und eines "Wiederentdeckens bzw. -auflebens" andererseits.

Schließlich kennzeichnet der besitzbedingte Regenerationskreislauf mit seinen $\mathrm{Zu}$ - und Abflüssen das aktuelle Marktgeschehen. Jeder Besitz von Antiquitäten in privater Iland entzieht dem Markt auf unbestimmte Zeit einen Teil seines Angebots. Das derartig latent gewordene Antiquitätenangebot kann jederzeit durch eine Verkaufsabsicht aktualisiert werden.

\subsection{Rahmenbedingungen des Antiquitätenmarktes}

$\mathrm{Zu}$ den Rahmenbedingungen des Antiquitätenmarktes zählen vor allem rechtlich-politische, ökonomische, sozial-kulturelle und kommunikative Gegebenheiten sowie die Entwicklung auf bedarfs- und nutzenverwandten Konkurrenzmärkten. In Abbildung 9 sind die wichtigsten Subvariablen ohne Anspruch auf Vollständigkeit und Unabhängigkeit zusammengestellt.

\footnotetext{
1 Vgl. Herchenröder, Chr., Die Kunstmärkte, a.a.o., s. 7 ff.; Wiegelmann, G., Anmerkungen zur Antiquitätenmode, unveröffentlichtes Manuskript eines Vortrages in Münster am 3. Juni 1977, S. 7 ff.; Meunier, E.F., Die "Bekehrung" Amerikas zur Kunst, Einführung in: Behrmann, S.N., Duveen und die Millionäre. Zur Soziologie des Kunsthandels in Amerika, München 1960, S. 9 ff.; o.V., Kaufen Sie antizyklisch. Interview mit $\mathrm{H}$. Hanstein, in: Capital, 18. Jg. (1979), Heft 1, s. 41.
} 
I. RECHTLICH - POLITISCHE RAHMENBEDINGUNGEN

Sondervorschriften (z.zt.) bei

Erwerb und Besitz

- Vermögenssteuer

- Erbschaftssteuer/Schenkungssteuer

- Mehrwertsteuer

- Zöllen

- Einkommens- bzw. Körperschaftssteuer (Absetzbarkeit) etc. gewerblicher Veräußerung

- Rabattgesetz

- Preisauszeichnungsverordnung

- Gewährleistung

- Versteigerungsverordnung etc.

II. ÖKONOMISCHE RAHMENBEDINGUNGEN

Konjunktur

- Konjunkturphase

- Erwartung der Entwicklung etc.

$\underline{\text { ins }}$

- Kapitalverzinsung

- Fremdkapitalkosten etc.
Geld

- Inflation

- Währungsstabilität

- Wechselkurse etc.

Lebensstandard/Liquidität - verfügbares Einkommen

- Kreditfinanzierung etc.

III. BEDARFS- UND NUTZENVERWANDTE KONKURRENZMÄRKTE

Imitationsmärkte

- Reproduktionen, Repliken

- Kopien

- Fälschungen etc.

Anlagemärkte und Börse

- Aktien / Rentenpapiere

- Sparen / Termingelder

- Immobilien

etc.

Einrichtungsmärkte

- Möbel

- Wohnaccessoires etc.
Zeitgenössische Kunstgewerbemärkte

- Schmuck

- Glas / Porzellan etc.

Freizeit - Hobbymärkte
Märkte für sonstige "speciality goods"

Abb. 9: Ausgewählte Rahmenbedingungen des Antiquitätenmarktes (Teil I) 
IV. KOMMUNIKATIVE RAHMENBEDINGUNGEN

Medienpublizität

- Marktdarstellungen

- "Sensationspresse" etc.

Kommunikationspolitisches Niveau (Markttransparenz)

- Anzahl von Messen - Märkten etc.

- Ausstellungen, Aktionen

- Medienwerbung etc.
Museumsaktivitäten

- Vorträge

- Ausstellungen

etc.

Literaturangebot

- Zeitschriften, Periodica

- Fachbücher

- Nachschlagwerke

\section{KULTURELLE RAHMENBEDINGUNGEN}

Kulturgeschichte

- Tradition

- Diskontinuität (z.B. Kriege)

- Kolonialmacht

etc.

Kultureller Lebensstil

- Werte

- Normen

- Einstellungen

- kulturelle Meinungsführung (siehe IV)

etc. etc.
Kulturpolitik

- materielle Kunst- und Kulturförderung (Museen, Stiftungen etc.)

- bildungspolitische Kunstund Kulturförderung

- Aus fuhrbeschränkungen

- Vorkaufsrechte

etc.

\section{SONSTIGE RAHMENBEDINGUNGEN}

Wohnungsbau

- Raumdimensionierung

- Klimatisierung

etc.

\section{Finanzierungsbereitschaft}

von Banken beim privaten Kunsterwerb und im kommerziellen Handel

Abb. 9:Ausgewählte Rahmenbedingungen des Antiquitätermarktes (Teil II) 
3. Analyse des Käuferverhaltens privater Antiquitätenkäufer

Der Bestandsaufnahme des Käuferverhaltens liegt eine zweigeteilte Klassifikation von Verhaltensmerkmalen zugrunde, die sich inhaltlich an der struktur des verhaltenswissenschaftlichen Erklärungsansatzes der Käufermotivation orientiert.

Zunächst werden die motivationsbestimmenden personalen Dispositionen der Antiquitätenkäufer vorgestellt. Daran anschließend rücken ihre motivationsgeleiteten kognitiven und konativen Verhaltensmerkmale in den Mittelpunkt der Betrachtung.

\section{1 Personale Dispositionen}

Die Charakterisierung personaler Dispositionen privater Antiquitätenkäufer läßt sich durch soziodemographische sowie psychographische Merkmale vornehmen.

Die Ergebnisse beider Analysebereiche dienen als Informationsgrundlage der Erklärung von Motivationen des Antiquitätenkäufers. Im einzelnen sind darin die empirisch erhobenen Merkmalsausprägungen als auch beispielhafte orientierungshypothesen über Bedeutung und Einflußrichtung personaler Dispositionen als Motivationsdeterminanten enthalten.

\subsection{Soziodemographische Merkmale}

Die empirisch abgefragten soziodemographischen Merkmale von Antiquitätenkäufern umfassen die demographischen Größen Geschlecht, Alter, Familienstand, HaushaltsgröBe, Religionszugehörigkeit, Wohnort sowie die sozioökonomischen Merk- 
male Einkommen, Anzahl der verdienenden Personen im Haushalt, Schulbildung, Beruf ${ }^{1}$.

Soziodemographische Merkmale können in ihrer individuellen Variation partielle Erklärungsbeiträge für die Motivation (Motivationsinhalte und -ausprägungen) von Antiquitätenkäufern besitzen. Beispielsweise ist hypothetisch zu erwarten, daB in Abhängigkeit

(1) von der Geschlechtszugehörigkeit rational-sachliche Motivationsinhalte (wie z.B. ökonomisch orientierte Motive) bei Männern und emotionalgefühlsbetonte Motivationsinhalte (wie z.B. Streben nach Ästhetik, Nostalgie) bei Frauen stärker ausgeprägt sind ${ }^{2}$.

(2) vom Alter, aufgrund typischer situationaler Gegebenheiten und $\mathrm{Zielorientierungen} \mathrm{in} \mathrm{bestimmten}$ Lebensabschnitten altersbedingte Motivationen (z.B. Antiquitäten zur Wohneinrichtung bei Gründung des Hausstandes, als Freizeitbeschäftigung im Ruhestand) anzutreffen sind ${ }^{3}$.

1 Das sozialstatistische Strukturprofil der befragten Antiquitätenkäufer gibt im Anhang die Abbildung 1 mit seinen demographischen und die Abbildung 2 mit seinen sozioökonomischen Komponenten als Ubersicht wieder.

2 Ob solche Motivationsunterschiede kausal auf geschlechtsbestimmte psychische Unterschiede oder auf sozial zugewiesene Rollenerwartungen zurückzuführen sind, ist nur im Einzelfall entscheidbar. Zur Rolle von Mann und Frau in familiären Kaufentscheidungsprozessen vgl. Dahlhoff, H.-D., KaufentscheidungsprozeB von Familien - Empirische Untersuchung zur Beteiligung von Mann und Frau an der Kaufentscheidung, Frankfurt a.M. 1980, S. $120 \mathrm{ff.}$.

3 In engem Zusammenhang mit dem Lebensalter sind auch mögliche Stadien des "Familienlebenszyklus" zu sehen, in denen phasentypische konsummuster und im engeren Sinne Konsummotive auftreten können. Zum Begriff des Familienlebenszyklus vgl. Meffert, H., Marketing, a.a.0., s. $130 \mathrm{ff.;}$ Kotler, Ph., Marketing Management,a.a.0., S. 109 ff.; Ruhfus, R.E., Kaufentscheidungen von Familien - Ein theoretischer Bezugsrahmen zur Analyse des kollektiven Entscheidungsverhaltens im privaten Haushalt, Diss. Münster 1975, S. $32 \mathrm{ff}$. und S. $159 \mathrm{ff}$. 
(3) vom Bildungsgrad die kognitiv diskriminatorischen Fähigkeiten der Antiquitätenkäufer variieren, so da. $B$ aufgrund unterschiedlich stark differenzierter Wahrnehmung der oigenen Bedürfnisse einerseits und der Antiquitätencharakteristika andererseitc unterschiedlich stark ausdifferenzierte Motivationsstrukturen auftreten.

(4) von der Einkommenshöhe Verstärkung bestimmter Motivationsinhalte (z.B. Repräsentation) möglich sind oder aber daneben im Extremen eigenständige ökonomische Motivationen (z.B. Geldanlage in Antiquitäten zur Wertsicherung) erst durch die Einkommensverhältnisse entstehen ${ }^{1}$.

(5) von der Schichtzugehörigkeit spezifische Motivationsinhalte (z.B. Prestige- und Statusdokumentation durch Antiquitäten) anzutreffen sind ${ }^{2}$.

Zur Prüfung und detaillierten Analyse von Zusammenhëncen zwischen Motivationen und soziodemographischen Merkmalen vergleiche die Ergebnisse der empirischen Untersuchung in Kapitel C. dieser Arbeit.

1 Die überdurchschnittlich hohen Einkommen lassen den Schluß zu, daß die Einkommensverhältnisse ihre Rolle als restriktive Nebenbedingung beim Kauf von Antiquitäten in der Regel verlieren. Auf Antiquitäten bezogene Motivationen können sich daher vermutlich relativ "frei" von ökonomischen Einschränkungen im Kaufakt verwirklichen.

2 Der "Schicht-Begriff" wird in der Literatur uneinheitlich interpretiert und operationalisiert. $\mathrm{Zu}$ Möglichkeiten der Schichtinterpretation und Bestimmung der Schichtzugehörigkeit von Konsumenten vgl. Engel, J.F., Blackwell, R.D., Kollat, D.T., Consumer Behavior, 3. Aufl., Hinsdale 1978, S. 278 f.; Scheuch, E.K., Daheim, H. , Sozialprestige und soziale schichtung, in: Glass, D.V., König, R. (Hrsg.), Soziale Schichtung und soziale Mobilität, Sonderheft 5 der Kölner Zeitschrift für Soziologie, 5. Jg. (1961), S. 65-103; Markin, J.R. (jr.), Consumer Behavior ..., a.a.o.,
S. $410 \mathrm{ff..}$ 
3.12 Psychographische Merkmale

Da in der Käuferverhaltensliteratur nur wenig Einigkeit über Anzahl, Struktur und Relevanz einzelner psychographischer Merkmalsklassen besteht, ist in der vorliegenden Untersuchung eine problembezogene Auswahl und Gliederung vorzunehmen. 1

Zur Psychographie von Antiquitätenkäufern sollen diejenigen intrapersonell bedingten psychischen Größen gezählt werden, von denen motivationsklärende Erkenntnisse sowohl für unterschiedliche Motivationsinhalte als auch -intensitäten erwartet werden können. Dabei erscheint eine Differenzierung der psychographischen Einflußgrößen in grundlegende Persönlichkeitsmerkmale (Charaktereigenschaften), Merkmale des Lebensstils und der Interessenentstehung sowie die allgemeine Vorstellung von Antiquitätenkäufern über die Instrumentalität und Leitbildwirkung eines Antiquitätenkaufs sinnvoll.

\subsection{Persönlichkeitsmerkmale}

Die Beschreibung von Persönlichkeitsmerkmalen der Antiquitätenkäufer konzentriert sich auf die Isolation und Messung genereller psychologischer Dispositionen, oft auch als Charakterzüge (traits) oder Persönlichkeitstypen (types) bezeichnet.

1 Vgl. als tberblick Kassarjian, H.H., Sheffer, M.J., Personality and Consumer Behavior: One More Time, in:

AMA Combined Proceedings 1975, Mazze, M. (ed.), S. 197201 und die dort angegebene Literatur. 
Die vorgenommene Operationalisierung der als relevant erachteten Persönlichkeitsmerkmale basiert auf einer Selosteinschätzung von Antiquitätenkäufern im Rahmen eines semantischen Differentials mit 16 vorgegebenen Einzelmerkmalen. Die Abbildung 10 gibt einen Ergebnisüberblick. Das gezeigte Profil kennzeichnet die durchschnittlichen Ausprägungen der Befragten als Ausdruck ihres psychographischen Selbstkonzepts bzw. Selbstimages. 2

\begin{tabular}{|c|c|c|c|}
\hline $3.8^{1)}$ & pessimistisch & $0 \longrightarrow-0$ & optimistisch \\
\hline 2.7 & risikofreudig & 0 & risikoscheu \\
\hline 2.9 & introvertiert & 0 & extravertiert \\
\hline 2.9 & impulsiv & $-\infty \longrightarrow$ & planend \\
\hline 2.3 & entscheidungsfreudig & $\longrightarrow$ & entscheidungsschwach \\
\hline 3.1 & rational & $\rightarrow-0$ & emotional \\
\hline 2.1 & kontaktfreudig & -0 & kontaktscheu \\
\hline 1.9 & geschmackssicher & $-0-0-0$ & geschmackiich unsicher \\
\hline 2.4 & musisch & $0-1)$ & nicht musisch \\
\hline 2.4 & konservativ & 0 & progressiv \\
\hline 2.2 & selbstsicher & $-1-0$ & unsicher \\
\hline 1.7 & kritisch & -0 & unkritisch \\
\hline 3.2 & fortschrittlich & -0 & traditionsbewust \\
\hline .3 .4 & modebewußt & $-a+0$ & modisch unabhängig \\
\hline 4.1 & gefühlsarm & 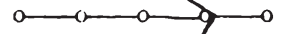 & gef ühl sbe tont \\
\hline 4.0 & angepatt & $0-0-0-0$ & individualistisch \\
\hline
\end{tabular}

Abb. 10: Semantisches Differential zur Kennzeichnung der psychographischen Selbsteinschätzung

1 Zur Methodik vgl. Mayntz, R., Holm, K., Hübner, P., Einführung in die Methoden der empirischen Soziologie, 3. Aufl., Opladen 1972, S. 50 ff..

$2 \mathrm{Zu}$ unterscheiden sind "actual, expected and ideal selfconcept". Vgl. Sanchez, H.T. et al., a.a.O., S. 225 ff.. Abgefragt wurde das aktuelle Selbstkonzept der Käufer. 
Antiquitätenkäufer schätzen sich insbesondere als kritisch, geschmacksicher, aber auch gefühlsbetont und individualistisch ein. Darüber hinaus neigen sie eher zu einer optimistischen Grundhaltung und sehen sich überwiegend als konservative, selbstsichere und entscheidungsfreudige Käuferpersönlichkeiten. Schließlich prägen auch die Merkmale musisch und kontaktfreudig das erhobene selbstkonzept.

Kann aus den genannten Eigenschaftsschwerpunkten ein erstes (grobes) Bild der Antiquitätenkäuferpersönlichkeit gewonnen werden, so bleibt der innere Zusammenhang der einzelnen psychographischen Merkmale unberücksichtigt. Es ist daher von zusätzlichem Interesse, nach dem strukturellen Grundmuster des Selbstkonzeptes von Antiquitätenkäufern zu fragen.

Eine faktorenanalytische Auswertung ${ }^{1}$, deren Ergebnis in Tabelle 2 zusammengestellt ist, zeigt die besondere Bedeutung (relativer Varianzerklärungsanteil 31,6 \%) einer psy= chischen Mobilität im Selbstbild der Antiquitätenkäufer.

(1) Geringe psychische Mobilität kennzeichnet dabei Käufer mit einem hohen Bewußtsein für Tradition. Die zugleich stark konservative Gedankenhaltung impliziert eine weitgehende Akzeptanz und Internalisierung überlieferter oder bestehender Normen und Wertvorstellungen, bei einer tendenziell geringen Bereitschaft zur Veränderung und Erneuerung ${ }^{2}$.

(2) Wohe Mobilität kennzeichnet daher eher Käufer, die ihren Wertbezug in der Gegenwart oder Zukunft finden (fortschrittlich). Sie sind damit auch tendenziell veränderungsbewußter gegenüber überlieferten oder bestehenden Normen und Werten (progressiv).

$1 \mathrm{Zu}$ Verfahren, Interpretation und Ziel der multivariaten Faktorenanalyse vgl. die allgemeinen Darstellungen in Punkt 1.22 dieses Kapitels.

2 Eine geringe psychische Mobilität fördert allgemein die Stabilität der gesamten Persönlichkeitsstruktur von Antiquitätenkäufern. Bei der tendenziell konservativen und traditionsbewußten selbsteinschätzung kommt dieses auch einer zeitstabilen Erklärungsrelevanz der "Persönlichkeitsmerkmale" zur Motivation zugute. 


\begin{tabular}{|c|c|c|c|}
\hline PERSONLICHKEITSDIMENSIONEN & $\begin{array}{l}\text { Faktor- } \\
\text { ladungen }\end{array}$ & $\begin{array}{l}\text { relativ } \\
\text { erklarter } \\
\text { Varianz- } \\
\text { anteil }\end{array}$ & $\begin{array}{l}\text { absolut } \\
\text { erklarter } \\
\text { Varianz- } \\
\text { anteil }\end{array}$ \\
\hline $\begin{array}{l}\frac{\text { PSYCHISCHE MOBILITAT }}{\text { konservativ }} \text { - progressiv } \\
\text { traditionsbewuBt - fortschrittlich }\end{array}$ & $\begin{array}{l}0,95 \\
0,64\end{array}$ & 31,6 & 14.2 \\
\hline $\begin{array}{l}\text { SOZIALE INTERAKTIONSEAHIGKEIT } \\
\text { introvertiert - extrovertiert } \\
\text { pessimistisch - optimistisch } \\
\text { unsicher - selbstsicher } \\
\text { kontaktscheu - kontaktfreudig }\end{array}$ & $\begin{array}{l}0,64 \\
0,56 \\
0,51 \\
0,50\end{array}$ & 21,2 & 9,5 \\
\hline $\begin{array}{l}\text { ENTSCHEIDUNGSSTIL } \\
\text { entscheidungsfreudig - entscheidungsschwach } \\
\text { risikofreudig }\end{array}$ & 0,69 & 17,3 & 7,8 \\
\hline $\begin{array}{l}\text { MODESTIL } \\
\text { modebewrat - modisch unabhangig } \\
\text { impulsiv - planend } \\
\text { emotional - rational }\end{array}$ & $\begin{array}{l}0,61 \\
0,54 \\
0,53\end{array}$ & 14,3 & 6,4 \\
\hline $\begin{array}{ll}\text { ASTHETISCHE KOMPETEN2 } & \\
\text { musisch } & \text { - nicht musisch } \\
\text { kritisch } & \text { - unkritisch } \\
\text { gefülsbetont } & \text { - gefülsarm } \\
\text { geschmackssicher - geschmacklich unsicher }\end{array}$ & $\begin{array}{l}0,58 \\
0,54 \\
0,37 \\
0,26\end{array}$ & 8,6 & 3.9 \\
\hline$\frac{\text { SOZIALE ABHANGIGKEIT }}{\text { angepaBt - individualistisch }}$ & 0,71 & 7,0 & 3,1 \\
\hline & & 100,08 & 44,98 \\
\hline
\end{tabular}

Tab. 2: Persönlichkeitsdimensionen von Antiquitätenkäufern Ergebnisüberblick einer Faktorenanalyse

Als zweite wichtige psychische Disposition im Selbstbild von Antiquitätenkäufern erweist sich der Grad ihrer sozialen Kontaktfähigkeit.

(1) Für hohe Kontaktfähigkeit legen die Persönlichkeitstendenzen Extravertiertheit, Optimismus und Selbstsicherheit die Voraussetzungen, wobei schließlich in einer expliziten Kontaktfreudigkeit die Bereitschaft und der Wunsch nach sozialer Interaktion am deutlichsten wird. 
(2) Für niedrige Kontaktfähigkeit sind dagegen die persönliche Unsicherheit der Käufer, gekoppelt mit einer gefühlsmäßigen Furcht vor sozialen Begegnungen (kontaktscheu) und einer tendenziell pessimistisch unterlegten Introvertiertheit ausschlaggebend.

Von annähernd gleicher Bedeutung ist auch der Entscheidungsstil der Käufer als Persönlichkeitsdimension. Der Entscheidungsstil in seinen extremen Ausprägungsmöglichkeiten entscheidungs- und risikofreudig vs. entscheidungsschwach/ risikoscheu, ist eine Variable, die insbesondere Rückschlüsse auf konkretes (konatives) Kaufverhalten von Antiquitätenkäufern erlaubt.

Gleiches gilt für den Modestil und das Modeverhalten von Antiquitätenkäufern. Individuelle Ausprägungen können auf dieser Dimension von einer stark modisch orientierten Persönlichkeit, wie sie durch hohes Modebewußtsein, Impulsivität und Emotionalität charakterisiert wird, bis zur eher unmodischen Persönlichkeit, wie sie durch hohe modische Unabhängigkeit, Planung und Rationalität charakterisiert wird, reichen ${ }^{1}$.

Die ästhetische Kompetenz als weitere Persönlichkeitsdimension verdichtet die Variablen musisch, kritisch, gefühlsbe-

1 Die Trennung von Entscheidungsstil und Modestil in statistisch unabhängige Dimensionen ist interessant, da viele Merkmale des Modestils (impulsiv, planend, emotional, rational) oft auch zur Kennzeichnung des allgemeinen Entscheidungsverhaltens benutzt werden. Die Differenzierung deutet auf den hohen EinfluB modischer Gesichtspunkte auf Motivation und Kaufentscheid bei Antiquitäten hin. Unterstützt wird diese Vermutung durch eine sehr ausgeprägte Zustimmung der Antiquitätenkäufer zu dem Statement: "Im Moment gibt es eine regelrechte Antiquitätenmode" . Auf einer 5-stufigen Skala mit den Kategorien "trifft sehr zu" (1) bis "trifft gar nicht zu" (5) liegt sie bei einem statistischen Mittel von 1,9. 
tont und geschmackssicher zu einer hohen Ausprägung und die Variablen nicht musisch, unkritisch, gefühlsarm und geschmacklich unsicher zu einer niedrigen Ausprägung der Kompetenz. Der scheinbarewiderspruch zwischen den Variablenpaaren musisch/gefühlsbetont und kritisch/geschmackssicher bestätigt in Wirklichkeit die individuell empfundene Kompetenz, da sich der Käufer einerseits als gegenüber musischen Dingen gefühlsmäßig aufgeschlossen bezeichnet und sich zugleich einen kritischen und geschmackssicheren "Beurteilungsmaßstab" zuschreibt.

AbschlieBend ist die Persönlichkeitsdimension "soziale Abhängigkeit" mit ihren Ausprägungspolen angepaßt - individualistisch als unabhängiger Bestandteil des Selbstkonzepts von Antiquitätenkäufern zu nennen. Von der Selbsteinschätzung des einzelnen Käufers als mehr oder weniger individualistisch bzw. angepaßt ist ebenso wie von der individuellen Einschätzung in Bezug auf alle anderen diskutierten Persönlichkeitsdimensionen eine Vielzahl von Verbindungen zur Motivation des Antiquitätenkaufs $z u$ erwarten.

Die nachfolgenden Beispiele dienen der Verdeutlichung denkbarer Zusammenhänge:

(1) Die Selbsteinschätzung im Sinne einer geringen psychischen Mobilität läßt auf hohe Ausprägungen bei tradierten und traditionsorientierten Motivationsinhalten (Bewahrung von Erinnerungen an die Familie, Empfinden eines kulturellen Auftrages etc.) schlieBen. Die implizierte Ablehnung "heutiger Werte" kann auch zusätzlich einen Rückgriff (Regression) auf "alte Werte" mit entsprechenden Motivationsinhalten (Reaktion auf moderne Kunst bzw. modernes Möbeldesign, Nostalgie etc.) fördern.

Hohe psychische Mobilität harmoniert andererseits eher mit zeittypischen und zeitbedingten Motivationsinhalten (Antiquitätenkauf als Konsumerlebnis etc.).

(2) Die Art der Selbsteinschätzung als mehr individualistisch einerseits oder angepaßt andererseits har- 
moniert mit mehr "innengerichteten" Motivationsinhalten (Selbstverwirklichung, Sammelleidenschaft etc.) oder mehr "außengerichteten" Motivationsinhalten (Repräsentation, Selbstdarstellung etc.).

(3) Eine zunehmend ausgeprägte Selbsteinschätzung in Bezug auf das Vorhandensein einer ästhetischen Kompetenz korreliert vermutlich mit zunehmender Bedeutung von Motivationsinhalten, die einen kunstwissenschaftlichen Hintergrund verlangen (Sammeln, finanzielle Spekulation etc.).

Eine detaillierte empirische Analyse der Zusammenhänge zwischen Persönlichkeitsmerkmalen und Motivationen erfolgt im Kapitel C. dieser Untersuchung.

\subsection{Lebensstil}

Zur zweiten Merkmalsklasse von psychographischen Eigenschaften der Antiquitätenkäufer zählen die Merkmale des Lebensstils. In der Literatur werden eine Vielzahl von Verhaltensmerkmalen zu diesem Konstrukt zusammengefaßt, ohne daß sich eine überschneidungsfreie Klassifikation (z.B. mit Einstellungen) ergibt ${ }^{1}$. Die Merkmale des Lebensstils von Antiquitätenkäufern können durch ihr Aktivitätsniveau in ausgewählten Lebensbereichen, ihre allgemeinen Wertorientierungen und ihren Wohnstil interpretiert und gemessen werden.

\subsection{Aktivitäten}

Abbildung 11 zeigt als Mittelwertanalyse die Ausprägung der Aktivitäten von Antiquitätenkäufern in unterschiedlichen Lebensbereichen als Bestandteil ihres Lebensstils.

1 Vgl. zum Begriff des Lebensstils die zusammenfassende Darstellung bei Bruhn, M., Das soziale BewuBtsein von Konsumenten, Wiesbaden 1978 , S. 88 ff.. 


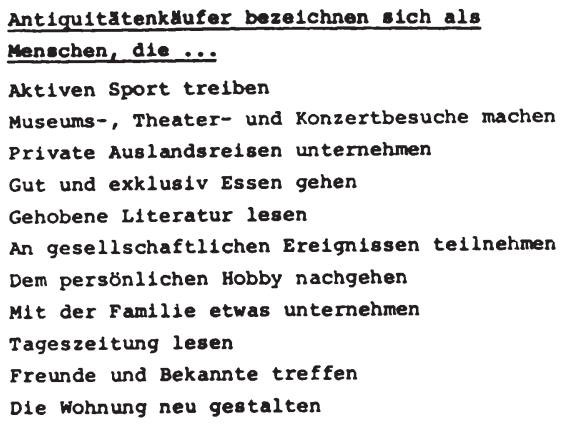

\begin{tabular}{|c|c|c|c|c|}
\hline $\begin{array}{l}\text { (1) } \\
\text { regel- } \\
\text { mäBig }\end{array}$ & $\begin{array}{c}\text { (2) } \\
\text { häufig }\end{array}$ & $\begin{array}{l}\text { (3) } \\
\text { gelegent- } \\
\text { l1ch }\end{array}$ & $\begin{array}{c}\text { (4) } \\
\text { selten }\end{array}$ & $\begin{array}{l}\text { (5) } \\
\text { nie }\end{array}$ \\
\hline 0 & 0 & 0 & 0 & 0 \\
\hline 0 & 0 & 0 & 0 & 0 \\
\hline 0 & 0 & 0 & 0 & 0 \\
\hline 0 & 0 & 0 & 0 & 0 \\
\hline 0 & 0 & 0 & 0 & 0 \\
\hline 0 & 0 & 3, 3,1 & 0 & 0 \\
\hline 0 & & 0 & 0 & 0 \\
\hline 0 & & 30 & 0 & 0 \\
\hline 0 & & 0 & 0 & 0 \\
\hline 0 & & 0 & 0 & 0 \\
\hline 0 & 0 & $\rightarrow 0$ & 0 & 0 \\
\hline 1 & itte & rte & & \\
\hline
\end{tabular}

Abb. 11: Aktivitätsniveaus in ausgewählten Lebensbereichen

Ohne auf alle Merkmale im einzelnen einzugehen, zeigt sich ein insgesamt hohes Aktivitätsniveau bei den Befragten. Das regelmäßige Lesen der Tageszeitung korrespondiert mit dem bereits erörterten hohen Bildungsniveau. Als soziale Aktivitäten sind insbesondere Interaktionen im engeren sozialen Umfeld, d.h. mit Freunden/Bekannten und der Familie bedeutsam. Die "Teilnahme an gesellschaftlichen Ereignissen" besitzt dagegen das geringste Aktivitätsniveau. Dem "persönlichen Hobby" wird durchschnittlich relativ viel Aufmerksamkeit unter den genannten Lebensbereichen gewidmet.

"Museums-, Theater- und Konzertbesuche", welche thematisch in engem zusammenhang mit einem Interesse an Antiquitäten gesehen werden können, nehmen nur einen mittleren Platz zwischen den abgefragten Aktivitäten ein. Eine detailliertere Betrachtung von relativen Häufigkeiten zeigt jedoch, daß immerhin ca. $19 \&$ der Antiquitätenkäufer regelmäßig 
und weitere ca. 31 \& häufig von den genannten kulturellen Angeboten Gebrauch machen.

\subsection{Allgemeine Wertorientierungen}

Abbildung 12 beschreibt die allgemeinen Wertorientierungen von Antiquitätenkäufern als weitere Merkmale ihres Lebensstils ${ }^{1}$.

\begin{tabular}{|c|c|c|c|c|c|}
\hline Allgemeine Wertorientierung & $\begin{array}{l}\text { (1) } \\
\text { trifft } \\
\text { sehr } \\
z u\end{array}$ & $\begin{array}{c}(2) \\
\text { trifft } \\
\text { ziemlich } \\
2 u\end{array}$ & $\begin{array}{c}(3) \\
\text { trifft } \\
\text { etwas } \\
2 u\end{array}$ & $\begin{array}{l}\text { (4) } \\
\text { trifft } \\
\text { kaum } \\
\text { zu }\end{array}$ & $\begin{array}{c}\text { (5) } \\
\text { trifft } \\
\text { gax nicht } \\
\text { zu }\end{array}$ \\
\hline $\begin{array}{l}\text { Ich möchte als Person von anderen anerkannt und } \\
\text { gemocht werden }\end{array}$ & & 0 & & $\mathbf{0}$ & 0 \\
\hline $\begin{array}{l}\text { Ich möchte mich deutlich vom Durchschnittsmensch } \\
\text { abheben }\end{array}$ & hen & & & 0 & 0 \\
\hline Ich mbchte mehr als bisher aus meinem Leben mach & hen & 0 & • & 0 & 0 \\
\hline Ich möchte gern meinen Freundeskreis erweitern & $\mathbf{0}$ & 0 & & 0 & 0 \\
\hline $\begin{array}{l}\text { Ich möchte mein eigenes Leben führen, was } \\
\text { andere dabei von mir denken, lst mir egal. }\end{array}$ & $\mathbf{0}$ & & & 0 & 0 \\
\hline $\begin{array}{l}\text { Ich möchte mein Leben genieBen und mich nicht } \\
\text { mit den Sorgen von morgen belasten }\end{array}$ & 0 & 0 & & 30 & 0 \\
\hline Ich möchte mehr materielle sicherheit erlangen & 0 & 0 & & 20 & 0 \\
\hline $\begin{array}{l}\text { Ich möchte elne unverwechselbare Persönlichkelt } \\
\text { darstellen }\end{array}$ & 0 & 0 & & 0 & 0 \\
\hline $\begin{array}{l}\text { Ich möchte, daB sich melne Freunde und Bekannten } \\
\text { auch bel mir zuhause wohlfulhlen }\end{array}$ & n 01,6 & & 0 & 0 & $\mathbf{0}$ \\
\hline Ich möchte intensiver meinen Hobbys nachgehen & 0 & & 0 & 0 & o \\
\hline $\begin{array}{l}\text { Ich möchte als elne gepflegte Erscheinung mit } \\
\text { sicherem Auftreten gelten }\end{array}$ & 0 & & & 0 & 0 \\
\hline Ich möchte beruflich weiterkommen & 0 & o & & 0 & 0 \\
\hline Ich möchte mehr von der Welt sehen & 0 & & 0 & 0 & 0 \\
\hline $\begin{array}{l}\text { Ich möchte gern für meine Zukunft und die mel- } \\
\text { ner Kinder vorsorgen }\end{array}$ & 0 & & 0 & 0 & 0 \\
\hline $\begin{array}{l}\text { Ich möchte mich in meiner wohnung besser als } \\
\text { bisher einrichten }\end{array}$ & 0 & 0 & &, 10 & 0 \\
\hline \multicolumn{6}{|c|}{ 1) Mittelwerte } \\
\hline
\end{tabular}

Abb. 12: Allgemeine Wertorientierungen von Antiquitätenkäufern

1 Vgl. die Diskussion um den "Werte-Begriff" und seine Stellung im Lebensstilkonstrukt bei Dahlhoff, H.-D., Individuelle Wertorientierung - Analyse und Aussagewert personenspezifischer Werthierarchien im Marketing, in: Meffert, H., (Hrsg.), Arbeitspapiere des Instituts für Marketing der Universität Münster, Nr. 23, Münster 1980, insbesondere S. $16 \mathrm{ff}$.; Bruhn, M., Das soziale Bewußtsein von Konsumenten, a.a.O., S. $88 \mathrm{ff}$.. 
Auffällig ist der überdurchschnittlich hohe wert, den die Käufer der eigenen Individualität und ihrer Anerkennung durch die soziale Umgebung beimessen. Entsprechend stark ist auch das Bestreben der Befragten, daß sich Freunde und Bekannte in dem ihnen "präsentierten" Zuhause des Käufers emotional wohlfühlen. Ebenso möchte der Käufer als Person akzeptiert und gemocht werden, ohne sich dabei allerdings von der Meinung der anderen abhängig zu machen. Im Gegenteil ist man eher bemüht, eine eigenständige und vom Durchschnitt abgehobene (individuelle) Persönlichkeit darzustellen.

Das insgesamt zum Ausdruck kommende Individualitätsbewußtsein von Antiquitätenkäufern findet seine produktspezifische Entsprechung in der Individualität von Antiquitäten als Einzelstück. Der Beweggrund für den Kauf einer Antiquität beinhaltet dabei zwei Teilaspekte: Erstens die Individualisierung der Persönlichkeit "nach innen" (individuelle Selbstverwirklichung) und zweitens ihre Dokumentation "nach auBen" (Selbstdarstellung).

Die niedrigen Mittelwerte bei den Wertorientierungen "mehr materielle Sicherheit", "bessere Wohnungseinrichtung" und "berufliches Fortkommen" sprechen für die hohe materielle Ausstattung des befragten Kreises. Die Annahme überwiegend "höherer" und zugleich psychologisch komplexstrukturierter Motivationsinhalte, die über primäre Grundbedürfnisse hinausgehen, scheint daher bezogen auf den Käuferkreis von Antiquitäten - die als "speciality good" hohen immateriellen Nutzen stiften können - realistisch ${ }^{1}$.

1 Die Uberlegung steht im Einklang mit einer Bedürfnishierarchie wie sie im Rahmen der dynamischen Motivtheorie von Maslow propagiert wird. Zur Darstellung der Theorie vgl. Maslow, A.M., A Theory of Human Motivation, in: PR, 1943, S. 370-396; derselbe, Motivation and personality, New York 1954, als Auszug wiedergegeben in: Levine, F.M. (Hrsg.), Theoretical readings in motivation: Perspectives on human behavior, Chicago etc. 1975, S. 358-379. Zur kritischen Diskussion der Aussagekraft für das Käuferverhalten vgl. Kroeber-Riel, W., Konsumentenverhalten, a.a.O., S. 10 f.; Wiswede, G., Motivation, a.a.0., S. $114 \mathrm{ff} .$. 


\subsection{Wohnstil}

Für die Erklärung der Beweggründe von Antiquitätenkäufern kann der Wohnstil als eine besonders aufschlußreiche Komponente des allgemeinen Lebensstils angesehen werden. Als Gründe hierfür lassen sich nennen:

(1) Kaum ein anderer Lebensbereich dokumentiert die individuelle Persönlichkeit so intensiv und sichtbar nach außen wie das "Wohnen". Antiquitäten tragen als Gebrauchs- oder Dekorationsgegenstände und integraler Bestandteil der Wohnungseinrichtung in besonderem Maße dazu bei.

(2) Hohe Identifikation der Person mit ihrer Wohnumgebung bedeutet, daß der bevorzugte Wohnstil Ausdruck individueller Wünsche, Bedürfnisse und Bestrebungen ist. Von diesem können intensive Beziehungen und Uberschneidungen zur Motivation von Antiquitätenkäufern erwartet werden.

(3) Die steigende Beliebtheit von Antiquitäten ist nicht zuletzt auf die zunehmende Bedeutung der Wohnungseinrichtung im Zuge einer stärkeren Privatisierung und Individualisierung des Konsumenten zurückzuführen 1 .

Abbildung 13 liefert erste Anhaltspunkte über die wohnliche Umgebung der befragten Antiquitätenkäufer.

1 Vgl. hierzu Meffert, H., Marketing in den 8oer Jahren, in: DBW 4o. Jg. (1980), S. $68 \mathrm{ff.;}$ o.V., Verbraucher 1978 - Individualisierungswünsche, in: asw 21. Jg. (1978), Heft 4, S. $38 \mathrm{ff.}$. Gruner + Jahr (Hrsg.), Wohnen und Leben - Marketinggerechte Strukturen der zentralen Lebensbereiche, Hamburg 1978, S. 22; Gruner + Jahr (Hrsg.), Der Wohnmöbelmarkt in der Bundesrepublik Deutschland von 1960 - 1990 - Eine Branchenanalyse, Symposiumsbericht, Hamburg-Saarbrücken 1978, S. 93; Noelle-Neumann, E. , Besitz-Konsum, in: Allensbacher Jahrbuch der Demoskopie, Bd. IV, 1974-1976, S. 234 . 


\begin{tabular}{|c|c|c|c|c|c|c|}
\hline $\begin{array}{l}\text { Ant1quitatenklufer } \\
\text { beze1chnen 1hre Wohn- } \\
\text { umgebung als ....... }\end{array}$ & $\begin{array}{l}\text { Mittel- } \\
\text { wort }\end{array}$ & $\begin{array}{l}(1) \\
\operatorname{tr} 1 f f t \\
\operatorname{sehr} \\
2 u\end{array}$ & $\begin{array}{l}\text { (2) } \\
\text { trifft } \\
\text { ziem- } \\
\text { lich } \\
\text { zu }\end{array}$ & $\begin{array}{l}(3) \\
\text { trifft } \\
\text { etwas } \\
\text { zu }\end{array}$ & $\begin{array}{c}(4) \\
\text { trifft } \\
\substack{\text { kaum } \\
20}\end{array}$ & $\begin{array}{l}(5) \\
\text { trifft } \\
\text { gar } \\
\text { nicht } \\
\text { zu }\end{array}$ \\
\hline nuchtern & 4.4 & 0 & o & 0 & 0 & 0 \\
\hline elegant & 3.5 & 0 & 0 & 0 & 0 & 0 \\
\hline individuell & 1.9 & 0 & $\infty$ & 0 & 0 & 0 \\
\hline dezent & 2.7 & 0 & 0 & 0 & 0 & 0 \\
\hline unkonventionell & 2.8 & 0 & 0 & 0 & 0 & 0 \\
\hline verspielt & 4.0 & 0 & 0 & 은 & 0 & $\circ$ \\
\hline gemultlich & 1.6 & 0 & $\sigma$ & 0 & 0 & 0 \\
\hline praktisch/funktionell & 2.4 & 0 & 0 & 0 & o & 0 \\
\hline exklusiv & 3.7 & 0 & 0 & 0 & 0 & $\circ$ \\
\hline rustikal & 3.9 & 0 & 0 & $\circ$ & 0 & 0 \\
\hline repräsentativ & 3.5 & o & 0 & 0 & 0 & o \\
\hline modern & 3.7 & o & o & 0 & 0 & $\circ$ \\
\hline aufwendig & 3.7 & o & $\circ$ & 0 & o & $\circ$ \\
\hline dekorativ & 2.7 & o & 0 & $\sigma$ & 0 & 0 \\
\hline zeltlos & 2.4 & 0 & & 0 & 0 & $\circ$ \\
\hline schon & 1.8 & 0 & 7 & 0 & 0 & 0 \\
\hline ausgefallen & 3.1 & 0 & 0 & $\infty$ & 0 & 0 \\
\hline
\end{tabular}

Abb. 13: Merkmale der Wohnumgebung von Antiquitätenkäufern

Die empirische Analyse zeigt, daß Antiquitätenkäufer ihr Wohnen in erster Linie als "gemütlich", "schön" und "individuell" bezeichnen.

Wie Tabelle 3 nach einer faktorenanalytischen Verdichtung einzelner Merkmale zu weitgehend unabhängigen Wohnstilkomponenten zeigt ${ }^{1}$, wird damit ein überwiegend von individueller Atmosphäre geprägtes Wohnen beschrieben ${ }^{2}$.

Als strukturell bedeutendste Stilkomponente zur Erklärung der Wohnumgebung von Antiquitätenkäufern kristallisiert sich in der Faktorenanalyse eine nach außen gerichtete (be-

$1 \mathrm{Zu}$ Zielen und Interpretationen von Ergebnissen der Faktorenanalyse vgl. Kapitel B.1.2. Ein gemeinsam durch die 6 extrahierten Faktoren der Tabelle 2 erklärter Varianzanteil von über $45 \&$ an der gesamten Ausgangsvarianz der 17 Einzelmerkmale ist als gut $z u$ beurteilen.

2 Interessant ist die (einzig) stark negative Korrelation (Faktorladung -0,49) zwischen "nüchtern" und der Stilkomponente "Atmosphäre". Kennzeichnend für "Atmosphäre" ist daher das semantische Gegenstück von "nüchtern", also z.B. "warm". 


\begin{tabular}{|c|c|c|c|c|}
\hline $\begin{array}{l}\text { KOMPONENTEN } \\
\text { DES WOHNSTILS }\end{array}$ & $\begin{array}{l}\text { Faktor- } \\
\text { ladungen }\end{array}$ & $\begin{array}{l}\text { relativ } \\
\text { erklarter } \\
\text { Varianz- } \\
\text { anteil }\end{array}$ & $\begin{array}{l}\text { absolut } \\
\text { erklarter } \\
\text { Varianz- } \\
\text { anteil }\end{array}$ & $\begin{array}{l}\text { gesamt } \\
\text { erklarter } \\
\text { Varianz- } \\
\text { anteil }\end{array}$ \\
\hline $\begin{array}{l}\text { DEMONSTRATIV } \\
\text { repräseniativ } \\
\text { exklusiv } \\
\text { elegant } \\
\text { aufwendig } \\
\text { dekorativ }\end{array}$ & $\begin{array}{l}0,83 \\
0,79 \\
0,68 \\
0,63 \\
0,34\end{array}$ & 41,3 & 18,6 & 18,6 \\
\hline $\begin{array}{l}\text { ATMOSPHARE } \\
\text { gemütlich } \\
\text { schön } \\
\text { nüchtern }\end{array}$ & $\begin{array}{r}0,65 \\
0,59 \\
(-) 0,49\end{array}$ & 20,8 & 9,4 & 28,0 \\
\hline $\begin{array}{c}\text { UNAUFFALLIG } \\
\text { dezent } \\
\text { zeitlos }\end{array}$ & $\begin{array}{l}0,67 \\
0,64\end{array}$ & 14,8 & 6,7 & 34.7 \\
\hline $\begin{array}{l}\text { AUTONOM } \\
\text { ausgefallen } \\
\text { verspielt } \\
\text { unkonventionell } \\
\text { individuell }\end{array}$ & $\begin{array}{l}0,79 \\
0,41 \\
0.41 \\
0,33\end{array}$ & 10,6 & 4,8 & 39,5 \\
\hline $\begin{array}{l}\text { SACHLICH } \\
\text { modern } \\
\text { praktisch/ } \\
\text { funktionell }\end{array}$ & $\begin{array}{l}0.64 \\
0.57\end{array}$ & 7,6 & 3,4 & 42,9 \\
\hline$\frac{\text { RUSTIKAL }}{\text { rustikal }}$ & 0.29 & 5,0 & 2,3 & 45,2 \\
\hline & & 100,08 & 45,28 & 45,28 \\
\hline
\end{tabular}

Tab. 3: Wohnstile von Antiquitätenkäufern Ergebnisüberblick einer Faktorenanalyse

wußte oder unbewußte) Demonstration heraus. Ein demonstrativer Wohnstil mit den Attributen "repräsentativ", "exklusiv", "elegant", "aufwendig" und "dekorativ" Iäßt eine hohe Instrumentalität des Wohnens bei Antiquitätenkäufern vermuten. Es erscheint plausibel, daß auch die Motivation des Kaufs von Antiquitäten durch die sozialen Demonstrationseffekte des Wohnstils in gleicher Richtung beeinflußt wird. 
Von relativ hoher (faktorenanalytischer) Bedeutung unter den ermittelten Wohnstilen ist andererseits die Unauffälligkeit der Wohnungseinrichtung von Antiquitätenkäufern. "Dezent" und "zeitlos" als Beschreibungsmerkmale kennzeichnen Einschätzungen, die sich auf eine "natürliche" bzw. "zurückhaltende" Eingliederung von Antiquitäten in die gesamte Wohnumgebung beziehen. Antiquitäten eignen sich aus der Sicht ihrer Käufer hierzu besonders, da sie ebenfallsals "dezent", "zeitlos" und als "zu jeder Einrichtung passend" gesehen werden.

Eine bewußte Autonomie des Einrichtungsstils ist dagegen eher Ausdruck einer gegenteiligen Wohnauffassung. "Ausgefallen", "verspielt", "unkonventionell" und "individuell" sind Beschreibungsmerkmale, denen eine Unabhängigkeit und Selbständigkeit gegenüber äußeren Einflüssen bei der Gestaltung der Wohneinrichtung gemeinsam ist. Insbesondere unterschiedlich ausgeprägte Autonomievorstellungen innerhalb des Wohnstils werden vermutlich mit bestimmten Motivationsinhalten und -intensitäten bei Antiquitätenkäufern korrelieren; z.B. ist die Verwirklichung einer "Individualität" im Wohnbereich durch den Kauf von Antiquitäten relativ leicht erreichbar.

Etwas geringere Bedeutung als eigenständiger Wohnstil von Antiquitätenkäufern besitzt die Dimension Sachlichkeit.

Sachlichkeit wird dokumentiert durch "moderne" und "praktisch/ funktionelle" Wohnungseinrichtungen: Antiquitäten können dabei entweder als bewußte stilistische Kontrastierung zu ansonsten modernen Einrichtungen oder auch als (immer noch) funktionelle Gebrauchsgegenstände dienen.

Von nur untergeordneter Bedeutung zur Stilbeschreibung des Wohnens von Antiquitätenkäufern ist abschließend die Dimension "rustikal". Aber auch von dieser stilkomponente sind, wie in Kapitel C. näher untersucht wird, korrelative Zusammenhänge zur Kaufmotivation zu erwarten. 


\subsection{Wahrgenommene Instrumentalität und Leitbild}

Weiteres psychographisches Merkmal der Antiquitätenkäufer ist ihr Vorstellungsbild von der Eignung eines Antiquitätenkaufs bzw. -besitzes zur Erreichung angestrebter Verhaltensziele und Motivationen.

"That is, there is cognitive association between a specific product class or the buying of a certain product from a set of product classes, and the expectation that the product contributes to the attainment of a goal or the satisfaction of a motivational dimension" ${ }^{1}$. Eine derart wahrgenommene Instrumentalität ${ }^{2}$ von Antiquitäten zur Motivbefriedigung ist - der ïberwiegenden Anzahl kognitiver Motivationsmodelle folgend ${ }^{3}$ - als wichtige psychische Einflußgröße der Käufermotivation anzusehen.

Ausgangspunkt der Entstehung und Beurteilung wahrgenommener Instrumentalitäten sind nicht nur Warenmerkmale, sondern ebenso das durch Attribuierung entstehende Vorstellungsbild von Merkmalen der Antiquitätenkäufer als typisierte Gruppe (Käuferbild bzw. -image).

1 v. Raaij, F., Wandwossen, K., Motivatıon - Need Theories..., a.a.0., s. 16 .

2 Die wahrgenommene Instrumentalität (perceived instrumentality) eines Produktes zur Motivbefriedigung ist neben der Wichtigkeit des Motivs für den Käufer (value importance) zweite Modellkomponente einer multiattributiven Einstellungsmessung nach ROSENBERG. Im Vordergrund steht dabei die Zweck-Mittel-Analyse (means-end-analysis), ohne daB auf differenzierte Eigenschaften und Merkmale des Einstellungsobjektes eingegangen wird. Enge Bezüge zwischen Motivations- und Einstellungsforschung werden hier besonders deutlich. Vgl. Rosenberg, M.J., Cognitive Structure and Attitudinal Affect, in: JoASP, Vol. 53 (1956), S. 367-372; derselbe, A Structural Theory of Attitude Dynamics, in: POQ, Vol. 24 (1960), S. 319-340; Bass, F.M., Wilkie, W.L., A Comparative Analysis of Attitudinal Predictions of Brand Preference, in: JoMR, Vol. 10 (1973), S. 262 ff..

$3 \mathrm{Vgl}$. Atkinson, J.W., Einführung in die Motivationsforschung, Stuttgart 1975, S. 441; v. Raaij, F., Wandwossen, K., Motivation - Need Theories .... a.a.O., S. 11. 
Solche generalisierten Vorstellungsbilder von "dem Käufer" können $z u$ abstrakten Leitbildern des individuellen Käuferverhaltens werden ${ }^{1}$. Voraussetzung dazu ist eine möglichst weitgehende Identifikation des einzelnen Käufers mit den (gedanklich vorgestellten) Leitbildeigenschaften ${ }^{2}$.

Zwei EinfluBrichtungen einer Leitbildidentifikation sind denkbar: entweder möchte der Käufer"auch so sein und wirken" wie das attraktiv erscheinende Leitbild, oder er möchte "nicht so sein und wirken" wie das als unattraktiv erscheinende Leitbild. Veränderungen bestehender Motivationen können dabei von nur geringen Modifikationen einzelner Motivinhalte und -ausprägungen bis zur geschlossenen Ubernahme bzw. "Aussonderung" vollständiger Motivstrukturen reichen.

Abbildung 14 zeigt die empirisch ermittelten Vorstellungsbilder der Käufer in ihrer durchschnittlichen Ausprägung. Auf ihre vollständige Kommentierung wird an dieser stelle zugunsten der späteren Motivationsanalyse (vgl. Kapitel C.) und aufgrund ihres hohen "Selbsterklärungswertes" verzichtet.

Hervorzuheben ist jedoch die ausgeprägte Zustimmung der Befragten $z u$ einer statusbezogenen Instrumentalität von Antiquitäten. Die zum Ausdruck kommende Bedeutung von Statusund Repräsentationsmotiven eines Antiquitätenkaufs kann vermutlich durch eine "selbstbekennende" Fragestellung ("Ich kaufe, weil ...") nicht in dieser Intensität offengelegt werden ${ }^{3}$.

1 Hohe Korrespondenz zwischen Warenmerkmalen und Leitbildmerkmalen macht eine empirisch trennscharfe operationalisierung nicht immer möglich.

2 Vgl. Wiswede, G., Motivation und Verbraucherverhalten, a.a.0., S. $178 \mathrm{ff.}$.

3 Unterstützt wird die Indikatorfunktion, da die zustimmung $z u$ einer Instrumentalität bzw. zu einem Leitbildmerkmal eine "unverbindliche" Projektion eigener Motivationsinhalte in die Antwort ermöglicht. 
Antiquititen sind eine ebenso sichere Geldanlage we ein Sparbuch

Antiquitaten sind genauso spekulationsobjekte we unsichere Aktien

Antiquitaten dienen vielen Menschen ebenso wie nauser, Autos oder teurer schmuck als Statussymbol

Antiquitatenkăufer sind ein ganz bestimmter Typ von Menschen

Antiquitaten verleihen Ihrem Besitzer eine individuelle Note

Antiquitaten demonstrieren elnen kultivierten Lebensst 11 Antiquităten sind Ausdruck eines Kunstverstandisses ihres Besitzers

Antiquitaten erinnern die Menschen an die gute alte zelt

Antiquitaten passen zu Jeder Einrichtung

Mit Antiquitgten kann wan sich whltuend von einer "norealen" Wohnungseinrichtung abheben

Menschen, die sich teure Antqiultaten leisten können, sind su beneiden

Es gibt viele Antlquitstenkaufer, die ohne Sachver-

stand kaufen, aber alt lhren Antiquitaten angeben

$\begin{array}{ccccc}\text { (1) } & \text { (2) } & \text { (3) } & \text { (4) } & \text { (5) } \\ \text { trifft } & \text { trifft } & \text { trifft } & \text { trifft } & \text { trifft } \\ \text { gehr } & \text { ziemlich } & \text { etwas } & \text { kaum } & \text { gar nicht } \\ \text { zu } & z u & z u & z u & z u\end{array}$

zu

1)

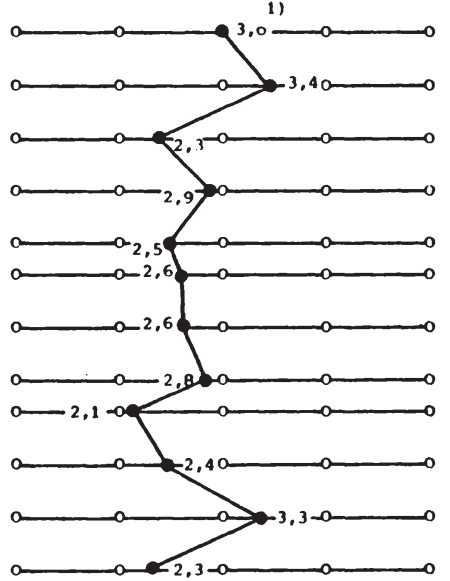

1) Mittelwert

Abb. 14: Wahrgenommene Instrumentalitäten und Leitbildmerkmale - Vorstellungsbilder privater Käufer

\subsection{Interessenentstehung}

AbschlieBend ist im Rahmen der Diskussion psychographischer Merkmale auf die historische Anamnese der Interessenentstehung für Antiquitäten einzugehen. "Interesse" ist als eine mentale und thematisch ausgerichtete Gedankenhaltung der Käufer zu verstehen, der in der Literatur ein (nicht unbestrittener) Stellenwert im KaufentscheidungsprozeB des Konsumenten zugewiesen wird ${ }^{1}$.

1 Zum "Interesse" als Stadium der Kaufbereitschaft von Konsumenten vgl. die Darstellungen bei Kotler, Ph., Marketing-Management, a.a.O., S. 618 ff.; Schulz, R., Kaufentscheidungsprozesse, a.a.O., S. $43 \mathrm{ff.;}$ Meffert, H., Marketing, a.a.O., S. 422 und S. 138; Hoepfner, F.G., Verbraucherverhalten, a.a.0., S. 55 ff.. 
Die Interessenentstehung durch soziale Interaktion (z.B. in Bezugsgruppen wie der Familie), durch die individuelle Persönlichkeitsentwicklung des Käufers oder durch situationsbedingte Umwelteinflüsse kann zugleich prägender Ausgangspunkt des "Erlernens" und "Entstehens" von Motivationsinhalten des Antiquitätenkäufers sein ${ }^{1}$.

Abbildung 15 zeigt die wichtigsten ermittelten Ausgangspunkte und Rahmenbedingungen der Interessenentstehung bei den Befragten im Überblick.

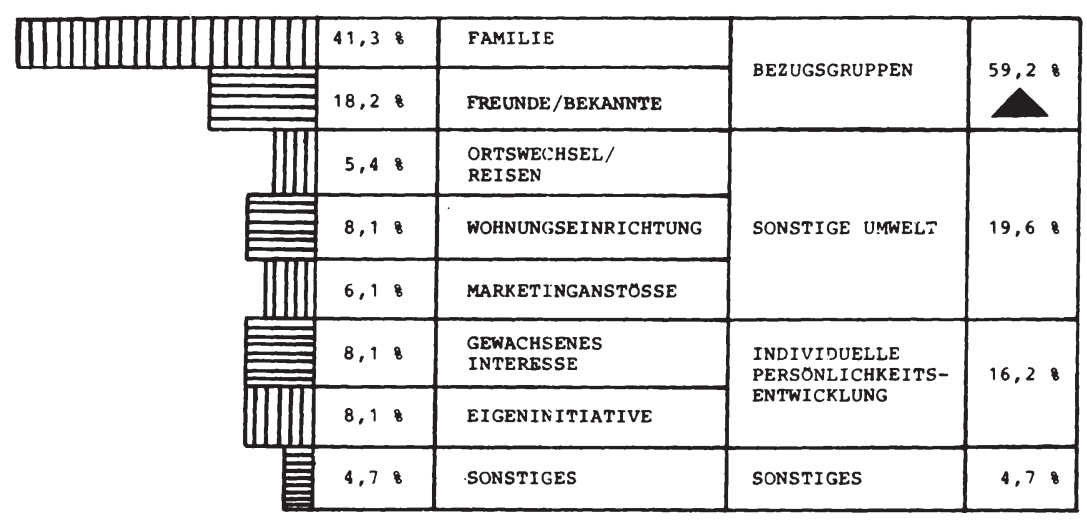

Abb. 15: Ausgangspunkte der Interessenentstehung für Antiquitäten

Die Interessenweckung an Antiquitäten findet zu einem erwartungsgemä $B$ hohen Prozentsatz von 59,5 \% in engeren Be-

1 Vgl. beispielhaft Wiswede, G., Motivation und Verbraucherverhalten, a.a.O., S. 175 f.; Wiswede, G., Rationalität und soziales Wertsystem, in: zfWirtsch.u.Soz.Wiss. $\mathrm{Nr} .4(1972)$, S. $67 \mathrm{ff} .$. 
zugs- bzw. Referenzgruppen ${ }^{1}$ des Antiquitätenkäufers statt. Eine herausragende stellung unter ihnen nimmt die Familie $(41,3 \%)$ ein. Das Elternhaus gibt mittelbar oder unmittelbar den ersten Anstoß für die Auseinandersetzung mit Antiquitäten. Es ist $z u$ vermuten, daß dabei komparative Maßstäbe und Normen für Empfinden, Beurteilung, Motivation und Verhalten gegenüber Antiquitäten vermittelt werden ${ }^{2}$.

Gleiches gilt für die zumeist intensiven und tendenziell zeitlich stabilen Interaktions- und Kommunikationsbeziehungen zu Freunden/Bekannten, die zu 18,2 \& als Ausgangspunkt des Interesses genannt wurden. Wegen seiner in der Regel hohen subjektiven Kompetenz- und Vertrauensgrundlage läßt sich ein derartiger Einfluß recht zutreffend mit einem Meinungsführungs- bzw. Meinungsfolgschaftsverhältnis umschreiben ${ }^{3}$.

Von insgesamt etwas geringerer Bedeutung sind sonstige Umweltbedingungen mit einer Nennungshäufigkeit von 19,6 \%

1 Zur Verhaltenswirksamkeit von Bezugs- und Referenzgruppen vgl. u.a. Hofstätter, P.R., Gruppendynamik, Beitrag in: Herrmann, T. et al., Handbuch .... a.a.O., S. 187; Festinger, L., A Theory of Social Comparison Processes, in: HR Vol. 7 (1954), S. 117-140; Hillmann, K.H., Soziale Bestimmungsgründe des Konsumentenverhaltens, stuttgart 1971, S. $50 \mathrm{ff}$. und S. $98 \mathrm{ff}$..

2 Die Ubernahme komparativer Maßstäbe wird oft im Zusammenhang mit gleichen oder ähnlichen Bewertungen von Produkten diskutiert. Für Antiquitäten ist die Ubernahmewahrscheinlichkeit von Maßstäben der Bezugsgruppen vermutlich besonders ausgeprägt, da objektive ("non-social") Vergleichsmaßstäbe ( $z . B$. objektive Qualität) für ihre Bewertung fehlen. Vgl. dazu die Wiedergabe der Hypothesen von Festinger, in: Kroeber-Riel, W., Konsumentenverhalten, a.a.O., S. 442 und die dort angegebene Literatur.

3 Zum Konzept der Meinungsführung vgl. Hummrich, U., Interpersonelle Kommunikation im Konsumgütermarketing. Erklärungsansätze und Steuerungsmöglichkeiten, in: Meffert, H. (Hrsg.), Schriftenreihe Unternehmensführung und Marketing, Bd. 8, Wiesbaden 1976, S. $42 \mathrm{ff}$. ; Markin, R.J. (jr.), Consumer Behavior, a.a.O., S. $322 \mathrm{ff}$. und S. $533 \mathrm{ff} .$. 
Von insgesamt etwas geringerer Bedeutung sind sonstige Umweltbedingungen mit einer Nennungshäufigkeit von $19.6 \%$.

Interessant ist, daB dabei nur $6,1 \%$ der Befragten absatzpolitische Aktivitäten des Antiquitätenhandels (Messen, Kataloge, Beratung, Schaufenster o.ä.) als Ursache der Interessenentstehung angeben. Diese Tatsache verweist auf eine nur begrenzte Reichweite des bisherigen Antiquitäten-Marketing bei der Akquisition unausgeschöpfter Käuferpotentiale. Als situativer AnstoB wird ferner zu 8,1 \& ein bestehendes Einrichtungsproblem genannt, wobei enge Bezüge zu Einrichtungs-, Gebrauchs- und Dekorationsmotiven eines Antiquitätenkaufs offensichtlich sind.

Abschließend glauben in ihrer eigenen Persönlichkeitsentwicklung weitere 16,2 \& den Ursprung ihres Interesses für Antiquitäten $z u$ sehen. Obwohl tiefer gehende inhaltliche Spezifizierungen nicht möglich sind ${ }^{1}$, liegt hierin ein Hinweis auf die allgemeine Bedeutung psychischer Dispositionen für die Motivation von Antiquitätenkäufern.

\subsection{Verhaltensmerkmale}

Die empirische Bestandsaufnahme von ausgewählten Aspekten des Entscheidungsverhaltens, des Informationsverhaltens und der Einkaufsstättenwahl dient als Informationsgrundlage der späteren Analyse motivationaler Erklärungsbeiträce zu den genannten Verhaltensaspekten. Beispielhaft aufgeführte Annahmer über Einflüsse der Käufermotivation auf spezifische Verhaltensmerkmale von Antiquitätenkëufern sind als explorativ begründete Orientierungshypothesen $z u$ verstehen.

1 Typische Antworten waren "aus eigenem Antrieb", "aus persc̈nlicher Neigung", "durch das Alter gewachsen" etc.. 


\subsection{Entscheidungsverhalten}

Das Entscheidungsverhalten von Antiquitätenkäufern läßt sich aus (eher) konativer Sicht durch eine Klassifikation unterschiedlicher Entscheidungstypen und aus (eher) kognitiver Sicht durch unterschiedliche, den Kaufentscheidungsprozeß begleitende Gedankenhaltungen der agierenden Käufer kennzeichnen.

\subsection{Typen und Träger der Kaufentscheidung}

In der Literatur zum Käuferverhalten werden zahlreiche Typologien des Entscheidungsverhaltens von Konsumenten vorgestellt, die sich weitgehend in Art, Anzahl, Generalisierungsgrad und Begrifflichkeit der typenbildenden Variablen unterscheiden ${ }^{1}$. Inhaltliche schnittmengen ergeben sich in einem besonderen Interesse für alternative Entscheidungsstile bei der Produktwahl bezogen auf Extensität und Kollektivität des Kaufentscheidungsprozesses.

Je extensiver Antiquitätenkäufer ihre Kaufentscheidung treffen, d.h. je mehr Prozeßphasen (z.B. Erleben eines Bedürfnisses, Alternativensuche, Alternativenbewertung, Kaufentscheidung, Bewertung der Entscheidungsfolgen) durchlaufen werden, und je umfangreicher die kaufbegleitenden Informationsgewinnungs - und -verarbeitungsaktivitäten ausfallen, um so mehr kann von einem planvollen, intellektuell kontrollierten Problenlösungsverhalten der Antiquitätenkäufer gesprochen werden . Je "verkürzter" die Entscheidungs-

1 Vgl. u.a. Ruhfus, R., Kaufentscheidungen, a.a.o., S. 20 ff.; Schulz, R., Kaufentscheidungsprozesse, a.a.O., S. 30 ff.; Wiswede, G., Motivation und Verbraucherverhalten, a.a.o., S. $108 \mathrm{ff.;}$ Raffée, H., Konsumenteninformation und Beschaffungsentscheidung des privaten Haushalts, Stuttgart 1969, S. $47 \mathrm{ff.;}$ Katona, G., Das Verhalten der Verbraucher und Unternehmer, Tübingen 1960, S. 57 f.; Howard, J.A., Consumer Behavior: Application of Theory, New York etc. 1977. S. $10 \mathrm{f}$. 
und je"eingeschränkter" die Informationsprozesse, um so eher ist die Produktwahl als ungeplantes reizkontrolliertes Impulsverhalten $z u$ typisieren ${ }^{1}$. Ferner ist als weitere Variante eines "begrenzten" Problemlösungsverhaltens ein habitueller, gewohnheitsmäßiger und damit "routinisierter" Antiquitätenkauf zumindest denkbar.

Der Grad an Kollektivität einer Entscheidungsfindung kann durch ein Kontinuum zwischen den Extremen eines überwiegend intraindividuell (autonomen) oder überwiegend kollektiven (interaktiven) Entscheidungsstils gekennzeichnet werden.

Als Determinanten alternativer Entscheidungsstile von Antiquitätenkäufern sind eine Vielzahl produkt-, situationsund personenbezogener Variablen relevant ${ }^{2}$. Auch für diese ergeben sich - ebenso wie für die Bestimmung und Abgrenzung der Entscheidungsstile - umfangreiche Probleme einer überschneidungsfreien Klassifikation und empirischen Operationalisierung.

Als besonders "griffig" im Hinblick auf die Ableitung von Tendenzhypothesen über mögliche Ausprägungen von Entscheidungsstilen werden produkt- bzw. warenbezogene Einflußgrößen angesehen. Nutzt man vorhandene Typologien ${ }^{3}$ als Denkraster, sollte bei Antiquitätenkäufern ein überwiegend planvolles und kollektives Entscheidungsverhalten anzutreffen sein. Hierfür sprechen im einzelnen

1 Vgl. u.a. Meffert, H., Marketing, a.a.O., S. $111 \mathrm{ff.;}$ Dahlhoff, H.-D., Ungeplantes und impulsives Kaufverhalten, Beitrag in: Meffert, H., Steffenhagen, H., Freter, H. (Hrsg.), Konsumentenverhalten und Information, Wiesbaden 1979 , S. $313 \mathrm{ff}$. und die Literaturangaben S. $330 \mathrm{f}$.; Kollat, D.T., Willett, R.P., Customer Impulse Purchasing Behavior, in: JoMR, Vol. 4 (February 1967), S. $21 \mathrm{ff.;}$ dieselben, Is Impulse Purchasing Really a Useful Concept for Marketing Decisions, in: JoM, Vol. 33, S. 79 ff..

2 Vgl. u.a. Kroeber-Riel, W., Konsumentenverhalten, a.a.o., S. $313 \mathrm{ff.;}$ Engel, J.F., Kollat, D.T., Blackwell, R.D., Consumer Behavior, New York 1968, S. 355 ff.; Markin, R.J. (jr.), Consumer Behavior ..., a.a.o., s. 501 f..

$3 \mathrm{Vgl}$. insbesondere Ruhfus, R., Kaufentscheidungen ..., a.a.o., S. $22 \mathrm{ff}$.. 
(1) die geringe Vergleichbarkeit der Auswahlalternativen (Einmaligkeit, Heterogenität, Individualität etc.);

(2) die ausgeprägte Informationsbedỉrftigkeit (künstlerische und kulturhistorische Beurteilung, Qualitäts-, Fälschungs- und Wertrisiko etc.);

(3) die hohen Anforderungen an Suchaktivitäten der Käufer lobjektbezogene und geographische Seltenheit, Raritä.t etc.);

(4) die ausgeprägte soziale Bedeutung und Sichtbarkeit (Status- und Prestigerelevanz, soziales Risiko etc.);

(5) die hohen Anforderungen an den zeitlichen Planungshorizont des Käufers (tradierte und langlebige Gebrauchsgüter, langfristige Geschmacksfestlegung und Kapitalbindung etc.);

(6) die geringe Kaufhäufigkeit (Luxusartikel, "aufschiebbarer" Konsum) und begrenzte Möglichkeit zur Erfahrungsbildung bezogen auf den KaufentscheidungsprozeB (Einzelkäufe, Unikate et . );

(7) die hohe "Betroffenheit" mehrerer Baushalts- bzw. Familienmitglieder sowohl im Rahmen der Verteilung des verfügbaren Haushaltsbudgets als auch im Hinblick auf "geschmackliche" Differenzen beim Wohnen mit Antiquitäten.

Im Gegensatz zu der "theoretisch" erwartbaren (durchschnittlichen) Ausprägung des antiquitätenspezifischen Entscheidungsstils stehen die ermittelten empirischen Ergebnisse, die in sbbildung 16 wiedergegeben sind.

Impulsiver Kaufentscheid

Ich entschließe mich meist spontan zum Kauf, wenn ich ein zusagendes Stück finde

ungeplanter Kaufentscheid

Auch wenn ich eigentlich nicht die Absicht hatte,

:- twas zu kaufen, kommt es schon vor, daß ich

einc Antiquität mit nach liause bringe

Geplanter Kaufentscheid

Ich plane einen Antiquitätenkauf ganz gezielt und informiere $\mathrm{mich}$ vorher gewissenhaft

Individueller Kaufentscheid

it: cntscheide in der Regel allein, wenn elne nntiquität angeschafft werden sol?

äms liarer Kaufentscheid

Eine Antiquität wird nur dann gekauft, wenn

auch die anderen Familienmitglieder einver-

standen sind

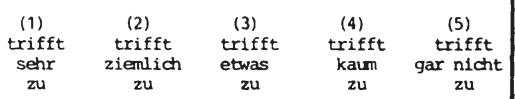

1)

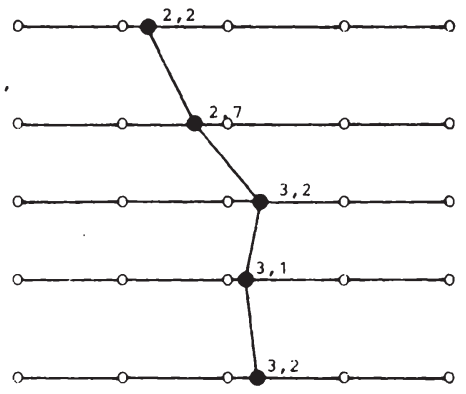

1) Mittelwert

Abb. 16: Entscheidungstypen beim Antiquitätenkauf 
Impulsives Entscheidungsverhalten ist als Kaufentscheidungsstil unter den Antiquitätenkäufern eindeutig am weitesten verbreitet $^{1}$. Im Sinne der empirischen operationalisierung beinhaltet der impulsive Kaufentscheid eine spontan ablaufende Verhaltensreaktion ${ }^{2}$. Folgt man der Ansicht, daß "ein Kauf ... impulsmäßig (ist), bei dem eine starke, häufig monothematische Motivation ohne Uberlegung auf offenes Kaufverhalten durchschlägt, ohne daß eine Kaufgewohnheit im Spiel war" ${ }^{3}$, liegt die Vermutung motivationaler Erklärungsbeiträge als "Korrektiv" zu rein warenbezogenen Erklärungsbeiträgen nahe (vgl. Kapitel C.). Letztendlich macht daher wohl erst das Zusammentreffen eines sehr intensiven motivationalen Bedürfnisses mit der Wahrnehmung einer als (zur Motivationsbefriedigung) äuBerst attraktiv empfundenen Antiquität den hohen Anteil von Impulskäufen verständlich.

Ungeplante Käufe haben mit Impulskäufen die fehlende Kaufabsicht gemeinsam. Im Gegensatz zum Impulskauf wird jedoch

1 Dem Mittelwert von 2,2 entspricht ein Anteil von 68 o der Käufer, die ein impulsives Kaufverhalten als "sehr oder ziemlich zutreffende" Beschrelbung für das eigene Verhalten einschätzen. Diese generelle Selbsteinschätzung ist für die jeweilig konkrete, d.h. orts- und zeitgebundene Verhaltenssituation $z u$ relativieren. Bezogen auf die der empirischen Erhebung zugrunde liegenden Messen und Märkte (vgl. die Beschreibung des Untersuchungsdesigns im Anhang), äuBerten nur ca. 7 \& eine bestehende Kaufabsicht vor dem Besuch, wobei schlieblich ca. 228, d.h. fast jeder 4 . Käufer, tatsächlich ein Stück erwarb.

2 Eine sehr ähnliche Operationalisierung findet sich in den Brigitte Frauen-Typologien (1973, 1975 und 1977) und bei Gröne und Dahlhoff (1977). In keinem Fall konnte ein annähernd hoher Prozentsatz von Impulskäufen ermittelt werden. Zu weiteren Detalls (Skalierung, Befragtenkreis, Einzeldaten etc.) vgl. beispielhaft o.V., Frauen-Typologie 1, Hrsg.: Brigitte, Gruner + Jahr AG \& Co, Hamburg 1973, S. 88 ; Gröne, A., Dahlhoff, H.-D., Das Käuferverhalten einer Stadtbevölkerung, in: Projektarbeit des Instituts für Marketing der Universität Münster, Meffert, H. (Hrsg.), Münster 1977 , S. 37.

3 Hoepfner, F.G., Beeinflussung des Verbraucherverhaltens, München 1975, S. 221. 
nicht ausgeschlossen, daß der Antiquitätenkäufer "vor Ort" (am point of purchase) einen - wenn auch begrenzten - Kaufentscheidungsprozeß durchläuft. In diesem Sinne wird oft von sogenannten "vereinfachten Entscheidungsprozessen" gesprochen, welche auf der Grundlage einer mittelgroßen Informationsmenge und einer mittleren Entscheidungsgeschwindigkeit getroffen werden ${ }^{1}$. Die empirische operationalisierung ungeplanter Antiquitätenkäufe zielt auf die Erfassung tatsächlich vollzogener Kaufakte, ohne daß eine Motivation bzw. eine Absicht vor dem Kauf erkannt wurde ${ }^{2}$. Auch der ungeplante Kauf besilzt als Entscheidungsstil der Antiquitätenkäufer eine unerwartet hohe Bedeutung. Als Erklärung bieten sich folgende hypothetische Überlegungen an:

(1) Die Wahrnehmung eines Entscheidungsdrucks führt über die Forcierung einer Motivationsaktualisierung vermutlich auch $\mathrm{zu}$ einer Beschleunigung des Kaufentscheidungsprozesses.

(2) Unter der Annahme einer starken Motivationsbedingtheit des Antiquitätenkaufes können insbesondere emotional-affektiv ausgerichtete Motivationsinhalte ungeplante Kaufhandlungen unterstützen.

(3) Ein insgesamt hohes und breit gefächertes "motivationales Engagement" prädisponiert Antiquitätenkäufer zu einer ausgeprägten aber zugleich unbewußt bleibenden Kaufbereitschaft. Trifft der Käufer in dieser Ausgangslage auf eine Antiquität, die sich in ihrem Erscheinungsbild weitgehend mit seiner motivational bedingten Produktanforderung deckt, wird ein affektiver, ungeplanter Kaufentscheid wahrscheinlich.

1 Die Operationalisierung bezieht sich auf einen Vorschlag von Kollat und Willett, die zugleich eine übersicht über alternative Vorgehensweisen im Rahmen einer "Kaufabsichts-Kaufergebnismatrix" geben. Vgl. Kollat, D.T., Willet, R.P., Customer..., a.a.O., S.

2 Als "Limited Problem-Solving" bezeichnet vgl. Markin, R.J. (jr.), Consumer Behavior, a.a.O., S. 499;

Howard, J.A., a.a.0., S. 10. 
(4) Einzelne Motivationsinhalte, wie z.B. bestehende familiäre Traditionen oder auch soziale Beweggründe eines Antiquitätenkaufs, beinhalten in ihrer (lerntheoretischen) Genese Tendenzen zur Habitualisierung und Gewohnheitsbildung im Kaufentscheidungsverhalten.

(5) Aufgrund des Unikatcharakters von Antiquitäten kann sich der Kaufentscheid in eine langfristige gedankliche "Vorplanung" bezogen auf die produktgruppe und einen als ungeplant und kurzfristig zu aktualisierenden Kaufentscheid bezogen auf das Einzelstück aufspalten. So wird z.B. ein Sammler, der bereits seit längerem den Kauf einer bestimmten Antiquität zur Abrundung seiner Sammlung plant, einen relativ schnellen und affektiven Kaufentscheid vollziehen, wenn ihm das gesuchte stück tatsächlich begegnet. Ein solches Verhalten steht zudem im Einklang mit motivationstheoretischen Uberlegungen, nach denen die Stärke (Intensität) einer Motivation in Abhängigkeit von der Zeitdauer der Motivationsdeprivation (Nichtbefriedigung, Unterdrückung) und damit schließlich auch die latente Handlungstendenz zunimmt ${ }^{1}$.

(6) Nicht zuletzt schafft der hohe Einkommensstandard von Antiquitätenkäufern die Voraussetzung, impulsive und ungeplante Käufe relativ "unbedenklich" realisieren zu können.

Geplante Käufe, die einen bewußten, kognitiv kontrollierten Entscheidungsstil auf der Grundlage extensiver Informationsaktivitäten widerspiegeln, besitzen empirisch die geringste Bedeutung. Im wesentlichen sprechen die genannten Argumente für die hohe Bedeutung ungeplanter und impulsiver Kaufentscheidungen zugleich gegen diejenige von geplanten Käufen.

1 Uber den funktionalen Verlauf des zusammenhangs bestehen unterschiedliche Auffassungen. "The other is that drive theorists have usually assumed a monotonic relationship between deprivation (or other drive-inducing operations) and drive level, and this suggests that behavior would be a monotonic function of drive level ... rather than be related to drive level by the inverted u-shaped function stresses so much by the activation theorists". Cofer, C.N., Appley, M.H., Motivation: Theory and Research, Sixth Printing, New York etc. 1968, S. 397. 
Der Grad an Kollektivität der Entscheidungsfindung bei Antiquitäten stellt sich in der Befragung in einem fast ausgeglichenen Verhältnis von individuell und familiär getroffenen Kaufentscheidungen dar. Der Anteil individueller Kaufentscheidungen liegt damit relativ hoch'.

In ihrer Gesamttendenz weisen die Diskrepanzen zwischen warenspezifisch erwarteter und empirisch ermittelter Entscheidungsfindung auf einen starken Einfluß intrapersoneller Bestimmungsgrößen bei der Auswahlentscheidung für Antiquitäten hin. Die tberproportionalität von spontaner und individueller Entscheidungsfindung hebt aus inhaltlicher sicht die Bedeutung der Käufermotivation als hypothetische Einflußvariable besonders hervor.

\subsection{Kaufbegleitende Kognitionen}

Kaufentscheidungsprozesse von Antiquitätenkäufern werden von unterschiedlichsten Kognitionen wie z.B. subjektivem Wissen, Wahrnehmungen, Bereitschaften und Anforderungshaltungen begleitet. Einerseits beeinflussen solche kaufbegleitende Kognitionen als Entscheidungsprämissen das faktische Verhalten der Käufer und sind deshalb - ebenso wie motivationale Verhaltenserklärungen - zu den partialanalytischen Erklärungsansätzen des Konsumentenverhaltens zu zählen.

1 Ein Vergleich dieses Ergebnisses mit anderen empirischen Untersuchungen ist nur begrenzt möglich. Eine Studie von Dahlhoff unterstützt insofern die Vermutung eines überdurchschnittlich hohen Anteils individueller Kaufentscheidungen bei Antiquitäten, indem er für Möbel die vom Warentyp Antiquitäten ähnlich sind - einen fast ausschließlich kollektiv (familiär) getroffenen Kaufentscheid ermittelt. Vgl. Dahlhoff, H.-D., Kaufentscheidungsprozesse, a.a.O., S. $190 \mathrm{ff}$. und S. $198 \mathrm{ff.}$ 
Andererseits ist anzunehmen, daß die kaufbegleitenden Kognitionen ihrerseits durch Motivationsinhalte und -konfigurationen beeinflußt und geprägt werden.

Die folgende Diskussion behandelt das Involvement von Antiquitätenkäufern, kognitive Konflikte und die Preisbereitschaft beim Kauf von Antiquitäten als sachlich abgrenzbare Teilbereiche.

\subsection{Involvement}

Das Verhaltenskonstrukt "Involvement" wird in der Literatur oft zur Umschreibung eines "inneren Engagements" oder auch einer "Ich-Beteiligung" von Konsumenten benutzt". Nach vorherrschender Meinung besitzt das Involvement neben affektiven und konativen zugleich stets kognitive Bestandteile bzw. Entwicklungsstadien ${ }^{2}$. Die kognitive Involvementkomponente umfaßt dabei Meinungen und Anschauungen der Antiquitätenkäufer, welche aus einer gedanklichen Beschäftigung mit Antiquitäten resultieren und als subjektives Wissen "gedanklich präsent" sind.

Die Ursache bzw. der Bezugspunkt der Entstehung eines Involvement kann unterschiedlich gesehen werden: entweder bezieht es sich auf eine spezifische Situation und ihre Fähigkeit, den Käufer zu engagieren (situation involvement), oder es wird als eine generelle Eigenschaft (Disposition) des Käufers verstanden, welche Ausdruck seiner momentanen

1 Vgl. Kroeber-Riel, W., Konsumentenverhalten, a.a.O., S.315.

2 Vgl. Rothschild, M.L., Involvement as a Determinant of Decision Making Styles, in: AMA Combined Proceedings 1975, Mazze, E.M. (ed.), S. 216; Cofer, C.N., Appley, M.H., a.a.O., s. $784 \mathrm{ff.}$; Bruhn, M., a.a.0., S. $67 \mathrm{f}$. und die dort genannte Literatur. 
und früheren kognitiven "Erfahrungen" ist (enduring involvement) ${ }^{1}$.

Der Versuch, das Involvement von Antiquitätenkäufern empirisch zu erfassen, orientiert sich an der letzteren Interpretation.

Dabei kann davon ausgegangen werden, daB ein derartiges Antiquitäten-Involvement mittelbar oder unmittelbar auf den Erfahrungen der Käufer mit ihrem bisherigen Antiquitätenbesitz basiert. Im Vordergrund der empirischen Analyse stand somit die Fragestellung, wie stark den Antiquitätenbesitzern eine "innere " Beziehung zu ihren Antiquitäten mental bewuBt ist. Als "Gradmesser" wurde dabei eine unterschiedlich starke Besitzbindung und -identifikation ("Besitzerstolz") der empirischen Operationalisierung zugrunde gelegt. Abbildung 17 gibt einen Uberblick uber die benutzten Fragestellungen und die ermittelten Durchschnittswerte.

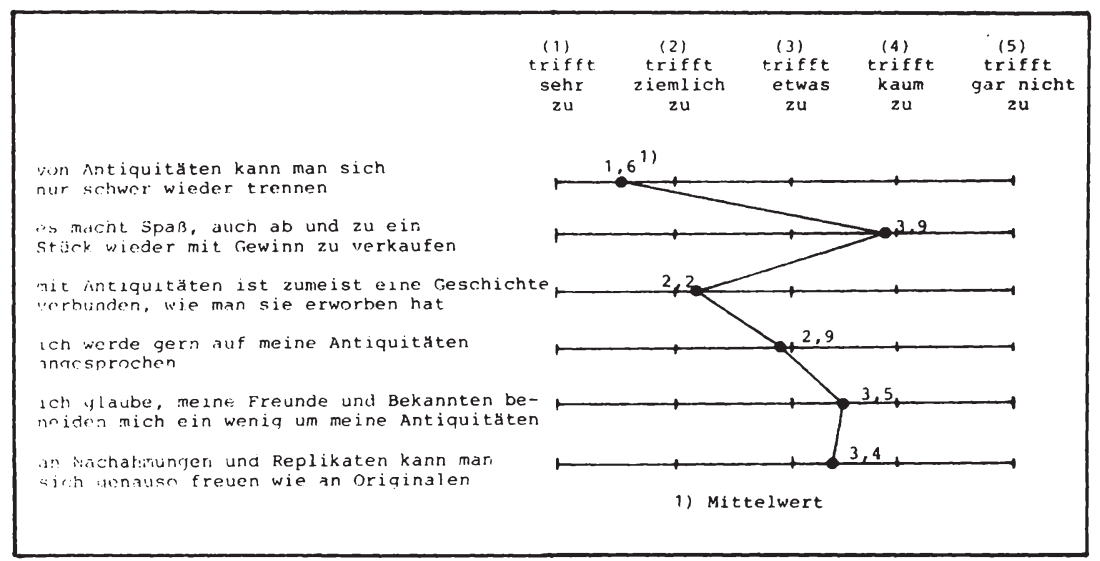

Abb. 17: Indikatoren für das Involvement gegenüber Antiquitäten

1 Je nach Blickrichtung sind die wichtigsten Bestimmungsgrößen der Invelvement-Intensität (stark vs. schwach) entweder eher situationaler oder eher personaler Herkunft. 
Die befragten Antiquitätenkäufer können sich von ihrem einmal erworbenen Besitz nur sehr schwer wieder trennen. Auch finanzielle Anreize bewegen sie hierzu kaum. Beides deutet auf eine insgesamt sehr intensive allgemeine Bindung des Käufers zur Antiquität. Eine inhaltlich spezifizierte Analyse zeigt, daB ein ausgeprägter "regressiver" Erlebniswert wichtiger Bestandteil der Besitzbindung ist: Der Käufer läBt sich gern durch einzelne Antiquitäten an Begleitumstände und situationale Gegebenheiten des zurückliegenden Kaufes erinnern. Antiquitäten bieten ihrem Käufer durch ihre besonderen Eigenschaften vielfältige Möglichkeiten, sich als (überdauernde) Träger individueller Erinnerungswerte auszuzeichnen.

Je ausgeprägter der Erinnerungewert einer Antiquität, um so intensiver ist vermutlich zugleich die Identifikation des Käufers mit ihr. Der empirische Befund zeigt, daß bei einer explorativen Operationalisierung der Besitzidentifikation durch den Wunsch, von anderen auf den eigenen Antiquitätenbesitz angesprochen zu werden, ein eher mittleres Identifikationsniveau unterstellt werden kann. Eine Aufschlüsselung des Mittelwertes durch die relative Häufigkeitsverteilung deutet jedoch auf ein sehr differenziertes Meinungsbild hin: ca. 30 \& der Befragten stimmen sehr oder ziemlich, weitere $29 \&$ etwas und restliche 32 \& kaum oder gar nicht der gestellten Frage zu.

Ähnliches gilt auch für die Einschätzung des Antiquitätenbesitzes als möglichen Gegenstand des Neides von Freunden und Bekannten: trotz eines unterdurchschnittlichen Mittelwertes von 3,5 auf der gewählten 5-stufigen Skala, stimmen immerhin noch $1 / 4$ der Befragten sehr oder ziemlich dieser Möglichkeit zu; ein relativ hoher Prozentsatz, wenn man die in der Fragestellung implizierte Wertung berücksichtigt. 
Abschließend zeigt das empirisch erhobene Mittelwertprofil, wie wichtig den Antiquitätenkäufern das "Original" als Grundlage einer Besitzbindung bei Antiquitäten ist. Die eher ablehende rialtung gegenüber Nachahmungen und Replikaten macht die Annahme wahrscheinlich, daB die käufer mehrheitlich ein bewußtes oder unbewußtes "Verständnis" für die besondere Bedeutung der Originalität von Antiquitäten als Kunstgegenstände entwickeln.

Die individuellen Ausprägungen des Antiquitäten-Involvement, beschrieben durch unterschiedliche Besitzbindungen und -identifikationen, besitzen einleuchtende Zusammenhänge zur Käufermotivation. Einzelne Motivinhalte können dabei sowohl in unterschiedlichem Ausma $B$ als auch in unterschiedlicher Richtung EinfluB auf die Stärke des Involvement ausüben.

Hypothetisches Beispiel für einen engen positiven Korrelationszusammenhang sind eine ausgeprägte Sammelleidenschaft des Käufers oder eine allgemeine Liebhaberei zum alten Kunsthandwerk als Beweggrund des Antiquitätenkaufs. In der Tendenz enge negative Korrelationen sind dagegen bei Motiven wie dem Kauf von Antiquitäten zur Wohneinrichtung oder als Wertanlage zu erwarten. Gerade der Wertanlageaspekt macht andererseits aber auch deutlich, daB - in Abhängigkeit von der inhaltlichen operationalisierung beide genannten Einflußrichtungen jeweils bezogen auf unterschiedliche Teilaspekte des Involvement auftreten können. So ist denkbar, daß sich "Wertanleger" weder durch eine intensive Besitzbindung noch -identifikation auszeichnen, wohl aber aufgrund eines hohen finanziellen Einsatzes stark sachlich informativ und mental engagiert sind.

\subsection{Kognitive Konflikte}

Kognitive Konflikte beschreiben wahrgenommene Spannungszustände und Ungleichgewichte in den kognitiven Strukturen der Antiquitätenkäufer. Konsistenztheoretisch ausgerichtete Verhaltensmodelle gehen davon aus, daß kognitive Konflikte als unangenehm erlebt werden und deshalb den Käufer zum 
Abbau bzw. zur Wiederherstellung eines als befriedigend empfundenen "kognitiven Gleichgewichtes" motivieren".

Kognitive Konflikte können in allen Phasen des Kaufentscheidungsprozesses auftreten. Wählt man den Zeitpunkt der Entscheidungsfindung bzw. des tatsächlichen Kaufaktes als Abgrenzungskriterium, so lassen sich "Vor-Kauf-Konflikte" und "Nach-Kauf-Konflikte" unterscheiden. In der Literatur zum Käuferverhalten wird zumeist das wahrgenommene Risiko stellvertretend für den ersten und die sog. kognitive Dissonanz stellvertretend für den zweiten Konflikttyp exemplarisch diskutiert.

Von einem wahrgenommenen Kaufrisiko ist $z u$ sprechen, wenn der individuelle Antiquitätenkäufer vor dem Kauf i.e.s. die Möglichkeit eines Nachteils oder Schadens als Folge seiner Kaufentscheidung antizipiert. Es ist unmittelbar einleuchtend, daß das wahrgenommene Risiko sowohl vom Grad der UngewiBheit als auch von Art und wichtigkeit der jeweiligen Handlungskonsequenzen bezogen auf die angestrebten Verhaltensziele abhängig ist ${ }^{2}$. Inhaltlich kann das wahrgenommene Risiko entweder als globale Kognition (Konstrukt) aufgefaßt oder in einzelne Dimensionen wie z.B. finanzielle, soziale, funktionale oder psychologische Risiken aufgespalten werden ${ }^{3}$.

1 Vgl. die Darstellung bei Markin, welcher zugleich die unterschiedlichen Aspekte und Entwicklungsstufen konsistenztheoretischer Ansätze anhand der Theorien von Heider, Newcomb, Osgood und Tannenbaum sowie Festinger nachzeichnet. Vgl. Markin, R.J. (jr.), Consumer Behavior, a.a.O., S. $130 \mathrm{ff}$. sowie die angegebenen Originalquellen.

2 Ebenda, S. $524 \mathrm{ff}$..

3 Roselius, T., Consumer Ranking of Risk Reduction Methods, in: JoM, Vol. 35 (1971), S. 56 ff.; Kaplan, L.B., Szybillo, C.J., Jacoby, J., Components of Perceived Risk in Product Purchase, in: JoAP, Vol. 59 (1975), S. 287291; Cunningham, S.M., The Major Dimensions of Perceived Risk, in: Cox, D.F. (Hrsg.), Risk Taking and Information Handing in Consumer Behavior, Boston 1967, S. 82-108. 
Einer umfassenden empirischen Operationalisierung des wahrgenommenen Risikos bei Antiquitätenkäufen steht der hohe Grad an "Individualität" des Risikoempfindens entgegen. Wahrnehmungsschwelle, -inhalt und -intensität variieren sowohl inter- als auch intrapersonell in Abhängigkeit von personalen, situativen, warenbezogenen und zeitbedingten Einflußgrößen. Forschungsökonomie und explorativer Charakter der vorliegenden Untersuchung bedingen eine globale und eindimensionale operationalisierung ${ }^{1}$ ausgewählter Inhaltsaspekte des vom Antiquitätenkäufer wahrgenommenen Risikos. Fragestellungen und empirische Ergebnisse zeigt die Tabelle 4.

\begin{tabular}{|c|c|c|c|c|c|}
\hline & \multicolumn{2}{|c|}{ Relative } & \multicolumn{2}{|c|}{ Häufigkeiten } & \multirow[b]{2}{*}{$\begin{array}{c}\text { (5) } \\
\text { trifft } \\
\text { gar nicht } \\
\text { zu }\end{array}$} \\
\hline & $\begin{array}{l}\text { (1) } \\
\text { trifft } \\
\text { sehr } \\
\text { zu }\end{array}$ & $\begin{array}{c}\text { (2) } \\
\text { trifft } \\
\text { ziemlich } \\
\text { zu }\end{array}$ & $\begin{array}{l}(3) \\
\text { trifft } \\
\text { etwas } \\
z u\end{array}$ & $\begin{array}{l}(4) \\
\text { trifft } \\
\text { kaum } \\
\text { zu }\end{array}$ & \\
\hline $\begin{array}{l}\text { ohne Fachkenntnisse kommt man beim } \\
\text { Antiquitătenkauf nicht aus }\end{array}$ & 39,1 & 42,4 & 11,9 & 4,6 & 2,0 \\
\hline $\begin{array}{l}\text { ein Antiquitatenkauf bleibt immer } \\
\text { ein finanzielles Wagnis }\end{array}$ & 4,6 & 27,2 & $35, i$ & 26,5 & 6,6 \\
\hline
\end{tabular}

Tab. 4: Ausgewählte Aspekte des wahrgenommenen Kaufrisikos relative Häufigkeitsverteilungen

1 Auch im Rahmen multidimensionaler Operationalisierungen können lediglich annäherungsweise Indikatorwerte zur empirischen "Messung" des wahrgenommenen Risikos ermittelt werden. Zur Problematik mehrdimensionaler Messung vgl. Zikmund, W.G., Scott, E.A., A Multivariate Analys is of Perceived Risk, Self-Confidence and Information Sources, in: AoCR, Ward, S., Wright, P. (eds.), Advances in Consumer Behavior, Vol. 1, 1974, s. 406-416. 
Es zeigt sich, daß den Antiquitätenkäufern ein "Kompetenzrisiko" sehr eindringlich bewußt ist. Ein solches "Kompetenzrisiko" erscheint als ein sinnvoller Indikator für das global wahrgenommene Kaufrisiko bei Antiquitäten, da es den Befragten einen individuellen Assoziationsspielraum zu den unterschiedlichsten Risikoarten gibt. Dominant sind darunter vermutlich Zweifel an der Richtigkeit des persönlichen Urteils bzw. der persönlichen Einschätzung über Merkmale und Eigenschaften zur Disposition stehender Antiquitäten (z.B. künstlerische und kunsthistorische Bedeutung, Qualität, Authentizität etc.).

Im Mittelpunkt der Untersuchung einzelner Risikoinhalte steht oftmals in der Literatur das wahrgenommene finanzielle Risiko eines Kaufs. Die Risikowahrnehmung wird dabei im engen $\mathrm{Zu}-$ sammenhang mit dem Erwerb höherwertiger Gebrauchsgüter, zu denen i.d.R. auch Antiquitäten zählen, gesehen. Nicht nur die häufig hohe finanzielle Mittelbindung, sondern ein breites Spektrum weiterer Eigenschaften von Antiquitäten und Bedingungen ihres Marktes (z.B. mode- und marktbedingte Preismanipulationen, geringe Krisenwerte etc.) weisen aus "objektivierender" Sicht auf das reale Bestehen einer finanziellen Verlustgefahr bei Antiquitätenkäufen hin. Die "subjektive" Wahrnehmung des Käufers scheint nicht gleichermaßen rational solche "objektiven" Gründe nachzuvollziehen; denn in den Antworten der befragten Antiquitätenkäufer kommt eine eher mittelstarke durchschnittliche Wahrnehmung finanzieller Risiken zum Ausdruck. Nur zu einem geringen Prozentsatz werden dabei extreme Meinungsstandpunkte vertreten.

Ein Vergleich der gewonnenen Ergebnisse zum globalen und finanziellen Risiko bestätigt in der Tendenz eine erwartete Relation: je inhaltlich spezifizierter das Risiko um so geringer seine durchschnittliche Bedeutung bei den Antiquitätenkäufern und vice versa. Dabei darf jedoch nicht über- 
sehen werden, daß die vorgenommenen Operationalisierungen der Komplexität der Risikowahrnehmung nur in sehr grober Annäherung gerecht werden können.

Verhaltensfolgen und-konsequenzen des wahrgenommenen Risikos zielen, entsprechend der konsistenztheoretischen Grundhypothese, auf eine Risikoreduktion. Neben der Annahme, daß die Beschaffung und Selektion von Informationen nach Art, Menge und Quelle wesentlich durch die Höhe des Risikos bestimmt wird ${ }^{1}$, werden u.a. auch die senkung von Anspruchsniveaus für die angestrebten ziele, verstärkte Rückgriffe auf eigene Erfahrungen (Routinisierung des Verhaltens) oder diejenige anderer (Imitationsverhalten) sowie Tendenzen zur Kollektivierung der Kaufentscheidung (Verantwortungsdelegation, "risky-shift"2) als Risikoreduzierungsstrategien postuliert ${ }^{3}$.

Andererseits wird dem psychologischen Ursachenkomplex des wahrgenommenen Risikos bislang nur wenig Aufmerksamkeit geschenkt ${ }^{4}$. Allgemein strukturierende Ansätze ${ }^{5}$ weisen auf die hohe Bedeutung der individuellen Käufermotivation hin. Im Fortgang der vorliegenden Untersuchung (vgl. Kapitel C) wird zu klären sein, ob und in welcher Form spezifische Mo-

1 Vgl. Meffert, H., Modelle, a.a.O., S. 338; Sheth, J.N., Venkatesan, M., Risk-Reduction Process in Repetetive Consumer Behavior, in: JoMR, Vol. 5 (1968), Nr. 3, S. 3o7310; Schroeder, H.M., Driver, M.J., Streufert, S., Menschliche Informationsverarbeitung. Die Strukturen der Informationsverarbeitung bei Einzelpersonen und Gruppen in komplexen sozialen Situationen, Weinheim 1975, S. $60 \mathrm{ff}$; ; Kroeber-Riel, W., Konsumentenverhalten, a.a.O., S. 253 ff..

2 Vgl. Rüttinger, B. et al., a.a.O., S. 144.

3 Vgl. Roselius, T., a.a.O., S. 56-61; Sheth, J.N. et al., a.a.o., S. $307 \mathrm{ff.;}$ Markin, R.J. (jr.), Consumer Behavior, a.a.0., S. $524 \mathrm{f}$. und die dort angegebene Literatur.

4 Vgl. Kroeber-Riel, W., Konsumentenverhalten, a.a.O., S. 352.

$5 \mathrm{Vgl}$. exemplarisch den Ursachenkatalog bei Markin, R.J. (jr.), Consumer Behavior, a.a.0., S. $524 \mathrm{f}$. und die dort angegebene Literatur. 
tivinhalte und Konfigurationen zur Erklärung des wahrgenommenen Kaufrisikos von Antiquitätenkäufern beitragen.

Kognitive Dissonanzen beschreiben Widersprüche in der "subjektiven Psycho-Logik" ${ }^{1}$ von Antiquitätenkäufern, die mittelbar oder unmittelbar aus Erfahrung und Umgang mit dem Antiquitätenbesitz, d.h. nach dem vollzogenen Kauf entstehen. Folgende generellen Typen von "Nach-Kauf-Erfahrungen", welche zugleich unterschiedliche Dissonanzarten ${ }^{2}$ von Antiquitätenkäufern abgrenzen, sind denkbar:

(1) Der Antiquitätenkäufer bezweifelt nachträglich die Richtigkeit seiner Entscheidung, da er z.B. eine Vielzahl von Kaufalternativen, die in einer oder mehreren Eigenschaften "besser" seiner motivationalen Anforderung entsprochen hätten, ablehnen mußte oder, da er zusätzliche "neue" Informationen erhält, die die abgelehnten Alternativen "aufwerten" ${ }^{3}$.

(2) Der Antiquitätenkäufer stellt nachträglich fest, daß seine in die gekaufte Antiquität gesetzten Erwartungen (z.B. funktionaler, finanzieller, repräsentativer oder künstlerischer Art) nicht erfüllt werden oder zumindest nicht seinen motivationalen Ansprüchen gerecht werden 4 .

1 Vgl. zum Begriff Kroeber-Riel, w., Konsumentenverhalten, a.a.O., S. $291 \mathrm{ff.}$.

2 Zur Theorie der kognitiven Dissonanz vgl. Festinger, C., A Theory of Cognitive Dissonance, 2nd ed., Stanford 1963, in deutscher Sprache; Irle, M. , Möntemann, V., Theorie der kognitiven Dissonanz, Bern etc. 1978; sowie als Uberblick Frey, D., Die Theorie der kognitiven Dissonanz, in: derselbe (Hrsg.), Kognitive Theorien der Sozialpsychologie, Bern etc. 1978, S. 243-294; Zu den Implikationen für die Käuferverhaitensforschung vgl. Meffert, H. , Modelle, a.a.O., S. 339 f.; Kroeber-Riel, W. Konsumentenverhalten, a.a.O., S. $222 \mathrm{ff}$. und S. $356 \mathrm{ff.;}$ Markin, R.J. (jr.), Consumer Behavior, a.a.0., s. $143 \mathrm{ff}$. sowie die jeweils zahlreichen Literaturangaben.

3 Fach FESTINGER könnte man diesen "Dissonanztyp" auch als "postdecional regret" bezeichnen; vgl. Festinger, L., A Theory, a.a.0., S. $16 \mathrm{ff}$. und S. $37 \mathrm{ff}$..

4 In Festingers Terminologie "disconfirmed expectancies". Vgl. ebenda. 
Die Wahrscheinlichkeit des Auftretens von Dissonanzen, ihre Inhalte und Intensitäten sind eng mit den Merkmalen der spezifischen Kaufsituation verflochten. Uberträgt man die in der Literatur genannten situativen Einflußfaktoren ${ }^{1}$ auf die Problematik des Antiquitätenkaufs, so muß eine tendenziell hohe Dissonanzwahrscheinlichkeit und -intensität für Antiquitäten prognostiziert werden. Hierfür spricht u.a.:

(1) Die z.T. unüberschaubar hohe Anzahl von relevanten Kaufalternativen zur Befriedigung der unterschiedlichsten Käufermotivationen;

(2) die breite Palette motivationsrelevanter (materieller und imaterieller) Wareneigenschaften des Einzelstücks, so daß i.d.R. jede gekaufte Antiquität neben motivationskongruenten auch eine Reihe motivationsdivergente Charakteristiken besitzt; gleiches gilt ferner auch für die abgelehnten Kaufalternativen;

(3) die besonderen Schwierigkeiten einer objektiven "Produktbewertung" (Qualität usw.) ;

(4) ein ausgeprägtes Involvement der Käufer gegenüber Antiquitäten (vgl. Kapitel B. 3.2121).

Der Versuch, den "theoretischen" Erwartungen eine empirische "Maßgröße" für die Dissonanz von Antiquitätenkäufern zum Vergleich gegenüberzustellen, birgt umfangreiche Operationalisierungsprobleme ${ }^{2}$. Als Indikatoren des Dissonanzempfindens dienen in der vorliegenden Untersuchung die in Tabelle 5 wiedergegebenen (jeweils eindimensionalen) Fragestellungen als direkte und indirekte "Maßgröße".

1 Einen Uberblick über dissonanzerzeugende Situationsfaktoren und die resultierenden Dissonanzerwartungen (hoch vs. niedrig) ist $\mathrm{zu}$ finden bei Holloway, R.J., An Experiment on Consumer Behavior Dissonance, in: JoM, Vol. 31 (January 1967), S. 40 f..

2 "At the present time no one has developed a method that will predict the situation or the type of individual who becomes cognitively dissonant. Another point is that the type of product, the individual, and the specific buying situation all interact to create a state of dissonance". Markin, R.J.(jr.), Consumer Behavior, a.a.O., S. 162. 


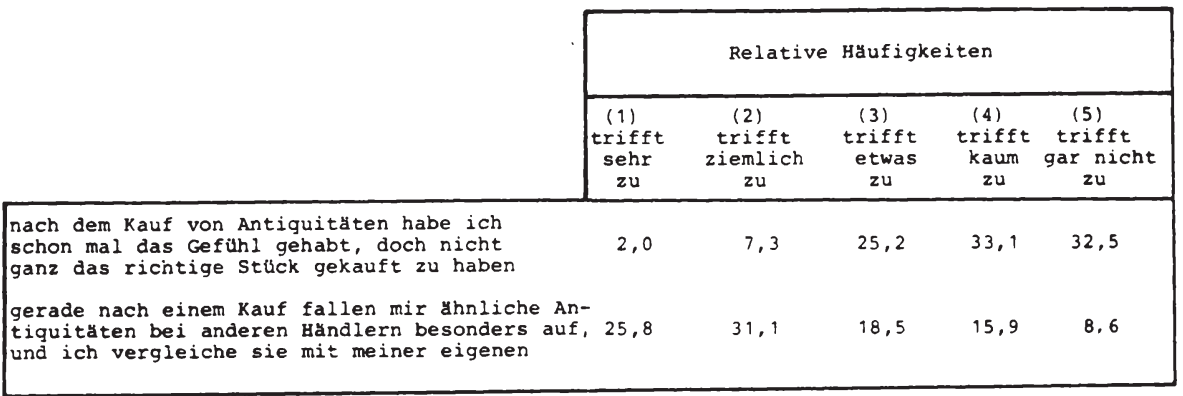

Tab. 5: Ausgewählte Aspekte der kognitiven Dissonanz relative Häufigkeitsverteilungen

Die direkte Fragestellung versucht, ein globales, inhaltlich nicht näher spezifiziertes Dissonanzgefühl der Antiquitätenkäufer $z u$ erfassen. Insofern umschließt es beide (weiter oben) als relevant herausgestellten Dissonanztypen.

Die Verteilung der relativen Antworthäufigkeiten spiegelt ein nur geringes Ausma $B$ an Nachkauf-Dissonanzen bei den Befragten wider. Dabei sind mögliche "Antwortverzerrungen" dadurch möglich, daß die Käufer nur ungern eine "Fehlentscheidung" vor sich selbst und anderen eingestehen.

Zu einer Relativierung des gewonnenen Ergebnisses kann die indirekte Abfrage der Dissonanz beitragen. Sie beruht auf der hypothetischen Annahme, daß Antiquitätenkäufer bei wahrgenommenen Dissonanzen aktiv und selektiv nach zusätzlichen Informationen zur Rechtfertigung ihrer Entscheidung und damit zur Dissonanzreduktion suchen ${ }^{1}$. Akzeptiert man

1 Zur Diskussion dieser und weiterer Reduktionsstrategien vg1. Kroeber-Riel, W., Konsumentenverhalten, a.a.O., S. 360 f.; Markin, R.J. (jr.), Consumer Behavior, a.a.o., S. $148 \mathrm{f}$. und die dort angegebenen Literaturstellen im Originaltext von Festinger; Holloway, R.J., a.a.O., S. $41 \mathrm{f}$.. 
diese - wenn auch aufgrund fehlender Validierung nicht unproblematische - Hypothese, dann weist die "überproportionale" Wahrnehmungs- und Vergleichsaktivität der Antiquitätenkäufer zugleich auf ein insgesamt höheres Dissonanzniveau als es in der direkten Fragestellung ermittelt werden konnte, hin.

Eine interessante Anfälligkeit zeigt das empirische Ergebnis durch die gleichzeitig ausgeprägte Zunahme einer Meinungspolarität. Unter den gesetzten Bedingungen kann dies als ein Anzeichen für das Bestehen stark individuell differenzierter Dissonanzempfindungen bei Antiquitätenkäufern interpretiert werden. Schließlich gibt insbesondere dieses breite Spektrum unterschiedlicher Dissonanzbeurteilungen der Käufer den $A n l a B$, nach ihren möglichen motivationalen Bestimmungsgrößen $\mathrm{zu}$ fragen (vgl. Kapitel C.).

\subsection{Preisbereitschaft}

Die Bereitschaft von Antiquitätenkäufern, für Einzelstücke nur einen begrenzten Preis zahlen zu wollen, kann als kognitive Gedankenhaltung Einfluß auf ihren Kaufentscheidungsprozeß ausüben.

Die preisbereitschaft ist inhaltlich nicht gleichzusetzen mit der finanziellen Kauffähigkeit (Zahlungsfähigkeit) im Sinne der "aus der Sicht eines Käufers verfügbaren Mittel" ${ }^{1}$. Ist die Kauffähigkeit oftmals eher in "exogenen"und kurzfristig nur wenig veränderbaren Bedingungen (Arbeitseinkommen etc.) verwurzelt, ist die individuelle Preisbereitschaft als selbstbestimmte kognitive "Restriktion" des Käufers zu verstehen. Insofern läßt sich vermuten, daß die Kauffähigkeit eher in der realen Kaufsituation, d.h.

1 Vgl. Hoepfner, G., Verbraucherverhalten, a.a.o., s. 92. 
zugleich erst "spät" im KaufentscheidungsprozeB, als "Regulativ" wirkt; andererseits die subjektive Preisbereitschaft alle Auswahlphasen von der Bedürfnisentstehung bis zur Auswahl und dem Erwerb einer Antiquität begleitet.

Beide "Konstrukte" sind dabei nicht unabhängig voneinander, so daß die Preisbereitschaft durch die Selbsteinschätzung der finanziellen Mittelausstattung speziell in der engeren Kaufsituation mitbestimmt wird. Daneben sind als hypothetische Determinanten das Vorstellungsbild von Antiquitäten (Qualität, Prestige- und statuswert, Sammlerwert etc.) und insbesondere psychisch personale Dispositionen wie die Kaufmotivation oder die Risikoneigung relevant.

Die letztgenannten Einflußgrößen gewinnen zusätzlich an Bedeutung, wenn die Preisbereitschaft nicht auf ein reales sondern auf ein "fiktives" (beliebiges) Einzelstück, d.h. letztlich auf die gesamte Warengruppe "Antiquitäten" bezogen wird. Gerade in dieser generalisierten Form besitzt die Preisbereitschaft der Antiquitätenkäufer einen hohen und voraussichtlich zeitlich stabilen Interpretationswert.

In der empirischen Erhebung wurde versucht, das allgemeine Preislimit der Antiquitätenkäufer durch die (offene) Frage: "Wieviel Geld würden Sie höchstens für eine einzelne Antiquität ausgeben?" als explorativen Anhaltspunkt ihrer warengruppenbezogenen Preisbereitschaft zu ermitteln. Einen ersten Ergebnisüberblick gibt die Abbildung 18. 


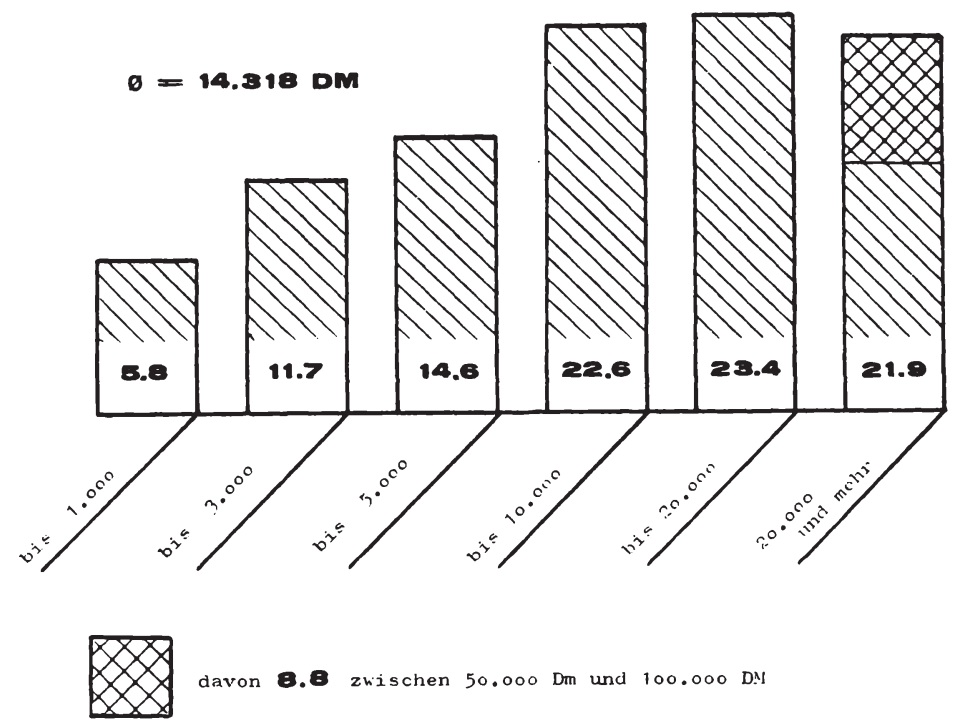

Anm.: Zahlenangaben in relativen Häufigkeiten

Abb. 18 : Preisbereitschaft von Antiquitätenkäufern in abgegrenzten Preisklassen

Bei einer Spannweite der genannten Preislimits von 100 DM bis 100.000 DM ergibt sich eine hohe (durchschnittliche) Preisbereitschaft von 14.318 DM. Sieht man darin zugleich einen "Maßstab" für die allgemeine Wertschätzung der Käufer gegenüber Antiquitäten, ist auch diese tendenziell als hoch zu bezeichnen. Auf der Basis der zugrundeliegenden Klassenteilung spricht hierfür ferner die fast durchgängige Zunahme der Käufernennungen in Richtung steigender "subjektiver" Preisgrenzen. 
Neben dieser orientierenden, klassenbezogenen Quantifizierung interessierte auch die Frage, bei welchen Preisen die individuellen Preisgrenzen liegen. Aus möglicherweise auftretenden Konzentrationen in ihrer Häufigkeitsverteilung sollte auf allgemeine "psychologische Preisschwellen" einzelner Käufergruppen geschlossen werden.

Abbildung 19 gibt das ermittelte Stabdiagramm wieder, wobei die Distanzen zwischen den Preisnennungen (in unterschiedlichen Maßstäben je Intervall) den effektiven Betragsdifferenzen (in DM) proportional sind.

Der empirische Befund weist den Betrag von 5.000 DM und den von $10.000 \mathrm{DM}$ als häufigste Preisgrenze und damit als wichtige allgemeine "Preisschwelle" bei Antiquitätenkäufern aus. Da zwischen ihnen nur eine geringe zahl von Einzelnennungen liegt, ist anzunehmen, daß Käufer bei einer Erhöhung ihres "subjektiven Preislimits" über 5.000 DM hinaus einen "großen Preisschritt" zum 10.000 DM Limit machen. Analoge 


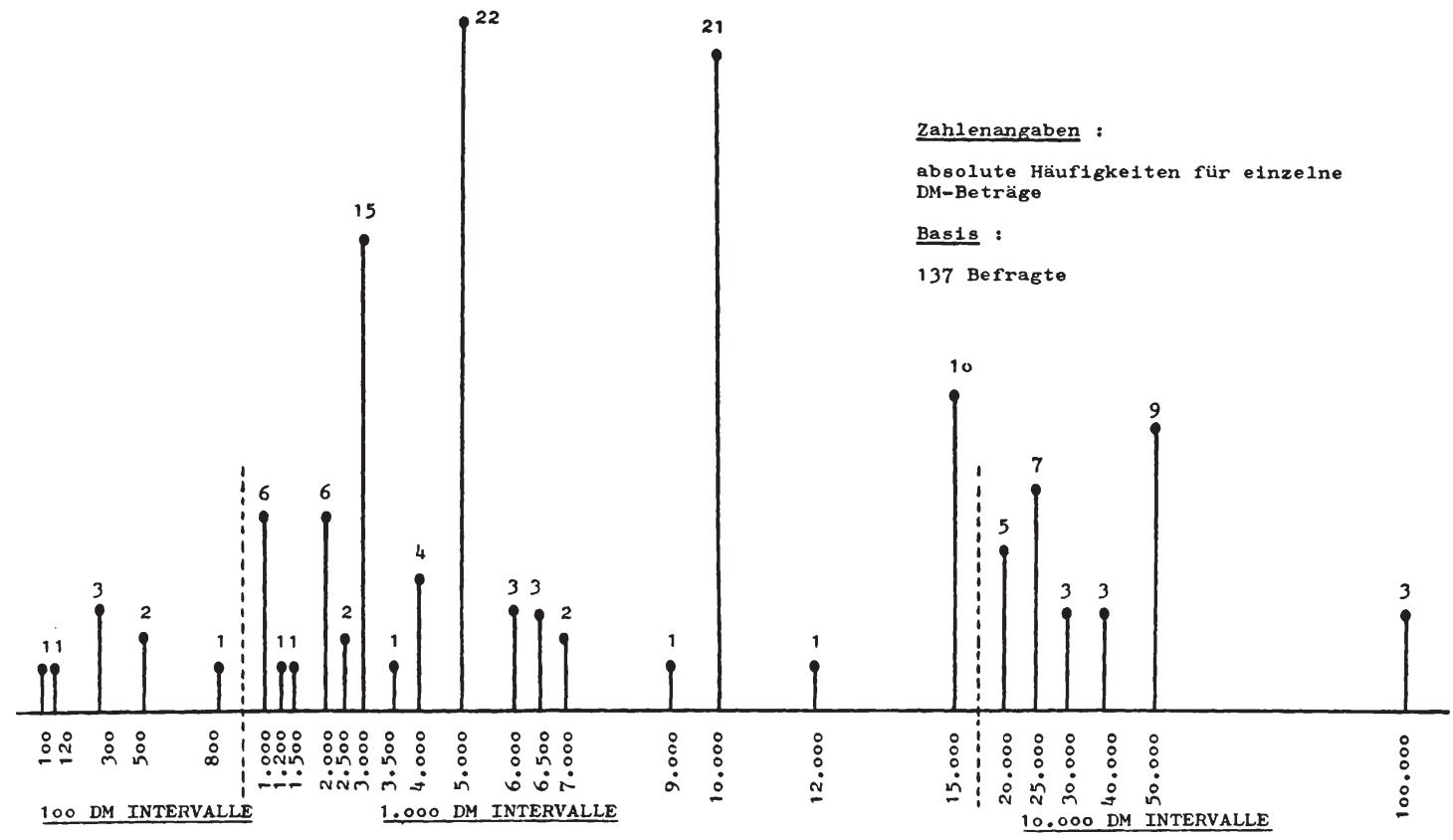

Abb. 19: Häufigkeitsverteilung individueller Preisgrenzen beim Kauf von Antiquitäten 
Uberlegungen lassen sich - bezogen auf kleinere absolute Intervalle - für den tbergang von der 3.000 DM zur 5.000 DM und von der 10.000 DM zur 15.000 DM "Preisschwelle" anstellen.

Die in der Abbildung aus Darstellungsgründen unterschiedlich gewählten Skalenintervalle dürfen nicht darüber hinwegtäuschen, daß bei zunehmender Preisbereitschaft jeweils auch die "Nennungszwischenräume" wachsen. Damit findet die Annahme Unterstützung, daß Antiquitätenkäufer mit höherer Preisbereitschaft auch (dem Betrag nach) in größeren preisrelationen denken.

Das erhobene Datenmaterial deutet insgesamt sowohl das Bestehen markt- und warentypischer Preisschwellen als auch individuell geprägter Preisgrenzen an. Damit wird u.a. die Frage nach den psychologisch-motivationalen Hintergründen und Erklärungsbeiträgen aufgeworfen. Dies gilt nicht nur für einzelne, inhaltlich spezifizierte Motivationen sondern auch für Motivationskonfigurationen als Repräsentanten unterschiedlicher Käufertypen.

\subsection{Einkaufsstättenwahl}

Jeder Erwerb von Antiquitäten umschließt die Wahl einer "geeignet erscheinenden" Einkaufsstätte als wichtige und notwendige Teilentscheidung des Käufers. Als Auswahlalternativen bieten sich dem privaten Käufer alle Mitglieder der in Kapitel B.2.2 bereits näher typisierten Gruppen von aktuellen und potentiellen Marktpartnern wie Handelsgeschäfte, Auktionshäuser, Messen etc.

Die Entscheidungsfindung für das "Wo" eines Antiquitätenkaufs vollzieht sich in einem mehr oder weniger ausgeprägten 


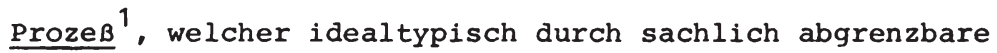

"kognitive Teilaktivitäten" (Phasen) strukturierbar ist. In Anlehnung an die Literatur zur Einkaufsstättenwahl ${ }^{2}$

läßt sich beispielsweise folgende phasenteilung für die

Wahl einer Antiquitäten-Einkaufsstätte postulieren:

- Entstehung bzw. Anregung eines Kaufanlasses für eine Antiquität z.B. durch die Aktualisierung warenspezifischer Motivationsinhalte ${ }^{3}$

- Suche nach Einkaufsstättenalternativen mit dem Ziel einer Bestandsaufnahme einer als ausreichend erachteten Anzahl "erreichbarer" Antiquitätengeschäfte, Auktionshäuser etc.

- Bewertung der Alternativen anhand eines individuellen Kriterienkataloges durch den Vergleich des persönlichen (allgemeinen) Anspruchsniveaus an Eigenschaften von Einkaufsstätten und den wahrgenommenen Ausprägungen im konkreten Einzelfall 4

- Entscheidung i.e.s. und realer Kontakt mit der gewählten Einkaufsstätte, z.B. durch Betreten des Ladenlokals, Besuch einer Antiquitätenmesse oder auch ein schriftliches/fernmündiches Auktionsgebot

$1 \mathrm{Vgl}$. auch die Ausführungen $\mathrm{zu}$ den Entscheidungstypen bei der Antiquitätenwahl in Kapitel B. 3.211.

2 Vgl. Heinemann, M., Einkaufsstättenwahl und Firmentreue der Konsumenten, Schriftenreihe Unternehmensführung und Marketing, Meffert, H. (Hrsg.), Bd. 6, Wiesbaden 1976, $\mathrm{S} .109 \mathrm{ff}$. und die dort angegebene Literatur.

$3 \mathrm{Vgl}$. auch Klenger, F., Krautter, J., Simulation des Käuferverhaltens, Teil 2, Analyse des Kaufprozesses, Wiesbaden 1972 , S. 79 .

4 Die Motivation eines Antiquitätenkaufs wird damit sowohl zum Ausgangspunkt der warenbezogenen als auch der einkaufsstättenbezogenen Entscheidungsprozesse. Die Einkaufsstättenwahl kann daneben auch durch nicht unmittelbar antiquitätenbezogene Motivationen wie z.B. die Freude am Einkaufserlebnis, die Neugierde an "ausgefallenen" Einkaufsformen (Auktionen, Messen) initiiert werden. Es bleibt daher i.d.R. offen, ob der Käufer "zuerst" seine Entscheidung für einen Antiquitätenkauf oder eine Einkaufsstätte trifft. Zu warenunabhängigen Einkaufsanlässen vgl. Tauber, E.M., Why do People Shop?, in: JoM, vol. 36 (October 1972), S. $46 \mathrm{ff}$.. 
- nachträgliche Bewertung der Einkaufsstätte, die sich in einer Zufriedenheit/Unzufriedenheit des Antiquitätenkäufers, basierend auf seinen gesammelten Erfahrungen, äußert.

Die genannte Phasenabfolge besitzt auch als zeitliche Reihenfolge eine - allerdings nur strukturelle - Plausibilität. In der Realität wird der Einkaufsstättenwahlproze $B$ durch viele zusätzliche kognitive "Schleifen", "Rückkopplungen" , "Sprünge" und "Gleichzeitigkeiten", insbesondere bezogen auf die begleitende Informationsgewinnung und-verarbeitung, charakterisiert. Vermutlich steht aus diesem Grund eine umfassende empirische Operationalisierung und Validierung des "Phasenmodells" sowohl allgemein als auch waren- bzw. produktspezifisch bislang aus.

Die empirische Erfassung der Einkaufsstättenwahl von Antiquitätenkäufern beschränkt sich auf zwei wesentliche Teilaspekte: Die Anforderungen der Käufer an ausgewählte Eigenschaften von Einkaufsstätten als Grundlage der Entscheidungsfindung und die Nutzungsfrequenz ausgewählter Einkaufsstättenformen als ihr "beobachtbares" Resultat.

\subsection{Anforderungen an Einkaufsstätten}

Die Erhebung des Anforderungsprofils von Antiquitätenkäufern an den Antiquitätenhandel (i.w.S.) verlangt zunächst die Auswahl eines problemorientierten Katalogs entscheidungsrelevanter Bewertungskriterien ${ }^{1}$. Die inhaltliche Abgrenzung basierte auf der Annahme, daB die Dimensionen der Einkaufsstättenbeurteilung hinreichend durch die Gestaltungsbereiche der absatzpolitischen Instrumente oder m.a.w. das Erscheinungsbild des Antiquitätenhandels ${ }^{2}$ repräsentiert

1 Denn diese sind keineswegs in Art und $\mathrm{Zahl}$ apriori gegebene, sondern personen-, situations- und warenspezifisch gebildete Größen. Vgl. beispielhaft Klenger, F., Krautter, J., a.a.0., S. $60 \mathrm{ff}$..

2 Vgl. dazu die Ausführungen in Kapitel B. 2.2. 
werden. Das in Abbildung 20 wiedergegebene Anforderungsprofil der befragten Käufer ist daher als Ausschnitt ihres "Ideal-Image" für den Antiquitätenhandel interpretierbar ${ }^{1}$.

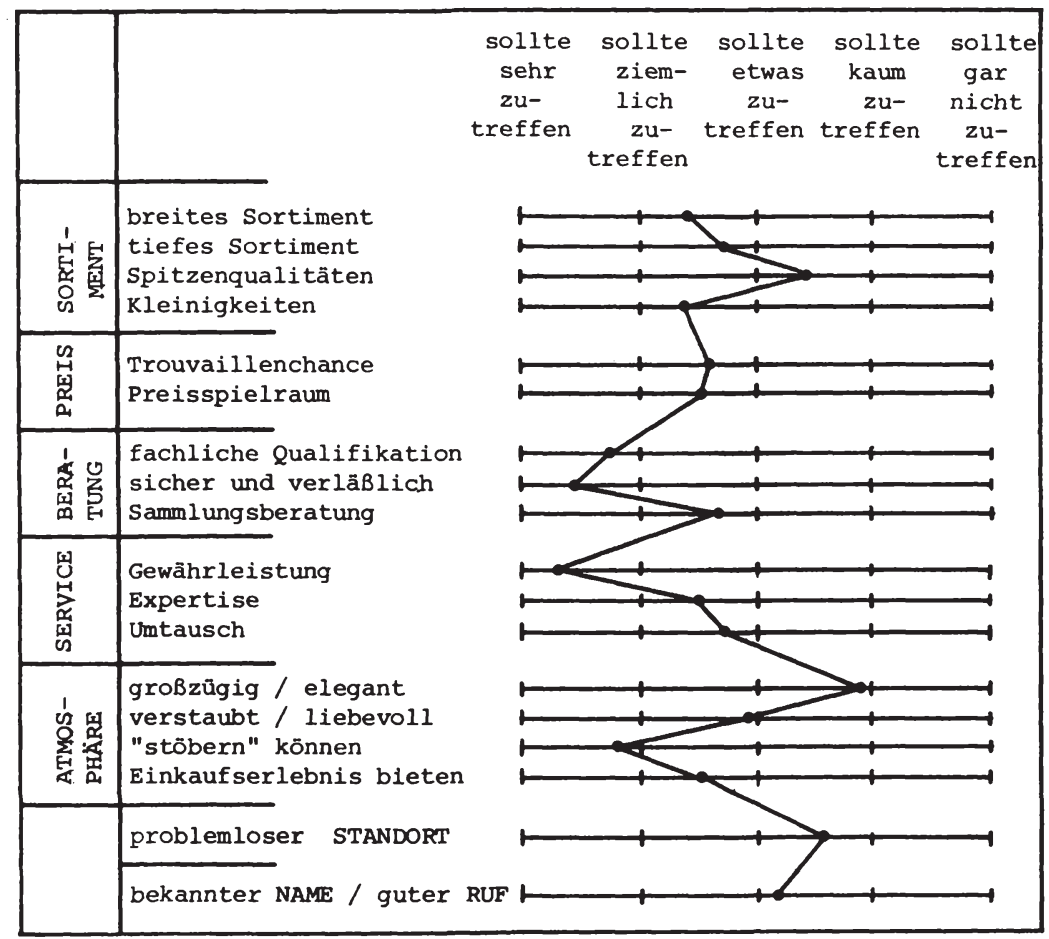

Abb. 20: Anforderungen an Einkaufsstätten

Unter den abgefragten Sortimentsanforderungen dominiert der Wunsch nach einer breit gefächerten, sich über mehrere Sach- und Sammelgebiete erstreckenden Auswahlmöglichkeit. Dabei möchten die Antiquitätenkäufer überwiegend nicht

1 Vgl. Heinemann, M., a.a.O., s. 157 f.. 
nur Stücke auf hohem und höchstem Qualitätsniveau, sondern verstärkt Kleinigkeiten, die sich z.B. als Geschenke eignen, im Angebot finden. Eine ausgesprochene Sortimentsspezialisierung, d.h. ein tiefes Sortiment in Spezialgebieten ist im Anforderungsprofil von eher mj.tlerer Bedeutung. Dies ist ein Hinweis, daß sich ein universell interessierter Kälfertyp gegenüber den "Spezialisten" in der Uberzahl befindet.

Bei der Preisstellung erwarten die Käufer von ihrer "idealen" Einkaufsstätte sowohl die Möglichkeit einer "passiv-sachlichen Preischance" in Form von Trouvaillen als auch eine "aktive Verhandlungschance" in Form eines Preisnachlasses oder zumindest einer Verhandlungsbereitschaft des Händlers.

Die höchsten durchschnittlichen Anforderungen stellen die Antiquitätenkäufer in den Bereichen Beratung und Service. Fast einhellig wird eine sichere und verläßliche Beratung bei hoher fachlicher Qualifikation des Verkaufspersonals erwartet. In Ubereinstimmung mit den geäußerten Sortimentsanforderungen kommt einer spezialisierten (Sammler-) $\mathrm{Be}-$ ratung dagegen nur geringere Bedeutung zu. Eindeutig wünschen die Käufer die Ubernahme einer Gewährleistung für die gekauften Antiquitäten. Dieser wird ein erheblich größerer "Wert" beigemessen als Expertisen oder der Möglichkeit eines Umtausches. Insgesamt verweist das hohe Anspruchsniveau an "risikoreduzierende" Service- und Beratungsmerkmale u.a. nochmals auf die Wahrnehmung kaufimmanenter Risiken durch Antiquitätenkäufer.

In einem interessanten Gegensatz stehen hierzu die erheblich geringeren Anforderungen an das allgemeine Geschäftsrenommee (bekannter Name / guter Ruf), das tendenziell ebenfalls zur subjektiven Sicherheit des Käufers beitragen 
könnte. Es erstaunt, daß fast 40 \% der Befragten ein Renommee als kaum oder gar nicht zutreffende Anforderung an eine Einkaufsstätte bezeichnen.

In die gleiche Richtung weisen auch die Erwartungen an die Atmosphäre der Warenpräsentation aufgrund der geringen Bedeutung einer "exklusiven" Ausstrahlung. Andererseits scheint für den Käufer ein "Freiraum", in dem er eigene "Suchaktivitäten" entfalten kann, besonders wichtig zu sein. Die "eigene Aktivität" ist vermutlich eng mit einer Art "Spannung" bzw. "Trouvaillenhoffnung" verbunden. Es liegt nahe, dies zugleich als Hintergrund des relativ hohen Anspruchsniveaus an die Vermittlung eines Einkaufserlebnisses durch die Einkaufsstätte anzusehen.

Abschließend ist $z$ u bemerken, daß die "subjektive Mühe", die Antiquitätenkäufer zum Erreichen des Einkaufsstättenstandortes aufwenden müssen, eine nur untergeordnete Rolle im Anforderungsprofil einnehmen.

Interessant ist im zusammenfassenden Gesamtüberblick, daß die Käuferansprüche an Service, Beratung (hoch), Geschäftsrenommee und Ausstrahlung der Einkaufsstättenatmosphäre ${ }^{1}$ (niedrig) eher "sachlich-rational" geprägt $z u$ sein scheinen; dagegen diejenigen an die preisgestaltung und "erlebnisbezogenen" Aspekte der Einkaufsatmosphäre eher "affektive" Uberbetonungen vermuten lassen.

Die genannten durchschnittlichen Anforderungen an Einkaufsstätten können das Ergebnis einer Aggregation der unterschiedlichsten individuellen Käufervorstellungen sein. Die-

1 Die "niedrigen" Ansprüche deuten darauf hin, daß das Geschäftsrenommee und andere "äußere" Einkaufsstättenmerkmale nicht als Surrogate für ein fehlendes Fachwissen der Käufer zur Warenbeurteilung herangezogen werden. 
se werden u.a. von zahlreichen personengebundenen Einflußgrößen, wie soziodemographischen Merkmalen (z.B. soziale Schicht, Alter ${ }^{1}$ ) oder psychographischen Merkmalen (z.B. multithematisch gebildete Käufertypen, tatsächliches oder ideales Selbst-Image ${ }^{2}$ ) bestimmt. Von besonderem Interesse ist die Fragestellung, ob und in welchem AusmaB die individuellen Anforderungsprofile mit bestimmten Motivinhalten oder -konfigurationen korrelieren, denn der subjektive Vorteilhaftigkeitsvergleich zwischen idealen Erwartungen an Eigenschaften von Einkaufsstätten und der Wahrnehmung ihrer konkreten Ausprägung im Einzelfall, stellt den wohl wichtigsten "Entscheidungsschritt" des Antiquitätenkäufers dar.

Partielle Erklärungsbeiträge zum kognitiven Anforderungsprofil können Ansatzpunkte für eire bedürfnis- und bedarfsgerechte Planung und Gestaltung absatzpolitischer Konzeptionen des Antiquitätenhandels liefern. Die tatsächliche Nutzung einer spezifischen Einkaufsstätte läßt sich nur unter Berücksichtigung zusätzlicher situativer "Korrekturfaktoren" und bezogen auf ein Set konkreter Einkaufsstättenalternativen (z.B. im Rahmen multidimensionaler Einstellungsmodelle) prognostizieren.

Der explorative und damit notwendig "breit" angelegte Ansatz der vorliegenden Untersuchung kann einer derartig spezifischen Aufgabenstellung nicht gerecht werden. Aus "vergröberter" Perspektive wurden daher die Nutzungen antiquitätenspezifischer Einkaufsstättentypen - wie sie bereits als die wichtigsten Marktpartner von privaten Antiquitätenkäufern vorgestellt wurden - analysiert.

$1 \mathrm{Vgl}$. Heinemann, M., a.a.O., s. $163 \mathrm{ff.}$.

2 Vgl. ebenda. 


\subsection{Nutzung ausgewählter Einkaufsstätten}

Für ihre bisherigen Antiquitätenkäufe nutzten die befragten Käufer die in Tabelle 6 zusammengestellten Formen von Einkaufsstätten in z.T. sehr unterschiedlichem Umfang.

\begin{tabular}{|c|c|}
\hline \multicolumn{2}{|l|}{ Fachhandel } \\
\hline Universalgeschäft & $49,7 \%$ \\
\hline Spezialgeschäft & 38,48 \\
\hline Trödler & 34,48 \\
\hline \multicolumn{2}{|l|}{ Nicht-Fachhandel } \\
\hline Nebensortiment Antiquitäten & $6,6 \%$ \\
\hline Versender & $1,3 \%$ \\
\hline \multicolumn{2}{|l|}{ Auktionshäuser } \\
\hline persönliches Gebot & $19,2 \%$ \\
\hline schriftliches Gebot & $13,2 \%$ \\
\hline Handels-Agglomerationen & • \\
\hline Kunst- und Antiquitätenmessen & 42,48 \\
\hline Antiquitätenmärkte & $43,7 \%$ \\
\hline Trödel- und Flohmärkte & $49,0 \%$ \\
\hline Privatpersonen & $40,4 \%$ \\
\hline
\end{tabular}

Tab. 6: Nutzung von Einkaufsstätten Häufigkeiten bei Mehrfachnennungen

Bevorzugt werden als Einkaufsquellen alle Formen des Fachhandels und der durch sie gebildeten Handels-Agglomerationen. Auffällig ist, daß Trödelgeschäften in der "Einzelbetrachtung" die geringste und in der "Agglomerationsbetrachtung" die höchste Bedeutung zukommt.

Als Begründungen bieten sich neben einer allgemeinen Dominanz von "floh- und trödelmarktspezifischen" Motivationen ("Nostalgie", "Einkaufserlebnis" etc.) sowohl die bereits an früherer Stelle vermutete "Durchlässigkeit" des Marktes in Richtung auf niedrige Preis- und Qualitätsstandards als 
auch eine möglicherweise überproportionale "Agglomerationswirkung" (der Käufer kann noch mehr "stöbern", "handeln", "suchen" und "hoffen") gerade dieses Einkaufsstättentyps an.

Uberraschend hoch fällt der Anteil von Käufen bei "anderen Privatleuten" aus, welcher dem Durchschnitt der Nutzung aller Fachhandelstypen fast exakt gleicht. Anscheinend wird hier ein bestimmter Prozentsatz "halbprofessioneller" und "schwarzer" Antiquitätenkäufe miterfaßt.

Eine mittlere Position unter den Einkaufsstättenalternativen nehmen die Auktionshäuser ein. Fast jeder 5. Befragte ist schon einmal Kunde eines Auktionators gewesen. Private Käufer sind damit fester Bestandteil des Kundenkreises von Auktionshäusern geworden,welcher noch vor wenigen Jahren überwiegend vom professionellen Handel dominiert wurde .

Eine relativ unbedeutende Stellung als Einkaufsstätten für Antiquitäten besitzen abschließend die abgefragten Nicht-Fachhandelstypen, d.h. zum einen Geschäfte, welche Antiquitäten nur im Nebensortiment (z.B. neben Einrichtungsgegenständen) oder zum anderen im Versandgeschäft verkaufen.

Es kann angenommen werden, daß zwischen einzelnen Motivationen bzw. Motivationskonfigurationen und der Nutzung unterschiedlicher Einkaufsstätten ebenfalls Abhängigkeiten bestehen. Hierfür spricht nicht zuletzt die relative Eigenständigkeit des Erscheinungsbildes der Geschäftstypen, welche unterschiedliche Erwartungen bezogen auf die Befriedigung der individuellen käufermotivation wecken können. 


\subsection{Informationsverhalten}

Informationsaufnahme, -verarbeitung , -speicherung und -weitergabe begleiten alle Phasen des Kaufentscheidungsprozesses von Antiquitätenkäufern. Operationalisierung und Einordnung einzelner Teilaspekte und -dimensionen des Informationsverhaltens werden in sehr unterschiedlicher weise vorgenommen'. Bezogen auf die spätere Analyse motivationaler Einflußgrößen von Antiquitätenkäufern bietet sich eine Trennung folgender Verhaltensbereiche an:

1. Merkmale des Informationsstils

2. Nutzungsintensitäten unterschiedlicher Informationsquellen

3. thematische Ausrichtung und Schwerpunkte des Informationsinteresses.

\subsection{Merkmale des Informationsstils}

Einen Uberblick über die erhobenen Merkmale zur Kennzeichnung des Informationsstils und ihre typischen Ausprägungen bei den befragten Antiquitätenkäufern gibt die Abbildung 21 .

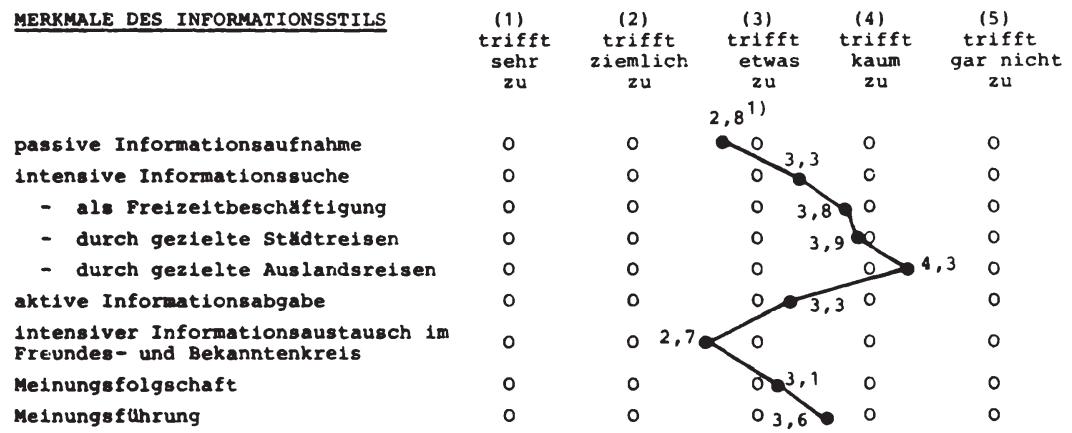$$
2,8^{1)}
$$$$
0 \quad 0 \quad 0,3,0
$$$$
0 \quad 0
$$$$
0 \quad 0
$$$$
0
$$$$
0
$$$$
0
$$$$
\text { o }
$$

0
0
0
0
0
0
0
0
0

Abb. 21: Merkmale des Informationsstils

1 Siehe Fußnote 1 folgende seite 
Unter den erhobenen Typen von Informationsaufnahmeaktivitäten zeigt sich eine deutlich ausgeprägte passive Informationsaufnahmebereitschaft. Intensive Informationssuchanstrengungen werden nur in geringerem Umfang unternommen. Unter Berücksichtigung zusätzlicher "Engagementindikatoren" wie z.B. "Einsatz von Freizeit" oder "gezielte Städte- und Auslandsreisen" nimmt die Suchbereitschaft weiter ab. Das durchschnittliche Niveau der (aktiven und passiven) Informationsaufnahmeaktivitäten "verdeckt" allerdings die individuellen Verhaltensunterschiede, die ihrerseits u.a. durch unterschiedliche käufermotivationen bedingt sein können.

Interessant ist, daß die befragten Antiquitätenkäufer im Vergleich $z u$ ihren Suchaktivitäten ein insgesamt höheres Niveau selbständiger Informationsweitergabe kennzeichnet ${ }^{1}$. Typisch ist dabei ein relativ hohes Austauschniveau, insbesondere im Freundes- und Bekanntenkreis.

Die jeweils zunehmende Bedeutung von Suche, Weitergabe und schließlich Austausch von Informationen über Antiquitäten deutet auf ihren hohen sozialen stellenwert und ihre Beliebtheit als Kommunikationsobjekt hin.

Das hohe Informationstauschniveau ist oftmals Hintergrund und Ausgangspunkt der Entstehung gerichteter Kommunikations-

1 Zum Informationsverhalten von Konsumenten vgl. exemplarisch Kroeber-Riel, W., Konsumentenverhalten, a.a.O., S. 226 f.; Hummrich, U., a.a.O. sowie die Beiträge zu neueren Forschungsergebnissen im Sammelband von Meffert, H., Freter, H., Steffenhagen, H. (Hrsg.), Konsumentenverhalten und Information, Wiesbaden 1979.

$1 \mathrm{Ob}$ aus der "Niveaudiskrepanz" zwischen Aufnahme- und Weitergabeaktivitäten auch auf eine "sachliche Kompetenzlücke" geschlossen werden kann, muß unbeantwortet bleiben. Allerdings wurde das Statement "Es gibt viele Antiquitätenkäufer, die ohne Sachverstand kaufen, aber mit ihren Antiquitäten angeben" von immerhin fast $2 / 3$ der befragten Antiquitätenkäufern als "sehr oder ziemlich zutreffend" angesehen. 
beziehungen im Sinne einer Meinungsführung bzw. Meinungsfolgschaft. Die im Durchschnitt ausgeprägtere Selbsteinstufung der Befragten als Meinungsfolger ist als Indiz für das Bestehen fachlicher Unsicherheit und das Bewußtsein für finanzielle und sozial bedingte Risiken bei Antiquitätenkäufen $z u$ verstehen. Demgegenüber bezeichnen sich die Befragten in etwas kleinerem Umfang als Meinungsführer für Antiquitäten. Die Selbsteinschätzung bezogen auf den Besitz einer fachlichen und sozialen Kompetenz mit der Folge einer informellen Meinungsführerschaft gegenüber anderen Antiquitätenkäufern ist daher vermutlich einem kleineren aber zugleich um so einflußreicheren Kreis von Käufern gemeinsam.

Motivationale Erklärungsbeiträge zur Meinungsführung und Informationssuche sind beispielhaft von Motivationsinhalten zu erwarten, deren Befriedigung eine hohe informative Kompetenz ("Informiertheit") voraussetzt (Sammeln etc.), oder welche eine selbständige ("natürliche") oder gezielte ("dokumentative") Ausstrahlung auf andere besitzen (Besitzerstolz, Streben nach Individualismus etc.). Motivationale Erklärungsbeiträge zur Meinungsfolgschaft sind dagegen insbesondere von Motivationen zu erwarten, bei denen die "Folgschaft" als ökonomisch oder sozial bedingte "Risikoreduzierungsstrategie" interpretiert werden kann (Wertanlage, Spekulation oder Repräsentation mit Antiquitäten etc.).

\subsection{Nutzungsintensitäten von Informationsquellen}

Die Abbildung 22 gibt Auskunft über die Nutzungsintensitäten verschiedener Informationsquellen bei Antiquitätenkäufen. 


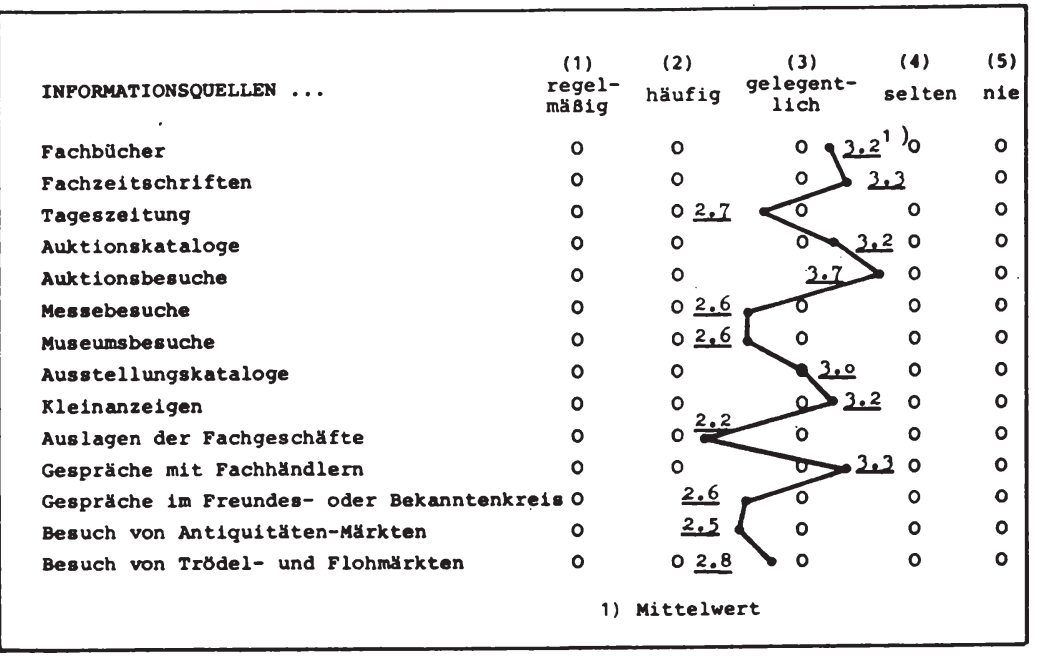

Abb. 22: Nutzung von Informationsquellen

Die im Gesamtüberblick festzustellende mittlere Nutzungsintensität, die weder eine "häufige Nutzung" einer spezifischen Informationsquelle überschreitet, noch aus entgegengesetzter Blickrichtung eine "gelegentliche Nutzung" wesentlich unterschreitet, harmoniert mit dem bereits erörterten mittleren Niveau der Informationssuchaktivitäten von Antiquitätenkäufern.

Für die Informationsgewinnung besitzen Auslagen von Fachgeschäften die relativ größte Bedeutung. Fast ebenso häufig werden Besuche von Märkten, Messen und Museen genannt. Für die Bedeutung bzw. Nutzungsintensität von Informationsquellen scheint die "Nähe zur Antiquität", d.h. die Möglichkeit eines "visuellen Kontakts" oder einer"physischen Warenbegutachtung" ausschlaggebend zu sein. Ferner ist für die am häufigsten genannten Informationsquellen (außer Museen) die Gleichzeitigkeit von einer Kaufaufforderung und Kaufmöglichkeit typisch. Ihre Nutzung begleitet daher in der Regel, insbesondere bei einer bereits latent vorhandenen Kaufabsicht, ein Spannungsverhältnis, welches durch die 
bewußte Wahrnehmung der Einmaligkeit und zeitlichen Begrenztheit der Erwerbschance (Entscheidungsdruck) verstärkt werden kann. Schließlich bieten Messen, Märkte und Museen einen hohen Informationsnutzen. Beispielsweise wird von Museumsbesuchen ein Lerneffekt im Sinne einer "Objektivierung" des "Blicks"für Güte und Qualität erwartet. Auch durch die besondere Angebotsbreite auf Messen und Märkten läßt sich die "Richtigkeit" des subjektiven "PreisQualitäts-Maßstabes" überprüfen und gegebenenfalls revidieren.

Hohe subjektive Nützlichkeit für die sachliche und emotionale "Fundierung" des individuellen Kaufentscheidungsprozesses besitzen ferner Gespräche mit Freunden und Bekannten, die zumeist aufgrund ihrer anscheinenden "Neutralität" als besonders zuverlässige Informationsquellen angesehen werden ${ }^{1}$. Entsprechend überdurchschnittlich ist ihre Nutzungsintensität. Die Verbindung zum bereits erörterten hohen Informationstauschniveau von Antiquitätenkäufern ist offensichtlich.

Allen gedruckten Medien wie Fachbüchern, Fachzeitschriften, Auktions- und Ausstellungskatalogen schenken Antiquitätenkäufer etwas weniger Aufmerksamkeit.

\subsection{Thematische Ausrichtung des Informationsinteresses}

Abschließend ist das Informationsverhalten von Antiquitätenkäufern durch seine thematische Ausrichtung zu kennzeichnen.

1 In dieses Bild paßt die nur geringe Bedeutung von Gesprächen mit Fachhändlern (vgl. Abb. 22), denen zwar i.d.R. eine höhere Sachkompetenz, aber zugleich auch "Informationsbefangenheit" unterstellt werden kann. 


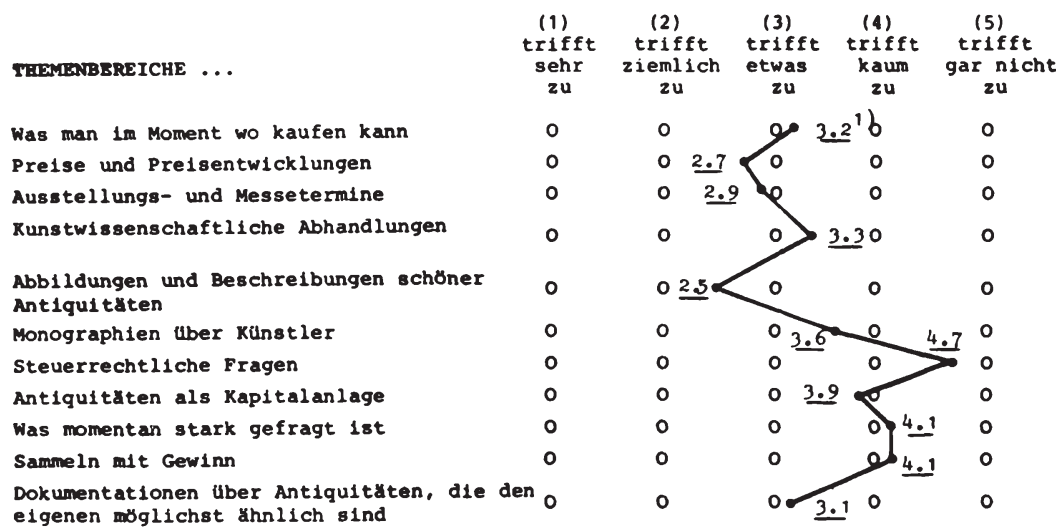

\begin{tabular}{|c|c|c|c|c|}
\hline $\begin{array}{l}\text { (1) } \\
\text { trifft } \\
\text { sehr } \\
\text { zu }\end{array}$ & $\begin{array}{c}\text { (2) } \\
\text { trifft } \\
\text { ziemlich } \\
2 u\end{array}$ & $\begin{array}{c}\text { (3) } \\
\text { trifft } \\
\text { etwas } \\
\text { zu }\end{array}$ & $\begin{array}{l}\text { (4) } \\
\text { trifft } \\
\text { kaum } \\
\text { zu }\end{array}$ & $\begin{array}{c}\text { (5) } \\
\text { trifft } \\
\text { gar nicht } \\
\text { zu }\end{array}$ \\
\hline 0 & 0 & & b & 0 \\
\hline 0 & $0 \quad 2.7$ & & 0 & 0 \\
\hline 0 & $0 \quad 2$. & & 0 & 0 \\
\hline 0 & 0 & & & 0 \\
\hline 0 & $0 \underline{2}$ & 0 & 0 & 0 \\
\hline 0 & 0 & & & .70 \\
\hline 0 & 0 & 0 & & 0 \\
\hline 0 & 0 & 0 & & 0 \\
\hline 0 & 0 & 0 & & 0 \\
\hline 0 & 0 & 0 & & 0 \\
\hline 10 & 0 & & 0 & 0 \\
\hline
\end{tabular}

1) Mittelwert

Abb. 2 3: Interesse an antiquitätenspezifischen Informationsinhalten

Hervorzuheben ist das ausgeprägte Interesse an "ästhetischen Informationsinhalten", das sich in einer hohen Aufmerksamkeit für Abbildungen und Beschreibungen schöner Antiquitäten wiederspiegelt. Obwohl speziell Preisen und Preisentwicklungen ein ebenfalls starkes Informationsinteresse entgegengebracht wird, verfolgt der Kăufer andere wertbezogene Themen wie "Antiquitäten als Kapitalanlage", "Sammeln mit Gewinn" oder "steuerrechtliche Fragen", wenn überhaupt, nur am Rande. Allen anderen abgefragten Themenbereichen wird - wie die Abbildung zeigt - eine durchschnittliche Aufmerksamkeit geschenkt.

Die Abbildung 24 gibt eine Antwort auf die Frage nach der Interessenausrichtung der befragten Antiquitätenkäufer in bezug auf unterschiedliche Sach- und Sammelgebiete. 


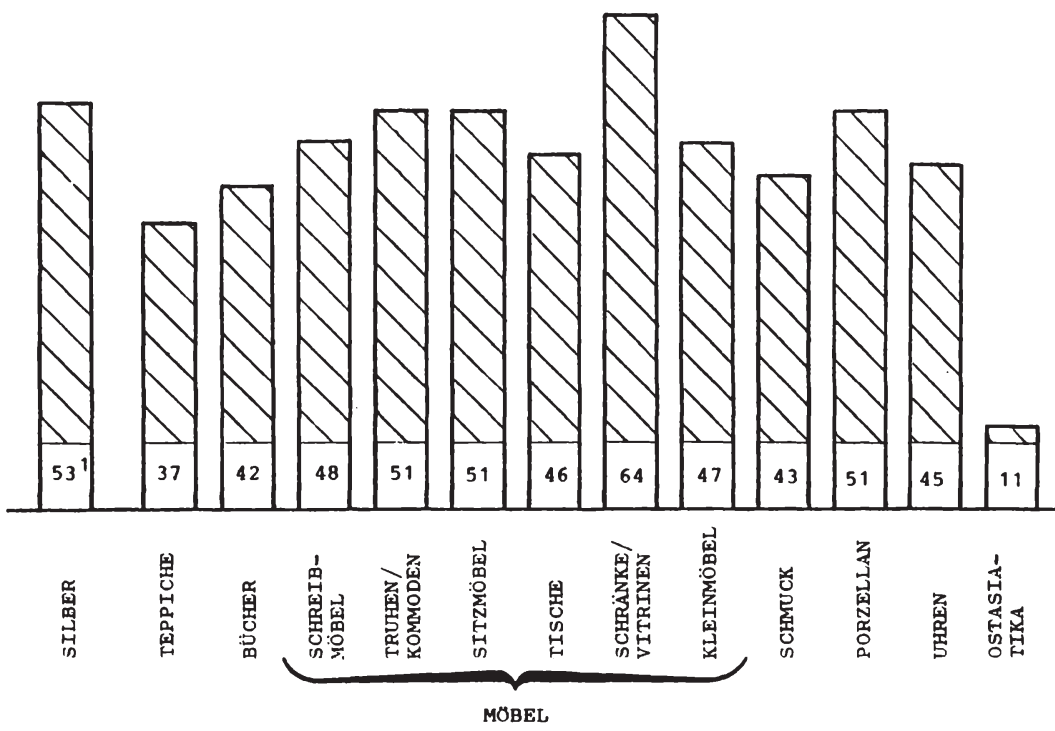

1) Kummulation der Antwortkategorien "sehr" und "ziemlich" auf die Frage nach der Hobe des Interesses an einzelnen Sachgebieten (Angaben in 8 ).

$\mathrm{Abb}, 24:$ Interesse an Sach- bzw. Sammelgebieten

Lediglich antike Möbel wurden als bedeutendstes Sachgebiet von Antiquitäten bei der Befragung in Teilbereiche aufgegliedert. Dabei zeigt der empirische Befund, daß auch innerhalb dieses Sachgebietes - abgesehen von einer leichten Dominanz des Interesses an Schränken und Vitrinen - eine sehr gleichmäßige Verteilung des Käuferinteresses anzutreffen ist. In einer AuBenseiterposition unter den gelisteten Sachgebieten befinden sich Ostasiatika, die vermutlich nur eine begrenzte und spezialisierte Käuferschaft besitzen ${ }^{1}$.

Zusammenfassend ist $z u$ bemerken, daß sich das sachbezogene Interesse auf einem insgesamt hohen Niveau bewegt. Dies deutet zugleich auf eine multithematische Ausrichtung unter den Antiquitätenkäufern hin.

1 In einer zusätzlichen offenen Antwortmöglichkeit konnten unter der Rubrik "Sonstiges" weitere Interessensgebiete genannt werden. Davon machten knapp $50 \%$ der Befragten Gebrauch. $\mathrm{Zu} \mathrm{ca}$. 208 wurden ausgesprochene Spezialinteressen (Apothekeninventar, Tabakdosen, Instrumente etc.) genannt. 
C. Die Motivation von Antiquitätenkäufern - Erklärungsansätze und ihre empirische Prüfung

Auf der Grundlage der vermittelten Kenntnisse über Charakteristiken von Antiquitäten, Antiquitätenmarkt und Antiquitätenkäufern, widmet sich das folgende Kapitel einer intensiven Analyse der Käufermotivation. Zur Verdeutlichung des methodischen Vorgehens und zur Abgrenzung der wichtigsten inhaltlichen Problemkreise dient der zunächst vorgestellte generelle Bezugsrahmen der Motivationsanalyse. Im Sinne eines programmatischen Leitfadens spiegelt er zugleich Aufbau und Ablauf der sich anschließenden Darstellung von empirischen Ergebnissen wider.

\section{Bezugsrahmen motivationaler Erklärungsansätze}

Der in Abbildung 25 gezeigte Bezugsrahmen ordnet die Käufermotivation in den Kontext der als problemrelevant abgegrenzten und empirisch untersuchten Merkmale von Antiquitätenkäufern ein. Dabei entsteht ein richtungsgebundenes Beziehungsgefüge von unabhängigen ("erklärenden") und abhängigen. ("zu erklärenden") Variablen: Motivationale Anreize warenbezogener und situativer Art bestimmen und beeinflussen die Käufermotivation, welche ihrerseits die motivationsgeleiteten Verhaltensweisen lenkt. Die Motivation selbst wechselt als "zentrales Konstrukt" des Erklärungsansatzes ihre Rolle als abhängige und unabhängige Variable.

Das Spektrum individueller Motivationen, verstanden als personen-, situations- und warenspezifisch aktualisierte Beweggründe des Kaufverhaltens, ist gerade bei Antiquitäten inhaltlich besonders vielschichtig. Diese Tatsache beruht im wesentlichen darauf, daß mit Antiquitäten - im Gegensatz zur überwiegenden Zahl "uniformer" Konsumgüter - 
Involvement

Kognitive Konflikte

Preisbereitschaft

Anforderungshal tungen

$\rightarrow$ Einkaufsstätten

$\rightarrow$ Kaurbedeutung von Warenmerkmalen

Interessenausrichtung

\begin{tabular}{|} 
KONATIVE VERHALTENSMERKMALE \\
Typen und Träger der \\
Kaufentscheidung \\
Nutzung von Einkaufs- \\
stätten \\
Informationsverhalten \\
$\rightarrow$ Informationsstil \\
$\rightarrow$ Informationsquelien \\
$\rightarrow$ Informationsinhalte
\end{tabular}

B: Univarlate Analysemethoden (VAR) = Einfache Varianzanalyse (CROSS) $=$ Kontingenzanalyse (Cross - tabs)

A: Multivariate Analysemethoden

(REG) = Multiple (stufenweise) Regressionsanalyse mit Vorgabe von Inklusionsparametern

(FAK) = Faktorenanalyse

$($ CLUS $)=$ Clusteranalyse

- hierarchisch - agglomerative Verfahren

- partitionierende Verfahren (IIIl-Climbing/Iterierte

- gemischtes "Optim.

$($ DISK $)=$ Diskriminanzanalyse

Abb. 25: Bezugsrahmen motivationaler Erklärungsansätze 
als "einzigartige" Individualgüter die unterschiedlichsten funktionalen, psychischen, sozialen und ästhetischen Nutzenvorstellungen und -erwartungen verknüpft sein können. Dabei stellen sich Motivationen als nicht direkt beobachtbare, hypothetische Konstrukte der Verhaltensexplikation dar, welche erst durch eine empirische Operationalisierung Bedeutungsgehalt gewinnen.

Als erster wichtiger Problembereich der Motivationsanalyse stellt sich daher die Aufgabe, Einzelmotivationen der Antiquitätenkäufer auf der Grundlage eines umfassenden "Motivationskatalogs" zu explorieren und ihre (durchschnittliche) Bedeutung bei den Antiquitätenkäufern zu ermitteln. Dies schließt die Frage nach einer möglichen "Verdichtung" von Einzelmotivationen auf eine geringere Anzahl "übergeordneter" und inhaltlich (statistisch) unabhängiger Motivationsdimensionen ein. Unter methodischen Gesichtspunkten bietet sich hierbei die Faktorenanalyse als adäquates Auswertungsverfahren an ${ }^{1}$.

Darauf aufbauend wird ein tiefergehendes Verständnis der erhobenen Einzelmotivationen von der Bestimmung der spezifischen Motivationsdeterminanten erwartet. Dabei ist zu klären, in welcher "Richtung" und "Intensität" die jeweiligen Kaufmotivationen in ihrer Kaufbedeutung verstärkt (gefördert) bzw. abgeschwächt (gehemmt) werden ${ }^{2}$.

$1 \mathrm{zu}$ zielsetzung, Verfahren und Ergebnisinterpretation faktorenanalytischer Auswertungen vgl. das Kapitel

B. 1.22 und die dort angegebene Literatur.

2 Die in der Abbildung 29 aufgeführten Gruppen von Bestimmungsgrößen fächern sich in der empirischen Analyse in mehr als 110 Einzelvariablen, deren Erklärungsbeiträge für die Motivexplikation unterschiedlich und zudem interdependent sind. 
Ein wichtiges Analyseproblem besteht daher in der Isolation eines überschaubaren Sets von Bestimmungsfaktoren, welches unter Berücksichtigung gegenseitiger Abhängigkeiten die wichtigsten Einflußgrößen jeder Einzelmotivation zusammenfast. Eine analoge zielsetzung verfolgt (rechentechnisch) die multiple, stufenweise Regressionsanalyse,die sich hierdurch als geeignetes statistisches Analyseverfahren ausweist ${ }^{1}$.

Trägt die Analyse von Einzelmotivationen in erster Linie $z u$ einem partiell-inhaltlichen Verständnis des Motivationskonstrukts bei, so ist andererseits die implizite Annahme eines ausschlieblich monothematisch motivierten Antiquitätenkäufers nur wenig realitätsnah; denn Antiquitätenkäufer besitzen in der Regel eine vielzahl unterschiedlicher Beweggründe, welche im gegenseitigen Wechselspiel, d.h. gemeinschaftlich, ihr Verhalten initiieren.

Das innere Verhältnis der Motive zueinander z.B.,im Sinne einer Unterscheidung von Haupt- und Nebenmotiven, kennzeichnet die Motivstruktur der Käufer ${ }^{2}$. Dabei erscheint die Annahme sinnvoll, daB zwar die individuelle Motivstruktur eines einzelnen Kăufers tendenziell einzigartig ist, sich aber gleichzeitig bestimmte warenspezifische strukturtypen herausbilden und empirisch abgrenzen lassen, die jeweils einer größeren Anzahl von Käufern zumindest annäherungsweise gemeinsam sind. Solche strukturtypen oder auch

1 Die statistischen Auswertungen wurden mit dem SPSSProgrammaket "REGRESSION", wie es im Rechenzentrum der Westf. Wilhelms-Universität Münster implementiert ist, gerechnet. Zur Programmbeschreibung vgl. Beutel, P. et al., SPSS 7, a.a.0.; S. 157 ff.; Kim, I.O., Kohout, F.J., Multiple Regression Analysis: Subprogram Regression, in: SPSS, Nie, N.H. et al., a.a.O., S. $320 \mathrm{ff.;}$ zur spezifischen Verfahrensweise der "stufenweisen" Regressionsanalyse vgl. insbesondere Schuchard-Ficher, Chr. et al., a.a.0., s. $76 \mathrm{ff.}$

2 Vgl. Wiswede, G., a.a.O., S. $89 \mathrm{ff..}$ 
Motivkonfigurationen werden demnach durch Käufergruppen (Motivationstypen) repräsentiert, deren Motivstrukturen innerhalb der Gruppen weitgehend homogen,im Gruppenvergleich jedoch weitgehend heterogen sind. Die empirische Motivationsanalyse verfolgt dabei zunächst die Fragestellung, ob sich aus dem erhobenen Datenmaterial inhaltlich sinnvoll abgrenzbare Motivationskonfigurationen (Typen) ableiten lassen. Unter methodischen Gesichtspunkten handelt es sich um ein klassifikatorisches Problem, zu dessen Lösung clusteranalytische Auswertungsverfahren beitragen können ${ }^{1}$.

Darüber hinaus stellt sich die Frage, ob und in welchem Ausmaß Motivationstypen auch hinsichtlich der untersuchten personen-, waren- und situationsspezifischen Motivationsdeterminanten unterschiedliche strukturen aufweisen.

Ebenso wie bei der Bestimmung der Einflußgrößen von Einzelmotivationen gilt es, dasjenige set von Bestimmungsfaktoren $\mathrm{zu}$ isolieren, durch welches - unter Berücksichtigung bestehender gegenseitiger Abhängigkeiten - die gefundenen Typen am eindeutigsten diskriminiert, d.h. voneinander abgegrenzt werden können. Methodisch handelt es sich um eine Aufgabenstellung der multivariaten, stufenweisen Diskriminanzanalyse ${ }^{2}$.

1 Die clusteranalytischen Auswertungen stützen sich überwiegend nicht auf die im SPSS implementierten Verfahren. $\mathrm{Zu}$ detaillierten Angaben vgl. Kapitel C. 3.1. $\mathrm{Zu}$ einem allgemeinen Uberblick über clusteranalytische Verfahren vgl. Steinhausen, D., Langer, K., Clusteranalyse, Berlin-New York 1977, S. 6o ff.; Schuchard-Ficher, Chr. et al., a.a.0., S. 107-150.

2 Für die diskriminanzanalytische Auswertung wurde wiederum auf das SPSS-Programmpaket und zwar auf das Subprogramm "DISCRIMINANT" zurückgegriffen. Vgl. Klecka, W.R., Discriminant Analysis, in: SPSS, Nie, N.H. et al., a.a.O., S. 434-467; Beutel, P., a.a.O., S. 174-181 sowie zu einer anwendungs orientierten Einführung Schuchard-Ficher, Chr., et al., a.a.0., s. 151-211. 
Letztendich dient die Analyse und Exploration von Einzelmotiven und Motivationskonfigurationen der Beantwortung der Frage nach dem "Warum", d.h. nach den wichtigsten motivationalen Determinanten des Verhaltens von Antiquitätenkäufern. Als hypothetisch motivationsgeleitete Verhaltensmerkmale wurden alle im Kapitel B. 3.2 bereits näher analysierten kognitiven und konativen Verhaltensbereiche angesehen ${ }^{1}$.

Die Ermittlung der Richtung und Stärke des motivationalen Einflusses beinhaltet zwei Untersuchungsebenen: Zum ersten die Analyse hypothetischer Ursache-Wirkungs-Zusammenhänge zwischen Einzelmotivationen und Verhaltensmerkmalen, sowie zweitens diejenigen zwischen Motivationskonfigurationen und Verhalten ${ }^{2}$. Eine vergleichende Darstellung der auf beiden Ebenen gewonnenen empirischen Ergebnisse bildet daher den AbschluB der Untersuchung motivationaler Erklärungsansätze des Verhaltens von Antiquitätenkäufern.

\section{Motivationen und ihre Bestimmungsfaktoren}

Bildet der theoriegeleitete Bezugsrahmen den Ausgangspunkt der empirischen Untersuchung, müssen die Käufermotivationen für das konkrete Forschungsvorhaben operationalisiert werden. Hierzu erfolgt zunächst eine Präzisierung der einzelnen Motivationsinhalte, ihrer Kaufbedeutungen und ihrer Inhaltsdimensionen.

1 Die in Abbildung 25 aufgefuhrten Variablengruppen fächern sich in ca. 120 erhobene Einzelvariablen auf.

2 In Abhängigkeit vom unterstellten MeBniveau (Skalenniveau) der Einzelmotive ("metrisch") bzw. der Motivationstypen ("nominal") bieten sich als geelgnete statistische Analyseinstrumente im ersten Fall multiple schrittweise Regressionsanalysen und im zweiten Fall einfache Varianzanalysen an. Vgl. u.a. Bleymüller, J., Gülicher, H., Gehlert, G., Statistik für Wirtschaftswissenschaftler, in: Wist 1978, S. $369 \mathrm{ff.;}$ Sachs, L., Angewandte Statistik, Planung und Auswertung, Methoden und Modelle, 4. Aufl., Berlin usw. S. 107; Yamane, T., Statistik, Frankfurt 1976, S. 331 ff.; Green, P.E., a.a.O., S. $50 \mathrm{ff.;}$ Schuchard-Ficher, Chr., a.a.o., s. $3 \mathrm{ff.}$. 


\subsection{Operationalisierung}

\subsection{Bedeutung einzelner Motivationsinhalte}

Abbildung 26 zeigt die zur Ermittlung der Bedeutung einzelner Motivinhalte verwendeten Motivumschreibungen in Form von "Kurztiteln" sowie ihre durchschnittliche "Kaufrelevanz" (vgl. durchgezogenes Mittelwertprofil) bei den befragten Antiquitätenkäufern ${ }^{1}$.

Im Gesamtüberblick fällt die enorme Spannweite der Kaufbedeutungen (Mittelwerte zwischen 2,03 und 4,83) einzelner Motivationsinhalte auf. Das Auftreten z.T. verhältnismäßig niedriger Mittelwerte spricht dafür, daß der abgefragte Motivkatalog auch spezifische, d.h. nur für kleinere Käufergruppen relevante Einzelmotive enthält ${ }^{2}$.

Als dominierende Einzelmotive des privaten Käufers weisen sich die "Lösung eines funktionalen Einrichtungsproblems", das "Bedürfnis nach Ästhetik", der "Wunsch mit Antiquitäten die eigene Wohnung zu dekorieren", der "Spaß bei der Suche nach einer Antiquität" und die "Freude an schönen und wertvollen Naturstoffen" aus. Der inhaltliche Zusammenhang dieser Einzelmotive zeigt deutlich, wie sehr Antiquitäten von ihren heutigen Käufern als integraler Bestandteil der Wohneinrichtung angesehen werden und wie stark das Wohnen

1 Die Fragestellung lautete: "Es gibt verschiedene Gründe, sich Antiquitäten zu kaufen. Wir nennen Ihnen im folgenden eine Reihe solcher Gründe. Bitte geben Sie an, wie wichtig diese Gründe für Sie persönlich bei einem Antiquitätenkauf sind". Zum vollen Wortlaut der Motivitems vgl. Frage 5 im Originalfragebogen im Anhang.

2 Gleichzeitig ist hierin ein Hinweis auf die "Vollständigkeit" des zugrundegelegten Motivkatalogs zu sehen. 


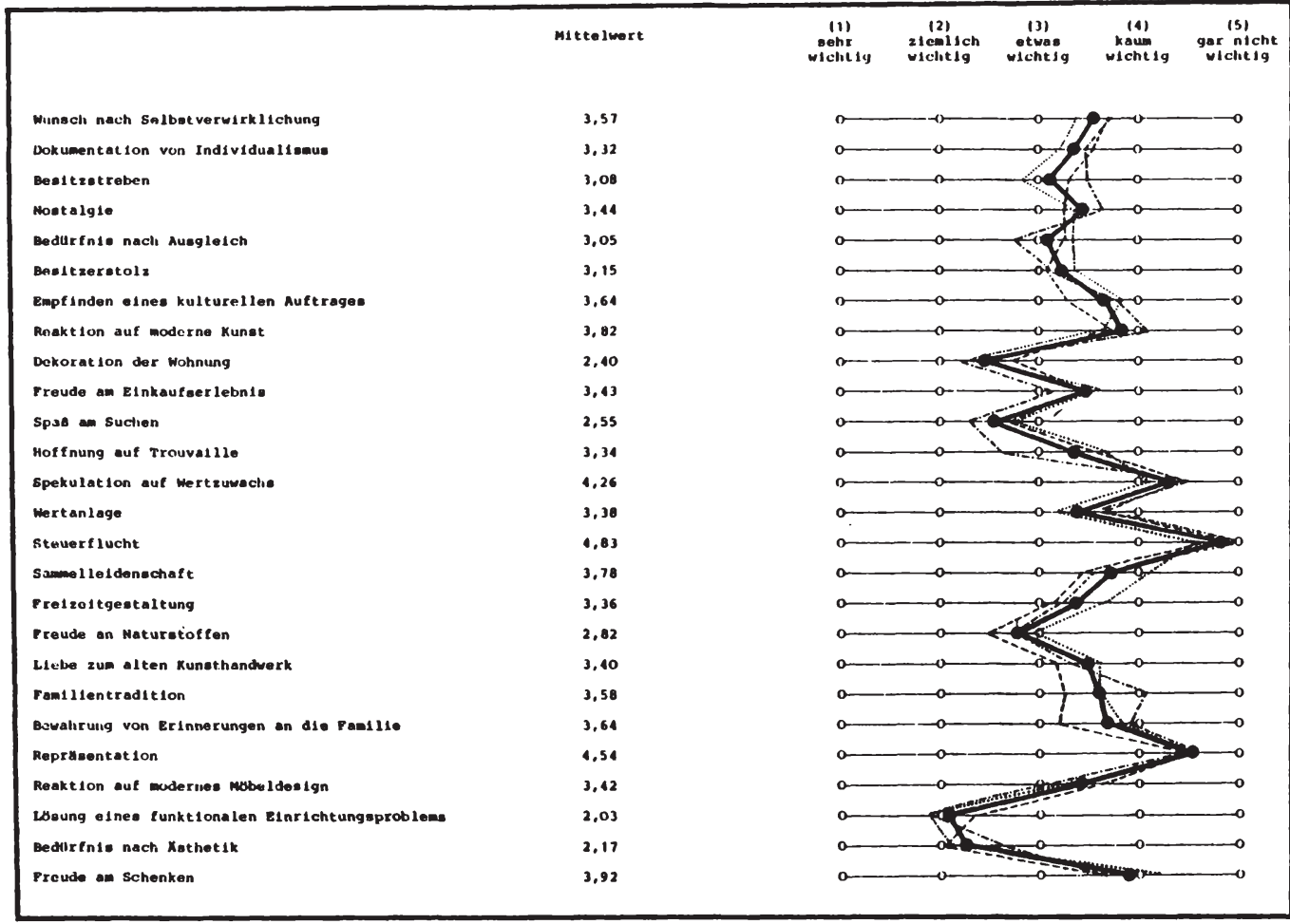

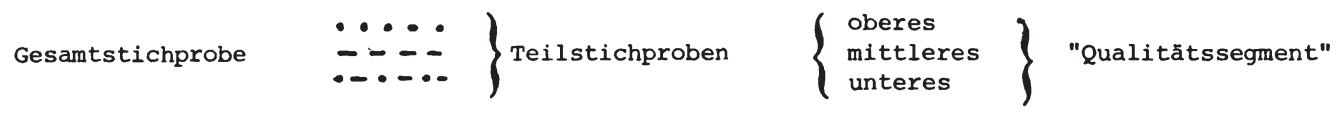

Abb. 26: Bedeutung einzelner Motivationsinhalte als Beweggrund eines Antiquitätenkaufs 
mit Antiquitäten als Kristallisationspunkt der Motivationsentstehung wirkt. Auf einen weiteren,grundsätzlichen Motivationshintergrund verweist das Motiv "Spaß am Suchen". Mit seiner wider Erwarten hohen Bedeutung hebt es die ausgeprägte "Eigenwertigkeit" des Antiquitätenkaufs im Sinne eines "Konsumerlebnisses" hervor.

Eine unterdurchschnittlich eingestufte Motivation ist die Sammelleidenschaft. Dies ist als überraschendes Ergebnis $z u$ werten, welches die häufig anzutreffende Gleichsetzung des "Kaufens" und "Sammelns" von Antiquitäten relativiert. Der private Käufer scheint hier eine zutreffendere Differenzierung vorzunehmen, da er sich nicht allein aufgrund des Besitzes u.U. mehrerer Antiquitäten schon als "Sammler" bezeichnet.

Geringe Bedeutung in der durchschnittlichen (Selbst-) Einschätzung der befragten Antiquitätenkäufer besitzen insbesondere die Motive "Freude am Schenken von Antiquitäten" und die materiell ausgerichteten Motive "Spekulieren auf einen hohen Wertzuwachs" und "Wahrnehmung steuerlicher Vorteile durch einen Antiquitätenkauf" (Steuerflucht) Unter $d \in m$ Gesichtspunkt einer Individualanalyse schliebt dies eine im Einzelfall dominante Kaufrelevanz der genannten Motivationsinhalte nicht aus.

Alle weiteren abgefragten Einzelmotive bewegen sich in einem mitteleren (durchschnittlichen) Zustimmungsbereich, wobei z.T. erhebliche individuelle Bedeutungsunterschiede durch die Mittelwertanalyse "verdeckt" werden. Ein Beispiel ist das "Wertanlagemotiv" mit einem Mittelwert von 3,4, welches von $7 \&$ als sehr, $22 \%$ als ziemlich, $23 \%$ als etwas, weiteren 24 \& als kaum und schließlich 25 als gar nicht wichtiger Kaufgrund angegeben wird. 
Zusätzlich zum Mittelwertprofil der Gesamtstichprobe sind in Analogie zum Vorgehen bei der Bestimmung der käuferbezogenen Begriffsauffassung ${ }^{1}$ - diejenigen der zugrundeliegenden Teilstichproben aufgezeigt. In Ergänzung zur bereits diskutierten Kongruenz der Käufervorstellungen über Antiquitäten zeigt sich hier eine ähnlich hohe Ubereinstimmung bezogen auf die Bedeutung einzelner Kaufmotivationen.

Die Homogenität der Einschätzungen in den Teilstichproben legt den Schluß nahe, daß die Käufer in einer "subjektiven Realität leben", die von den "objektiven Gegebenheiten", insbesondere der Qualität der gekauften Antiquitäten, tendenziell unabhängig ist. So kann beispielsweise angenommen werden, daß die Käufer von "Trödel" und die Käufer von "hochklassigen Antiquitäten" überwiegend in ihrer Einschätzung der Kaufobjekte übereinstimmen und auch weitgehend ähnliche Kaufmotivationen besitzen. Hierin liegt ein deutlicher Hinweis auf die stark motivational geleitete und subjektiv verzerrte Wahrnehmung der Befragten von Antiquitäten; eine Erscheinung, die zumeist bei einer intensiven emotionalen (affektiven) Beziehung zu einem Meinungsgegenstand anzutreffen ist.

Die gezeigten Mittelwertprofile dienen einer ersten $\mathrm{Ab}-$ schätzung der allgemeinen Bedeutung einzelner Inhaltsaspekte der Käufermotivation. Dabeibleibt die Frage ausgeklammert, inwieweit die semantischen Motivumschreibungen inhaltlich unabhängige Teilaspekte der Käufermotjvation erfassen. In einem weiteren Analyseschritt soll daher versucht werden, übergeordnete Inhaltsdimensionen der Käufermotivation durch eine faktorenanalytische Verdichtung des Motivkataloges zu ermitteln.

1 Vgl. Kapitel B. 1.23. 


\subsection{Dimensionen der Motivation}

Auf der Grundlage korrelativer Beziehungen lassen sich die 26 abgefragten Einzelmotive faktorenanalytisch auf neun Inhaltsdimensionen (Faktoren) reduzieren, welche knapp die Hälfte der durch die Einzelmotive gegebenen Ausgangsinformationen (Ausgangsvarianz) reproduzieren (vgl. Tabelle 7)! Die Höhe des gemeinsamen faktoriellen Erklärungsbeitrages ist als eindeutiges zeichen dafür zu werten, daß es grundsätzlich möglich und sinnvoll ist, zentrale Inhaltsbereiche der Käufermotivation abzugrenzen.

Betrachtet man die Gruppierung der Einzelvariablen und ihre Korrelationen $\mathrm{zu}$ den jeweiligen Inhaltsdimensionen ( $\mathrm{vgl}$. Faktorladungen), so lassen sich relativ gut abgrenzbare Beschreibungen für die extrahierten Dimensionen finden. Die folgende Dimensionsinterpretation führt damit zugleich zu einem tiefergehenden Einblick und Verständnis genereller Aspekte der Käufermotivation.

Mit einem relativen Erklärungsanteil von fast 35 of erweist sich das Streben nach Selbstverwirklichung und Selbstdarstellung als die strukturell bedeutendste Inhaltsdimension. Die Begriffsfindung lehnt sich im wesentlichen an die beiden Einzelmotive "Wunsch nach Selbstverwirklichung" und "Dokumentation von Individualismus" an, welche die eindeutig engsten Beziehungen zur übergeordneten

1 Die Varianzerklärungsanteile der Tabelle 7 beziehen sich auf die vollständige, rotierte Faktorladungsmatrix, welche hier nur im Auszug,d.h. unter Berücksichtigung der aufgrund hoher Faktorladungen diminierenden Einzelmotive je Inhaltsdimension, wiedergegeben ist. Die Vielzahl extrahierter Inhaltsdimensionen sowie die tendenziell ausgeglichenen "relativen" Varianzerklärungsanteile der Dimensionen 2,3 und 4 bis 8 weisen auf das insgesamt breite Spektrum relevanter Kaufmotivationen auch aus struktureller sicht hin. 


\begin{tabular}{|c|c|c|c|}
\hline \multicolumn{2}{|c|}{ DIMENSIONEN DER MOTIVATION VON ANTIQUITÄTENKÄUFERN } & $\begin{array}{l}\text { relativ } \\
\text { erklarter } \\
\text { Vartanz- } \\
\text { anteil in } 8 \\
\end{array}$ & $\begin{array}{l}\text { gesamt } \\
\text { erklärter } \\
\text { Varianz- } \\
\text { antell in }\end{array}$ \\
\hline \multicolumn{2}{|l|}{$\begin{array}{l}\text { SREBEN NACH SELBSTVERWIRKLICHUNG } \\
\text { UND SELBSTDARSTELLUNG }\end{array}$} & \multirow[b]{2}{*}{$34,8 \quad 8$} & \multirow[b]{2}{*}{17,228} \\
\hline $\begin{array}{l}\text { Wunsch nach Selbstverwirklichung } \\
\text { Dokumentation von Individualismus } \\
\text { Besitzstreben } \\
\text { Nostalgie } \\
\text { Bedurinis nach Ausgleich } \\
\text { Besitzerstolz } \\
\text { Empfinden aines kulturellen Auftrags } \\
\text { Reaktion auf noderne Kunst } \\
\text { Dekoration der Wohnung }\end{array}$ & $\begin{array}{c}\text { Faktor ladungen } \\
0,74 \\
0,61 \\
0,50 \\
0,50 \\
0,42 \\
0,40 \\
(0,38) \\
(0,36) \\
(0,27)\end{array}$ & & \\
\hline \multicolumn{2}{|l|}{ STREBEN NACH KONSUMERLEBNIS } & \multirow[b]{2}{*}{13,98} & \multirow[b]{2}{*}{24,108} \\
\hline $\begin{array}{l}\text { Freude am Elnkaufserlebnis } \\
\text { SpaB am Suchen } \\
\text { Iloffnung auf eine Trouvalle }\end{array}$ & $\begin{array}{c}\text { Faktorladungen } \\
0,77 \\
0,63 \\
0,51\end{array}$ & & \\
\hline \multicolumn{2}{|l|}{ ANTIQUITATENKAIJF ALS OKONOMISCHES KALK!IL } & \multirow[b]{2}{*}{12,68} & \multirow[b]{2}{*}{$30,34 \%$} \\
\hline $\begin{array}{l}\text { Spekulation auf wertzuwachs } \\
\text { Wertanlage } \\
\text { Steuerflucht }\end{array}$ & $\begin{array}{c}\text { Faktor ladungen } \\
0,90 \\
0,65 \\
0,47\end{array}$ & & \\
\hline \multicolumn{2}{|c|}{$\begin{array}{l}\text { SAMMELN VON ANTIQUITATEN ALS FREIZEITGESTALTENDES } \\
\text { HOBBY }\end{array}$} & \multirow[b]{2}{*}{9,38} & \multirow[b]{2}{*}{34,958} \\
\hline $\begin{array}{l}\text { Sammelleldenschaft } \\
\text { Frelzeitgestaltung }\end{array}$ & $\begin{array}{c}\text { Faktorladungen } \\
0,85 \\
0,65 \\
\end{array}$ & & \\
\hline
\end{tabular}

Tab. 7: Inhaltsdimensionen der Motivation von Antiquitätenkäufern Ergebnisüberblick einer Faktorenanalyse (Teil I) 


\begin{tabular}{|c|c|c|c|}
\hline DIMENSIONEN DER MOTIVATION VON ANTIO & NKÄUFERN & $\begin{array}{l}\text { relativ } \\
\text { erklärter } \\
\text { varianz- } \\
\text { anteil in }\end{array}$ & $\begin{array}{l}\text { gesamt } \\
\text { erkläter } \\
\text { Varianz- } \\
\text { anteil in }\end{array}$ \\
\hline \multicolumn{2}{|l|}{ LIEBHABEREI } & \multirow[b]{2}{*}{7,48} & \multirow[b]{2}{*}{38,598} \\
\hline $\begin{array}{l}\text { Freude an Naturstof fen } \\
\text { L1ebe zum alten kunsthandwerk }\end{array}$ & $\begin{array}{c}\text { Faktorladungen } \\
0,64 \\
0,58\end{array}$ & & \\
\hline \multicolumn{2}{|l|}{ FAMILIAR BFDINGTE MOTIVATION } & \multirow[b]{2}{*}{6,88} & \multirow[b]{2}{*}{41,968} \\
\hline $\begin{array}{l}\text { Famillentradition } \\
\text { Rewahrung von Erinnerungen } \\
\text { an die Familie }\end{array}$ & $\begin{array}{c}\text { Faktorladungen } \\
0,71 \\
0,70\end{array}$ & & \\
\hline \multicolumn{2}{|l|}{ REPRASENTATION } & \multirow[b]{2}{*}{6,1 웅 } & \multirow[b]{2}{*}{44,988} \\
\hline Repräsentation & $\frac{\text { Faktor ladung }}{0,96}$ & & \\
\hline \multicolumn{2}{|l|}{ ENDOGENER GESCHMACKSWANDEL } & \multirow[b]{2}{*}{$5,2 \because$} & \multirow[b]{2}{*}{47,568} \\
\hline $\begin{array}{l}\text { Reaktion auf inodernes Möbeldesign } \\
\text { Lösung eines funktionalen } \\
\text { Einrichtungsproblems }\end{array}$ & $\begin{array}{c}\text { Faktor ladungen } \\
0,83 \\
0,16\end{array}$ & & \\
\hline \multicolumn{2}{|l|}{ STREBEN NACH ASTHETIK } & \multirow[b]{2}{*}{3,98} & \multirow[b]{2}{*}{49,548} \\
\hline \multirow[t]{2}{*}{$\begin{array}{l}\text { Bedurfnis nach Asthetik } \\
\text { Freude am Schenken }\end{array}$} & $\begin{array}{c}\text { Faktor ladungen } \\
0,44 \\
(0,371\end{array}$ & & \\
\hline & & $100,00 \%$ & $49,54 \%$ \\
\hline
\end{tabular}

Tab. 7: Inhaltsdimensionen der Motivation von Antiquitätenkäufern Ergebnisüberblick einer Faktorenanalyse (Teil II) 
Dimension aufweisen. Darüber hinaus sind die vier weiteren

Einzelmotive "Besitzstreben", "Nostalgie", "Bedürfnis nach Ausgleich" und "Besitzerstolz" interpretationsrelevant". Die Dimension "Streben nach Selbstverwirklichung und Selbstdarstellung" zeigt sich damit als komplex strukturiert und inhaltlich breit gefächert. Eine mögliche Erklärung ist darin zu sehen, daß es sich um ein "hochorganisiertes" bzw. "abstraktes" menschliches Grundmotiv handelt, dessen individual-psychologische Bedeutung weit über den betrachteten warenspezifischen Zusammenhang hinausgeht.

Das Streben nach Konsumerlebnis trägt als zweitbedeutendste Inhaltsdimension knapp $14 \%$ Varianzerklärungsanteil. Sie faßt ein Set von Einzelmotiven zusammen, welche einen offensichtlichen und eher monothematischen Zusammenhang aufweisen: die "Freude am Einkaufserlebnis" konkretisiert sich im "Spaß am Suchen" und der "Hoffnung auf eine Trouvaille". Der Einkauf von Antiquitäten beinhaltet anscheinend eine eigenständige "Wertigkeit", wobei die motivierende "Kraft" auf der Erlebniserwartung der Käufer beruht.

1 Faktorladungen $\leqslant 0,40$ wurden, dem üblichen statistischen Vorgehen folgend, nicht zur Interpretation herangezogen. I.d.R. besitzen Einzelmotive mit niedrigen Faktorladungen zugleich Korrelationen mit mehreren Motivdimensionen, wodurch die Aussagekraft eingeschränkt wird. Andererseits zeigt sich hierbei nochmals der Unterschied zwischen "Kaufbedeutung" und "struktureller (dimensionaler) Bedeutung" von Motivationsinhalten. Gerade diejenigen Einzelmotive mit der höchsten Kaufrelevanz "Lösung eines funktionalen Einrichtungsproblems", "Dekoration der Wohnung" und "Bedürfnis nach Ästhetik" (vgl. Abbildung 28) besitzen nur relativ geringe Zusammenhänge zu den extrahierten Inhaltsdimensionen. Bei entsprechend hohen Einzelrestfaktoren-Varianzen bzw. niedrigen Kommunalitäten (zwischen 0,44 und 0,21 ) weist dies auf ein sehr differenziertes Verhältnis dieser Einzelmotive zu den extrahierten Inhaltsdimensionen hin. 
Mit ca. 13 \& Erklärungsanteil ist das mit einem Antiquitätenkauf verbundene ökonomische Kalkül eine ebenfalls überdurchschnittlich bedeutsame Inhaltsdimension.

Zwei Teilaspekte materiell-ökonomisch ausgerichteter Beweggründe eines Antiquitätenerwerbs werden in den Einzelmotivationen sichtbar: Die "Spekulation auf Wertzuwachs" welche zugleich die Motivdimension inhaltlich dominiert zielt tendenziell auf die Absicht einer Vermögensmehrung, während die "Wertanlage" tendenziell auf den Wunsch einer Vermögenssicherung hindeutet.

Das Sammeln von Antiquitäten als freizeitgestaltendes Hobby, die Liebhaberei, die familiär bedingten Motivationen und die Repräsentation gehören zu einer im strukturellen Erklärungswert fast gleichbedeutenden Gruppe unabhängiger Inhaltsdimensionen. Die jeweils geringe Anzahl relevanter Einzelmotive und deren uberwiegend hohe Korrelation $z u$ den extrahierten Motivdimensionen sorgen für hohe Interpretationstransparenz. Auf eine detaillierte Darstellung kann daher an dieser Stelle verzichtet werden.

Von etwas geringerer struktureller Bedeutung ist mit einem relativen Varianzerklärungsanteil von etwas mehr als 5 \& der endogene Geschmackswandel. Der endogene Geschmackswandel bezieht sich im wesentlichen auf die Nutzung von Antiquitäten als Einrichtungs- bzw. Gebrauchsgegenstände ${ }^{1}$. Sein Ursprung ist dabei schwergewichtig in einer geschmacklichen Bevorzugung von Antiquitäten als "Reaktion auf das moderne Möbeldesign" zu suchen.

1 Das Einzelmotiv "Lösung eines funktionalen Einrichtungsproblems" besitzt hier seine höchste positive Faktorladung. 
AbschlieBend ist das Streben nach Ästhetik als Inhaltsdimension der Käufermotivation zu nennen. Einschränkungen des Aussagegehalts ergeben sich, da weder ein wesentlicher Varianzerklärungsbeitrag, noch eine hohe Korrelation zwischen Dimension und Einzelmotiven ermittelt werden konnte.

Die vorgenommene faktorenanalytische Auswertung erfüllt mit ihrem bislang vorgestellten Teilergebnissen überwiegend inhaltlich strukturierende und deskriptive Funktionen ${ }^{1}$.

Für die Analyse der Käufermotivation und ihrer Bestimmungsgrößen empfiehlt es sich, die extrahierten Inhaltsdimensionen als Bezugsrahmen zu nutzen, im übrigen jedoch die Einzelmotivationen isoliert zu untersuchen.

\subsection{Analyse der Käufermotivationen}

Einen Ergebnisüberblick über die zur Analyse der Käufermotivationen gerechneten Regressionsanalysen gibt Tabelie $8^{2}$.

1 Durch die Ermittlung von Faktorenwerten als Schätzwerte für die Ausprägungen der Befragten auf den unabhängigen Inhaltsdimensionen kann darüber hinaus eine wesentliche Datenverdichtung erreicht werden.

Gegen dieses Vorgehen im Rahmen der vorliegenden Untersuchung sprechen folgende Gesichtspunkte:

(1) Die zu Inhaltsdimensionen "verdichteten" Einzelmotive sind zu abstrakt und"synthetisch", um wichtige Nuancen der Käufermotivation, die u.U. sehr verschiedenartige Verhaltenswirkungen besitzen können, zu erfassen.

(2) Bei einem (gesamten) Varianzerklärungsanteil von ca. $50 \%$,bezogen auf die Ausgangsinformation, entsteht bei der Verwendung von Faktorenwerten in weiterführenden (statistischen) Analysemethoden ein Informationsverlust in mindestens gleicher Höhe.

2 Fußnote s. S. 137. 


\begin{tabular}{|c|c|c|c|}
\hline MOTIVATION & $\begin{array}{l}\text { ANZAHL EINBE- } \\
\text { ZOGENER } \\
\text { ERKLÄARENDER } \\
\text { VARIABLEN }\end{array}$ & $\begin{array}{l}\text { MULTIPLER } \\
\text { KORREIATIONS- } \\
\text { KOEFFIZIENT }\end{array}$ & $\begin{array}{l}\text { MULTIPLES } \\
\text { BESTIMMT- } \\
\text { HETTSMASS }\end{array}$ \\
\hline $\begin{array}{l}\text { LÖSUNG ETNES FUNKTIONALEN } \\
\text { ETNRICHIUNGSPROBLEMES }\end{array}$ & $\begin{array}{l}18^{1} \\
13^{2}\end{array}$ & $\begin{array}{l}0,78 \\
0,75\end{array}$ & $\begin{array}{l}60,47 \\
56,66\end{array}$ \\
\hline DEKORATION DER WOHNUNG & $\begin{array}{l}15 \\
10\end{array}$ & $\begin{array}{l}0,74 \\
0,71\end{array}$ & $\begin{array}{l}55,10 \\
50,44\end{array}$ \\
\hline DOKIMENTATION VON INDIVIDUALISMUS & S $\begin{array}{l}21 \\
21\end{array}$ & $\begin{array}{l}0,71 \\
0,71\end{array}$ & $\begin{array}{l}50,43 \\
50,43\end{array}$ \\
\hline WERIANLAGE & $\begin{array}{l}15 \\
13\end{array}$ & $\begin{array}{l}0,77 \\
0,76\end{array}$ & $\begin{array}{l}58,71 \\
57,15\end{array}$ \\
\hline SPEKULATION AUF WERTZUWACHS & $\begin{array}{l}13 \\
10\end{array}$ & $\begin{array}{l}0,68 \\
0,65\end{array}$ & $\begin{array}{l}45,55 \\
42,13\end{array}$ \\
\hline WUNSCH NACH SEIBSTVERWIRKIICHUNG & $\begin{array}{l}15 \\
11\end{array}$ & $\begin{array}{l}0,67 \\
0,63\end{array}$ & $\begin{array}{l}44,26 \\
39,94\end{array}$ \\
\hline BESITZSTREBEN & $\begin{array}{l}10 \\
10\end{array}$ & $\begin{array}{l}0,64 \\
0,64\end{array}$ & $\begin{array}{l}41,32 \\
41,32\end{array}$ \\
\hline SAMMEILIEIDENSCHAFT & $\begin{array}{l}15 \\
11\end{array}$ & $\begin{array}{l}0,71 \\
0,69\end{array}$ & $\begin{array}{l}50,87 \\
47,34\end{array}$ \\
\hline BEDURFNIS NACH ASTHETIK & $\begin{array}{r}10 \\
8\end{array}$ & $\begin{array}{l}0,62 \\
0,61\end{array}$ & $\begin{array}{l}39,98 \\
36,97\end{array}$ \\
\hline FREUDE AM SCHENKEN & $\begin{array}{l}20 \\
12\end{array}$ & $\begin{array}{l}0,66 \\
0,58\end{array}$ & $\begin{array}{l}43,13 \\
33,51\end{array}$ \\
\hline REAKIION AUF MODERNES MÖBEIDESIGN & N $\begin{array}{l}12 \\
12\end{array}$ & $\begin{array}{l}0,65 \\
0,65\end{array}$ & $\begin{array}{l}42,66 \\
42,66\end{array}$ \\
\hline STEUERFWCHT & $\begin{array}{l}12 \\
12\end{array}$ & $\begin{array}{l}0,59 \\
0,59\end{array}$ & $\begin{array}{l}34,31 \\
34,31\end{array}$ \\
\hline NOSTALGIE & $\begin{array}{l}13 \\
13\end{array}$ & $\begin{array}{l}0,63 \\
0,63\end{array}$ & $\begin{array}{l}40,12 \\
40,12\end{array}$ \\
\hline FREIZETTGESTALTUNG & $\begin{array}{l}18 \\
16\end{array}$ & $\begin{array}{l}0,67 \\
0,65\end{array}$ & $\begin{array}{l}44,87 \\
42,49\end{array}$ \\
\hline
\end{tabular}

Tab. 8 : Erklärungsbeiträge motivationaler Bestimmungsfaktoren für die Kaufbedeutung einzelner Motivationsinhalte - Ergeb nisiuberblick multipler stufenweiser Regressionsanalysen (Teil I) 


\begin{tabular}{|c|c|c|c|}
\hline MOT IVATION & $\begin{array}{l}\text { ANZAHL ETNBE- } \\
\text { ZOGENER } \\
\text { ERKIL̈RENDER } \\
\text { VARIABLEN }\end{array}$ & $\begin{array}{l}\text { MUTIPLER } \\
\text { KORREIATIONS- } \\
\text { KOEFFIZIENT }\end{array}$ & $\begin{array}{l}\text { MULTIPLES } \\
\text { BESTIMMT- } \\
\text { HEITSMASS }\end{array}$ \\
\hline HOFFNUNG AUF TROUVAIILE & $\begin{array}{l}26 \\
23\end{array}$ & $\begin{array}{l}0,83 \\
0,81\end{array}$ & $\begin{array}{l}68,12 \\
65,65\end{array}$ \\
\hline SPASS AM SUCHEN & $\begin{array}{l}19 \\
13\end{array}$ & $\begin{array}{l}0,67 \\
0,61\end{array}$ & $\begin{array}{l}44,75 \\
37,69\end{array}$ \\
\hline FREUDE AM EINKAUFSERLEBNIS & $\begin{array}{l}15 \\
13\end{array}$ & $\begin{array}{l}0,68 \\
0,67\end{array}$ & $\begin{array}{l}46,36 \\
44,37\end{array}$ \\
\hline FAMILIENIRADITION & $\begin{array}{r}15 \\
8\end{array}$ & $\begin{array}{l}0,68 \\
0,60\end{array}$ & $\begin{array}{l}46,17 \\
35,63\end{array}$ \\
\hline BESITZERSTOLZ & $\begin{array}{l}14 \\
12\end{array}$ & $\begin{array}{l}0,65 \\
0,64\end{array}$ & $\begin{array}{l}42,33 \\
40,31\end{array}$ \\
\hline REPRÄSENTATION & $\begin{array}{l}14 \\
13\end{array}$ & $\begin{array}{l}0,68 \\
0,67\end{array}$ & $\begin{array}{l}45,95 \\
45, \infty\end{array}$ \\
\hline BEDURENIS NACH AUSGLEICH & $\begin{array}{l}15 \\
15\end{array}$ & $\begin{array}{l}0,67 \\
0,67\end{array}$ & $\begin{array}{l}44,70 \\
44,70\end{array}$ \\
\hline $\begin{array}{l}\text { EMPFINDEN EINES KULIUREIJEN } \\
\text { AUFTRAGES }\end{array}$ & $\begin{array}{l}17 \\
11\end{array}$ & $\begin{array}{l}0,67 \\
0,62\end{array}$ & $\begin{array}{l}45,14 \\
38,02\end{array}$ \\
\hline REAKTION AUF MODERNE KUNST & $\begin{array}{l}16 \\
15\end{array}$ & $\begin{array}{l}0,72 \\
0,71\end{array}$ & $\begin{array}{l}51,04 \\
50,15\end{array}$ \\
\hline $\begin{array}{l}\text { BENAHRUNG VON ERINRERUINGEN } \\
\text { AN DIE FAMILIE }\end{array}$ & $\begin{array}{r}15 \\
7\end{array}$ & $\begin{array}{l}0,65 \\
0,58\end{array}$ & $\begin{array}{l}42,75 \\
33,76\end{array}$ \\
\hline IIEBE ZUM ALTEN KUNSTHANDWERK & $\begin{array}{l}20 \\
15\end{array}$ & $\begin{array}{l}0,70 \\
0,66\end{array}$ & $\begin{array}{l}48,47 \\
43,52\end{array}$ \\
\hline FREUDE AN NATURSTOFFEN & $\begin{array}{l}15 \\
12\end{array}$ & $\begin{array}{l}0,60 \\
0,57\end{array}$ & $\begin{array}{l}35,78 \\
32,45\end{array}$ \\
\hline \multicolumn{4}{|c|}{$\begin{array}{l}\text { 1. ohne nominalskalierte Variablen } \\
\text { 2. der jeweils erste Zahlenwert gilt für die Voreinstellumgen: } \\
\text { a.) Maximale Anzahl von Auswahlschritten }=30 \\
\text { b.) Inklusionsniveau (F-Wert) für erklärende Variablen }=2,0 \\
\text { c.) Toleranzniveau }=0,5 \\
\text { Der jeweils zweite Zahlenwert gilt für ein "strengeres" Inklusions- } \\
\text { niveau (F-Wert) = } 2,5 \\
\text { Güte aller Regressionsgleichungen : Signifikanzniveau } 99,9 \text { \& }\end{array}$} \\
\hline
\end{tabular}

Tab. 8: Erklärungsbeiträge motivationaler Bestimmungsfaktoren für die Kaufbedeutung einzelner Motivationsinhalte - Ergebnisüberblick multipler stufenweiser Regressionsanalysen (Teil II) 
Dabei zeigt sich, daß die einbezogenen personalen, situativen und warenbezogenen Motivationsdeterminanten - in der Regel zwischen 10 und 20 Variablen - in eindrucksvoller Weise geeignet sind, die Motivationen von Antiquitätenkäufern zu erklären. Bei multiplen Korrelationskoeffizienten (MULT R) bis zu Werten über 0,80 schwanken die Erklärungsanteile zwischen $32 \%$ und $68 \%$ (vgl. multiple Bestimmtheitsmaße) ${ }^{1}$.

Die folgende Detailanalyse der Einzelmotivationen und ihrer spezifischen Einflußgrößen stützt sich auf empirisch faßbare "Je-desto-Beziehungen" im Sinne hypothetischer UrsacheWirkungszusammenhänge. Zwei Varianten der Beeinflussungsrichtung sind denkbar: Entweder kann eine Motivation durch eine zunehmende Ausprägung ihrer Bestimmungsgrößen

2 Fußnote von S. 135. In der vorliegenden Studie uberschritt die Anzahl von Bestimmungsfaktoren die Beschränkungen des SPSS-Programms für unabhängige Variablen bei multiplen Regressionen. Daher wurden zusätzlich Regressionen zur Voroptimierung bzw. Variablenreduktion gerechnet. Zum einen bildeten dabei die situationalen und die warenbezogenen Determinanten (Anreize) und zum anderen die personalen Determinanten einen getrennten Datensatz unabhängiger Variablen. Uber entsprechende Voreinstellungen der Inklusionsparameter des Rechenprogramms wurde die Zahl der in die Hauptoptimierung $\mathrm{zu}$ übernehmenden Bestimmungsfaktoren auf ein rechentechnisch verarbeitbares Maß reduziert. Dies hat zum Folge, daß der Dateninput für die Hauptoptimierung von Motivation zu Motivation variiert.

Als wesentliche Ergebnisse der Voroptimierung (vgl. Tabelle 1 im Anhang) sind zu nennen:

1. Sowohl motivationale Reize als auch personale Dispositionen liefern bereits getrennt gute Erklärungsbeiträge zur Käufermotivation.

2. Die Erklärungsbeiträge beider Variablengruppen sind i.d.R. relativ ausgeglichen.

3. Die simultane Berücksichtigung beider Variablengruppen (vgl. Tabelle 8) führt z.T. zu nochmals wesentlich verbesserten Erklärungsbeiträgen, so daß beide Variablengruppen als "motivationsrelevant" anzusehen sind. 
in ihrer Kaufbedeutung gefördert (verstärkt) oder gehemmt (gemindert) werden ${ }^{1}$. Die inhaltliche Analyse solcher Beziehungen "füllt" das "hypothetische Motivationskonstrukt" mit einem empirischen Bedeutungsgehalt und dient einem intensiveren Verständnis des personen-, waren- und situationsbezogenen Hintergrundes der Käufermotivation.

\subsection{Streben nach Selbstverwirklichung und Selbstdar- stellung}

Es entspricht einem inneren Bedürfnis eines jeden Menschen, die bestehenden Einstellungen, Werte, Ziele, Gedanken und Gefühle mit den ausgeführten Handlungen, Verhaltensweisen und schließlich auch dem persönlichen Besitz in Einklang $z u$ bringen. Der Kauf von Antiquitäten als Ausdruck eines Strebens nach Selbstverwirklichung stellt in diesem Sinne einen "Baustein" der Realisierung einer individuell befriedigenden Existenz dar.

Erkennt der Käufer dabei zugleich in Antiquitäten einen imagebildenden Faktor, tritt der Antiquitätenbesitz bzw. -erwerb in ein Mittel-Zweck-Verhältnis zur bewußten und unbewußten Persönlichkeitsdarstellung.

Dem Streben nach Selbstverwirklichung ordnen sich als inhaltliche Bezugspunkte schwerpunktartig die Motivinhalte "Nostalgie", "Bedürfnis nach Ausgleich", "Empfinden eines

1 Als statistische "Maßzahl" für "Stärke" und "Richtung" des Einflusses dienen im folgenden die "Höhe" und das "Vorzeichen" der standardisierten Regressionskoeffizienten. Zur Interpretation vgl. Schuchard-Ficher, Chr. et al., a.a.O., S. 74 f.. Die standardisierten Regressionskoeffizienten werden in den folgenden Abbildungen 31-38 sowie 40 und 41 unter der Bezeichnung "beta" ausgewiesen. Die Standardisierung macht die Wirkungen der motivationalen Bestimmungsfaktoren auf die Motivation untereinander vergleichbar. Die standardisierten Regressionskoeffizienten werden in den folgenden Abbildungen 27 - 34 sowie 36 und 37 unter der Bezeichnung "beta" ausgewiesen. Die Standardisierung macht die Wirkungen der motivationalen Bestimmungsaktoren auf die Motivation untereinander vergleichbar. 
kulturellen Auftrags" und "Reaktion auf moderne Kunst" zu. Das "Besitzstreben", der "Besitzerstolz", die "Dekoration der Wohnung" sowie insbesondere die "Dokumentation von Individualismus" akzentuieren dagegen eher das streben nach Selbstdarstellung.

Die empirisch erhobenen Bestimmungsfaktoren der Einzelmotivationen zeigt die Abbildung 27 !

Aufgrund hoher Korrelation und sprachlicher Identität mit der übergeordneten Inhaltsdimension ist die Einzelmotivation "Wunsch nach Selbstverwirklichung" von besonderem Interesse.

Erwartungsgemäß weist die Regressionsanalyse personale Variablen als die wichtigsten und einflußreichsten Bestimmungsgrößen der angesprochenen Motivation aus. Dominant ist das ziel "mehr als bisher aus dem Leben zu machen", welches auf ein motivationsförderndes "Defizitempfinden" des Käufers,bezogen auf die Einschätzung der gegenwärtigen und das Anspruchsniveau an die angestrebte "Lebenssituation; hinweist.Ein Nebenziel ist der Wunsch, eine "eigenständige und unverwechselbare Persönlichkeit" durch den Antiquitätenbesitz und seine Ausstrahlung darzustellen.

1 Bei der Interpretation der folgenden regressionsanalytischen Ergebnisse ist $z u$ beachten, daß für Variablen mit "gegenläufiger" Skalierung zur Motivation (im wesentlichen das "Einkommen") ein negativer Regressionskoeffizient als "motivationsfördernd" $\mathrm{zu}$ interpretieren ist. Um Einheitlichkeit zu wahren, besitzt in der graphischen Darstellung das Vorzeichen des Koeffizienten bei der zuordnung stets Priorität. Im Einzelfall auftretende "Gegenläufigkeiten" werden im Text erläutert.

Die angeführten Regressionskoeffizienten bewegen sich im übrigen auf einem Signifikanzniveau von $95 \%$. 
MOTIVATION: WUNSCH NACH SELBSTVERWIRKLICHUNG

Die erklărende Variable ...

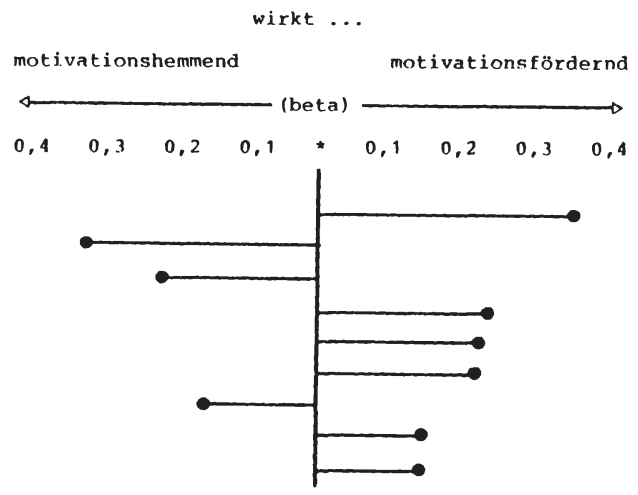

mehr aus dem Leben machen

Freunde bei mir wohlfithlen

gehobene Literatur lesen

oriqina 1

kontaktfreudig - kontaktscheu

Auslandsreisen

mehr Freunde

pessimistisch - optimistisch

unverwechselbare Persionlichkeit

MOTIVATION: DOKUMENTATION VON INDIVIDUALISMUS

Die erk]arende Variable ...

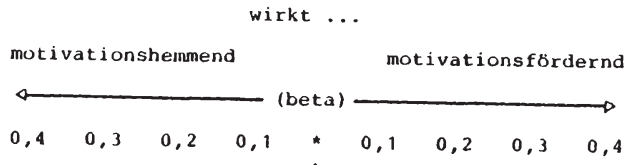

wertbestandig

Freunde bei mir wohlfühlen

wiederverkăuflich

zeitlos

Daseinsgenu 8

ausgefallen

Neid der Freunde

Antiquitaten sind Mode

individue 11

unverwechselbare Persönlichkeit

individuelle Note

Schwarzmarkt fur Antiquitaten

Ausl andsreisen

Neid auf teure Antiquitaten

bessere Wohneinrichtung

dezent

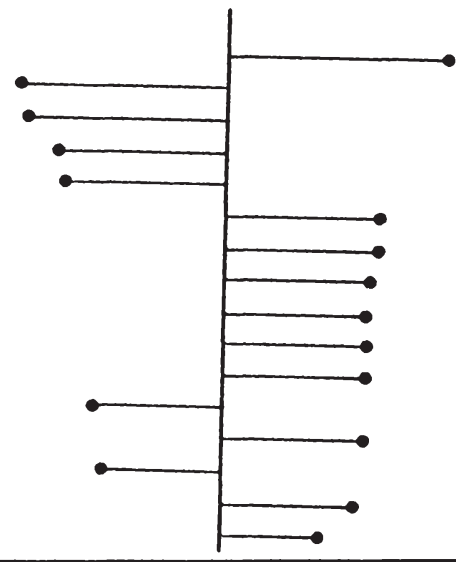

$\mathrm{Abb}$. 27: Bestimmungsfaktoren der Motivationsdimension "Streben nach Selbstverwirklichung und Selbstdarstellung (Teil I) 
MOTIVATION: EMPFINDEN EINES KULTURELLEN AUFTRAGS

Die erklärende Variable ...

wirkl ...

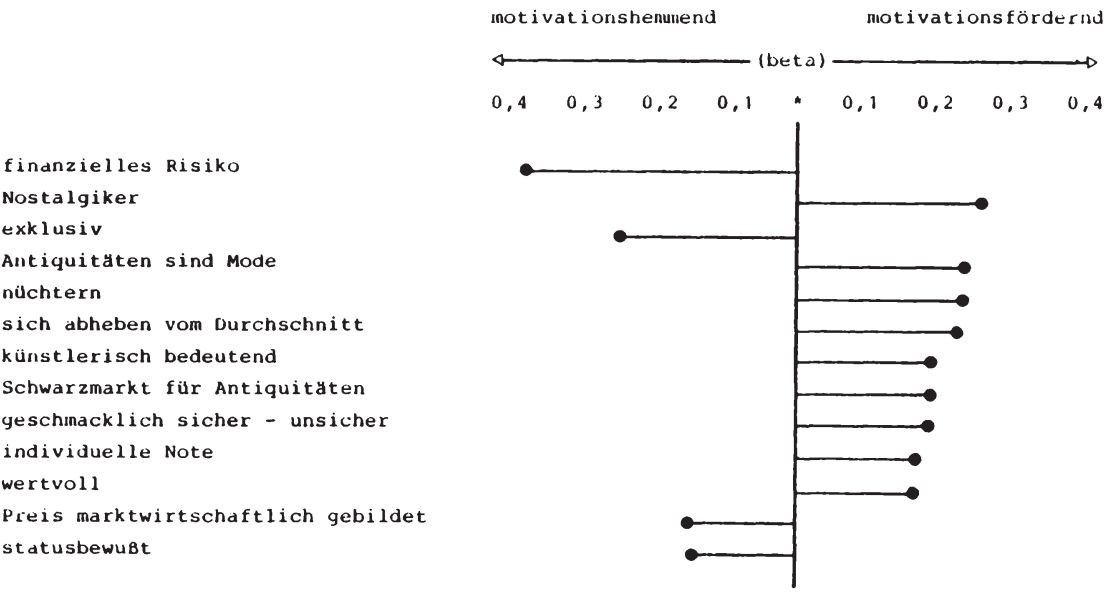

MO'TIVATION: REAKTION AUF MODERNE KUNST

Die erklarende Variable ...

wirkt ...

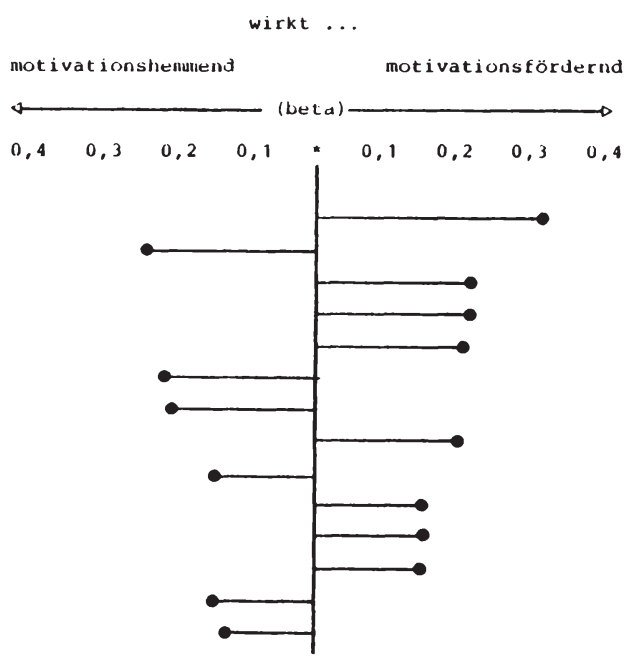

kultivierter lebensstil

spektakulare Preisentwicklungen

rustikal

stilecht

sinkendes Angebot, steigende Preise

modebewußt - modisch unabhangig

wertvoll

dezent

oriyinal

alt

monatl. Netto-Einkomnen im

Spekulationsobjekt

fortschrittlich - traditionsbewuBt

Menschentyp

Abb.27 : Bestimungsfaktoren der Motivationsdimension " Streben nach Selbstverwiklichung und Selbstdarstellung"(Teil II) 
MOTIVATION: DEKORATION DER WOHNUNG

Die erklarende Variable ...

wirkt ...

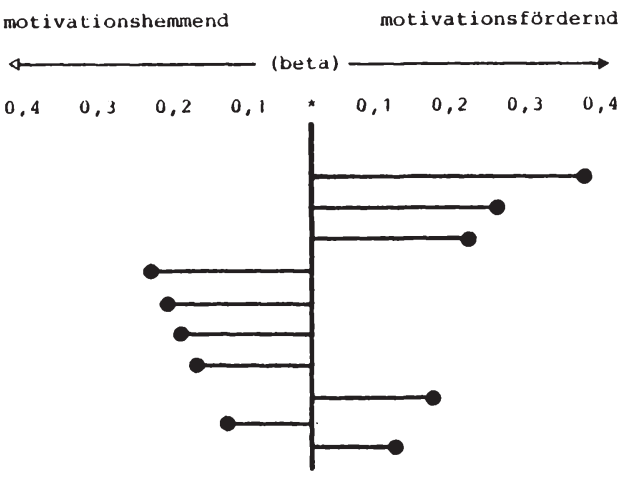

MOTIVATION: BESITZERSTOLZ

Die erklarende Variable ... wirkt ...

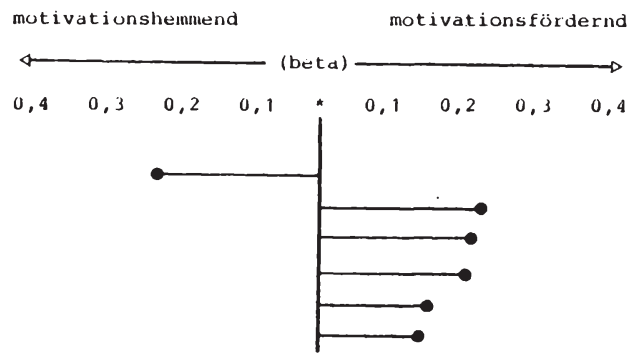

Daseinsgenus

Nostalgiker

monatl. Netto-Einkounmen im нн

anziehend

unverwechselbare Personlichkeit

rustikal

MOTIVATION: BESITZSTREBEN

Die erklarende Variable ...

wirkt ...

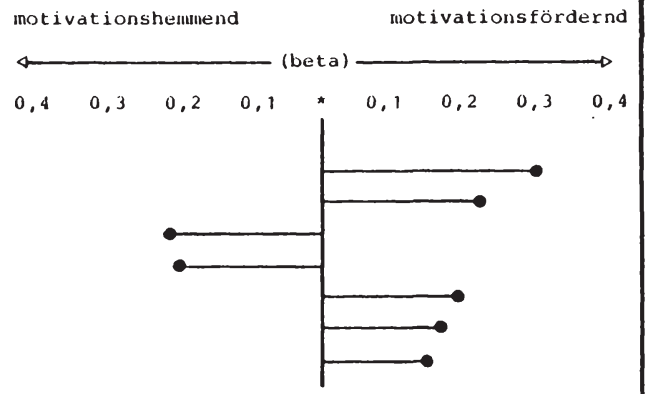

exklusiv

Neid der Freunde

solide gearbeitet

gut exklusiv essen

dezent

Nostalgiker

rustikal

Abb, 27 : Bestimmmgsfaktoren der Motivationsdimension "Streben nach Selbstverwirklichung und Selbstdarstellung" (T'eil III) 
MOTIVATION: NOSTALGIE

Die erklarende Variable...

Nostalgiker

fortschrittlich - traditionsbewußt

finanzielles Risiko

Fachkenntnisse notwendig

yebrauchsfahig

Inan inus schnell zuyreifen

pessimistisch - optimistisch

rustikal

MOTIVATION: BEDURFNIS NACH AUSGI.EICH

Die erklärende Variable...

Neid der Freunde

entscheidunysfreudig/-schwach

aufwendig

kontaktfreudig - kontaktscheu

ausdrucksstark

funktional/prakt isch

Wohnung abheben

pessimistisch - optimistisch

Neid auf teure Antiquitaten

Unabhanyigkeit.

anziehend wirkt ...

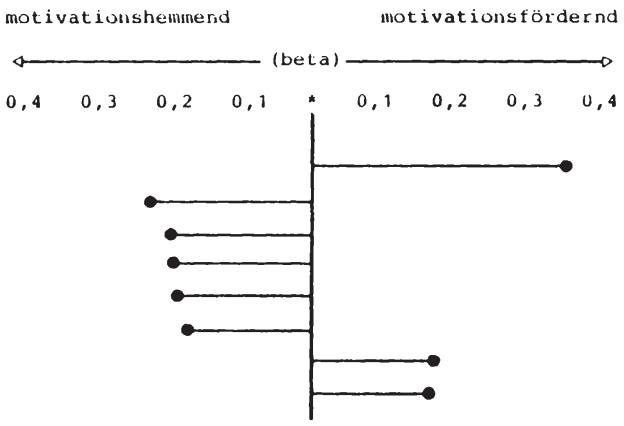

wirkt ...

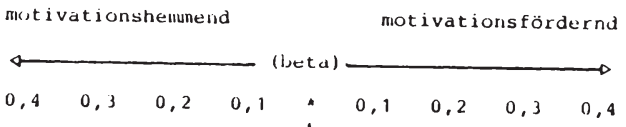

$0,4 \quad 0,3 \quad 0,2 \quad 0,1 \quad * \quad 0,1 \quad 0,2 \quad 0,3 \quad 0,4$

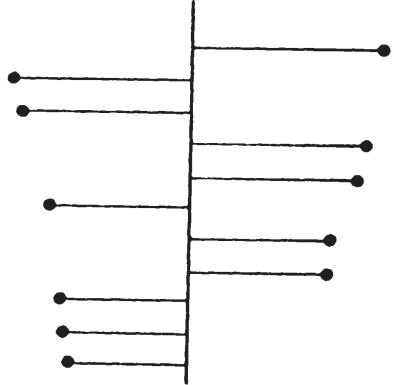

Abb. 27 : Bestimmungfaktoren der Motivationsdimension "Streben nach Selbstverwirklichung und Selbstdarstellung"(Teil IV) 
Andererseits stellt sich ein geringer "Außenbezug", wie er in einem Desinteresse an Freunden und in einer ausgeprägten Selbsteinschätzung als "Kontaktscheu" zum Ausdruck gebracht wird, als motivationsbestimmendes Persönlichkeitsmerkmal heraus.

Eine positive Korrelation besteht zwischen dem Wunsch nach Selbstverwirklichung und der Häufigkeit von Auslandsreisen als Merkmal des Lebensstils. Im Einklang hierzu steht eine signifikant höhere Motivationsausprägung bei denjenigen Käufern, deren Interesse an Antiquitäten speziell auf Auslandsreisen geweckt wurde ${ }^{1}$.

Unter den warenbezogenen Determinanten besitzt die "Originalität" motivationalen Anreiz. Dabei läßt sich ein motivationswirksames Wechselspiel zwischen angestrebter personaler und vorgestellter warenspezifischer "Individualität" beim Zustandekommen eines Kaufentscheids mutmaßen.

Signifikante Einflüsse soziodemographischer Variablen auf die Motivation "Wunsch nach Selbstverwirklichung" konnten nicht ermittelt werden.

Eine beachtenswerte Einzelmotivation ist u.a. auch die "Nostalgie". Nostalgie im Sinne einer Handlungsmotivation beim Kauf von Antiquitäten beschreibt ein inneres Bedürfnis zur Rückbesinnung auf althergebrachte kulturelle, gesellschaftliche, individuelle und materielle Werte bzw. Ideale, für die Antiquitäten Symbolfunktionen übernehmen können.

1 Für alle Einzelmotivationen wurde der Einfluß ausgewäh1ter nominal und ordinal skalierter Merkmale und der Variablen "Entstehung des Interesses an Antiquitäten" durch parallel durchgeführte Varianzanalysen geprüft. Eine Einbeziehung dieser Bestimmungsfaktoren in die multiplen Regressionsanalysen ist in Form sog. "Dummy-Variablen" generell möglich, bereitet aber oft Interpretationsschwierigkeiten. Zu den varianzanalytischen Ergebnissen vgl. die Tabellen 2 bis 7 im Anhang; hier insbesondere Tabelle 7 . 
Diese Interpretation von "Nostalgie" bestätigt sich in einem empirisch belegten zusammenhang zur Käufereinschätzung,"daß Antiquitäten die Menschen an die gute alte zeit erinnern ( Nostalgiker )". Die motivationale Bedeutung der "Nostalgie" gründet damit anscheinend in "emotionalen Defiziten", deren Ursprung in einer Ablehnung bzw. Unzufriedenheit mit heutigen und zukünftig erwarteten Entwicklungen zu suchen ist.

Die Kaufmotivation "Nostalgie" sollte daher auch eher für traditionsbewußte als für fortschrittlich orientierte Käufer typisch sein. Der empirische Befund zeigt jedoch das Gegenteil. Ein Erklärungsansatz bietet sich, wenn Nostalgie zugleich als ein "Zeitphänomen", eine momentane "Kulturstimmung" und damit nicht zuletzt als eine "Modeerscheinung" angesehen wird. Der motivationale Reiz eines Antiquitätenkaufs liegt dann u.a. darin, sich gegenüber den in einer "Progressivität verharrenden" Menschen durch eine "demonstrative Rückorientierung" (Regression) als besonders fortschrittlich auszuzeichnen. Dies kann objektiviert nur in einer relativ frühen Phase einer aufkommenden Antiquitätenmode gelingen, in der der Käufer eine innovative Funktion übernimmt. Ein "Nostalgiekauf" im Sinne einer Anpassung an eine gesellschaftlich bereits vorherrschende und breit akzeptierte Modeerscheinung stellt andererseits einen - dem einzelnen Käufer zumeist unbewußt bleibenden - Anachronismus dar.

Auch die Erklärungsansätze für die weiteren Einzelmotivationen führen zu ähnlich detaillierten und "abgerundeten" Aussagen. Im Vergleich der motivationsspezifisch isolierten Bündel von Bestimmungsfaktoren werden dabei z.T. sehr verschiedenartige Schwerpunkte und Akzente deutlich (zu Einzelanalysen vgl. die in Abbildung 27 graphisch dargestellten Beziehungen). 


\subsection{Streben nach Konsumerlebnis}

Ein Antiquitätenkauf - ob beim Fachhandel, auf einer Messe oder Flohmarkt, beim Trödler oder auf einer Auktion beinhaltet subjektive Erlebnisinhalte, welche eine eigenständige Kaufmotivation darstellen können. Die allgemeine Freude am Einkaufserlebnis, der $\mathrm{Spa} \beta$ an einer Antiquitätensuche und die Hoffnung auf das Entdecken einer Trouvaille sind Teilaspekte dieser Kaufmotivation. Ihre empirisch ermittelten Bestimmungsfaktoren sind in der Abbildung 28 zusammengefaßt.

Die Einzelmotivation "Freude am Einkaufserlebnis" wird wesentlich durch die Einschätzung bestimmt, daß Antiquitäten ihrem Besitzer eine individuelle Note verleihen. Folgerichtig liegt der Schwerpunkt der Käufervorstellung auf den "dekorativen" Eigenschaften von Antiquitäten. Unter den Persönlichkeitsmerkmalen erweisen sich die Selbstbeurteilungen als "geschmackssicher" und "optimistisch" sowie auch der Wunsch nach einem größeren Freundeskreis als motivationsfördernd.

Voraussetzung für eine "unbeschwerte" Freude am Antiquitätenkauf ist anscheinend die Vermutung eines "realistischen", d.h. eines qualitätsbestimmten preises. Sie gibt dem Käufer eine subjektive Sicherheit, welche durch ein verstärktes Auftreten dieser Motivation bei Käufern mit geringeren Einkommen an zusätzlicher Bedeutung gewinnt.

Die weiteren Beziehungen, die in der Abbildung 28 für die Motivationen "Spaß am Suchen" und "Hoffnung auf Trouvaille" aufgezeigt werden, lassen sehr differenzierte Schlußfolgerungen $z u$. So erweist sich z.B. erwartungsgemä $\beta$ das Wissen um "einen Schwarzmarkt für Antiquitäten" neben den Persönlichkeitsmerkmalen "optimistisch", "unkritisch", "nicht unsicher" und "modebewußt" für die erstgenannte Einzel- 
MOTIVATION: FREUDE AM EINKAUFSERIEBNIS

Die erklarende variable ...

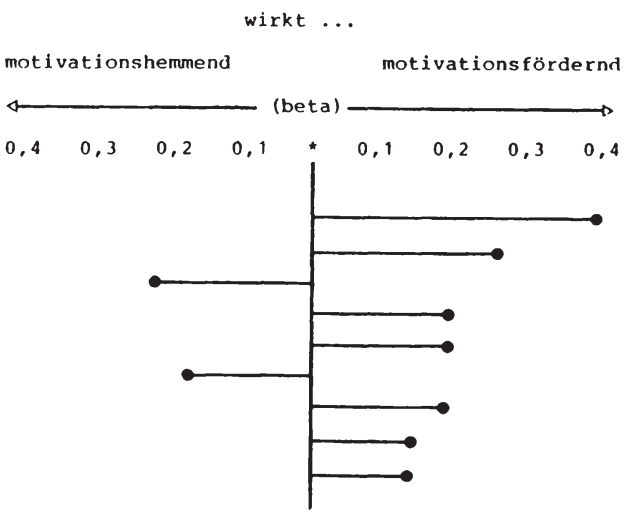

\section{MOTIVATION: SPASS AM SUCHEN}

Die erklurende Variable... wirkt ...

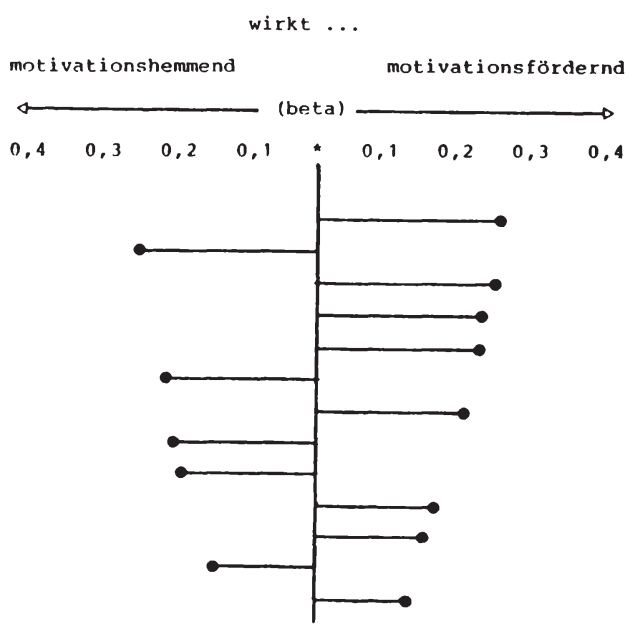

individuel le Note

kultivierter Lebensstil

kunstverstandig

Schwarzmarkt filr Antiquitaten

eleyant

zeitlos

kritisch - unkritisch

gehobene literatur lesen

Museum, Theater, Konzert

unkonventione ll

musisch - nicht musisch

modebewuBt - modisch unabhangi t

pessimistisch - optimistisch

Abb. 28: Bestimmungsfaktoren der Motivationsdimension "Streben nach Konsumerlebnis" (Teil I) 
MOTIVATION: HOFFNUNG AUF TROUVAILLE

Die erklarende Variable...

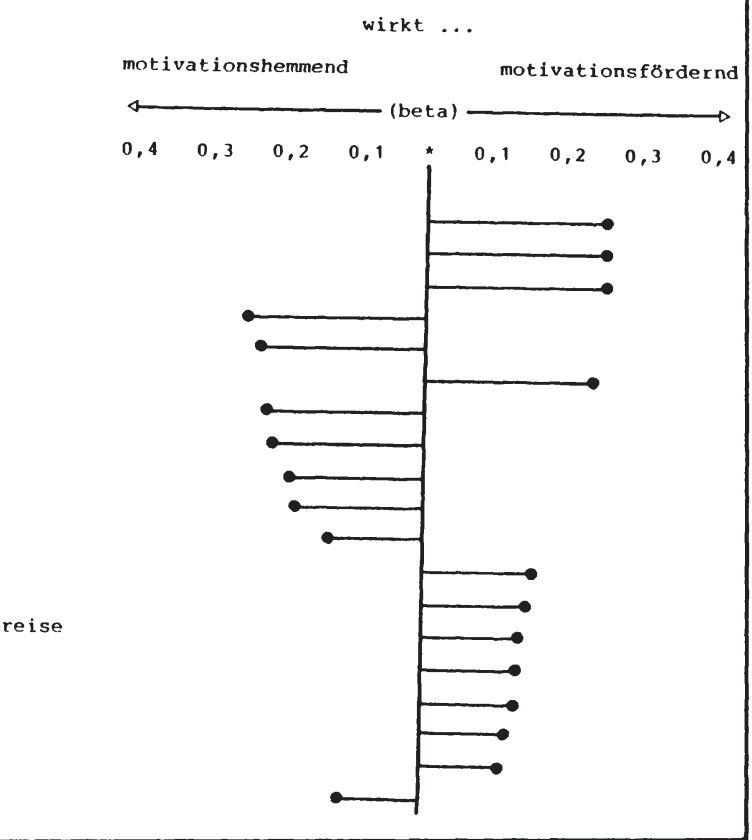

nüchtern

kontaktfreudig - kontaktscheu

individuell

kunstverstăndig

kritisch - unkritisch

Trouvaillen möglich

modern

mehr Freunde

Museum, Theater, Konzert

zeitlos

funktional/praktisch

ausge fallen

pessimistisch - optimistisch

sinkendes Angebot, steigende Preise

Antiquitaten sind Mode

musisch - nicht musisch

passen zu jeder Einrichtung

Nostalgiker

impulsiv - planend

Abb. 28: Bestimmungsfaktoren der Motivationsdimension

"Streben nach Konsumerlebnis" (Teil II) 
motivation oder die Einschätzung "eines stetig sinkenden Angebots bei steigenden Antiquitätenpreisen" und "der Möglichkeit von Trouvaillen" neben mehreren Wohnstilmerkmalen ("nüchtern", "individuell") für die zweitgenannte Einzelmotivation als relevant.

Erstaunlich gering ist die Bedeutung der assoziierten Eigenschaften und Merkmale von Antiquitäten als Bestimmungsfaktoren. Nur das Vorstellungsbild "ausgefallen" besitzt durchgängig motivationalen Anreizwert. Hierin ist ein Hinweis zu sehen, daß es sich bei der Inhaltsdimension "Streben nach Konsumerlebnis" um eine vom Kaufaspekt relativ unabhängige und eher situations- und persönlichkeitsbedingte Motivationsdimension handelt ${ }^{1}$.

\subsection{Antiquitätenkauf als ökonomisches Kalkül}

In Zeiten inflationärer Geldentwertung ist eine "Flucht in Sachwerte" typisch. Antiquitäten werden von ihren Käufern gerade in den letzten Jahren als eine Möglichkeit zur Wertanalge und z.T. darüber hinaus als Chance eines Wertzuwachses angesehen, da

- das Angebot an Antiquitäten einer natürlichen, d.h. substanzbedingten Auszehrung unterliegt und ihr "Seltenheitswert" steigt

- eine starke private Nachfrage, sowie der damit erhöhte private Antiquitätenbesitz die Marktregeneration erschwert und $\mathrm{zu}$ einer Marktverknappung führt

- ein relativ konstanter Nachfrageüberhang das Preisniveau, insbesondere für Antiquitäten höherer Qualität beständig steigen läßt.

- ein weitgespannter "Schwarzmarkt" und der "private Charakter" von Antiquitätenkäufen "verdeckte" Kapitalanlagen erleichtem.

1 Daneben konnten auch Einflüsse soziodemographischer Variablen ermittelt werden. Vgl. dazu Tabellen 4,5,6 und 7 im Anhang. 
Abbildung 29 zeigt die regressionsanalytisch ermittelten personen-, situations- und warenbezogenen Bestimmungsfaktoren der Einzelmotive bzw. Teilaspekte eines Antiquitätenkaufs als "ökonomisches Kalkül".

Ein erstes interessantes Resultat ist, daß die Kaufrelevanz des Wertanlagemotivs mit der Markteinschätzung auch zukünftig steigender Preise zunimmt, wobei die Käufer jedoch nicht an ein marktwirtschaftliches Zustandekommen des Preises glauben. Auch die Vorstellung von Antiquitäten als "selten" weist eher in Richtung auf ein "irrationales" Vertrauen in eine ständig zunehmende Marktverknappung. Daneben werden Antiquitäten generell als "wertbeständig" angesehen. Man sieht in ihnen eine ebenso sichere Geldanlage wie z.B. in einem sparbuch.

Unter den Persönlichkeitsmerkmalen fördern das "Traditionsbewußtsein", ein "planender" Charakter und der Wunsch, als gepflegte Erscheinung mit sicherem Auftreten zu gelten, das Wertanlagemotiv.

Einen motivationsfördernden Einfluß übt im übrigen die Vermutung eines Neides der Freunde und Bekannten auf den eigenen Antiquitätenbesitz aus. Da auch die genannten Persönlichkeitsziele auf eine "äußere Wirkung" angelegt sind, deutet dies auf einen latenten Zusammenhang von "Wertanlage" und "materieller Demonstration" hin.

Erwartungsgemäß gewinnt das Wertanlagemotiv mit steigendem Einkommen an Kaufrelevanz. Wie die gesonderte varianzanalytische Auswertung zeigt, besteht eine signifikante Dominanz des Wertanlagemotives bei Männern ${ }^{1}$.

1 Vgl. Tabellen 2 und 6 im Anhang. 


\section{MOTIVATION: SPEKULATION AUP WERTZUWACHS}

Die erklarrende Variable ...

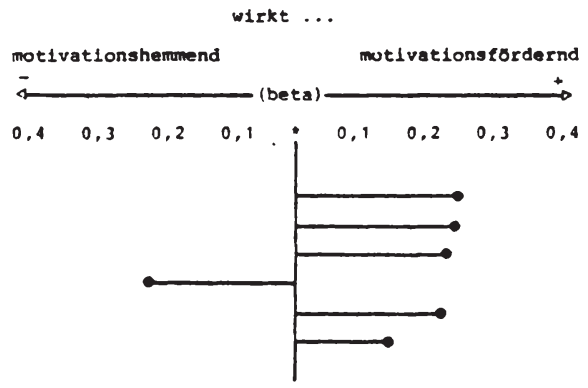

materiel'e sicherheit

ausgefallen

wertbestandig

konservat $1 v$ - progressiv

Neld der Freunde

angepast - individual lstisch

\section{MOTIVATION: WERTANI.AGE}

Die erklarende Variable ...

rustikal

selten

ausere Erscheinung pflegen

sinkendes Angebot, stelgende Preise

aktiver Sport

wertbast Indig

Cortschrittlich - traditionsbewrut

Neid der Freunde

exklusiv

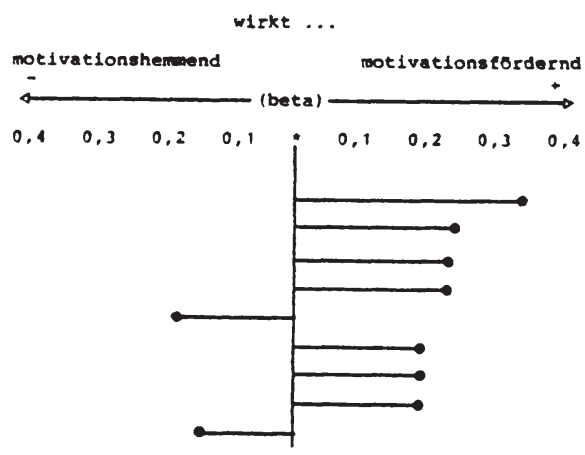

\section{MOTIVATION: STEUERFLUCHT}

Die erkigrende Variable...

wirkt ...

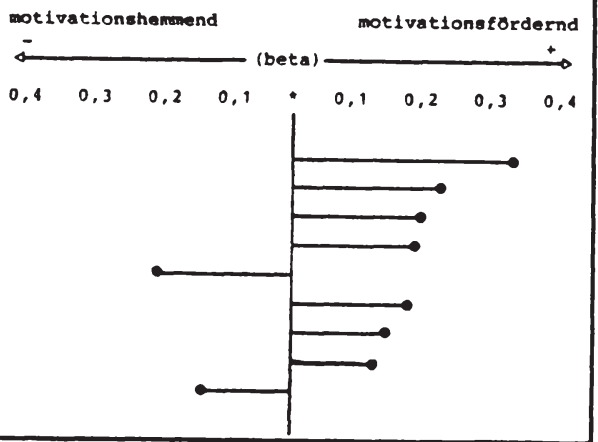

$\mathrm{Abb}$. 29: Bestimmungsfaktoren der Motivationsdimension "Antiquitätenkauf als ökonomisches Kalkül" 
Gleiches trifft auch für die "Spekulation auf Wertzuwachs" als Kaufmotiv $z u$, welches sich bereits durch ein erheblich kleineres Bündel von Bestimmungsfaktoren fast ebensogut erklären läßt wie die "Wertanlage". Interessant ist, daß gerade bei dem "aggressiveren" ökonomischen Kalkül einer Spekulation auf Wertzuwachs das persönliche ziel einer materiellen Sicherheit als Einflußgröße dominiert.

Ansonsten zeigen sich Parallelen zum "Wertanlagemotiv", wobei allerdings in der Charakterisierung durch Persönlichkeitsmerkmale eine bemerkenswerte und anscheinend folgerichtige Nuancierung festzustellen ist: Die Selbsteinschätzung traditionsbewußt/planend wechselt zu konservativ/individualistisch.

Als abschließendes ökonomisches Kalkül wurde das Motiv einer "Steuerflucht" im Sinne der bewußten Nutzung steuerlicher Vorteile durch einen Antiquitätenkauf auf seine Einflußgrößen untersucht. Obwohl es sich bei der "Steuerflucht" um das relativ am schlechtesten (regressionsanalytisch) erklärbare Einzelmotiv handelt, ist der absolute Erklärungsbeitrag ( MULT $R=0,59$ ) immer noch als hoch einzuschätzen.

Uberraschend dominieren Persönlichkeitsmerkmale (individualistisch, entscheidungsschwach und zugleich optimistisch) als Bestimmungsfaktoren. Daneben weisen sich erwartungsgemäß die Einschätzung - und mutmaßlich gleichzeitig die Kenntnis und Nutzung - eines umfangreichen "Schwarzmarktes" für Antiquitäten sowie ein (steigendes) Einkommen als motivationsfördernd aus.

\subsection{Sammelleidenschaft}

Das "Sammeln" von Kunst und Antiquitäten erscheint zunächst als ein "natürliches" und transparentes Motiv von Antiquitätenkäufern. 
Nützlich ist bei der Analyse des "Sammelmotivs" jedoch die Unterscheidung zwischen "Zwecksammeln" und "Sammeln aus Selbstzweck" 1 .

Im ersten Fall verbindet der Käufer mit dem Sammeln, d.h. $d \in m$ Kauf und Besitz von Antiquitäten,bestimmte zielsetzungen, z.B. persönlichkeitsbezogener, materieller oder sozialer Art. Die gesammelten Antiquitäten besitzen in diesem Fall eine instrumentelle Wertigkeit (z.B. Gebrauchsfunktion, Demonstrationseffekt etc.)? Bei einem "Sammeln aus Selbstzweck" ist dies nicht der Fall. Hier dominiert ausschließlich das streben, ein individuell gesetztes Anspruchsniveau,bezogen auf den zahlenmäßigen Umfang oder die Vollständigkeit einer "Sammlung" in einem abgegrenzten Sach-bzw. Sammelgebiet, zu erreichen.

Die Ubergänge zwischen einem "Zwecksammeln" und einem "Sammeln aus Selbstzweck" bleiben in der Realität zumeist unsichtbar, da sich die spezifischen Motivationsinhalte des "Zwecksammelns" in unterschiedlichem Ausmaß zu einem "Sammeln aus Selbstzweck" verselbständigen können.

Die Frage, ob und inwieweit die Sammelleidenschaft von Antiquitätenkäufern auf mögliche "Hintergrundmotive" zurückgeführt werden kann, ist in der empirischen Analyse von besonderem Interesse. Einen ersten Ansatzpunkt liefert die

1 Vgl. dazu Wiswede, G., a.a.O., S. $36 \mathrm{ff.}$.

2 Fast alle durch die empirische Studie erfaßten Einzelmotive sind in diesem weiten Begriffsverständnis zugleich psychische Beweggründe des Zwecksammelns. 
bereits diskutierte faktorenanalytische Auswertung, indem sie einen inhaltlichen Zusammenhang zwischen "Sammelleidenschaft" und "Freizeitgestaltung" aufdeckte. Das "Sammeln" von Antiquitäten besitzt demnach zugleich einen maßgeblichen Freizeitwert (Hobby). Interessant ist, daß auch die Freizeitgestaltung ein relativ warenunabhängiger Motivationsinhalt ist, welcher sich durchaus mit dem "Sammeln als Selbstzweck" verbinden läßt.

In der getrennten Analyse verdichtet sich diese Vermutung, da für beide Einzelmotive der "Wunsch intensiver dem persönlichen Hobby nachgehen $\mathrm{zu}$ wollen"als signifikante Einflußfaktoren isoliert werden konnten (vgl. Abbildung 30). Insgesamt macht die empirische studie damit auf einen Zusammenhang aufmerksam, welcher bislang in Theorie und Praxis keine Beachtung gefunden hat.

Bei detailierter Betrachtung liegt ein weiteres Indiz für einen hohen Selbstzweck des Sammelns von Antiquitäten im gegenläufigen Zusammenhang zwischen der Käufervorstellung von Antiquitäten als "funktional/praktisch" und "exklusiv". Der Gebrauchs- und Demonstrationswert steht für Sammler vermutlich im Hintergrund z.B. gegenüber der "Ausgefallenheit" als Merkmal sammlungswürdiger und -attraktiver Einzelstücke.

Auffallend ist ferner die durchgängig hohe motivationale Bedeutung der Käufereinschätzung "von anderen um den eigenen Antiquitätenbesitz beneidet zu werden!" Für die Einzelmotivation "Sammelleidenschaft" erweist sich sogar ein "aktives" Neidgefühl auf Menschen, die sich teure Antiquitäten leisten können, als wichtig. Ob hieraus letztlich doch auf ein latentes Prestige- und Statusstreben als Zweck des Sammelns zu schließen ist,oder ob es sich um eine eher "objektive" Beurteilung (z.B. Bestehen einer 
MOTIVATION: SAMMELLEIDENSCHAFT

Die erklarende Variable ... wirkt ...

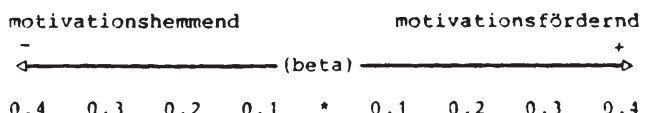

Neid der Freunde

funktional /praktisch

ausgefallen

exklusiv

Neid auf teure Antiquitaten

Anerkennung

Dase insgenuB

introvertiert - extrovertiert

intensiver Hobby nachgehen

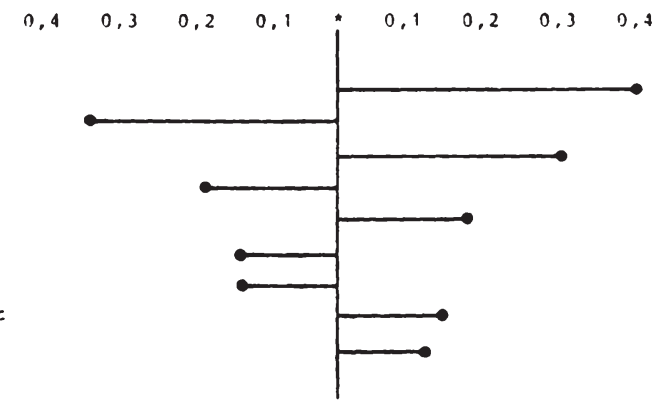

\section{MOTIVATION: FREIZEITGESTALTUNG}

Die erklarende variable ...

wirkt ...

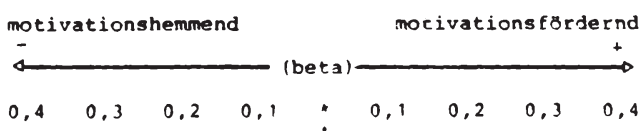

aufwendig

Neid der Freunde

bessere Wohneinrichtung

impulsiv - planend

Angabe mit Antiquitaten

elegant

intensiver Hobby nachgehen

selbstsicher - unsicher

verspielt

nüchtern

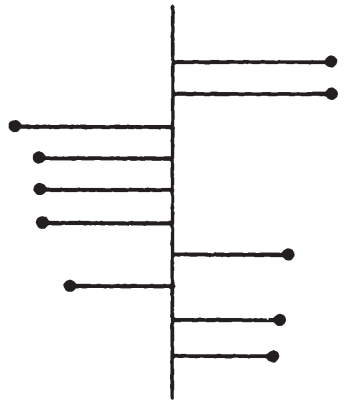

Abb. 30: Bestimmungsfaktoren der Motivationsdimension

"Sammeln von Antiquitäten als freizeitgestaltendes Hobby" 
besonders attraktiven Sammlung) handelt,kann an dieser Stelle nicht endgültig beurteilt werden. Gegen eine Zweckgebundenheit spricht vor allem eine abnehmende Sammelleidenschaft bei einem zunehmenden Streben nach persönlicher Anerkennung.

Für das Einzelmotiv "Freizeitgestaltung" ist auf die Relevanz wohnbezogener Einflußgrößen (Wohnstilmerkmale: aufwendig, elegant, verspielt, nüchtern) hinzuweisen, die in einem inhaltlich sehr ambivalenten Verhältnis zur Motivation stehen. Bei der varianzanalytischen Prüfung soziodemographischer Einflußgrößen erweist sich lediglich der "Beruf" als bedeutsam. Eine gegenüber anderen Berufsgruppen signifikant ausgeprägte "Freizeitmotivation" besitzen erwartungsgemäß die Rentner" ${ }^{1}$.

\subsection{Liebhaberei}

Die Liebhaberei umfaßt die "Freude an Naturstoffen" und die "Liebe zum alten Kunsthandwerk" als Einzelmotive. Mit der Wahl des Begriffes "Liebhaberei" zur Kennzeichnung dieser Inhaltsdimension soll zum Ausdruck gebracht werden, daB ihre motivierende Kraft in einem eher affektiv-emotionalen Verhältnis des Käufers zu Antiquitäten begründet ist ${ }^{2}$.

1 Vgl. die Tabelle 5 im Anhang. Auch für andere soziodemographische Variablen ergaben sich signifikante "Gruppenunterschiede", die sich jedoch nicht auf einem varianzanalytisch signifikanten Niveau bewegen. Vgl. Tabelle 2 bis Tabelle 7 im Anhang.

2 In Abgrenzung $z u$ den übrigen Kaufmotivationen lassen sich die Einzelmotive der "Liebhaberei" nur unzureichend in der Kategorie eines "Strebens nach ...", sondern besser in derjenigen einer "Freude bzw. Liebe an ..." semantisch beschreiben. 
Zur Erklärung einer "Liebhaberei" bezogen auf Antiquitäten ist deshalb tendenziell nicht nach möglichen Nutzenerwartungen der Käufer, sondern nach den Merkmalen und Eigenschaften von Antiquitäten zu fragen, die ihren Ausgangspunkt bzw. Bezugspunkt bilden.

Einen ersten Hinweis geben die unter dieser Inhaltsdimension zusammengefaßten Einzelmotive, welche sich auf die beiden wesentlichen (materiellen) strukturmerkmale "Naturstoffe" und "kunsthandwerkliche Fertigung" beziehen ${ }^{1}$. Eine "Liebhaberei" zu Antiquitäten ist dabei insbesondere als Anzeichen zu werten, daß Käufern ein emotional befriedigender "Erlebnisbesitz"wichtiger als ein faktischer "Warenbesitz" ist.

In der empirischen Analyse stellt sich erwartungsgemä $B$ die Käufervorstellung von Antiquitäten als "solide gearbeitet" als wichtige Einflußgröße sowohl der Einzelmotivation "Liebe zum alten Kunsthandwerk" als auch der "Freude an Naturstoffen" heraus. Daneben unterstreichen auch die Vorstellungen "individuell" und "zeitlos" das bislang gewonnene Bild (vgl. Abbildung 31).

1 Bevor auf die empirisch ermittelten Einflußgrößen eingegangen wird, ist auf zwei zeitbedingte Rahmenbedingungen hinzuweisen, welche die Bedeutung beider Kaufmotivationen beeinflussen können. Antiquitäten sind ihrem Ursprung nach Gebrauchsgegenstände, welche in vielen Fällen (z.B. Möbel, Wohnaccessoires) zeitgenössische "Pendants" bzw. Kaufalternativen besitzen. Es kann vermutet werden, daß sowohl das starke Vordringen "künstlicher" Materialien als auch die serielle "Massenproduktion" mit z.T. subjektiv empfundener und objektiv geringerer Fertigungsqualität und Ausstrahlungskraft die "Liebe" zu kunstvolien und aus Naturstoffen gefertigten Handwerksstücken fördert. 
MOTIVATION: FREUDE AN NATURSTOFFEN

Die erklärende Variable ... wirkt ...

motivationshemmend motivationsfordernd

$$
-
$$
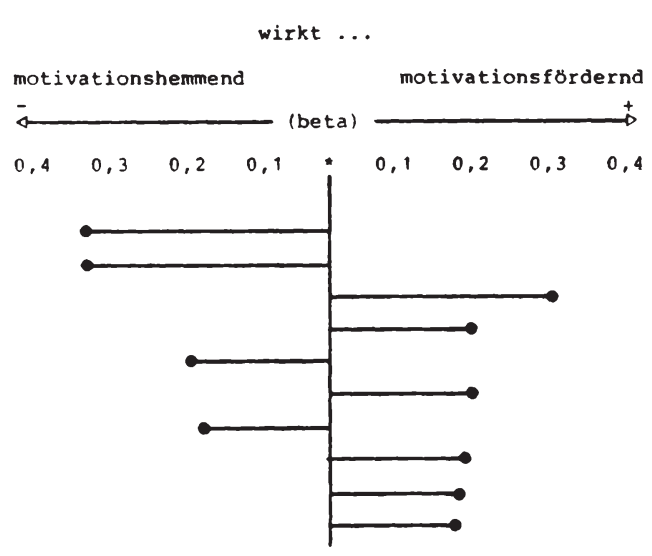

individuelle Note

spektakulare freisentwicklungen

solide gearbeitet

mobil

Museum, Theater, Konzert

wohnung abheben

Angabe mit Antiguitaten

zeitlos

statusbewuBt

sinkendes Angebot, steigende Preise

MOTIVATION: LIEBE ZUM ALTEN KUNSTHANDWERK.

vie erklarende variable...

wirkt ...

motivationshemmend

motivationsfordernd

$$
-
$$
(beta)

$\stackrel{+}{\rightarrow}$

$\begin{array}{lllllllll}0,4 & 0,3 & 0,2 & 0,1 & * & 0,1 & 0,2 & 0,3 & 0,4\end{array}$

pessimistisch - ofitimistisch

solide gearbeitet

individuell

Nostalaiker

finanzielles Risiko

sinkendes Angebot, steigende Preise

Fachkenntnisse notwendig

schon

geschmacklich sicher - unsicher

Museum, Theater, Konzert

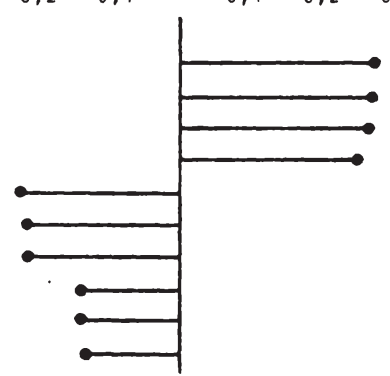

Abb. 31: Bestimmungsfaktoren der Motivationsdimension "Liebhaberei" 
Für die "Liebe zum alten Kunsthandwerk" erweist sich zusätzlich die Käufervorstellung "nostalgisch" als wichtige Bestimmungsgröße. Hierzu spiegelt sich ein wertender, zeitlicher Rückbezug der Käufer auf ảie "gute alte Zeit". Die zum Ausdruck kommende affektive Aufwertung des "früheren" steht im Einklang mit einer Ablehnung von zeitgenössischen Entwicklungen (Massenfertigung, Kuststoffe etc.). DaB die "Liebe zum alten Kunsthandwerk" eine überwiegend emotionale und damit tendenziell auch weniger "sachlich kompetente" Grundlage besitzt, zeigt sich u.a. in der Zunahme (Abnahme) dieser Motivation, je geringer (höher) das finanzielle Risiko und die Notwendigkeit von Fachkenntnissen bei einem Antiquitätenkauf eingeschätzt wird ${ }^{1}$.

\subsection{Familiär bedingte Motivationen}

Die angesprochene Motivdimension umschließt die Einzelmotive "Familientradition" und "Bewahrung von Erinnerungen an die Familie" zusammen (vgl. Abbildung 32 ).

Unter den regressionsanalytisch ermittelten Bestimmungsfaktoren der Motivation "Familientradition" sind Variablen des Lebensstils der Antiquitätenkäufer in der Überzahl. Dadurch findet die Annahme Unterstützung, daß diese Motivation - wie viele andere Verhaltensweisen, Gedankenhaltungen, Einstellungen etc. - eine dem Käufer unbewußt

1 Die varianzanalytische Prüfung des Einflusses soziodemographischer Käufermerkmale erbrachte für keines der beiden diskutierten Einzelmotive signifikante Ergebnisse. Zu teilweise sehr aufschlußreichen Gruppenunterschieden, wie z.B. eine signifikant höhere "Freude an Naturstoffen" bei den 20 - 34 jährigen im vergleich $z u$ den 35 - 49jährigen Käufern, vgl. im einzelnen die Tabellen 2 bis 7 im Anhang; (hier Tabelle 3). 
MOTIVATION: FAMILIENTRADITION

Die erklarende variable...

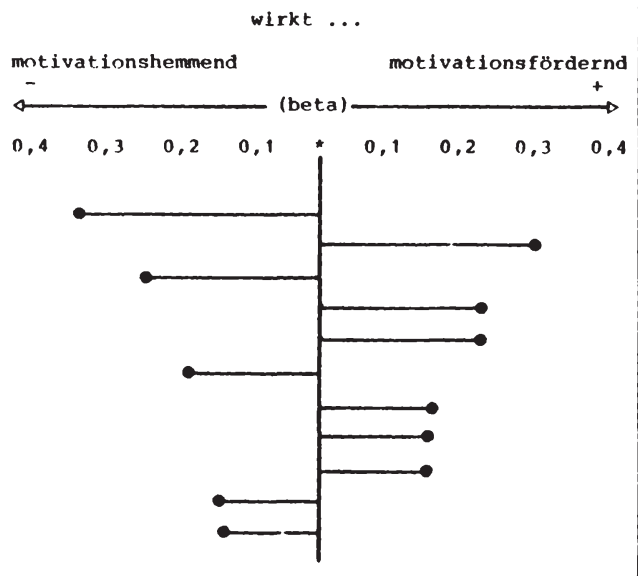

Anerkennung

gehobene Literatur.

dekorativ

reprasentativ

schön

Unabhangigkeit

Fachkenntnisse notwendiq

anziehend

dezent

Antiquitaten sind Mnde

pessimistisch - optimistisch

MOTIVATION: BEWAHRUNG VON ERINNERUNGEN AN DIE FAMILIE

Die erklarende variable ...

wirkt ...

Nostalgiker

wiederverkyuf $1 \mathrm{ich}$

kuristverstindig

Alter.

rustikal

fortschrittlich - traditinnsbewunt

motivationsfördernd

motivationshemmend

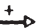

$0,4 \quad 0,3 \quad 0,2 \quad 0,1$ (beta)

$0,1 \quad 0,2 \quad 0,3 \quad 0,4$

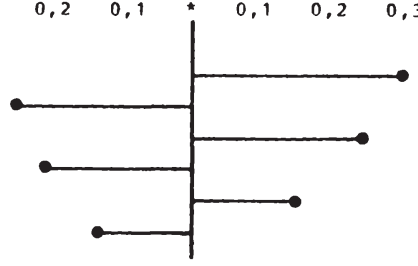

Abb. 32: Bestimmungsfaktoren familiär bedingter Motivationen 
bleibende familiäre "Lerngeschichte" besitzt. In diesem Kontext ist ein Antiquitätenkauf als eher habitualisierter und "selbstverständlicher" Bestandteil des tradierten (allgemeinen) Lebensstils der Käufer zu sehen.

Besonders enge Verknüpfungen lassen sich zu einigen Wohnstilvariablen ("repräsentativ", "schön", "dezent" und "dekorativ") aufzeigen. Die unterschiedlichen EinfluBrichtungen spiegeln dabei eine nuancierte, aber zugleich auch akzentuierte Einschätzung der Befragten wider. Im Uberblick zeichnet sich ein "gehobener", "kultivierter" und "unabhängiger" Lebensstil als wesentlich motivationsfördernd aus.

Wie oftmals bei traditionsorientierten Menschen ist die Grundhaltung der durch familiäre Tradition motivierten Antiquitätenkäufer eher pessimistisch. Ihr Verhältnis zu Antiquitäten ist vermutlich überwiegend affektiv-unbewuBter Natur, da sich einzig die Vorstellung "Antiquitäten sind anziehend" als direkt warenbezogene Bestimmungsgröße herausstellt.

Die vorgenommenen varianzanalytischen Prüfungen des Einflusses soziodemographischer Variablen weisen signifikante Ergebnisse beim Geschlecht (Frauen (+)) und Alter (hohes Alter (+)) auf ${ }^{1}$. Als "Kontrollvariable" eignet sich im Zusammenhang mit der Motivation "Familientradition" die Frage nach der Entstehung des Interesses, welche eindrucksvolle Signifikanzen bei den Antwortkategorien "Familie" und "Erbschaft" besitzt. 2

Die gleichen soziodemographischen Variablen sind bei der Variablen "Bewahrung von Erinnerungen an die Familie" als

$1 \mathrm{Vgl}$. Tabellen 2 und 3 im Anhang.

2 Vgl. Tabelle 7 im Anhang. 
Bestimmungsfaktoren erkennbar (eher Frauen, eher ältere Menschen). Die regressionsanalytische Untersuchung zeigt, daß die Einschätzung "Antiquitäten erinnern die Menschen an die gute alte zeit" am stärksten motivationsfördernd, die "Wiederverkäuflichkeit" am stärksten motivationshemmend wirkt. Der hohe "Erinnerungswert" und die ausgeprägte "Symbolfunktion" von Antiquitäten im Zusammenhang mit der angesprochenen Einzelmotivation wird damit eindrucksvoll unterstrichen.

\subsection{Repräsentationsstreben}

Antiquitätenkäufe sind zu einem nicht unerheblichen Teil demonstrativer Konsum, von dem sich der Käufer eine soziale Repräsentationswirkung verspricht. Prestigegewinn und Statusstreben als soziale Bedürfnisse von Konsumenten werden dabei als Handlungsanstöße indirekt sichtbar. Die sozialen "Zielsetzungen" der Antiquitätenkäufer können dabei in Abhängigkeit von der bestehenden und angestrebten sozialen "Position" erheblich differieren. Formal sind u.a. folgende Varianten abgrenzbar:

(1) Der Antiquitätenkäufer möchte durch einen Antiquitätenkauf die zugehörigkeit zu seiner sozialen Schicht oder Gruppe festigen und nach außen demonstrieren. Er unterwirft sich damit den Normund Wertvorstellungen dieser Schicht (Gruppe) und erfüllt durch den Antiquitätenbesitz gruppenspezifische Rollenerwartungen. Seine motivationalen Zielvorstellungen könnten beispielsweise durch eine angestrebte "Sicherheit durch soziale Integration", ein "Anerkennungs- und Kontaktstreben" oder ein "Identifikationsbedürfnis" umschrieben werden. Im Extremfall besteht sogar die Möglichkeit, daß sein Verhalten als "sozialer Gehorsam" die Folge eines übermäßig stark empfundenen Konformitätsdruckes ist.

(2) Der Antiquitätenkäufer möchte durch den Antiquitätenkauf seine "Stellung" im Sozialgefüge ändern. So kann er einerseits versuchen, sich durch 
den Besitz des "Statusobjektes" Antiquität gesellschaftlich oder auch nur von seinem engeren sozialen Umfeld abzuheben. Dieses "soziale Differenzierungsstreben" braucht dabei zunächst nicht unbedingt richtungsgebunden $\mathrm{zu}$ sein. In den meisten Fällen kann andererseits der Wunsch nach einer "Verbesserung" der sozialen Stellung angenommen werden. Ein solches "soziales Aufstiegsstreben" fördert u.a. die Ubernahme (Imitation) des Konsumverhaltens sozialer Gruppierungen, deren Stellung im Sozialgefüge als "höher" und "erstrebenswert" empfunden werden.

Neben den beispielhaft genannten Zielsetzungen von Antiquitätenkäufern ist es für die Entstehung einer Kaufmotivation "Repräsentation" wichtig, daß sich der Käufer von einem Antiquitätenbesitz einen Beitrag zur Erreichung seiner ziele verspricht. Wesentliche Voraussetzung ist, daB Antiquitäten - als "Symbole" - statusrelevante Eigenschaften (z.B. Wohlstand, Kunstkenntnis, Ästhetik, Geschmack etc.) repräsentieren können.

In diesem Sinne war die Frage nach den empirisch erfaßbaren Bestimmungsfaktoren des Einzelmotivs "Repräsentation" von besonderem Interesse. Zu den Ergebnissen vgl. Abbildung 33.

Der empirische Befund bestätigt die Bedeutung eines Zusammenwirkens von persönlicher Zielsetzung (der Käufer legt auf sein "äuBeres Erscheinungsbild" besonderen Wert) und Prestigegehalt von Antiquitäten (Antiquitäten wirken "repräsentativ") bei der Motivationsentstehung.

Ein Hinweis auf die hohe "Sensibilität" der Befragten, ein Repräsentationsstreben für die eigene Person zuzugeben, ist darin $z u$ sehen, daß eine positive Korrelation $z u$ der Einschätzung "ich kenne Leute, die in Gesprächen ganz schön mit ihren Antiquitäten prahlen" und eine negative zu "es gibt viele Antiquitätenkäufer, die ohne Sachverstand 
MOTIVATION: REPRXSENTATION

Die erklurende variable ... wirkt ...

motivaticnshemmend

motivationsfordernd

$$
\text { - }
$$

$\begin{array}{lllllllll}0,4 & 0,3 & 0,2 & 0,1 & * & 0,1 & 0,2 & 0,3 & 0,4\end{array}$

auBere Erschelnung pflegen

reprasentativ

intensiver Hobby nachgehen

prahlen mit Antiquitäten

zeltlos

wertbeständig

nuchtern

reprasentativ

exklusiv

beruflich weiterkommen

Angabe mit Antiquitäten

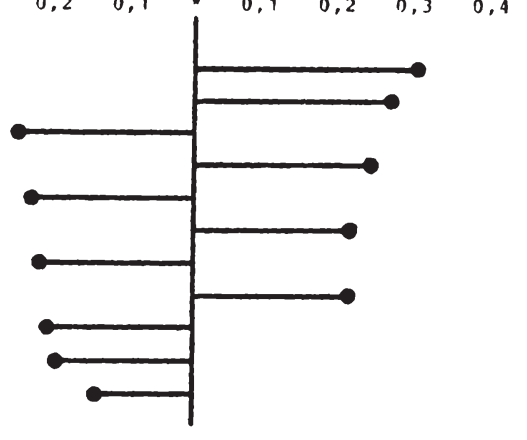

Abb. 33: Bestimmungsfaktoren der Motivation "Repräsentation"

kaufen, aber mit ihren Antiquitäten angeben" ermittelt werden konnte. Im konkreten, d.h. personell eingegrenzten Fall wird eine "Angabe" mit Antiquitäten konstatiert, wohingegen im allgemeinen Fall, in den sich der Käufer eher "projektiv" mit einbezieht, diese gleichzeitig verneint wird.

Darüber hinaus legen die empirischen Ergebnisse den Schluß nahe, daß zu "Repräsentationsantiquitäten" nur wenig "innere Beziehungen" bestehen, denn es zeigen sich sowohl negative Zusammenhänge $z u$ einer als "nüchtern" eingeschätzten Wohnatmosphäre (zu der ja auch der Antiquitätenbesitz i.d.R. beiträgt) und zum "Hobbywunsch" der Käufer. 
Abschließend fällt auf, daß das Repräsentationsstreben durch ein zunehmendes berufliches Engagement gehemmt zu werden scheint. Vermutlich handelt es sich dabei jedoch um eine "Scheinkorrelation" für die eine zunehmende Befriedigung mit dem bereits erreichten beruflichen status verantwortlich ist ${ }^{1}$. Die Bedeutung berufsorientierter Variablen wird im übrigen durch eine signifikant höhere Ausprägung des Repräsentationsstrebens bei männlichen Antiquitätenkäufern erklärbar ${ }^{2}$.

\subsection{Endogener Geschmackswandel}

Viele neuere Einrichtungsgegenstände sind unter streng funktionalen (z.B. ergonometrischen), praktischen (z.B. Pflege) und produktionstechnischen (Serienproduktion) Gesichtspunkten konzipiert. Hand in Hand mit der Architektur neigt das heutige Möbeldesign zu klaren, sachlichen Formen, geraden Linien und Flächen und damit zur Schmucklosigkeit und "Sterilität". Durch das Verkümmern einer Formvielfalt entsteht ein ästhetisches Defizit, welches ein geschmackliches Umschlagen zugunsten dekorativer und verspielterer objekte provoziert.

Während die varianzanalytische Untersuchung keine deutlichen Tendenzen aufzeigt, konnten im Rahmen der multiplen Regressionsanalyse die in Abbildung 34 dargestellten motivationalen Bestimmungsfaktoren isoliert werden.

1 Hierfür spricht insbesondere auch, daß das Repräsentationsstreben bei den ältesten Befragten signifikant höher ausgeprägt ist als bei den jüngsten Befragten. Vgl. Tabelle 3 im Anhang.

2 Vgl. Tabelle 2 im Anhang. 
MOTIVATION: REAKTION AUF MODERNES MOBELDESIGN

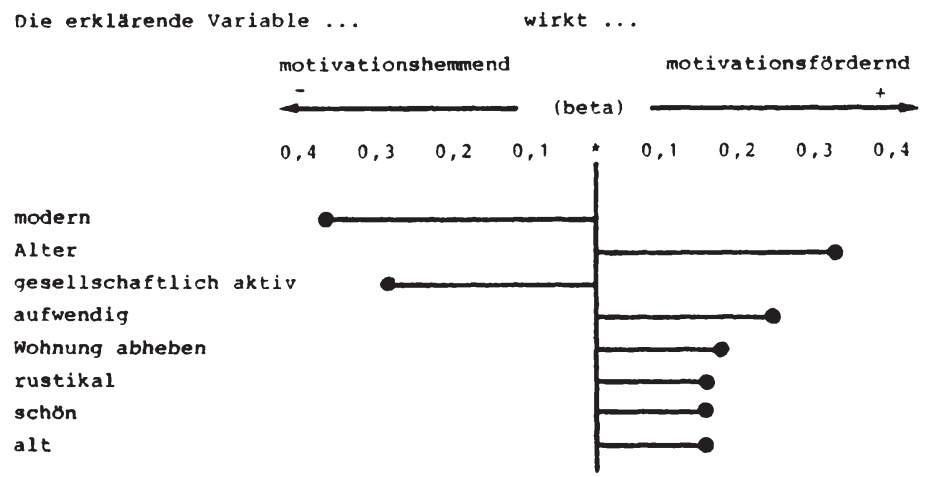

MOTIVATION: LOSUNG EINES FUNKTIONALEN EINRICHTUNGSPROBLEMS

Die erklarende Variable... wirkt...

gebrauchsfahig

reprasentativ

Freunde bei mir wohlfuhlen

aufwendig

Unabhangigkeit

kunstverstandig

ausgefallen

man muB schnell zugreifen

dekorativ

kritisch - unkritisch

rational - emotional

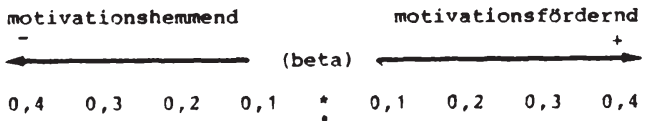

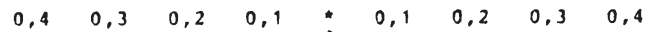

Abb. 34: Bestimmungsfaktoren der Motivationsdimension

"Endogener Geschmackswande1" 
Für die Käufermotivation "Reaktion auf modernes Möbeldesign" ergeben sich plausible Erklärungen: eine negative Korrelation ergibt sich $z u$ dem Bestimmungsfaktor "moderne Wohnatmosphäre"; positive Beziehungen sind zu einem "rustikalen", "aufwendigen" und "sich vom Durchschnitt abhebenden Wohnstil" erkennbar. Als Vorstellung von Antiquitäten dominieren die Assoziationen "alt" und "schön". Diese Einstellung, sowohl zum Wohnen als auch zu Antiquitäten, trifft tendenziell für ältere Menschen zu.

Die Motivation "Lösung eines funktionalen Einrichtungsproblems" liegt insbesondere bei Käufern vor, welche Antiquitäten als "gebrauchsfähig" und "repräsentativ", andererseits nicht als "ausgefallen" und "dekorativ" empfinden. Die Selbsteinschätzung der Befragten läßt den SchluB zu, daß "unkritisch" und "emotional" orientierte Käufer sich am ehesten mit Antiquitäten als Gebrauchsgegenständen einrichten. Je mehr sie glauben, durch den Besitz von Antiquitäten als Kunstverständige zu gelten und je eher sie das ziel verfolgen, daß sich Freunde in ihrer Wohnung wohlfühlen, desto ausgeprägter ist die angesprochene Motivation.

\subsection{Streben nach Ästhetik}

In der Faktorenanalyse hat sich die Motivationsdimension "Streben nach Ästhetik" als inhaltlich sehr heterogen herausgestellt. Die empirischen Ergebnisse ihrer Einzelmotive werden daher getrennt vorgestellt.

Ästhetik ist eine subjektive "Wahrnehmungskategorie" (Sinnesempfindung) des Antiquitätenkäufers. Seit langem gibt es die unterschiedlichsten philosophischen, kulturellen,kunsthistorischen, physiologischen und psychologischen 
Begriffsbestimmungen, die sowohl methaphysischer oder empirischer als auch analytisch-deskriptiver, formalistischer oder normativer Art sind. Für die Zwecke der Motivationsanalyse von Antiquitätenkäufern bietet sich eine allgemein gehaltene "Definition" an: "Here aesthetics is taken to refer to the entire realm in which people feel interest, pleasure, and emotion at the presence or absence of beauty." 1

Das "Bedürfnis nach Ästhetik" beschreibt sich damit als eine kognitive, affektive und verhaltensbezogene Reaktion der Käufer, welche unabhängig von objektiven ästhetischen Kriterien (z.B. normativ gesetzten Form- und Strukturidealen) ist ${ }^{2}$. Als wesentliche Bestimmungsgrößen eines "ästhetischen Motiviertseins" sind die individuellen "ästhetischen Maßstäbe" und der "ästhetische Wert" der Objekte (Antiquitäten) anzusehen. Eine modellhafte Einordnung des "ästhetischen Wertes" in die üblicherweise diskutierten "funktional und "psychisch/sozial bedeutsamen Warennutzen zeigt Abbildung 35. 3

Es erscheint plausibel, daß das Vorstellungsbild der "ästhetisch motivierten" Antiquitätenkäufer von Antiquitäten tendenziell "hoch" in der gezeigten "Pyramide" angesiedelt ist. Dabei ist gleichzeitig anzunehmen, daß Antiquitätenkäufer mit anderen Motivationen (z.B. "Einrichter") Antiquitäten auch an anderer Stelle (z.B. in Richtung Gebrauchsgegenstand) positionieren.

1 Levy, S.J., Czepiel, I.A., Marketing and Aesthetics, in: AMA Combined Proceedings 1974, Curhan, R.C. (ed.), 1975 , S. 387.

$2 \mathrm{Vgl}$. Holbrook, M.B., Some Preliminary Notes on Research in Consumer Esthetics, in: Advances in Consumer Research, Proceedings of the Association of Consumer Research, Olson, I.C. (ed.), San Francisco 1979, S. 104 .

3 Abbildung in Anlehnung an die "Functional-PsychologicalAesthetic Pyramid" von Levy, S.J., Czepiel, I.A., a.a.o., s. 387 . 


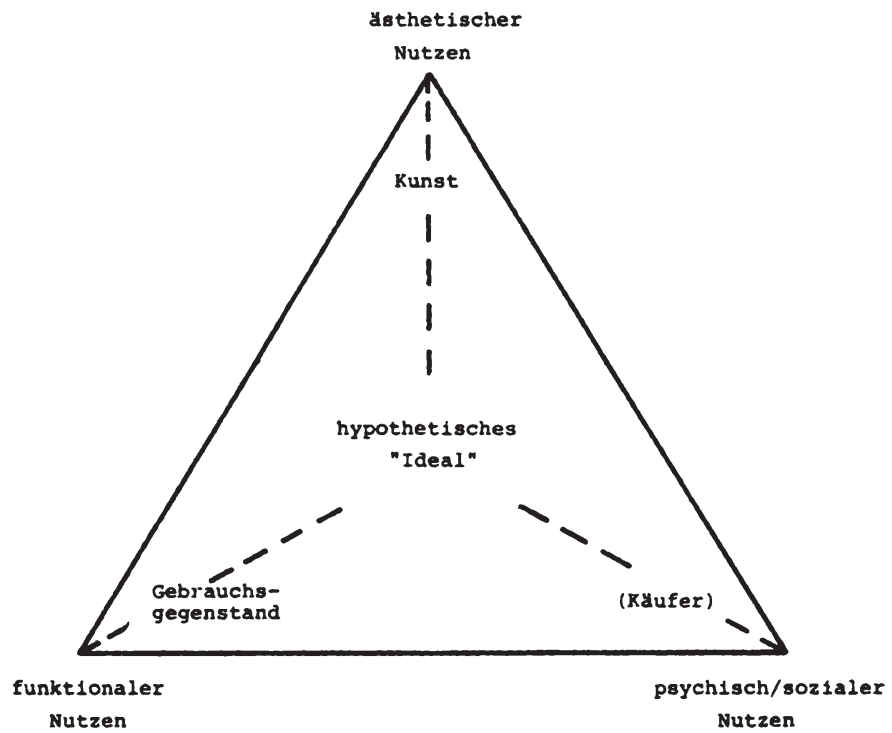

Abb. 35: Das "Aesthetik-Funktion-Psyche-Dreieck" zur Kennzeichnung struktureller Nutzenwerte von Antiquitäten

Ferner ist eine für Antiquitäten typische, zeitbedingte "Wanderung" von einem ursprünglich dominanten Funktionsnutzen zu mehr "ästhetischem und psychisch-sozialem" Nutzen denkbar ${ }^{1}$.

Der empirische Befund der Abbildung 36 zeigt, daB unter den

1 "In moving from bare function through varying degrees of integration of artistic design, aesthetic self-consciousness increases until pride in the beauty of the object may take precedence over its utility. It is no longer a practical object that happens also to look good, but it has become an art object that happens also to work", Levy, S.J., Czepiel, I.A., a.a.O., S. 388. 


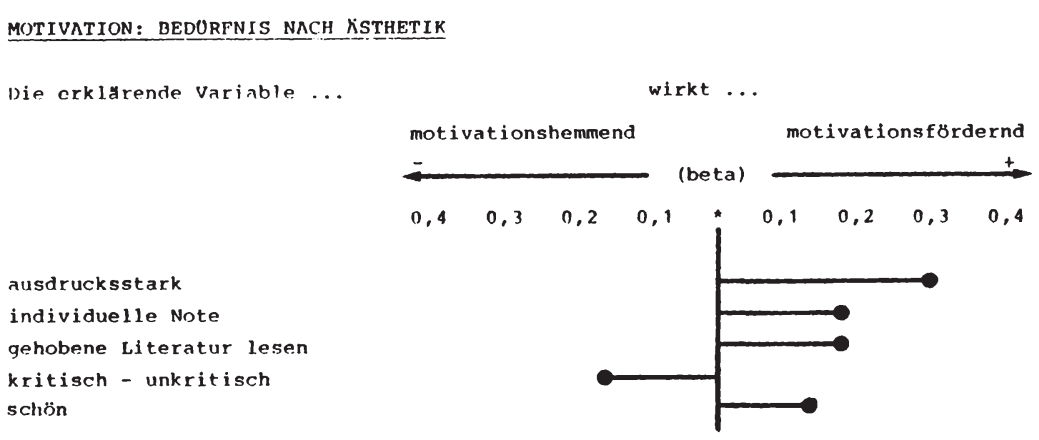

Abb. 36: Bestimmungsfaktoren der Motivation "Streben nach Ästhetik"

abgefragten Antiquitäteneigenschaften die "Ausdrucksstärke" und"Schönheit" die engsten Beziehungen zur Kaufmotivation "Bedürfnis nach Ästhetik" besitzen, wobei erstere erstaunlicherweise einen doppelt so hohen Erklärungsbeitrag leistet. Daneben besteht u.a. auch eine enge Beziehung zur Käufermeinung, daß "Antiquitäten ihrem Besitzer eine individuelle Note verleihen". "Ästhetik" scheint in den Augen der Käufer eine neue standardisierbare Wahrnehmungskategorie mit engen Bezügen zur individuellen Persönlichkeit und ihrer Ausstrahlung auf die soziale Umgebung zu sein.

Das "Bedürfnis nach Ästhetik" ist bei den weiblichen Antiquitätenkäufern signifikant höher ausgeprägt als bei den männlichen ${ }^{1}$. Eine gleichgerichtete Signifikanz kann für Käufer festgestellt werden, deren Interesse an Antiquitäten durch eine Erbschaft entstanden ist. Auffällig dagegen ist, daß die durch absatzpolitische Aktivitäten des Handels gewonnenen Antiquitäteninteressenten bei der genannten Motivation unterrepräsentiert sind ${ }^{2}$. Insgesamt zeigt sich hierin, "daß ein Ästhetizismus bei Antiquitäten"eine überwiegend familiär bedingte Genese besitzt.

1 Vgl. Tabelle 2 im Anhang.

2 Vgl. Tabelle 7 im Anhang. 
Das abschließend $z u$ diskutierende Einzelmotiv "Freude am Schenken" bedarf keiner inhaltlichen Präzisierung. Vielmehr steht im Mittelpunkt die Frage nach den personalen-, warenbezogenen- und situativen Bestimmungsgrößen dieser Motivation (vgl. Abbildung 37).

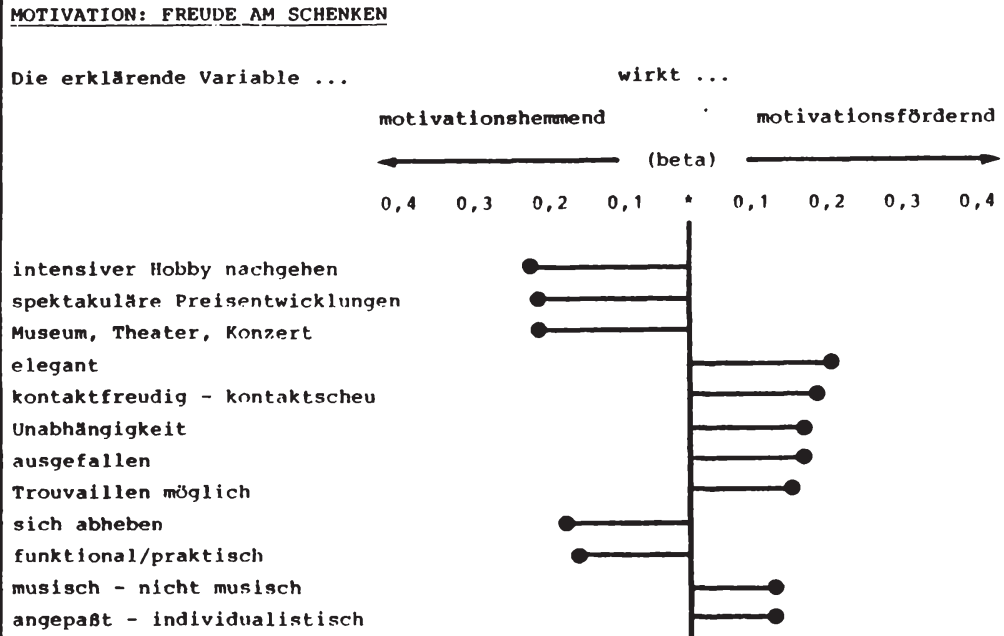

Abb. 37: Bestimmungsfaktoren der Motivation "Freude am Schenken"

Erwartungsgemäß sind die personenbezogenen Variablen in der Uberzahl. Inhaltlich wird dabei eine vielschichtige und nicht vollständig widerspruchsfreie Persönlichkeitsstruktur sichtbar. Interessant sind u.a. die Selbsteinschätzungen "individualistisch", "kontaktscheu" in Verbindung mit dem Ziel "persönliche Unabhängigkeit", ohne sich dabei allzusehr vom "Durchschnitt" abzuheben. Dieses Persönlichkeitsbild gibt AnlaB, im "Schenken von Antiquitäten" eine 
"soziale Uberbrückungs- und Anknüpfungsfunktion" zu vermuten.

Daß die "Käufer" nur im geringen Umfang die Vorstellung von Antiquitäten als "funktional/praktisch" besitzen, deckt sich mit der Intention ihrer Motivation, dem Beschenkten eine "ungebundene" (z.B. optische, ästhetische) Freude zu bereiten ${ }^{1}$.

Die bisherige Analyse der Motivation von Antiquitätenkäufern hat gezeigt, daß sich inhaltlich abgrenzbare und durch personen-, waren- und situationsbezogene Bestimmungsfaktoren weitgehend erklärbare Einzelmotivationen empirisch erfassen lassen. Nur im Ausnahmefall kann jedoch davon ausgegangen werden, daß das Verhalten von Antiquitätenkäufern durch monothematische "Motivationseinheiten" bestimmt wird. Der folgende Untersuchungsabschnitt wendet sich daher der Frage $z u$, ob sich für bestimmte Antiquitätenkäufergruppen typische Motivationskonfigurationen ermitteln und gegeneinander abgrenzen lassen.

3. Motivationstypen (Motivationskonfigurationen) und ihre Bestimmungsfaktoren

Die Untersuchung von Motivationstypen bzw. Motivationskonfigurationen erfolgt in zwei Schritten. Zunächst werden zur Verdeutlichung der empirischen operationalisierung inhaltliche und methodische Ansatzpunkte einer Typenbildung sowie die strukturellen Implikationen der Typenlösung (Dimensionalität) diskutiert. Darauf aufbauend schließt sich eine detaillierte Analyse einzelner Motivationstypen an.

$1 \mathrm{Zu}$ den signifikanten Unterschieden in der Motivationsausprägung einzelner soziodemographischer Variablen vgl. Tabellen 5, 6 und 7 im Anhang. Varianzanalytisch signifikante Einflüsse konnten dabei nicht nachgewiesen werden. 


\subsection{Operationalisierung}

\subsection{Ansatzpunkte und Vorgehensweise bei der Typenbildung}

Ausgangspunkt des Typenkonzeptes bildet die Annahme, daß bei einer statischen "Momentaufnahme" des motivationalen Geschehens für jeden Antiquitätenkäufer ein multithematisches und individuelles Beziehungsgefüge einzelner Motivationsinhalte offensichtlich wird. Solche individuellen Motivationsstrukturen kommen empirisch in einer differenzierten und abgestuften Einschätzung der Kaufbedeutung von Einzelmotivationen zum Ausdruck.

Auf dieser Grundlage läßt sich die Zielsetzung der Typenbildung präzisieren: Gesucht wird eine Zusammenfassung einzelner Antiquitätenkàufer zu Käufergruppen, deren Mitglieder sich durch eine weitgehende Ubereinstimmung ihrer individuellen Motivationsstrukturen auszeichnen. Untereinander sollen sich die Käufergruppen andererseits möglichst unähnlich sein. Die durchschnittlichen Motivationsstrukturen in den Käufergruppen repräsentieren die gruppenspezifischen Motivationskonfigurationen, die Gruppenmitglieder den Motivationstyp. Zur Ermittlung der Motivationstypen und -konfigurationen stellen clusteranalytische Verfahren das statistisch-methodische Instrumentarium ${ }^{1}$.

Die wichtigsten Entscheidungsbereiche bei der Planung der clusterungsstrategie umfassen ${ }^{2}$ :

1 Die im Rahmen der Käuferverhaltensforschung eingesetzten Clusteranalysen stützen sich dabei überwiegend auf heuristische Strategien, welche zu lokalen Optima bei der Typenbildung führen. Die Auswahl eines problemadäquaten Verfahrens beinhaltet deshalb eine nicht unwesentliche "Vorentscheidung" für die spätere Typenlösung. Vgl. Steinhausen, D., Langer, K., a.a.O., S. 19 u. 101; Schuchard-Ficher, Chr. et al., a.a.O., S. 149.

2 Vgl. Steinhausen, D., Langer, K., a.a.O., S. 19 ff.; Berekoven, L., Eckert, W. , Ellenrieder, P., Marktforschung, Methodische Grundlagen und praktische Anwendung, Wiesbaden 1977, S. 170 ff.. 
(1) Die Festlegung der typenbildenden Variablen

(2) Die Festlegung einer "Maßgröße" (Ähnlichkeitsfunktion) zur Bestimmung der Ähnlichkeiten zwischen den individuellen Motivationsstrukturen

(3) Die Festlegung eines Clusteralgorithmus, d.h. einer Verfahrensregel für die Bildung der Motivationstypen auf der Grundlage der ermittelten Ähnlichkeiten und unter Berücksichtigung einer Zielfunktion

(4) Die Festlegung einer geeigneten Typenzahl.

Die typenbildenden Variablen leiten sich direkt aus der problemstellung $a b$ und ergeben sich als die empirisch erhobenen individuellen Kaufbedeutungen einzelner Motivationsinhalte.

Aufgrund des als metrisch unterstellten Skalenniveaus und möglicher Korrelationen zwischen den typenbildenden Variablen wurde die "Mahalanobis-Distanz" als Ähnlichkeitsmaß bzw. -funktion gewählt. Sie unterdrückt bestehende Korrelationen durch eine $(k-)$ Transformation der Ausgangsdaten und schließt somit die Gefahr von "verzerrten" clusterlösungen weitgehend aus ${ }^{1}$.

Als clusteralgorithmus wurde ein gemischtes "Optimalverfahren" aus einer Abfolge eines hierarchisch-agglomera-

1 Die "Mahalanobis-Distanz" weist damit für die vorliegenden Ausgangsdaten Vorteile gegenüber den häufiger benutzten Ahnlichkeitsfunktionen "Euklidsche-Distanz" oder "City-block-Metrik" auf. Vgl. Steinhausen, D., Langer, K., a.a.O., S. $58 \mathrm{ff}$.. 
tiven und zwei weiterführenden partitionierenden Verfahren zusammengestellt'. Das Zielkriterium bildete jeweils das Varianzkriterium für transformierte Daten ${ }^{2}$.

Die Festlegung der "optimalen" Clusterzahl erfolgte in heuristischer Vorgehensweise, indem alternative Clusterzahlen (zwischen 2 und 10) dem Rechenalgorithmus vorgegeben wurden $^{3}$. Die Entscheidung für eine 9-Clusterlösung fiel

1 Das Vorgehen und die Programmierung in Fortran IV lehnt sich an die Empfehlungen von Steinhausen und Langer an. Unter der Vielzahl hierarchisch-agglomerativer Verfahren wurde aufgrund seines Zielkriteriums (Varianzkriterium) das "Wardverfahren" gewählt. Seine Ergebnisse liefern den Input für das erste partitionierende Verfahren und zwar das "Iterierte Minimaldistanzverfahren". Ziel des Vorgehens ist eine Voroptimierung der Anfangspartition für das abschließende Austauschverfahren "Hill-climbing". Das in Fortran IV geschriebene Clusteranalyseprogramm ist in Abbildung 2 im Anhang wiedergegeben. Es ermöglicht sowohl die isolierte Rechnung einzelner Verfahren als auch Kombinationen mit 7 weiteren hierarchisch-agglomerativen "Startverfahren" wie u.a. "Single-, Completeoder Average Linkage. Neben den Subroutines für die unterschiedlichen Verfahren ("AGLOM", "MINDS 1", "HLCMB 1") werden solche für die Datentransformation ("KTRANS"), die Erstellung von Dendogrammen ("DENDRO") sowie die Berechnung der Gruppencentroide und Varianzen ("MEANS") benötigt. Zur detaillierten Darstellung der "Programmbausteine" vgl. Steinhausen, D., Langer, K., a.a.0., S. 75-138.

2 vgl. ebenda, s. $105 \mathrm{f}$..

3 Da kein allgemeingültiger Vorschlag zur Lösung des Clusterzahlproblems existiert und die Bestimmung "optimaler" Clusterzahlen stets approximativ ist, werden oft spezielle "HilfsgröBen" zur Entscheidungsfindung herangezogen. Hierzu gehört u.a. die Veränderung des zielwertes (Varianzkriterium) bei unterschiedlichen Clusterlösungen. Vgl. Schuchard-Ficher, Chr. et al., a.a.0., S. 143 oder die Errechnung zusätzlicher Optimierungskriterien (im Clusterprogramm als "FCRIT" bezeichnet). Vgl. Steinhausen, D. , Langer, K., a.a.O., S. 171. Beide Kriterien leisten im vorliegenden Fall keinen Beitrag zur Entscheidungsfindung. Vgl. Tabelle 8 im Anhang, in der zugleich auch die Ergebnisse alternativer Verfahren- und Verfahrenskombinationen zusammengestellt sind. Teilweise stützen sich diese "Alternativrechnungen" auf verfügbare Clusterprogramme des Rechenzentrums der Universität Münster ("CAM-Cluster-Analyse-Münster" und "Clusterprogramm als lokale Erweiterung des SPSS-8") Vgl. Steinhausen, D., Neue Prozedur Cluster im SPSS-8 als lokale Erweiterung, in: inforum 1980, Heft 2, S. $27 \mathrm{ff}$. 
aufgrund sachlogischer tberlegungen, insbesondere der Clustergröße und -aussagefähigkeit und einer diskriminanzanalytischen Reklassifikationsprüfung ${ }^{1,2}$.

Eine graphische Darstellung des Gruppierungsvorganges, d.h. der Verschmelzung einzelner Käufer zu den 9 Motivationstypen ist für das voroptimierende, die "Startpartitionierung" errechnende, agglomerative Verfahren möglich ${ }^{3}$.

Abbildung 38 zeigt den Ablauf der Typenbildung und die "Startpartitionierung" in Form eines Dendogramms. Dabei repräsentieren kleine $\mathrm{ziffern}$ die befragten Antiquitätenkäufer und große Ziffern Typennummern. An der Skala unterhalb des Dendogramms ist das Distanzniveau der jeweiligen "Verschmelzungspunkte" als Ausdruck des Heterogenitätszuwachses einer Typenfusion ablesbar 4 .

Der "Schnitt" durch das Dendogramm auf dem Niveau der ausgewählten 9-Typenlösung zeigt, daß bis zu dieser Gruppierungsstufe relativ geringe Heterogenitätszuwächse (z.B. im Verhältnis $z u$ einer 2 oder 3-Clusterlösung) bei einer (erwünscht) starken Reduzierung der Gruppenzahl auftreten.

1 Für jede Clusterlösung wurde eine Diskriminanzanalyse mit den typenbildenden Einzelmotivationen als unabhängige Variablen gerechnet. Einzig die 9-Clusterlösung erreichte eine Reklassifikation von $100 \%$. Hierin ist ein Hinweis zu sehen, daß diese Lösung die trennschärfste darstellt.

2 Interessant ist, daß damit ebensoviele Cluster als Ausdruck unterschiedlicher Motivationskonfigurationen wie Faktoren als Ausdruck unabhängiger Inhaltsdimensionen ermittelt wurden.

3 "Startpartitionierung"bedeutet, daß die durch das agglomerative Ward-Verfahren aufgrund der vorgegebenen clusterzahl errechnete Gruppierungen als "Anfangslösung" in die weiterführenden Verfahren übernommen werden.

4 Vgl. Steinhausen, D., Langer, K., a.a.O., s. 93. 


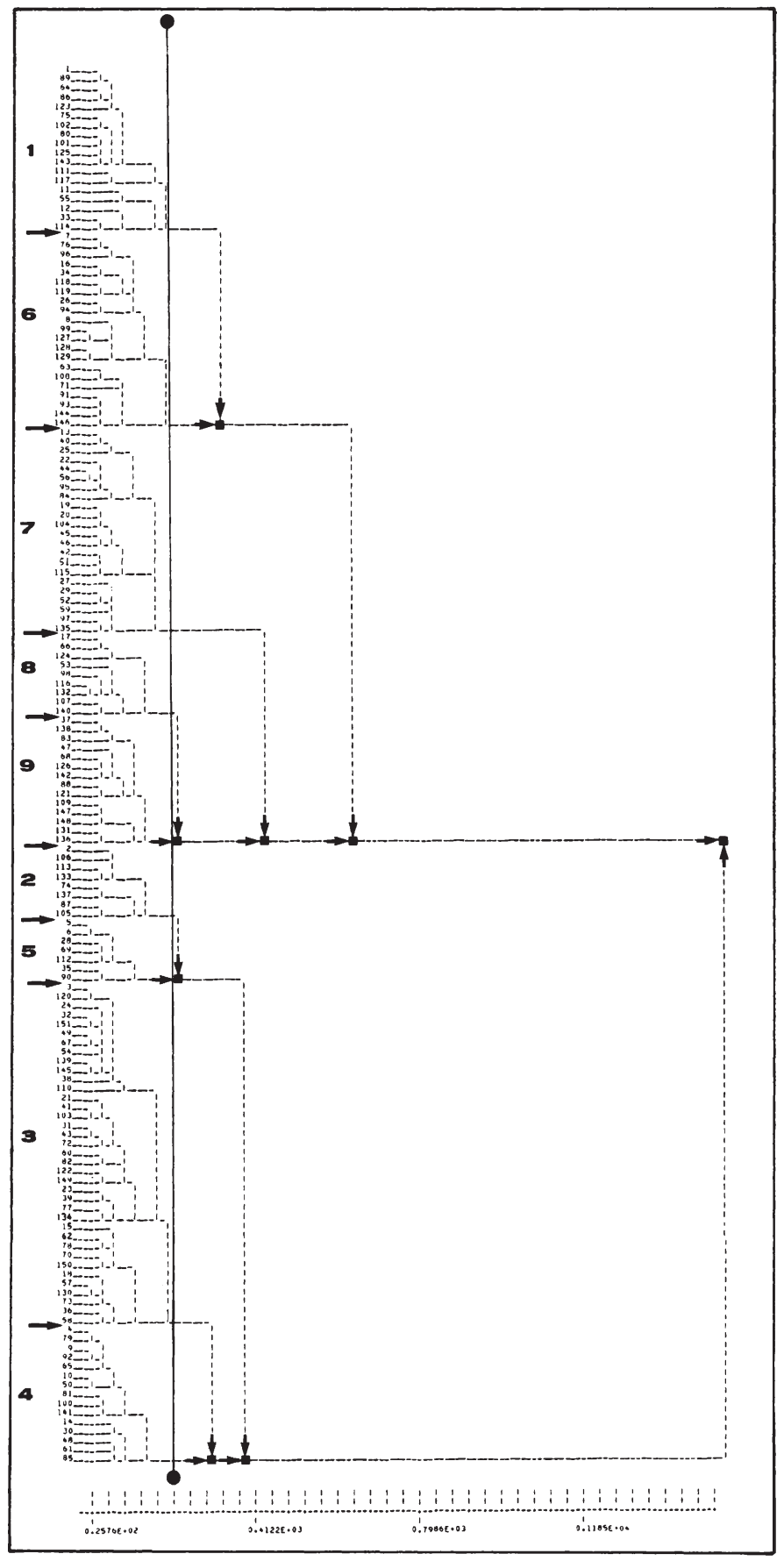

Abb. 38: Dendogramm für das voroptimierende, agglomerative "Ward-Verfahren" 245 graphigghger3-631-75024-7 Darstellung der Clusterbildung

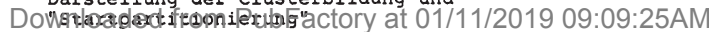


Schon die "Startpartitionierung" weist damit auf relativ homogene Motivationsstrukturen innerhalb der Motivationstypen hin.

Tabelle 9 faßt schließlich die Ergebnisse nach Durchlauf des vollständigen Clusterprogramms zusammen.

\begin{tabular}{|c|c|c|c|c|}
\hline $\begin{array}{c}\text { Beziehung der } \\
\text { tionstypen }\end{array}$ & $\begin{array}{c}\text { Motiva- } \\
\mathrm{NR}\end{array}$ & $\begin{array}{l}\text { Ujbereinstimmung } \\
\text { mit } \\
\text { "Startpartition" }\end{array}$ & $\begin{array}{l}\text { Anteil an der be- } \\
\text { fragten Antiqui- } \\
\text { tätenkäuferschaft } \\
\text { in } 8\end{array}$ & $\begin{array}{l}\text { Typenhomoge- } \\
\text { nität } \\
\text { (Clustervarianz) }\end{array}$ \\
\hline Nostalgiker & 1 & 938 & $10,0 \%$ & 1,2 \\
\hline Materialist & 6 & $85 \&$ & 13,28 & 1,1 \\
\hline Individualist & 7 & $78 \%$ & 15,28 & 0,8 \\
\hline Liebhaber & 8 & 588 & 8,08 & 1,1 \\
\hline Erlebniskäufer & 9 & 758 & 10,68 & 0,8 \\
\hline Rationalist & 3 & 868 & 19,98 & 0,9 \\
\hline Traditionalist & 4 & $64 \%$ & 11,38 & 1,4 \\
\hline Sammlex & 2 & $93 \%$ & $4,6 \%$ & 1,3 \\
\hline Einrichter & 5 & 658 & $7,3 \%$ & 0,8 \\
\hline
\end{tabular}

Tab. 9: Motivationstypen im tberblick

Eine nachträgliche Einzelfallauszählung kommt zu dem Ergebnis, daß sich die "Startpartitionierung" schon relativ gut an die endgültig ermittelten Motivationstypen annähert. Für die Motivationstypen mit hoher Ubereinstimmung (z.B. "Nostalgiker" oder "Sammler") kann das gezeigte Dendogramm daher die "Verwandtschaftsverhältnisse" der Typenmitglieder sowie auch der Motivationstypen untereinander annäherungsweise repräsentieren.

Obgleich im Rahmen der vorgestellten Lösung auch kleinere Motivationstypen erfaßt werden, kristallisiert sich andererseits kein Typ als besonders umfangreich bzw. dominant he- 
raus ${ }^{1}$. Dies legt den Schluß nahe, daß es einen vorherrschenden "Durchschnittstyp" unter den Antiquitätenkäufern anscheinend nicht gibt, sondern im Gegenteil sehr differenzierte "Individualtypen" die Regel bilden. $\mathrm{zu}$ ähnlichen Schlußfolgerungen hatte u.a. auch die hohe zahl faktorenanalytisch extrahierter Inhaltsdimensionen der Käufermotivation geführt.

Clustervarianzen sind als Kennziffern der Ähnlichkeit (Homogenität) der zu einem Typ zusammengefaßten individuellen Motivationsstrukturen interpretierbar. Die Verteilung der Typenhomogenitäten scheint im vorliegenden Fall nicht oder nur wenig von der Clustergröße abhängig zu sein; denn die "Individualisten", "Erlebniskäufer" und "Einrichter" bilden gemeinsam eine Gruppe tendenziell homogener Motivationstypen, deren Mitglieder geringere Abweichungen von der typenspezifischen Motivationskonfiguration (repräsentiert durch den clustercentroid) aufweisen. Relativ heterogen sind dagegen die individuellen Motivationsstrukturen der "Traditionalisten" und der "Sammler".

Typennamen sind kelne a-priori gegebenen oder unmittelbar aus dem Clusterprogramm folgende Beschreibungsmerkmale. Sie basieren auf der clusterinterpretation und sind daher inhaltlich $z u$ begründen. Aufgrund der Komplexität der 9-Typenlösung erscheint es zweckmäßig, zunächst die grundsätzliche struktur und die darin enthaltenen inhaltlichen Implikationen zu untersuchen.

1 Unter dem Gesichtspunkt ihrer quantitativen Marktbedeutung lassen sich die "Rationalisten", "Individualisten" und "Materialisten" zu den eher größeren und die "Liebhaber" "Einrichter" und "Sammler" zu den eher kleineren Käufergruppen zählen. 


\subsection{Dimensionalität der Typenlösung}

Die Bestimmung der Dimensionalität der gefundenen Typenlösung verfolgt das ziel, die grundsätzlichen strukturellen Unterschiede zwischen den isolierten Motivationstypen bzw. Motivationskonfigurationen aufzudecken und interpretatorisch zu nutzen.

Hierzu bietet es sich an, die Motivationstypen einer multivariaten Diskriminanzanalyse als Gruppierung vorzugeben und die Kaufbedeutungen der Einzelmotivationen auf ihren isolierten und gemeinschaftlichen Beitrag zur Typentrennung (-diskrimination) zu untersuchen ${ }^{1}$. Letztlich steht die Frage im Mittelpunkt, ob sich die gefundenen Motivationstypen signifikant hinsichtlich der Gesamtstruktur der typenbildenden Einzelmotivationen unterscheiden.

Die zur Beantwortung dieser Frage notwendigen diskriminanzanalytischen Ergebnisse sind in der Tabelle 10 zusammengestellt?

Zur Trennung der 9 Motivationstypen isoliert die Diskriminanzanalyse ein Set von 19 aus 26 einbezogenen Einzelmotivationen. Dieser set von Einzelmotivationen erweist sich als sehr gut geeignet, die Motivationstypen hinsichtlich

1 Schuchard-Ficher et al., sehen hierin einen "bisher weitgehend vernachlässigten Anwendungsbereich der Diskriminanzanalyse". Schuchard-Ficher, Chr. et al., a.a.o., S. 154 .

2 Die angegebenen Werte beziehen sich auf die in Tabelle 9 im Anhang charakterisierte MOTDISC 4. Dort werden die Inklusionsparameter der Diskriminanzanalyse spezifiziert und die Ergebnisvariationen bei Veränderungen dieser Parameter (MOTDISC 1 - MOTDISC 8) dargestellt. Zur Interpretation der Parameter vgl. Beutel, P. et al., a.a.O., S. $174 \mathrm{ff.} ;$ Klecka, W.R., Discriminant Analysis, Beitrag in: SPSS, Nie, N.H. et al., a.a.O., S. $447 \mathrm{f}$. und S. $452 \mathrm{ff..}$ Zur Interpretation der Parameter vgl. Beutel, P., et al., a.a.O., s. $174 \mathrm{ff.;}$ Klecka, W.R., Discriminant Analysis, Beitrag in: SPSS, Nie, N.H. et al., a.a.o., S. $447 \mathrm{f}$. und $452 \mathrm{ff}$.. 


\begin{tabular}{|c|c|c|c|c|c|c|c|c|}
\hline \multirow[t]{2}{*}{ MOTIVATION } & \multirow[t]{2}{*}{$\begin{array}{l}\text { WIL.KS' } \\
\text { LAMBDA }\end{array}$} & \multicolumn{2}{|c|}{$\begin{array}{l}\text { STANDARDISIERTE } \\
\text { DISKRIMINANZ- } \\
\text { KOEFFIZIENTEN DER } \\
\text { DISKRIMINANZFUNKTION }\end{array}$} & \multicolumn{2}{|c|}{$\begin{array}{l}\text { RELATIVE GEUEUTUNG } \\
\text { DER MOTIVATION IN } \\
\text { DISKRIMINANZFUNKTION }\end{array}$} & \multirow[t]{2}{*}{$\begin{array}{l}\text { GEWICUTETE } \\
\text { DISKRIMINANZ- } \\
\text { KOEFFIZIENTEN }\end{array}$} & \multicolumn{2}{|c|}{$\begin{array}{l}\text { RELATIVE BEDEUTUHU } \\
\text { DER MOTIVATIONEN } \\
\text { BEI DER DISKRIMINATION }\end{array}$} \\
\hline & & 1 & 2 & 1 & - & & in & RANG \\
\hline $\begin{array}{l}\text { Bewahrung von Erinnerungen } \\
\text { an die ramille }\end{array}$ & 0,1632 & $+0,11$ & $\oplus 0,72$ & 2,61 & 16,12 & 0,2361 & 5,77 & 9 \\
\hline Freizeityestal tung & 0,2316 & $\oplus 0,40$ & $-0,12$ & 9,63 & 2,58 & $0,2824 \triangleleft$ & $\triangleright 6,90$ & \\
\hline $\begin{array}{l}\text { Dokumentation von } \\
\text { Individuall smus }\end{array}$ & 0.1413 & $+0,33$ & $-0,17$ & 8,03 & 3.72 & $0,2617 \triangleleft$ & $D_{6,40}$ & \\
\hline $\begin{array}{l}\text { Liebe zum } \\
\text { alten Kunsthandwerk }\end{array}$ & 0,0909 & $+0,19$ & $\oplus 0,48$ & 4,64 & 10,79 & 0,2490 & 6,09 & 6 \\
\hline Bedurfnis nach Ausgleich & 0.0641 & $+0,32$ & +0.09 & 7.86 & 2,06 & 0,2345 & 5,73 & 10 \\
\hline Spas am Suchen & 0,0456 & $+0,19$ & $+0,19$ & 4,68 & 4,26 & 0,1841 & 4,50 & 13 \\
\hline Freude an Naturstoffen & 0,0340 & $-0,02$ & $-0,23$ & 0,57 & 5,11 & 0,1822 & 4,45 & 14 \\
\hline Desitzstreben & 0,0252 & $+0,15$ & $-0,15$ & 3,73 & 3,29 & 0,1635 & 4,00 & 16 \\
\hline Familientradition & 0,0196 & $-0,11$ & $\oplus 0,48$ & 2,57 & 10,64 & 0,1941 & 4.75 & 12 \\
\hline Besitzerstolz & 0,0152 & $\oplus 0.41$ & $+0,12$ & 9,93 & 2,60 & $0,2687 \triangleleft$ & $D 6,57$ & \\
\hline
\end{tabular}

$\rightarrow$ Motivationen mit der höchsten relativen Bedeutung insgesamt

- Motivationen mit der höchsten relativen Bedeutung in den einzelnen Diskriminanzfunktionen

Tab. 10: Die Bedeutung von Einzelmotivationen zur Diskrimination von Motivationstypen Ergebnisüberblick einer multiplen schrittweisen Diskriminanzanalyse (Teil I) 


\begin{tabular}{|c|c|c|c|c|c|c|c|c|}
\hline \multirow[t]{2}{*}{ MOT IVAT ION } & \multirow[t]{2}{*}{$\begin{array}{l}\text { WII.KS' } \\
\text { LAMBDA }\end{array}$} & \multicolumn{2}{|c|}{$\begin{array}{l}\text { STANDARDISIERTE } \\
\text { DISKRIMINANZ- } \\
\text { KOEFFIZIENTEN DER } \\
\text { DISKRIMINANZFUNKTION }\end{array}$} & \multicolumn{2}{|c|}{$\begin{array}{l}\text { RELATIVE BEDEUTUNG } \\
\text { DER MOTJVATION IN } \\
\text { DISKRIMINAJZFUNKTION }\end{array}$} & $\begin{array}{l}\text { GEWICHTETE } \\
\text { DISKRIMINANZ- } \\
\text { KOETFIZIENTEN }\end{array}$ & \multicolumn{2}{|c|}{$\begin{array}{l}\text { RELATIVE BEDEUTUNG } \\
\text { DER MOTIVATIONEN } \\
\text { BEI DER DISKRIMINATION }\end{array}$} \\
\hline & & 1 & 2 & 1 & 2 & & in 8 & RANG \\
\hline Hoffnung duf Trouvaille & 0,0124 & +0.31 & $-0,19$ & 7,58 & 4,34 & 0,2481 & 6,07 & 7 \\
\hline Wertanlage & 0,0102 & $+0,17$ & $-0,36$ & 4,19 & 8,06 & 0,2004 & 4,90 & 11 \\
\hline $\begin{array}{l}\text { Lobsurg eines funktionalen } \\
\text { Einrichtungsproblems }\end{array}$ & 0,0085 & $-0,07$ & $+0,22$ & 1,81 & 5,01 & 0,1746 & 4,27 & 15 \\
\hline Sammelleidenschaft. & 0,0071 & $+0,01$ & $-0,18$ & 0,21 & 4,11 & 0,1625 & 3,97 & 17 \\
\hline $\begin{array}{l}\text { Empfinden eines } \\
\text { kulturellen Auftrags }\end{array}$ & 0,0059 & +0.26 & +0.31 & 6,39 & 6,95 & 0,2410 & 5,89 & 8 \\
\hline - vekoration der Wohnung & 0.0050 & $+0,36$ & $-0,12$ & 8,78 & 2,70 & $0,2517 \triangleleft$ & $D 6,15$ & \\
\hline$\rightarrow$ Reaktion auf moderne Kunst & 0,0042 & $+0,37$ & $-0,28$ & 9,04 & 6,36 & 0,27154 & $D 6,64$ & \\
\hline Nostalgie & 0,0035 & $+0,19$ & $+0,02$ & 4,55 & 0,39 & 0,1624 & 3,97 & 17 \\
\hline Freude am Schenken & 0,0031 & $+0,13$ & $-0,04$ & 3,19 & 0,91 & 0,1217 & 2,98 & 18 \\
\hline & & & & & & 4,0902 & 100,00 & \\
\hline
\end{tabular}

- Motivationen mit der höchsten relativen Bedeutung insgesamt

Motivationen mit der höchsten relativen Bedeutung in den einzelnen Diskriminanzfunktionen Insgesamt erreichter Zielwert Wilks' Lambda

Tab. 10 : Die Bedeutung von Einzelmotivationen zur Diskrimination von Motivationstypen Ergebnisüberblick einer multiplen schrittweisen Diskriminanzanalyse (Teil II) 
ihrer motivationalen Sturktur zu trennen (vgl. den erreichten Zielwert Wilk's Lambda von 0,0031$)^{1}$. Dies läßt den Schluß $z u$, daß die Motivationstypen sowohl eine hohe "innere" Homogenität als auch eine hohe "außere" Heterogenität besitzen.

Die aus den gewichteten Diskriminanzkoeffizienten abgeleitete relative Bedeutung einzelner Motivationsinhalte für die Typentrennung weist annähernd gleich hohe Prozentanteile aus ${ }^{2}$. Alle aufgeführten Einzelmotivationen können damit als wichtige Bestandteile der Gruppentrennung angesehen werden. Zugleich kommt hierin zum Ausdruck, daß sich die Motivationstypen durch eine außerordentlich komplexe und gesamtheitlich zu betrachtende Motivationsstruktur unterscheiden.

Gerade unter diesem Gesichtspunkt gewinnt die Frage nach der (motivationalen) Dimensionalität der Typenlösung besondere Bedeutung. Ansatzpunkte zur Analyse solcher Strukturdimensionen bieten die ermittelten Diskriminanzfunktionen (Trennfunktionen) und die in ihnen schwerpunktartig zusammengefaßten Trennbeiträge einzelner Motivationsinhalte (vgl. die standardisierten Diskriminanzkoeffizienten bzw. die relative Bedeutung der Einzelmotivationen in den Diskriminanzfunktionen) 3,4 .

1 Wilk's Lambda kann Werte zwischen 0 und 1 annehmen. Je geringer dieser Wert, umso besser ist die Diskriminanzstärke der ermittelten Diskriminanzfunktionen $\mathrm{zu}$ beurteilen. Vgl. Schuchard-Ficher, Chr. et al., a.a.0., S. 174; Das Wilk's Lambda bewegt sich auf einem 99,98 Signifikanzniveau.

2 Die Ermittlung der gewichteten Diskriminanzkoeffizienten und die Ermittlung der relativen Bedeutung einzelner unabhängiger Variablen ist nicht im SPSS-Programmpaket "DISCRIMINANT" implementiert. Zur Berechnung vgl. SchuchardFicher, Chr. et al., a.a.O., S. 195.

3 Aus Gründen der Uberschaubarkeit und der anschließenden graphischen Darstellung der Motivationstypen beschränkt sich die Analyse auf die beiden wichtigsten Diskriminanzfunktionen. Gemeinsam tragen sie $2 / 3$ (46 \& Fkt. 1 und 20 \& Fkt.2) der Trenninformation aller Diskriminanzfunktionen und weisen mit kanonischen Korrelationskoeffizienten von 0,92 und 0,83 enge Beziehungen zu den Gruppierungsvariablen auf. Zur Kennzeichnung der weiteren Diskriminanzfunktionen vgl. Tabelle1o im Anhang. 
Die erste Funktion läßt sich dabei sinnvoll als (motivationales) Engagement gegenüber Antiquitäten interpretieren. Hierfür sprechen im wesentlichen zwei Argumente: Zunächst besitzen die standardisierten Diskriminanzkoeffizienten fast durchgängig relativ hohe und positive Werte, so daß hohe (niedrige) Ausprägungen der Kaufbedeutung einzelner Motivationen $z u$ gleichgerichteten hohen (niedrigen) Diskriminanzwerten bzw. Positionen auf dieser Diskriminanzfunktion führen; m.a.W.: Ein einzelner Käufer mit einer multithematisch und stark (positiv) ausgeprägten individuellen Motivationsstruktur wird durch einen höheren Wert auf dieser Dimension gekennzeichnet als ein Käufer mit einer monothematisch und niedrig ausgeprägten Motivationsstruktur ${ }^{1}$. Daneben zeigen die standardisierten Diskriminanzkoeffizienten, daß insbesondere ein zeitliches Engagement ("Freizeitgestaltung") und ein warenbezogenes Engagement ("Besitzerstolz") prägende Einzelmotivationen dieser Strukturdimension sind. Das "Engagement" trägt ca. 70 \% der gemeinsamen Trenninformation und ist damit die wesentlich bedeutendere Dimension.

Die zweite Funktion läßt sich sinnvoll als "Tradition" der Motivation interpretieren, wie die hohe diskriminatorische Bedeutung der Einzelmotivationen "Bewahrung von Erinnerungen an die Familie" $(+0,72)$, "Liebe zum alten Kunsthandwerk" $(+0,48)$ und "Familientradition" $(+0,48)$ zeigen. Insgesamt trägt die "Tradition" weitere $30 \%$ der gemeinsamen Trenninformation beider Dimensionen

4 Als methodisches Beispiel vgl. Johnson, R.M., Market Segmentation - A Strategic Management Tool, in: JoMR, Vol. 9 (February 1971), S. 13-18.

1 Zwischen den genannten Extremen liegen "Mischformen", welche $\mathrm{zu}$ gleichen Werten des Engagements führen können (z.B. monothematisch / hoch vs. multithematisch / schwach). 
Uber die Bestimmung ihrer Diskriminanzwerte lassen sich damit sowohl die einzelnen befragten Käufer als auch die Motivationskonfigurationen (clustercentroide) im 2-dimensionalen, motivationalen Diskriminanzraum positionieren (vgl. Abbildung 39).

Die gezeigten Clustergrenzen schließen als "Hilfslinien" alle zu einem Motivationstyp gehörenden Antiquitätenkäufer (vgl. Punkte) ein ${ }^{1}$. Daß eine solche überschneidungsfreie "Grenzziehung" im 2-dimensionalen Diskriminanzraum überhaupt möglich ist, weist auf die außerordentlich hohe Diskriminationsfähigkeit der beiden ermittelten Dimensionen hin.

Die "Ausdehnung" der Motivationstypen und die Lage einzelner Käufer zu "ihrer" Motivationskonfiguration (Centroid) geben Anhaltspunkte zur "visuellen" Beurteilung der Typenhomogenität ${ }^{2}$. Parallelen zu den quantitativen Maßzahlen (vgl. Tabelle 9) sind dabei z.B. für die "Sammler" oder "Traditionalisten" als relativ heterogene Typen oder die "Individualisten" und "Erlebniskäufer" als relativ homogene Typen offensichtlich.

Von besonderer Bedeutung für die inhaltliche Charakterisierung der Motivationstypen ist die Lage ihrer Motivationskonfiguration (Centroide) im Diskriminanzraum ${ }^{3}$.

1 Die "wahren" Verläufe der geometrischen clustergrenzen sind generell nicht bestimmbar, da die Typenzugehörigkeiten durch Wahrscheinlichkeitsverteilungen gekennzeichnet sind.

2 Die "Nachbarschaftsverhältnisse" der einzelnen Käufer untereinander bieten zudem die Grundlage detaillierter Einzelfallanalysen, da eine individuelle Identifikation und damit zugleich ein Rückgriff auf das originäre Datenmaterial (Fragebogen) grundsätzlich möglich ist.

$3 \mathrm{zu}$ den $\mathrm{Zahlenwerten} \mathrm{vgl.} \mathrm{Tabelle} 11$ im Anhang. 


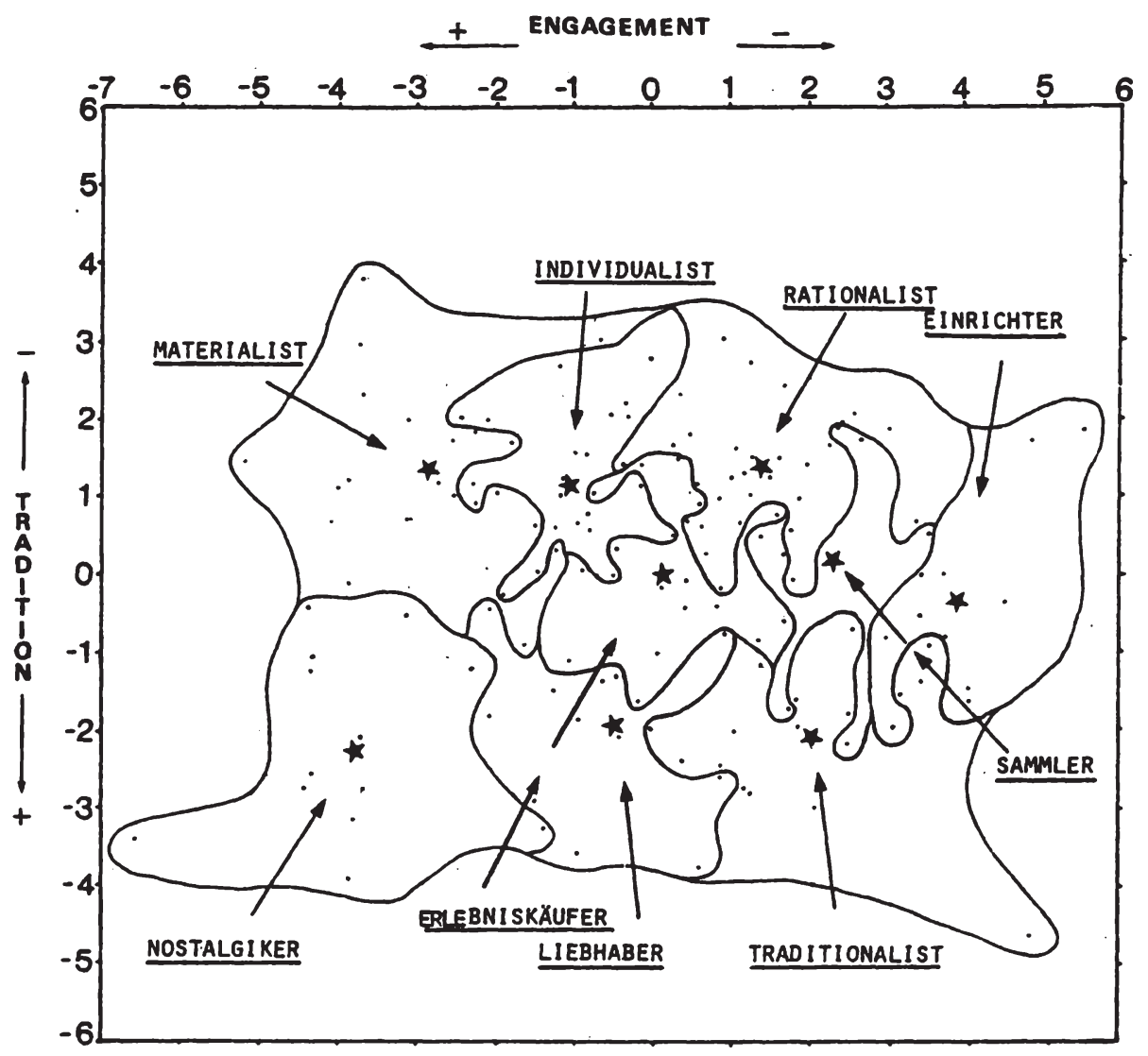

Clustercentroide

Abb. 39: Motivationstypen und -konfigurationen im 2-dimensionalen ("reduzierten") Diskriminanzraum 
Der empirische Befund (vgl. Abbildung 39) zeigt, daß sich unter dem Gesichtspunkt einer "Traditionsgebundenheit" der Motivationskonfigurationen tendenziell drei Untergruppen bilden lassen:

(1) "Materialisten", "Individualisten" und "Rationalisten" erweisen sich als eher traditionsunbeeinflubte Konfigurationen

(2) "Erlebniskäufer", "Sammler" und "Einrichter" erweisen sich als eher traditionsneutrale Konfigurationen

(3) "Nostalgiker", "Liebhaber" und - erwartungsgemäB "Traditionalisten" erweisen sich als traditionsgebundene Konfigurationen

Unter dem Gesichtspunkt ihres motivationalen Engagements lassen sich tendenziell zwei Untergruppen bilden:

(1) "Nostalgiker", "Materialisten", "Individualisten" und "Liebhaber" zeichnen sich durch überdurchschnittliches (starkes) motivationales Engagement

(2) "Erlebniskäufer", "Rationalisten", "Traditionalisten", "Sammler" und "Einrichter" zeichnen sich dagegen durch unterdurchschnittliches (schwaches) motivationales Engagement aus.

Motivationale "Antipoden" bilden demnach:

(1) "Nostalgiker" (hohes Engagement / hohe Traditionsgebundenheit) und "Rationalisten" (schwaches Engagement / schwache Traditionsgebundenheit)

(2) "Traditionalisten" (schwaches Engagement / hohe Traditionsgebundenheit) und "Materialisten" (hohes Engagement / schwache Traditionsgebundenheit).

Uberdies besitzen die "Erlebniskäufer" als einzige eher mittelbar warenbezogene Motivationskonfiguration interessanterweise eine "neutrale" Position unter den ansonsten eher unmittelbar auf Antiquitäten ausgerichteten Motivationskonfigurationen. 
Typenbildende Clusteranalyse und typenstrukturierende Diskriminanzanalyse liefern damit bereits im strukturellen Uberblick éin klares, geschlossenes und plausibles Bild unterschiedlicher Motivationstypen im Antiquitätenmarkt. Zu einer weiteren Verfeinerung des Verständnisses dient die folgende Detailanalyse.

\subsection{Analyse der Motivations-Typen}

Die Detailanalyse der Motivations-Typen erfolgt auf drei Ebenen:

(1) Beschreibung der Motivationstypen anhand ihrer spezifischen Motivationskonfiguration

(2) Beschreibung der Motivationstypen anhand eines diskriminanzanalytisch bestimmten Bündels personenund warenspezifischer Motivationsdeterminanten

(3) Beschreibung der Motivationstypen anhand soziodemographischer Merkmale.

Der Ablauf der Analyse folgt der strukturellen Klassifikation in stark engagierte und in schwach engagierte Motivationstypen $^{\top}$. Innerhalb beider Untergruppierungen beginnt die Analyse mit dem jeweils am stärksten engagierten Motivationstyp.

1 Die Diskriminanzfunktion 1 (Engagement) wurde gewählt, da sie gegenüber der Diskriminanzfunktion 2 - wie bereits erwähnt - eine erheblich höhere Trenninformation beinhaltet. 


\subsection{Stark engagierte Typen}

\subsection{Nostalgiker}

Die Motivationskonfiguration "Nostalgiker" zeigt entsprechend dem hohen motivationalen Engagement dieses Käufertyps für fast alle Einzelmotivationen eine gegenüber dem Gesamtdurchschnitt aller Käufer höhere, d.h. überdurchschnittliche Ausprägung (vgl. Abbildung 40) ${ }^{1}$. Für Mittelwertanalysen auf der Grundlage 5-stufiger Skalen sehr aussagekräftige Abweichungen von z.T. weit über 1,5 Skaleneinheiten sind für diese Konfiguration dabei keine Seltenheit. Es erstaunt daher nicht, daß die "Nostalgiker" auch absolut für fast die Hälfte aller Einzelmotivationen die höchste Ausprägung besitzen ${ }^{2}$.

Eine interessante Ausnahme besteht für das Einzelmotiv "Lösung eines funktionalen Einrichtungsproblems", welches als einziges eine unterdurchschnittliche Bedeutung aufweist. Im Zusammenhang mit einer gegenläufigen Beurteilung der "Dekoration der Wohnung" kommt darin eine sehr differenzierte Haltung der "Nostalgiker" bezogen auf das Wohnen mit Antiquitäten zum Ausdruck.

1 Legende der Abbildungen 40,43,46,55, 58, 61 und 64 1.) $+(-)=\Delta \geq 0,5$ $++(--)=\Delta \geq 1,0\}$ bezogen auf den Gesamtdurch$++(--)=\Delta \geq 1,5]$ schnitt

2.)+(-)=$ stärkste (schwächste) + Motivationsausprägung $\begin{aligned}++ & =2 . \text { stärkste (schwächste) } \\ +++(--) & =3 . \text { stärkste (schwächste) }\end{aligned}$ bezogen auf alle

3.) * (********) : signifikant $(\alpha=0,95)$ unterschiedliche Motivationsausprägung gegenüber $5(6,7,8)$ anderen Typen (Least-significant-difference-Test)

2 Im Intergruppenvergleich der je Motivationstyp erreichten Anzahl absolut höchster (niedrigster) Ausprägungen von Einzelmotivationen sowie im Intergruppenvergleich der gewogenen Gesamtmittelwerte über alle 26 Einzelmotivationen findet die Interpretation der Diskriminanzfunktion "Engagement" nachträglich eine eindrucksvolle Bestätigung, da

(1) die Anzahl höchster (niedrigster) Motivationsausprägungen mit zunehmendem(abnehmendem) Engagement tendenziell steigt

(2) die Gesamtmittelwerte-bis auf eine geringfügige Ausnahmemit zunehmendem (abnehmendem) Engagement monoton sinken

Zum quantitativen Beleg vgl. Tabelle 12 im Anhang. 
Lösung eines funktionalen Einrichtungsproblems

Dekoration der Wohnung

Dokumentation von Individualismus

Wertanlage

Spekulation auf Wertzuwachs

Wunsch nach Selbstverwirklichung

Besitzstreben

Sammelleidenschaft

Bedürfnis nach Ästhet1k

Freude am Schenken

Reaktion auf modernes Mobeldesign

Steuerflucht

Nostalgie

Freizeltgestaltung

Yoffnung auf Trouvaille

SpaB am Suchen

Freude am Einkaufserlebnis

Familientrad 1 ¿ion

Besitzerstolz

Repräsentation

Beaürfnis nach Ausgleich

Empfinder elnes

kulturellen Auftrags

Reaktion auf moderne kunst

Bewahrung von

Erinnerungen an die Familie

Liebe zum alten Kunsthandwerk

Freude an Naturstoffen

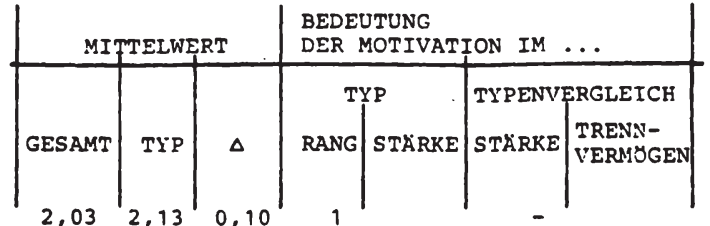

$2,032,13 \quad 0,10$

$2,40 \quad 1,73 \quad 0,66 \quad 16$

$3,32 \quad 2,20 \quad 1,12$

$\begin{array}{llll}3,38 & 3,00 & 0,38 & 20\end{array}$

$\begin{array}{llll}4,26 & 4,27 & 0,01 & 2\end{array}$

$3,57 \quad 2,27 \quad 1,30 \quad 5$

$\begin{array}{llll}3,08 & 2,33 & 0,75 & 15\end{array}$

$3,7 \varepsilon \quad 2,73 \quad 1,04 \quad 11$

$\begin{array}{llll}2,17 & 1,60 & 0,57 & 17\end{array}$

$\begin{array}{llll}3,92 & 3,87 & 0,05 & 21\end{array}$

$\begin{array}{llll}3,42 & 2,53 & 0,89 & 13\end{array}$

$\begin{array}{llll}4,83 & 4,80 & 0,03 & 22\end{array}$

$3,44 \quad 1,53 \quad 1,91 \quad 1$

$3,36 \quad 2,20 \quad 1,16 \quad 8$

$\begin{array}{llll}3,36 & 2,93 & 0,43 & 19\end{array}$

$2,55 \quad 1,73 \quad 0,82 \quad 14$

$\begin{array}{llll}3,43 & 2,53 & 0,90 & 12\end{array}$

$3,58 \quad 2,13 \quad 1,45$

$3,15 \quad 1,93 \quad 1,22$

$\begin{array}{llll}4,54 & 4,00 & 0,54 & 18\end{array}$

$\begin{array}{llll}3,05 & 1,33 & 1,71 \quad 2\end{array}$

$3,64 \quad 2,07 \quad 1,57$

$3,82 \quad 2,73 \quad 1,10 \quad 10$

$3,64 \quad 1,73 \quad 1,91$

$3,40 \quad 2,20 \quad 1,20$

$2,82 \quad 2,80 \quad 0,02 \quad 23$

Abb. 40: Motivationskonfiguration "Nostalgiker" (Teil I) 
(1)

sehr

(2)

ziemlich

(3)

etwas

(4)

kaum

wichtig

wichtig

wichtig

wichtis

(5)

gar nicht

wichtig

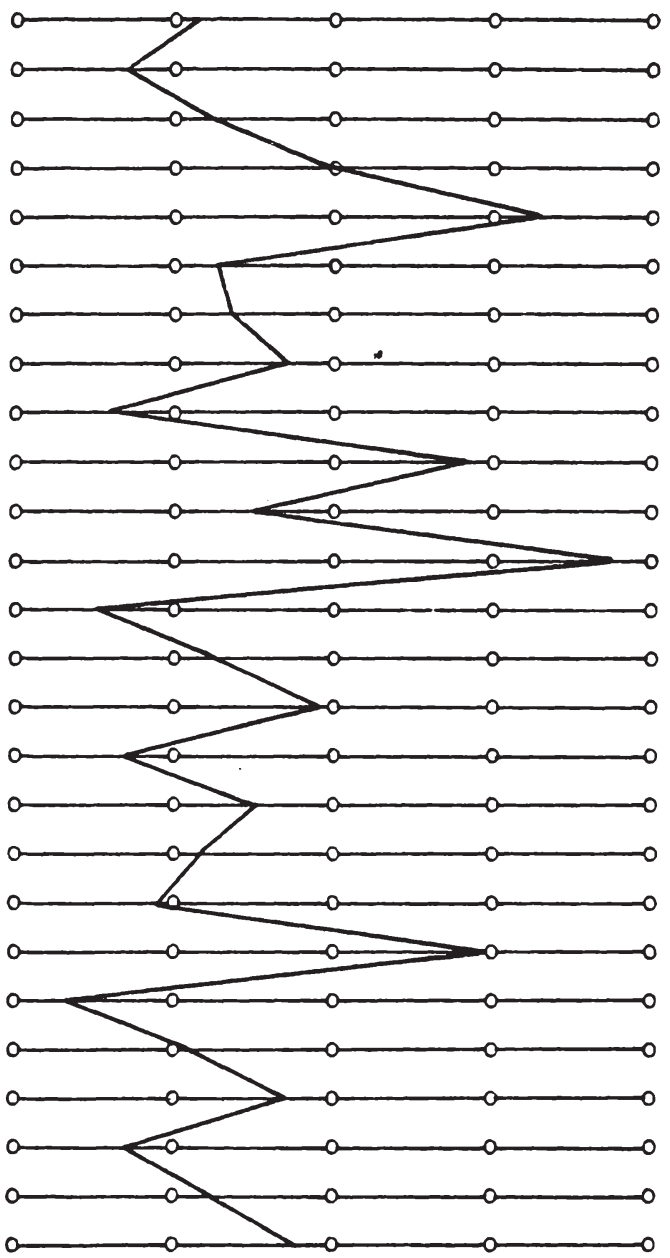

Abb. 40: Motivationskonfiguration "Nostalgiker" (Teil II) 
Im "relativen" Vergleich zur Gesamtheit aller befragten Antiquitätenkäufer bilden die Einzelmotivationen "Nostalgie", "Bewahrung von Erinnerungen an die Familie", das "Bedürfnis nach Ausgleich" die Schwerpunkte der Motivationskonfiguration. Der innere zusammenhang der Einzelmotivationen ist offensichtlich, so daB sich bereits hier ein relativ "abgerundetes" motivationales Bild dieses Typs zeigt.

Im (absoluten) Vergleich $z u$ allen anderen isolierten Motivationstypen erweisen sich neben den bereits genannten Einzelmotiven der "Wunsch nach Selbstverwirklichung", das "Empfinden eines kulturellen Auftrags" sowie die "Dokumentation von Individualismus" als besonders trennscharf. Ihre Kaufbedeutung ist gegenüber allen acht, zumindest aber gegenüber 7 weiteren Typen signifikant höher.

Neben den typenbildenden Variablen zeigen auch die wichtigsten diskriminanzanalytisch ermittelten "passiven", d.h. zusätzlich typenbeschreibenden Variablen spezifische Schwerpunkte für das "Nostalgikercluster" (vgl. Abbildung 41) 1,2.

1 Die diskriminanzanalytisch untersuchten, typenbeschreibenden Variablen sind personen-, waren- und situationsbezogene Bestimmungsfaktoren der käufermotivation (vgl. Abbildung 25). Ziel war, ein überschaubares set motivationaler Bestimmungsfaktoren $\mathrm{zu}$ isolieren, welches "am besten" zur Trennung der vorgegebenen Motivationstypen geeignet ist. Hierzu wurden Diskriminanzanalysen mit unterschiedlichen Anforderungen an die Inklusionsparameter gerechnet. Diese Ergebnisse sind in Tabelle $13 \mathrm{im} \mathrm{Anhang}$ zusammengestellt. Die zur Interpretation ausgewählte Analyse (PRA DISC 2) ermittelt 14 motivationale Bestimmungsfaktoren, die zur weiteren Typenbeschreibung genutzt werden. Ihr singulärer und gemeinschaftlicher Beitrag zur Diskrimination ist der Tabelle 14 im Anhang zu entnehmen. Die Tabelleninterpretation kann in Analogie zur Texttabelle 8 (vgl. Kapitel C. 3.12) vorgenommen werden. Wichtige übergeordnete Ergebnisse der Diskriminanzanalyse sind:

(1) Die 14 isolierten motivationalen Bestimmungsfaktoren eignen sich gemeinschaftlich sehr gut zur Trennung der Motivationstypen (Wilk's Lambda $=0,05$ )

(2) Die Trennbeiträge der einzelnen Bestimmungsfaktoren sind tendenziell ausgeglichen, so daß sie für die Typentrennung relativ gleichbedeutend sind.

2 Die Skalierung der Variablen von (1) "trifft sehr zu" bis (5) 'trifft gar nicht zu" hat zur Folge, daß negative Mittelwertabweichungen einer höheren Ausprägung entsprechen. 


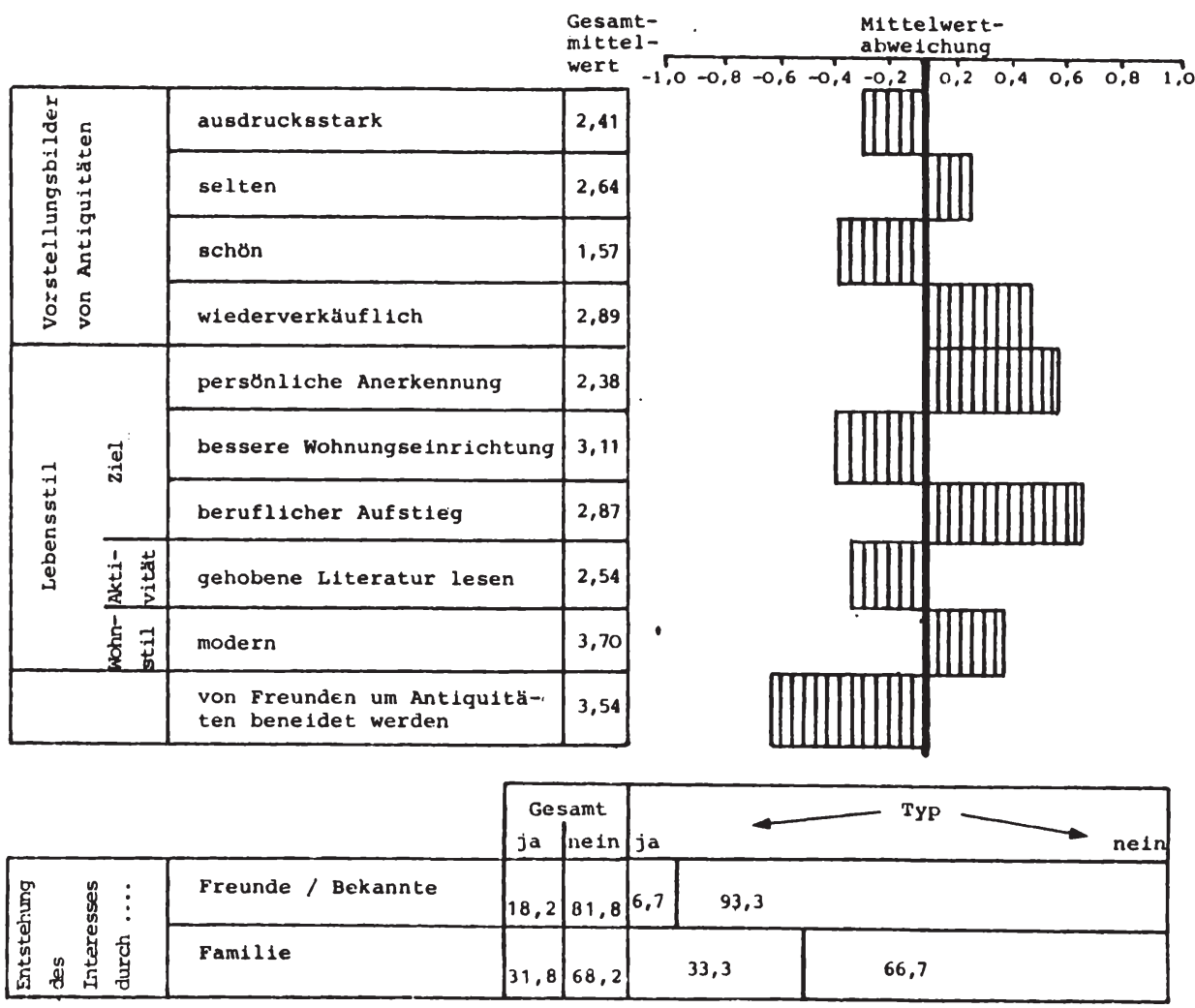

Abb. 41: Ausprägungen motivationaler Bestimmungsfaktoren im Motivationstyp "Nostalgiker" 


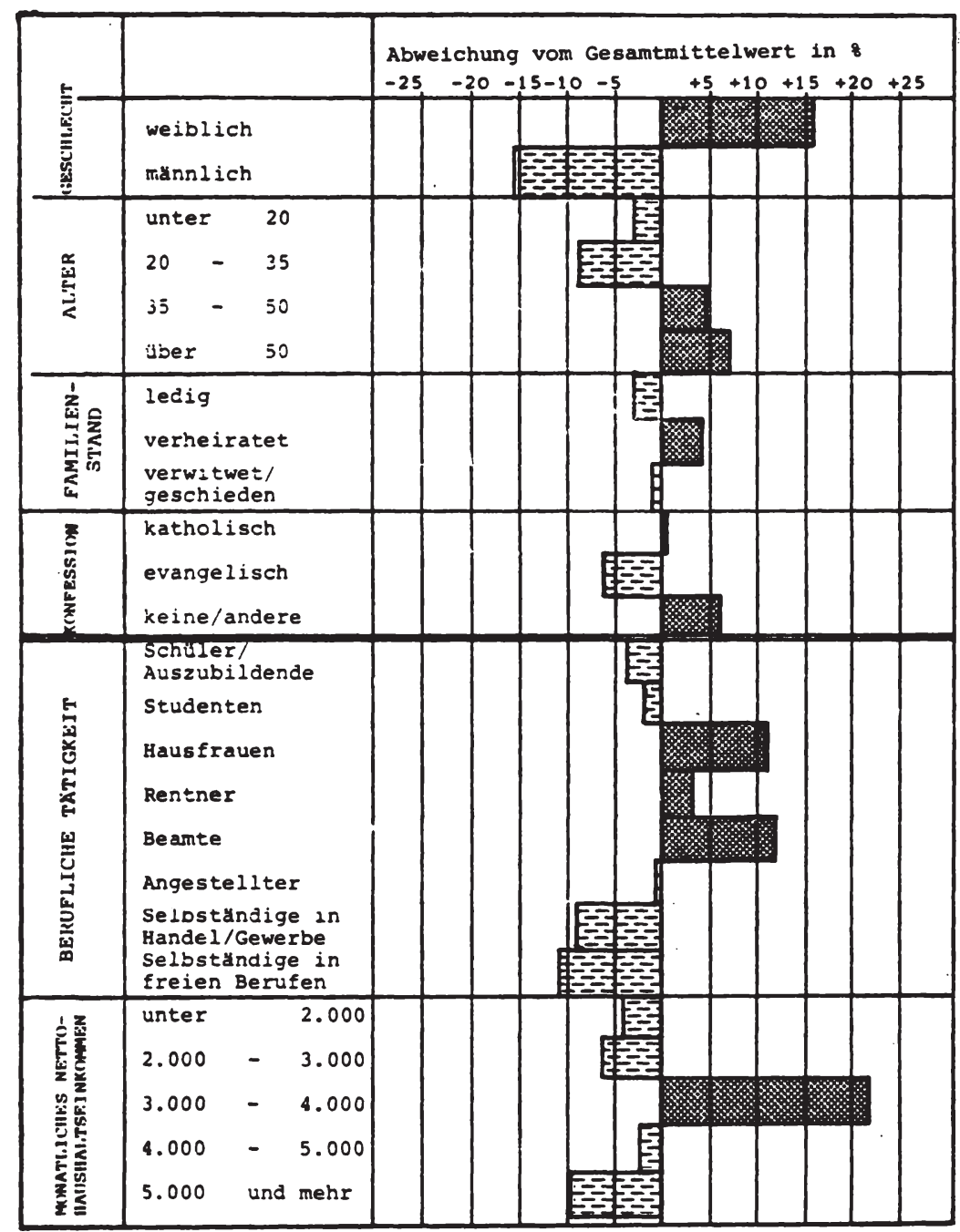

Abb. 42: Soziodemographie der "Nostalgiker" 
tberproportional intensiv verbinden die "Nostalgiker" die Warenmerkmale "schön" und "ausdrucksstark" in ihrer Vorstellung mit Antiquitäten. An eine "Seltenheit" oder "Wiederverkäuflichkeit" denken sie dagegen weniger. Auffällig gering ist das Interesse an einem beruflichen Fortkommen und an einer persönlichen Anerkennung durch die soziale Umwelt.

Der Wunsch nach einer besseren Wohnungseinrichtung ist schließlich überdurchschnittlich stark, wobei die Art des bisherigen Wohnstils der "Nostalgiker" eher als "nicht modern" einzuschätzen ist. Schließlich besitzt der Einfluß des Elternhauses bei der Entstehung eines Interesses an Antiquitäten nur eine "normale" Bedeutung; die Rolle von Freunden und Bekannten ist als sehr gering zu werten.

Das soziodemographische Profil ${ }^{1}$ der "Nostalgiker" weist erhebliche Dominanzen bei den Antiquitätenkäuferinnen gehobenen Alters, den Berufsgruppen Hausfrau, Rentner und Beamte sowie mittleren Einkommensklassen auf (vgl. Abbildung 42).

\subsection{Materialist}

Für die "Materialisten" unter den Antiquitätenkäufern ist ein fast ebenso hohes motivationales Engagement kennzeichnend, wie für die "Nostalgiker". Bezogen auf den motivationalen Ursprung ihres Engagements stehen diese beiden Motivationstypen in einem deutlichen Gegensatz, da die "Materialisten" die am wenigsten traditionsorientierte Käufer-

1 Die soziodemographischen Clusterbeschreibungen dienen ausschließlich deskriptiven Zwecken, so daß auch bei hohen Abweichungen von der "Durchschnittsverteilung" der Gesamtstichprobe nicht auf signifikante Einflüsse soziodemographischer Merkmale auf die Motivkonfigurationen geschlossen werden kann. 
gruppe darstellen. Die Motivkonfiguration der "Materialisten" zeigt ebenfalls überdurchschnittlich ausgeprägte Kaufbedeutung einer Vielzahl von Einzelmotiven,wobei die inhaltlichen Akzente jedoch sehr von denen der "Nostalgiker" abweichen (vgl. Abbildung 43).

So besitzt die "Spekulation auf Wertzuwachs" im Typenvergleich hier ihre absolut höchste Ausprägung. Die Ausprägungsdifferenzen $\mathrm{z} u$ den anderen clustern bewegen sich dabei auf einem durchgängig signifikanten Niveau, so daß dieses Einzelmotiv eine besonders hohe Trennschärfe besitzt. ErwartungsgemäB nimmt auch die "Wertanlage" im Rahmen der angesprochenen Motivationskonfiguration einen wichtigen Platz ein.

Interessant ist die enge Verbindung ökonomisch ausgerichteter Motivationsinhalte mit einem Streben nach Konsumerlebnis. Die "Brücke" zwischen diesen beiden Motivationskomponenten ist vor allem in der "Hoffnung auf eine Trouvaille" als Einzelmotiv zu sehen: Zum einen harmoniert dieses Motiv mit dem spekulativ-ökonomischen Reiz eines Antiquitätenkaufs; zum anderen aber auch mit dem "Spannungsreichtum" der Einkaufssituation selbst. Dagegen erweisen sich die besitzbezogenen Einzelmotivationen "Besitzstreben" und "Besitzerstolz" erwartungsgemäB als relevante Bestandteile der Motivationskonfiguration "Materialist".

"Freude am Einkaufserlebnis" und "Freizeitgestaltung" fügen sich damit gut in die Charakterisierung der "Materialisten" unter den Antiquitätenkäufern.

Wohnbezogene Motivationen sowohl dekorativer als auch funktionaler Art korrelieren eher negativ mit dem angesprochenen Käufertyp. Abbildung 44 zeigt die Ausprägungen der personen- und warenbezogenen motivationalen Bestimmungsfaktoren. Sämtliche Vorstellungsbilder von Antiquitäten besitzen ein gegenüber dem Gesamtdurchschnitt z.T. deutlich 
Lossung eines funktionalen Elnrichtungsproblems

Dekoration der wohnung

Dokumentation von Individualismus

Hertanlage

spekulation auf Wertzuwachs

Wunsch nach Selbstverwirklichung

Besitzstreben

Sammelleidenschaft

Bedurfnis nach Ästhetik

Freude am Schenken

Reaktion auf modernes Mrbeldesign

Steuerflucint

Nostalgie

Frelzeitgestaltung

Hoffnung auf Trouvaille

SpaB am suchen

Freude am Elnkaufserlebnis

Famflientradition

Besitzarstolz

Repräsentation

Bedürfnis nach Ausgleich

Empfinden eines

kulturellen Auftrags

Reaktion auf moderne Kunst

Bewahrung von

Erinnerungen an die Familie

Liebe zum alten Kunsthandwerk

Freude an Naturstoffen

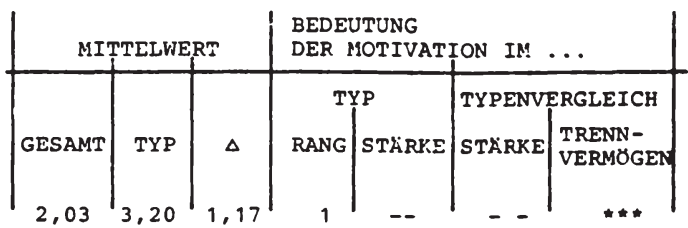

$2,40 \quad 2,00 \quad 0,40 \quad 13$

$3,32 \quad 2,90 \quad 0,42 \quad 12$

$\begin{array}{llll}3,38 & 2,45 & 0,93 & 6\end{array}$

$\begin{array}{llll}4,26 & 3,50 & 0,76 \quad 8\end{array}$

$\begin{array}{llll}3,57 & 3,05 & 0,52 & 11\end{array}$

$\begin{array}{llll}3,08 & 1,95 & 1,13 \quad 5\end{array}$

$3,78 \quad 2,85 \quad 0,93 \quad \epsilon$

$\begin{array}{llll}2,17 & 2,00 & 0,17 & 17\end{array}$

$3,92 \quad 3,20 \quad 0,72 \quad 9$

$\begin{array}{llll}3,42 & 3,75 & 0,33 & 2\end{array}$

$\begin{array}{llll}4,83 & 4,65 & 0,18 & 16\end{array}$

$3,44 \quad 3,15 \quad 0,28 \quad 14$

$\begin{array}{llll}3,36 & 1,80 & 1,56 & 1\end{array}$

$3,36 \quad 1,90 \quad 1,46 \quad 2$

$\begin{array}{llll}2,55 & 1,75 & 0,80 & 7\end{array}$

$3,43 \quad 2,20 \quad 1,23 \quad 3$

$\begin{array}{llll}3,58 & 3,85 & 0,27 & 3\end{array}$

$3,15 \quad 2,00 \quad 1,15 \quad 4$

$\begin{array}{llll}4,54 & 4,35 & 0,19 & 15\end{array}$

$\begin{array}{llll}3,05 & 2,55 & 0,50 & 12\end{array}$

$3,64 \quad 3,00 \quad 0,64 \quad 1 c$

$\begin{array}{llll}3,82 & 3,30 & 0,52 \quad 11\end{array}$

$3,64 \quad 3,90 \quad 0,26 \quad 4$

$3,40 \quad 3,55 \quad 0,15 \quad 5$

$\begin{array}{llll}2,82 & 2,90 & 0,08 & 6\end{array}$

Abb. 43: Motivationskonfiguration "Materialist" (Teil I) 
(1)

sehr wichtig
(2)

$21 \mathrm{em} 1 \mathrm{ch}$ wichtig
(3)

etwas wichtig
(4)

kaum wichtig
(5)

gar nicht

wichtig

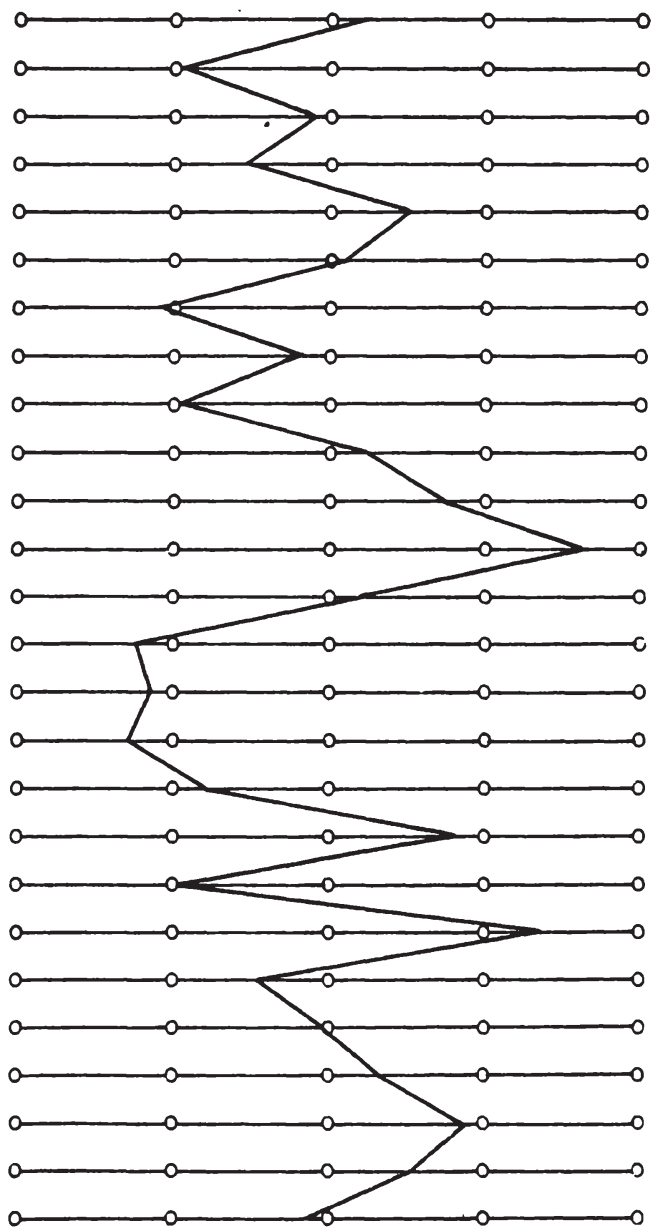

Abb. 43: Motivationskonfiguration "Materialist" (Teil II) 


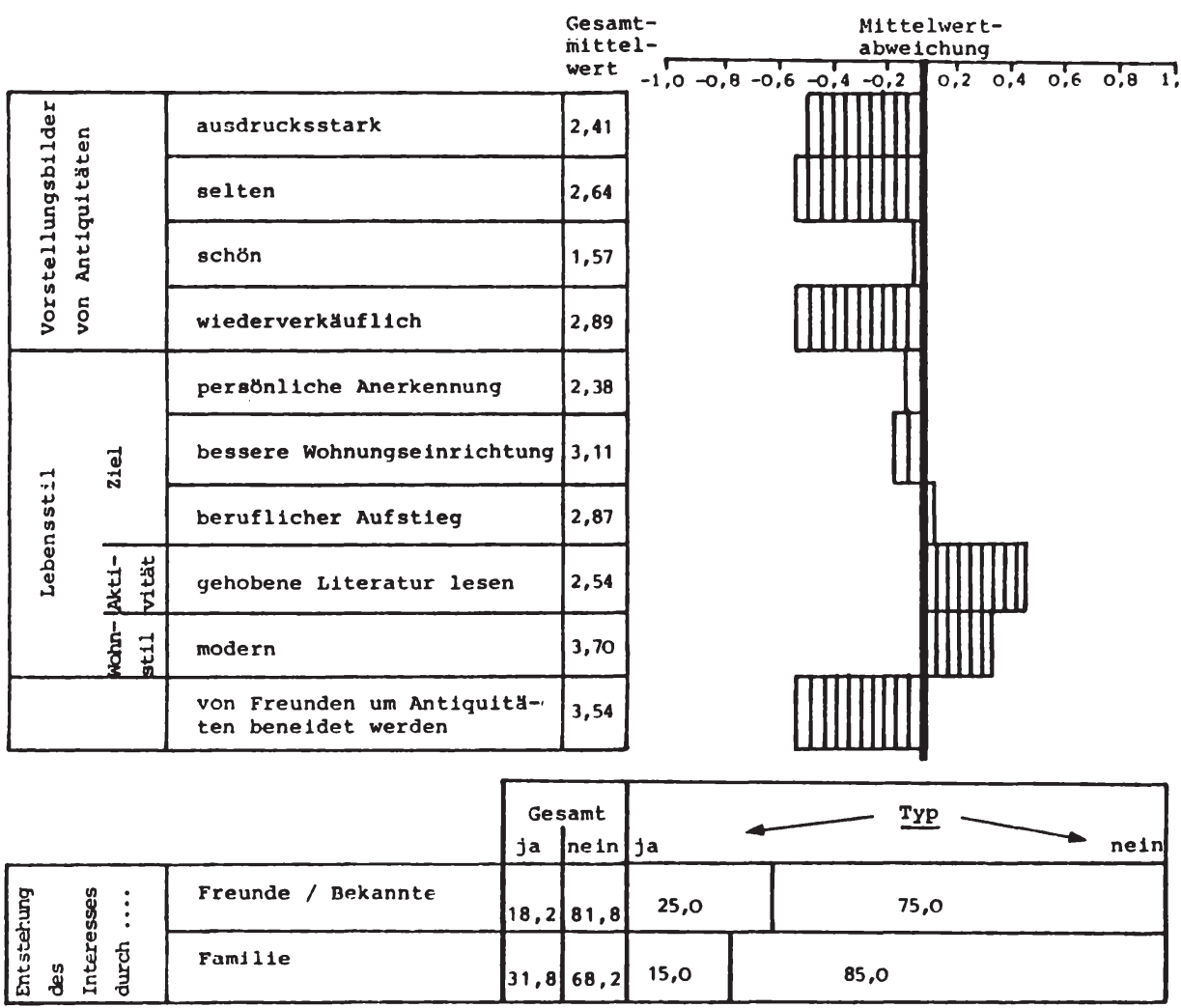




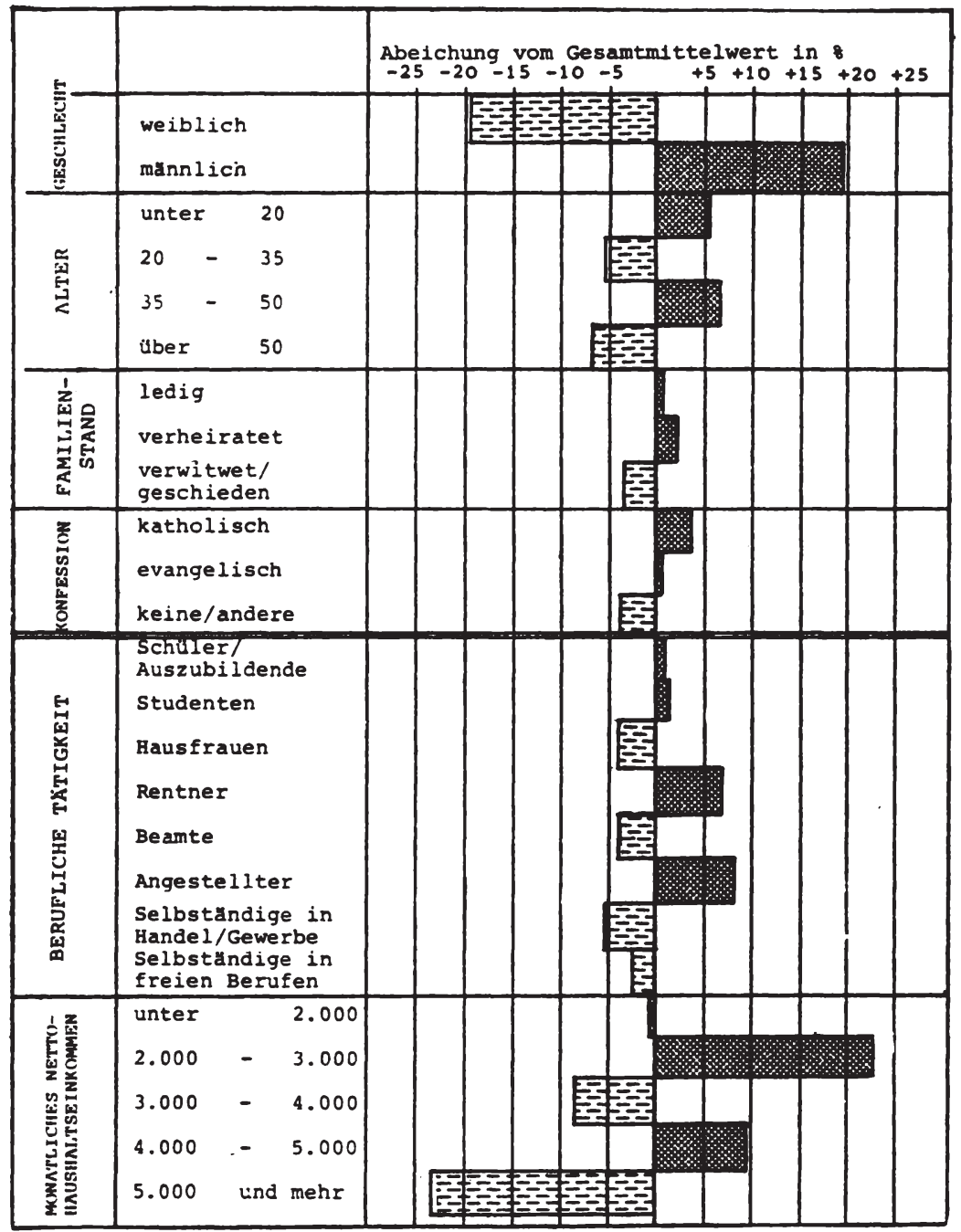

Abb. 45: Soziodemographie der "Materialisten" 
höheres Gewicht. Die Images "Seltenheit" und "Wiederverkäuflichkeit" bringen zum Ausdruck, daß speziell die "Materialisten" mit einem zunehmenden Seltenheitswert von Antiquitäten die Chance steigender Wiederverkaufspreise und die Möglichkeit ihrer Realisierung im Markt verbinden.

Die typendiskriminierenden Persönlichkeitsziele zeigen keine interpretationsrelevanten Auffälligkeiten. Ein stark unterdurchschnittliches Interesse an gehobener Literatur unterstreicht dagegen das Bild einer wenig ausgeprägten oder auch "versachlichten" Beziehung der "Materialisten" zur Kunst allgemein.

Die Entstehung des Interesses an Antiquitäten wurzelt im Verhältnis zur Gesamtkäuferschaft überproportional im Freundes-und Bekanntenkreis. Auf der anderen Seite ist der familiäre EinfluB, der in der Tendenz emotionale und intensive Beziehungen zu Antiquitäten fördert, erwartungsgemäB gering.

Unter soziodemographischen Gesichtspunkten (vgl. Abbildung 45) unterstreicht insbesondere der hohe Anteil von Männern unter den "Materialisten" die Charakterisierung dieses Motivationstyps.

\subsection{Individualist}

Die Stellung der "Individualisten" im Diskriminanzraum deutet bereits ein gehobenes motivationales Engagement und eine schwächere Traditionsgebundenheit dieser Motivationskonfiguration im Vergleich zum Gesamtdurchschnitt aller Käufer an. Die "Individualisten" vertreten eine der größten Käufergruppen.

Aufgrund ihres Trennvermögens weisen sich die Einzelmotiva- 
tionen "Dokumentation von Individualismus" und "Besitzstreben" als besonders charakteristisch für die "Individualisten" aus. Ferner erreicht das "Bedürfnis nach Asthetik" bei diesem Motivationstyp seine relativ höchste Kaufrelevanz. Es wird damit zugleich auch aus "typeninterner", d.h. absoluter sicht zum bedeutendsten Einzelmotiv. (vgl. das Mittelwertprofil in Abbildung 46).

Ergänzt wird das Bild über die Motivationskonfiguration schließlich durch einen überdurchschnittlichen Einfluß der Beweggründe "Wertanlage", "Wunsch nach Selbstverwirklichung" und "Reaktion auf modernes Möbeldesign" auf den Kaufentscheid der "Individualisten".

Eine zusammenfassende Kennzeichnung dieser Käufergruppe fällt durch seine multithematisch ausgerichtete Motivationsstruktur schwer. In der Tendenz erkennbar ist eine dualistische Ausrichtung: Einerseits dienen Antiquitäten rein personenbezogenen, individuellen Bedürfnissen, andererseits soll ihr Besitz eine Dokumentationsfunktion nach "außen" erfüllen. Gerade die Synthese der genannten Ziele in der Motivationsstruktur der "Individualisten" scheint besonderes Merkmal dieses clusters zu sein.

Weiteren Aufschluß geben die typendiskriminierenden Variablen, da die "Individualisten" überwiegend höhere Ausprägungen als der Durchschnitt zeigen (vgl. Abbildung 47). Das Verhältnis zu Antiquitäten ist durch eine überdurchschnittliche Betonung sämtlicher - diskriminierender Vorstellungsbilder gekennzeichnet. Inhaltlich wird hierin ansatzweise eine ebenfalls differenzierte Einschätzung von Antiquitäten deutlich.

Daß die "Individualisten" ihre angestrebten Persönlichkeitsziele nicht ausschließlich"aus sich selbst heraus"definieren, sondern u.a. auch auf das Urteil und die Anerkennung ihrer Umwelt angewiesen sind, ist aus ihrer Zielkombination ableitbar: sowohl der "berufliche Aufstieg" als 
Lobsung eines funktionaler Einrichtungsproblems

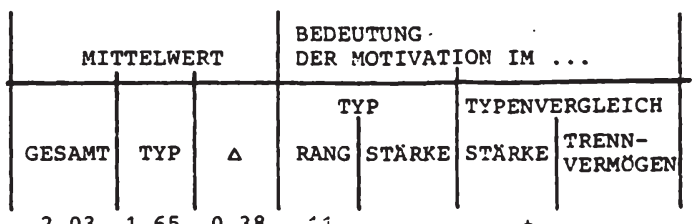
Dekoration der wohnung $2,03 \quad 1,65 \quad 0,38 \quad 1$ $2,40 \quad 1,91 \quad 0,48 \quad 8$

Dokumentation von Individualismus

$3,32 \quad 1,96 \quad 1,36$ Wertanlage

$3,38 \quad 2,78 \quad 0,60$

$4,26 \quad 4,09 \quad 0,17 \quad 14$

Spekulation auf Wertzuwachs

$3,57 \quad 3,00 \quad 0,57$

Wunsch nach Selbstverwirklichung

$\begin{array}{lll}3,08 & 2,13 & 0,95\end{array}$

Besitzstreben

$3,78 \quad 3,35 \quad 0,43 \quad 10$

Sammelleidenschaft

$2,17 \quad 1,52 \quad 0,64$

Bedürfnis nach Ästhetik

$\begin{array}{llll}3,92 & 3,65 & 0,27 & 13\end{array}$

Freude am Schenken

Reaktion auf modernes Möbeldesign

$3,42 \quad 2,65 \quad 0,77$

Steuerflucht

$\begin{array}{llll}4,83 & 4,74 & 0,09 & 15\end{array}$

Nostalgie

Freizeitgestaltung

$3,44 \quad 3,00 \quad 0,44 \quad 9$

$3,36 \quad 3,57 \quad 0,20 \quad 5$

Hoffnung auf Trouvaille

$\begin{array}{llll}3,36 & 3,74 & 0,38 & 2\end{array}$

SpaB am Suchen

Freude an Elnkaufserlebnis

$\begin{array}{llll}2,55 & 2,78 & 0,23 \quad 4\end{array}$

$3,43 \quad 3,61 \quad 0,18 \quad 6$

Familientradition

$\begin{array}{llll}3,58 & 3,83 & 0,24 & 3\end{array}$

Besitzerstolz

Repräsentation

Bedurfnis nach Ausgleich

Empfinden eines

kulturellen Auftrags

Reaktion auf moderne Kunst

Bewahrung von

Erinnerungen an die Familie

Liebe zum alten Kunsthandwerk

$\begin{array}{llll}3,15 & 3,09 & 0,07 & 16\end{array}$

$\begin{array}{llll}4,54 & 4,26 & 0,28 & 12\end{array}$

$3,05 \quad 3,09 \quad 0,04 \quad ?$

$3,64 \quad 3,57 \quad 0,07 \quad 16$

$\begin{array}{llll}3,82 & 2,87 & 0,95 \quad 2\end{array}$

$3,64 \quad 4,22 \quad 0,58 \quad 1$

$3,40 \quad 3,13 \quad 0,27 \quad i 3$

$2,82 \quad 2,30 \quad 0,52 \quad 7 \quad+$

Freude an Naturstoffen

Abb. 46: Motivationskonfiguration "Individualist" (Teil I) 
(1)

sehr wichtig
(2)

ziemlıch wichtig
(3)

etwas wicht1g
(4)

kaum wichtig
(5)

gar nicht wichtig

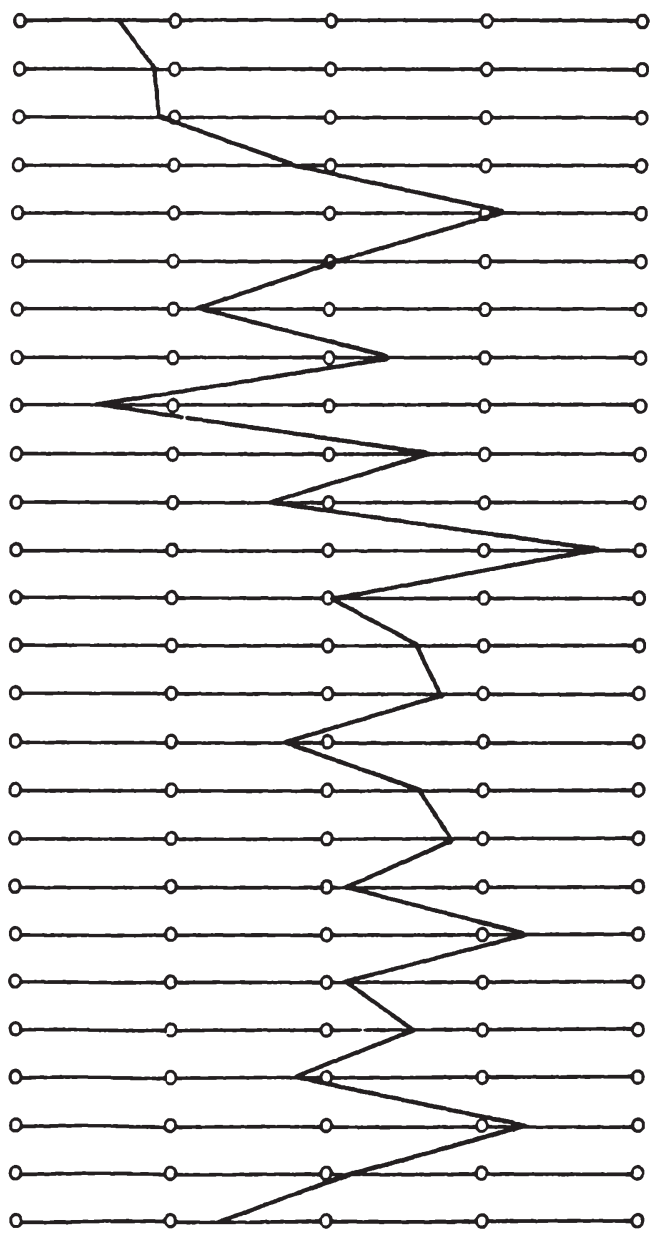

Abb. 46: Motivationskonfiguration "Individualist" (Teil II) 


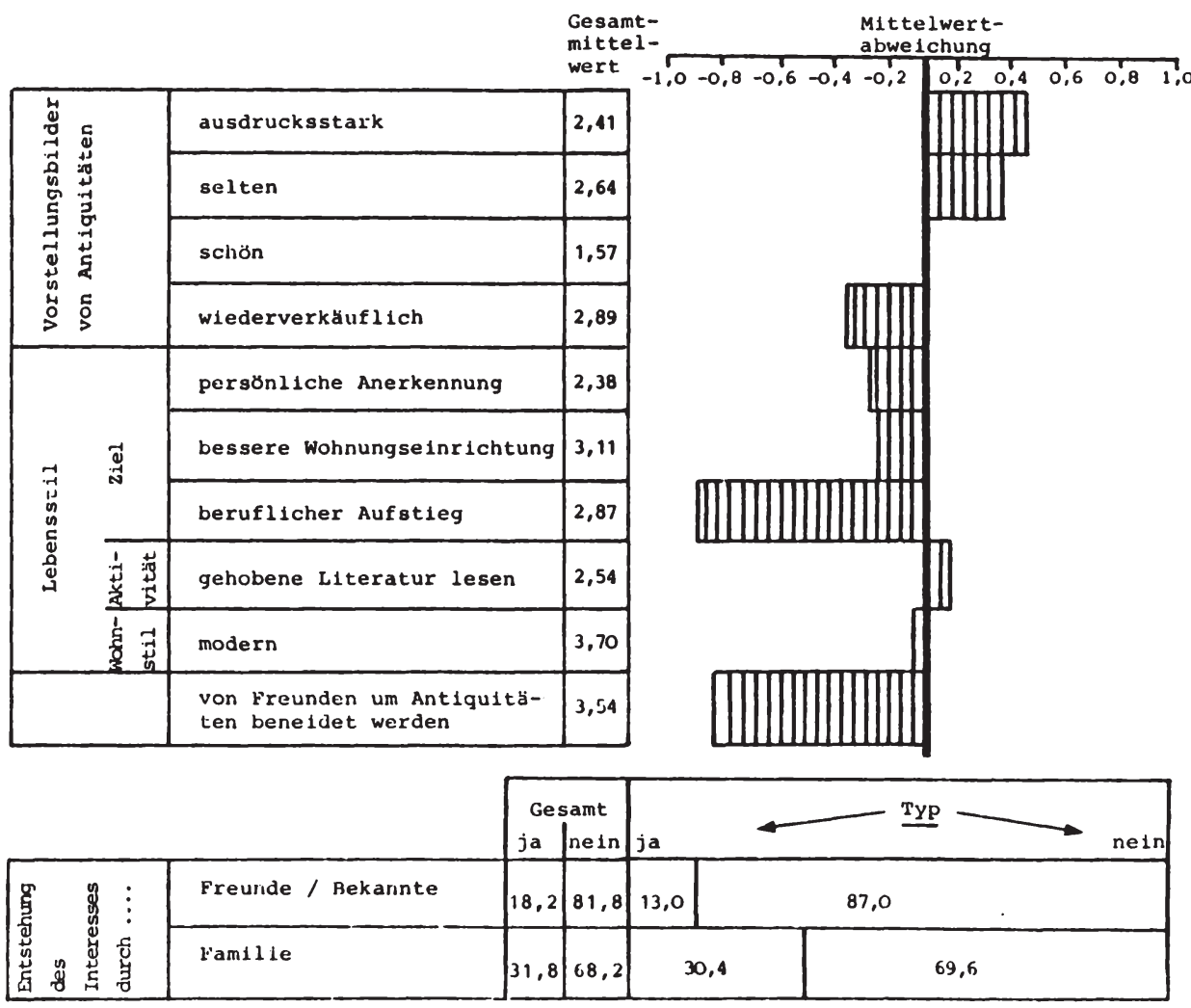

Abb. 47: Ausprägungen motivationaler Bestimmungsfaktoren im Motivationstyp "Individualist" 


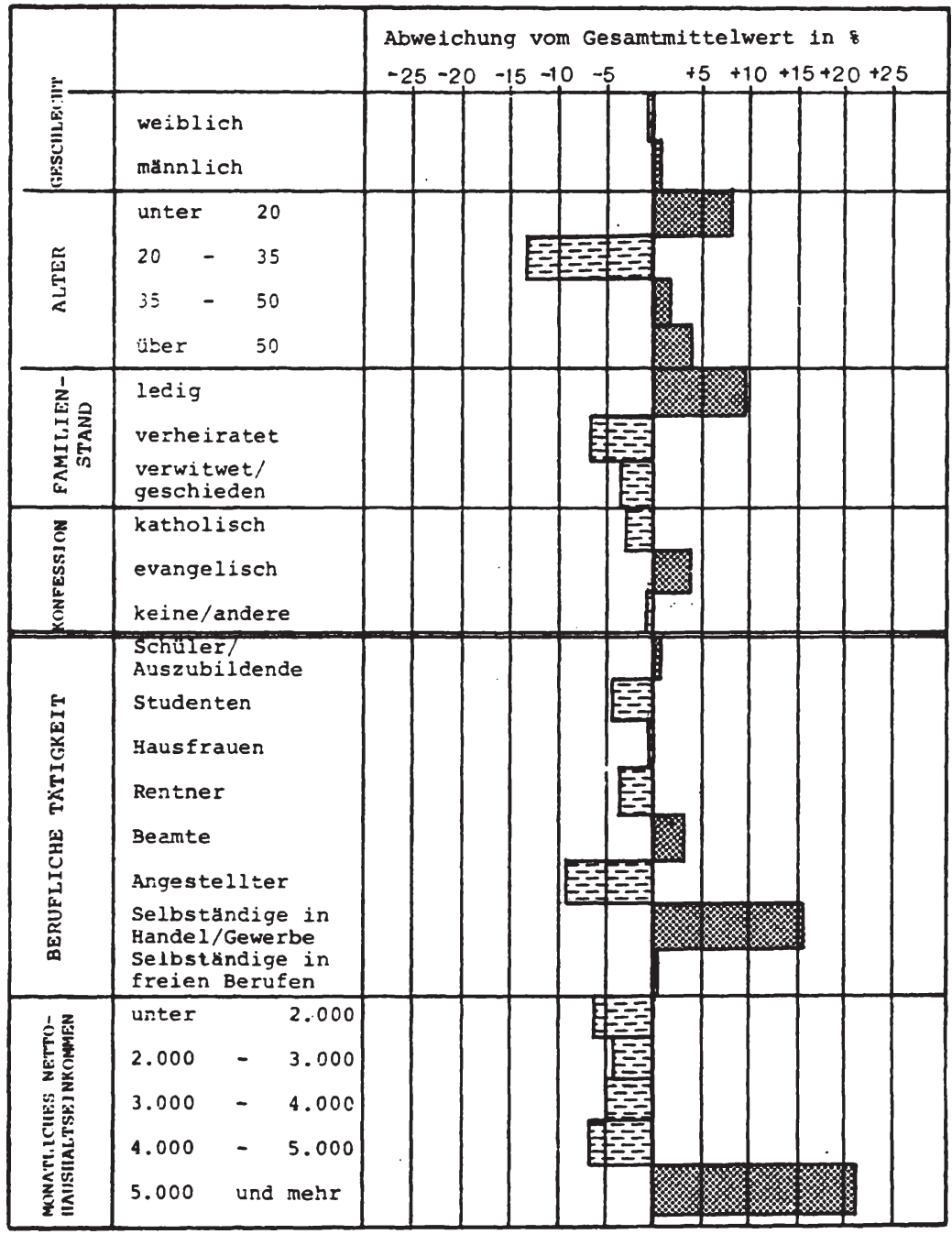

Abb. 48: Sozioàemographie der "Individualisten" 
auch der Wunsch nach "persönlicher Anerkennung" und einer "verbesserten Wohnungseinrichtung" besitzen für die "Individualisten" hohe Priorität. Darüber hinaus erweist sich die Einschätzung "daß man um den eigenen Antiquitätenbesitz von Freunden und Bekannten ein wenig beneidet wird" mit seiner absolut höchsten Zustimmung als auBerordentlich charakterisitisch für den angesprochenen Motivationstyp.

Aus soziodemographischer sicht ist auffällig, daß die "Individualisten" als einziges cluster kaum geschlechtsspezifische Abweichungen vom Gesamtdurchschnitt aufweisen (vgl. Abbildung 48). Uberproportional vertreten sind im übrigen relativ junge und relativ alte Käuferschichten. Unter dem Aspekt der beruflichen Tätigkeit sind die "Individualisten" eher selbständig in Handel und Gewerbe, wodurch mutmaßlich die Einkommensverteilung ihren Schwerpunkt in den obersten Einkommen ( 5.000 DM und mehr) besitzt.

\subsection{Liebhaber}

Die "Liebhaber" unter den Antiquitätenkäufern repräsentieren eine Motivationskonfiguration, deren Schwerpunkt durch ein nur noch geringfügig überdurchschnittliches motivationales Engagement, dafür aber durch eine ausgeprägt traditionsorientierte Disposition der Motivation charakterisiert wird.

Die Interpretation der in Abbildung 49 gezeigten Motivkonfiguration als "Liebhaber" basiert im wesentlichen auf der Einzelmotivation "Liebe zum alten Kunsthandwerk". Sie besitzt in dieser Käufergruppe die relativ höchste Kaufbedeutung und führt damit $z u$ einer signifikanten Trennung gegenüber sechs weiteren clustern.

Inhaltlich am "nächsten" steht ihr - wie die faktorenanalytische Verdichtung der Einzelmotive gezeigt hat - die 
MOTIVATION

Lösung eines funktionalen Einrichtungsproblems

Dekoration der Wohnung

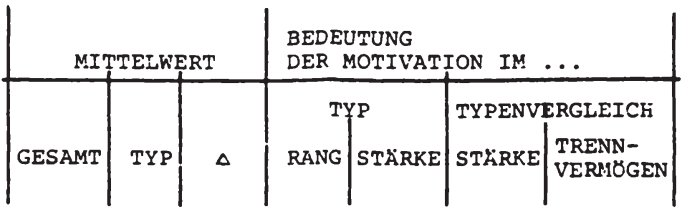

Dokumentation von Individualismus

$2,03 \quad 1,58 \quad 0,45 \quad 4$

$2,40 \quad 2,83 \quad 0,44 \quad 5$

Wertanlage

$\begin{array}{llll}3,32 & 3,50 & 0,18 & 11\end{array}$

Spekulation auf Wertzuwachs

$3,38 \quad 3,83 \quad 0,46 \quad 3$

wunsch nach Selbstverwirklichung

$\begin{array}{llll}4,26 & 4,67 & 0,41 \quad 6\end{array}$

$\begin{array}{llll}3,57 & 3,83 & 0,26 \quad 9\end{array}$

Besitzstreben

$3,08 \quad 3,08 \quad 0,00 \quad-$

Sammelleidenschaft

Bedürfnis nach Ästhetik

Freude am Schenken

$\begin{array}{llll}3,78 & 4,83 & 1,06 & 1\end{array}$

$2,17 \quad 1,83 \quad 0,33 \quad 8$

$3,92 \quad 3,92 \quad 0,00 \quad-$

Reaktion auf modernes Möbeldesign

Steuerflucht

$\begin{array}{llll}3,42 & 2,75 & 0,67 \quad 6\end{array}$

$\begin{array}{llll}4,83 & 4,92 & 0,09 & 13\end{array}$

Nostalgie

Freizeitgestaltung

$3,44 \quad 2,75 \quad 0,69 \quad 5$

$3,36 \quad 4,00 \quad 0,64 \quad 2$

Hoffnung auf Trouvaille

$\begin{array}{llll}3,36 & 3,67 & 0,30 \quad 7\end{array}$

SpaB am Suchen

$\begin{array}{llll}2,55 & 2,83 & 0,28 & 8\end{array}$

Freude am Einkaufserlebnis

$3,43 \quad 3,25 \quad 0,18 \quad 11$

Familientradition

$\begin{array}{llll}3,58 & 3,75 & 0,17 & 12\end{array}$

Besitzerstolz

$\begin{array}{llll}3,15 & 2,83 & 0,32 & 9\end{array}$

Repräsentation

$\begin{array}{llll}4,54 & 4,75 & 0,21 \quad 10\end{array}$

Bedürfnis nach Ausgleich

$\begin{array}{llll}3,05 & 2,08 & 0,96 \quad 4\end{array}$

Empinden eines

kulturellen Auftrags

$\begin{array}{llll}3,64 & 3,33 & 0,30 & 10\end{array}$

Reaktion auf moderne kunst

Bewahrung von

Erinnerungen an die Familie

$\begin{array}{llll}3,82 & 3,25 & 0,57 & 7\end{array}$

$3,64 \quad 2,17 \quad 1,48 \quad 1$

Liebe zum alter. Kunsthandwerk

Freude an Naturstoffen

$3,40 \quad 2,00 \quad 1,40 \quad 2$

$2,82 \quad 1,58 \quad 1,24 \quad 3$

Abb. 49: Motivationskonfiguration "Liebhaber" (Teil I) 
(1) sehr wichtig
(2)

$21 \mathrm{em} 11 \mathrm{ch}$ wichtig
(3)

etwas wichtig
(4)

kaum wichtig
(5)

gar nicht wichtig
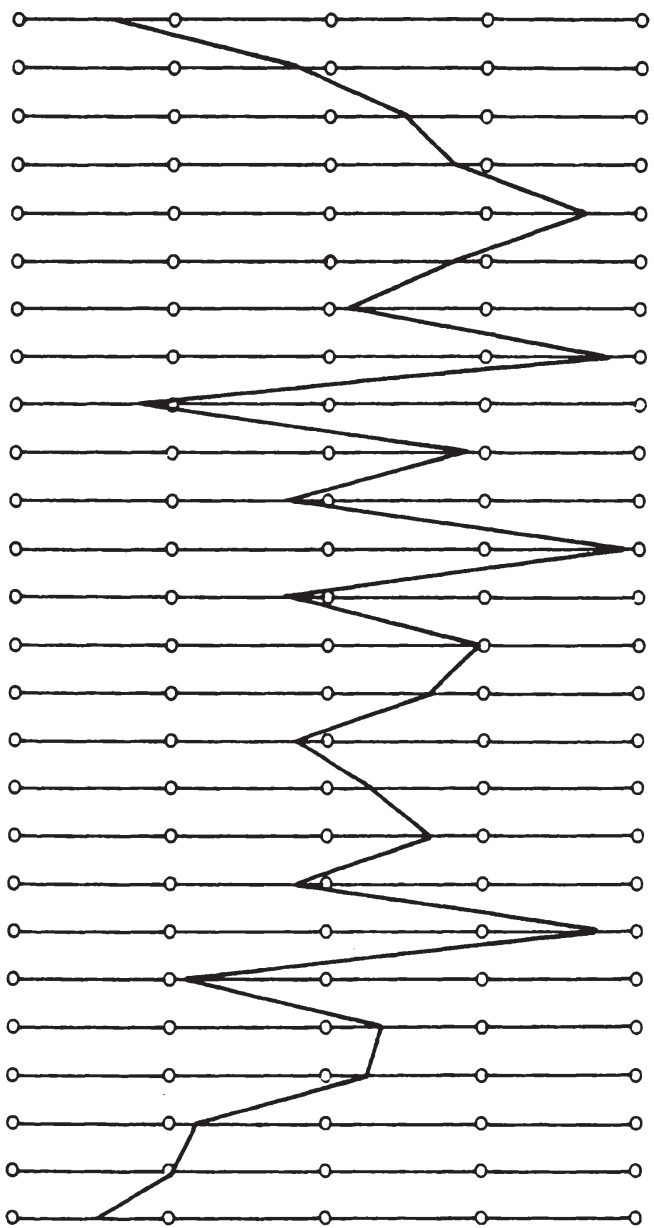

Abb. 49: Motivationskonfiguration "Liebhaber" (Teil II) 
"Freude an Naturstoffen". Auch sie besitzt bei den "Liebhabern", sowohl bezogen auf den Gesamtmittelwert $(-1,24)$ als auch im clustervergleich, eine überdurchschnittliche Ausprägung.

Typisches Kennzeichen der Motivstruktur sind darüber hinaus das "Bedürfnis nach Ausgleich", die "Bewahrung von Erinnerungen an die Familie", die "Reaktion auf moderne Kunst", sowie mit gewissen Einschränkungen beim Trennvermögen die "Nostalgie" und "Lösung eines funktionalen Einrichtungsproblems".

Die aufgezeigten Schwerpunkte der Motivationskonfiguration deuten auf eine insgesamt eher affektiv-betonte Kaufmotivation der "Liebhaber" hin, die inhaltlich in einem relativ engen und geschlossenen zusammenhang $\mathrm{zu}$ stehen scheint.

Interessant ist abschließend die wider Erwarten geringe Bedeutung einer Sammelleidenschaft im Rahmen der "Liebhaberei". Als Erklärung bietet sich die Notwendigkeit eines eher "planvollen" Vorgehens beim Sammeln und einer eher "zufällig" entstehenden Beziehung beim Antiquitätenkauf aus Liebhaberei an.

Unter den typendiskriminierenden Variablen der Abbildung 50 fällt das ausgeprägte "Streben nach persönlicher Anerkennung" sowie das Interesse der "Liebhaber" an der Lektüre gehobener Literatur auf. Eine nicht ausschließlich auf Antiquitäten beschränkte Beziehung $z u$ anderen Teilgebieten der Kunst ist daher wahrscheinlich. Mehr als in anderen Käufergruppen besitzt der angesprochene Motivationstyp die Vorstellung, daß Antiquitäten "ausdrucksstark" sind. Es ist $z$ u erwarten, daß speziell von Einzelstücken, die dieses Warenmerkmal besitzen, ein besonderer motivationaler Anreiz ausgeht. 


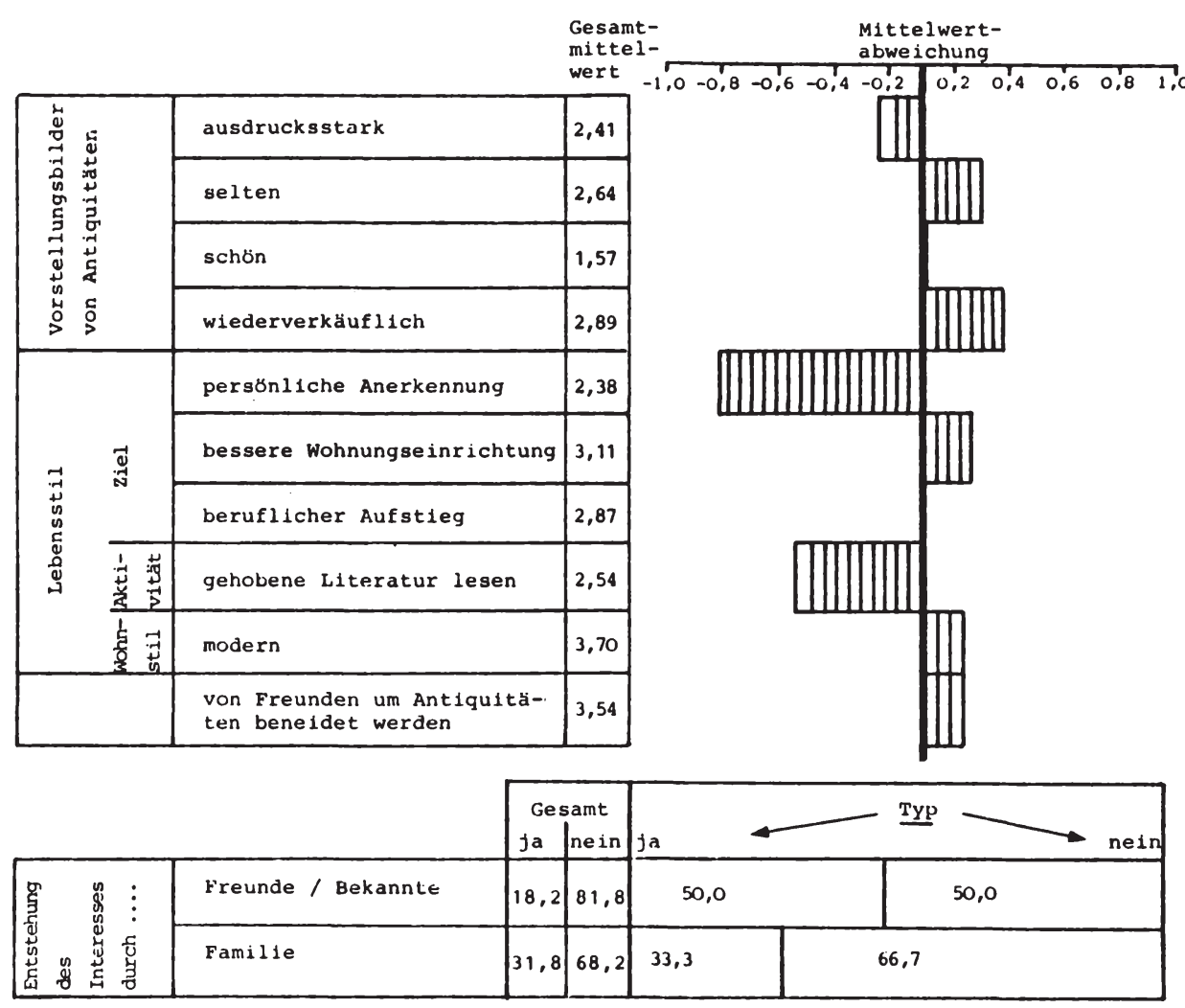




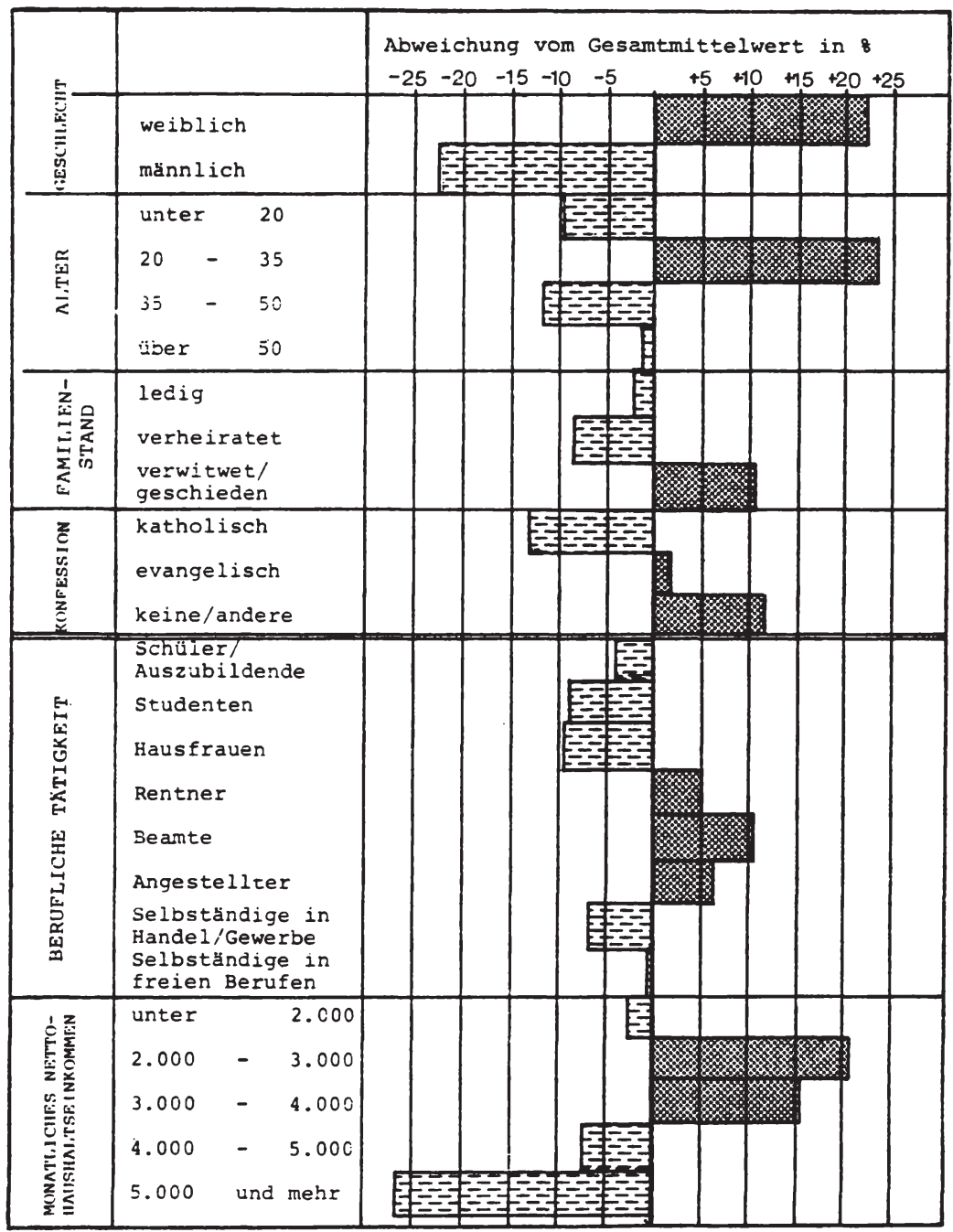

Abb. 51: Soziodemographie der "Liebhaber" 
Im übrigen fällt ein Kontrast zwischen der überwiegend durch Freunde und Bekannte bedingten Entstehung des Interesses für Antiquitäten und der überproportionalen Kaufrelevanz des Motives"Bewahrung von Erinnerungen an die Familie" auf.

Die in Abbildung 51 gezeigte Soziodemographie der "Liebhaber" harmoniert gut mit ihrer motivationalen Struktur.Wesentlich überrepräsentiert sind in diesem Käufersegment die Frauen. Eine Liebhaberei für Antiquitäten scheint darüber hinaus tendenziell für die jüngeren Käufer (zwischen 20 bis 35) und die unteren und mittleren Einkommen typisch. Beamte, Angestellte und Rentner stellen schlieblich die überdurchschnittlich vertretenen beruflichen Tätigkeiten der "Liebhaber" dar.

\subsection{Schwach engagierte Typen}

\subsection{Erlebniskäufer}

Im zweidimensionalen diskriminanzanalytischen Raum besetzt die Käufergruppe mit der Motivationskonfiguration "Erlebniskäufer" die zentrale Position. Der Clusterschwerpunkt läßt insofern kaum Schlußfolgerungen auf ein über- oder unterdurchschnittliches motivationales Engagement, noch eine über- oder unterdurchschnittliche traditionsbedingte Motivationsausprägung $\mathrm{zu}$.

Gegenüber dem Gesamtdurchschnitt aller befragten Antiquitätenkäufer, besitzen insbesondere die Motivationen "Spaß am Suchen", "Hoffnung auf eine Trouvaille", "Freude am Einkaufserlebnis" und "Liebe zum alten Kunsthandwerk" eine höhere Kaufbedeutung (vgl. Abbildung 52). 
Lösung eines funktionalen Einrichtungsproblems

Dekoration der Wohnung

Dokumentation von Individualismus

Wertanlage

Spekulation auf Wertzuwachs

Wunsch nach Selbstverwirklichung

Besitzstreben

Sammelleidenschaft

Bedürfnis nach Ästhetik

Freude am Schenken

Reaktion auf modernes Möbeldesign

steuerflucht

Nostalgie

Freizeitgestaltung

Hoffnung auf Trouvaille

SpaB am Suchen

Freude am Einkaufserlebnis

Familientradition

Besitzerstolz

Repräsentation

Bedürfnis nach Ausgleich

Empfinden eines

kulturellen Auftrags

Reaktion auf moderne Kunst

Bewahrung von

Erinnerungen an die Familie

Llebe zum alten Kunsthandwerk

Freude an Naturstoffen

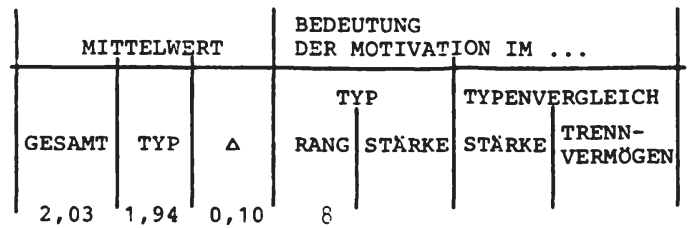

$2,40 \quad 2,44 \quad 0,04 \quad 16$

$3,32 \quad 4,13 \quad 0,81 \quad 5$

$3,38 \quad 4,38 \quad 1,00$

$4,26 \quad 4,44 \quad 0,18 \quad 13$

$\begin{array}{llll}3,57 & 3,69 & 0,12 & 15\end{array}$

$3,08 \quad 4,00 \quad 0,92 \quad 4$

$\begin{array}{llll}3,78 & 4,19 & 0,41 & 8\end{array}$

$2,17 \quad 2,44 \quad 0,27 \quad 11$

$\begin{array}{llll}3,92 & 4,31 & 0,39 & 9\end{array}$

$3,42 \quad 3,63 \quad 0,20 \quad 12$

$\begin{array}{llll}4,83 & 4,81 & 0,02 & 9\end{array}$

$3,44 \quad 4,06 \quad 0,62 \quad 6$

$3,36 \quad 3,00 \quad 0,36 \quad 6$

$3,36 \quad 2,56 \quad 0,80 \quad 3$

$2,55 \quad 1,50 \quad 1,05 \quad 1$

$3,43 \quad 2,81 \quad 0,62 \quad 4$

$\begin{array}{llll}3,58 & 4,56 & 0,98 & 2\end{array}$

$3,15 \quad 3,31 \quad 0,16 \quad 14$

$\begin{array}{llll}4,54 & 4,88 & 0,33 & 10\end{array}$

$\begin{array}{llll}3,05 & 3,31 & 0,27 & 11\end{array}$

$3,64 \quad 3,44 \quad 0,20 \quad 7$

$\begin{array}{llll}3,82 & 4,31 & 0,49 & 7\end{array}$

$3,64 \quad 4,56 \quad 0,92 \quad 3$

$3,40 \quad 2,44 \quad 0,96 \quad 2$

$2,82 \quad 2,25 \quad 0,57 \quad 5$

Abb. 52: Motivationskonfiguration "Erlebniskäufer" (Teil I) 


$\begin{array}{ccccc}\text { (1) } & \text { (2) } & \text { (3) } & \text { (4) } & \text { (5) } \\ \text { sehr } & \text { 21emlich } & \begin{array}{c}\text { etwas } \\ \text { wichtig }\end{array} & \begin{array}{c}\text { kaum } \\ \text { wichtig }\end{array} & \begin{array}{c}\text { gar nicht } \\ \text { wichtig }\end{array} \\ \text { wichtig } & \text { wichtig }\end{array}$

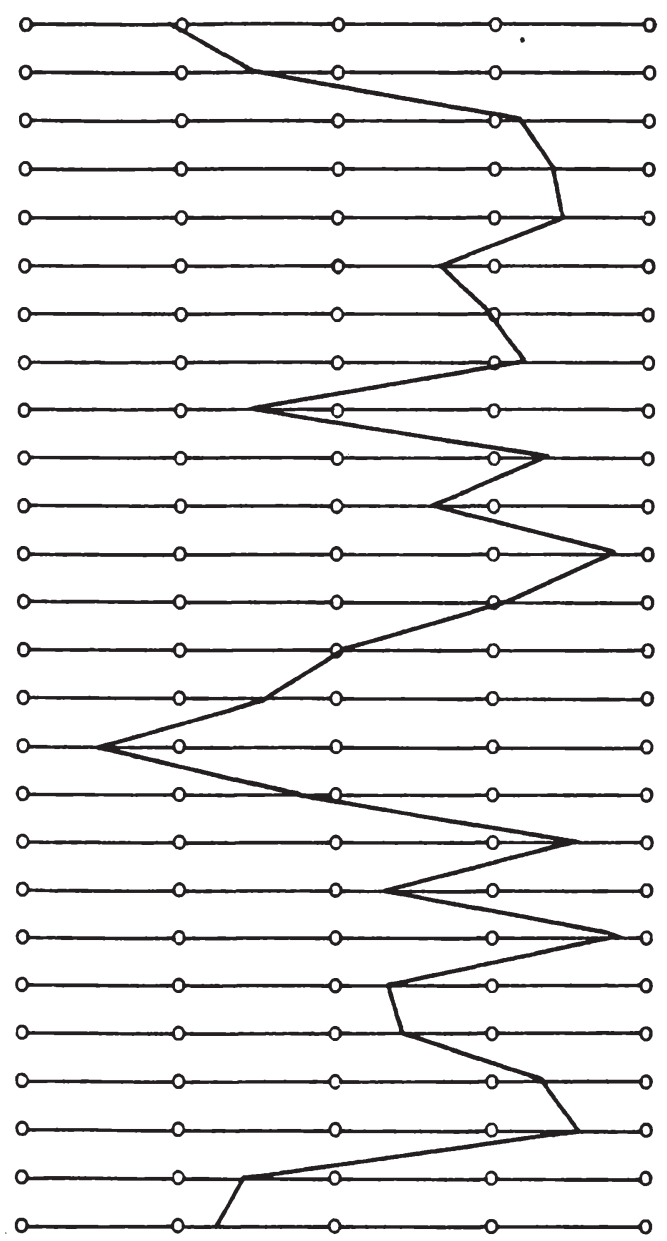

Abb. 52: Motivationskonfiguration "Erlebniskäufer" (Teil II) 
Bereits die faktorenanalytische Untersuchung der Einzelmotivationen hatte gezeigt, daß die drei erstgenannten Einzelmotivationen unter der Inhaltsdimension "Streben nach Konsumerlebnis" zusammengefaßt werden können. Die dort vorgenommene Charakterisierung dieser Motivdimension kann daher weitgehend analog für die Beschreibung der Motivationskonfiguration "Erlebniskäufer" herangezogen werden ${ }^{1}$. Dies gilt insbesondere, da auch aus "typeninterner" sicht eine weitgehende Dominanz dieser Inhaltsdimension vorliegt (vgl. das Mittelwertprofil).

Daneben unterstreicht die unterdurchschnittliche Motivationsausprägung des "Besitzstrebens" die eher geringe Bedeutung der Antiquität als selbständig motivierendes Kaufobjekt für die "Erlebniskäufer".

Auch der empirische Befund bei den typendiskriminierenden Vorstellungsbildern über Antiquitäten läßt diese Schlußfolgerung $z u$, indem eine durchgängig unterdurchschnittliche Assoziation mit den Merkmalen "schön", "ausdrucksstark" und "selten" erhoben wurde (vgl. Abbildung 53).

Da sich ähnliche Tendenzen bei den psychographischen Bestimmungsfaktoren der Käufermotivation aufzeigen lassen, scheint die Persönlichkeitsstruktur für den "Erlebniskäufer" ebenfalls geringen Erklärungswert zu besitzen. Offensichtlich liegt eine wichtige Begründung für diesen empirischen Befund in der Situationsgebundenheit dieser Motivationskonfiguration.

Die Abbildung 54 zeigt in der soziodemographischen Struktur der "Erlebniskäufer" ausgeprägte Schwerpunkte unter den männlichen und den jüngeren (bis 35 Jahre) Antiquitätenkäufern. Selbständige sowohl in Handel und Gewerbe als auch in freien Berufen sind dagegen unterrepräsentiert. In engem $\mathrm{Zu}-$

1 Vgl. Kapitel C. 2.22. 


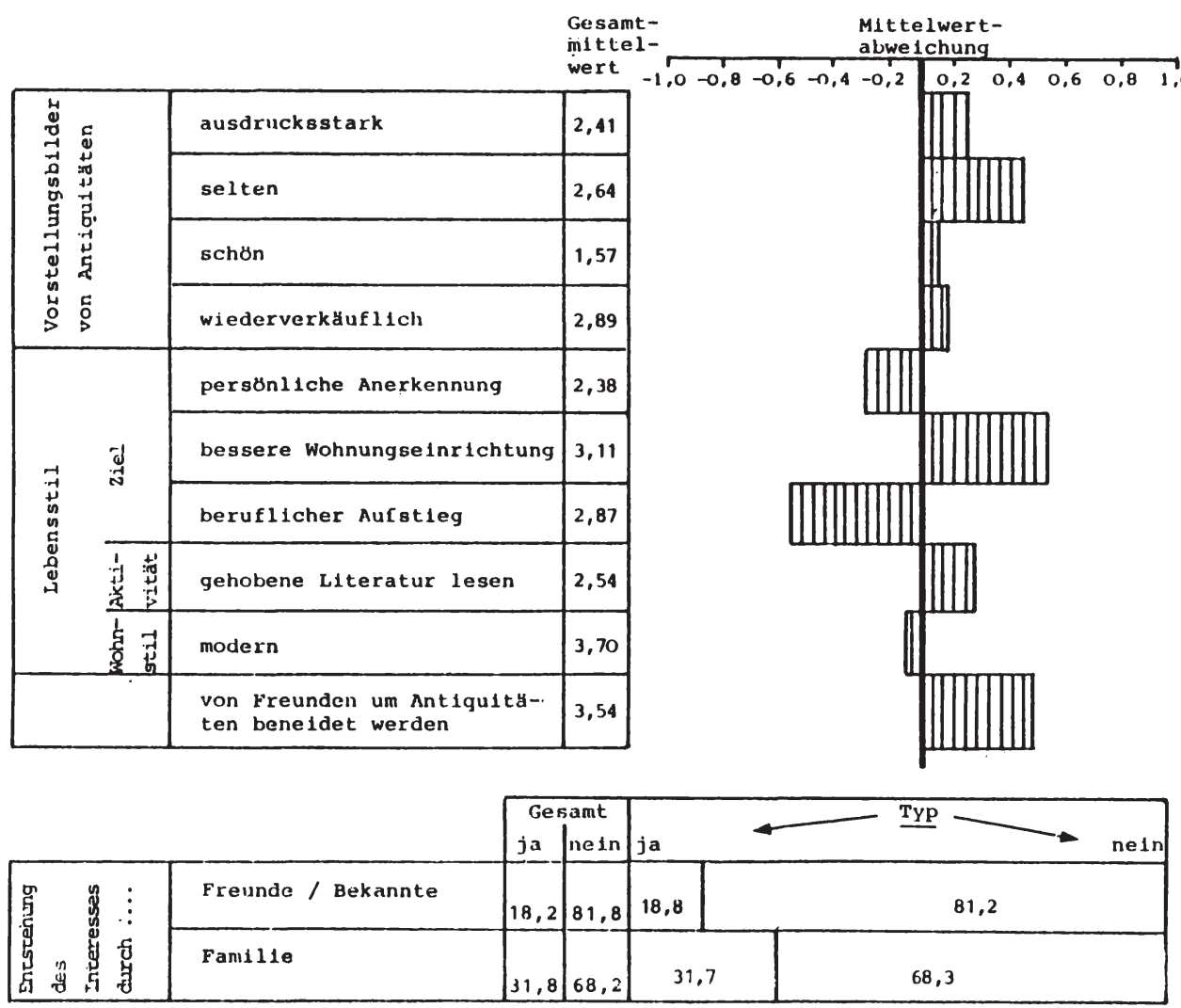

Abb. 53: Ausprägungen motivationaler Bestimmungsfaktoren im Motivationstyp "Erlebniskäufer" 


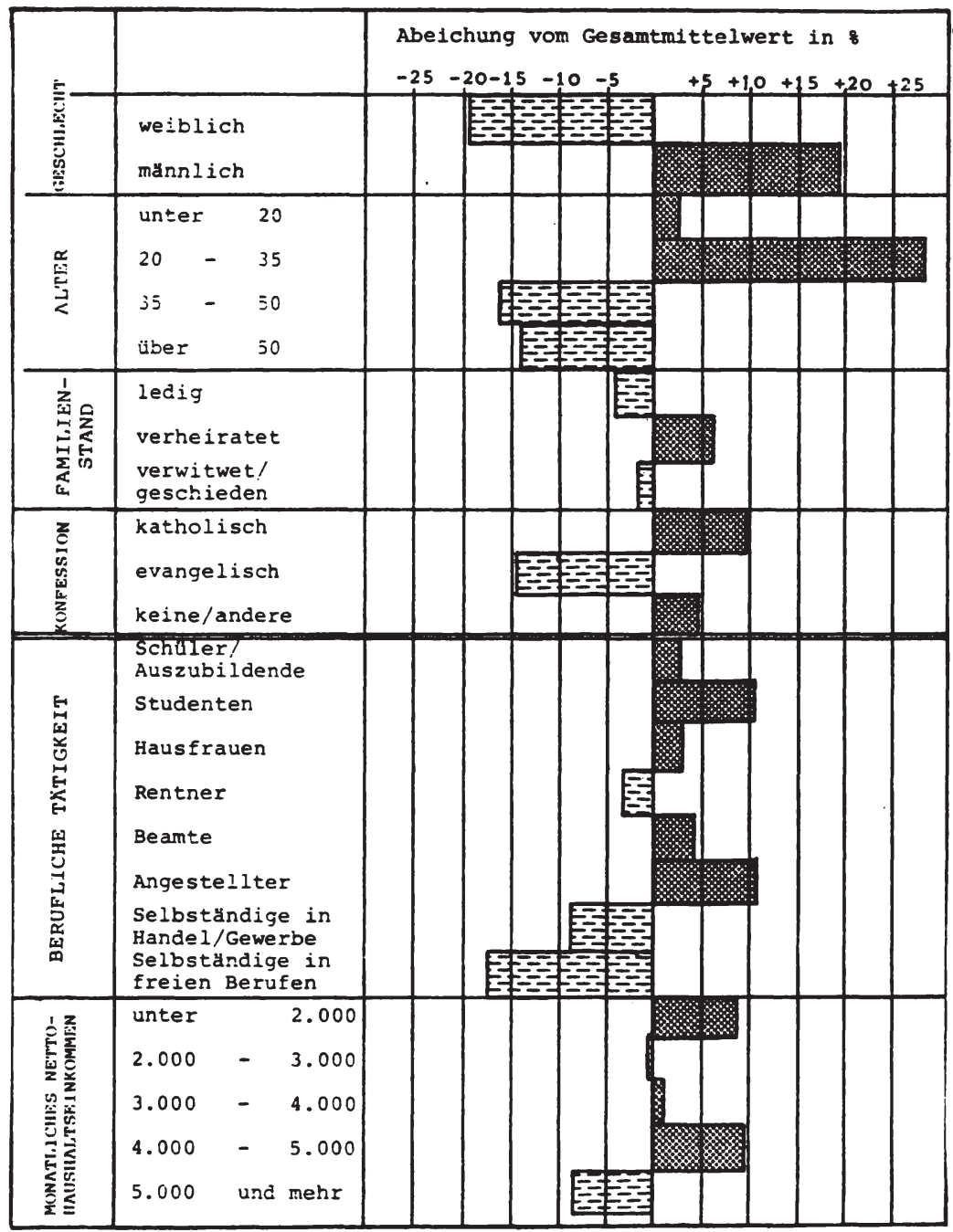

Abb. 54: Soziodemographie der "Erlebniskäufer" 
sammenhang, damit ist die geringere Anzahl von "Spitzenverdienern" in diesem cluster.zu sehen.

\subsection{Rationalist}

Gruppengröße, Homogenität und eher geringe Abweichungen der Motivkonfiguration vom Gesamtdurchschnitt lassen sich als Hinweisedeuten, daß die "Rationalisten" am ehesten einen "Durchschnittstyp" unter den Antiquitätenkäufern vertreten. Die feststellbaren Diskrepanzen weisen entsprechend der Lage des "Centroids"auf der Diskriminanzfunktion "Engagement" fast ausschließlich negative Abweichungen auf.

Als "Rationalist" erweist sich die vorgestellte Käufergruppe daher in erster Linie durch die geringe Bedeutung einer "Liebe zum alten Kunsthandwerk", "Freude an Naturstoffen", eines "Bedürfnis nach Ästhetik" und einer "Bewahrung von Erinnerungen an die Familie", welche z.T. in diesem Cluster die absolut niedrigsten Ausprägungen erreichen (vgl. Abbildung 55). Schlieblich zeigt die Struktur des Mittelwertprofiles bei absoluter Betrachtung, daB für "Rationalisten" die Nutzung von Antiquitäten als funktionale Einrichtungsgegenstände einen sehr wichtigen Kaufgrund darstellt.

Insgesamt verdichten sich die aus der Motivationskonfiguration ableitbaren Einzelaspekte zu einer eher "affektionsarmen" und "sachlichen" Motivationshaltung der "Rationalisten" gegenüber Antiquitäten.

In dieses Bild fügen sich die in Abbildung 56 dargestellten Ausprägungen der ermittelten diskriminierenden waren- und personenbezogenen Bestimmungsfaktoren; insbesondere die unterdurchschnittlich anzutreffenden Vorstellungsbilder von 


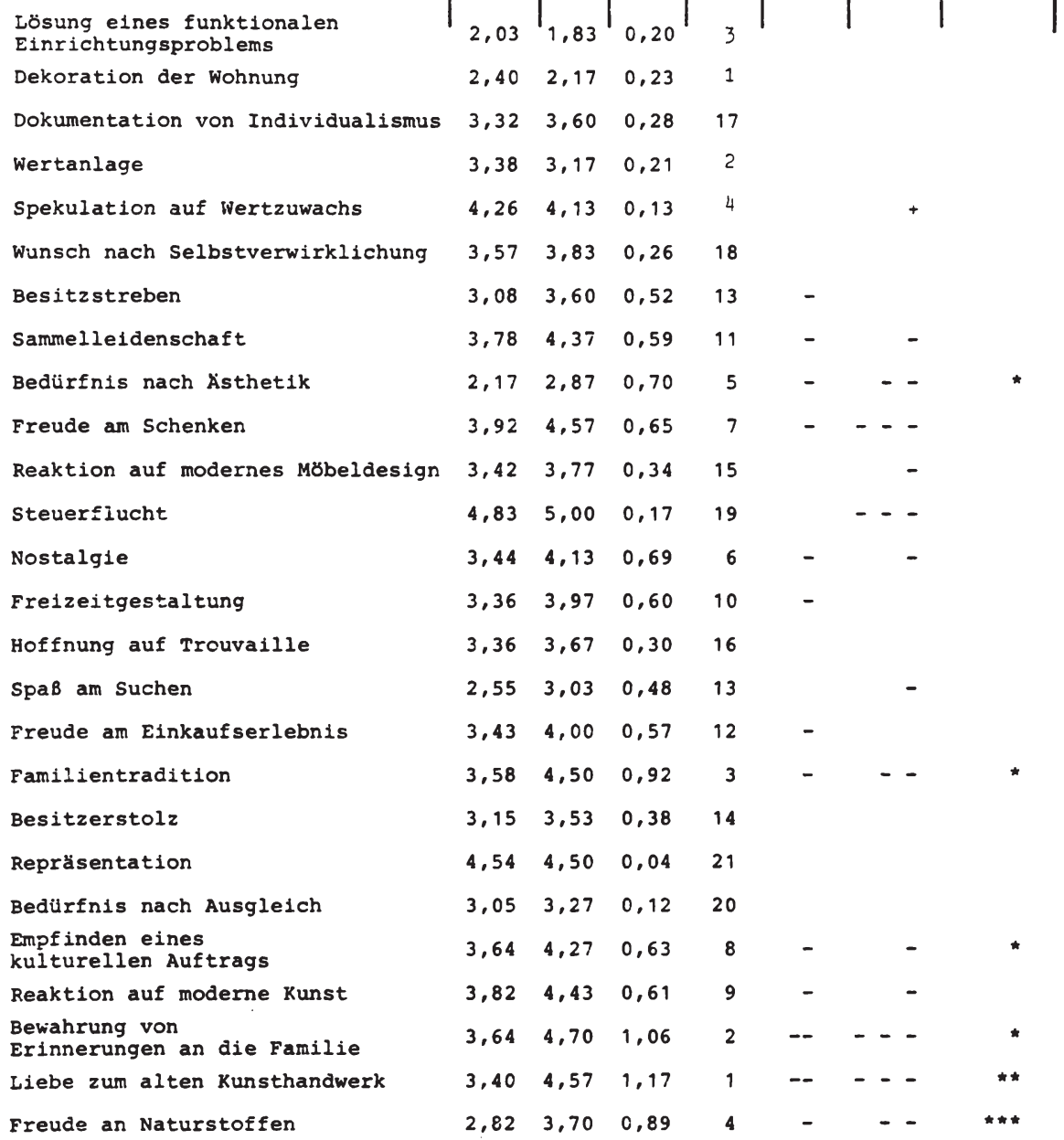

Abb. 55: Motivationskonfiguration "Rationalist" (Teil I) 


\begin{tabular}{clccc} 
(1) & \multicolumn{1}{c}{$(2)$} & (3) & (4) & (5) \\
sehr & zlemilch & etwas & kaum & gar nicht \\
wichtig & wichtig & wichtig & wichtig & wichtig
\end{tabular}

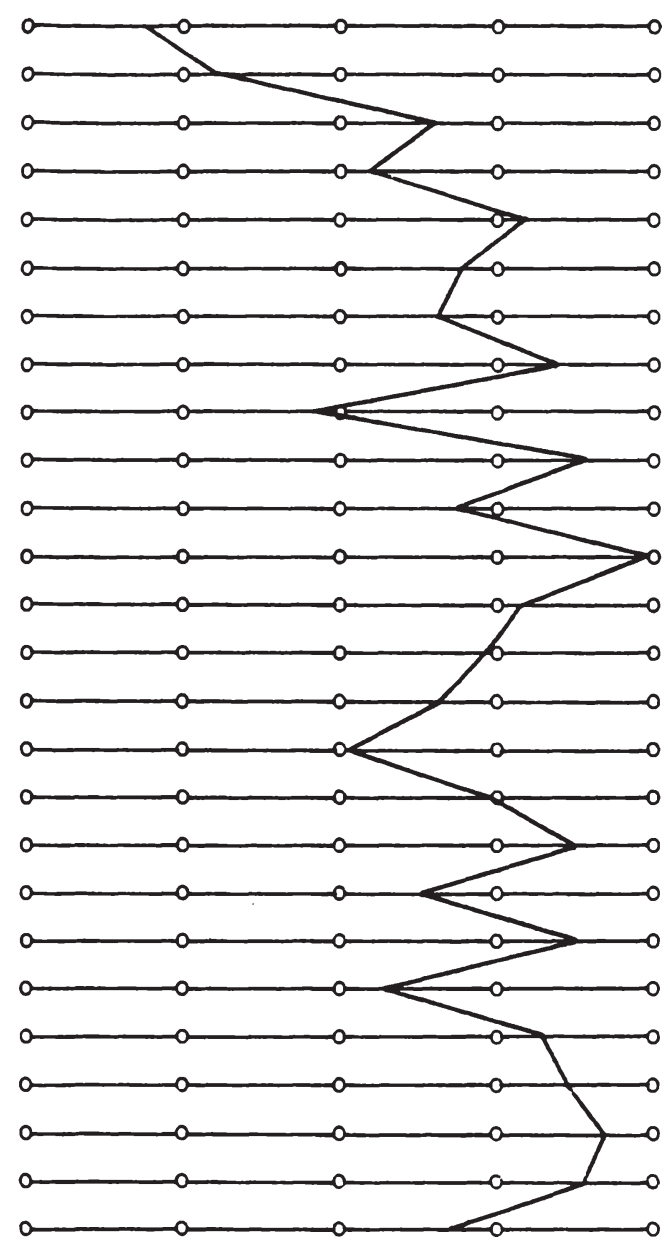

Abb. 55: Motivationskonfiguration "Rationalist" (Teil II) 

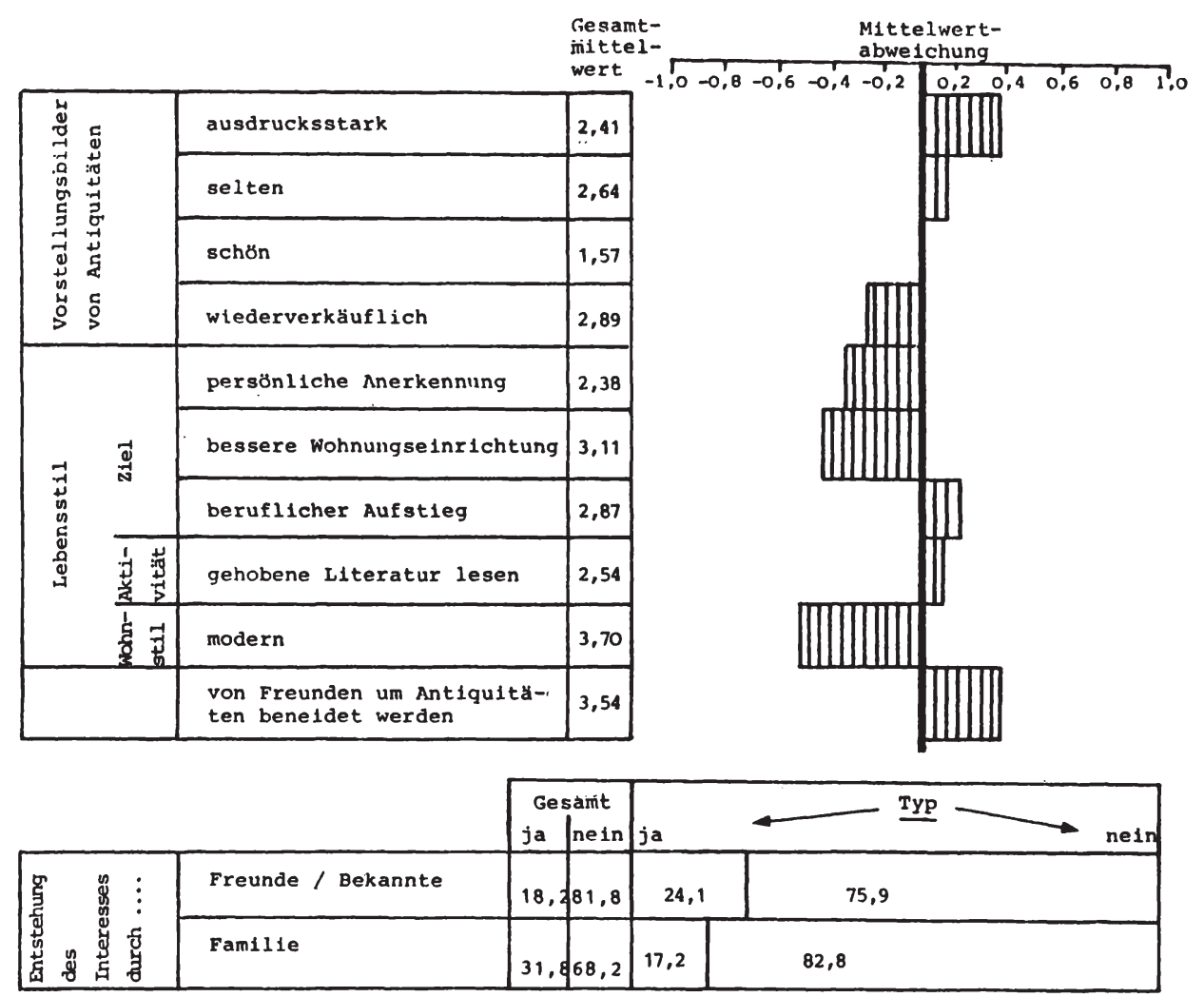


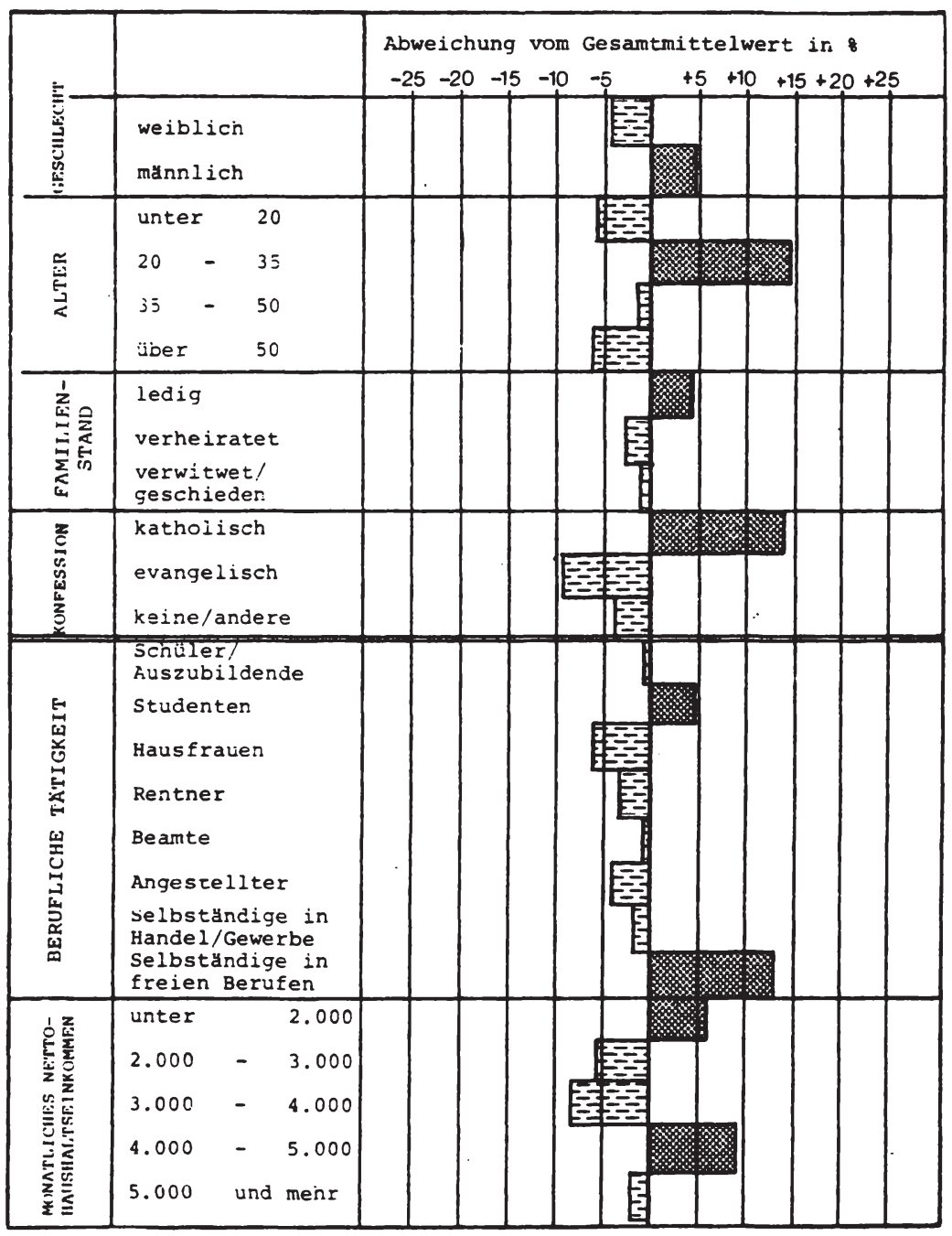

Abb. 57: Soziodemographie der "Rationalisten" 
Antiquitäten als "ausdrucksstark" und "selten". Die enge Assoziation zur "Wiederverkäuflichkeit" steht im Einklang mit einer überdurchschnittlichen Kaufbedeutung der Einzelmotivation "Spekulation auf Wertzuwachs", der geringen motivationalen Traditionsgebundenheit sowie auch der "affektionsarmen" Beziehung der "Rationalisten" zu Antiquitäten.

Weitere Prägnanz gewinnt die Typencharakterisierung u.a. durch ein überdurchschnittliches Engagement der "Rationalisten" für eine"bessere Wohnungseinrichtung", wobei der Wohnstil bezeichnenderweise als ausgesprochen "modern" eingeschätzt wird. Vermutlich dienen Antiquitäten dabei in erster Linie als bewußt gesetzte "Kontrastpunkte" zum ansonsten modernen Mobiliar. Dabei ist es möglich, daß gerade die "Rationalisten" ein kombiniertes Einrichten mit "alten" und "neuen" Möbeln an sich als eine zeitgemäße Art des Wohnens bzw. Einrichtens sehen.

Aus sozialstatistischer Sicht sind erwartungsgemä $\beta$ die männlichen Antiquitätenkäufer unter den "Rationalisten" in der Uberzahl. (vgl. Abbildung 57). Interessant ist die hohe Dominanz junger Käufer zwischen 20 und 35 Jahren. In der Tendenz scheinen sich diese in zweì Untergruppen aufzufächern: zum einen in studenten, welche sich noch in der Ausbildung befinden und nur über ein geringes Haushaltseinkommen verfügen und zum anderen in bereits beruflich etablierte Selbständige mit erheblich gehobenen Einkommen.

\subsection{Traditionalist}

Der Traditionalist zeigte im Spektrum der Antiquitäten-Käufergruppen eine geringe Ausprägung auf der Diskriminanzfunktion "motivationales Engagement" und eine hohe Aus- 
prägung auf der Diskriminanzfunktion "Tradition".

Wie bei den anderen schwach engagierten Motivationstypen lassen sich tendenziell geringe Mittelwerte im strukturprofil dieses Clusters erkennen (vgl. Abbildung 58).

Seine Schwerpunkte liegen eindeutig bei den Einzelmotivationen "Familientradition" und "Bewahrung von Erinnerungen an die Familie". Eine signifikante Trennung ergibt sich für beide Motive jeweils gegenüber sechs weiteren Motivationstypen.

Darüber hinaus scheinen "Traditionalisten" aus "typeninterner Sicht" ein ausgeprägtes "Bedürfnis nach Ästhetik" durch einen Antiquitätenkauf befriedigen $z u$ wollen. Die unterdurchschnittliche Kaufrelevanz eines "Bedürfnisses nach Ausgleich" überrascht in diesem Zusammenhang. Eine hypothetische Erklärung bietet die Annahme, daß "Traditionalisten" womöglich stark "in sich selbst ruhen".

Die Analyse der diskriminierenden Persönlichkeitsziele und -merkmale erhärtet die Hypothese, da für diesen Käufertyp die "persönliche Anerkennung" durch die soziale Umwelt, der "berufliche Aufstieg" und eine "verbesserte Wohnungseinrichtung" unterdurchschnittliche Bedeutung besitzen. Auch glauben "Traditionalisten" kaum, von anderen um "den eigenen Antiquitätenbesitz" beneidet $z \mathrm{u}$ werden und zeigen sich damit tendenziell "unabhängig" bzw. "indifferent" gegenüber ihrer Umwelt (vgl. Abbildung 59).

Ferner fällt der besonders hohe Einfluß der Familie auf die Entstehung des Interesses an Antiquitäten auf. Demgegenüber ist der EinfluB der Freunde und Bekannten als engere soziale Bezugsgruppen gleich null (keine Nennung). Da sich im Besitz der "Traditionalisten" offensichtlich zahlreiche Erbstücke befinden, assoziieren die Befragten die Wareneigenschaft "Wiederverkäuflichkeit" nur in sehr geringem Umfang mit Antiquitäten. 
Lobsung eines funktionalen Einrichtungsproblems

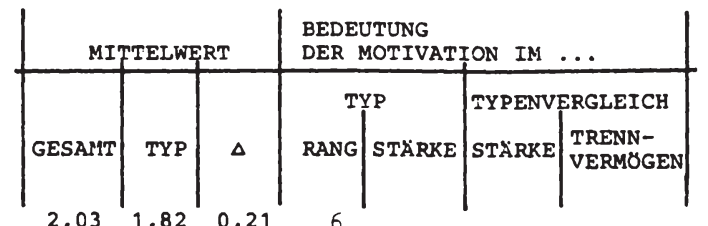
Dekoration der wohnung

$2,40 \quad 2,82 \quad 0,43 \quad 12$

Dokumentation von Individualismus

$3,32 \quad 3,94 \quad 0,62 \quad 9$

vertanlage

$\begin{array}{llll}3,38 & 3,53 & 0,15 & 16\end{array}$

Spekulation auf Wertzuwachs

$\begin{array}{llll}4,26 & 4,47 & 0,21 & 15\end{array}$

Wunsch nach Selbstverwirklichung

$\begin{array}{llll}3,57 & 4,18 & 0,61 \quad 10\end{array}$

Besitzstreben

$\begin{array}{llll}3,08 & 3,18 & 0,10 \quad 17\end{array}$

Sammelleidenschaft

$\begin{array}{llll}3,78 & 4,29 & 0,52 \quad 12\end{array}$

Bedurfnis nach Ästhetik

$2,17 \quad 1,88 \quad 0,28 \quad 3$

Freude am Schenken

$3,92 \quad 3,65 \quad 0,27$

Reaktion auf modernes Mobbeldesign

$\begin{array}{llll}3,42 & 4,00 & 0,58 & 1\end{array}$

Steuerflucht

$4,83 \quad 4,71 \quad 0,12$

Nostalgie

Freizeitgestaltung

$3,44 \quad 4,24 \quad 0,79 \quad 5$

$3,36 \quad 4,41 \quad 1,05 \quad 3$

Hoffnung auf Trouvaille

$3,36 \quad 4,35 \quad 0,99$

SpaB am Suchen

$2,55 \quad 2,29 \quad 0,26 \quad 5$

Freude am Einkaufserlebris

Familientradition

$\begin{array}{llll}3,43 & 4,12 & 0,69 & 7\end{array}$

$3,58 \quad 2,06 \quad 1,52 \quad 1$

Besitzerstolz

$3,15 \quad 3,65 \quad 0,50 \quad 13$

Repräsentation

Bedürfnis nach Ausgleich

$\begin{array}{llll}4,54 & 4,83 & 0,28 & 14\end{array}$

Empfinden eines

kulturellen Auftrags

$3,05 \quad 4,29 \quad 1,25 \quad 2$

$3,64 \quad 3,94 \quad 0,31 \quad 13$

Reaktion auf moderne kunst

Bewahrung von

Erinnerungen an die Familie

Liebe zum alten Kunsthandwerk

$\begin{array}{llll}3,82 & 4,47 & 0,65 & 8\end{array}$

$3,64 \quad 2,29 \quad 1,35$

$3,40 \quad 4,18 \quad 0,78$

$2,82 \quad 4,18 \quad 1,36 \quad 1$

Freude an Naturstoffen

Abb. 58: Motivationskonfiguration "Traditionalist" (Teil I) 


$\begin{array}{ccccc}\text { (1) } & (2) & \text { (3) } & \text { (4) } & \text { (5) } \\ \text { wichtig } & \begin{array}{c}\text { ziemilch } \\ \text { wichtig }\end{array} & \begin{array}{c}\text { etwas } \\ \text { wichtig }\end{array} & \begin{array}{c}\text { xaum } \\ \text { wichtig }\end{array} & \begin{array}{c}\text { garnicht } \\ \text { wichtig }\end{array}\end{array}$

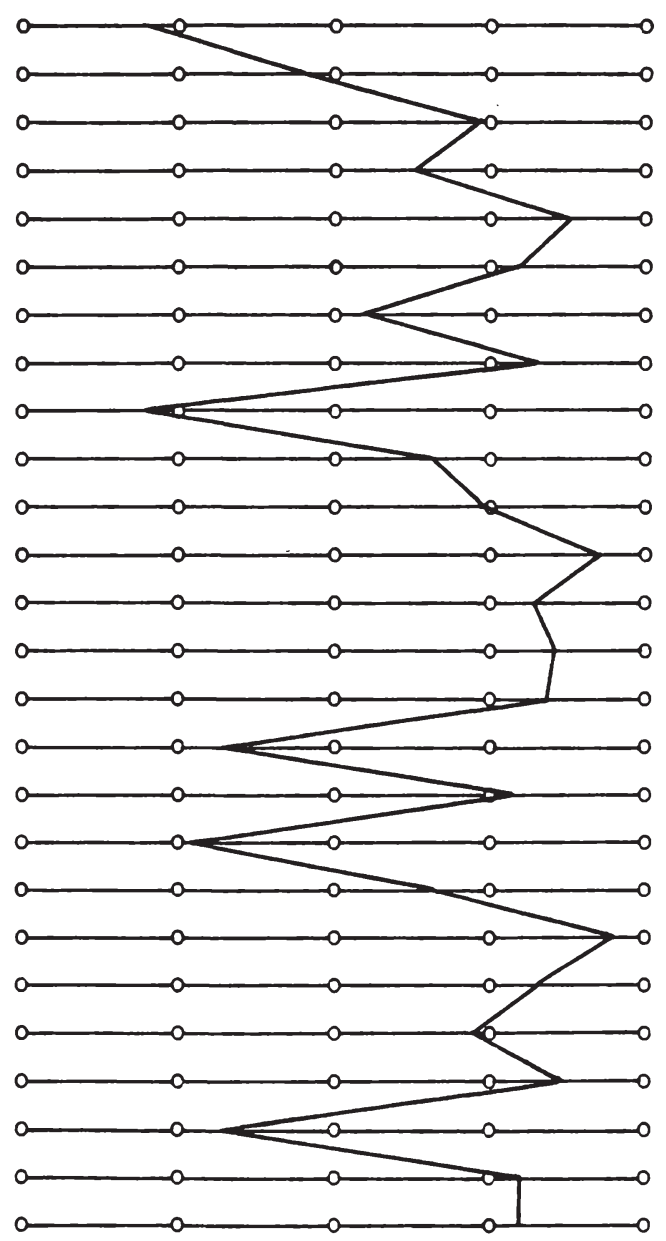

Abb. 58: Motivationskonfiguration "Traditionalist" (Teil II) 


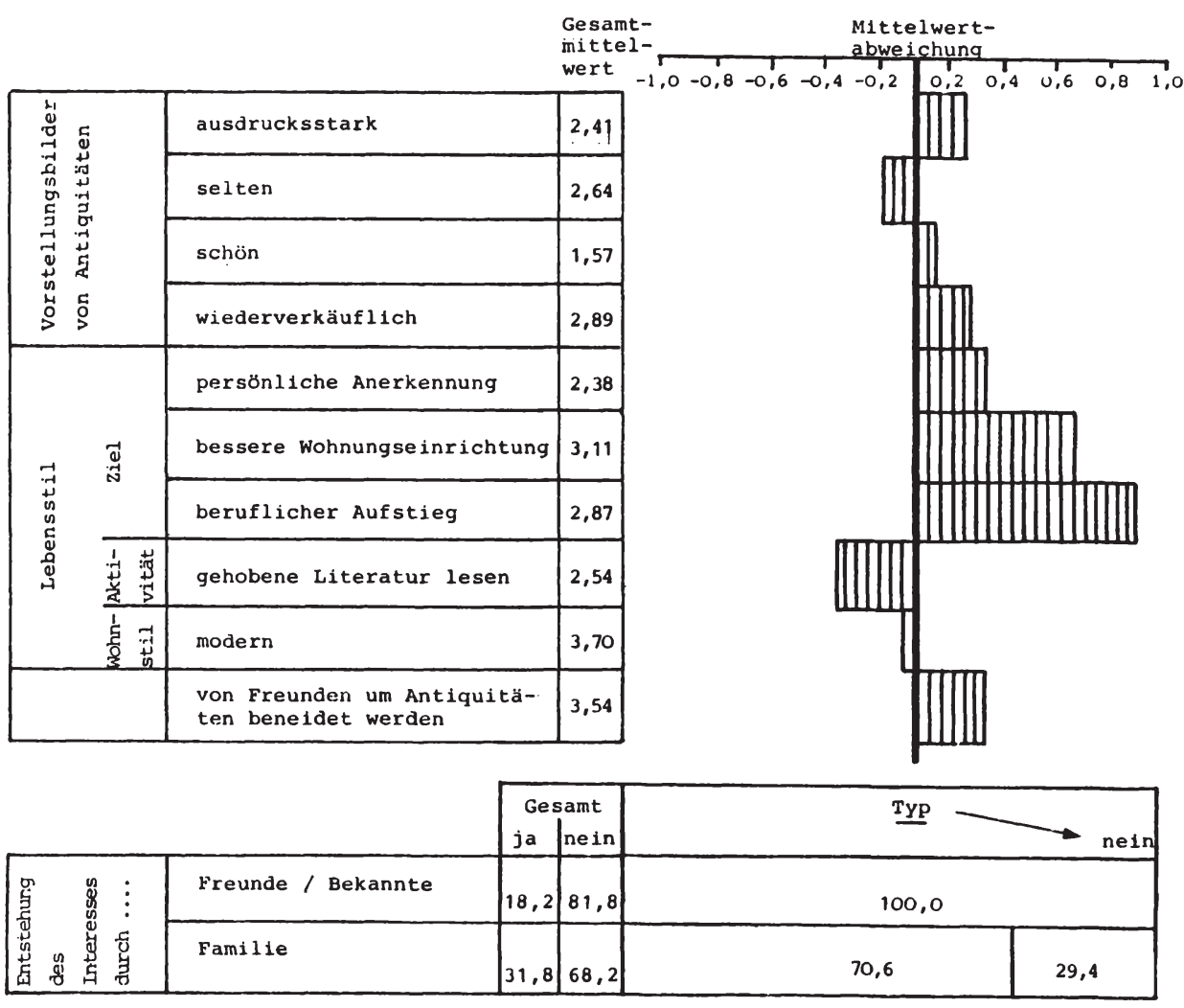

Abb. 59: Ausprägungen motivationaler Bestimmungsfaktoren im Motivationstyp "Traditionalist" 


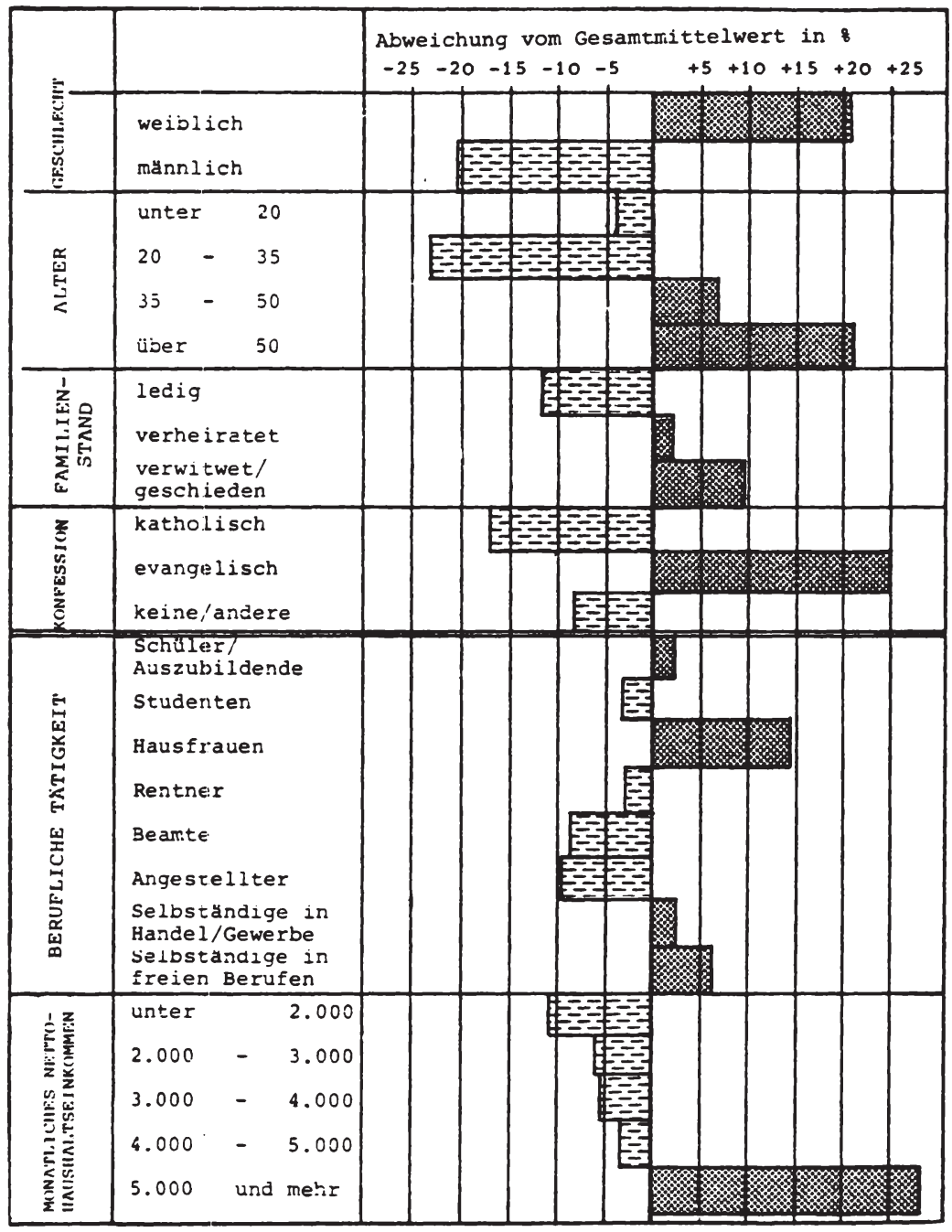

Abb. 6o: Soziodemographie der "Traditionalisten" 
Die interpretatorische Konsistenz des angesprochenen Motivationstyps wird schließlich in dem sozialstatistischen Profil der Abbildung 60 deutlich. So sind "Traditionalisten" u.a. erwartungsgemä $B$ ältere Menschen. Ihr Haushaltseinkommen bewegt sich zu einem hohen Prozentsatz im Bereich über 5.000,- DM, und sie üben neben hausfraulichen Tätigkeiten überwiegend selbständige Berufe aus.

\subsection{Sammler}

Die Position des Clusterschwerpunktes für die "Sammler" läßt sich durch eine mittlere stellung auf der Diskriminanzfunktion "Tradition" und eine relativ niedrige Stellung auf der Diskriminanzfunktion "Engagement" beschreiben. Die "Sammler" stellen die kleinste Käufergruppe in der ermittelten clusterlösung dar."

Das unterdurchschnittliche motivationale Engagement der "Sammler" erscheint zunächst als Widerspruch, da das Sammeln von Antiquitäten zumeist mit einem "besonderen Einsatz" oder allgemeiner mit einem besonderen ausgeprägten "inneren Engagement" für das jeweilige Sammelgebiet assoziiert wird.

Im Sinne der vorliegenden Untersuchung ist das motivationale Engagement der Sammler niedrig, weil sich ihre Motivkonfiguration als fast ausschließlich monothematisch ausgeprägt zeigt (vgl. Abbildung 61). Die Kaufbedeutung

1 Ihre Homogenität, d.h. die Ähnlichkeit der individuellen Motivstrukturen gehört im Gruppenvergleich $\mathrm{zu}$ den schlechtesten. Dies deutet die in Abbildung 39 ablesbare "Flächenstreuung" der einzelnen Clustermitglieder und die daraus resultierenden "Verflechtungen" mit den "benachbarten" Käufergruppen an. 
Lösung eines funktionalen Einrichtungsproblems

Dekoration der Wohnung

Dokumentation von Individualismus

Wertanlage

Spekulation auf wertzuwachs

Wunsch nach Selbstverwirklichung

Besitzstreben

Sammelleidenschaft

Bedürfnis nach Asthetik

Freude am Schenken

Reaktion auf modernes Mobbeldesign

steuerflucht

Nostalgie

Freizeitgestaltung

Hoffnung auf Trouvajile

SpaB am Suchen

Freude am Einkaufserlebnis

Familientradition

Besitzerstolz

Repräsentation

Bedürfnis nach Ausgleich

Empfinden eines

kulturellen Auftrags

Reaktion auf moderne Kunst

Bewahrung von

Erinnerungen an die Familie

Liebe zum alten Kunsthandwerk

Freude an Naturstoffen

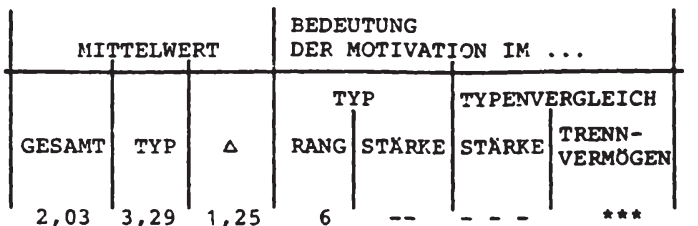

$2,40 \quad 3,71 \quad 1,32 \quad 2$

$3,32 \quad 4,43 \quad 1,11 \quad 10$

$3,38 \quad 4,29 \quad 0,91 \quad 12$

$4,26 \quad 5,00 \quad 0,74 \quad 14$

$3,57 \quad 4,86 \quad 1,29$

$3,08 \quad 4,71 \quad 1,63$

$3,78 \quad 1,71 \quad 2,06$

$2,17 \quad 3,43 \quad 1,25$

$\begin{array}{llll}3,92 & 3,86 & 0,06 & 7\end{array}$

$\begin{array}{llll}3,42 & 3,71 & 0,29 & 17\end{array}$

$\begin{array}{llll}4,83 & 4,86 & 0,03 & 18\end{array}$

$3,44 \quad 4,57 \quad 1,13 \quad 9$

$3,36 \quad 1,86 \quad 1,51$

$\begin{array}{llll}3,36 & 3,29 & 0,08 & 6\end{array}$

$2,55 \quad 3,86 \quad 1,31 \quad 3$

$3,43 \quad 4,57 \quad 1,14$

$\begin{array}{llll}3,58 & 2,43 & 1,15 & 3\end{array}$

$3,15 \quad 4,29 \quad 1,13 \quad 9$

$\begin{array}{llll}4,54 & 4,86 & 0,31 & 16\end{array}$

$3,05 \quad 4,29 \quad 1,24 \quad 7$

$3,64 \quad 4,71 \quad 1,08 \quad 11$

$\begin{array}{llll}3,82 & 4,72 & 0,89 & 13\end{array}$

$\begin{array}{llll}3,64 & 3,57 & 0,07 & 5\end{array}$

$\begin{array}{llll}3,40 & 3,72 & 0,32 & 15\end{array}$

$2,82 \quad 2,72 \quad 0,11 \quad 4$

Abb. 61: Motivationskonfiguration "Sammler" (Teil I) 
(1)

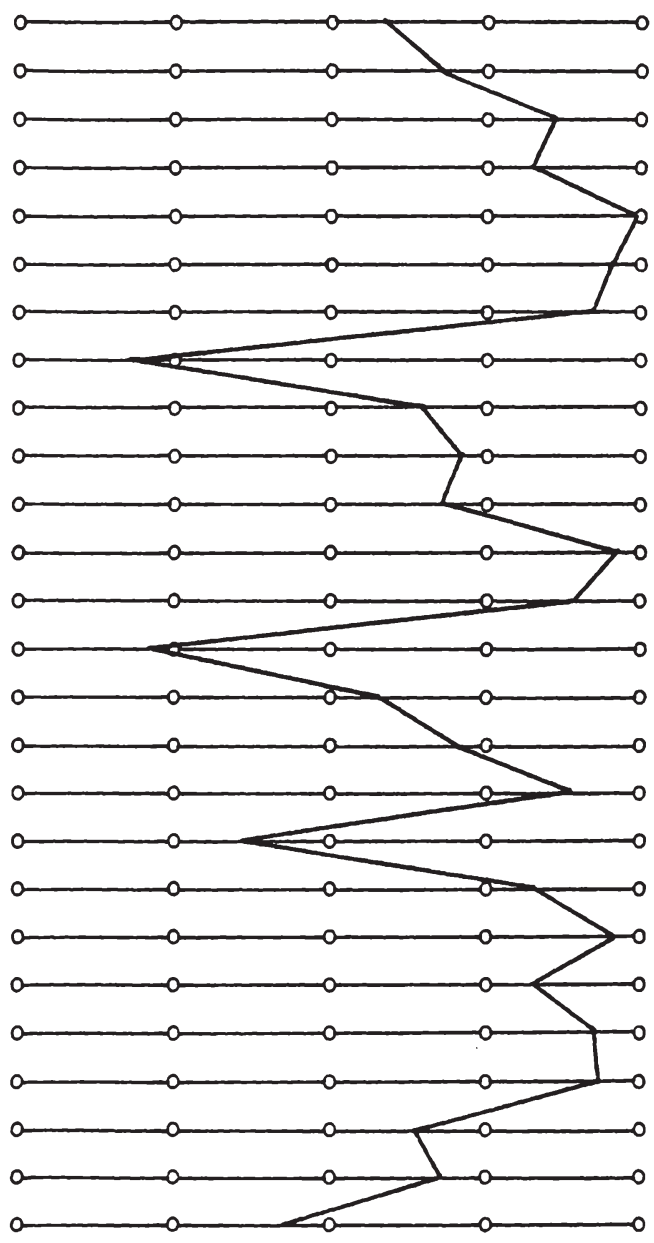

Abb. 61: Motivationskonfiguration "Sammler" (Teil II) 


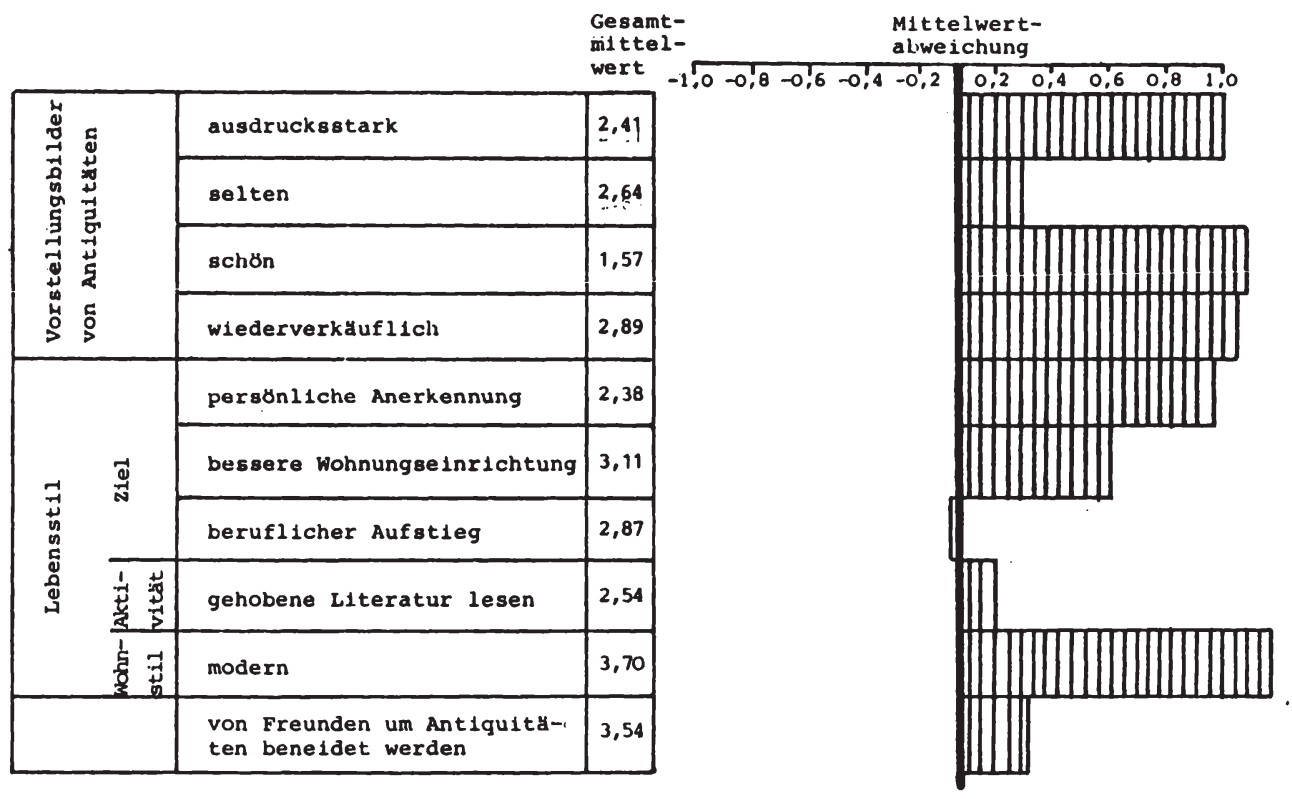

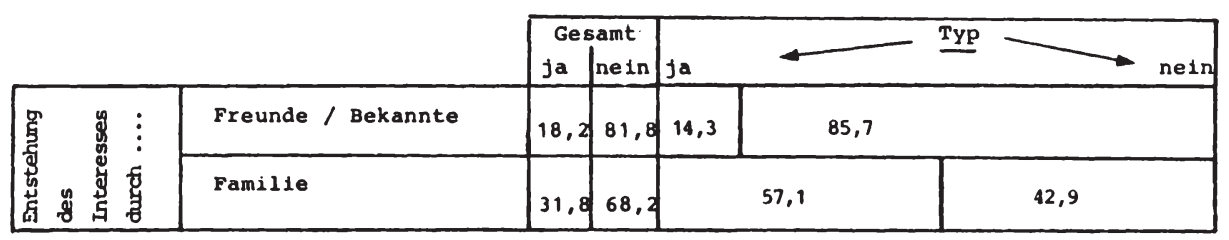




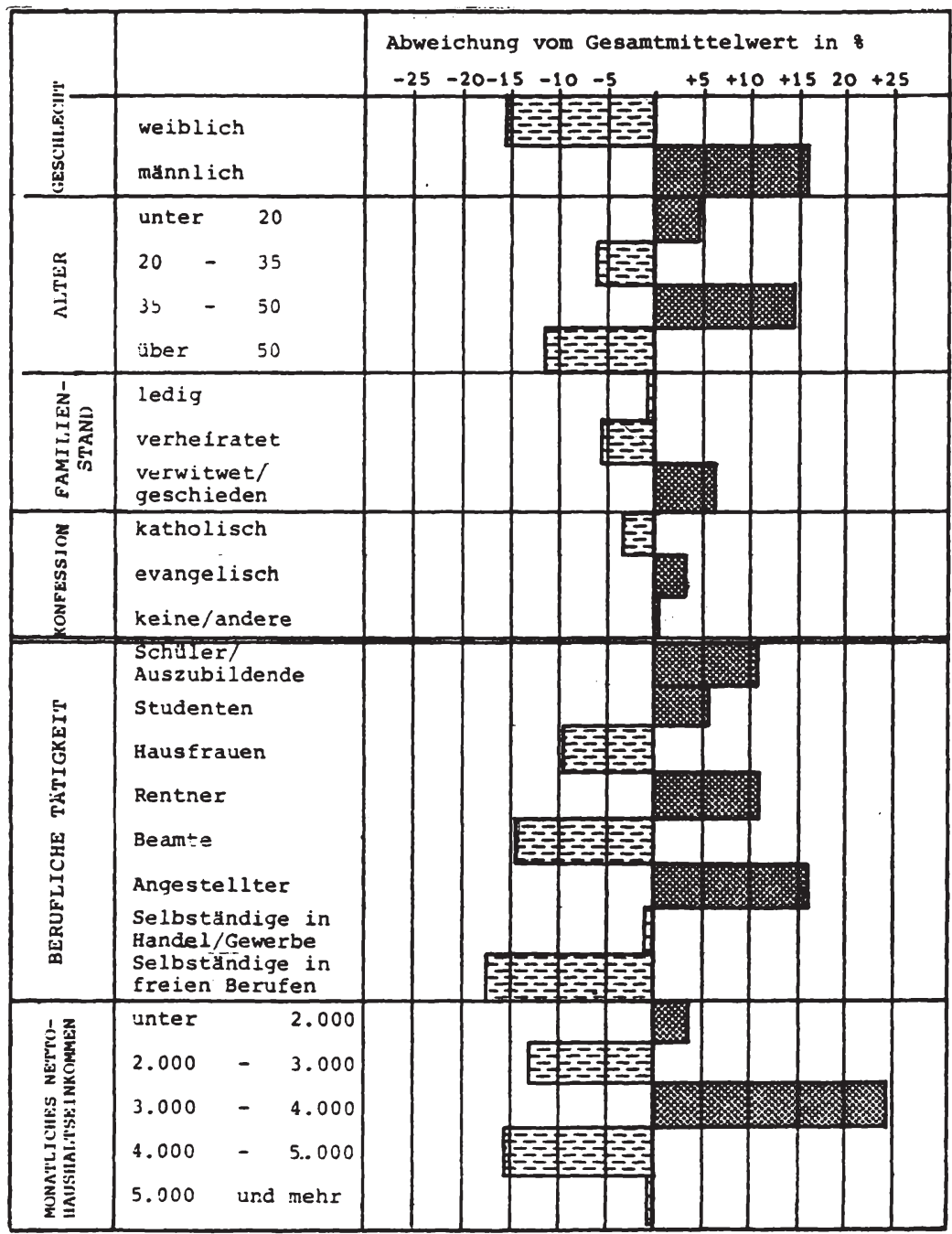

Abb. 63: Soziodemographie der "Sammler" 
einzelner Motivationen, sowohl in Richtung einer zustimmung als auch im besonderen einer Ablehnung, ist uberaus "kategorisch". Hierdurch formt sich ein sehr deutliches und transparentes Bild dieser Motivationskonfiguration.

Folgt man den vorgestellten faktorenanalytischen Ergebnissen, wird die Motivationskonfiguration "Sammler" durch nur eine Inhaltsdimension und zwar das "Sammeln von Antiquitäten als freizeitgestaltendes Hobby" dominiert. Insbesondere die Einzelmotivation "Sammelleidenschaft" ist mit einer Mittelwertabweichung von uber zwel skalenstufen, die zu einer signifikanten Trennung gegenuber sieben weiteren clustern führt, hervorzuheben.

Eine uberdurchschnittliche Relevanz kommt daneben einzig der "Familientradition" zu. Im zusammenhang mit der "Sammelleidenschaft" ist hierin weniger ein gleichbedeutendes Elnzelmotiv als vielmehr der Hinweis auf den "Ursprung" der "Sammelleidenschaft" zu sehen. Unterstützt wird diese Annahme durch den ausgeprägten familiăren EinfluB bel der Interessenentstehung, wie ihn die Analyse der typendiskriminierenden Variablen ausweist (vgl. Abbildung 62).

Die Kaufbedeutung fast aller welteren Elnzelmotive wird von den Sammlern, sowohl im Vergleich zum Gesamtmittelwert der Stichprobe als auch im Vergleich zu den Mittelwerten der ubrigen Motivationstypen, uberdurchschnittlich stark negiert.

Durch die starke "Polarisierung" innerhalb der Motivationskonfiguration besteht der seltene Fall, daB nicht nur eine relative, sondern auch absolute Dominanz der clusterbildenden-Motivationen festgestellt werden kann (vgl. das Mittelwertprofil).

Die Ausschließlichkeit der Sammelmotivation ist vermutlich mit individuellen und selektiven vorstellungen uber 
das Sammelobjekt verknüpft. Hierdurch werden die z.T. sehr gering ausgeprägten Vorstellungsbilder "schön", "ausdrucksstark" und "selten" erklärbar. Die geringe Assoziation zur "Wiederverkäuflichkeit" steht dagegen in Einklang mit den Vorstellungen und zielen der Sammler.

Das sozialstatistische Portrait der "Sammler", welches gerade für dieses cluster sehr geringen Umfangs in seiner Aussagekraft nicht überbewertet werden darf, gibt die Abbildung 63 wieder. Neben der Feststellung, daß Sammler eher unter den männlichen Antiquitätenkäufern und in mittleren Einkommensklassen anzutreffen sind, zeigt sich eine tendenziell heterogene soziodemographische Zusammensetzung.

\subsection{Einrichter}

Der abschließend zu diskutierende Motivationstyp "Einrichter" ist von besonderem Interesse, da Wohn- und Einrichtungsmotive für die Gesamtkäuferschaft von Antiquitäten zwar zu den bedeutendsten Kaufmotivationen zählen, sie aber andererseits faktorenanalytisch nicht als eigenständige Inhaltsdimension extrahiert werden konnte. Die Clusteranalyse erweist sich speziell in diesem zusammenhang als das "feinere" und offensichtlich "realitätsnähere" Analyseinstrument.

Der Clusterschwerpunkt weist die "Einrichter" als durchschnittlich am wenigsten motivational engagierte Käufer aus. In Einklang mit seiner Lage im Diskriminationsraum steht die fast durchgängig geringe Bedeutung der überwiegenden Anzahl von Einzelmotivationen (vgl. Abbildung 64). Viele von ihnen besitzen bei den "Einrichtern" den geringsten absoluten Mittelwert aller vorgestellten Motivationstypen. Die Mittelwertdifferenzen für die Motivationsinhalte "Frei- 
Lösung elnes funktionalen Einrichtungsproblems

Dekoration der Wohnung

Dokumentation von Individualismus

Wertanlage

Spekulation auf Wert:zuwachs

wunsch nach Selbstverwirklichung

Bes1tzstreben

Sammelleidenschaft

Bedurfnis nach Àsthetik

Freude am Schenken

Reaktion auf modernes Mobeldesign

steuerflucht

Nostalg1e

Fre1zeitgestaltung

Hoffnung auf Trouvallle

SpaB am Suchen

Freude am Einkaufserlebnis

Familientradition

Bes1tzerstolz

Repräsentation

Bedurfnis nach Ausgleich

Empfinden elnes

kulturelien Auftrags

Reaktion auf moderne Kunst

Bewahrung von

Erinnerungen an die Familie

Liebe $z$ un alten Kunsthandwerk

Freude an Naturstoffen

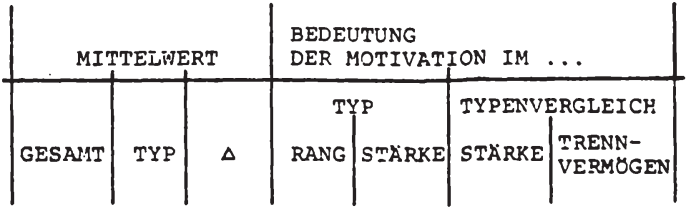

$\begin{array}{lllll}2,03 & 1,27 & 0,76 & 2 & +\end{array}+++$

$2,40 \quad 3,64 \quad 1,24 \quad 6 \quad \ldots \quad-$

$3,32 \quad 4,64 \quad 1,3$

$3,38 \quad 4,64 \quad 1,26$

$4,26 \quad 4,82 \quad 0,56 \quad 15$

$\begin{array}{llll}3,57 & 4,55 & 0,98 & 11\end{array}$

$3,08 \quad 4,18 \quad 1,10 \quad 9$

$3,78 \quad 4,91 \quad 1,13 \quad 8$

$2,17 \quad 2,27 \quad 0,11 \quad 20$

$3,92 \quad 4,00 \quad 0,08 \quad 22$

$\begin{array}{llll}3,42 & 4,09 & 0,67 & 14\end{array}$

$\begin{array}{llll}4,83 & 5,00 & 0,17 & 19\end{array}$

$3,44 \quad 3,55 \quad 0,10 \quad 21$

$3,36 \quad 4,91 \quad 1,55$

$3,36 \quad 4,36 \quad 1,00 \quad 10$

$2,55 \quad 4,09 \quad 1,54 \quad 2$

$3,43 \quad 4,27 \quad 0,84 \quad 12$

$\begin{array}{llll}3,58 & 3,55 & 0,04 & 3\end{array}$

$3,15 \quad 4,64 \quad 1,48 \quad 3$

$\begin{array}{llll}4,54 & 5,00 & 0,46 & 16\end{array}$

$\begin{array}{llll}3,05 & 3,82 & 0,77 & 13\end{array}$

$3,64 \quad 4,82 \quad 1,18$

$3,82 \quad 4,91 \quad 1,10$

$3,64 \quad 4,09 \quad 0,45 \quad 17$

$3,40 \quad 3,64 \quad 0,24 \quad 18$

$2,82 \quad 1,55 \quad 1,28 \quad 1$

Abb. 64: Motivationskonfiguration "Einrichter" (Teil I) 
(1)

(2)

(3)

(4)

kaum

(5)

sehr

iemlich

wichtig

gar nicht wichtig

wicht:g
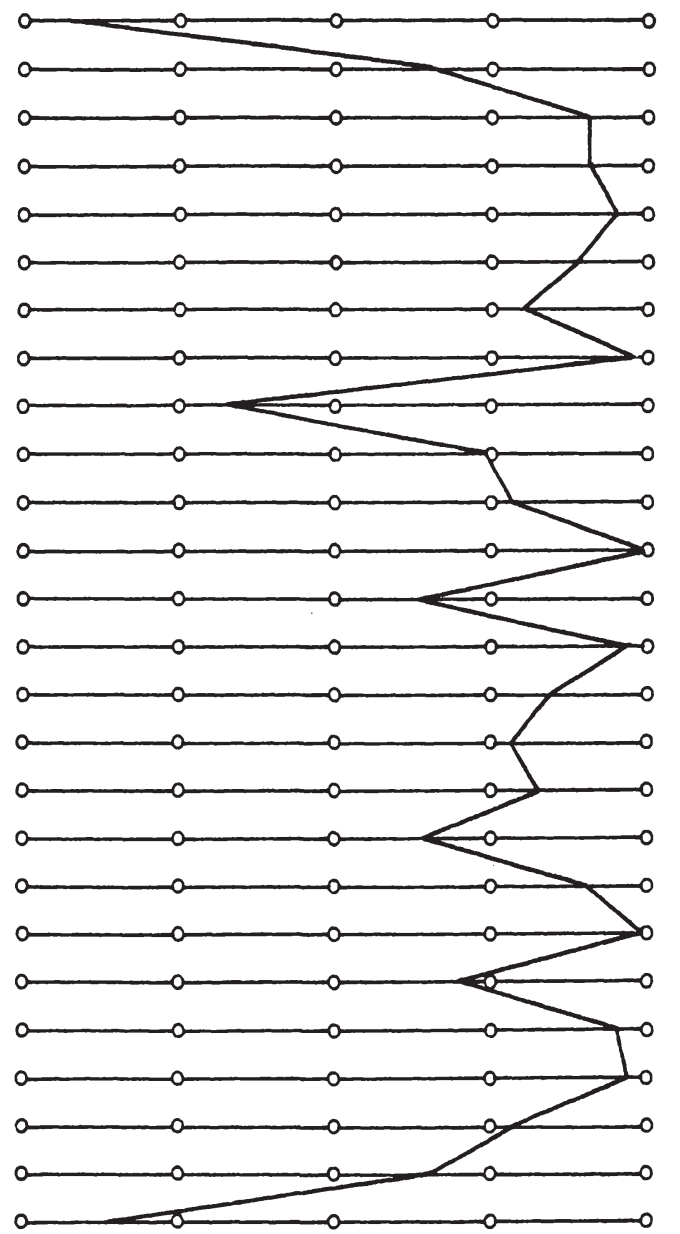

Abb. 64: Motivationskonfiguration "Einrichter" (Teil II) 
zeitgestaltung", "Spaß am Suchen" und "Besitzerstolz" sind dabei zu jewej.ls sieben weiteren Käufergruppen signifikant, so daß sie ein hohes Trennvermögen besitzen.

Inhaltliches Profil gewinnt die Motivationskonfiguration "Einrichter" durch die überdurchschnittlich ausgeprăgte Kaufrelevanz der Motivationen "Lösung eines funktionalen Einrichtungsproblems" und "Freude an Naturstoffen" und in geringem Umfang durch das "Bedürfnis nach Ästhetik". Augenfällig wird ihre Dominanz in der "typeninternen" Betrachtung.

Das "elnseitige" Interesse an Antiquitäten als schöne und funktionstüchtige Gebrauchsgegenstände charakterisiert die "Einrichter" als eine, der ursprünglichen "Erstkäufern " motivational verwandte Käufergruppe; denn die heutigen Antiquitäten sollten zum Zeitpunkt ihrer Entstehung (als "zeitgenössisches Kunsthandwerk") gerade diese Nutzenkombination von ästhetisch-schöner und zuweilen prunkvoll-repräsentativer Form und Gebrauchsfähigkeit erfullen.

Erwartungsgemäß überwiegt unter den Vorstellungsbildern uber Antiquitäten in der Käufergruppe "Einrichter" das Warenattribut "schön" (vgl. Abbildung 65). Weiterhin ist unter den typendiskriminierenden Variablen die vorherrschende Einschätzung cles Wohnstils als "nicht modern" beachtenswert. Im Gegensatz zu den "Rationalisten", welche Antiquitäten eher in eine moderne Wohnumgebung als "Stilmittel" integrieren, zeigt sich beim "Einrichter" eine eher durchgängige und ausschließliche Wohnungsgestaltung mit alten stücken.

$\mathrm{DaB}$ den als "trennscharf" ermittelten Persönlichkeitszielen "persönliche Anerkennung", "berufliches Fortkommen" und "bessere Wohnungseinrichtung" nur eine geringe Bedeutung beigemessen wird, findet u.a. eine Erklärung in der Soziodemographie der "Einrichter" (vgl. Abbildung 66). Hier dominieren 


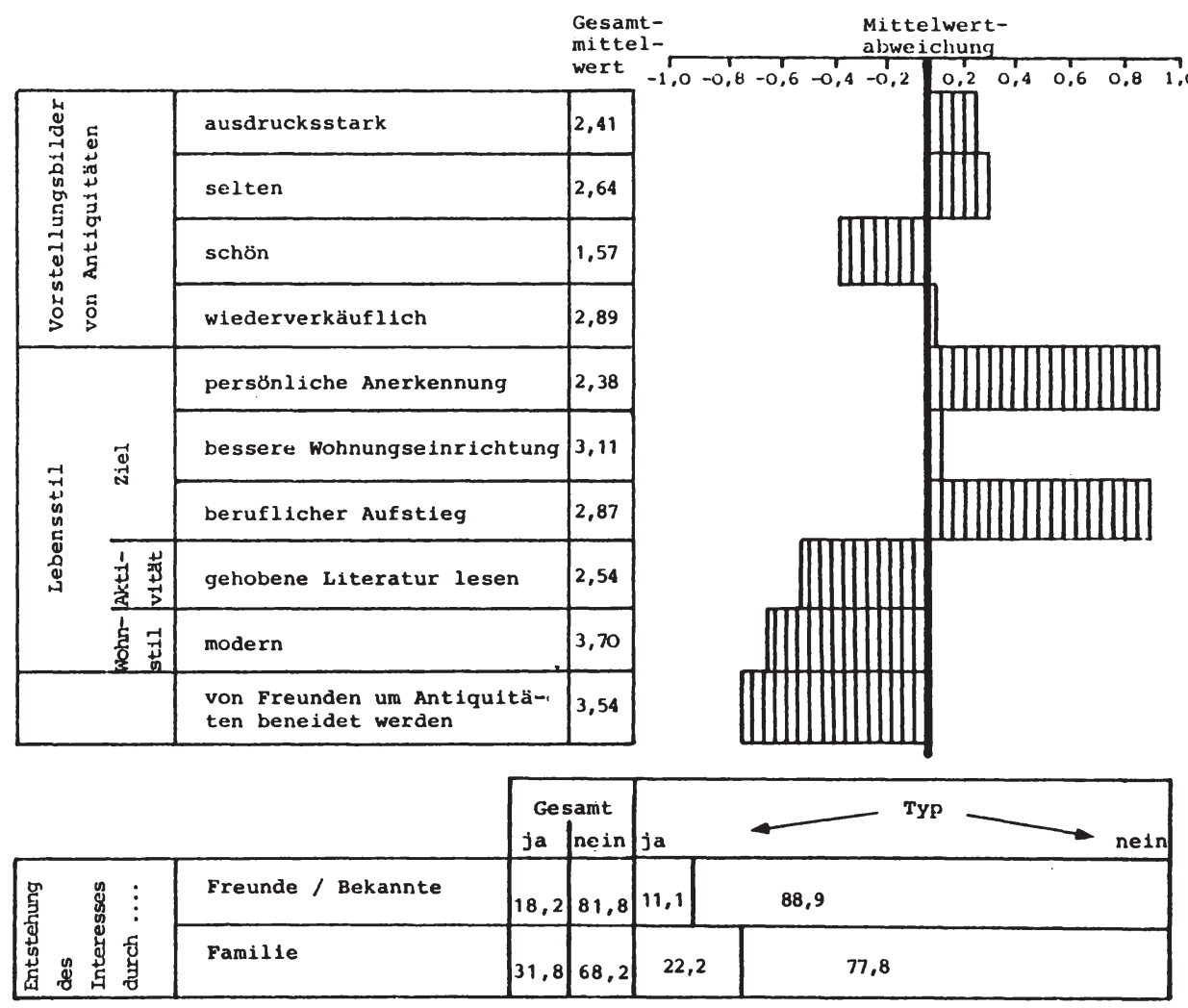




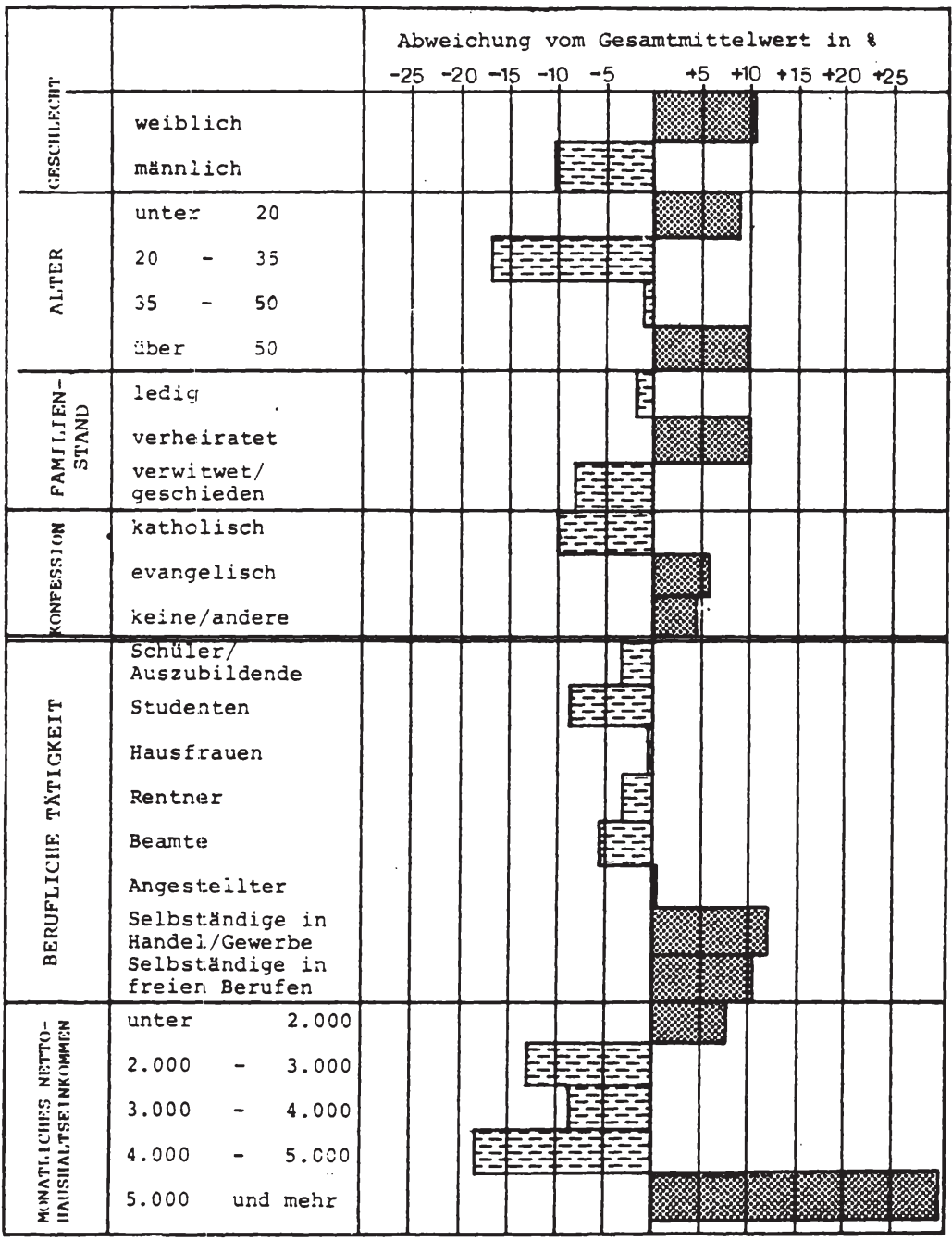

Abb. 66: Soziodemographie der "Einrichter" 
die Spltzeneinkommen (über 5.000,-DM), die selbständigen Berufsgruppen, sowie die älteren Käufer (über 50 Jahren). Daneben läßt sich $1 \mathrm{~m}$ kleineren Umfang ein uberproportionaler Antell sehr junger Kăufer (unter 20 Jahren) und geringerer Einkommen (unter $2.000,-D M$ ) feststellen.

\subsection{Analyse der Typenzugehörlgkeiten}

Die typenspezifischen Motivationskonfigurationen bildeten die wichtigste Interpretationsgrundlage der vorausgegangenen Detallanalyse einzelner Motivationstypen von Antiquitätenkăufern. Motivationskonfigurationen sind das Resultat einer Durchschnittsbildung der zu einem Motivationstyp zusammengefaßten individuellen Motivstrukturen einzelner Käufer. Die im Rahmen der clusteranalytischen Klassifikation festgelegte Typenzugehörigkeit bestimmt damit letztendlich die Motivationskonfiguration. Dabei wird stillschweigend davon ausgegangen, daß die Typenzugehörigkelt des einzelnen Käufers stets eindeutig und ausschließlich ist.

Die folgenden Ausfuhrungen berühren zwe1 Problemkreise im zusammenhang mit der Typenzugehörigkeit:

(1) Die Möglichkeit einer Erfassung von Wahrscheinlichkeiten für Typenzugehörigkeiten auf der Grundlage Individueller Motivationsstrukturen der Antiquitätenkăufer.

(2) Die Möglichkeit einer Prognose von Typenzugehörigkelten auf der Grundlage der individuellen Strukturen motivationaler Bestimmungsfaktoren. 


\subsection{Wahrscheinlichkeit der Typenzugehörigkeit}

Obwohl clusteranalysen die Typenzugehörigkeit von Antiquitätenkäufern im Sinne eines Ja/Nein-Kriteriums bestimmen, kann davon ausgegangen werden, daß einzelne Antiquitätenkäufer die typenspezifische Motivationskonfiguration unterschiedlich gut repräsentieren.

Für die beiden Strukturdimensionen "Engagement" und "Tradition" kommt dies in einer unterschiedlichen "Nähe" der Käufer zu ihrem Clustercentroid zum Ausdruck (vgl. Abbildung 43$)^{1}$. Die Abbildung zeigt zugleich, daß einige Käufer in "Randzonen", d.h. in einer engen "Nachbarschaft" zu anderen Motivationskonfigurationen liegen. Diese realistischerweise fließenden Ubergänge zwischen den Motivationstypen lassen sich durch diskriminanzanalytisch ermittelte Typenzugehörigkeitswahrscheinlichkeiten reproduzieren ${ }^{2}$. Die Tabelle 11 gibt einen Ergebnisüberblick über die empirischen Werte.

Es zeigt sich, daß knapp $80 \%$ aller befragten Antiquitätenkäufer mit einer Wahrscheinlichkeit zwischen 95 ind 100 \% auf der Grundlage individueller Motivationsstrukturen einem spezifischen Motivationstyp (Motivationskonfiguration) zugeordnet werden können ${ }^{3}$. Zugehörigkeitswahrscheinlichkeiten unter $80 \&$ bestehen dagegen für nur knapp 16 \& aller Käufer.

$1 \mathrm{Bei}$ der Betrachtung aller typenbildenden Motivationen drückt sich der Tatbestand in einer mehr oder weniger starken Kongruenz der individuellen Motivationsstrukturen mit den typenspezifischen Motivationskonfigurationen aus.

2 Im Rahmen der Deskription von Zugehörigkeitswahrscheinlichkeiten bilden die Einzelmotivationen die unabhängigen Variablen und die Motivationstypen die abhängigen Variablen der Diskriminanzanalyse. zur Interpretation der Wahrscheinlichkeiten vgl. Klecka, W.R., Discriminant Analysis, a.a.O., S. 445 f.; Beutel, P. et al., a.a.0., S. 178 .

3 Die Ermittlung der Typenzugehörigkeitswahrscheinlichkeiten erfolgte im Rahmen der MOT DISC 4. $\mathrm{Zu}$ den Voreinstellungen der Inklusionsparameter vgl. Tabelle 9 im Anhang. 


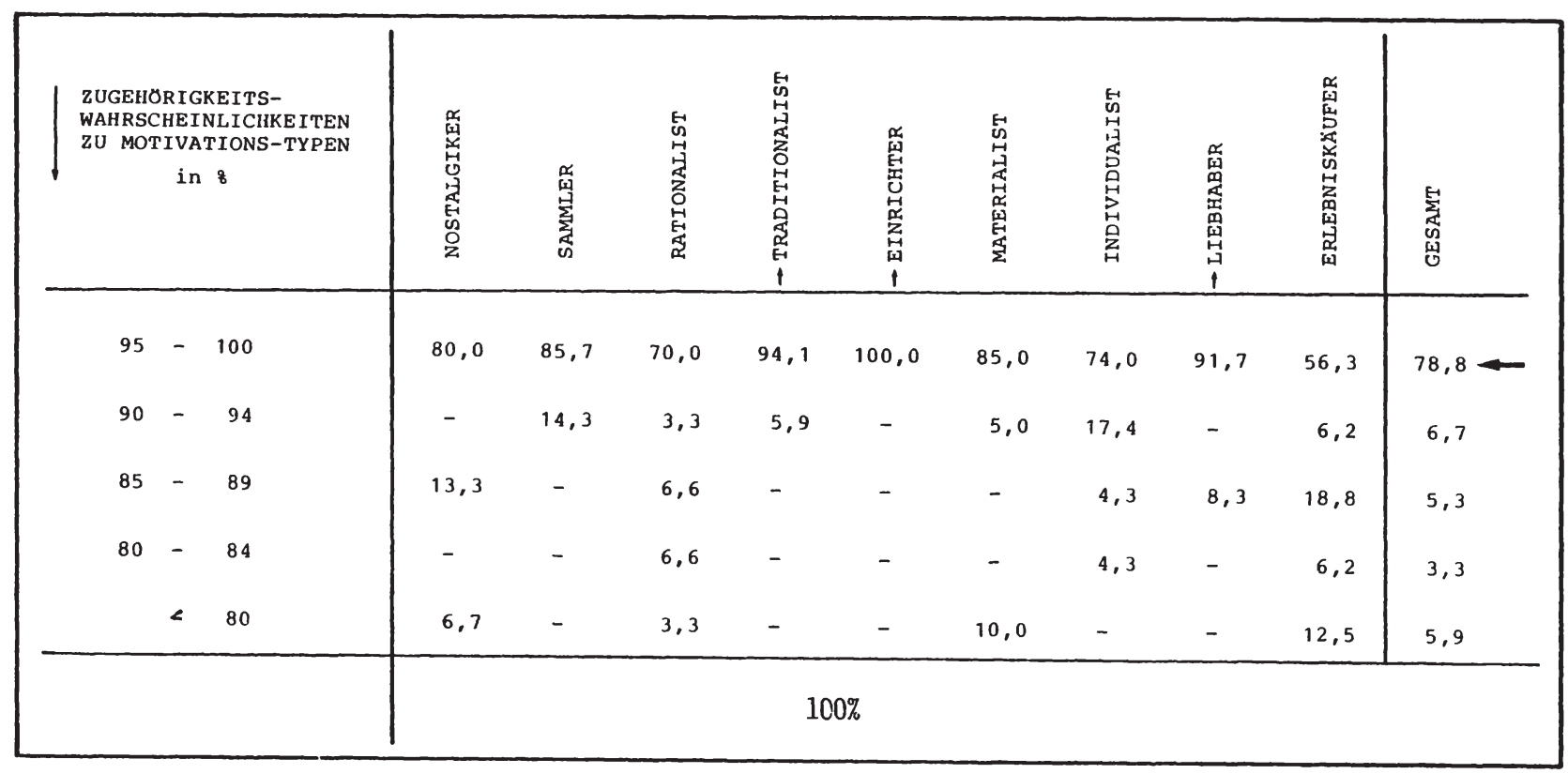

Tab. 11: Zugehörigkeitswahrscheinlichkeiten zu Motivationstypen 
Unter den Einzeltypen erweisen sich die "Einrichter", "Materialisten" und "Sammler" als diejenigen Cluster, deren Mitglieder sowohl am eindeutigsten ihren Motivationstyp repräsentieren, als auch die geringsten Ähnlichkeiten zu den anderen Typen aufweisen. Etwas größere "Randgruppen", d.h. Anteile von Käufern mit niedrigen Zugehörigkeitswahrscheinlichkeiten, besitzen insbesondere die "Rationalisten" und "Erlebniskäufer".

Das insgesamt hohe Niveau der diskriminanzanalytisch bestimmten Wahrscheinlichkeiten dokumentiert schlieBlich die ausgeprägte Trennschärfe, bzw. "innere Homogenität" und "äußere Heterogenität" der clusteranalytischen Typenlösung.

\subsection{Prognose der Typenzugehörigkeit}

Die Prognose cler Typenzugehörigkeit stellt sich als ein Klassifikationsproblem: Es wird gefragt, zu welchem Prozentsatz sich die Antiquitätenkäufer ihrem "richtigen", d.h. clusteranalytisch ermittelten Motivationstyp zuordnen lassen, wenn ausschließlich die Ausprägungen ihrer motivationalen Bestimmungsfaktoren bekannt sind ${ }^{1}$.

1 Methodisch entspricht dies einer ebenfalls diskriminanzanalytischen Fragestellung: auf der Grundlage der trennschärfsten motivationalen Bestimmungsfaktoren (unabhängige Variablen) wird über die Bestimmung von Diskriminanzfunktionen und Diskriminanzwerten eine retrograde Zuordnung der einzelnen Antiquitätenkäufer zu den Motivationstypen (abhängige Variable) vorgenommen. 
Die Anzahl empirisch "richtiger" Klassifikationen im Vergleich zur maximalen Zufallswahrscheinlichkeit "richtiger" Klassifikationen, gibt Hinweise für die Güte einer Trennung (Diskrimination) der Motivationstypen durch die personen-, waren- und situationsbezogenen Bestimmungsfaktoren der Käufermotivation ${ }^{1}$.

Die in Tabelle 12 gezeigte Matrix "richtiger" und "falscher" Typenzugehörigkeiten stüzt sich auf 46 als besonders trennscharf ermittelte Bestimmungsfaktoren und eine Reklassifikation auf der Basis von acht Diskriminanzfunktionen ${ }^{2}$.

Die Gesamtzahl von knapp 83 \& richtig zugeordneter Antiquitätenkäufer kann unter Berücksichtigung der großen Zahl von Motivationstypen und einer maximalen Zufallswahrscheinlichkeit von knapp 20 ols sehr gut bezelchnet werden ${ }^{3}$.

Ähnlich gut sind die Ergebnisse für den ubberwiegenden Tell einzelner Motivationstypen zu beurteilen.

Die seltenen Konzentrationen von "Fehlklassifikationen" zwischen zwei Motivationstypen deuten an, daß sich das jeweils betrachtete Typen-Paar nicht exakt durch unterschiedliche Strukturen in den motivationalen Bestimmungsfaktoren der Antiquitätenkäufer abgrenzen lassen, bzw. ihre Prognose-

1 Die maximale Zufallswahrscheinlichkeit liegt bei 19,9 \%. Sie entspricht dem Umfang des größten Motivationstyps; denn würden alle Antiquitätenkäufer bei der Reklassifikation ausschlieblich diesem Typ zugeordnet, ergeben sich "richtige" Klassifikationen in maximal gleicher Höhe.

2 Grundlage bildet die Diskriminanzanalyse PRA DISC 3, deren weitere Ergebnisse und Inklusionsparameter der Ubersichtstabelle $13 \mathrm{im}$ Anhang entnommen werden können.

3 Dabei ist auf einen der Diskriminanzanalyse immanenten "Stichprobenfehler" hinzuweisen, der sich aus einerwe1tgehenden Stichprobengleichheit bei der Ermittlung der Diskriminanzfunktionen und der Reklassifikation ergibt. In der vorliegenden Untersuchung wird er dadurch gemindert, daß in dem ersten Analyseschritt aufgrund "missing values" nur knapp 88 \& der Urteile aller Antiquitätenkäufer einbezogen wurden. Die restlichen 12 g gehen dagegen in die Reklassifikation ein und stellen damit eine zusätzliche stichprobe dar. Vgl. Schuchard-Ficher, Chr.et al., a.a.0., s. 176 f.. 


\begin{tabular}{|c|c|c|c|c|c|c|c|c|c|}
\hline $\overrightarrow{\text { MOTIVATIONS-TYP }}$ & 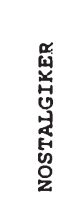 & 㑁 & 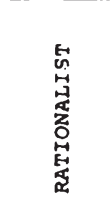 & 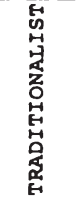 & 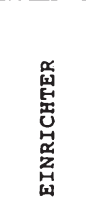 & 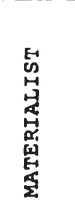 & 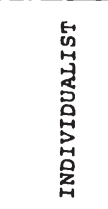 & 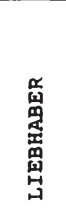 & 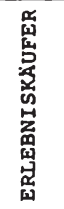 \\
\hline NOSTALGIKER & 66,7 & & $\rightarrow 20,0$ & 6.7 & & & 6,7 & & \\
\hline SAMMLER & & 85,7 & & & & 14,3 & & & \\
\hline RATIONALIST & 3.3 & & 86,7 & 3.3 & 3.3 & & 3.3 & & \\
\hline TRADITIONALIST & & & & 94,1 & 5,9 & & & & \\
\hline EINRICHTER & & & & 9,1 & 54,5 & 9,1 & $\rightarrow 18,2$ & 9,1 & \\
\hline MATERIALIST & & & & & & 85,0 & 10,0 & 5,0 & \\
\hline INDIVI DUALIST & & & 8,7 & & & 8,7 & 82,6 & & \\
\hline LIEBHABER & 8,3 & & & & & & & 91,7 & \\
\hline ERLEBNISKAUYFER & & & 12,5 & & & & & & 87,5 \\
\hline
\end{tabular}

Tab. 12: Diskriminanzanalytische Klassifikationsergebnisse für die Typenzugehörigkeit auf der Basis motivationaler Bestimmungsfaktoren 
fähigkeit eingeschränkt bleibt. Beispiel sind die Käufer des "Nostalgiker-Clusters", welche zu 20 \& den "Rationalisten", sowie die "Einrichter", die zu 18,2 \& den "Individualisten" zugeordnet werden.

Resümierend ist die Prognosefähigkeit motivationaler Bestimmungsfaktoren für die Typenzugehörigkeit prinzipiell zu bejahen. Wenn auch die diskriminanzanalytische Klassifikation im statistischen Sinne keine Abhängigkeitsbeziehungen ausdrückt, so legen ihre Ergebnisse doch den Schluß nahe, daß unterschiedliche Strukturen motivationaler Bestimmungsfaktoren in engem zusammenhang mit der Typenzugehörigkeit und den implizierten Motivationskonfigurationen stehen.

\section{Vergleichende Analyse der Erklärungsbeiträge von Motivationen und Motivationskonfigurationen zum Verhalten}

Vor dem Hintergrund der vorausgegangenen Motivationsanalyse und der dort vermittelten Kenntnisse uber Motivationsinhalte und-bestimmungsfaktoren werden im folgenden motivationale Verhaltenswirkungen auf ausgewählte kognitive und konative Verhaltensmerkmale der Antiquitätenkäufer untersucht.

Grundlage der empirischen Erfassung von Erklärungsbeiträgen der Käufermotivation sind die erhobenen individuellen Verhaltensvariationen der Antiquitätenkäufer ${ }^{1}$, welche auf ihre Abhängigkeiten von einzelnen Motivationsinhalten bzw. gesamtheitlichen Motivationskonfigurationen $z u$ prüfen sind ${ }^{2}$.

1 Vgl. zur Verhaltenscharakterisierung der Antiquitätenkäufer Kapitel B. 3.2.

2 Zur Auswahl des methodischen Instrumentariums vgl. die Ausführungen in Kapitel C. 1. 


\subsection{Erklärungsbeiträge zu kognitiven Verhaltensmerkmalen}

\subsection{Kaufrelevanz von Antiguitäten-Merkmalen}

Im Mittelpunkt der Analyse von Beziehungen zwischen Einzelmotivationen bzw. Motivationskonfigurationen und der Kaufbedeutung von Warenmerkmalen steht die Frage, ob und inwieweit Kaufbedeutungen motivational bedingte (kognitive) Anspruchshaltungen an Antiquitäten darstellen.

Die regressionanalytische Prüfung des Erklärungsbeitrages einzelner Motivationsinhalte weist für eine Vielzahl von Antiquitätenmerkmalen überdurchschnittliche Varianzerklärungsanteile aus. Die Höhe der multiplen Korrelationskoeffizienten schwankt bei i.d.R. 6-10 einbezogenen Einzelmotiven überwiegend zwischen 0,40 und $0,60^{1}$.

Von motivational bedingten Anspruchshaltungen kann insbesondere im Zusammenhang mit den Warenmerkmalen "Repräsentativität", "Stilechtheit", "Seltenheit", "Alter", "Dekorationswert", "Wertbeständigkeit", "Funktionalität" und "Gebrauchsfähigkeit" gesprochen werden, deren Ausgangsvarianz z.T. weit über 30 \& erklärt wird ${ }^{2}$.

1 Vgl. die Ergebnisübersicht in der Tabelle 15 im Anhang.

2 Motivationale Erklärungsansätze für abhängige Variablen hier Kaufrelevanzen von Antiquitätenmerkmalen - mit Varianzerklärungsanteilen (Bestimmtheitsma $B$ ) unter $30 \%$, bzw. multiplen Korrelationskoeffizienten unter 0,55 , werden im folgenden als nicht interpretationsrelevant angesehen. Auf ihre Darstellung wird verzichtet, obwohl auch aus solchen "schlechteren" Einzelergebnissen interessante Tendenzaussagen abgeleitet werden können. Die gewählte "Interpretationsgrenze" setzt ein insgesamt relativ hohes Niveau der dargestellten motivationalen Erklärungsansätze voraus. 
Tabelle 13 gibt einen detaillierten Uberblick der empirischen Einzelergebnisse.

Unter Berücksichtigung der jeweils wichtigsten erklärenden Motivationsinhalte lassen sich schlaglichtartig folgende Feststellungen treffen:

(1) Die Kaufrelevanz der "Repräsentativität" wächst insbesondere in Abhängigkeit von den Kaufmotivationen "Repräsentation", "Dekoration der Wohnung" und "Bedürfnis nach Ästhetik".

(2) Die Kaufrelevanz der "Stilechtheit" wächst insbesondere in Abhängigkeit von den Kaufmotivationen "Wunsch nach Selbstverwirklichung", "Familientradition" und "Bedürfnis nach Ästhetik".

(3) Die Kaufrelevanz der "Seltenheit" wächst insbesondere in Abhängigkeit von den Kaufmotivationen "Sammelleidenschaft" und "Spaß am Suchen".

(4) Die Kaufrelevanz des "Alters" wächst insbesondere in Abhängigkeit von den Kaufmotivationen "Wertanlage","Empfinden eines kulturellen Auftrags" und der "Sammelleidenschaft".

(5) Die Kaufrelevanz des "Dekorationswertes" wächst in Abhängigkeit von den Kaufmotivationen "Dekoration der Wohnung" und "Bedurfnis nach Asthetik".

(6) Die Kaufrelevanz der "Wertbeständigkeit" wächst insbesondere in Abhängigkeit von den Kaufmotivationen "Wertanlage", "Familientradition" und "Freude an Naturstoffen".

(7) Die Kaufrelevanzen von "Funktionalität" und "Gebrauchsfähigkeit" wachsen insbesondere in Abhängigkeit von der Kaufmotivation "Lösung eines funktionalen Einrichtungsproblems" und sinken insbesondere in Abhängigkeit von der Kaufmotivation "Sammelleidenschaft". 
Erklärende Motivationer ...

Dekoration der Wohnung

Repräsentation

Frelzeitgestaltung

Wertanlage

Bedirfnis nach Asthetik

Freude am Schenken

Nostalgie

Lösung eines funktionaien Einrichtungsproblems

\section{STILECHTHEIT}

Erklärende Motivationen ...

Familientradition

Wertanlage

Bedürfnis nach fisthetik

Empfinden eines kulturelien Auftrags

Liebe zum alten Kunsthandwerk

Dokumentation von Individualismus

Wunsch nach Selbstverwirklichung

Repräsentation

Reaktion auf moderne kunst

Besitzerstolz

Sammelleidenschaft

\begin{tabular}{|l|l|r|r|l|} 
MULT R & $\begin{array}{c}\text { BESTIMMT- } \\
\text { HEITSMASS }\end{array}$ & EINFACH R & BETA & RANG \\
\hline 0.380 & 0.144 & 0.380 & 0.222 & 2 \\
0.452 & 0.204 & 0.340 & 0.280 & 1 \\
0.476 & 0.227 & -0.109 & -0.163 & 6 \\
0.500 & 0.251 & 0.290 & 0.167 & 4 \\
0.517 & 0.267 & 0.226 & 0.205 & 3 \\
0.539 & 0.289 & -0.111 & -0.164 & 5 \\
0.551 & 0.304 & 0.001 & -0.142 & 7 \\
0.563 & 0.317 & -0.055 & -0.125 & 8
\end{tabular}

\section{SELTENHEI?}

Erklarenaie Motivationen ...

\begin{tabular}{|l|c|c|c|c|} 
MULT R & $\begin{array}{l}\text { BESTIMMT- } \\
\text { HEITSMASS }\end{array}$ & EINFACH R & BETA & RANG \\
\hline 0.290 & 0.084 & 0.290 & 0.240 & 2 \\
0.401 & 0.161 & 0.272 & 0.178 & 6 \\
0.449 & 0.202 & 0.275 & 0.208 & 5 \\
0.467 & 0.218 & 0.228 & 0.140 & 8 \\
0.496 & 0.246 & -0.125 & -0.231 & 3 \\
0.510 & 0.260 & 0.063 & -0.258 & 1 \\
0.536 & 0.287 & 0.238 & 0.218 & 4 \\
0.551 & 0.304 & 0.189 & 0.175 & 7 \\
0.561 & 0.315 & 0.178 & 0.129 & 10 \\
0.572 & 0.327 & 0.108 & -0.139 & 9 \\
0.583 & 0.340 & 0.221 & 0.122 & 11
\end{tabular}

Stejerflucht

SpaB am Suchen

Sammelleidenschaft

Wertanlage

Freizeitgestaltung

Freude am Schenken

Reaktion auf mcdernes Möbeldesign

Bewahrung von Erinnerungen an die Eamilie

Emofinden eines kulturellen Auftrags

Liebe zum alten Kunsthandwerk

\begin{tabular}{|l|c|r|r|r|} 
MULT R & $\begin{array}{c}\text { BESTIMNT- } \\
\text { HEITSMASS }\end{array}$ & EINFACH R & BETA & RANG \\
\hline 0.291 & 0.085 & 0.291 & 0.183 & 4 \\
0.390 & 0.152 & 0.279 & 0.227 & 3 \\
0.443 & 0.196 & 0.266 & 0.347 & 1 \\
0.473 & 0.224 & 0.275 & 0.161 & 7 \\
0.503 & 0.253 & 0.104 & -0.261 & 2 \\
0.524 & 0.275 & 0.204 & 0.176 & 6 \\
0.545 & 0.297 & -0.126 & -0.135 & 9 \\
0.561 & 0.315 & -0.084 & -0.145 & 8 \\
0.578 & 0.334 & 0.241 & 0.179 & 5 \\
0.588 & 0.346 & -0.099 & -0.116 & 10
\end{tabular}

ALTER

Erklärende Motivationen ...

Wertanlage

ampfinden eines kulturelien Auftrags

Sammelleidenschaft

Familientradition

Dokumentation von Individualismus

Dekoration der Nohnung

Freude am Schenken

Bedürfnis nach Ausgleich

\begin{tabular}{|l|c|r|r|l|} 
MULT R & $\begin{array}{c}\text { SESTIMMT- } \\
\text { HEITSMASS }\end{array}$ & EINFACH R & BETA & RANG \\
\hline 0.371 & 0.137 & 0.379 & 0.315 & 1 \\
0.459 & 0.211 & 0.347 & 0.256 & 2 \\
0.506 & 0.256 & 0.305 & 0.197 & 5 \\
0.532 & 0.283 & 0.208 & 0.212 & 4 \\
0.558 & 0.311 & 0.046 & -0.220 & 3 \\
0.579 & 0.335 & 0.210 & 0.150 & 6 \\
0.591 & 0.350 & -0.042 & -0.127 & 7 \\
0.599 & 0.359 & 0.211 & 0.108 & 8
\end{tabular}

Tab. 13: Motivationale Erklärungsbeiträge zur Kaufrelevanz von Antiquitätenmerkmalen - Ergebnistableaus multipler schrittweiser Regressionsanalysen (Teil I) 
DEKCRATIONSWERT

Erklärende Motivationen ...

\begin{tabular}{|l|c|r|r|l|} 
MULT R & $\begin{array}{c}\text { BESTIMMT- } \\
\text { HEITSMASS }\end{array}$ & EINFACH R & BETA & RANG \\
\hline 0.557 & 0.310 & 0.557 & 0.479 & 1 \\
0.587 & 0.344 & 0.315 & 0.192 & 2 \\
0.595 & 0.354 & -0.053 & -0.124 & 4 \\
0.606 & 0.368 & 0.272 & 0.126 & 3
\end{tabular}

Dekoration der Wohnung

Bedürfnis nach Ästhetik

Freude am Schenken

Frelde am Einkaufserlebnis

\begin{tabular}{|l|c|r|r|c|} 
MULT R & $\begin{array}{c}\text { BESTIMMT- } \\
\text { HEITSMASS }\end{array}$ & EINFACH R & BETA & RANG \\
\hline 0.499 & 0.249 & 0.499 & 0.475 & 1 \\
0.526 & 0.276 & -0.109 & -0.330 & 2 \\
0.555 & 0.308 & 0.065 & 0.253 & 3 \\
0.583 & 0.340 & 0.096 & 0.160 & 4 \\
0.597 & 0.356 & 0.100 & 0.157 & 5 \\
0.614 & 0.377 & 0.302 & 0.134 & 6 \\
0.622 & 0.388 & 0.239 & 0.112 & 7
\end{tabular}

\section{EJNKTIONALITÄT/PRAKTIKABILITAT}

Erklärende Motivationen ...

Lösung eines funktionalen Einrichtungspr.

Sammelleidenschaft

Empfinden eines kulturelien Aurtrags

Freude am Einkaufserlebnis

Besitzerstolz

Steuerflucht

Ereude am Schenken

\begin{tabular}{|l|c|r|r|l|} 
MULT R & $\begin{array}{l}\text { BESTIMMT- } \\
\text { HEITSMASS }\end{array}$ & EINFACH R & BETA & RANG \\
\hline 0.646 & 0.418 & 0.646 & 0.585 & 1 \\
0.661 & 0.436 & -0.301 & -0.172 & 2 \\
0.675 & 0.456 & 0.055 & 0.131 & 4 \\
0.685 & 0.470 & -0.260 & -0.144 & 3 \\
0.694 & 0.482 & -0.025 & 0.118 & 5 \\
0.700 & 0.490 & 0.125 & 0.101 & 6 \\
0.707 & 0.499 & -0.156 & -0.098 & 7
\end{tabular}

GEBRAUCHSFAHIGKEIT

\begin{tabular}{|c|c|c|c|c|c|}
\hline Erklärende Motivationen ... & MULT $R$ & $\begin{array}{l}\text { BESTIMMT- } \\
\text { HEITSMASS }\end{array}$ & EINFACH R & BETA & RANG \\
\hline $\begin{array}{l}\text { Lösung eines funktionalen Einrichtungspr. } \\
\text { Sammelleidenschaft } \\
\text { Spekulation auf Wertzuwachs } \\
\text { peaktion auf modernes Möbeldesign } \\
\text { Bedürfnis nach Ausgleich } \\
\text { Freude an Naturstoffen }\end{array}$ & $\begin{array}{l}0.629 \\
0.668 \\
0.680 \\
0.688 \\
0.700 \\
0.709\end{array}$ & $\begin{array}{l}0.396 \\
0.446 \\
0.463 \\
0.474 \\
0.490 \\
0.502 \\
\end{array}$ & $\begin{array}{r}0.629 \\
-0.379 \\
0.084 \\
0.151 \\
-0.160 \\
0.217\end{array}$ & $\begin{array}{r}0.523 \\
-0.244 \\
0.138 \\
0.141 \\
-0.144 \\
0.116\end{array}$ & $\begin{array}{l}1 \\
2 \\
5 \\
4 \\
3 \\
6\end{array}$ \\
\hline
\end{tabular}

Tab. 13: Motivationale Erklärungsbeiträge zur Kaufrelevanz von Antiquitätenmerkmalen - Ergebnistableaus multipler schrittweiser Regressionsanalysen (Teil II) 
Die hohe inhaltliche Konsistenz der aufgezeigten motivationalen Einflüsse bedarf keiner weiteren Erläuterungen. Ergänzende Detailaussagen z.B. Höhe und Verhältnis der Erklärungsbeiträge einzelner Motivationsinhalte oder auch einfache Korrelationsbeziehungen können der angesprochenen Tabelle 13 entnommen werden.

Für insgesamt 14 Antiquitätenmerkmale besteht ein varianzanalytisch signifikanter Einfluß der Typenzugehörigkeit auf ihre Kaufbedeutung bei Anschaffungsentscheidungen privater Antiquitätenkäufer (vgl. Tabelle 14) ${ }^{1}$.

Aus der Fülle erwähnenswerter Einzelergebnisse kann an dieser Stelle nur eine geringe Auswahl exemplarisch vorgestellt werden ${ }^{2}$ :

(1) Bezogen auf die "Gebrauchsfähigkeit" kommt es zu einer fast vollständigen "Marktspaltung". Die "Sammler", "Materialisten" und "Nostalgiker" messen dieser Wareneigenschaft jeweils eine signifikant geringere Kaufbedeutung bei als die "Einrichter", "Liebhaber", "Erlebniskäufer", "Individualisten" und "Rationalisten".

1 Der Aufbau der Tabelle 14 gleicht den Tabellen 2-7 im Anhang. Die angegebenen Mittelwerte beziehen sich auf eine 5-stufige Skala von (1) sehr wichtig bis (5) gar nicht wichtig. Eine zeilenweise Tabelleninterpretation zeigt die Ergebnisse von Varianzanalysen, wobei in der Vorspalte die jeweilig abhängige Variable und in der Kopfspalte die Motivationskonfiguration als unabhängige Variable angegeben wird. Die Ermittlung der signifikanten Gruppentrennungen basiert auf einem "Least-significantdifference-Test", wie er im SPSS-Subprogramm ONEWAY abrufbar ist $(\alpha=0,05)$. Eine spaltenweise Tabelleninterpretation erlaubt eine deskriptive Beschreibung einzelner Motivationstypen durch die Mittelwerte der jeweils betrachteten kognitiven und konativen Verhaltensmerkmale.

2 Die angefühten Beispiele dienen zugleich als "Lesebeispiele" der Tabelle 14 und der folgenden, gleichartig aufgebauten Tabellen. 


\begin{tabular}{|c|c|c|c|c|c|c|c|c|c|c|c|c|c|}
\hline $\begin{array}{l}\text { EICENSCHAFTEN } \\
\text { VON ANTIOUITXTEN }\end{array}$ & GESAMT & 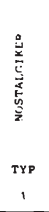 & 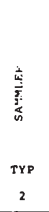 & 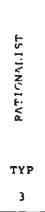 & 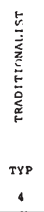 & 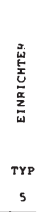 & 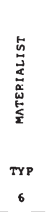 & 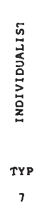 & 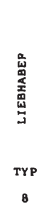 & 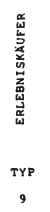 & $\begin{array}{l}\text { SIGNIF } \\
\text { EINFAC } \\
\text { VARIAN }\end{array}$ & $\begin{array}{l}\text { IANZNIVEAU } \\
\text { R RALYSE }\end{array}$ & \begin{tabular}{|l} 
GRUPPEN MIT \\
SIGNIFIKANT \\
UNTESCHIEDICHER \\
MOTIVATIONSAUSPRGCUNG
\end{tabular} \\
\hline Dekorat 1 onsuert & 2,28 & & 2,86 & & 2,59 & 2,62 & 1.95 & 1.87 & & & 0.153 & ws & $7,2,5,4 \quad 5,6$ \\
\hline Gebrauchsfanigkelt & 2,43 & 3,07 & 3,57 & 2,33 & 2,35 & 1,91 & 3,45 & 2,00 & 1,58 & 1.94 & 0,000 & .... & $5,8,9,7,3+2,6,1 \quad 6,2,4,3,8$ \\
\hline Seltenhe1t & 2,91 & 3.07 & 3.29 & 2,83 & 2,76 & 3,73 & 2,30 & 2,70 & 3,42 & 3,00 & 0.013 & .. & $6 \times 9,1,2,8,5 \quad 5,7,4,3>18$ \\
\hline Wertbestundigkeit & 2,64 & 2,53 & 3,71 & 2,30 & 2,94 & 2,73 & 2.10 & 2,30 & 3,00 & 3.44 & 0,001 & $\cdots$ & $6,4,8,9,2 \quad 9,2+3,7,1$ \\
\hline Exklugivitat & 3,47 & 3.67 & 4.00 & 3.35 & 3,94 & 4,00 & 3,25 & 2,78 & 3,67 & 3.50 & 0,041 & .. & $7,5,2,4,8,1$ \\
\hline Anziehungskraft & 2,56 & 1.67 & 3.86 & 2,73 & 2,65 & 3.09 & 2,45 & 2,39 & 2,33 & 2,56 & 0,008 & $\ldots$ & $1,2,5,3,4,9=2,1,8,7,6,9,4,3$ \\
\hline zeltlosigkeit & 2,76 & 2,60 & 3,86 & 2,77 & & & 2,35 & 2,56 & 2,50 & 3,31 & 0.196 & NS & $2,6,8,7,1,3 \quad 6 * 9,2$ \\
\hline Individualityt & 2,54 & 2,33 & 3,86 & 2,75 & 2,29 & 3,09 & 2,35 & 2,13 & 2,33 & 2,63 & 0,024 & .. & $7,2,5=2,7,4,1,8,6,9,3$ \\
\hline Orlginalityt & 2,46 & & 3.29 & & & 3,09 & 2,10 & 2,13 & & & 0.210 & ws & $6.7+2,5$ \\
\hline wert & 2.78 & 2.40 & $3,7:$ & 2,66 & 2,71 & 3,36 & 2,40 & 2,57 & 2,92 & $3,3:$ & 0.014 & .. & $1,6,7+2,5,9 \quad 3+9,2+42$ \\
\hline Schonhert & 1,52 & 1,53 & 2,29 & 1,53 & 1,35 & 1,18 & & & 1,25 & $1,5 \mathrm{c}$ & 0.244 & Ns & $2+5,8,4,9,1,3$ \\
\hline Reprysentativitat & 3,56 & & 4.14 & & 3,82 & 4.18 & & 3,00 & & & 0,171 & Ns & $7 \cdot 5,2,4$ \\
\hline solide Verarbeltung & 2,09 & 2.27 & 2,71 & 2,43 & 2,06 & 1,81 & 2,10 & 1.96 & 1,58 & 1,81 & 0,064 & - & $8,9,2,3 \quad 5,2$ \\
\hline kunst lerische Bedeutung & 2,92 & 2,47 & 3.29 & 3,43 & 2,47 & 3,09 & 2,55 & 2,70 & 3,00 & 3,31 & 0.035 & 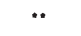 & $3,9 \neq 1,4,6 \quad 3,7$ \\
\hline Ausdrucksstyrke & 2,40 & 2.13 & 3,14 & 2,83 & 2,29 & 2,27 & 2,15 & 2,04 & 2,33 & 2,63 & 0,098 & - & $7,1,6,2,3$ \\
\hline Ausgefallenheit & 3,20 & 3,33 & 3,29 & 3,30 & 3,65 & 4,00 & 2,80 & 2.78 & 3,42 & 2,75 & 0,048 & .. & $9,7,6 \times 5,4$ \\
\hline st1lecheheit & 2,25 & & & 2,60 & & & 1,80 & & & & 0,222 & ns & $6: 3$ \\
\hline Wlederverkguf $11 \mathrm{chke} 1 \mathrm{t}$ & 3,34 & 3,27 & 4.43 & 3,07 & 3,65 & 3.55 & 3,10 & 2.87 & 3.75 & 3.69 & 0.064 & - & $7,2,8,9,4 \quad 3,6,1,2$ \\
\hline Mob111tat & 3.21 & & 4,29 & 3,10 & & 2,82 & 3.20 & 3.04 & 3.00 & & 0,317 & Ns & $5,8,7,3,6,2$ \\
\hline Alter & 2,79 & 1,93 & 3,42 & 3.07 & 2,71 & 3.45 & 2,15 & 2,70 & 3.00 & 3,19 & 0.001 & $\ldots$ & $1,6,5,2,9,3,8 \quad 1,4,7 \quad 7,5$ \\
\hline Funktionalitut & 2,72 & 3,07 & 4,00 & 2,60 & 2,59 & 2,18 & 3,50 & 2,57 & 2,08 & 2,31 & 0.002 & $\cdots$ & $8,5,9,7,4,3 \neq 2,6 \quad B$ \\
\hline
\end{tabular}

Tab. 14: Der Einfluß von Motivationskonfigurationen (Typenzugehörigkeiten) auf die Kaufrelevanz von Antiquitätenmerkmalen - Ergebnisübersicht einfacher Varianzanalysen 
(2) Der varianzanalytisch hoch signifikante Einfluß der Typenzugenörigkeit auf die Kaufbedeutung der "Wertbeständiqkeit" läßt sich dahingehend spezifizieren, daß für die "Materialisten" diese Wareneigenschaft signifikant wichtiger ist als für die "Traditionalisten", "Liebhaber", "Erlebniskäufer" und "Sammler". Gleiches gilt ferner für die "Rationalisten", "Individualisten" und "Nostalgiker" gegenüber den "Sammlern" und "Erlebniskäufern".

(3) Außer gegenüber den "Individualisten" besitzt das "Alter" bei den "Nostalgikern" eine durchgängig höhere Kaufrelevanz als in allen anderen Typen.

Unter Berücksichtigung der weiteren, überwiegend signifikanten Ergebnisse der Tabelle 14 erweist sich die Typologisierung individueller Motivationsstrukturen als ein "sensibles" und aussagekräftiges Konzept zur Explikation von Kaufrelevanzen einzelner Antiquitäten-Merkmale. Es kann damit einen wesentlichen Beitrag zum Verständnis der "internen Entscheidungsprämissen" von Antiquitätenkäufern leisten.

\subsection{Wahrgenommenes Risiko und kognitive Dissonanz}

Wahrgenommenes Risiko und kognitive Dissonanz werden als "Vor-Kauf-Konflikte" und "Nach-Kauf-Konflikte" von Antiquitätenkäufern beschrieben und voneinander abgegrenzt ${ }^{1}$.

Im Hinblick auf die vorgenommene Operationalisierung des wahrgenommenen Kaufrisikos durch die Teilaspekte eines "fachlichen Kompetenzrisikos" und eines "finanziellen Risikos" bleiben die regressionsanalytisch ermittelten Er-

1 Vgl. im einzelnen Kapitel B. 3.2122. 
klärungsbeiträge von Einzelmotivationen unbefriedigend. Die erklärten Varianzanteile erreichen bei einer Inklusion von sechs bzw. einer Motivation(en) $21 \%$ und $2 \%$.

Die varianzanalytische Prüfung des Einflusses von Motivationstypen läßt die folgenden Schlüsse zu: "Sammler" besitzen gegenüber der Mehrzahl anderer Motivationstypen eine signifikant unterdurchschnittliche Wahrnehmung eines finanziellen Risikos. Zusammen mit den "Materialisten" vertreten sie andererseits in besonderem Maße die Meinung, daß "man ohne Fachkenntnisse beim Antiquitätenkauf nicht auskommt".

Dabei ist zu vermuten, daß das implizite "Kompetenzrisiko" für den "Sammler" eher in der Möglichkeit eines kunsthistorischen und für den "Materialisten" in der Möglichkeit eines materiell-wertbezogenen Fehlurteiles zu sehen ist. Signifikante Unterschiede bestehen dabei für beide Typen gegenüber den "Einrichtern" und "Liebhabern", welche entsprechend ihrer Kaufintention nur ein geringes "Kompetenzrisiko" empfinden.

Ein inhaltlich konsistentes und zugleich weiterführendes Ergebnis ist, daß sich bei den "Materialisten" unter allen Motivationstypen am häufigsten Dissonanzen nach einem Antiquitätenkauf einstellen und sie darüber hinaus das ausgeprägteste dissonanztypische Informationsverhalten zeigen: für das selektiv vergleichende Informationsinteresse nach einem Kauf ${ }^{1}$ konnte ein varianzanalytisch hochsignifikanter Einfluß der Typenzugehörigkeit ermittelt werden.

1 Das Originalitem lautete: "Gerade nach einem Kauf fallen mir ähnliche Antiquitäten bei anderen Händlern besonders auf, und ich vergleiche sie mit meiner eigenen". 
Neben den "Materialisten" verhalten sich auch die "Individualisten" in diesem Sinne dissonanztypisch. Den Gegenpol bilden die "Traditionalisten" und "Sammler", für die ein höheres "Nachkauf-Selbstbewußtsein" charakteristisch zu sein scheint.

Es bleibt zu erwähnen, daß weder für das Dissonanzempfinden noch das dissonanztypische Informationsverhalten tragfähige Erklärungsansätze auf der Grundlage von Einzelmotivationen ermittelt werden konnten ${ }^{1}$.

\subsection{Involvement}

Das Involvement stellt eine kaufbegleitende Kognition von Antiquitätenkäufern dar, welche häufig als "Ich-Beteiligung" oder "Engagement" umschrieben wird. Die empirische Analyse motivationaler Erklärungsbeiträge stützt sich auf die in Kapitel B. 3.2121 vorgenommene inhaltliche Operationalisierung ausgewählter Teilaspekte des Antiquitäten-Involvement ${ }^{2}$.

Einen Überblick über die regressionsanalytischen Erklärungsbeiträge von Motivationen für das Involvement vermittelt Tabelle 15.

Es zeigt sich, daß insbesondere die Involvement-Komponente "Ich glaube, meine Freunde und Bekannten beneiden mich ein wenig um meine Antiquitäten" dem als interpretationsrelevant erachteten Grenzwert von 30 o erklärtem Varianzanteil nahekommt. Ohne auf Details einzugehen, ist erwähnenswert, daß dieser Erklärungswert weitgehend von einer

1 Bei vier (sieben) einbezogenen Einzelmotivationen wurden Varianzerklärungsanteile von $10 \%(20 \%)$ für das Dissonanzempfinden (Informationsverhalten) erreicht.

2 Hiervon abzugrenzen ist das motivationale Engagement als Strukturdimension des motivationalen Diskriminanzraumes, welches auch als Motivations-Involvement bezeichnet werden kann. 


\begin{tabular}{|c|c|c|c|}
\hline $\begin{array}{l}\text { INVOLVEMENT GEGENUBER } \\
\text { ANTIQUITÁTEN-BESIT'Z }\end{array}$ & $\begin{array}{l}\text { Anzahl } \\
\text { einbezogener } \\
\text { erklärender } \\
\text { Motivationen }\end{array}$ & $\begin{array}{l}\text { Multipler } \\
\text { Korrelations- } \\
\text { koeffizient }\end{array}$ & $\begin{array}{l}\text { erklärter } \\
\text { Varianz- } \\
\text { antell in } \\
8\end{array}$ \\
\hline $\begin{array}{l}\text { von Antiquitäten kann man sich } \\
\text { nur schwer wieder trennen }\end{array}$ & 4 & 0,36 & 0,13 \\
\hline $\begin{array}{l}\text { mit Antiquität ist zumeist } \\
\text { ejne Geschichte verbunden, wie } \\
\text { inan sie erworben hat }\end{array}$ & 7 & 0.45 & 0,20 \\
\hline $\begin{array}{l}\text { Ich werde gern auf meine } \\
\text { Antiquitäten angesprochen }\end{array}$ & 6 & 0,51 & 0,26 \\
\hline $\begin{array}{l}\text { Ich glaube, meine Freunde } \\
\text { und Bekannte beneiden mich } \\
\text { ein wenig um meine Antiqui- } \\
\text { täten }\end{array}$ & 8 & 0,54 & 0,29 \\
\hline $\begin{array}{l}\text { Es macht Spaß, auch ab und } \\
\text { zu ein Stück wieder mit } \\
\text { Gewinn zu verkaufen }\end{array}$ & 7 & 0,52 & 0.27 \\
\hline
\end{tabular}

Güte der multiplen Regressionsgleichungen: Signifikanzniveau $>99,9 \%$

Tab. 15: Erklärungsbeiträge von Motivationen zum Involvement gegenüber Antiquitäten - Ergebnisüberblick multipler schrittweiser Regressionsanalysen

"Sammelleidenschaft", einem "Besitzstreben", sowie von einigen einrichtungsbezogenen Motivationen getragen wird. Sieht man in der genannten Involvement-Komponente einen "Gradmesser" für die Besitzbindung und-identifikation von Antiquitätenkäufern, erscheinen die erklärenden Motivationen als plausible Bestimmungsfaktoren.

Aufschlußreicher sind dagegen die varianzanalytischen Untersuchungen des Einflusses der Typenzugehörigkeit auf das Involvement (vgl. Tabelle 16). 


\begin{tabular}{|c|c|c|c|c|c|c|c|c|c|c|c|c|c|}
\hline $\begin{array}{l}\text { INVOLVEMENT GEGENUBER } \\
\text { ANTI QUITATEN-BESITZ }\end{array}$ & 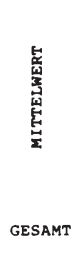 & 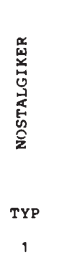 & 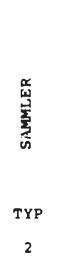 & 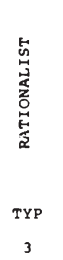 & 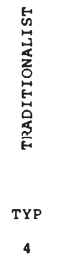 & 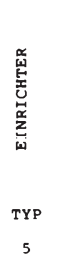 & 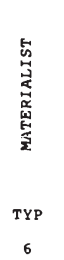 & 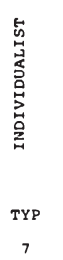 & 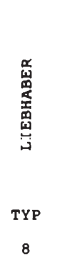 & 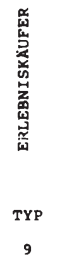 & $\begin{array}{l}\text { SIGNIF } \\
\text { EINFAC } \\
\text { VARIAN2 } \\
\end{array}$ & $\begin{array}{l}\text { AN2NIVEAU } \\
\text { R } \\
\text { NALYSE }\end{array}$ & $\begin{array}{l}\text { GRUPPEN MIT } \\
\text { SIGNIFIKANT } \\
\text { UNTERSCHEDLICHER } \\
\text { MOTIVATIONSAUSPRAGUNG }\end{array}$ \\
\hline $\begin{array}{l}\text { von Antiquitaten } \\
\text { kann man sich nur schwer } \\
\text { wieder trennen }\end{array}$ & 1,57 & & & 1,80 & & & & & 1,25 & & 0,250 & NS & $8 \neq 3$ \\
\hline $\begin{array}{l}\text { mit Antiquitaten ist zumeist } \\
\text { eine Geschichte verbunden, } \\
\text { wie man sie erworben hat }\end{array}$ & 2,16 & 1,73 & 2,71 & 2,63 & 1,94 & 2,09 & 1,65 & 2,57 & 1,92 & 1,94 & 0,005 & $\cdots *$ & $6,1 \neq 2,3,7 \quad 8,9,4 \neq 3$ \\
\hline $\begin{array}{l}\text { ich werde gern } \\
\text { auf meine Antiquitaten } \\
\text { angesprochen }\end{array}$ & 2,89 & 2,40 & 3,71 & 3,00 & 3,41 & 3,91 & 2,40 & 2,52 & 3,00 & 2,56 & 0,002 & $\cdots$ & $6,1,7,9 \neq 5,2,4 \quad 3 \neq 5$ \\
\hline $\begin{array}{l}\text { ich glaube, meine freunde bzw. } \\
\text { Bekannten beneiden mich ein } \\
\text { wenig um meine Antiquitaten }\end{array}$ & 3,54 & 2,93 & 3,86 & 3,90 & 3,88 & 4,27 & 3,00 & 2,78 & 3,75 & 4,00 & 0,000 & $\cdots * *$ & $7 \neq 5,9,3,4,2,8 \quad 1,6 \neq 5,9,3,4$ \\
\hline $\begin{array}{l}\text { teure Antiquitaten sollte man } \\
\text { sich erst leisten, wenn man } \\
\text { sonst alles Notwendige hat }\end{array}$ & 2,74 & 2,53 & 4,00 & 2,73 & 2,82 & 3,36 & 2,60 & 2,91 & 2,00 & 2,38 & 0,065 & * & $8 \neq 2,5 \quad 9,1,6,3,4 \neq 2$ \\
\hline $\begin{array}{l}\text { es macht Spas, } \\
\text { auch ab und zu ein Stuck } \\
\text { wieder mit Gewinn zu verkaufen }\end{array}$ & 3,93 & 4,07 & 4,43 & 3,80 & 4,35 & 4,55 & 3,15 & 3,83 & 4,42 & 3,69 & 0,013 & *. & $6 \neq 5,2,8,4,1,3$ \\
\hline
\end{tabular}

Tab. 16: Der Einfluß von Motivationskonfigurationen (Typenzugehörigkeiten) auf das Involvement gegenüber Antiquitäten - Ergebnisübersicht einfacher Varianzanalysen 
Signifikant höher als z.B. "Sammler" und "Rationalisten" verbinden "Nostalgiker" und "Materialisten" mit dem Besitz von Antiquitäten zugleich "eine Geschichte, wie man sie erworben hat." Die Äußerung "Ich werde gern auf meine Antiquitäten angesprochen" ist ebenfalls besonders kennzeichnend für "Nostalgiker" aber auch für "Materialisten"; "Einrichter" und "Sammler" stimmen dagegen dieser Äußerung signifikant weniger $\mathrm{zu}$.

\begin{abstract}
"Individualisten" besitzen ein Antiquitäten-Involvement insofern, als sie signifikant ausgenrägter als fast alle anderen Motivationskonfigurationen einen Neid ihrer Freunde und Bekannten auf ihren Antiquitätenbesitz vermuten. Hierin ist ein weiterer Beleg dafür zu sehen, daß "Individualisten" die Dokumentationsfunktion von Antiquitäten nutzen und gerade hierauf ihre "Bindung" zu Antiquitäten beruht.
\end{abstract}

Im Gesamtüberblick fördert wohl speziell eine Motivationskonfiguration wie die der "Individualisten" und "Nostalgiker" ein hohes und tendenziell unbewußt-affektives Involvement gegenüber Antiquitäten. Obgleich sich auch die "Materialisten" durch hohe Zustimmungen auszeichnen, muß hier eher von einem bewußten und "sachlichen" Involvement ausgegangen werden; denn den "Materialisten" macht es erfahrungsgemäß besonderen Spaß, "auch ab und zu ein Stück wieder mit Gewinn zu verkaufen".

\title{
4.14 Preisbereitschaft
}

Die individuelle Preisbereitschaft wurde als derjenige DMBetrag operationalisiert, den die Antiquitätenkäufer maximal für eine einzelne Antiquität auszugeben bereit sind (persönliches Preislimit) ${ }^{1}$.

1 Vgl. Kapitel B. 3.2123. 
Eine regressionsanalytisch ermittelte Gruppe von 10 Einzelmotiven konnte keinen wesentlichen Erklärungsbeitrag $z u$ dieser kognitiven Entscheidungsrestriktion der Antiquitätenkäufer leisten (Bestimmtheitsmaß $=0,24$ ). Die varianzanalytische Prüfung des Einflusses der Typenzugehürigkeit auf die preisbereitschaft zeigt dagegen einen $\mathrm{Zu}$ sammenhang auf einem 90\%igen Signifikanzniveau. Dieses Ergebnis ist im wesentlichen durch das außerordentlich hohe Preislimit der "Traditionalisten" mit ca. 30 TDS DM bedingt. Sie unterscheiden sich damit signifikant von den "Rationalisten", "Sammlern" (beide ca. 12 TSD DM), den "Nostalgikern" und "Erlebniskäufern" (ca. 11 TSD DM), sowie den "Liebhabern" (ca. 10 TSD DM), "Materialisten" (ca. 9 TSD DM) und schließlich den "Einrichtern. (ca. 8 TSD DM). Nicht signifikant ist der Unterschied zu den "Individualisten" mit einer Preisbereitschaft von annähernd 18 TSD DM.

\section{15 Interessenausrichtung}

Motivationen können Auskunft auf die Frage nach der Interessenausrichtung der Antiquitätenkäufer in Hinblick auf unterschiedliche Sach- und Sammelgebiete geben. Die empirischen Befunde dokumentieren einen (im Durchschnitt hohen) Erklärungsbeitrag einzelner Motivationsinhalte ${ }^{1}$.

Wie Tabelle 17 zeigt, erklären 10 Motivationen mit einem Varianzerklärungsanteil von 31,4 \& (vgl. dazu das multiple Bestimmtheitsmaß) das Interesse an antiken Tischen. Diese Einrichtungsgegenstände stellen bei einer pauschalen Interpretation der Einzelmotivationen in erster Linie Ge-

1 Vgl. dazu die Ubersichtstabelle Tabelle 16 im Anhang. 
INTERESSE AN TISCHEN

\begin{tabular}{|c|c|c|c|c|c|}
\hline Erklärende Motivationer ... & MULT R & $\begin{array}{l}\text { BESTIMMT- } \\
\text { HEITSMASS }\end{array}$ & EINFACH $R$ & BETA & RANG \\
\hline $\begin{array}{l}\text { Lösung eines funktionalen Einrichtungs } \\
\text { Repräsentation } \\
\text { Dekoration der Wohnung } \\
\text { SpaB am Suchen } \\
\text { Bedürnis nach Asthetik } \\
\text { Wertanlage } \\
\text { Freude an Naturstoffen } \\
\text { Reaktion auf modernes Möbeldesign } \\
\text { Besitzstreben } \\
\text { Liebe zum alten Kunsthandwerk }\end{array}$ & $\begin{array}{l}0.307 \\
0.356 \\
0.419 \\
0.449 \\
0.479 \\
0.504 \\
0.523 \\
0.536 \\
0.550 \\
0.560\end{array}$ & $\begin{array}{l}0.094 \\
0.127 \\
0.176 \\
0.202 \\
0.230 \\
0.254 \\
0.274 \\
0.287 \\
0.302 \\
0.314\end{array}$ & $\begin{array}{r}0.307 \\
-0.148 \\
0.113 \\
-0.157 \\
0.185 \\
0.106 \\
0.196 \\
0.215 \\
-0.021 \\
-0.076\end{array}$ & $\begin{array}{r}0.249 \\
-0.302 \\
0.158 \\
-0.170 \\
0.188 \\
0.216 \\
0.195 \\
0.188 \\
-0.131 \\
-0.126\end{array}$ & $\begin{array}{r}2 \\
1 \\
8 \\
7 \\
6 \\
3 \\
4 \\
5 \\
9 \\
10\end{array}$ \\
\hline
\end{tabular}

INTERESSE AN SILBER

Erklärende Motivationen ...

Sammelleidenschaft

Besitzerstolz

Bewahrung von Erinnerungen an die Fam.

Hoffnung auf Trouvaille

Bedürfnis nach Ausgleich

Freude an Naturstoffen

Freizeitgestaltung

Bedürfnis nach Asthetik

Liebe zum alten Kunsthandwerk

Empfinden eines kulturellen Auftrags

\begin{tabular}{|l|l|r|r|r|r|} 
MULT R & $\begin{array}{c}\text { BESTIMMT- } \\
\text { HEITSMASS }\end{array}$ & EINFACH R & BETA & RANG & \\
\hline 0.248 & 0.061 & -0.248 & -0.113 & 10 & \\
0.376 & 0.141 & 0.231 & 0.288 & 1 & \\
0.432 & 0.187 & 0.236 & 0.211 & 2 & \\
0.466 & 0.217 & -0.239 & -0.127 & 7 \\
0.489 & 0.239 & -0.114 & -0.201 & 3 \\
0.512 & 0.262 & 0.179 & 0.189 & 4 & \\
0.530 & 0.280 & -0.226 & -0.187 & 5 \\
0.543 & 0.295 & 0.185 & 0.118 & 9 & \\
0.553 & 0.306 & -0.041 & -0.137 & 6 & \\
0.563 & 0.317 & 0.129 & 0.124 & 8 &
\end{tabular}

Tab. 17: Motivationale Erklärungsbeiträge zur Interessenausrichtung von Antiquitätenkäufern - Ergebnistableaus multipler schrittweiser Regressionsanalysen

brauchsmöbel und weniger Dekorationsmöbel dar. Dies zeigt sich insbesondere in einer hohen positiven Korrelation zur Einzelmotivation "Lösung eines funktionalen Einrichtungsproblems"; die Motivation "Repräsentation" hingegen liefert zwar den höchsten Erklärungsbeitrag, weist jedoch in eine negative Beeinflussungsrichtung. Trotzdem spiegelt sich in dieser Interessensausrichtung zugleich ein gewisses $M a ß$ an ästhetischer Wertschätzung für alte Möbel wieder, indem "Freude an Naturstoffen", "Bedürfnis nach Ästhetik" und "Reaktion auf modernes Möbeldesign" als gut erklärende Motivationen eine geschmackliche Bevorzugung antiker Tische zum Ausdruck bringen.

Für Silber interessiert man sich vor allem, um einen ausgeprägten Besitzerstolz zu befriedigen. Die "Bewahrung von Erinnerungen an die Familie" als ebenfalls einflußreicher 
Bestimmungsfaktor des Interesses ist im Zusammenhang mit der üblichen Weitergabe des "Familiensilbers" von Generation zu Generation zu sehen.

Auch bei der Motivationstypen-Betrachtung kann das Interesse für Silber und Tische varianzanalytisch signifikant erklärt werden (vgl. Tabelle 18). Dabei zeigt sich, daß besonders "Sammler" kaum Interesse an Tischen haben; "Traditionalisten" und "Liebhaber" interessieren sich für Silber.

Eine entsprechende Typenuntersuchung läßt sich auch für die anderen Sach- und Sammelgebiete vornehmen, wobei allerdings nur für Truhen/Kommoden ein - über signifikante Interessensunterschiede zwischen einzelnen Käufergruppen hinausgehender - varianzanalytischer Einfluß ermittelt werden konnte?

$1 \mathrm{Zu}$ den jeweiligen Interessendominanzen vgl. Tabelle 18. 


\begin{tabular}{|c|c|c|c|c|c|c|c|c|c|c|c|c|c|c|}
\hline \multirow{16}{*}{ 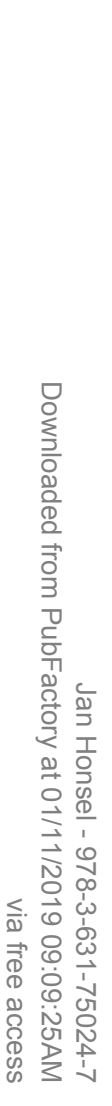 } & $\begin{array}{l}\text { INTERESSE AN SACH- } \\
\text { BZW. SAMMELGEBIETEN }\end{array}$ & 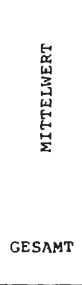 & 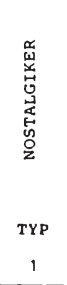 & 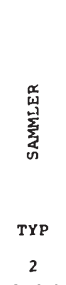 & 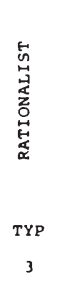 & 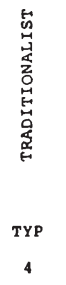 & 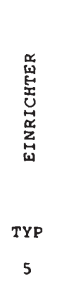 & 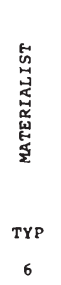 & 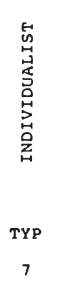 & 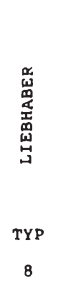 & 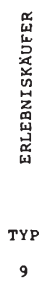 & $\begin{array}{l}\text { SIGNIFI } \\
\text { EINFCA } \\
\text { VARIANL }\end{array}$ & $\begin{array}{l}\text { A VZNIVEAU } \\
\text { NALYSE }\end{array}$ & $\begin{array}{l}\text { GRUPPEN MIT } \\
\text { SIGNIFI KANT } \\
\text { UNTERSCIEDLICHER } \\
\text { MOTIVAT IONSAUSPRAGUNG }\end{array}$ \\
\hline & Gemalde & 2,68 & 2,20 & 4,43 & 2,47 & 2,59 & 2,55 & 2,70 & 2,35 & 2,92 & 3,25 & 0,008 & $\ldots$ & $1,7,3 \neq 2,9 \quad 5,4,6,8,9 \neq 2$ \\
\hline & Graphik & 2,99 & 2,67 & $4,: 4$ & 2,93 & & & 2,80 & 2,83 & & & 0,453 & NS & $1,6,7,3 \neq 2$ \\
\hline & silber & 2,55 & 2,27 & 3,29 & 3,07 & 2,00 & 2,18 & 2,95 & 2,61 & 1,75 & 2,38 & 0,032 & ** & $8,4 \neq 2,3,6$ \\
\hline & Teppiche & 3,15 & 2,73 & 3,86 & 3,37 & & & & 2,57 & & 3,81 & 0,185 & NS & $7 \neq 2,9,3 \quad 1 \neq 9$ \\
\hline & Bücher & 2,94 & 2,33 & & 3,27 & & & & & & & 0,592 & NS & $1 \neq 3$ \\
\hline & Schreibmöbe 1 & 2,67 & & 3,57 & & & & 3,20 & 2,13 & & & 0.153 & NS & $7 \neq 2,6$ \\
\hline & Truhen/Kommoden & 2,52 & 2,33 & 4,14 & 2,80 & 2,24 & 2,18 & 2,75 & 2,13 & 2,33 & 2,38 & 0,016 & ** & $7 \neq 2,3 \quad 5,4,1,8,9,6,3 \neq 2$ \\
\hline & Sitzmöbel & 2,71 & & 3,57 & & & & & 2,39 & & & 0,559 & NS & $7 \neq 2$ \\
\hline & Tische & 2,81 & 3,13 & 4,00 & 2,77 & 2,41 & 2,36 & 3,15 & 2,43 & 2,50 & 3,19 & 0.089 & $*$ & $5,4,7,8,3 \neq 2$ \\
\hline & Schränke/Vitrinen & 2,28 & & 3,14 & & 1,94 & & & 2,00 & & & 0,474 & NS & $4,7 \neq 2$ \\
\hline & KLeinmöbe 1 & 2,76 & & & & & 3,36 & & 2,30 & & & 0,404 & NS & $7 \neq 5$ \\
\hline & Schmuck & 2,85 & & & 3,37 & 2,35 & & 2,50 & & & & 0,206 & NS & $4,6 \neq 3$ \\
\hline & Porzellan & 2,65 & 2,73 & 3.43 & 3,10 & 2,06 & 2,60 & 2,05 & 2,61 & 2,33 & 3.06 & 0,080 & * & $6,4 \neq 2,3,9$ \\
\hline & uhren & 2,77 & & & 3,17 & 2,35 & & 2,30 & & & & 0,181 & NS & $6,4 \neq 3$ \\
\hline & Ostasiatika & 4,26 & & 3,43 & & 4,59 & 4,73 & 3,70 & & & 4,50 & 0,143 & NS & $2,6 \neq 5,4,9$ \\
\hline
\end{tabular}

Tab. 18: Der Einfluß von Motivationskonfigurationen (Typenzugehörigkeiten) auf die Interessensausrichtung von Antiquitätenkäufern - Ergebnisüberblick einfacher Varianzanalysen 


\subsection{Anforderungen an Einkaufsstätten für Antiquitäten}

Die Anforderungen von Antiquitätenkäufern an Antiquitätenanbieter sind als individuelle Bewertungsmaßstäbe wesentlicher Bestandteil der Einkaufsstättenwahl 1. Das Anforderungsprofil der Käufer und die Analyse seines motivationalen Hintergrundes besitzt praxisnahen Aussagewert, da die Gegenstandsbereiche der Bewertung bzw. Anspruchssetzung im wesentlichen mit den absatzpolitischen Gestaltungsbereichen des Handels zusammenfallen.

Die in der empirischen Untersuchung jeweils kriterienspezifisch isolierten Bündel von Einzelmotivationen besitzen bei globaler Betrachtung einen mittleren Erklärungswert. Die multiplen Korrelationskoeffizienten bewegen sich in der Mehrzahl zwischen 0,45 und 0,60 . Bei durchschnittlich 5 - 10 einbezogenen Einzelmotivationen erreichen die erklärten Varianzanateile Werte im Bereich von $20 \%$ bis knapp $358^{2}$.

Als besonders relevant erweisen sich die in Tabelle 19 wiedergegebenen Explikationen der Käuferanforderungen an ein breites Sortiment, sowie an eine "Trouvaillenchance" ${ }^{3}$.

1 Zur Präzisierung der Einkaufsstättenwahl als Entscheidungsproze $B$ von Antiquitätenkäufern vgl. die Ausführungen in Kapitel B. 3.22.

2 Zur detaillierten Ergebnisübersicht vgl. Tabelle 17 im Anhang.

3 Die Trouvaillenchance besitzt, obwohl sie dem Gestaltungsbereich "Preispolitik" zugeordnet wurde, fließende übergänge zur "Sortimentspolitik". "Preischance" und "Objektchance" sind gleichbedeutende Blickwinkel der Trouvaille und nicht inhaltlich voneinander trennbar. 


\section{BREITES SORTIMENT}

Erklärende Motivationen ...

Hoffnung auf Trouvaille

Sammelleidenschaft

Dekoration der Wohnung

Freude am Schenken

Nostalgie

Repräsentation

Freizeitgestaltung

Besitzerstolz

SORTMIENT MIT CHANCEN FUR TROUVAILLEN

Erklärende Motivationen ...

Hoffnung auf Trouvallle

Sammelleidenschaft

Llebe zum alten Kunsthandwerk

Dekoration der Wohnung

Iösung eines funktionalen Einrichtungsproblems

\begin{tabular}{|c|c|c|c|c|}
\hline MULT $R$ & $\begin{array}{l}\text { BESTIMMT- } \\
\text { HEITSMASS }\end{array}$ & EINFACH R & BETA & RANG \\
\hline $\begin{array}{l}0.385 \\
0.441 \\
0.480 \\
0.515 \\
0.528 \\
0.542 \\
0.552 \\
0.566\end{array}$ & $\begin{array}{l}0.148 \\
0.194 \\
0.230 \\
0.266 \\
0.279 \\
0.294 \\
0.304 \\
0.320 \\
\end{array}$ & $\begin{array}{r}0.385 \\
-0.085 \\
0.215 \\
-0.162 \\
0.145 \\
-0.103 \\
-0.022 \\
0.158\end{array}$ & $\begin{array}{r}0.450 \\
-0.106 \\
0.216 \\
-0.169 \\
0.129 \\
-0.182 \\
-0.179 \\
0.143\end{array}$ & $\begin{array}{l}1 \\
8 \\
2 \\
5 \\
7 \\
3 \\
4 \\
6\end{array}$ \\
\hline
\end{tabular}

\begin{tabular}{|c|c|c|c|c|}
\hline MULT $R$ & $\begin{array}{l}\text { BESTIMMT- } \\
\text { HEITSMASS }\end{array}$ & EINFACH R & BETA & RANG \\
\hline $\begin{array}{l}0.488 \\
0.520 \\
0.542 \\
0.559 \\
0.571\end{array}$ & $\begin{array}{l}0.239 \\
0.271 \\
0.293 \\
0.313 \\
0.327\end{array}$ & $\begin{array}{r}0.488 \\
-0.018 \\
0.225 \\
0.168 \\
0.050\end{array}$ & $\begin{array}{r}0.525 \\
-0.160 \\
0.147 \\
0.160 \\
0.123\end{array}$ & $\begin{array}{l}1 \\
2 \\
3 \\
2 \\
4\end{array}$ \\
\hline
\end{tabular}

Tab. 19: Motivationale Erklärungsbeiträge für Anforderungen von Antiquitätenkäufern an Einkaufsstätten - Ergebnistableaus multipler schrittweiser Regressionsanalysen 
Die individuellen Anspruchsniveaus lassen sich in beiden Fällen zu ca. $32 \%$ auf die Ausprägungen der aufgeführten Motivationen zurückführen.

Das Motiv "Hoffnung auf eine Trouvaille", das faktorenanalytisch der Inhaltsdimension "Streben nach Konsumerlebnis" zugeordnet ist, dominiert die Erklärungsansätze deutlich. Als Schlußfolgerung liegt die Vermutung nahe, daß insbesondere eine breite Auswahl und die subjektiv empfundene "Trouvaillenchance" dem Antiquitätenkauf eine eigenständige motivationale "Wertigkeit" verleihen können.

Die "Sammelleidenschaft" besitzt für beide Anforderungshaltungen an Antiquitätenanbieter eine negative Korrelation. Hierin liegt ein Hinweis auf eine ausgeprägte Spezialisierung und kritische Markteinschätzung der sammelnden Antiquitätenkäufer.

Ein fast identisches Bild zeigen die Varianzanalysen zwischen der Typenzugehörigkeit und den genannten Anforderungen an Einkaufsstätten für Antiquitäten. Zusätzlich

deckt der typologische Erklärungsansatz den erwartbaren Einfluß ökonomischer Motivationen ("Materialist") auf den Wunsch nach einem"breiten Sortiment"und einer "Trouvaillenchance" auf (vgl. Tabelle 20).

Für eine Vielzahl weiterer Anspruchsniveaus bestehen signifikante oder hochsignifikante Zusammenhänge, die z.T. sehr detaillierte Aussagen zulassen. Einige exemplarische Beispiele sind:

(1) Der Wunsch, mit dem Händler um einen Preisnachlaß verhandeln zu können ist aus der Typenzugehörigkeit bzw. der Motivationskonfiguration der Käufer erklärbar. Bis auf den "Einrichter", der ein mittleres Anspruchsniveau besitzt, teilen sich die Käufer in zwei "Lager" bzw. Gruppen. 


\begin{tabular}{|c|c|c|c|c|c|c|c|c|c|c|c|c|c|}
\hline $\begin{array}{l}\text { ANFURDERUNGEN } \\
\text { AN EINKAUFSSTATTEN } \\
\text { FUR ANTIQUITATEN }\end{array}$ & 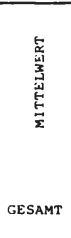 & 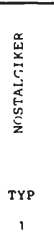 & 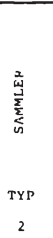 & 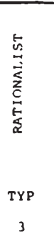 & 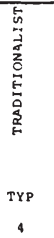 & 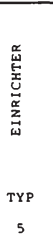 & 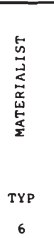 & 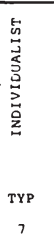 & 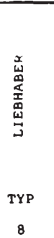 & 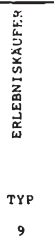 & $\begin{array}{l}\text { SIGNIFI } \\
\text { EINFACH } \\
\text { VARIANZ }\end{array}$ & $\begin{array}{l}\text { ANZNIVEAU } \\
\text { RALYSE }\end{array}$ & $\begin{array}{l}\text { GRUPPEN MIT } \\
\text { SERIFI FIKAT } \\
\text { UNTERSCHIEDLICHER } \\
\text { MOTIVATIUNSAUSPRAGGUNG }\end{array}$ \\
\hline breites Sortiment & 2,44 & 2,40 & 3,57 & 2,73 & 2,65 & 2,45 & 1.90 & 2,65 & 1,92 & 1,94 & 0,018 & .. & $6,8,9 \neq 2,3 \quad 6 \neq 7 \quad 1 \neq 2$ \\
\hline $\begin{array}{l}\text { tiefes Sortiment } \\
\text { in Spezialgebieten } \\
\text { nur mit Stücken } \\
\text { von Spitzenqualitat }\end{array}$ & 2,73 & 2,33 & 3,43 & & 3,06 & & & 3,13 & 3,92 & 3,81 & $\begin{array}{l}0,479 \\
0,131\end{array}$ & $\begin{array}{l}\text { NS } \\
\text { NS }\end{array}$ & $\begin{array}{l}1 \neq 2 \\
4,7 \neq 2,8 \quad 4 \neq 9 \quad 1 \neq 2\end{array}$ \\
\hline $\begin{array}{l}\text { auch mit Kleinigkeiten } \\
\text { als Geschenk }\end{array}$ & 2,40 & 2,53 & 4,00 & 2,47 & 2,53 & 2,27 & 2,10 & 2,22 & 2,17 & 2,25 & 0,066 & • & $6,8,7,9,5,3,4,1 \neq 2$ \\
\hline $\begin{array}{l}\text { mit Chancen } \\
\text { fur Trouvaillen }\end{array}$ & 2,65 & 2,27 & 3,86 & 2,83 & 3,47 & 2,27 & 2,00 & 2,87 & 2,67 & 2,06 & 0,001 & $\cdots$ & $6,9 \neq 2,4,7,3 \quad 1,5 \neq 2,4 \quad 2 \neq 8,3$ \\
\hline Bereitschaft zum Handeln & 2,59 & 2,07 & 3,14 & 2,97 & 3,18 & 2,45 & 1,85 & 3.04 & 2,17 & 2,19 & 0,001 & $\ldots *$ & $6,1,8,9 \neq 4,2,7,3 \quad 6,1 \neq 2$ \\
\hline $\begin{array}{l}\text { hohe fachliche } \\
\text { Qualifikation }\end{array}$ & 1,79 & & 2,57 & 1.53 & 1,53 & & & & 1,42 & & 0,306 & NS & $8,4,3 \neq 2$ \\
\hline sicher und verlablich & 1,38 & & 2,00 & 1,23 & 1,18 & & 1,65 & 1,22 & 1,08 & & 0,123 & NS & $8,4,7,3 \neq 2 \quad 8 \neq 6$ \\
\hline $\begin{array}{l}\text { Anleitung beim Aufbau } \\
\text { einer sammlung }\end{array}$ & 2,70 & 2.80 & 2,43 & 2,50 & & 3,64 & & & 2,17 & & 0,464 & NS & $8,3 \neq 5$ \\
\hline $\begin{array}{l}\text { Dekannter Name/ } \\
\text { guter Ruf }\end{array}$ & 3.15 & 2,93 & 4,14 & 3,07 & 2,71 & 3,55 & 3.05 & 2,74 & 3,08 & 4,00 & 0.021 & $*$ & $4,7,1,6,3 \neq 2,9$ \\
\hline Reprasentativitat & 3,91 & & 4,43 & & & & 3.55 & & & 4,25 & 0,349 & NS & $6 \neq 9,2$ \\
\hline $\begin{array}{l}\text { verstaubte, } \\
\text { liebevolle Atmosphare }\end{array}$ & 2,96 & & & & & & 2,55 & 3,35 & & & 0,226 & NS & $6 \neq 7$ \\
\hline $\begin{array}{l}\text { Möglichkeit } \\
\text { zum "Stöbern" bieten }\end{array}$ & 1,73 & 1,40 & 2,57 & 1,67 & & 1,55 & 1,65 & 2,09 & 1,42 & 1,56 & 0,140 & NS & $1 \neq 2,7 \quad 8,5,9,6,3 \neq 2$ \\
\hline Einkaufserlebnis bieten & 2,52 & 1,67 & 4,29 & 2,73 & 2,82 & 2,36 & 2,10 & 2,70 & 2,00 & 2,56 & 0,001 & $\ldots$ & $1 \neq 2,4,3,7,9 \quad 8,6,5,9,7,3,4 \neq 2$ \\
\hline $\begin{array}{l}\text { Gewahrleistung } \\
\text { ubernehmen }\end{array}$ & 1,28 & 1,53 & 2,00 & 1,13 & 1,12 & 1,27 & 1,45 & 1,17 & 1.08 & 1,25 & 0,047 & $*$ & $8,4,3,7,9,5 \neq 2$ \\
\hline Expertisen geben & 2,50 & 2,87 & 3,00 & 2,10 & 2,24 & 2,64 & 2,60 & 2,13 & 2,42 & 3.31 & 0,065 & • & $3,7,4 \neq 9$ \\
\hline Stücke zurücknehmen & 2,73 & 2,73 & 4,57 & 2,63 & 3,06 & 2,55 & 2,25 & 2,39 & 2,92 & 2,81 & 0,008 & $\cdots *$ & $6,7,5,3,1,9,8,4 \neq 2$ \\
\hline $\begin{array}{l}\text { problemlos } \\
\text { erreichbarer Standort }\end{array}$ & 3,63 & & & 3,50 & 4,12 & & 3,30 & & & & 0,296 & NS & $6,3 \neq 4,2$ \\
\hline
\end{tabular}

Tab. 20: Der Einfluß von Motivationskonfigurationen (Typenzugehörigkeiten) auf Anforderungen an Einkaufsstätten - Ergebnisübersicht einfacher Varianzanalysen 
Alle Mitglieder der einen Gruppe unterscheiden sich dabei signifikant von allen Mitgliedern der anderen. Die "Händlertypen" unter den privaten Käufern sind dabei die "Materialisten", "Nostalgiker", "Liebhaber" und "Erlebniskäufer"; die "Nicht-Händlertypen" sind die "Traditionalisten", "Sammler", "Individualisten" und "Rationalisten".

(2) "Antitypen" in bezug auf den Wunsch, durch die Atmosphäre der Einkaufsstätte (z.B. Trödelmarkt, Fachgeschäft, Auktion) einen Antiquitätenkauf als "Erlebnis" empfinden zu wollen, sind die "Sammler" und "Nostalgiker". Durch ihre extrem unterschiedlichen Ansprüche - "Sammler" besonders gering vs. "Nostalgiker" besonders hoch - unterscheiden sie sich nicht nur signifikant untereinander, sondern jeweils zugleich gegenüber der Mehrzahl aller anderen Typen.

(3) "Sammler" erweisen sich erneut als ein "entscheidungssicherer" Käufertyp, da ihr Anspruch an den Handel "Stücke zurückzunehmen" gegenüber allen anderen Typen signifikant niedriger ist. Sie nehmen damit eine exponierte Sonderstellung ein und verzichten auf eine wesentliche Serviceleistung des Handels.

(4) Schließlich stellen"Sammler" und "Erlebniskäufer" motivationsbedingt nur geringe Anforderungen an das allgemeine Renommée (bekannter Name / guter Ruf). Sie unterscheiden sich damit signifikant von den "Materialisten", "Individualisten", "Traditionalisten" und "Nostalgikern".

Der hohe Erklärungsbeitrag zu typenspezifischen Anforderungshaltungen gegenüber dem Antiquitätenhandel zeichnet die Motivationstypologie nicht nur als Instrument der Verhaltensexplikation, sondern auch als wichtige Informationsgrundlage einer gezielten Marktansprache durch den Handel aus. 


\subsection{Erklärungsbeiträge $\mathrm{zu}$ konativen Verhaltensmerkmalen}

\subsection{Typen und Träger der Kaufentscheidung}

Die Typisierung des Entscheidungsverhaltens als konative Verhaltenskomponente von Antiquitätenkäufern stützt sich auf die Merkmalsdimensionen "Entscheidungsstil" und "Entscheidungskollektivität". Bei den Entscheidungsstilen sind impulsives, ungeplantes, sowie geplantes und bei der Kollektivität individuelles und familiäres Entscheidungsverhalten zu unterscheiden ${ }^{1}$.

Einen Überblick über die regressionsanalytisch ermittelten Erklärungsbeiträge von Einzelmotivationen zu den genannten Merkmalen des Kaufentscheids bei Antiquitäten gibt Tabelle 21. Insgesamt wird in keinem Fall ein als interpretationsrelevant angesehener Varianzerklärungsanteil von mindestens $30 \%$ erreicht. Uberraschend gering sind die Erklärungsbeiträge der Einzelmotivationen zum impulsiven und zum familiären Kaufentscheid, obwohl eine Reihe von Einflüssen z.B. einkaufserlebnisorientierter Motivationen im ersten Fall oder einrichtungsbezogener Motivationen im zweiten Fall, erwartet wurden.

\begin{tabular}{|l|c|c|c|}
\hline $\begin{array}{l}\text { Merkmale des } \\
\text { Kaufentscheidungsprozesses }\end{array}$ & $\begin{array}{l}\text { Anzahl } \\
\text { einbezogener } \\
\text { erklärender } \\
\text { Motivationen }\end{array}$ & $\begin{array}{l}\text { Multipler } \\
\text { Korrelations- } \\
\text { Koeffizient }\end{array}$ & $\begin{array}{c}\text { erklarter } \\
\text { Varianz- } \\
\text { anteil in }\end{array}$ \\
\hline geplanter Kaufentscheid & 3 & 0,41 & 16,48 \\
ungeplanter Kaufentscheid & 4 & 0,26 & 7,02 \\
\hline impulsiver Kaufentscheld & 5 & 0,52 & 26,62 \\
familiärer Kaufentscheld & 6 & 0,48 & 23,08 \\
\hline
\end{tabular}

Tab. 21: Motivationale Erklärungsbeiträge zu Stil und Kollektivität der Kaufentscheidung bei AntiquitätenErgebnisüberblick multipler schrittweiser Regressionsanalysen 
Zu ähnlichen Schlußfolgerungen kommt die varianzanalytische Prüfung des Zusammenhangs zwischen Typenzugehörigkeit und Merkmalen des Kaufentscheids. Da in keinem Fall ein zumindest 958iges Signifikanzniveau erreicht wurde, kann auf eine detaillierte Darstellung verzichtet werden.

\subsection{Informationsverhalten}

Die Darstellung empirischer Ergebnisse der Analyse motivationaler Erklärungsbeiträge zum Informationsverhalten von Antiquitätenkäufern folgt der umfassenden Charakterisierung dieses Verhaltensaspektes im ersten Teil der vorliegenden Untersuchung ${ }^{1}$.

\subsection{Merkmale des Informationsstils}

Zu den Merkmalen des Informationsstils von Antiquitätenkäufern liefern sowohl Einzelmotivationen als auch Motivationstypen j.m Verhältnis $\mathrm{zu}$ allen untersuchten Verhaltensweisen die höchsten Erklärungsbeiträge.

Im Rahmen der Regressionsanalyse erreichen Sets von überwiegend sieben oder acht Einzelmotivationen Erklärungsbeiträge zwischen $20 \%$ und $40 q^{2}$. Eine "negative" Ausnahme bildet die aktive Informationsabcabe, welche nur unzureichend erklärt werden kann; eine "positive" Ausnahme bildet die intensive Informationssuche über Antiquitäten, welche sich als überdurchschnittlich motivationsgeleitet darstellt.

1 Vgl. Kapitel B. 3.23.

2 Vgl. die Ergebnisübersicht in Tabelle 18 im Anhang. 
Tabelle 22 zeigt die auf dem üblichen Niveau des Bestimmtheitsmaßes interpretationsrelevanten Ergebnisse.

Im überblick fällt die durchgängig hohe Bedeutung der "Sammelleidenschaft" für den Informationsstil von Antiquitätenkäufern auf. Úberwiegend treten dazu die ökonomisch orientierten Motivationsinhalte "Spekulation auf Wertzuwachs", "Wertanalge" und "Steuerflucht" auf. Für sammelnde Käufer und solche mit finanziellen zielsetzungen ist dabei eine hohe Informationsaktivität charakteristisch. Diese Feststellung steht im Einklang mit den an anderer Stelle vorgetragenen Verhaltenswirkungen dieser Motivationsinhalte wie z.B. auf das Dissonanz- und Risikoempfinden.

Inhaltiche Nuancen werden in den Erklärungsansätzen einzelner Merkmale des Informationsstils im wesentlichen durch die jeweils "nebengeordneten" Motivationsinhalte deutlich. So beeinflussen z.B. der "Wunsch nach Selbstverwirklichung" und die "Reaktion auf moderne Kunst" die intensive Informationssuche allgemein; die "Freizeitgestaltung" und "das Bedürfnis nach Ästhetik" dagegen die Informationssuche als spezielles Hobby von Antiquitätenkäufern.

Interessant ist die Konsistenz der Erklärungsansätze, die in einer negativen Korrelation - der ansonsten das Informationsniveau fördernden Einzelmotivationen - zur passiven Informationsaufnahme zum Ausdruck kommen. Lediglich die "Freude am Einkaufserlebnis" besitzt positiven Erklärungswert. In der Formulierung des Originalitems "ich überlasse es dem Zufall, Neues über Antiquitäten zu erfahren" wird der inhaltliche Zusammenhang besonders deutlich.

Eine informelle Meinungsführerschaft ist schließlich neben der "Sammelleidenschaft" besonders durch eine "Dokumen- 
INTENSIVE INFORMATION:SSUCHE

Erklärende Motivationen ...

Sammelleidenschaft

Wunsch nach Selbstverwirklichung

Steuerflucht

Reaktion auf moderne kiunst

Spekulation auf Wertzuwachs

Freude am Einkaufserlebnis

Reaktion auf modernes Möbeldesign

Freude am Schenken

\begin{tabular}{|l|c|c|c|c|} 
MULT R & $\begin{array}{c}\text { BESTIMMT- } \\
\text { HEITSMASS }\end{array}$ & EINFACH R & BETA & RANG \\
\hline 0.516 & 0.266 & 0.516 & 0.436 &. \\
0.576 & 0.332 & 0.345 & 0.202 & $?$ \\
0.608 & 0.369 & 0.236 & 0.120 & 7 \\
0.633 & 0.407 & 0.321 & 0.254 & 2 \\
0.649 & 0.421 & 0.297 & 0.151 & 4 \\
0.660 & 0.435 & 0.052 & -0.144 & 5 \\
0.668 & 0.446 & 0.021 & -0.124 & 6 \\
0.677 & 0.459 & 0.165 & 0.117 & 8
\end{tabular}

INFORMATIONSSUCHE ALS HOBBY

Erklärende Motivationen ...

Sammelleidenschaft

Wunsch nach Selbstverwirklichung

Wertanlage

Dekoration der Wohnung

Fre1zeitgestaltung

Familientradition

Besitzerstolz

Empfinden eines kulturelien Auftrags

Freude am Schenken

Spekulation auf Wertzuwachs

Bedürnis nach Asthetik

Reaktion auf moderne kunst

Besitzstreben

Freude an Naturstoffen

Spaß am Suchen

PASSIVE INRORMATIONSAUENAHME

Erklärende Motivationen ...

Sammelieidenschaft

Steuerflucht

Freude am Einkaufserlebnis

Freizeitaestaltung

Spekulation auf Wertzuwachs

Familientradition

Redürfnis nach Ausgleich

MEINUNGSF'JHRUNG

Erklärende Motivationen ...

Sammelleidenschaft

Dokumentation von Individualismus

Freude am Schenken

Besitzerstolz

Spekulation auf Wertzuwachs

Familientradition

Empfinden eines kulturellen Auftrags

ilebe zum alten Kunsthandwerk

\begin{tabular}{|l|r|r|r|r|} 
MULT R & $\begin{array}{r}\text { BESTIMMT- } \\
\text { HEITSMASS }\end{array}$ & EINFACH R & \multicolumn{1}{|c|}{ BETA } & RANG \\
\hline & 0.365 & 0.604 & 0.311 & 2 \\
0.604 & 0.409 & 0.313 & 0.124 & 12 \\
0.639 & 0.431 & 0.266 & 0.110 & 14 \\
0.657 & 0.450 & -0.073 & -0.150 & 8 \\
0.671 & 0.539 & 0.327 & 1 \\
0.686 & 0.471 & 0.244 & 0.156 & 7 \\
0.700 & 0.489 & 0.116 & -0.198 & 3 \\
0.714 & 0.510 & 0.301 & 0.127 & 11 \\
0.724 & 0.524 & 0.020 & -0.114 & 13 \\
0.733 & 0.537 & 0.267 & 0.183 & 4 \\
0.738 & 0.545 & 0.142 & 0.177 & 5 \\
0.744 & 0.554 & 0.244 & 0.160 & 6 \\
0.750 & 0.562 & 0.166 & -0.135 & 10 \\
0.756 & 0.571 & -0.116 & -0.139 & 9 \\
0.763 & 0.583 & 0.111 & -0.099 & 15 \\
0.769 & $0.59 !$ & & &
\end{tabular}

\begin{tabular}{|l|c|c|c|c|} 
MULT R & $\begin{array}{c}\text { BESTIMMm- } \\
\text { HEITSMASS }\end{array}$ & EINFACH R & BETA & FA:IG \\
\hline 0.509 & 0.259 & -0.059 & -0.335 & 1 \\
0.549 & 0.301 & -0.222 & -0.140 & 5 \\
0.560 & 0.313 & 0.014 & 0.198 & 2 \\
0.578 & 0.334 & -0.420 & -0.197 & 3 \\
0.589 & 0.346 & -0.270 & -0.155 & 4 \\
0.002 & 0.363 & -0.205 & -0.136 & 6 \\
0.611 & 0.373 & -0.151 & -0.170 & 7
\end{tabular}

\begin{tabular}{|l|c|r|r|r|} 
MULT R & $\begin{array}{r}\text { BESTIMMT- } \\
\text { HEITSMASS }\end{array}$ & EINFACH R & \multicolumn{1}{|c|}{ BETA } & RANG \\
\hline 0.421 & 0.177 & 0.421 & 0.339 & $i$ \\
0.486 & 0.236 & 0.303 & 0.244 & 3 \\
0.520 & 0.271 & 0.249 & 0.158 & 6 \\
0.542 & 0.294 & 0.015 & -0.251 & 2 \\
0.564 & 0.318 & 0.206 & 0.166 & $\vdots$ \\
0.579 & 0.335 & 0.225 & 0.136 & 7 \\
0.592 & 0.351 & 0.253 & 0.173 & 4 \\
0.605 & 0.365 & -0.036 & -0.126 & 8
\end{tabular}

Tab. 22: Motivationale Erklärungsbeiträge zum Informationsstil - Ergebnistableaus multipler schrittweiser Regressionsanalysen 
tation von Individualismus" bestimmt. Ob es sich im ersten Erklärungszusammenhang eher um eine fachlich begründete und im zweiten eher um eine "zur Schau gestellte" Meinungsführerschaft handelt, kann dabei nicht abschließend beurteilt werden.

Die varianzanalytische Auswertung bestätigt den hohen motivationalen Einfluß - hier in Form der Typenzugehörigkeit bzw. unterschiedlicher Motivationskonfigurationen - auf die Merkmale des Informationsstils. Auch die inhaltliche Interpretation der in Tabelle 23 zusammengestellten Ergebnisse deckt sich weitgehend mit den regressionsanalytischen Aussagen. Dies gilt u.a. für den Informationsstil der "Sammler" und "Materialisten".

Darüber hinaus deckt das Typenkonzept eine Grundstruktur hinsichtlich des Informationsstiles auf, die regressionsanalytisch nicht abgeleitet werden kann. Die neun unterschiedlichen Motivationskonfigurationen teilen die Antiquitätenkäuferschaft bezogen auf ihre Informationssuche in nur zwei, sich signifikant unterscheidende Informationstypen: die "Sammler", "Individualisten", "Materialisten" und "Nostalgiker" als aktive Informationssucher und die "Rationalisten", "Traditionalisten", "Einrichter", "Liebhaber" und "Erlebniskäufer" als nicht aktive Informationssucher. Zeichnet sich die erste Gruppe entsprechend eher durch überdurchschnittlichen Freizeiteinsatz, Reiseaufwand, Informationsaustausch im Freundes- und Bekanntenkreis, sowie die Selbsteinschätzung einer informellen Meinungsführerschaft aus, ist für die zweite Gruppe eher eine passive und zufällige Informationsaufnahme typisch ${ }^{1}$.

$1 \mathrm{zu}$ den einzelnen Signifikanzen zwischen den Motivationstypen vgl. die Tabelle 23 . 


\begin{tabular}{|c|c|c|c|c|c|c|c|c|c|c|c|c|c|}
\hline $\begin{array}{l}\text { MERKMALE } \\
\text { DES INFORMATIONSUERHALTENS } \\
\text { VON ANTIOUITKTENKXUFERN }\end{array}$ & GESAMT & 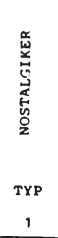 & 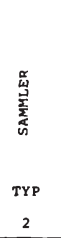 & 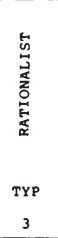 & 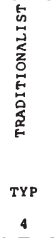 & 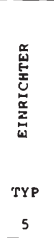 & 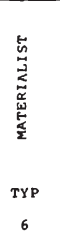 & 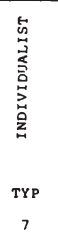 & 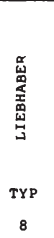 & 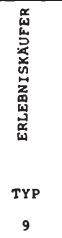 & $\begin{array}{l}\text { SIGNIFI } \\
\text { EINFACH } \\
\text { VARIAN } 2 \\
\end{array}$ & $\begin{array}{l}\text { AN2NIVEAU } \\
\text { R } \\
\text { NALYSE } \\
\text { SYMBOL }\end{array}$ & $\begin{array}{l}\text { GRUPPEN MIT } \\
\text { SIGNIFIKANT } \\
\text { UNTERSCHIDLICHER } \\
\text { MOTIVATIONSAUSPRKGUNG }\end{array}$ \\
\hline $\begin{array}{l}\text { Intensive } \\
\text { Informationssuche }\end{array}$ & 3,28 & 2,80 & 2,43 & 3.57 & 3,71 & 4,36 & 2,60 & 2,57 & 3,75 & 3,88 & 0.000 & $\cdots$ & $2,76,1+5,9,8,4,3$ \\
\hline $\begin{array}{l}\text { Informat ionssuche } \\
\text { als Hobby }\end{array}$ & 3,84 & 3,00 & 3,00 & 4.03 & 3,94 & 5,00 & 3,35 & 3,35 & 3.70 & 4.17 & 0,000 & $\cdots \cdots$ & $1 * 5,9,8,3,4 \quad 2,6 \neq 5,9,8,3 \quad 7,4,3 \neq 5$ \\
\hline $\begin{array}{l}\text { Informationssuche durch } \\
\text { gezielte Stldtereisen }\end{array}$ & 3,87 & 3,73 & 3,43 & 4,17 & 3,41 & 4,82 & 3,50 & 3,48 & 4,08 & 4,38 & 0,012 & $*$ & $4,7 \neq 5,9,3 \quad 6 \neq 5,9 \quad 2,1 \neq 5$ \\
\hline $\begin{array}{l}\text { Informationssuche durch } \\
\text { gezlelte Auslandsreisen }\end{array}$ & 4,30 & 4,20 & 3,00 & 4,50 & 3,88 & 5,00 & 4,10 & 4.17 & 4,50 & 4,81 & 0.002 & $\cdots$ & $2 \neq 5,9,8,3,1,7,6 \quad 4,6 \neq 5,9 \quad 7 \neq 5$ \\
\hline $\begin{array}{l}\text { passive } \\
\text { Informat Ionsauf nahme }\end{array}$ & 2,79 & 3,53 & 4,29 & 2,70 & 2,59 & 2,00 & 3,15 & 2,87 & 2,42 & 2,06 & 0,000 & $\cdots *$ & $5,9 \neq 2,1,6,7 \quad 8,4,3 \neq 2,1 \quad 7,6 \neq 2$ \\
\hline $\begin{array}{l}\text { aktive } \\
\text { Informat ionsabgabe }\end{array}$ & 3,30 & & 2,43 & 3,67 & 3.71 & & & 2,87 & & 3,56 & 0,126 & Ns & $2 \neq 4,3,9 \quad 7 \neq 4,3$ \\
\hline $\begin{array}{l}\text { intensiver Informationstausch } \\
\text { im Freundes- und Bekanntenkreis }\end{array}$ & 2,71 & 2,20 & 1,86 & 2,93 & 3,00 & 3,55 & 2,40 & 2,52 & 3,08 & 2,63 & 0.004 & *.* & $2,1 * 5,8,4,3 \quad 6,7,9 * 5$ \\
\hline Meinungsfolgschaft & 3.07 & 3,40 & 3.57 & & 3,59 & & 2,40 & & & 3,31 & 0.164 & ws & $6+4,2,1,9$ \\
\hline Me1nunga fuhrung & 3.60 & 3,20 & 2,86 & 4,13 & 3,53 & 4,18 & 3.05 & 3.00 & 4.00 & 4,25 & 0,000 & $\cdots$ & $2,7,6,1+9,5,3,8 \quad 4 \neq 9$ \\
\hline Informationsinteresse & 3.74 & 4,20 & 4.71 & 3,57 & 3,76 & 2,73 & 4,25 & 3,70 & 3,67 & 3,38 & 0,019 & $*$ & $5 \neq 2,6,1,4,7 \quad 3 \neq 2,6 \quad 3+2$ \\
\hline
\end{tabular}

Tab. 23: Der Einfluß von Motivationskonfigurationen (Typentuqehörigkeiten) auf den Informationsstil - Ergebnisüberblick einfacher Varianzanalysen 
4.22 Thematische Ausrichtung des Informationsinteresses

Das Interesse von Antiquitätenkäufern an spezifischen Informationsinhalten im Zusammen̈hang mit Antiquitäten läßt sich allgemein gesehen überdurchschnittlich gut durch Kombinationen von Einzelmotivationen erklären. Multiple Korrelationskoeffizienten zwischen 0 ,50 und 0 ,70 weisen auf Varianzerklärungsanteile zwischen $25 \%$ und $50 \%$ hin. Die Anzahl erklärender Einzelmotivationen schwankt dabei zum Teil erheblich ${ }^{1}$.

Betrachtet man die in Tabelle 24 zusammengestellten Informationsinhalte, für die besonders hohe Erklärungsanteile (vgl. Bestimmtheitsmaß) ermittelt wurden, so fällt ihr durchgängig materieller bzw. finanzieller Inhaltsbezug auf.

Übereinstimmend sind es die ökonomischen Einzelmotivationen "Spekulation auf Wertzuwachs", "Wertanalge" und "Steuerflucht", von denen die höchsten partiellen Einflüsse ausgehen: Interessant ist ihre wechselnde Bedeutungsrangfolge in den Erklärungsansätzen, welche auf ein sehr differenziertes Informationsinteresse auch innerhalb einer insgesamt ökonomisch motivierten Käuferschaft hindeutet. Die Zuordnungen von Motivationsinhalten $z u$ Informationsinhalten sind dabei folgerichtig und plausibel (vgl. dazu die spalte "Rang" in den Ergebnistableaus).

Gleiches trifft für die weiteren erklärungsrelevanten Motivationsinahlte zu. Ein Beispiel ist der positive Zusammenhang zwischen dem "Empfinden eines kulturellen Auftrages" und "steuerrechtlichen Fragen", da es gerade bei einem kulturellen Engagement für Antiquitäten (z.B. Sammlungen, Stiftungen, Schenkungen etc.) eine Reihe steuerrechtlicher Sondervorschriften zu beachten gilt.

$1 \mathrm{Vgl}$. Tabelle 19 im Anhang. 
SAMMELN MIT GEWINN

Erklärende Variablen ...

Spekulation auf Wertzuwachs Sammelleidenschaft

Bedurfnis nach Ausgleich

Liebe zum alten Kunsthandwerk

Besitzstreben

Spas am Suchen

\begin{tabular}{l|l|l|r|r|r|} 
MULT R & $\begin{array}{c}\text { BESTIMMT- } \\
\text { HEITSMASS }\end{array}$ & EINFACH R & BETA & RANG \\
\hline 0.588 & 0.346 & 0.588 & 0.551 & 1 & \\
0.612 & 0.374 & 0.267 & 0.193 & 2 \\
0.629 & 0.395 & -0.143 & -0.130 & 3 \\
0.641 & 0.423 & 0.179 & 0.126 & 4 \\
0.650 & 0.423 & 0.179 & 0.126 & 5 & \\
0.656 & 0.439 & -0.017 & -0.094 & 6 &
\end{tabular}

STEUERRECHTLICHE FRAGEN

Erkiarende variablen ...

Steverflucht

Empfinden eines kulturellen Auftrags

Reaktion auf modernes Möbeldesign

Freude am Schenken

Besitzerstolz

Wertanlage

Spekulation auf wertzuwachs

Bewahrung von Erinnerung an die Familie

Familientradition

\begin{tabular}{|c|c|c|c|c|}
\hline MULT $\quad R$ & $\begin{array}{l}\text { BEST IMMT - } \\
\text { HEITSMASS }\end{array}$ & EINFACH R & BETA & RANG \\
\hline 0.586 & 0.343 & 0.586 & 0.608 & 1 \\
\hline 0.600 & 0.360 & 0.219 & 0.226 & 4 \\
\hline 0.614 & 0.378 & -0.144 & -0.114 & 9 \\
\hline 0.624 & 0.390 & -0.048 & -0.146 & 8 \\
\hline 0.633 & 0.401 & -0.019 & -0.153 & 7 \\
\hline 0.647 & 0.419 & 0.263 & 0.297 & 2 \\
\hline 0.663 & 0.439 & 0.218 & -0.243 & 3 \\
\hline 0.673 & 0.453 & -0.062 & -0.216 & 5 \\
\hline 0.691 & 0.478 & 0.038 & 0.186 & 6 \\
\hline
\end{tabular}

\section{ANTIQUITATEN ALS KAPITALANLAGE}

Erklärende Varlablen ...

Spekulation auf wertzuwachs

Wertanlage

Steuerfluch

Sarmelleidenschaft

Freude am Schenken

Besitzstreben

\begin{tabular}{|l|c|c|c|c|} 
MULT R & $\begin{array}{c}\text { BESTIMMT- } \\
\text { HEITSMASS }\end{array}$ & EINFACH R & BETA & RANG \\
\hline 0.583 & 0.340 & 0.583 & 0.250 & 2 \\
0.641 & 0.411 & 0.575 & 0.323 & 1 \\
0.665 & 0.442 & 0.400 & 0.218 & 3 \\
0.678 & 0.460 & 0.222 & 0.123 & 4 \\
0.685 & 0.469 & -0.053 & -0.112 & 5 \\
0.692 & 0.479 & 0.252 & 0.109 & 6
\end{tabular}

Tab. 24: Motivationale Erklänungsbeiträge zum thematischen Informationsinteresse Ergebnistableaus multipler schrittweiser Regressionsanalysen 
Der Einfluß der Motivationskonfiguration von Antiquitätenkäufern auf das Interesse an spezifischen Themen ist für eine ganze Reihe von Einzelaspekten signifikant (vgl. Tabelle 25).

Erwähnenswerte Ergebnisse sind:

(1) "Individualisten" und "Materialisten" zeichnen sich durch ein überdurchschnittlich breit gefächertes und hohes Informationsinteresse aus. Betrachtet man die Einzelaspekte, lassen sich in vielen Fällen signifikante Unterschiede $\mathrm{zu}$ anderen Motivationstypen feststellen. Für die "Einrichter" gilt in der Tendenz das Gegenteil.

(2) An kunstwissenschaftlichen Themen ("kunstwissenschaftliche Abhandlungen", "Monographien über Künstler") zeigen sich insbesondere die "Liebhaber" und "Erlebniskäufer" wenig interessiert.

(3) "Sammler", die am Kapitalanlageaspekt kaum interessiert sind, besitzen motivationsbedingt das ausgeprägteste Interesse an "Preisen und Preisentwicklungen".

(4) Der signifikante Einfluß der Typenzugehörigkeit auf das Interesse am "aktuellen Modetrend" bei Antiquitäten läßt sich dahingehend spezifizieren, daß die "Materialisten", "Individualisten" und "Rationalisten" besonders aufmerksam verfolgen, "was im Moment stark gefragt ist".

Die jeweils signifikanten Gruppentrennungen und weitere Einzelergebnisse können der Tabelle 25 entnommen werden. 


\begin{tabular}{|c|c|c|c|c|c|c|c|c|c|c|c|c|c|}
\hline $\begin{array}{l}\text { THEMATISCHES INTERESSE } \\
\text { VON ANTIOUITKTENKXUFERN } \\
\text { IM UUAMMENHANG MIT } \\
\text { ANTIOUITKTEN }\end{array}$ & GESAMT & 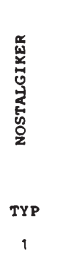 & 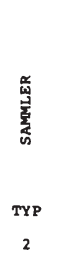 & 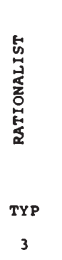 & 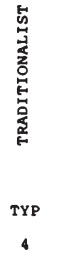 & 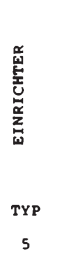 & 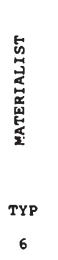 & 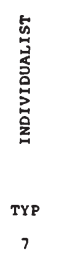 & 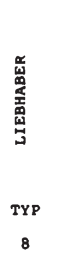 & 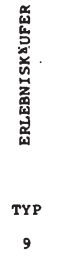 & $\begin{array}{l}\text { SICNIFI } \\
\text { EINFACH } \\
\text { VARIANZ } \\
\end{array}$ & $\begin{array}{l}\text { ANZNIVEAU } \\
\text { R } \\
\text { NALYSE } \\
\text { SYMBOL }\end{array}$ & $\begin{array}{l}\text { GRUPPE MIT } \\
\text { SIGNIFIKANT } \\
\text { UNERSCHIEDLICHER } \\
\text { MOTIVATIONSAUSPRAGUNG }\end{array}$ \\
\hline $\begin{array}{l}\text { was man im Moment } \\
\text { wo kaufen kann }\end{array}$ & 3,17 & & & & & 3,82 & 2,80 & 2,83 & & & 0.423 & NS & $6,7 \bowtie 5$ \\
\hline $\begin{array}{l}\text { Preise und } \\
\text { Preisentwicklungen }\end{array}$ & 2,70 & 2,87 & 2,00 & 2,80 & 3,00 & 3,55 & 2,25 & 2,17 & 2,75 & 3,00 & 0.034 & "* & $7 \neq 5,9,4 \quad 2,6 \neq 5$ \\
\hline $\begin{array}{l}\text { Ausstellungs- } \\
\text { und Messetermine }\end{array}$ & 2,93 & & & 3,30 & & & & 2,61 & & & 0,498 & NS & $7 \neq 3$ \\
\hline $\begin{array}{l}\text { kunstw1s senschaft } 11 \text { che } \\
\text { Abhandlungen }\end{array}$ & 3,29 & 2,93 & 3,57 & 3,40 & 3.06 & 3,82 & 2,95 & 2,83 & 4,00 & 3,75 & 0.035 & ** & $7,6 \neq 8,5,9 \quad 1,4 \neq 8$ \\
\hline $\begin{array}{l}\text { Abblldung und Beschre1- } \\
\text { bungen von Ant1quitaten }\end{array}$ & 2,53 & 2.47 & 2,43 & 2,73 & 2,35 & 2,64 & 2,15 & 2,04 & 3,00 & 3,19 & 0,055 & • & $7 * 9,8,3 \quad 6 \neq 9,8 \quad 4 \neq 9$ \\
\hline Monographien Uber Kunstler & 3,64 & 2,93 & 4,00 & 3,87 & 3,29 & 3.55 & 3,45 & 3,30 & 4,08 & 4,56 & 0.006 & $\cdots *$ & $1 \neq 9,8,3 \quad 4,7,6,5 \neq 9$ \\
\hline steuerrechtliche Fragen & 4,68 & & & 4,83 & & & & 4,43 & & & 0,439 & ns & $7+3$ \\
\hline $\begin{array}{l}\text { Antiquitaten } \\
\text { als Kapitalanlage }\end{array}$ & 3,94 & 4,00 & 4,57 & 3,97 & 4,18 & 4,82 & 3,30 & 3,39 & 4,08 & 4,19 & 0,002 & $\cdots *$ & $6 \neq 5,2,9,4,8,3 \quad 7 \neq 5,2,9,4,3 \quad 1 \neq 5$ \\
\hline $\begin{array}{l}\text { was momentan stark } \\
\text { gefragt let (Modetrend) }\end{array}$ & 4,13 & 4,07 & 4,71 & 4,00 & 4,76 & 4,64 & 3,35 & 3,91 & 4,58 & 4,13 & 0,002 & ** & $6+4,2,5,8,9,1,3 \quad 7,3 \neq 4$ \\
\hline Sammeln mit Gewinn & 4,05 & 4,20 & 3,86 & 3,83 & 4,35 & 4,64 & 3,35 & 3,77 & 4,67 & 4,50 & 0,002 & $\cdots$ & $6 \neq 8,5,9,1,4 \quad 7,3 \neq 8,5,9$ \\
\hline $\begin{array}{l}\text { Dokumentation von Anti- } \\
\text { quityten, die den elgenen } \\
\text { moglichst dhnlich sind }\end{array}$ & 3,08 & 2,40 & 2,71 & 3,30 & 3,06 & 4,36 & 2,75 & 2,35 & 3,50 & 3,75 & 0.001 & $\cdots * *$ & $7,1 \neq 5,9,8,3 \quad 6 \neq 5,9 \quad 2,4,3 \neq 5$ \\
\hline
\end{tabular}

Tab. 25: Der Einfluß von Motivationskonfigurationen (Typezugehörigkeiten) auf die Interessensausrichtung - Ergebnisübersicht einfacher Varianzanalysen 


\subsection{Nutzung von Informationsquellen}

Die Bedeutung der Einzelmotivationen zur Erklärung der Nutzungsintensitäten von Informationsquellen über Antiquitäten bewegt sich im Gesamtüberblick auf einem durchschnittlichen Niveau. Die durch individuelle Verhaltensvariationen entstehenden Ausgangsvarianzen können überwiegend durch 5 oder 6 regressionsanalytisch isolierte Einzelmotive $\mathrm{zu} \mathrm{ca} .15$ bis 20 \& erklärt werden (multiple Korrelationskoeffizienten zwischen 0,40 und 0,45$)^{1}$.

Unterdurchschnittlich sind die Erklärungsbeiträge für die nicht unmittelbar antiquitätenbezogenen Quellen "Tageszeitung" (10 \%) und "Gespräche mit Freunden und Bekannten" (11 8 ). Dieses Resultat ist verständlich, da beide genannten Informationsquellen ein weit über den Bereich "Antiquitäten" hinausgehendes Informationsinteresse der Käufer befriedigen, so daß warenspezifische Motivationen einen entsprechend reduzierten und partiellen Erklärungswert besitzen.

Indirekt bestätigt sich diese Annahme im hohen Einfluß der abgefragten Motivationen auf die Nutzung von "Fachbüchern" (vgl. Tabelle 26).

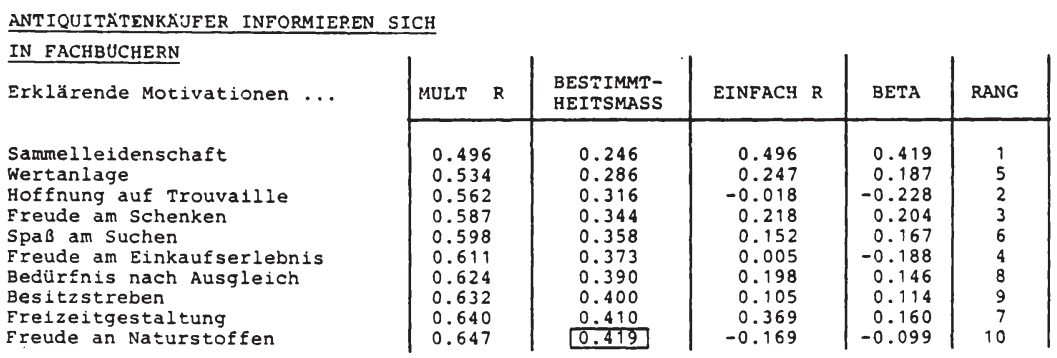

Tab. 26: Motivationaler Erklärungsbeitrag zur Nutzung von Fachbüchern als Informationsquelle - Ergebnistableau einer multiplen schrittweisen Regressionsanalyse

1 Eine Ergebnisübersicht gibt Tabelle 20 im Anhang. 
Der erreichte Varianzerklärungsanteil ist mit 42 \& als hoch zu beurteilen (vgl. multiples Bestimmtheitsmaß). Er wird zu einem wesentlichen Prozentsatz von der "Sammelleidenschaft" getragen: je ausgeprägter diese ist, umso intensiver ist die Nutzung von Fachbüchern. Gleichgerichtete Beziehungen bestehen ferner zur "Wertanlage" und zur "Freude am Schenken"; gegenläufig zu den konsumerlebnisorientierten Motivationen "Hoffnung auf Trouvaille" und "Freude am Einkaufserlebnis". Parallelitäten zu den motivationalen Bestimmungsfaktoren des Informationsstils, speziell der aktiven Informationssuche sind dabei erkennbar. Die varianzanalytische Prüfung des Einflusses unterschiedlicher Motivationskonfigurationen führt zu analogen Schlußfolgerungen: "Sammler" und "Materialisten" informieren sich u.a. über Antiquitäten gegenüber "Erlebniskäufern" signifikant häufiger in Fachbüchern (vgl. Tabelle 27).

Auf einem varianzanalytischen Signifikanzniveau von 958 und 998 können darüber hinaus Einflüsse der Motivationskonfigurationen auf die Nutzung von Messen und Märkten als Informationsquellen festgestellt werden. Dabei zeigt sich, daß für Antiquitätenmärkte und Flohmärkte ein tendenziell gleichgerichteter motivationaler Einfluß besteht: "Nostalgiker", "Erlebniskäufer", "Liebhaber" und "Materialisten" sind eher als "Nutzer", demgegenüber "Individualisten" und "Traditionalisten" als"Nicht-Nutzer" zu bezeichnen ${ }^{1}$.

Durch Messebesuche, bei denen sich der Käufer oftmals einem hochwertigen Angebot gegenübersieht, informieren sich u.a. die "Individualisten". Speziell die "Einrichter" machen gerade von dieser Informationsquelle einen gegenüber fast allen anderen Motivationskonfigurationen signifikant geringeren Gebrauch.

$1 \mathrm{Zu}$ signifikanten Gruppentrennungen vgl. im einzelnen die Angaben der Tabelle 27. 


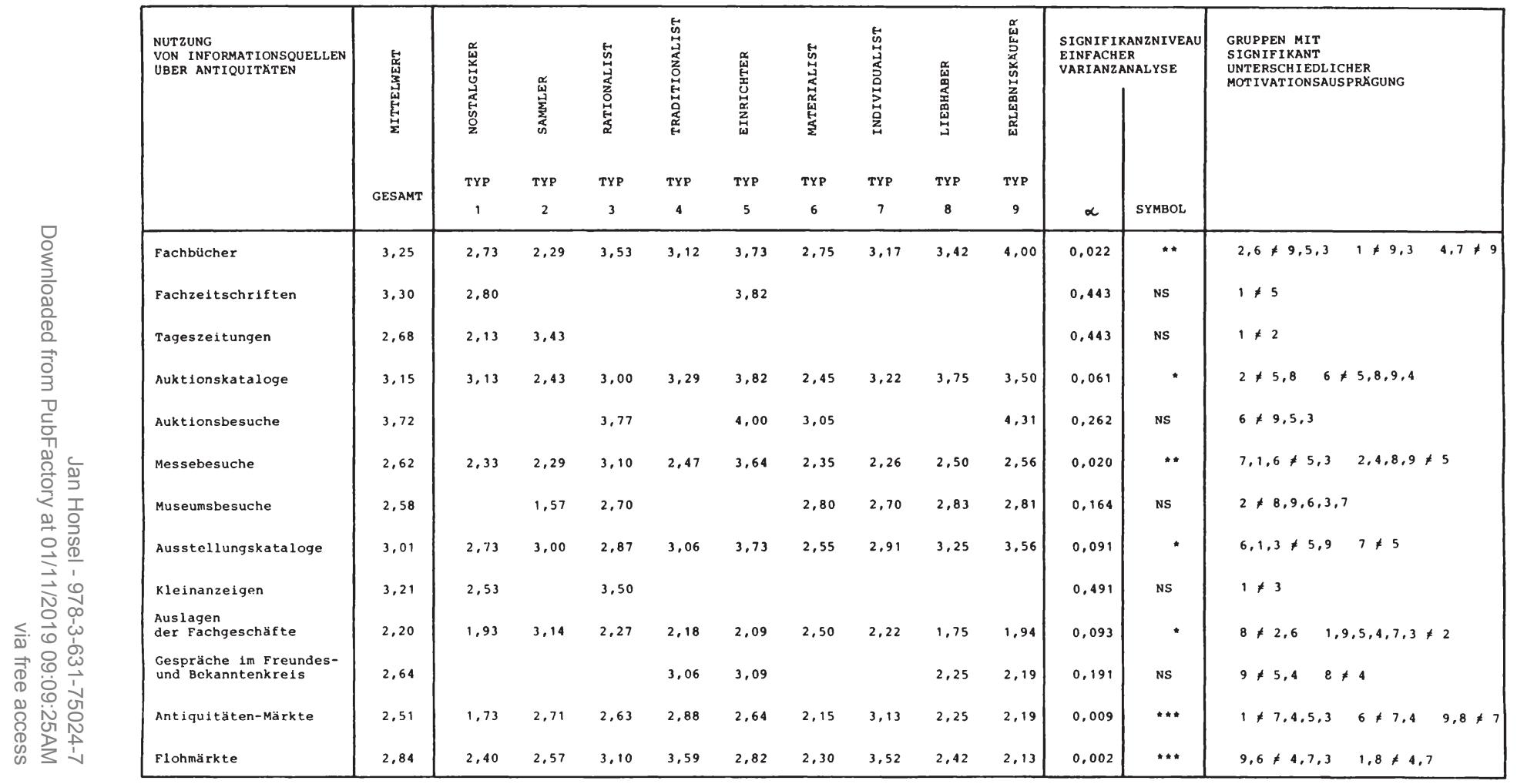

Tab. 27: Der Einfluß von Motivationskonfigurationen (Typenzugehörigkeiten) auf die Nutzung von Informationsquellen - Ergebnisüberblick einfacher Varianzanalysen 
Dienen Einkaufsstätten wie z.B. Messen und Märkte dem Käufer als Informationsquellen, bedeutet dies nicht, daß er sie auch für seine Kaufabschlüsse präferiert. Die Frage des motivationalen Einflusses auf die Nutzung von Einkaufsstätten als Einkaufsquelle ist Gegenstand des folgenden und abschließenden Analysebereiches.

\subsection{Nutzung von Einkaufsstätten}

Einzelmotivationen können Auskunft über die Nutzung alternativer Einkaufsstätten für Antiquitäten geben. Die Tabelle 28 zeigt einen Ergebnisüberblick interpretationsrelevanter zusammenhänge auf der Grundlage von Kontingenzanalysen $^{1}$.

Folgende Abhängigkeiten sind ohne Anspruch auf Vollständigkeit exemplarisch hervorzuheben :

(1) Sammelnde Käufer und solche mit ökonomisch ausgerichteten Motivationen präferieren Auktionen. Mit zunehmender "Wertanlage" und "Spekulationsmotivation" steigt der Anteil persönlicher Auktionsgebote, wohingegen bei zunehmender "Sammelleidenschaft" der Anteil schriftlicher Auktionsgebote steigt. Es kann angenommen werden, daß gerade die ökonomisch orientierten Käufer die Flexibilität und den Chancenreichtum einer persönlichen Anwesenheit auf einer Auktion schätzen. Sammelnde Käufer bevorzugen möglicherweise die schriftliche form als "Selbstschutz" vor zu hohen und spontanen Geboten z.B. für wichtige sammlungsergänzende Stücke. Die thematische Spezialisierung einer Sammelleidenschaft drückt sich im übrigen in einer Bevorzugung spezialisierter Antiquitätengeschäfte aus.

1 Die Nutzung von Einkaufsstätten wurde in der vorliegenden Untersuchung nominal skaliert, d.h. durch eine Ja/Nein-Frage erhoben. Weder der Einfluß einzelner Kaufmotivationen noch der Einfluß von Motivationskonfigurationen kann daher sinnvoll auf der Grundlage multivariater Analyseverfahren ermittelt werden. Die im folgenden dargesteliten Ergebnisse stützen sich auf Kreuztabulierungen, die mit dem SPSS-Unterprogramm CROSSTABS gerechnet wurden. Vgl. Beutel, P., et al., a.a.O., S. 112 ff.; Rogge, H.J., a.a.O., S. $233 \mathrm{ff.;}$ Nie, N.H. et al., a.a.O., S. $218 \mathrm{ff} .$. Zur Signifikanzprüfung wurde der Chi-Quadrat-Unabhängigkeitstest verwendet. Vgl. Bleymüller, J. et al., a.a.0.,s.126 ff.. 


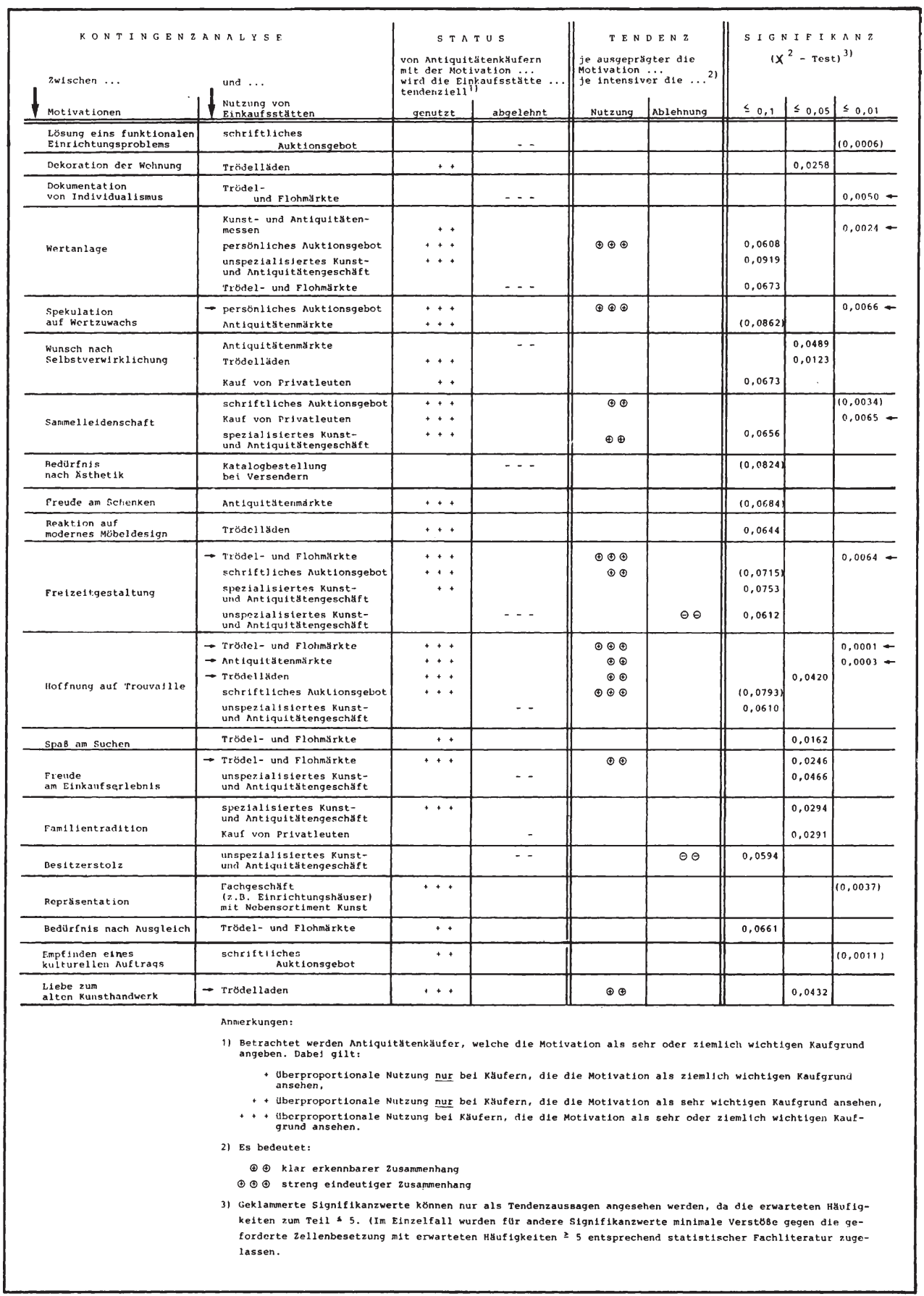


(2) Konsumerlebnisorientierte Motivationen stehen in engem Zusammenhang mit einer Präferenz für Handelsagglomerationen, insbesondere denjenigen mit niedrigerem Angebotsgenre: je ausgeprägter die Motivationsinhalte "Freizeitgestaltung", "Freude am Einkaufserlebnis" und "Hoffnung auf Trouvaille", je intensiver werden Trödel-, Floh- und Antiquitätenmärkte als Einkaufsstätte genutzt. Vermutlich sind die Ausstrahlung solcher Marktveranstaltungen, ihre breite Auswahl und die Möglichkeit einer "Eigeninitiative" der Käufer ("stöbern" etc.) wichtige Grundlage dieses Zusammenhanges.

Die Kontingenzanalyse zwischen Typenzugehörigkeiten und Nutzung von Einkaufsstätten zeigt nur für zwei Konfigurationen signifikante Zusammenhänge auf (vgl. Tabelle 29).

Während "Nostalgiker", "Erlebniskäufer" und "Materialisten" Trödel- und Flohmärkte für ihre Einkäufe bevorzugen, lehnen "Individualisten" und "Traditionalisten" diese Einkaufsquellen eher ab. "Erlebniskäufer" und "Materialisten" haben zugleich eine hohe Affinität zum Kauf auf Auktionen durch schriftliches Angebot. Inhaltlich bestätigt die Abhängigkeitsanalyse zwischen Motivationstypen und der Nutzung spezifischer Einkaufsstätten die bereits auf der Grundlage von Einzelmotivationen erkennbaren Präferenzen.

Mit der Diskussion motivationaler Erklärungsbeiträge zu kognitiven und konativen Verhaltensmerkmalen der Antiquitätenkäufer wurde ansatzweise versucht, Verbindungen zwischen dem Verhaltenskonstrukt "Motivation" und realen Verhaltensweisen von Antiquitätenkäufern aufzuzeigen. Beim gegenwärtigen Stand der Motivationsforschung ist eine Integration der vorgelegten empirischen Ergebnisse zu einem geschlossenen Erklärungsansatz nicht möglich. Die vorgestellten Resultate können jedoch als Anregungsinformationen für die weitere Forschung und zugleich auch für die Gestaltung eines Antiquitätenmarketings dienen. 


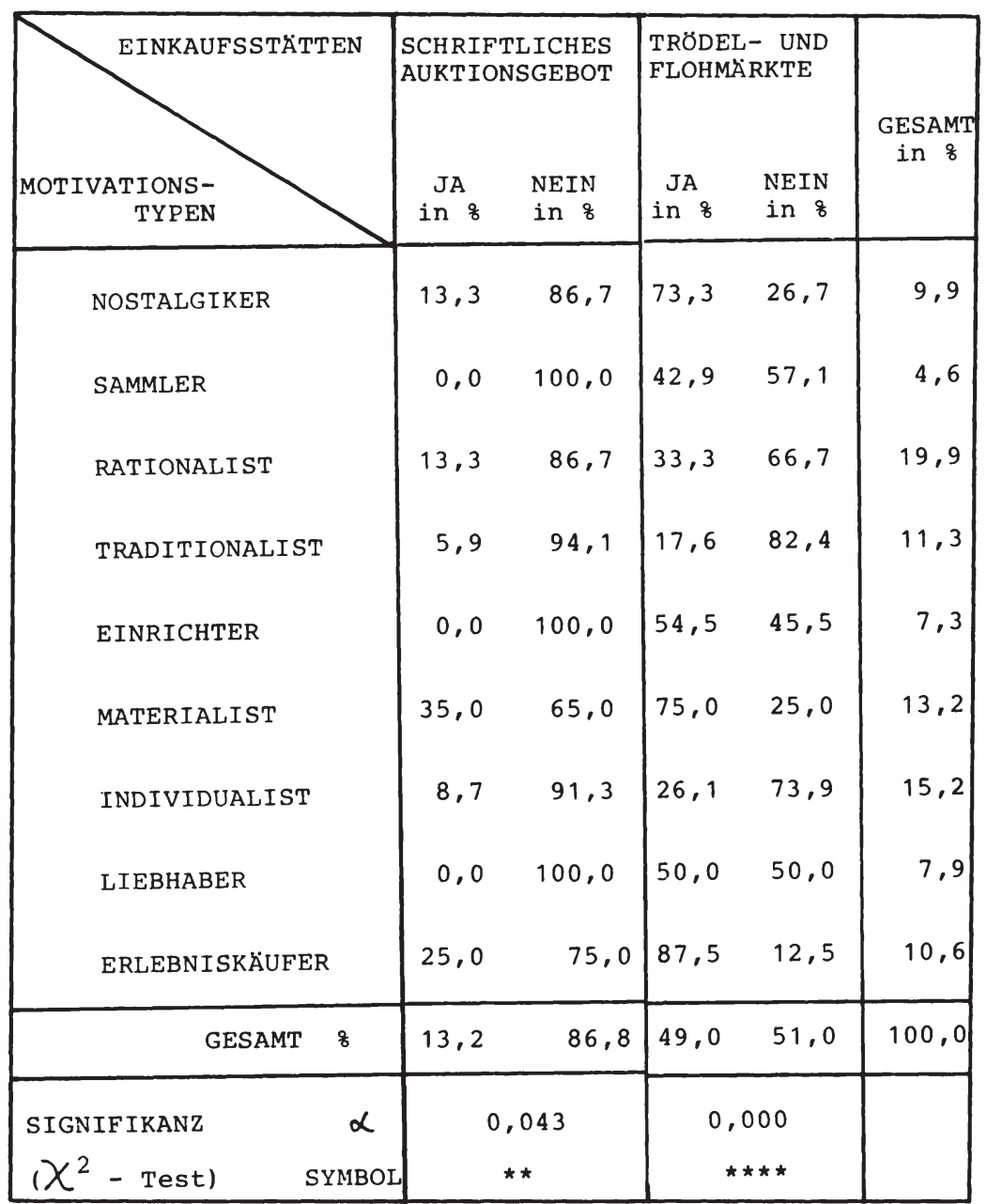

Tab. 29: Motivationskonfigurationen (Typenzugehörigkeiten) und Nutzung von Einkaufsstätten - Kreuztabulierungen 


\section{Implikationen der Motivationsanalyse für ein Antiqui- tätenmarketing}

\section{Implikationen für die weitere Forschung}

Die empirische Untersuchung von Motivationen von Antiquitätenkäufern hat den Aussagewert dieses hypothetischen Konstrukts für die Beschreibung und Erklärung des Kaufverhaltens im Antiquitätenmarkt unter Beweis gestellt. Ausgehend von einem wenig befriedigenden Stand der theoretischen und empirischen Motivationsforschung wurde daher in der vorliegenden Pilot-Studie versucht, unter Heranziehung eines theoriegeleiteten Bezugsrahmens motivationaler Erklärungsansätze sowohl Ausprägungen und strukturen unterschiedlicher Motivationsinhalte als auch deren Einfluß auf das Kaufverhalten am Beispiel von Antiquitätenkäufern zu erhellen.

Für die theoretische Diskussion sowie die weitere empirische Forschung können insbesondere die folgenden Ergebnisse hervorgehoben werden:

(1) Motivationen und ihre Bestimmungsfaktoren

Aus der empirischen Analyse geht hervor, daB der Kauf von Antiquitäten durch ein breites spektrum individueller Motivationen bestimmt wird. Im Rahmen einer differenzierten Analyse motivationaler Bestimmungsfaktoren konnten inhaltliche Nuancen bei der Erklärung von Einzelmotivationen - auch wenn sie derselben faktorenanalytischen Inhaltsdimension zuzurechnen sind - aufgedeckt und damit ein wesentlicher Beitrag zur Explikation und warenspezifischen Konkretisierung des theoretischen Konstrukts "Motivation" geleistet werden.

Die ermittelten personalen, situativen und warenbezogenen Bestimmungsfaktoren zeichneten ein überwiegend ge- 
schlossenes Bild des psychischen Hintergrundes eines Antiquitätenkaufs. Scheinbare Widersprüche in den Beziehungen zwischen Einzelmotivationen und ihren Bestimmungsfaktoren gaben Anlaß, nach "tieferliegenden" $\mathrm{Zu}$ sammenhängen $\mathrm{zu}$ fragen und neue, explorativ begründete Akzente bei der Inhaltsinterpretation zu setzen.

(2) Abgrenzung und Beschreibung von Motivationstypen Ausgehend von der Uberlegung, daß die Motivation von Antiquitätenkäufern nicht monothematisch ist, sondern ein multithematisch-komplexes Beziehungsgefüge einzelner Motivationsinhalte darstellt, wurden Ansätze zur Bildung von Motivationstypen bzw. Motivationskonfigurationen auf der Grundlage individueller Motivationsstrukturen diskutiert.

Dabei zeigte sich, daß eine größere Anzahl von Motivationstypen mit weitgehend homogenen Motivationsstrukturen ihrer Mitglieder gebildet werden konnten. Zugleich zeigte der empirische Befund, daß sich die Typenlösung trennscharf diskriminieren ließ und damit der idealtypischen Anforderung einer weitgehenden Heterogenität der Typen untereinander nahe kam.

Dieses Erfordernis wurde sowohl im Hinblick auf die typenbildenden individuellen Motivationsstrukturen als auch auf die typenbeschreibenden motivationalen Bestimmungsfaktoren erfüllt. Damit war die Voraussetzung gegeben, die Verhaltensrelevanz des Typenkonzeptes durch eine Beziehungsanalyse zwischen Typenzugehörigkeit (Motivationskonfigurationen) und konkreten Verhaltensausprägungen der Antiquitätenkäufer empirisch zu überprüfen. Zugleich legt die Typologie die Informationsgrundlage einer psychographischen Marktsegmentierung und zielgruppenadäquaten Marktansprache. 
(3) Zusammenhänge zwischen Einzelmotivationen, Motivationskonfigurationen und Käuferverhalten

Theoretische Verhaltenskonstrukte verfügen zumeist über eine nur partielle Erklärungs- und Prognoserelevanz für das konkrete Kaufverhalten. Die Untersuchung von Zusammenhängen zwischen "Ausprägungen eines Verhaltenskonstruktes" und "konkretem Verhalten" erbringen aufgrund des hohen Komplexitätsgrades dieses Beziehungsgeflechts daher in der Regel Erkenntnisse nur beschränkten Aussagewertes ${ }^{1}$. In der vorliegenden explorativen Studie wurde dieser Zusammenhang auf zwei Ebenen untersucht:

- durch die regressionsanalytische Isolation besonders erklärungsstarker Bündel von Einzelmotivationen und die Ermittlung ihres Erklärungsbeitrages für einzelne Verhaltensaspekte,

- durch die clusteranalytische Aggregation individueller Motivstrukturen $\mathrm{zu}$ abgrenzbaren Motivationstypen und die varianzanalytische Bestimmung des Erklärungsbeitrages der Typenzugehörigkeit für einzelne Verhaltensaspekte.

Dabei hat sich insbesondere das Typenkonzept zur Operationalisierung der Käufermotivation bewährt und als tragfähiger Forschungsansatz zur Verhaltensexplikation erwiesen. Dies gilt in überdurchschnittlichem Maße für Einzelaspekte des Informationsverhaltens der Antiquitätenkäufer sowie ihre motivationalen Anspruchsniveaus an Merkmale und Eigenschaften von Antiquitäten. Unbefriedigend blieben dagegen die Erklärungsbeiträge für das wahrgenommene Kaufrisiko, obwohl gerade hier enge zusammenhänge erwartet wurden.

Die empirischen Ergebnisse beinhalten Anregungen für weitere

1 Vgl. zu dieser Problematik ausführlich Kroeber-Riel, W., a.a.O., S. $174 \mathrm{ff.;}$ Meffert, H., Modelle..., a.a.O., S. $328 \mathrm{ff}$. 
Forschungen in theoretischer, empirischer und methodischer Hinsicht. Dies gilt auch für die motivationalen Bestimmungsfaktoren, obwohl die ausgewählten personalen-, warenbezogenen und situativen Variablen fast durchgängig hohe Erklärungsbeiträge zur Kaufbedeutung einzelner Motivationsinhalte lieferten. So ist daran zu denken, durch Elimination nicht erklärungsrelevanter und Aufnahme weiterer Determinantengruppen oder auch eine verfeinerte Operationalisierung tiefergehende Erkenntnisse zu gewinnen.

Das Typenkonzept zeichnete ein geschlossenes Bild motivational "definierter" Käufersegmente im Antiquitätenmarkt. Die Operationalisierung der Motivationstypen über die Aggregation individueller Motivationsstrukturen zu segmentspezifischen Motivationskonfigurationen ließe sich dahingehend fortentwickeln, daß die gezeigten Typenzugehörigkeitswahrscheinlichkeiten als Gewichtungsfaktoren bei der Typenbildung berücksichtigt werden. Damit ließe sich das idealtypische Erfordernis der clusterbildung, in sich homogene und untereinander heterogene Gruppen abzubilden, in höherem Maße erfüllen, da clusterrandgruppen entsprechend ihrer (geringeren) Bedeutung bei der Clusterbildung berücksichtigt werden.

Damit wäre vermutlich auch eine verbesserte Verhaltensexplikation bei Antiquitätenkäufern zu erreichen. Die tendenziell beschränkte Aussagefähigkeit der partiellen (motivationalen) Erklärungsansätze des Käuferverhaltens könnten dadurch graduell gesteigert werden. Zugleich ließe sich damit ein verbesserter Baustein für komplexe Modelle des Käuferverhaltens größerer Reichweite bereitstellen.

Die theoretische und empirische Anlage der vorliegenden Untersuchung besitzt den Charakter einer Pilotstudie, die den grundlegenden Bezugsrahmen für ein Antiquitäten-Marketing 
aufspannte. Die Klärung differenzierterer Fragestellungen sowohl im Hinblick auf die Antiquitätenanbieter als auch im Hinblick auf die Antiquitätenkäufer muß weiteren, auf den vorgestellten Ergebnissen aufbauenden Detailstudien vorbehalten bleiben.

Dieser Kennzeichnung entsprechend lassen sich einige grundlegende Implikationen der Untersuchungsergebnisse für Planung und Umsetzung absatzpolitischer Maßnahmen im Antiquitätengewerbe ableiten.

\section{Implikationen für ein Antiquitätenmarketing}

Eine Integration der empirischen Ergebnisse in die Informationssammlung (Marktanalyse) von Auktionshäusern, Antiquitätenhändlern (Fachhandel / Nicht-Fachhandel) und Messeveranstaltern (Verbärde, Händlerzusammenschlüsse etc.) trägt. zu einer differenzierten, warenspezifischen Kenntnis von Antiquitätenkäufern bei. Diese erhöht den Wissensstand von Antiquitätenanbietern und ermöglicht eine verbreiterte Planungsgrundlage für Strategien der Marktbearbeitung, insbesondere in Form eines zielgruppenspezifischen Einsatzes der Marketinginstrumente.

Die Motivationsanalyse hat gezeigt, daß sich Antiquitätenkäufer aufgrund ihrer individuellen Motivationsstrukturen zu geschlossenen und untereinander abgrenzbaren Käufersegmenten aggregieren lassen. Die Motivationstypen erfüllen damit weitgehend die Forderungen, die an eine Marktsegmentierung als Instrument der Definition aktueller und potentieller Zielgruppen gestellt werden ${ }^{1}$. Die bewußte Ausrichtung der absatzpolitischen Aktivitäten von Antiquitätenanbietern

1 Vgl. Meffert, H., Marketing, a.a.o., s. $213 \mathrm{ff..}$ 
auf die motivationalen Grundstrukturen der vorgestellten Zielgruppen, sowie die Berücksichtigung ihrer motivationsbedingten Verhaltensunterschiede kann die Effizienz der Marktansprache steigern, indem sie zur Verminderung von Streuverlusten beiträgt.

Dies gilt sowohl unter dem Gesichtspunkt einer strategischen Entscheidung für eine konzentrierte oder differenzierte Marktbearbeitung als auch für die eher taktische Maßnahmenplanung des absatzpolitischen Instrumentariums.

Eine konzentrierte Marketingstrategie, bei der die absatzpolitischen Aktivitäten auf einen einzelnen Motivationstyp beschränkt bleiben, scheint vor allem für "Newcomer" in einem aus Handelssicht speziell in den höheren Qualitätsgenres relativ "festgefügtem" und "konkurrenzintensivem" Antiquitätenmarkt von Vorteil ${ }^{1}$.

Ein erstes Beispiel ist die Konzentration der Marketinganstrengungen auf den Motivationstyp "Sammler". Da sich die Sammler als ein thematisch spezialisierter Käuferkreis herausgestellt haben, ließe sich durch ein bislang im Antiquitätenhandel nicht anzutreffendes, thematisch abgestimmtes Spezialsortiment möglicherweise eine Marktnische aufdecken. Dabei würde zugleich gewährleistet, daß die empirisch ermittelte, motivationsbedingte Präferenz der "Sammler" zu spezialisierten Kunst- und Antiquitätengeschäften eine

1 Hierfür ist nicht nur die traditionsgebundene struktur dieses Handelszweiges allgemein, sondern auch die Notwendigkeit einer kunsthistorischen Fachkenntnis des Händlers, das Problem der physischen und preisgerechten Warenakquisition, sowie die hohe und zumeist langfristige Kapitalbindung im Warenlager verantwortlich. 
adäquate Umsetzung findet. Diese Sortimentsstruktur sollte mit einer ausgeprägten "Sammlungsbetreuung" durch Beratung, Suche, Ankaufvorschläge oder die Zurücknahme nicht mehr in den Gesamtzusammenhang einer Sammlung "passender" Stücke einhergehen.

Ein zweites Beispiel ist die Konzentration der Marketinganstrengungen auf den Motivationstyp "Einrichter". Dabei wären im Rahmen der Sortimentsplanung insbesondere die motivationsbedingten Anforderungen an Antiquitäten als Einrichtungsgegenstände (Dekorativität, Gebrauchsfähigkeit, solide Verarbeitung etc.) zu berücksichtigen. Es wurde deutlich, daß für die Einrichter der kunsthistorische/ künstlerische Aspekt, oder auch in Abgrenzung zu den "Sammlern" die "Sammlungswürdigkeit" gegenüber dem "Wohnwert" von Antiquitäten zurücktritt. Anstelle einer "Sammlungsberatung" hat der Handel gegenüber den "Einrichtern" eher eine innenarchitektonische "Einrichtungsberatung" mit entsprechender Berücksichtigung stilistischer und geschmacklicher Gesichtspunkte zu leisten.

Auch im Rahmen einer differenzierten Marktbearbeitungsstrategie, die sich durch einen segmentspezifischen Einsatz der Marketingaktivitäten zur Erfassung des gemeinsamen Absatzpotentials aller Motivationstypen auszeichnet, ermöglicht die Kenntnis von Strukturen und Ausprägungen der segmentspezifischen Motivationskonfiguration ein effizienteres Antiquitätenmarketing .

Ein möglicher Ausgangspunkt für eine derartig kundenorientierte Marketingpolitik ist u.a. das Wissen um den Zusammenhang zwischen Bedürfnissen und Wünschen einzelner Käufersegmente und der Nutzung alternativer Einkaufsstätten.

So ergab beispielsweise die empirische Untersuchung, daß "Nostalgiker" und "Erlebniskäufer" Messe- und Marktveran- 
staltungen präferieren. Hier ließen sich z.B. die Erlebniswerte durch ein breiteres Angebot steigern.

Zudem führte eine Selektion der Messeantiquitäten unter dem Gesichtspunkt der typenspezifischen Warenanforderungen (Anziehungskraft, Ausdrucksstärke, Alter etc.) oder auch eine typengerechte Warenpräsentation ("stöbern können") zu einer effizienteren Ansprache der "Nostalgiker".

Auktionatoren, die eher mit "Materialisten"zu rechnen haben, könnten die Instrumente der Kontrahierungspolitik,insbesondere der Lieferungs- und Zahlungsbedingungen, gezielter einsetzen. So wäre eine erhöhte Attraktivität und eine gesteigerte "Kaufsicherheit" durch eine - bisher nur vom Fachhandel gewährte - Ubernahme von Gewährleistungsansprüchen anzustreben. In Verbindung mit einem konsequenten "Qualitätsmarketing" bei Vorselektion des Auktionsmateriales könnte dieses Vorgehen die Anbieter-Käuferbeziehung mit weiteren Vertrauenselementen fundieren. Wichtige Selektionskriterien stellen auch die motivationsbedingten Anforderungen an Eigenschaften und Merkmale von Antiquitäten (Seltenheit, Wertbeständigkeit, Originalität) dar. Darüber hinaus erscheinen auch Spezialauktionen, die sich nicht an Sachgebietsabgrenzungen, sondern an den Motivationsstrukturen einzelner Käufergruppen (z.B. "Anlegerauktionen") orientieren eine beachtenswerte Möglichkeit im Rahmen der zielgruppenadäquaten Marktansprache.

Schließlich lassen sich auch für den Fachhandel, der tendenziell ein breiteres Spektrum von Motivationstypen anspricht, eine Vielzahl absatzpolitischer Implikationen nennen. Hier könnte z.B. ein motivationsadäquates Angebot von Serviceleistungen dazu dienen, die bislang weniger stark motivational engagierten Käufergruppen wie u.a. "Traditionalisten", 
"Rationalisten" und "Einrichter" auf ein höheres "Motivationsniveau" zu heben. Geeignet erscheinen in diesem $\mathrm{Zu}$ sammenhang Rückkaufgarantien, Restaurations-, Pflege- und Versicherungshinweise sowie Gutachten oder Zertifikate als unentgeltliche Nebenleistungen. Auch das persönliche Verkaufsgespräch, bei dem versucht wird, ein über die engere Motivation der Käufersegmente (z.B. "Einrichter") hinausgehendes allgemeines Interesse durch die Vermittlung des kunsthistorischen Hintergrundes von Antiquitäten zu schaffen, kann demselben Zweck dienen. Eine daraus resultierende intensivere Wertschätzung könnte die Anbahnung oder Festigung einer persönlichen Beziehung $z u$ Antiquitäten und ein damit verbundenes höheres motivationales Engagement fördern.

Zusammenfassend zeigen die empirischen Ergebnisse der vorliegenden Studie und ihre exemplarisch angeführten Implikationen, daB theoretisch-inhaltliche Uberlegungen einerseits sowie methodisch-statistische Instrumente andererseits geeignet sind, Bedeutung und Einflußrichtung von Motivationen beim Kaufverhalten von Konsumenten in ausgewählten Produktbereichen zu erfassen und Hinweise für die Ausgestaltung eines strategischen und taktischen Antiquitätenmarketings zu liefern.

Somit ist die Auffassung, "Motivationen als schaffende und bewegende Kräfte gehören zum Bereich des Unbewußten und entziehen sich daher der Erforschung $z u$ wirtschaftlichen Zwecken" ${ }^{1}$, zu relativieren.

1 Kropff, H.F.J., Motivforschung. Methoden und Grenzen, Essen 1960, S. 39. 


\section{A n h a n g I}

\section{Untersuchungsdesign und Stichprobenstruktur}

Zur Analyse der Motivation von Antiquitätenkäufern, der personalen, warenspezifischen und situationalen Motivationsdeterminanten sowie der motivationalen Erklärungsbeiträge $\mathrm{zu}$ kognitiven und konativen Verhaltensweisen lag bis 1981 in der Bundesrepublik Deutschland noch keine wissenschaftlich-empirische Untersuchung vor. Entsprechend der im vorstehenden Teil dieser Arbeit diskutierten Problemstellungen wurde eine Befragung zu diesem Themenkreis bei Besuchern der Münchener Kunst- und Antiquitäten-Messe, der Internationalen AntiquitätenAusstellung in Essen und des Antiquitätenmarktes in Münster durchgeführt. Das Design der Untersuchung hatte dabei verschiedene Anforderungen zu erfüllen. So sollte die Studie auf der Basis einer schriftlichen Befragung

(1) ảie subjektiv käuferbezogene Begriffsauffassung von Antiquitäten ermitteln und damit die Grundlage eines Verständnisses für die wichtigsten Inhaltsdimensionen von Antiquitäten als Kaufobjekte mit motivationalem Anreiz legen. 
(2) ausgehend von der käuferbezogenen "Antiquitäten-Definition" eine inhaltlich möglichst umfassende quantitative Ermittlung

- antiquitätenspezifischer Motivationsinhalte und -inhaltsdimensionen im Sinne aktualisierter Beweggründe eines Antiquitätenkaufes

- personaler, warenbezogener und situativer Bestimmungsfaktoren der Käufermotivation

- motivationsgeleiteter kognitiver und konativer Verhaltensweisen von Antiquitätenkäufern im Zusammenhang mit einem Erwerb von Antiquitäten

gewährleisten.

(3) Ansatzpunkte einer Typologisierung von Antiquitätenkäufern auf der Grundlage ihrer individuellen Motivationsstrukturen aufzeigen und eine problemadäquate und differenzierte Typenlösung ermöglichen

(4) eine ausreichende Datenbasis für Beziehungsanalysen zwischen motivationalen Bestimmungsfaktoren und Motivation, sowie Motivation (Einzelmotivationen und Motivationstypen) und Verhaltensmerkmalen von Antiquitätenkäufern liefern

(5) durch Datenumfang (Stichprobengröße), Fragebogen- und Fragenkonstruktion sowie Skalierung der Variablen die sinnvolle Anwendung multivariater Analysemethoden im Rahmen einer EDV-Auswertung ermöglichen

(6) Repräsentativitätsanforderungen im Sinne einer explorativen $\mathrm{Pilot-Studie}$ erfüllen, so daß einerseits fundierte Tendenzaussagen insbesondere im Zusammenhang mit Beziehungsanalysen zwischen den einbezogenen Variablengruppen abgeleitet und andererseits bislang unbeachtete Ansatzpunkte für weiterführende Forschungen aufgezeigt werden können

(7) hinsichtlich des Fragebogendesigns (Umfang, Dauer und Verständlichkeit) die Befragten trotz des breiten Informationsinteresses der Untersuchung nicht überfordern und eine hinreichende Rücklaufquote gewährleisten

Durch eine entsprechende Umsetzung der Problemstellung 
innerhalb des Fragebogens und die Operationalisierung der Variablen ${ }^{1}$ wurde versucht, die genannten Anforderungen zu berücksichtigen. Aufgrund der methodischen Beschränkungen auf der einen (insbesondere Verständlichkeit der Fragen, Umfang des Fragebogens) und der inhaltlichen Notwendigkeiten auf der anderen Seite ergaben sich bei der Fragebogenkonstruktion folgende ausgewählte Fragenkomplexe:

(1) Motivationsinhalte (1 Frage, 26 Items)

(2) Motivationale Bestimmungsfaktoren

- warenbezogen (1 Frage, 22 Items)

- situativ (2 Fragen, 10 Items)

- personal-psychographisch (6 Fragen, 71 Items)

- personal-soziodemographisch (10 Fragen)

(3) Motivationsgeleitete Verhaltensmerkmale

- kognitiv ( 8 Fragen, 49 Items)

- konativ (4 Fragen, 50 Items)

Der Fragebogen enthielt damit für die vorliegende Studie 32 Fragen, darunter 1 offene. Bei einem Pretest mit $\mathrm{n}=8$ Antiquitätenkäufern wurde besonders die verständlichkeit und Vollständigkeit der Fragen überprüft. Der Fragebogen erhielt daraufhin seine endgültige Fassung. Die Befragung selbst erfolgte schriftlich und wurde im Herbst 1980 durchgeführt. Tabelle 30 gibt einen Uberblick über den jeweiligen Stichprobenumfang und die Rücklaufquoten an den einzelnen Befragungsorten sowie Hinweise über den zeitlichen Ablauf der Befragung. Aus insgesamt 600 verteilten Fragebögen ergab sich ein auswertbarer Netto-Rücklauf von 151 Stück $(25,2$ 8), welches einer Non-Response-(2uote von knapp 75 \& entspricht.

1 Grundlage bildete dazu ein Fragebogen, welcher im Rahmen einer Vorstudie auf der Westdeutschen Kunst- und Antiquitätenmesse in Düsseldorf getestet wurde. Die Verteilung von 190 Fragebögen führte verbunden mit einer ca. 2-5minütigen persönlichen Ansprache zu einer Rücklaufquote von 58 . 


\begin{tabular}{|c|c|c|c|c|c|c|c|c|}
\hline$\downarrow_{\text {FRAGEBÖGEN }}^{\text {BEFRAGUNG }}$ & $\begin{array}{r}\text { Mü } \\
\text { Stück }\end{array}$ & $\begin{array}{l}\text { hen } \\
8\end{array}$ & $\begin{array}{r}\text { E } \\
\text { Stück }\end{array}$ & sen & $\begin{array}{r}\text { Mün } \\
\text { Stück }\end{array}$ & $\begin{array}{r}\text { ster } \\
8\end{array}$ & $\begin{array}{l}\text { Gesa } \\
\text { Stück }\end{array}$ & int \\
\hline verteilt & 300 & 100,0 & 150 & 100,0 & 150 & 100,0 & 600 & 100,0 \\
\hline Brutto-Rücklauf & 76 & 25,3 & 47 & 31,3 & 36 & 24,0 & 159 & 26,5 \\
\hline nicht auswertbar & 3 & 1,0 & 4 & 2,7 & 1 & 0,7 & 8 & 1,3 \\
\hline Netto-Rücklauf & 73 & 24,3 & 43 & 28,6 & 35 & 23,3 & 151 & 25,2 \\
\hline Befragungszeitraum & \multicolumn{8}{|c|}{ Oktober bis November 1980} \\
\hline Rücklaufzeitraum & \multicolumn{8}{|c|}{ Oktober 1980 bis Januar 1981} \\
\hline
\end{tabular}

Tab. 30: Kennzeichnung der Stichprobe

Trotz der als "Pilot Study" angelegten Untersuchung, die Tendenzaussagen über tatsächliches motivgeleitetes verhalten von Antiquitätenkäufern liefern und Hypothesen über alternative Gestaltungsmöglichkeiten eines kundenorientierten Antiquitätenmarketing stützen soll, war eine angemessene Stichprobenauswahl bzw. -repräsentativität sicherzustellen. Dabei stellen Antiquitätenkäufer schon aufgrund der begrifflich uneinheitlichen Definition von Antiquitäten eine nur unter einschneidenden Prämissen gedanklich abgrenzbare Grundgesamtheit dar, für die es im exakt statistischen sinn eine repräsentative stichprobe nicht geben $k_{a n n}{ }^{1}$.

$1 \mathrm{Zu}$ Anforderungen und Auswahlverfahren vgl. Rogge, H.J., a.a.O., S. $162 \mathrm{ff.;}$ Bleymüller, J. et al., a.a.O., s. $71 \mathrm{f}$.. 
Deshalb wurde versucht, durch bewußte Auswahl des Befragtenkreises und tendenziell zufällige Auswahl der Einzelpersonen eine Stichprobe aufzubauen, die bezogen auf Motivation und motivationsgeleitetes Verhalten durch sachlogische Uberlegungen als hinreichend repräsentativ angesehen werden kann.

(1) Befragt wurden ausschlieBlich Besucher von Antiquitätenmessen bzw. -märkten, wobei sich die Antiquitätenkäufer durch eine Frage zu ihrem persönlichen Antiquitätenbesitz und der Nutzung von Einkaufsstätten zu identifizieren hatten.

(2) Die Befragten dokumentierten durch ihren Messebesuch überdurchschnittliches Interesse an Antiquitäten. Ihnen ist hinsichtlich der Untersuchungsmerkmale insbesondere ihrer Motivation ein besonderes Gewicht (Ausgeprägtheit) zu unterstellen 1 .

(3) Messen gewährleisten aufgrund ihrer spezifischen Eigenschaften eine ausgeprägte "Streuung" wichtiger Untersuchungsmerkmale der Befragten

- durch breites Angebot in einer Vielzahl von Sachund Sammelgebieten finden sich unter den Messebesuchern Antiquitätenkäufer unterschiedlichster Interessenausrichtung

- durch ihren zusätzlichen "Ausstellungscharakter" bieten Messen und Märkte am ehesten die Möglichkeit, ein nicht an spezielle Einkaufsstätten gebundenes (z.B. ausschließliche Auktionskäufer) Publikum zu erfassen

- durch überregionale Einzugsgebiete wird eine "geographische Verzerrung" (z.B. mit der Konsequenz spezieller Interessenschwerpunkte der Käufer) weitgehend vermieden

1 Ein ähnliches Vorgehen bewußter Stichprobenkonstruktion findet sich bei der Auswahl nach dem "Cut-off-Verfahren" (Konzentrationsprinzip) und der "typischen Auswahl" vgl. Berekoven, L. et al., a.a.0., S. $123 \mathrm{f} .$. 
- durch die Kaufmöglichkeit der Messeobjekte wird der gewünschte Bezug zur konkreten Kaufsituation - welcher gerade für die Erhebung von Motivationsinhalten bedeutsam erscheint - gewährleistet

- durch unterschiedliche Angebotsgenres sind Messen und Märkte ein Spiegelbild der qualitativen Angebotsschichten des Marktes und der ihnen zuzuordnenden Käufergruppen.

Durch die Aufspaltung der Gesamtbefragung in drei Teilstichproben auf Messen und Märkte mit unterschiedlichen Qualitätsstandards und unterschiedlicher Thematik (Sachund Sammelgebiete) der angebotenen Verkaufsware konnten typische Käufer aller Qualitätssegmente und Interessensausrichtungen in die Befragung einbezogen werden. In Verbindung hiermit wurde zugleich eine weitgehende Erfassung aller soziodemographischen und sozioökonomischen strukturen im Antiquitätenmarkt erwartet.

Das sozialstatistische strukturprofil der befragten Antiquitätenkäufer gibt die Abbildung 67 mit seinen demographischen und die Abbildung $68 \mathrm{mit}$ seinen sozioökonomischen Komponenten als Ubersicht wieder. 


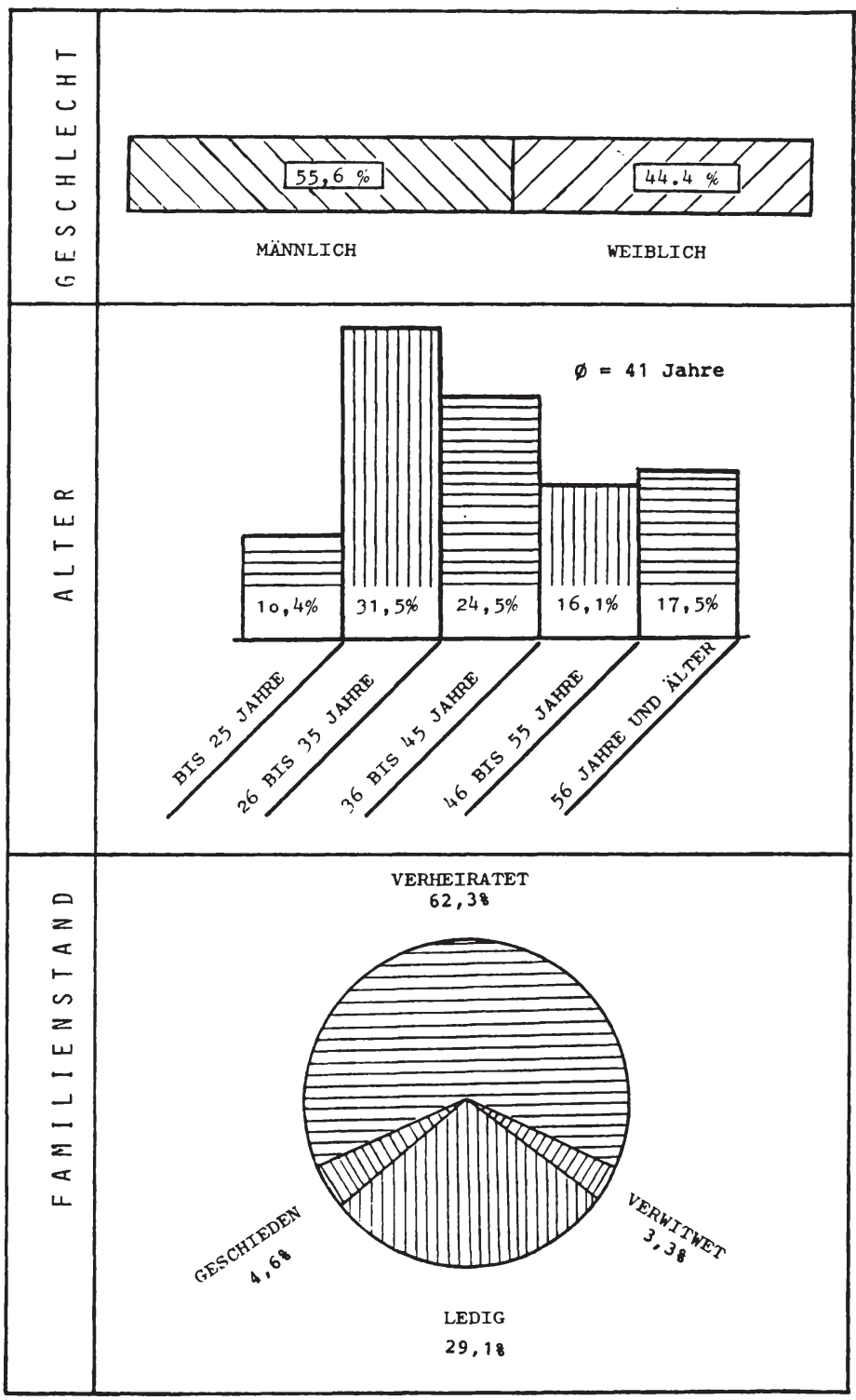

Abb. 67: Demographische Struktur der befragten Antiquitätenkäufer (Teil I) 


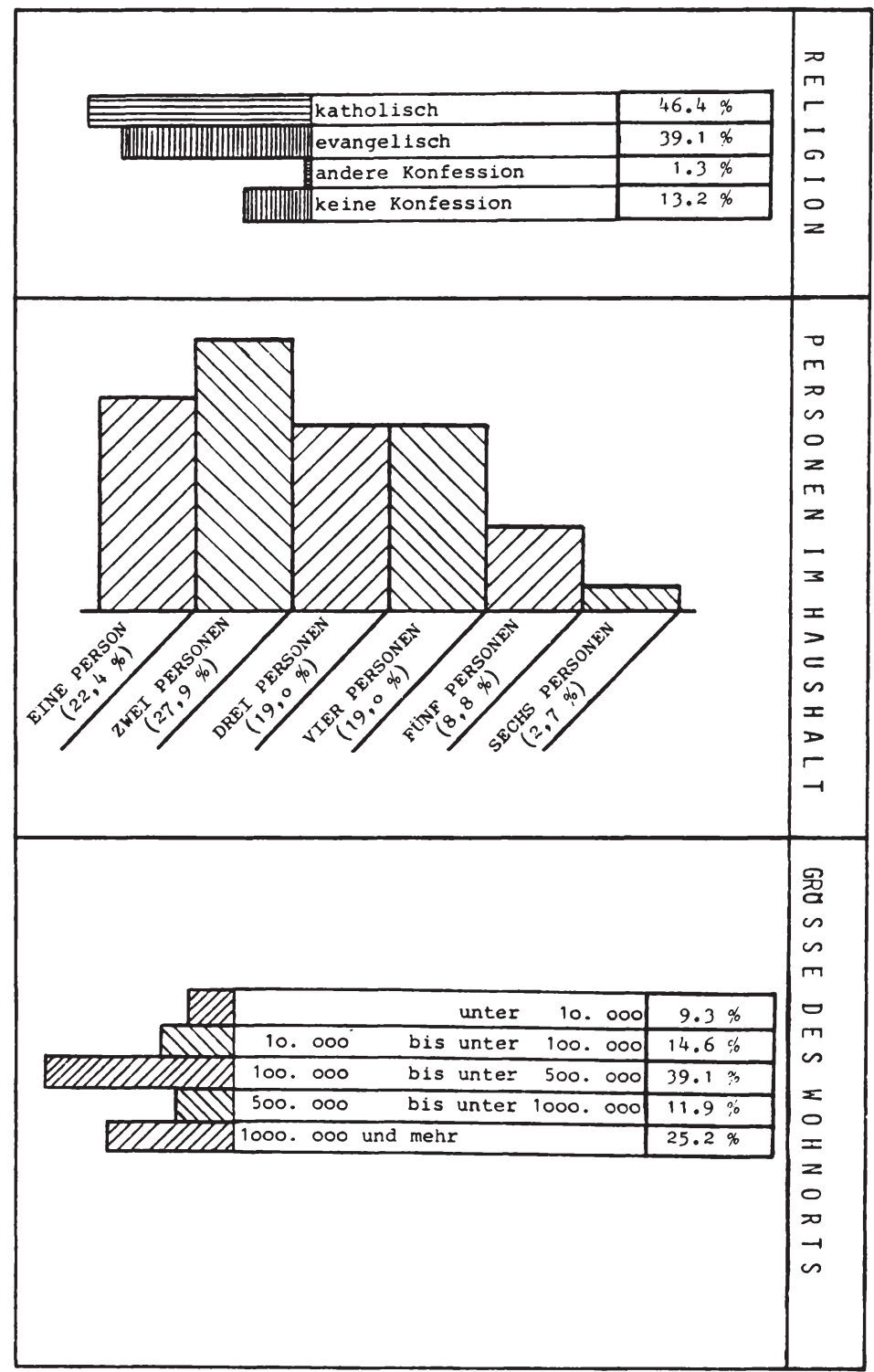

Abb. 67: Demographische Struktur der befragten Antiquitätenkäufer (Teil II) 
5000 und mehr

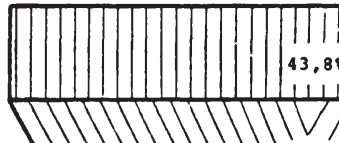

3000 bis unter 5000

1000 bis unter 3000
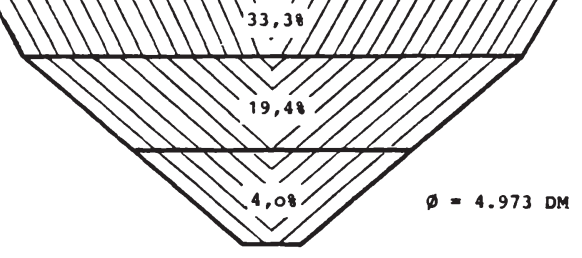

unter 1000

ABGESCHLOSSENESCHULBILDUNG
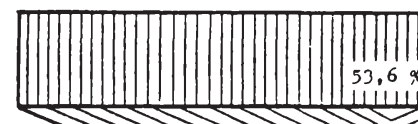

ABITUR, REIFEPRÜFUNC

WEITERFIJHRENDE SCHULE DHNE ABITUR

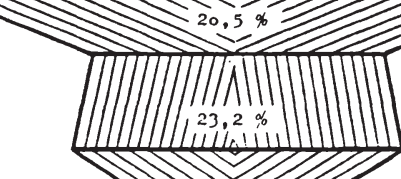

VOLKSSCHULE MIT ABGESCHLOSSENER LEHRE

VOLKSSCHULE OHNE ABGESCLOSSENE LEHRE

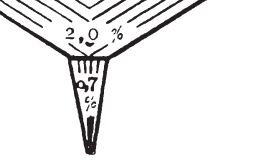

Abb. 68: Sozioökonomische Struktur der befragten Antiquitätenkäufer ( Teil I) 
PERSONEN MIT EI GENEMEINKOMIIE iV

I M HAUSHALT
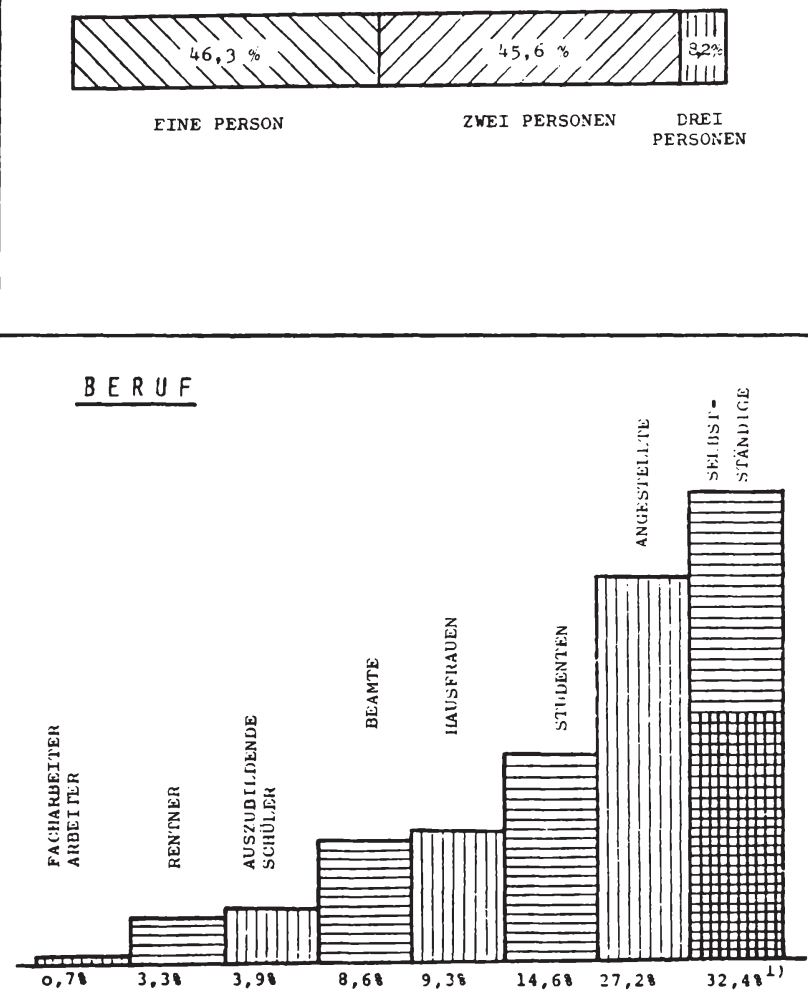

1) $=17,2$ in freien Berufen

Abb. 68: Sozioökonomische Struktur der befragten Antiquitätenkäufer (Teil II) 
Ohne auf die erhobenen Variablen detailliert einzugehen, können folgende demographische Besonderheiten und Schwerpunkte festgehalten werden:

(1) Eine Uberproportionalität männlicher Antiquitätenkäufer, die unter Berücksichtigung des unterproportionalen Anteils von Männern an der Gesamtbevölkerung zusätzliche Bedeutung gewinnt.

(2) Eine stark ausgeprägte Käuferschicht im Alter zwischen 26 und 35 Jahren, einer Entwicklungsphase, in der die meisten Ausbildungswege abgeschlossen sind,und eine familiäre und berufliche Etablierung stattindet.

(3) Eine nicht unbedeutende Käuferschicht bei den über 56 Jahre alten Menschen, einem Lebensabschnitt, in dem in der Regel ein Rückzug aus dem beruflichen in ein privates Leben mit entsprechenden Zeit- und Interessenumverteilungen (Hobby) stattfindet.

(4) Ein hoher Anteil verheirateter und in Mehrpersonenhaushalten lebender Antiquitätenkäufer, wodurch sich auf die Familie als warenspezifisches Umfeld von Antiquitätenkäufern schließen läßt.

Ergänzend zeigt der empirische Befund für die sozioökonomischen Variablen folgende Auffälligkeiten:

(1) Eine eindrucksvolle Dominanz hochverdienender Antiquitätenkäufer mit einem Durchschnittseinkommen (Netto-Haushaltseinkommen) von annähernd $5.000 \mathrm{DM}$ monatlich.

(2) Ein im gesamten Bevölkerungsvergleich sehr hohes Bildungsniveau mit über 50 \& Hochschulabsolventen und weiteren ca. $21 \%$ Abiturienten.

(3) Ein ebenso herausragendes berufliches Gesamtniveau unter den Antiquitätenkäufern, wobei Selbständige $(32,4 \%)$ und darunter insbesondere selbständige in freien Berufen (Ärzte, Rechtsanwälte etc. 17,2 \%) eine besondere Bedeutung besitzen. 
A n h a $\mathrm{n}$ II

A. Abbildungen

Seite

Abb. 1: PL/I Steuerprogramm zum Ausdruck von Faktorwerten und Faktor-Mittelwerten einzelner Käufergruppen

Abb. 2: Fortran IV - Clusterungsprogramm

B. Tabellen

Tab. 1: Isolierte Erklärungsbeiträge motivationaler Dispositionen und motivationaler Reize für einzelne Motivationsinhalte - Ergebnisüberblick (voroptimierender) multipler stufenweiser Regressionsanalysen

Tab. 2: Der Einfluß der "Geschlechtszugehörigkeit" auf die Käufermotivation - Ergebnisüberblick varianzanalytischer Prüfung

Tab. 3: Der Einfluß von "Altersstufen" auf die Käufermotivation - Ergebnisüberblick varianzanalytischer Prüfung

Tab. 4: Der Einfluß der "Schulbildung" auf die Käufermotivation - Ergebnisüberblick varianzanalytischer Prüfung

Tab. 5: Der Einfluß der "beruflichen Tätigkeit" auf die Käufermotivation - Ergebnisüberblick varianzanalytischer Prüfung

Tab. 6: Der Einfluß von "Einkommensklassen" auf die Käufermotivation - Ergebnisüberblick varianzanalytischer Prüfung 
Tab. 7: Der Einfluß der "Entstehung des Interesses" auf die Käufermotivation - Ergebnisüberblick varianzanalytischer Prüfung

Tab. 8: Kennzeichnung alternativer Clusterverfahren und ihrer Ergebnisse bei der Bildung von Motivationstypen

Tab. 9: Diskrimination von Motivationstypen auf der Grundlage von Einzelmotivationen - Ergebnisüberblick multipler schrittweiser Diskriminanzanalysen bei Variation der Inklusionsparameter

Tab. 10: Bedeutung einzelner Diskriminanzfunktionen zur Trennung von Motivationstypen auf der Grundlage von Einzelmotivationen (MOT DISC 4)

Tab. 11: Positionskoordinaten der Motivationskonfigurationen im 2-dimensionalen ("reduzierten") Diskriminanzraum

Tab. 12: Kennzahlen der Motivationskonfigurationen

Tab. 13: Diskrimination von Motivationstypen auf der Grundlage motivationaler Bestimmungsfaktoren Ergebnisüberblick multipler schrittweiser Diskriminanzanalysen bei Variation der Inklusionsparameter

Tab. 14: Die Bedeutung personaler-, waren- und situationsbezogener motivationaler Bestimmungsfaktoren zur Diskrimination von Motivationstypen - Ergebnisüberblick einer multiplen schrittweisen Diskriminanzanalyse (PRÄ DISC 2)

Tab. 15: Erklärungsbeiträge von Motivationen zur Kaufrelevanz von Antiquitäten-Merkmalen - Ergebnisüberblick multipler schrittweiser Regressionsanalysen 
Tab. 16: Erklärungsbeiträge von Motivationen zum Interesse an Sach- und Sammelgebieten - Ergebnisüberblick multipler schrittweiser Regressionsanalysen

Tab. 17: Erklärungsbeiträge von Motivationen für Anforderungen von Antiquitätenkäufern an Einkaufsstätten - Ergebnisüberblick multipler schrittweiser Regressionsanalysen

Tab. 18: Erklärungsbeiträge von Motivationen zum Informationsstil - Ergebnisüberblick multipler schrittweiser Regressionsanalysen

Tab. 19: Erklärungsbeiträge von Motivationen zum thematischen Informationsinteresse - Ergebnisüberblick multipler schrittweiser Regressionsanalysen

Tab. 20: Erklärungsbeiträge von Motivationen zur Nutzung von Informationsquellen - Ergebnisüberblick multipler schrittweiser Regressionsanalysen 


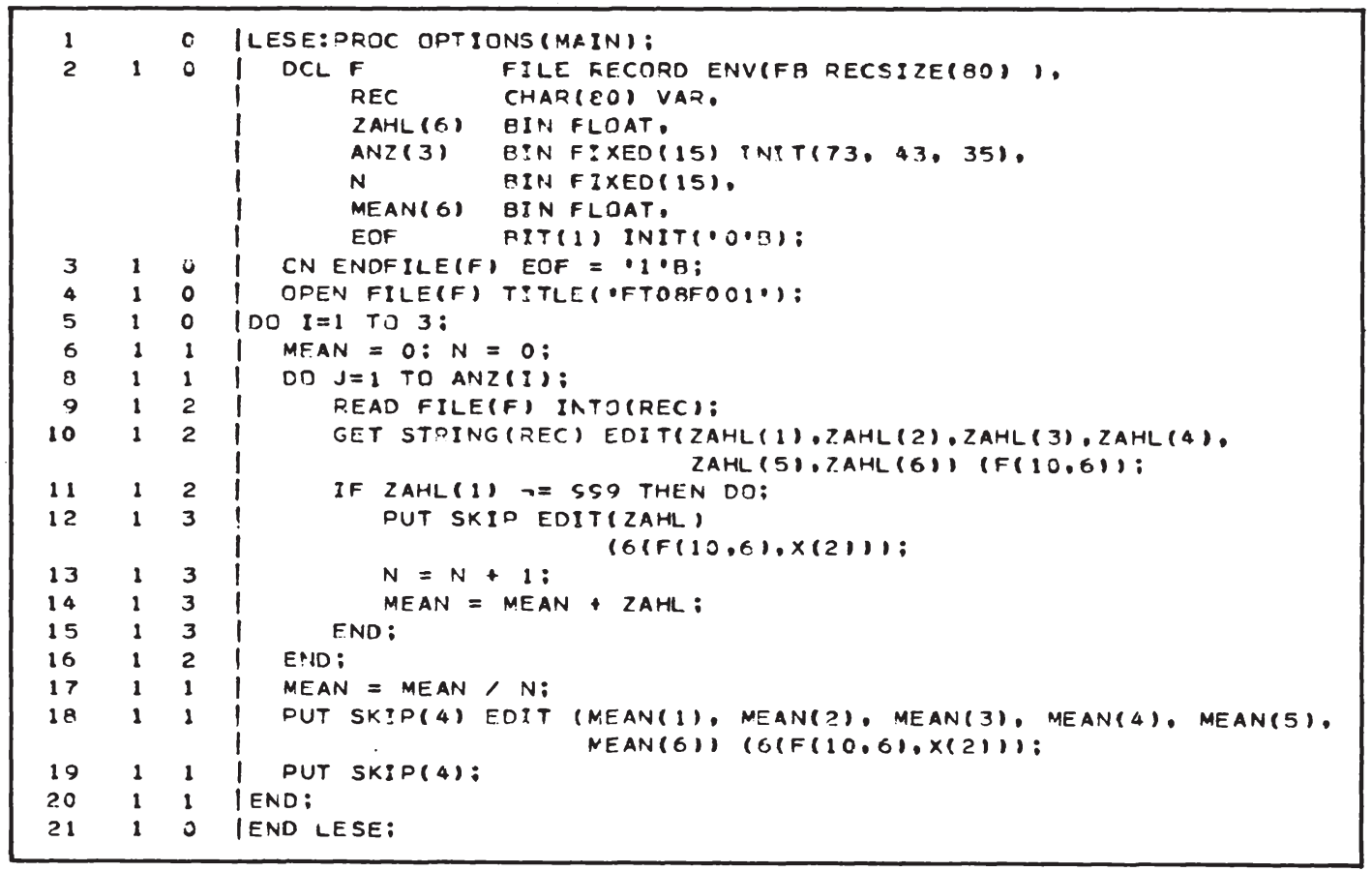

Abb. 1: PL/I Steuerprogramm zum Ausdruck von Faktorwerten und Faktorwert-Mittelwerten einzelner Käufergruppen 
KEAL C $(155), 0(16000), X(4000), C M(600), C V(600)$

KEAL $Y(4000), S 1(30)$, EW $(30), V(900), R(500), 0 S[G(30)$

KEAL FWERT

INTEGER NI (155), N2 (155), V $3(155), N 4(155)$, NS (155), D INN (9000)

INTEGER NP(155),KCT(151), VARFUN(20), SPSS, GRENLE/0/, VEK(2U)

INTEGER ZUGE (50)

LUGICAL N(155)

C

C EIHGAUE:

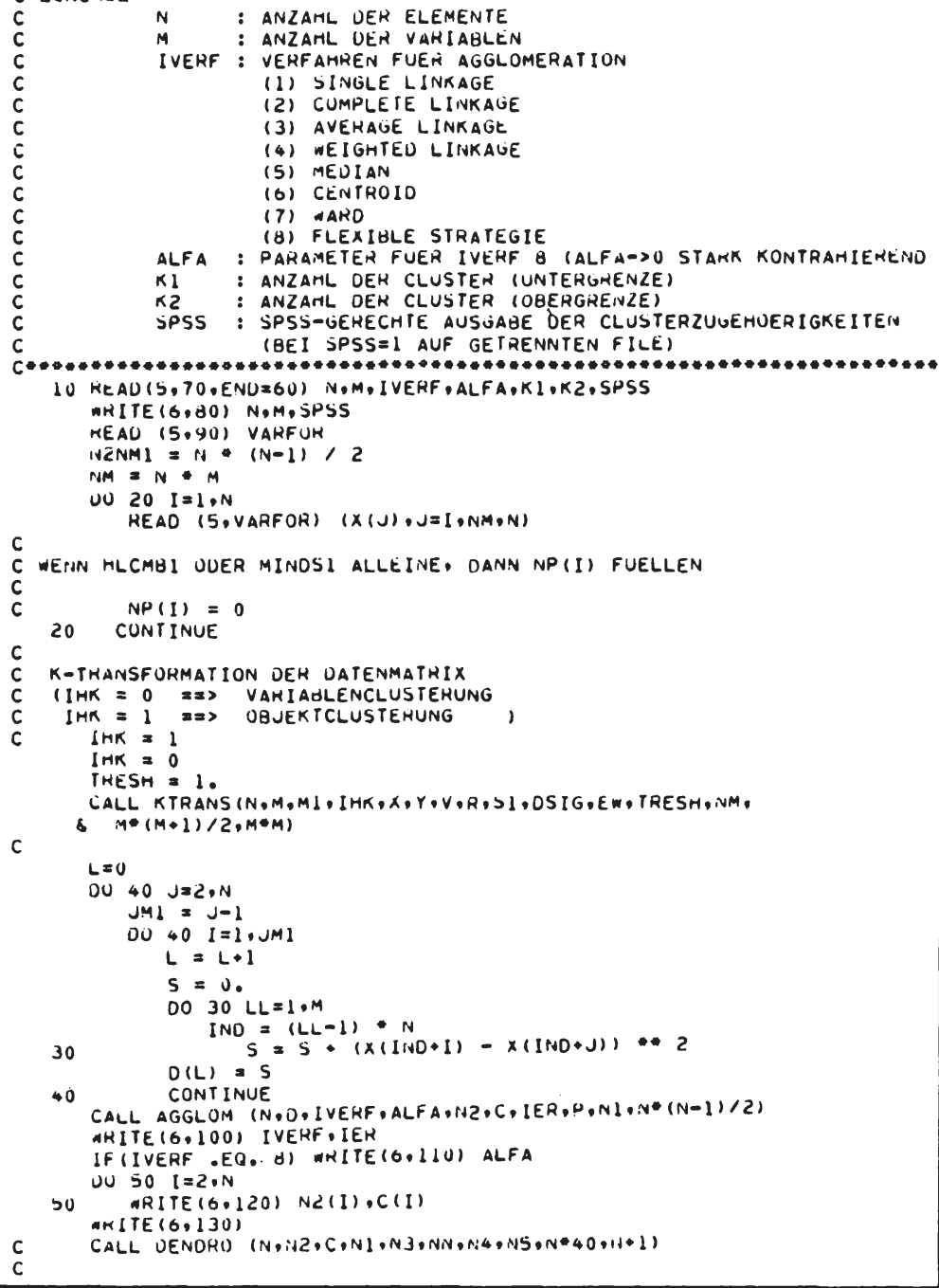

Abb. 2: Fortran IV - Clusterprogramm (Teil I) 
C FALLJ CLUSTERUNG DER VAKIABLEN

CALL DENDHO $(M, N 2, C, N 1, N 3, N N$, IV $4, N 5, M * 40, N+1)$

IF (1.EO. 1) GOTO OO

$M M=M \oplus N$

$M K=M * K$

UO $200 \quad K=K 1, K 2$

CALL GHOUP (H,N2,C,NI,N3,N4,NP,K,LEVEL, N+ I)

CALL MINOSI (N,M,K, $, O N P, C, H, C V, K C T, S P W, I N M, M K)$

CALL HLCME1 (N,M,K,X,NP,CM,CV,KCT,SPW,NM,MK)

WRITE $(6,140) \mathrm{K}$

DO $5000 \quad I 1=1, K$

KSUM $=0$

GRENZE $=0$

WRITE $(6,5100)$ II

DO $6000 \mathrm{Jl}=1, \mathrm{~N}$

IF (NPIJI) .NE. I1) GDIO 6000

KSUM $=$ KSUM 1

GRENZE = GRENZE • ।

VEK (GRENLE) = Jl

IF (GHENLE .LT. 20$)$ GOTO 6000

WRITE $(0,5110)$ (VEK $(I 2), 12=1,20)$

OU00 GRENZE $=0$

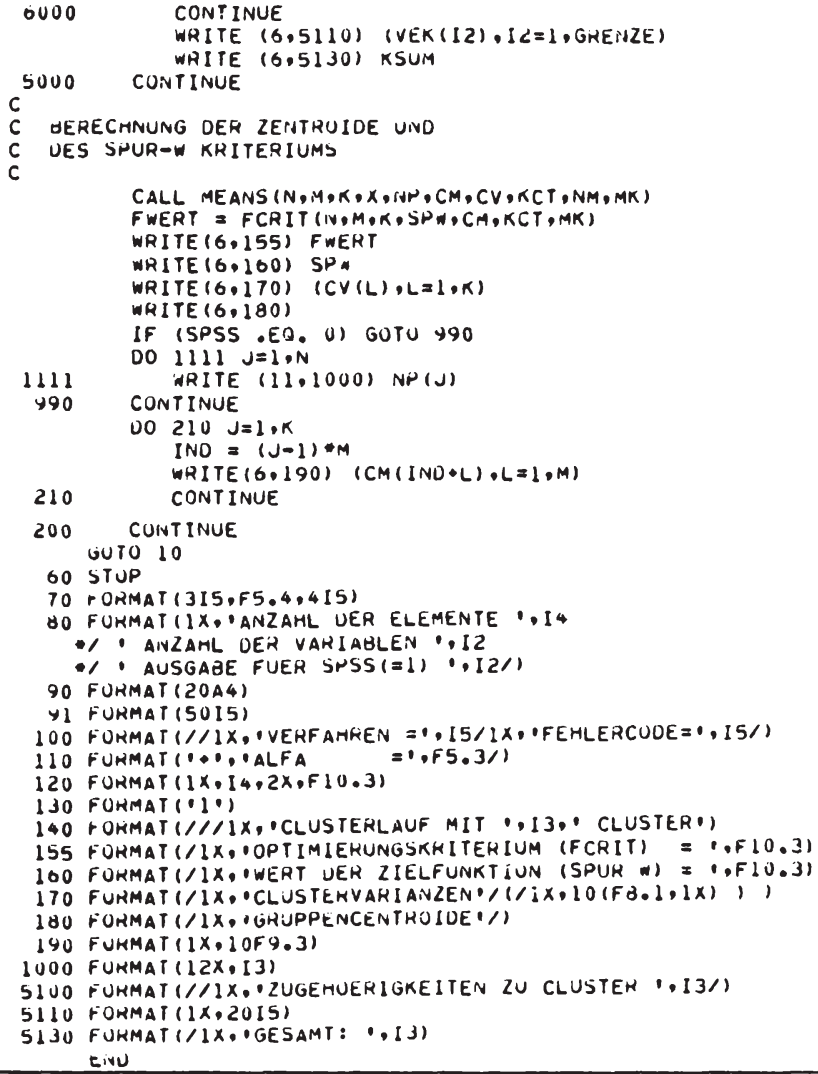

Abb. 2: Fortran IV - Clusterprogramm (Teil II) 


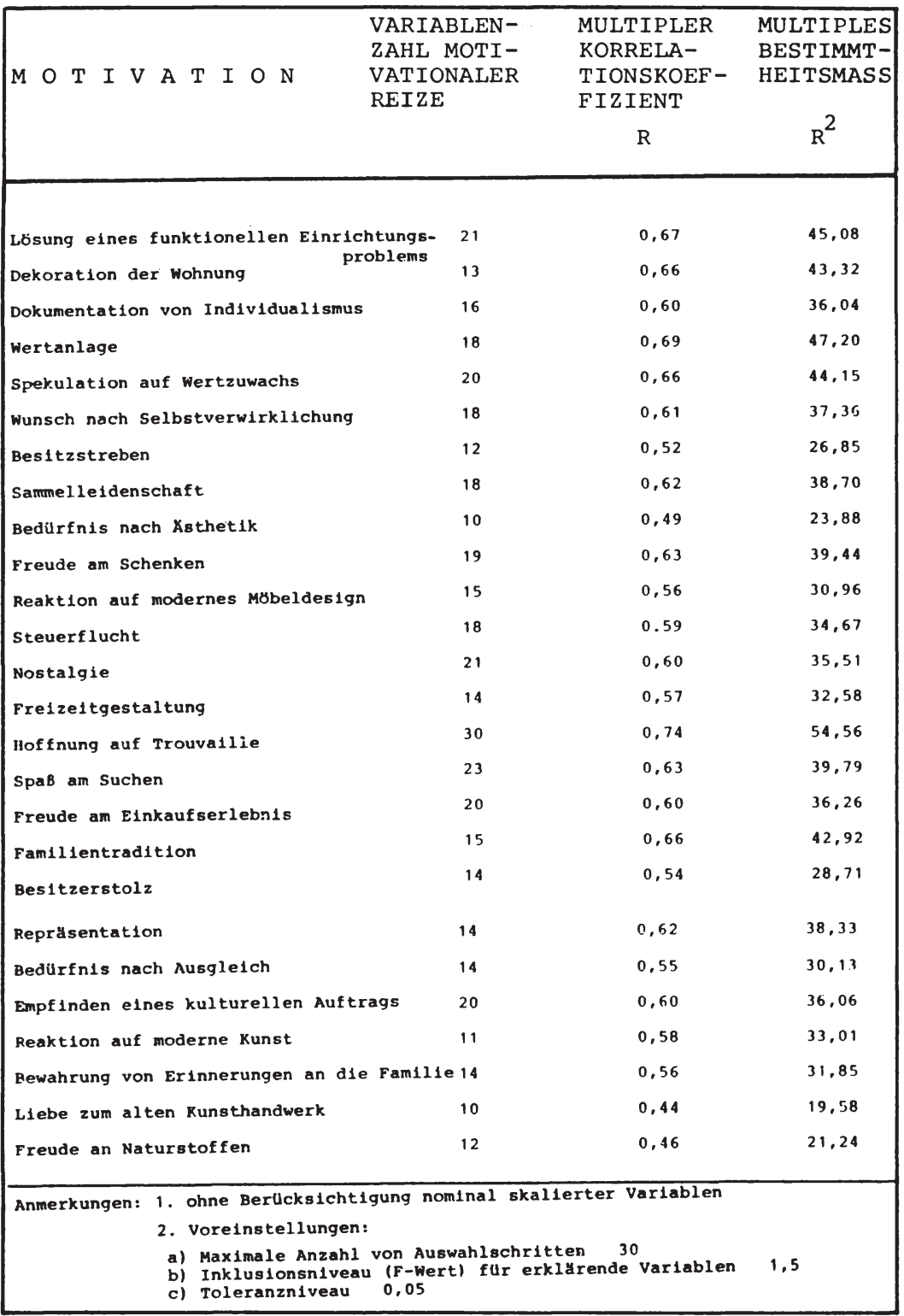

Tab. 1: Isolierte Erklärungsbeiträge motivationaler Dispositionen und motivationaler Reize für einzelne MotivationsinhalteErgebnisüberblick (voroptimierender) multipler stufenweiser Regressionsanalysen (Teil I) 


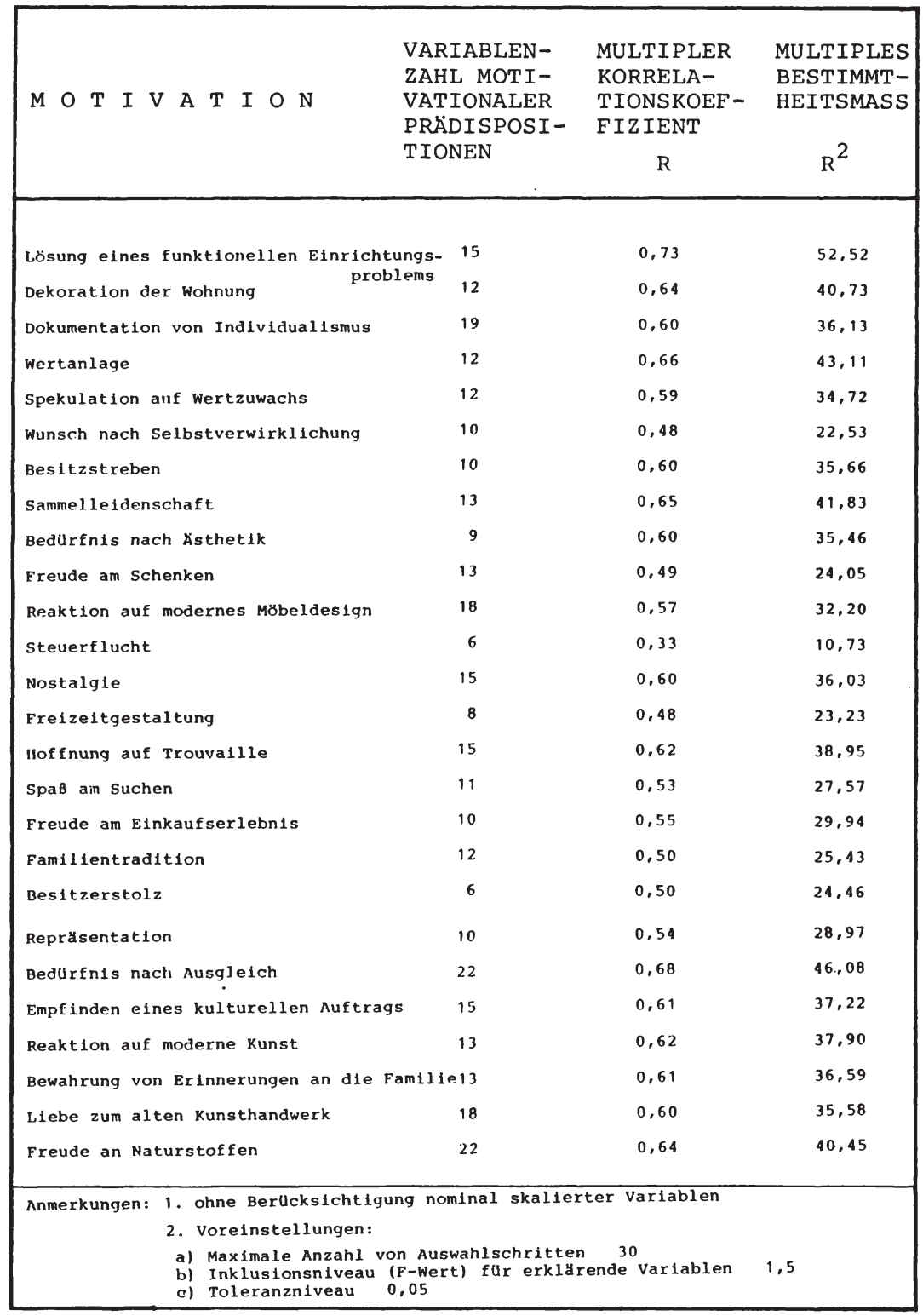

Tab. 1: Isolierte Erklärungsbeiträge motivationaler Dispositionen und motivationaler Reize für einzelne Motivationsinhalte Ergebnisüberblick (voroptimierender) multipler stufenweiser Regressionsanalysen (Teil II) 


\begin{tabular}{|c|c|c|c|c|c|}
\hline GESCHLECHT & 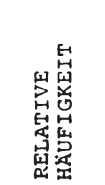 & 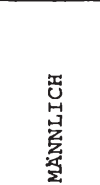 & 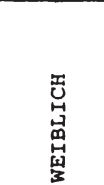 & \multirow{2}{*}{\multicolumn{2}{|c|}{$\begin{array}{l}\text { SIGNIFIKANZNIVEAU } \\
\text { EINFACHER } \\
\text { VARIANZANALYSEN }\end{array}$}} \\
\hline MOTIVATION & & 57,6 & 42,4 & & \\
\hline MITTELWERTE ${ }^{1)}$ & GESAMT & $\begin{array}{c}\text { GRUPPE } \\
1\end{array}$ & $\begin{array}{c}\text { GRUPPE } \\
2\end{array}$ & $\ldots \infty$ & SYMBOL $^{21}$ \\
\hline $\begin{array}{l}\text { Rewahrung von Erinnerungen } \\
\text { an die Familie }\end{array}$ & 3,64 & 3,98 & 3,22 & 0,001 & $\star \star \star \star \star$ \\
\hline Wertanlage & 3,38 & 3,11 & 3,72 & 0,003 & * * * \\
\hline $\begin{array}{l}\text { Spekulation } \\
\text { auf Wertzuwaclis }\end{array}$ & 4,26 & 4,08 & 4,48 & 0,007 & *** \\
\hline Familientradition & 3,58 & 3,82 & 3,28 & 0,022 & $\star \star$ \\
\hline Bedürfnis nach Ästhetik & 2,17 & 2,36 & 1,93 & 0,029 & * * \\
\hline Repräsentation & 4,54 & 4,44 & 4,67 & 0.096 & * \\
\hline
\end{tabular}

1) Die Mittelwerte beziehen sich auf eine 5-stufige Skalierung von (1) sehr wichtig bis (5) gar nicht wichtig.

2) $\quad a=$ Irrtumswahrscheinlichkeiten

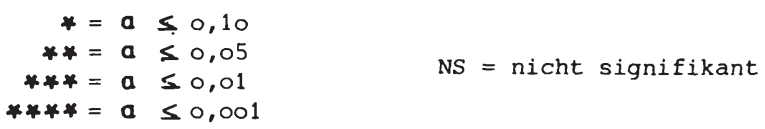

Tab. 2: Der Einfluß der "Geschlechtszugehörigkeit" auf die Käufermotivation - Ergebnisüberblick varianzanalytischer Prüfung 


\begin{tabular}{|c|c|c|c|c|c|c|c|c|c|}
\hline 4 & 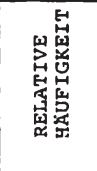 & $\begin{array}{l}\sigma \\
\stackrel{2}{\infty} \\
\stackrel{\infty}{\infty}\end{array}$ & 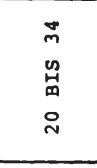 & 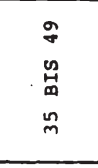 & $\begin{array}{l}\text { : } \\
\text { on } \\
\text { 㽞 } \\
\text { in }\end{array}$ & $\begin{array}{l}n \\
0 \\
\\
\end{array}$ & \multirow{2}{*}{\multicolumn{2}{|c|}{$\begin{array}{l}\text { SIGNIFIKANZNIVEAU } \\
\text { EINFACHER } \\
\text { VARIANZANALYSE }\end{array}$}} & \multirow[t]{2}{*}{$\begin{array}{l}\text { GRUPPEN MIT } \\
\text { SIGNIFIKANT } \\
\text { UNTERSCH IEDLICHER } \\
\text { MOTIVATIONSAUSPRXGUNG }{ }^{31}\end{array}$} \\
\hline & & 9,9 & 35,1 & 28,5 & 21,2 & 5,3 & & & \\
\hline MI'TTELWERTE ${ }^{1)}$ & GESAMT & $\begin{array}{c}\text { GRUPPE } \\
1\end{array}$ & $\begin{array}{c}\text { GRUPPE } \\
2\end{array}$ & $\begin{array}{c}\text { GRUPPE } \\
3\end{array}$ & $\begin{array}{c}\text { GPUPPE } \\
4\end{array}$ & $\begin{array}{c}\text { GRUPPE } \\
5\end{array}$ & & SYMBOL ${ }^{21}$ & \\
\hline Dekoration der Wohnung & 2,40 & 2,80 & 2,04 & 2,65 & 2,53 & 2,13 & 0,067 & * & $2 \neq 1,3^{41}$ \\
\hline Besitzstreben & 3,08 & & 3,36 & & & 2,13 & 0,142 & NS & $2 \neq 5$ \\
\hline Sammelleldenschaft. & 3,77 & & 4,04 & 3,40 & & & 0,252 & NS & $2 \neq 3$ \\
\hline Familientradition & 3,58 & 3,40 & 4,04 & 3,58 & 3,09 & 2,88 & 0,022 & $\star \star$ & $2 \neq 4,5$ \\
\hline Reprăsentation & 4,54 & 4,80 & & & & 4,00 & 0,288 & NS & $1 \not 5$ \\
\hline Reaktion auf moderne Kunst & 3,82 & & 3,92 & 4,07 & & 2,88 & 0,173 & NS & $5 \neq 2,3$ \\
\hline $\begin{array}{l}\text { Bewahrung von Erinnerungen } \\
\text { an die Fam1lie }\end{array}$ & 3,64 & 3,67 & 4,19 & 3,72 & 2,88 & 2,63 & 0,000 & $\star \star \star * \star$ & $4,5 \neq 2,3$ \\
\hline Freude an Naturstoffen & 2,82 & & 2,55 & 3,23 & & & 0,117 & NS & $2 \neq 3$ \\
\hline
\end{tabular}

Tab. 3: Der Einfluß von "Altersstufen" auf die Käufermotivation - Ergebnisüberblick varianzanalytischer Prüfung

Anmerkungen $\mathrm{zu}$ den Tabellen $3,4,5,6,7$

1) und 2) vgl. Tabelle 2

3) auf der Grundlage eines Least-Significant-Difference-Test (Signifikanzniveau 95\%)

4) Lesebeispiel: Bei Antiquitätenkäufern der Gruppe 2 (Alter zwischen 20 bis 34 Jahre) ist die Motivation "Dekoration der Wohnung" signifikant geringer als Kaufgrund ausgeprägt (vgl. Mittelwerte) als bei Käufern der Gruppen 1 (Alter bis 19 Jahre) und 3 (Alter zwischen 35 und 49 Jahre) 


\begin{tabular}{|c|c|c|c|c|c|c|c|c|c|}
\hline \multirow[t]{2}{*}{$\begin{array}{l}\text { ABGESCHLOSSENE } \\
\text { SCHULBILDUN }\end{array}$} & \multirow[t]{2}{*}{ 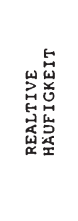 } & 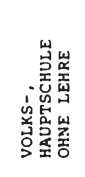 & 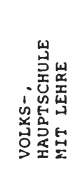 & 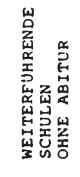 & 总 & 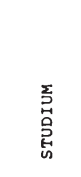 & \multirow{2}{*}{\multicolumn{2}{|c|}{$\begin{array}{l}\text { SIGNIFIKANZNIVEAU } \\
\text { EINFACHER } \\
\text { VARIANZANALYSE }\end{array}$}} & \multirow[t]{2}{*}{$\begin{array}{l}\text { GRUPPEN MIT } \\
\text { SIGNIFIKANT } \\
\text { UNTERSCHIEDLICHER } \\
\text { MOTIVATIONSAUSPRAGUNG }\end{array}$} \\
\hline & & 0.7 & 2,0 & 22,5 & 20,5 & 54,3 & & & \\
\hline MITTELWERTE $"$ & GESAMT & $\begin{array}{c}\text { GRUPPE } \\
1\end{array}$ & $\begin{array}{c}\text { GRUPPE } \\
2\end{array}$ & $\begin{array}{c}\text { GRUPPE } \\
3\end{array}$ & $\begin{array}{c}\text { GRUPPE } \\
4\end{array}$ & $\begin{array}{c}\text { GRUPPE } \\
5\end{array}$ & $\alpha$ & SYMBOL 21 & \\
\hline $\begin{array}{l}\text { Lösung eines funktionalen } \\
\text { Einrichtungsproblems }\end{array}$ & 2,03 & 1,00 & 1.33 & 2,56 & 2,03 & 1,85 & 0.053 & * & $5 \neq 3^{41}$ \\
\hline Wertanlage & 3,38 & & 1,67 & 3,26 & 3,42 & 3,46 & 0.162 & NS & $2 \neq 3,4,5$ \\
\hline $\begin{array}{l}\text { Spekulation } \\
\text { auf Wertzuwachs }\end{array}$ & 4,26 & 5,00 & 2,67 & 4,06 & 4,25 & 4,39 & 0,008 & $\cdots *$ & $2 \neq 1,3,4,5$ \\
\hline Besitzstreben & 3.08 & & & 2,65 & & 3,26 & 0,126 & NS & $3 \neq 5$ \\
\hline Nostalgie & 3.44 & 1,00 & & 3,15 & 3,84 & & 0,128 & NS & $4 \neq 1,3$ \\
\hline Freizeltgestaltung & 3,36 & & & 2,97 & & 3,55 & 0,215 & NS & $3 \neq 5$ \\
\hline Hof fnung auf Trouvallle & 3,36 & & 2,00 & & & 3.57 & 0,116 & NS & $2 \neq 5$ \\
\hline$S_{\text {paB }}$ am Suchen & 2,55 & 1,00 & 1,33 & 2,26 & 2,90 & 2,60 & 0,086 & * & $4 \neq 2,3$ \\
\hline $\begin{array}{l}\text { Freude am } \\
\text { Einkaufserlebnis }\end{array}$ & 3,43 & 1,00 & 2,33 & 3,18 & 3,23 & 3.68 & 0,033 & $" *$ & $1 \neq 5$ \\
\hline Familicntradition & 3,58 & & 5,00 & 3.29 & & & 0,185 & NS & $2 \neq 3$ \\
\hline Besitzerstolz & 3.15 & & & 2.79 & & 3,40 & 0,112 & NS & $3 \neq 5$ \\
\hline $\begin{array}{l}\text { Empfinden eines } \\
\text { kulturellen Auftrags }\end{array}$ & 3,64 & 3,00 & 3,00 & 3,35 & 3,32 & 3,90 & 0,094 & * & $5 \neq 3,4$ \\
\hline $\begin{array}{l}\text { Keaktion auf moderne kunst } \\
\text { Bewahrung von Erinnerungen } \\
\text { an die Familie }\end{array}$ & 3.82 & 1,00 & 4,33 & 3.41 & 3,71 & 4,02 & 0.159 & NS & $\begin{array}{l}3 \neq 5 \\
1 \neq 2\end{array}$ \\
\hline
\end{tabular}
Tab. 4: Der Einfluß der "Schulbildung" auf die Käufermotivation - Ergebnisüberblick
varianzanalytischer Prüfung 


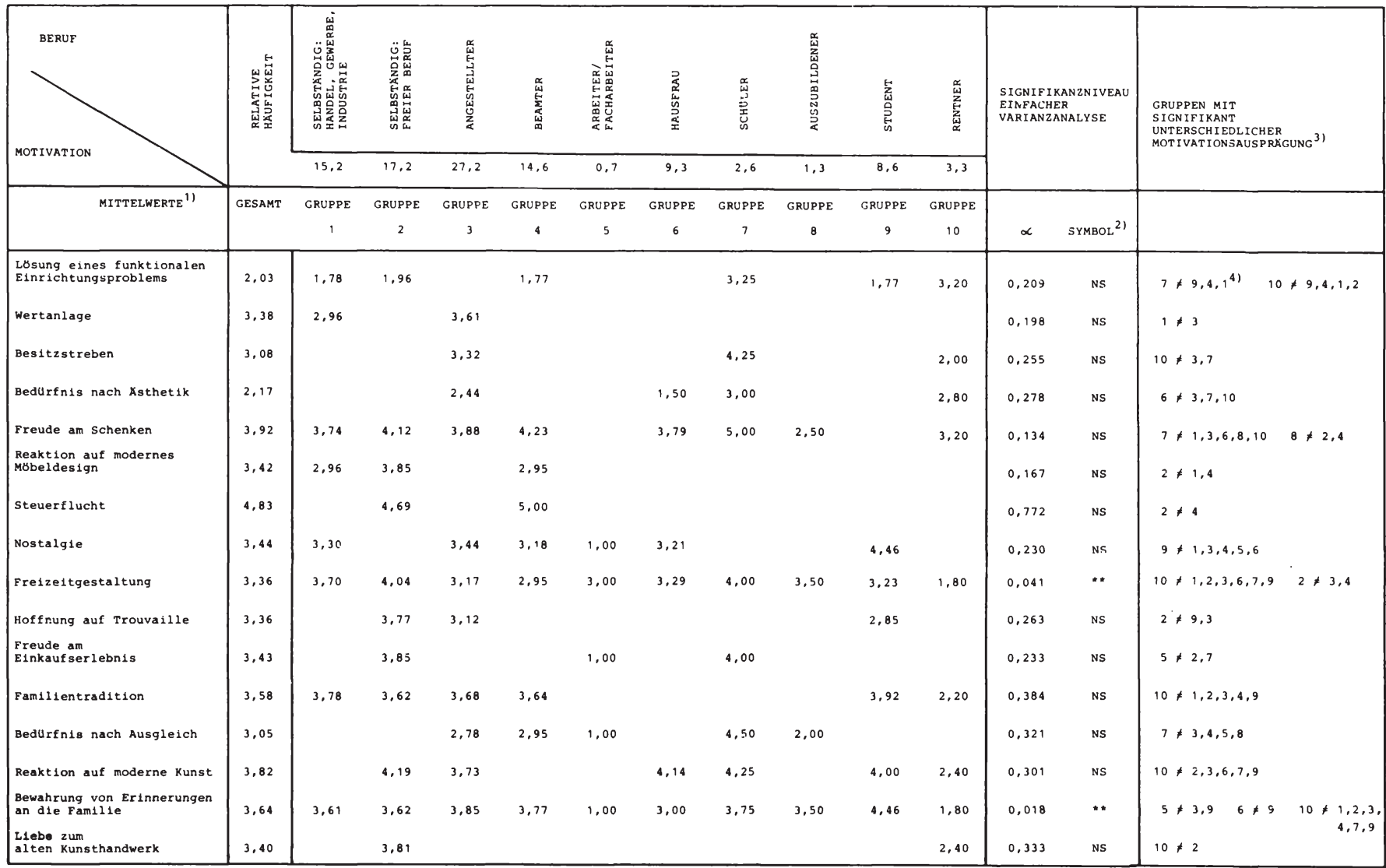

Tab. 5: Der Einfluß der "beruflichen Tätigkeit" auf die Käufermotivation - Ergebnisüberblick varianzanalytischer Prüfung 


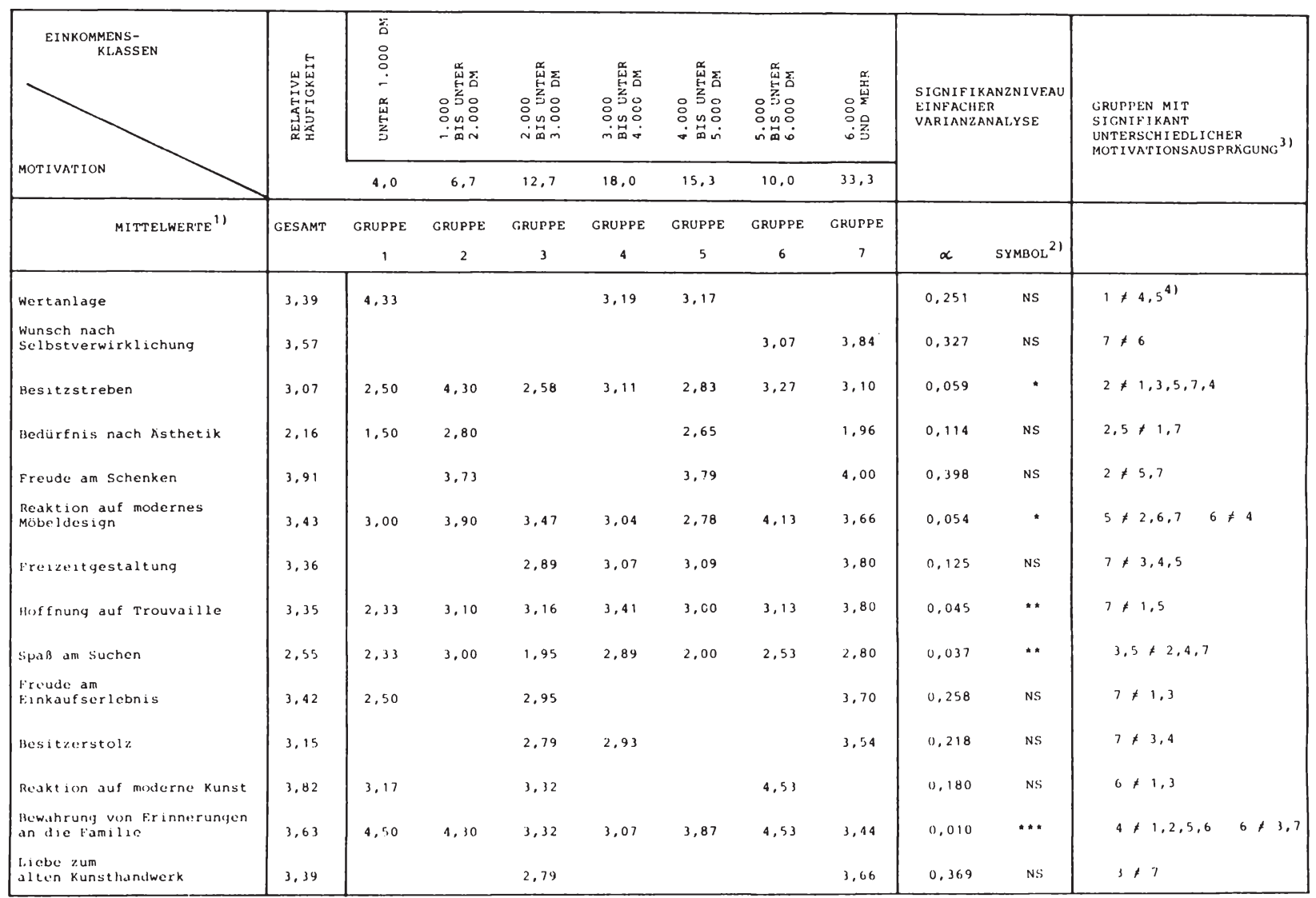

Tab. 6: Der Einfluß von "Einkommensklassen" auf die Käufermotivation - Ergebnisüberblick varianzanalytischer Prüfung 


\begin{tabular}{|c|c|c|c|c|c|c|c|c|c|c|c|c|c|}
\hline $\begin{array}{c}\text { ENTSTEHUNG DES } \\
\text { INTERESSES AN } \\
\text { ANTIOUITKTEN }\end{array}$ & 点 & 离 & 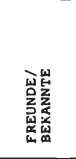 & 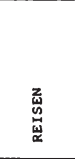 & 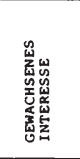 & 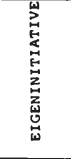 & 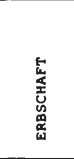 & 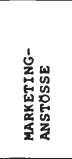 & 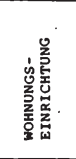 & $\begin{array}{l}\text { 岕 } \\
\text { 点 } \\
\text { 总 } \\
0\end{array}$ & \multirow{2}{*}{\multicolumn{2}{|c|}{$\begin{array}{l}\text { SIGNIFIKANZNIVEAU } \\
\text { EINFACHER } \\
\text { VARIANZANALYSE }\end{array}$}} & \multirow{2}{*}{$\begin{array}{l}\text { GRUPPEN MIT } \\
\text { SIGNIFI IANT } \\
\text { UNTERSCHIDLICHER } \\
\text { MOTIVATIONSAUSPRGUNG }\end{array}$} \\
\hline MOTIVATION & & 31,8 & 18,2 & 5,4 & 8,1 & 8,1 & 9,5 & 6,1 & 8,1 & 4,7 & & & \\
\hline MITTELWERTE 11 & GESAMT & $\begin{array}{c}\text { GRUPPE } \\
1\end{array}$ & $\begin{array}{c}\text { GRUPPE } \\
2\end{array}$ & $\begin{array}{c}\text { GRUPPE } \\
3\end{array}$ & $\begin{array}{c}\text { GRUPPE } \\
4\end{array}$ & $\begin{array}{c}\text { GRUPPE } \\
5\end{array}$ & $\begin{array}{c}\text { GRUPPE } \\
6\end{array}$ & $\begin{array}{c}\text { GRUPPE } \\
7\end{array}$ & $\begin{array}{c}\text { GRUPPE } \\
8\end{array}$ & $\begin{array}{c}\text { GRUPPE } \\
9\end{array}$ & $\alpha$ & SYMBOL $^{21}$ & \\
\hline Dekoration der wohnung & 2,39 & 2,64 & & & & 1,67 & & & & 2,86 & 0,280 & NS & $5 * 1,9^{41}$ \\
\hline wertanlage & 3,35 & & & 2,50 & & & & 3,78 & & & 0,471 & NS & $3 \neq 7$ \\
\hline $\begin{array}{l}\text { Spekulation } \\
\text { auf wertzuwachs }\end{array}$ & 4,24 & 4,32 & & 3,88 & 3,67 & & 4,71 & & 4,42 & & 0,191 & NS & $4 \neq 1,8 \quad 6 \neq 3,4$ \\
\hline $\begin{array}{l}\text { Wunsch nach } \\
\text { Selbstverwirk11chung }\end{array}$ & 3,54 & 3,87 & 3,70 & 2,63 & 3,33 & 3,33 & 3,07 & 4,00 & 3,00 & 3,71 & 0,065 & • & $1 * 3,6,8 \quad 3 \neq 2,7$ \\
\hline Besitzstreben & 3,04 & & & 2,88 & & & 2,93 & 4,00 & & & 0,652 & Ns & $7 * 3,6$ \\
\hline Sammelleidenschaft & 3,75 & & 4,11 & & & & 3,14 & 4,44 & 3,17 & & 0,206 & NS & $6 * 2,7 \quad 7 * 8$ \\
\hline Bedurfnis nach Asthetik & 2,18 & & & & & & 1,71 & 2,78 & & & 0,534 & NS & $6 * 7$ \\
\hline Freude am Schenken & 3,91 & & 4,04 & & & 4,17 & 3,21 & 4,56 & & 4,29 & 0,173 & Ns & $6+2,5,7,9$ \\
\hline Freizeltgestaltung & 3,35 & & & & 2,83 & 4,00 & & & & & 0,356 & NS & $4 * 5$ \\
\hline Hof fnung auf Trouvallle & 3,37 & 3,68 & 3.64 & 3,75 & 2,58 & 3,58 & 3,14 & 2,89 & 4.00 & 3,14 & 0,066 & • & $4+1,3,8$ \\
\hline Spar an Suchen & 2,53 & & & & 1.83 & 3.17 & & & 2,92 & 1.71 & 0,196 & Ns & $5 * 4,9 \quad 8 * 4,9$ \\
\hline $\begin{array}{l}\text { Freude am } \\
\text { Einkauf serlebn1s }\end{array}$ & 3,41 & 3,75 & & 2.75 & 2,83 & & & 2,78 & & & 0,193 & NS & $1,3,4,7$ \\
\hline Familientradition & 3,61 & 2,83 & 4,33 & 3,88 & 3,83 & 4,08 & 2,57 & 4,22 & 4,50 & 4,29 & 0,000 & $\cdots$ & $\begin{array}{l}1 \neq 2,3,4,5,7,8,9 \\
6 \neq 2,3,4,5,7,8,9\end{array}$ \\
\hline Bes1tzerstolz & 3,14 & & & & 2,50 & & & & & 3,86 & 0,255 & Ns & $4 * 9$ \\
\hline $\begin{array}{l}\text { Empfinden eines } \\
\text { kulturelien Auftrags }\end{array}$ & 3,61 & & 3,82 & & 2,92 & & & & & & 0,472 & NS & $2 \neq 4$ \\
\hline $\begin{array}{l}\text { Bewahrung von Erinnerungen } \\
\text { an die Familie }\end{array}$ & 3,62 & 3,30 & 3,96 & 2,88 & 4,25 & 4,17 & 2,57 & 3,67 & 4,42 & 3,86 & 0,005 & $\cdots$ & $\begin{array}{l}1: 2,4,8,3 * 4,5,8 \\
6: 2,4,5,8,9\end{array}$ \\
\hline Freude on Naturstof $f$ en & 2,83 & & 2,59 & & & & & 2,11 & 2,50 & 3,86 & 0,340 & NS & $9 \neq 2,7,8$ \\
\hline
\end{tabular}

Tab. 7: Der Einfluß der "Entstehung des Interesses" auf die Käufermotivation Ergebnisüberblick varianzanalytischer Prüfung 


\begin{tabular}{|c|c|c|c|c|c|c|c|}
\hline \multicolumn{8}{|c|}{$\begin{array}{c}\text { OPTIMIERUNGSKRITERIEN FUR } \\
\text { CLUSTERZAHLEN } \\
\end{array}$} \\
\hline ALGORITHMUS & $\begin{array}{c}\text { PROGRAMM- } \\
\text { PAKET/-SPRACHE }\end{array}$ & $\begin{array}{l}\text { GENERALI- } \\
\text { SIERUNGS- } \\
\text { GRAD }\end{array}$ & SPUR-W & F-CRIT & $\begin{array}{l}\text { CLUSTER- } \\
\text { ZAHL }\end{array}$ & $\begin{array}{l}\text { CLUSTER- } \\
\text { GRÖSSEN / } \\
\text { HOMOGENITÄT }\end{array}$ & $\begin{array}{l}\text { INTERPRETIER- } \\
\text { BARKEIT }\end{array}$ \\
\hline SINCLE LINKAGE & & 24,92 & 3702 & 1,22 & 49 & - & - \\
\hline COMPLETE LINKAGE & & 21,34 & 4563 & 8.05 & 6 & $\varnothing$ & $\varnothing$ \\
\hline AVERAGE LIIJKAGE & CI.USTER- & 24,83 & 4711 & 6.89 & 6 & $\varnothing$ & $\varnothing$ \\
\hline $\begin{array}{l}\text { WEIGHTED AVERAGE } \\
\text { I,INKAGE }\end{array}$ & ANALYSE- & 20,03 & 3809 & 4,78 & 16 & - & - \\
\hline MEDIAN & $\begin{array}{l}\text { MUNS'TER } \\
\text { (CAM) }\end{array}$ & 22,20 & 3957 & 1,97 & 30 & - & - \\
\hline WARD & & 20,50 & 4697 & 11,81 & 4 & + & $\varnothing$ \\
\hline $\begin{array}{l}\text { FLEXIBLE } \\
\text { STRATEGIE }(\alpha=0,65)\end{array}$ & & 18,70 & 5271 & 3,87 & 5 & + & $\varnothing$ \\
\hline CENTROID & & 23,60 & 3530 & 1,99 & 38 & - & - \\
\hline
\end{tabular}

Tab. 8: Kennzeichnung alternativer Clusterverfahren und ihrer Ergebnisse bei der Bildung von Motivationstypen (Teil I) 


\begin{tabular}{|c|c|c|c|c|c|c|c|}
\hline \multicolumn{8}{|c|}{$\begin{array}{l}\text { OPTIMIERUIJGSKRITERIEN FUR } \\
\text { CLUS'I'ERZAHLEN }\end{array}$} \\
\hline AI.GORITHMUS & $\begin{array}{l}\text { PROGRAMM- } \\
\text { PAKET/-SPRACHE }\end{array}$ & $\begin{array}{l}\text { GENERALI- } \\
\text { SIERUNGS- } \\
\text { GRAD }\end{array}$ & SPUR-W & F-CRIT & $\begin{array}{l}\text { CLUSTER- } \\
\text { ZAHL }\end{array}$ & $\begin{array}{l}\text { CLUSTER- } \\
\text { GRÖSSEN / } \\
\text { HOMOGENITÄT }\end{array}$ & $\begin{array}{l}\text { INTERPRETIER- } \\
\text { BARKEIT }\end{array}$ \\
\hline $\begin{array}{l}\text { WARD } \rightarrow \\
\text { ITERIERTES MINIMAL- } \\
\text { DISTANZVERFAHREN } \\
\rightarrow \text { HILI,-CLIMBING- } \\
\text { AUS'I'AUSCH- } \\
\quad \text { VERFAHREN }\end{array}$ & FORTRAN & entfällt & $\begin{array}{l}2650 \\
2553 \\
2482 \\
2404 \\
2332 \\
2259\end{array}$ & $\begin{array}{l}\text { nicht } \\
\text { pro- } \\
\text { gram- } \\
\text { miert }\end{array}$ & $\begin{array}{r}5 \\
6 \\
7 \\
8 \\
9 \\
10\end{array}$ & + & \\
\hline $\begin{array}{l}\text { COIAPLEY'E L.INKAGE } \\
\text { WE.RD }\end{array}$ & $\begin{array}{c}\text { CLUSTER- } \\
\text { ANALYSE- } \\
\text { MUNSTER } \\
\text { (CAM) }\end{array}$ & $\begin{array}{r}23,13 \\
9,77\end{array}$ & $\begin{array}{l}5205 \\
4490\end{array}$ & $\begin{array}{r}8,95 \\
18,43\end{array}$ & $\begin{array}{l}5 \\
9\end{array}$ & $\begin{array}{l}\varnothing \\
+\end{array}$ & $\begin{array}{l}\varnothing \\
+\end{array}$ \\
\hline $\begin{array}{l}\text { HILLL-CLIMBING- } \\
\text { AUSTAUSCHVERFAHREN }\end{array}$ & SPSS & entfällt & $\begin{array}{l}4895 \\
4701 \\
4664 \\
4502 \\
4374\end{array}$ & $\begin{array}{l}\text { nicht } \\
\text { imple- } \\
\text { men- } \\
\text { tiert }\end{array}$ & $\begin{array}{l}5 \\
6 \\
7 \\
8 \\
9\end{array}$ & + & $\begin{array}{l}\varnothing \\
- \\
\bar{\emptyset} \\
+\end{array}$ \\
\hline $\begin{array}{l}\text { WARD } \rightarrow \\
\text { ITERIERTES MINIMAL- } \\
\text { DISTANZVERFAHREN } \\
\rightarrow \text { HILL-CLIMBING- } \\
\text { AUSTAUSCHVERFAHREN } \\
\text { (K-TRANSFORMATION) }\end{array}$ & FORTRAN & entfällt & $\begin{array}{l}4897 \\
4726 \\
4579 \\
4461 \\
4345 \\
4214\end{array}$ & $\begin{array}{r}11,83 \\
10,79 \\
9,99 \\
9,27 \\
8,74 \\
8,44\end{array}$ & $\begin{array}{r}5 \\
6 \\
7 \\
8 \\
9 \\
10\end{array}$ & + & $\begin{array}{r}\emptyset \\
- \\
\emptyset \\
\varnothing \\
++ \\
-\end{array}$ \\
\hline
\end{tabular}

Tab. 8: Kennzeichnung alternativer Clusterverfahren und ihrer Ergebnisse bei der Bildung von Motivationstypen (Teil II) 


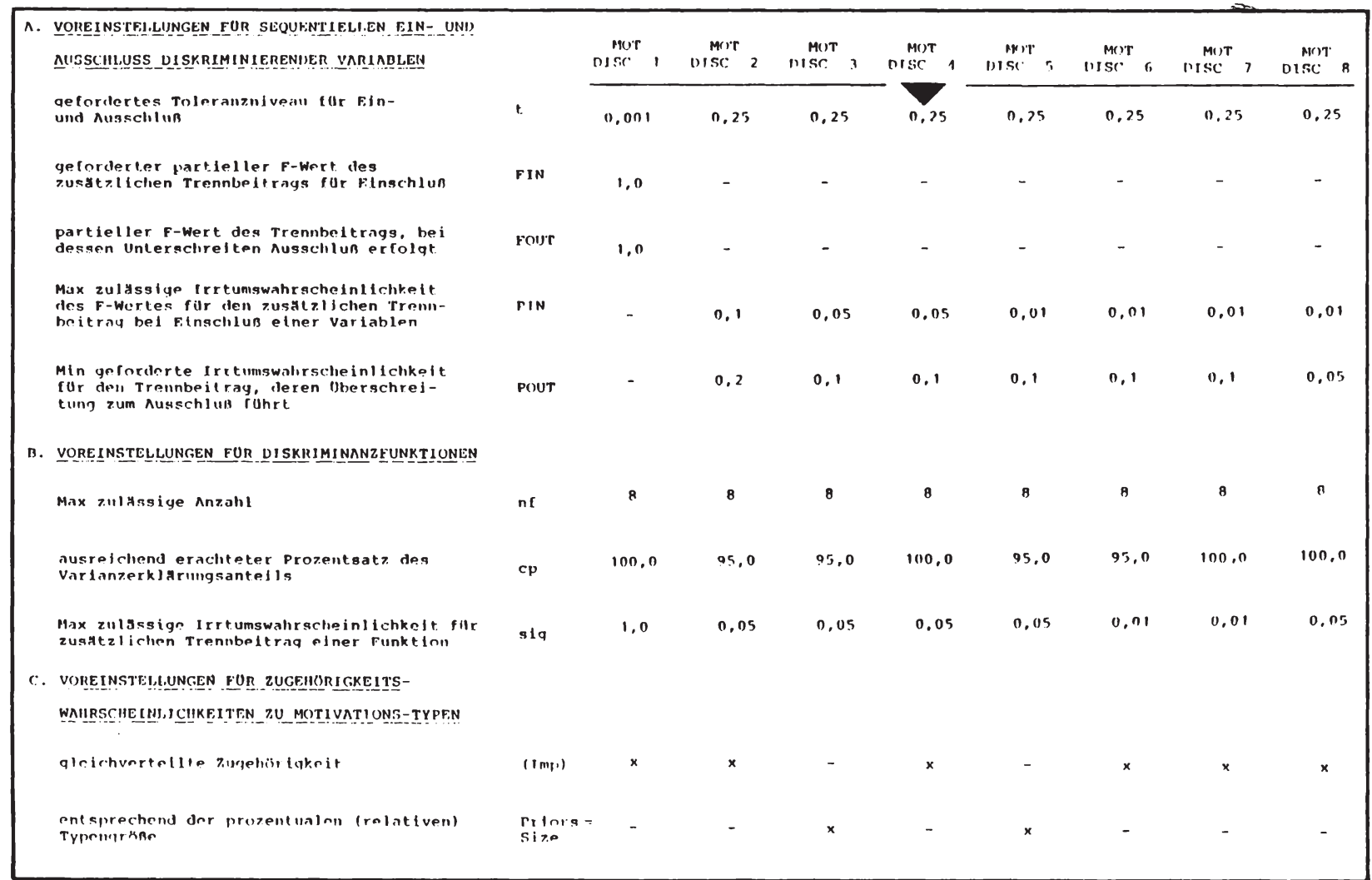

Tab. 9: Diskrimination von Motivationstypen auf der Grundlage von Einzelmotivationen Ergebnisüberblick multipler schrittweiser Diskriminanzanalysen bei Variation der Inklusionsparameter (Teil I) 


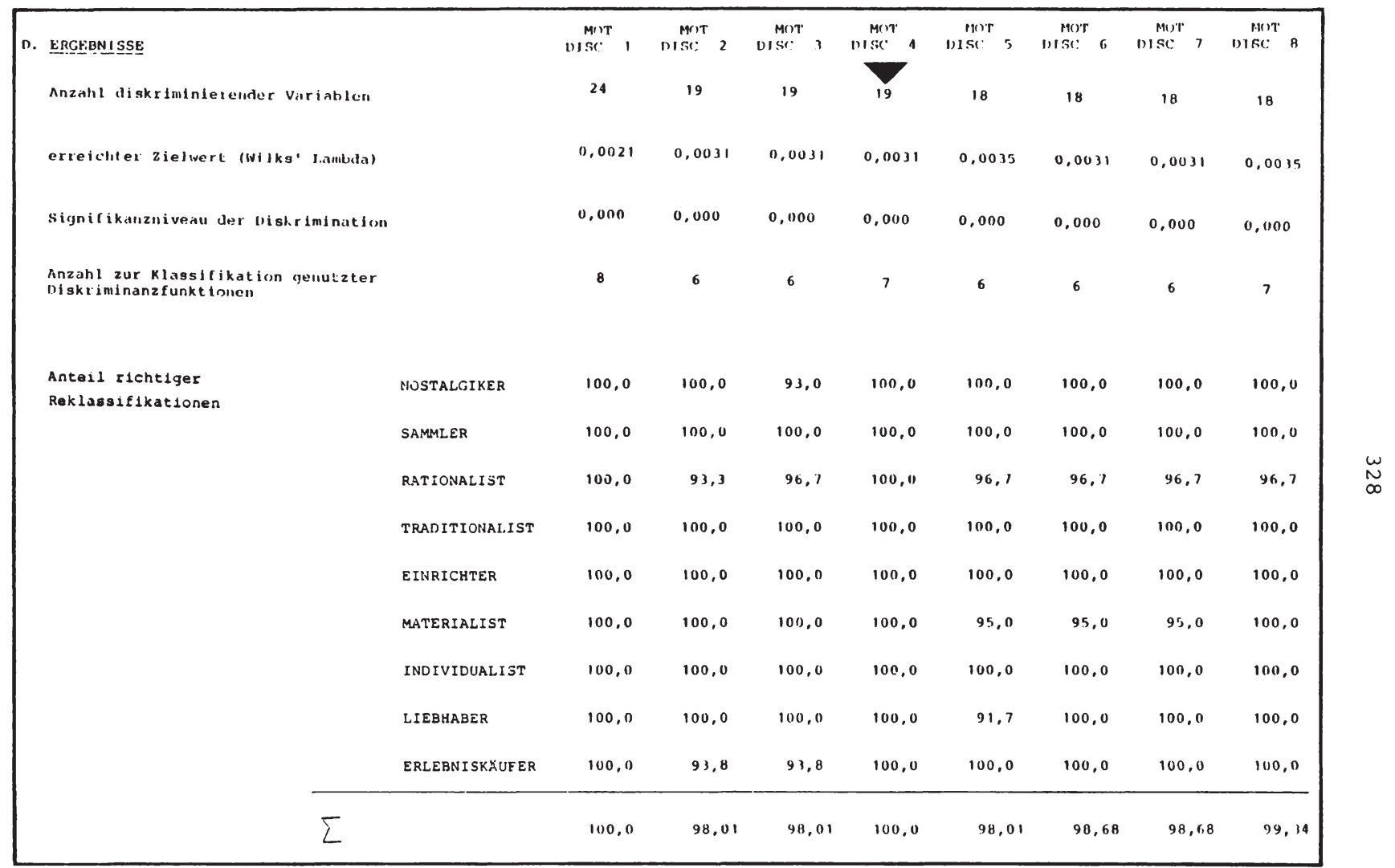

Tab. 9: Diskrimination von Motivationstypen auf der Grundlage von Einzelmotivationen Ergebnisüberblick multipler schrittweiser Diskriminanzanalysen bei Variation der Inklusionsparameter (Teil II) 


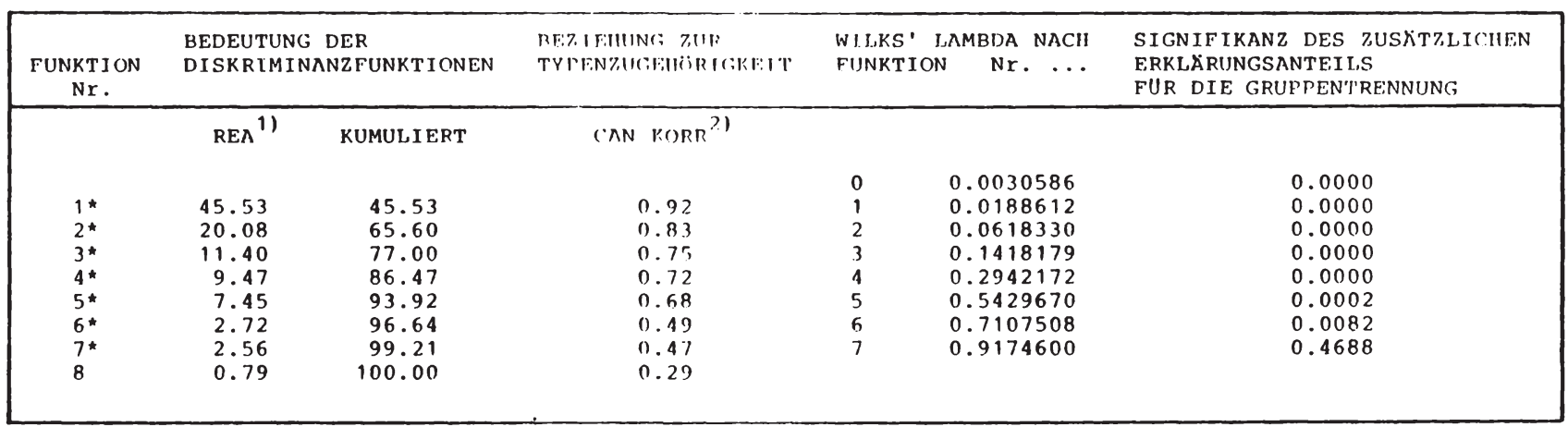

\footnotetext{
* = zur Reklassifikation genutzte Diskriminanzfunktionen

1) REA = relativer Eigenwertanteil
}

2) CAN KORR = Kanonischer Korrelationskoeffizient

Tab. 10: Bedeutung einzelner Diskriminanzfunktionen zur Trennung von Motivationstypen auf der Grundlage von Einzelmotivationen (MOT DISC 4) 1

1 Vgl. auch Tabelle 9 im Anhang. 


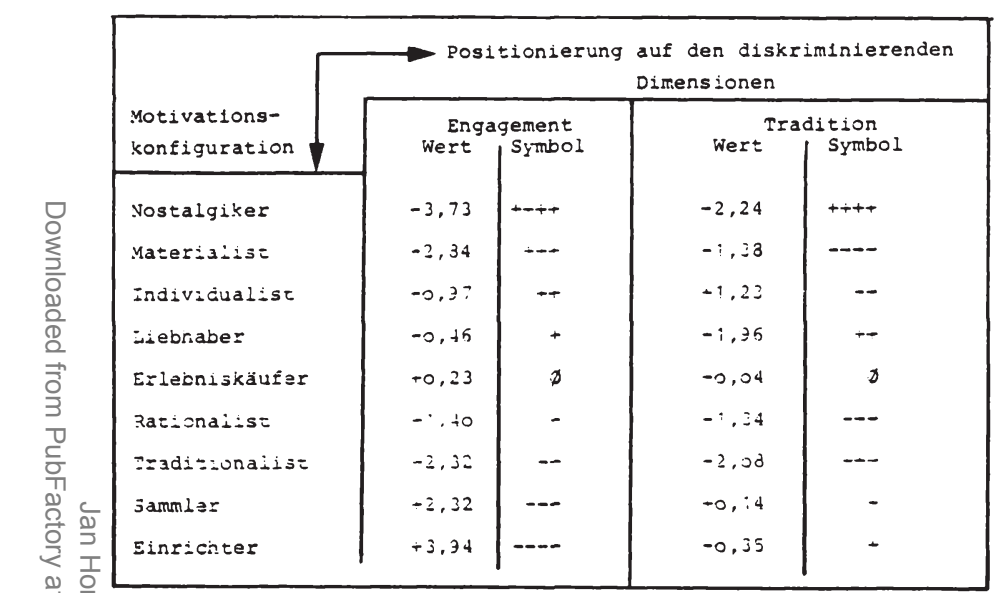

\begin{tabular}{|c|c|c|c|c|}
\hline Motivationstyp & Engagement & $\begin{array}{l}\quad \text { Anzahl a } \\
\text { höchster } \\
\text { Motiva- } \\
\text { tionsaus- } \\
\text { prägungen }\end{array}$ & $\begin{array}{l}\text { bsolut } \\
\text { niedrigster } \\
\text { Motivations- } \\
\text { aus- } \\
\text { prägungen }\end{array}$ & $\begin{array}{c}\text { Gesamt- } \\
\text { mittel- } \\
\text { wert }\end{array}$ \\
\hline Nostalgikar & ++++ & 10 & - & 2.5 \\
\hline Maceriaisst & + & 3 & - & 2,3 \\
\hline Indivicuaisse & $\div+$ & 2 & - & $3,:$ \\
\hline Liebhaber & - & 1 & - & 3,2 \\
\hline Erlebniskäufier & 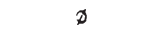 & & 1 & 3,5 \\
\hline kationaizst & - & - & + & 2,3 \\
\hline Rニadit:onaijse & -- & & . & $\therefore, ?$ \\
\hline Sammler & -- & & 3 & $3, j$ \\
\hline Einrichter & --- & 2 & 12 & 4,0 \\
\hline
\end{tabular}

Tab. 11 : Positionskoordinaten der Motivationskonfigurationen im 2-dimensionalen ("reduzierten") Diskriminanzraum
Tab. 12: Kennzahlen der Motivationskonfigurationen 
A. VOREINSTELLUNGEN FOR SEOUENTIELLEN EIN- UNII AUSSCIIIUSS DISKRIMINIERENDER VARIADLEN gefordertes Toleranziveau fir Elnuncl Ausschlus

gelorderter partieller f-wert des zusatzlichen Trennbeitrags fur Einschlun

partleller F-wert des Trennbeltrags, be deasen Unterschrcten Augschlun erfolat

Max zulassige 1 rrtumswahrschelnliclike it. des $F$-Wrtes fur den zusatzllchen Trennbeitrac bei Einschiub einer variablen

Min geforderte Irrtumswahrscheinllchkelt fllr den Trennbeltrag, deren Uberschre1tung zum Ausschlus fuhrt

B. VOREINSTELLUNGEN FUR DISKRIMINANZFUNKTIONE Max zulassige Anzahl

ausreichend erachteter prozentsatz des varianzerk1arungsanteilg

Max zulassige Irrtumswahrscheinlichkelt fur z.usatzllichen Trennbeitrag einer Funktion

C. VORFINSTELIIINGEN FUR ZUGFHURIGKEITS-

WAIIRSCHEINLICHKEITEN_ZU MOTIVATIONS-TYPEN

ginichuerteilte zugehisriukelt

entsprechend der promentualen (relativen) Typenqriske

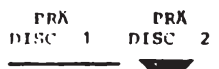

$\operatorname{Risc} 3$

TRK
DISC

DISC

PIR
DISC

t

o, 0

0,001

0,001

0.5

0,5

0,5

FIN

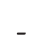

1,0

1.0

1.5

1,75

2.0

Fout

$$
-
$$$$
-
$$

1,0

1,0

1,0

1.0

1,5

PIN

0.1

0,05

rour

0.2

0,1

$-$

nr

cp

95.0

95,0

100,0

95,0

95,0

sig

0,05

0.05

1,0

1,0

0,05

0,05

0,05

priors = size

Tab. 13: Diskrimination von Motivationstypen auf der Grundlage motivationaler Bestimmungsfaktoren - Ergebnisüberblick multipler schrittweiser Diskriminanzanalysen bei Variation der Inklusionsparameter (Teil I) 


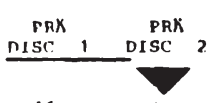

16 PRC
DISC

erreichler zielwert (Wilks' Latnbda)

Signifikanznlucau der Diskrimination

Anzahl zur Klassifikation genutzte Diskrinithanz funktionen

\section{Antell richtlger}

Reklasilfikationen

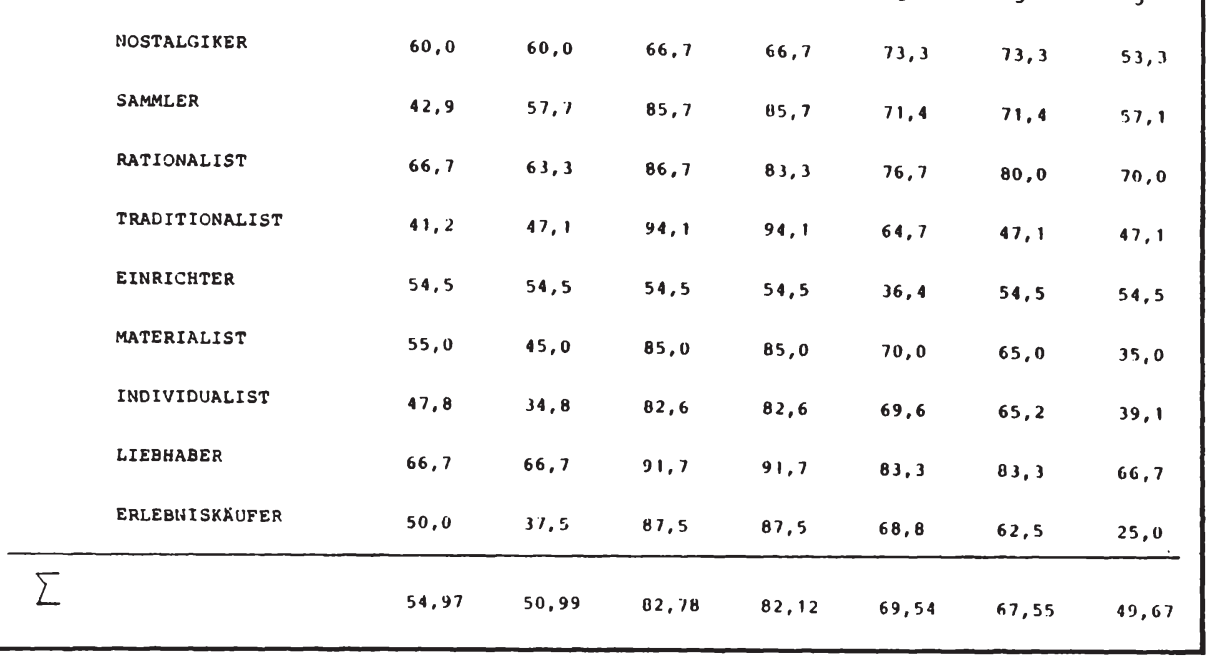

Tab. 13: Diskrimination von Motivationstypen auf der Grundlage motivationaler Bestimmungsfaktoren - Ergebnisüberblick multipler schrittweiser Diskriminanzanalysen bei Variation der Inklusionsparameter (Teil II) 


\begin{tabular}{|c|c|c|c|c|c|c|c|c|c|c|c|c|c|}
\hline \multirow[t]{2}{*}{ DISKRIMINIERENDE V } & \multirow[t]{2}{*}{ ARIABLEN } & \multirow[t]{2}{*}{$\begin{array}{l}\text { IRRTUMS- } \\
\text { WAHRSCHEIN- } \\
\text { LICHKET DES } \\
\text { F-WERTES }\end{array}$} & \multirow[t]{2}{*}{$\begin{array}{l}\text { TOLERANZ- } \\
\text { NIVEAU }\end{array}$} & \multirow[t]{2}{*}{$\begin{array}{l}\text { WILKS' } \\
\text { LAMBDA }\end{array}$} & \multirow[t]{2}{*}{$\begin{array}{l}\text { SIGNIFIKANZ- } \\
\text { NIVEAU DER. } \\
\text { DISKRIMINATION }\end{array}$} & \multicolumn{5}{|c|}{$\begin{array}{c}\text { RELATIVE BEDEUTUNG DER DISKRIMINIERENDEN } \\
\text { VARIABLEN IN DEN DISKRIMINANZFUNKTIONEN } \ldots \\
\vdots\end{array}$} & \multirow[t]{2}{*}{$\begin{array}{l}\text { GEWICHTETE } \\
\text { DISKRIMINANZ- } \\
\text { XOEFFIZIENTEN }\end{array}$} & \multicolumn{2}{|c|}{$\begin{array}{l}\text { RELATIVE BEDEUTUNG } \\
\text { DER DISKRIMINIERENDEN } \\
\text { VARIABLEN }\end{array}$} \\
\hline & & & & & & 1 & 2 & 3 & 4 & 5 & & in 1 & RANG \\
\hline WOHNSTIL & modern & 0,000 & 0.78 & 0,7710 & 0.0001 & 3,91 & 17,56 & 7,93 & 9,97 & 12,87 & 0,3255 & $9,72$. & 1 \\
\hline I NVOLVEMENT & $\begin{array}{l}\text { von Freunden un Anti- } \\
\text { quitaten beneidet werden }\end{array}$ & 0,001 & 0,80 & 0,6241 & 0,0000 & 19,57 & 8,81 & 0,11 & 1,57 & 2,90 & 0,2728 & 8,14 & 3 \\
\hline SOZ IODEMOGRAPHIE & $\begin{array}{l}\text { monatliches Netto- } \\
\text { Haushaltseinkommen }\end{array}$ & 0,003 & 0.81 & 0,5067 & 0,0000 & 6.01 & 0,52 & 15,32 & 6.73 & 0,52 & 0,2210 & 6,60 & 9 \\
\hline $\begin{array}{l}\text { ANSTOSS } \\
\text { FUR INTERESSE }\end{array}$ & Familie & 0,001 & 0,80 & 0,4173 & 0,0000 & 0,09 & 8,46 & 8,82 & 19,34 & 1,58 & 0,2439 & 7,28 & 7 \\
\hline ANTIQUITATENIMAGE & selten & 0,012 & 0,85 & 0,3455 & 0,0000 & 11,90 & 3,14 & 6,34 & 5,29 & 12,51 & 0,2557 & 7,63 & 4 \\
\hline 2 IEL & beruflicher Aufstieg & 0,041 & 0,75 & 0,2970 & 0.0000 & 0,63 & 10,05 & 3,16 & 5,66 & 9,82 & 0,1673 & 4,99 & 14 \\
\hline SOZIODEMOGRAPHIE & $\begin{array}{l}\text { weiterfuhrende } \\
\text { schulen ohne Abitur }\end{array}$ & 0,010 & 0,83 & 0,2557 & 0,0000 & 10,55 & 3,64 & 0,62 & 9,00 & 17,69 & 0.2375 & 7,09 & 8 \\
\hline ANTIQUITATENIMAGE & ausdrucksstark & 0,004 & 0,68 & 0,2165 & 0,0000 & 19,28 & 0,22 & 5,94 & 3,13 & 9,82 & 0,2819 & 8,41 & 2 \\
\hline $2 \mathrm{IEL}$ & personliche Anerkennung & 0.039 & 0,83 & 0,1889 & 0,0000 & 2,84 & 5,22 & 12,68 & 3,53 & 7,73 & 0,2097 & 6,26 & 11 \\
\hline $\begin{array}{l}\text { ANSTOSS } \\
\text { FUR INTERESSE }\end{array}$ & Ereunde/Bexannte & 0,008 & 0,73 & 0,1643 & 0,0000 & 2,25 & 2.01 & 18,23 & 2,53 & 3,04 & 0,2038 & 6,08 & 13 \\
\hline AKTIVITÄT & $\begin{array}{l}\text { gehobene } \\
\text { Literatur lesen }\end{array}$ & 0,010 & 0,77 & 0,1404 & 0,0000 & 4,11 & 19,00 & 4,71 & 4,22 & 3,17 & 0,2514 & 7,50 & 6 \\
\hline $2 \mathrm{IEL}$ & $\begin{array}{l}\text { bessere } \\
\text { wohnungseinrichtung }\end{array}$ & 0.029 & 0.77 & 0.1210 & 0,0000 & 4,28 & 5,84 & 6.19 & 13,71 & 4,50 & 0,2223 & 6,64 & 10 \\
\hline ANTIOUITATENIMAGE & schon & 0,049 & 0,70 & 0.1057 & 0,0000 & 11,94 & 0,89 & 7,70 & 6.83 & 11,13 & 0,2542 & 7,59 & 5 \\
\hline ANTIQUITATENIMAGE & wiederverkbuflich & 0.050 & 0,85 & 0,0922 & 0,0000 & 2,63 & 14,54 & 2,25 & 8,48 & 2,72 & 0,2072 & 6.19 & 12 \\
\hline
\end{tabular}

Tab. 14: Die Bedeutung personaler-, waren- und situationsbezogener motivationaler Bestimmungsfaktoren zur Diskrimination von Motivationstypen - Ergebnisüberblick einer multiplen schrittweisen Diskriminanzanalyse (PRÄ - DISC 2) 


\begin{tabular}{|c|c|c|c|c|}
\hline \multirow{2}{*}{$\begin{array}{l}\text { Eigenschaften von Antiquitäten } \\
\text { Dekorationswert }\end{array}$} & \multirow{2}{*}{$\begin{array}{l}\text { Anzahl } \\
\text { einbezogener } \\
\text { erklărender 11 } \\
\text { Motivat1onen }\end{array}$} & \multirow{2}{*}{$\begin{array}{l}\begin{array}{l}\text { Multipler } \\
\text { Korrelations- } \\
\text { Koeffizient }\end{array} \\
0,61\end{array}$} & \multicolumn{2}{|c|}{$\begin{array}{l}\text { erklärter } \\
\text { Varianz- } \\
\text { anteil in } 8\end{array}$} \\
\hline & & & $\triangleright$ & 36,76 \\
\hline Qualıtät & 9 & 0,49 & & 23,91 \\
\hline Gebrauchsfahigkeit & 6 & 0,71 & & 50,28 \\
\hline Seltenhe1t & 10 & 0,59 & $>$ & 34,61 \\
\hline Wertbeständigkelt & 7 & 0,62 & $\triangleright$ & 38,78 \\
\hline Exklusivität & 11 & 0,53 & & 27,85 \\
\hline Anziehungskraft & 5 & 0,48 & & 23,06 \\
\hline Zeitlosigkeit & 7 & 0,45 & & 20,56 \\
\hline Individual ităt & 7 & 0,45 & & 19,83 \\
\hline Originalität & 4 & 0,42 & & 17,69 \\
\hline Wert & 5 & 0,50 & & 25,24 \\
\hline Schönheit & 3 & 0,38 & & 14,61 \\
\hline Recräsentativităt & 8 & 0,56 & $\nabla$ & 31,69 \\
\hline Solide Verarbeitung & 6 & 0,44 & & 19,78 \\
\hline Künstlerische Bedeutung & 6 & 0,54 & & 29,52 \\
\hline Ausdrucksstärke & 3 & 0,34 & & 11.28 \\
\hline Ausgefallenheit & 6 & 0,49 & & 24,00 \\
\hline Stilechtheit & 10 & 0,58 & $>$ & 33,96 \\
\hline Wiederverkäuflichkeit & 4 & 0,53 & & 28,16 \\
\hline Mobilität & 7 & 0,49 & & 24,45 \\
\hline Nlter & 8 & 0,60 & $>$ & 35,95 \\
\hline Funktionalität/Praktıkab1lität & 7 & 0,71 & 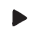 & 49,92 \\
\hline
\end{tabular}

Güte der multiplen Regressionsgleichungen: Signifikanzniveau $>99,9$ \&

Tab. 15: Erklärungsbeiträge von Motivationen zur Kaufrelevanz von Antiquitätenmerkmalen - Ergebnisüberblick multipler schrittweiser Regressionsanalysen

1) Voreinstellungen (Inklusionsparameter)

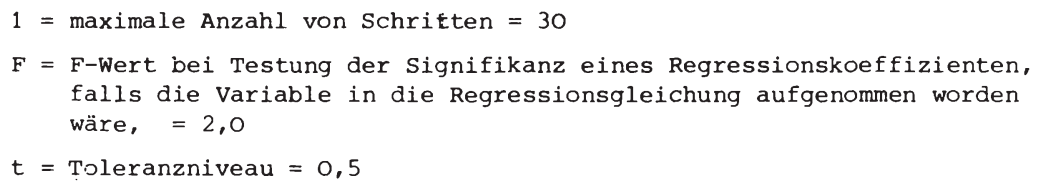
falls die Variable in die Regressionsgleichung aufgenommen worden wäre, $=2,0$

$t=$ Toleranzniveau $=0,5$ 


\begin{tabular}{|c|c|c|c|c|c|}
\hline $\begin{array}{l}\text { Interesse an } \\
\text { Sach- bzw. Sammelgebieten }\end{array}$ & $\begin{array}{l}\text { Anzahl } \\
\text { elibezogener } \\
\text { erkl.yrencler } \\
\text { Mntlval Ionen }\end{array}$ & $\begin{array}{l}\text { Mulilpjer } \\
\text { Kotrolatlons- } \\
\text { Knefrizient }\end{array}$ & $\begin{array}{l}\text { erklarter } \\
\text { Vartanz- } \\
\text { anteil in }\end{array}$ & $\begin{array}{c}\text { Gute } \\
\text { Regres } \\
\text { F-Wert }\end{array}$ & $\begin{array}{c}\text { ex multiplen } \\
\text { ionsgleicliung } \\
\text { signtfikanz- } \\
\text { niveau }\end{array}$ \\
\hline Gemylde & 9 & 0,52 & 27,08 & 5.65 & $\star \star \star \star$ \\
\hline Graph1k & 5 & 0,32 & 9,98 & 3,12 & $\star \star$ \\
\hline silber & 10 & 0.56 & $>31.73$ & 6,32 & $\star \star \star \star$ \\
\hline Tepplche & 1 & 0,32 & 10.50 & 4,17 & $* *$ \\
\hline Bucher & 5 & 0.37 & 13,65 & 4,46 & $\star \star \star$ \\
\hline Schreibmöbel & 5 & 0,50 & 24.91 & 9,35 & $\star \star \star *$ \\
\hline Truhen/Kommoden & B & 0,53 & 28,30 & 6.81 & $\star \star * \star *$ \\
\hline S1tzmöbel & 6 & 0.53 & 28.42 & 9.26 & $\star \star \star \star$ \\
\hline Tische & 10 & 0,56 & $\rightarrow 31.41$ & 6.23 & $\star \star * \star$ \\
\hline Schranke/vitrinen & 7 & 0,17 & 22,52 & 5,77 & $\star \star * \star$ \\
\hline Klelnmöbel & 5 & 0,42 & 17,69 & 6.06 & $\star \star \star \star$ \\
\hline Schmuck & 9 & 0.43 & 18,68 & 3.50 & $\star \star \star \star$ \\
\hline Porzellan & 1 & $0,3.3$ & 11.03 & 4,40 & $* \star *$ \\
\hline Uhren & 9 & 0,13 & 18,28 & 3,41 & $\star \star \star \star *$ \\
\hline Ostaslatika & 9 & 0.54 & 29.55 & 6,38 & $\star \star \star \star$ \\
\hline
\end{tabular}

Tab. 16: Erklärungsbeiträge von Motivationen zum Interesse an Sach- und Sammelgebieten - Ergebnisüberblick multipler schrittweiser Regressionsanalysen

$1 \quad *=$ Signifikanzniveau $=90 \%$

$* * *=$ Signifikanzniveau $=99 \%$

$* *$ Signifikanzniveau $=95 \%$

$* * * *=$ Signifikanzriveau $=99,9 \%$ 


\begin{tabular}{|c|c|c|c|}
\hline $\begin{array}{l}\text { Arforderungen } \\
\text { an Einkaufstatten } \\
\text { für Antiquitaten }\end{array}$ & $\begin{array}{l}\text { Anzahl } \\
\text { einbezogener } \\
\text { erklarender } \\
\text { Motivationen }\end{array}$ & $\begin{array}{l}\text { Multiple: } \\
\text { Korrelation }- \\
\text { Koeffizient }\end{array}$ & $\begin{array}{l}\text { eriduati: } \\
\text { Varianz:. } \\
\text { anteil in } 8\end{array}$ \\
\hline breites Sortiment & 8 & 0.57 & 32,00 \\
\hline $\begin{array}{l}\text { tiefes Sortiment } \\
\text { in Spezialgebieten }\end{array}$ & 8 & 0,50 & 25,18 \\
\hline $\begin{array}{l}\text { nur mit Stücken } \\
\text { von spitzenqualitat }\end{array}$ & 4 & 0,33 & 11,12 \\
\hline $\begin{array}{l}\text { auch mit Kleinigkeiten } \\
\text { als Geschenk }\end{array}$ & 5 & 0.47 & 22,34 \\
\hline $\begin{array}{l}\text { mit Chancen } \\
\text { für Trouvallien }\end{array}$ & 5 & 0,57 & 32.67 \\
\hline Bereitschaft zum llandeln & 4 & 0,44 & 19,13 \\
\hline hohe fachliche Qualifikation & 5 & 0,36 & 12,79 \\
\hline sicher und verlasilich & 3 & 0,35 & 12,36 \\
\hline $\begin{array}{l}\text { Anleitung beim Aufbau } \\
\text { einer Sammlung }\end{array}$ & 2 & 0,24 & 5,65 \\
\hline $\begin{array}{l}\text { bekannter Name/ } \\
\text { guter Ruf }\end{array}$ & 8 & 0,47 & 22,06 \\
\hline Repräsentativităt & 3 & 0,12 & 17.44 \\
\hline $\begin{array}{l}\text { verstaubte, } \\
\text { ljebevolle Atmosphäre }\end{array}$ & 7 & 0,48 & 22,85 \\
\hline $\begin{array}{l}\text { Mögl ichkejt } \\
\text { zum "Stöbern" bieten }\end{array}$ & 10 & 0.51 & 25,78 \\
\hline Einkaufserlebnis bieten & 5 & 0.41 & 16,97 \\
\hline Gewahrleistung übernehmen & 8 & 0,46 & 21,39 \\
\hline Expertisen geben & 6 & 0,49 & 24,00 \\
\hline Stücke zurücknehmen & 6 & 0.51 & 25.67 \\
\hline Stlicke besorgen & 1 & 0.34 & 11,63 \\
\hline $\begin{array}{l}\text { problemlos erreichbarer } \\
\text { Standort }\end{array}$ & 4 & 0.30 & 9.19 \\
\hline
\end{tabular}

Tab. 17: Erklärungsbeiträge von Motivationen für Anforderungen von Antiquitätenkäufern an Einkaufsstätten - Ergebnisüberblick multipler schrittweiser Regressionsanalysen 


\begin{tabular}{|c|c|c|c|c|c|}
\hline $\begin{array}{l}\text { Merkinale } \\
\text { des Informationsverhaltens } \\
\text { von Antigultatenkdufern }\end{array}$ & $\begin{array}{l}\text { Anzahl } \\
\text { einbezogener } \\
\text { erklärender } \\
\text { Mot ivationen }\end{array}$ & $\begin{array}{l}\text { Multipler } \\
\text { Korrelations- } \\
\text { Koeffizlent }\end{array}$ & $\begin{array}{l}\text { erklatier } \\
\text { Vartanz- } \\
\text { anceil in }\end{array}$ & $\begin{array}{c}\text { Gute } \\
\text { Regres } \\
\text { F-Wert }\end{array}$ & $\begin{array}{l}\text { er multiplen } \\
\text { lonsgleichung } \\
\text { signifikanz- } \\
\text { niveau }\end{array}$ \\
\hline $\begin{array}{l}\text { Intensive } \\
\text { Inforinationssuche }\end{array}$ & 8 & 0,68 & $\Rightarrow \quad 45,86$ & 14,51 & \#\#\#* \\
\hline - alsi Hohby & 15 & 0,77 & $\Rightarrow 59,08$ & 12,51 & $\star \star \star * *$ \\
\hline - durch gezielte stadterelsen & 8 & 0,59 & $\rightarrow 35,23$ & 9,32 & $* \star \star *$ \\
\hline - durch gezlelte Auslandsreisen & 7 & 0,53 & 28,35 & 7,80 & $\star \star \star \star *$ \\
\hline $\begin{array}{l}\text { passive } \\
\text { Informat I onsauf nahine }\end{array}$ & 7 & 0.61 & $\Rightarrow 37,35$ & 11.75 & $\star \star \star \star \star$ \\
\hline $\begin{array}{l}\text { aktive } \\
\text { rnforimat fonsalsgabe }\end{array}$ & 2 & 0,33 & 10,17 & 8,63 & $\star \star \star \star \star$ \\
\hline $\begin{array}{l}\text { Iintensiver Informationsaustausch } \\
\text { liil Freundes- und Bekanntenkreis }\end{array}$ & 5 & 0,54 & 29.10 & 11.49 & 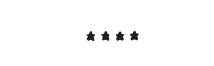 \\
\hline Meinungs fulyschaft & 8 & $0,4:$ & 20,02 & 4,29 & $\star \star \star * *$ \\
\hline Mei nungs fithrung & 8 & 0,60 & -36.55 & 9,86 & $\star \star \star \star *$ \\
\hline
\end{tabular}

1 Vgl. Tab. 16 (Anhang)

Tab. 18: Erklärungsbeiträge von Motivationen zum Informationsstil Ergebnisüberblick multipler schrittweiser Regressionsanalysen 


\begin{tabular}{|c|c|c|c|c|c|}
\hline $\begin{array}{l}\text { Thematisches Interesse } \\
\text { von Antiquitatenkaufern } \\
\text { im zusammenhang mit } \\
\text { Antiquitaten }\end{array}$ & $\begin{array}{l}\text { Anzahl } \\
\text { einbezogener } \\
\text { erklärender } \\
\text { Mot1vationen }\end{array}$ & $\begin{array}{l}\text { Multipler } \\
\text { Kurrelations- } \\
\text { Koetfizlent }\end{array}$ & $\begin{array}{l}\text { erklärter } \\
\text { Vartanz- } \\
\text { Antell in } 8\end{array}$ & $\begin{array}{r}\text { Gute } \\
\text { Regres } \\
\text { F-Wert }\end{array}$ & $\begin{array}{l}\text { er nultiplen } \\
\text { ionsgleichung } \\
\text { Signifikanz-1 } \\
\text { niveau }\end{array}$ \\
\hline $\begin{array}{l}\text { Was man in Moment } \\
\text { wo kaufen kann }\end{array}$ & 4 & 0,38 & 14,31 & 6,06 & $\star \star \star \star *$ \\
\hline $\begin{array}{l}\text { Preise und } \\
\text { Preisentwicklungen }\end{array}$ & 5 & 0.52 & 27,13 & 14,91 & $\star \star \star \star$ \\
\hline $\begin{array}{l}\text { Ausstellungs- } \\
\text { und Messetermine }\end{array}$ & 6 & 0,42 & 17,23 & 4,96 & $\star \star \star \star$ \\
\hline $\begin{array}{l}\text { Kunstwissenschaftl tche } \\
\text { Abhandlungen }\end{array}$ & 11 & 0,51 & 26,20 & 4,45 & $\star \star \star \star \star$ \\
\hline $\begin{array}{l}\text { Abbildungen und Beschreibungen } \\
\text { schöner Antiquitäten }\end{array}$ & 3 & 0.35 & 12,12 & 6,71 & $\star \star \star \star$ \\
\hline Monographien uber Kunstler & 7 & 0,50 & 25,03 & 6,77 & $\star \star \star \star \star$ \\
\hline Steuerrechtliche Fragen & 9 & 0,69 & $-47,77$ & 14,23 & $\star \star \star \star$ \\
\hline $\begin{array}{l}\text { Antiquitaten } \\
\text { als Kapitalanlage }\end{array}$ & 6 & 0.69 & - 47,92 & 21.93 & $\star \star \star \star \star$ \\
\hline $\begin{array}{l}\text { Was momentan } \\
\text { stark gefragt ist }\end{array}$ & 4 & 0,50 & 25,44 & 12,37 & $\star \star \star \star \star$ \\
\hline Sanmeln mit Gewinn & 6 & 0,66 & $\checkmark 43,09$ & 18,05 & $\star \star \star \star \star$ \\
\hline
\end{tabular}

Tab. 19: Erklärungsbeiträge von Motivationen zum thematischen Informationsinteresse - Ergebnisüberblick multipler schrittweiser Regressionsanalysen

TVgI. Tab. 16 (Anhang) 


\begin{tabular}{|c|c|c|c|c|c|}
\hline $\begin{array}{l}\text { Nutzung von } \\
\text { Informationsquellen } \\
\text { uber Antigutaten } \\
\text { durch Antiquitytenkaufer }\end{array}$ & $\begin{array}{l}\text { Anzinh) } \\
\text { elinhezngener } \\
\text { eik\} Grander } \\
\text { Mni luationen }\end{array}$ & $\begin{array}{l}\text { Multipler } \\
\text { Korrelations- } \\
\text { Koeflizient }\end{array}$ & $\begin{array}{l}\text { erklinter } \\
\text { Varianz- } \\
\text { anteil in }\end{array}$ & $\begin{array}{c}\text { Gute } \\
\text { Regres } \\
\text { F-Wert }\end{array}$ & $\begin{array}{l}\text { er multiplen } \\
\text { ionsglofching } \\
\text { signtffikanz- } \\
\text { njveau }\end{array}$ \\
\hline Fachbucher & 10 & 0,65 & $-41,89$ & 9,80 & $\star * \star *$ \\
\hline Fachzeitschriften & 1 & 0.17 & 21,90 & 9,96 & $\star \star \star \star$ \\
\hline Tageszeit tungen & 5 & 0,32 & 10,00 & 3,13 & ** \\
\hline Auktionskata loge & 5 & 0.47 & 22.07 & 7.99 & $* * * *$ \\
\hline Auktionsbesuche & 6 & 0.40 & 15.81 & 4.38 & $\star * \star *$ \\
\hline Messebesuche & 6 & 0.13 & 18,64 & 5.35 & $* \star * *$ \\
\hline Museumsbesuche & 6 & 0.45 & 20,03 & 5,84 & $\star \star \star \star$ \\
\hline Ausstellungskataloge & 6 & 0.40 & 16,31 & 4,55 & $* \star \star *$ \\
\hline Kleinanzelgen & 5 & 0.39 & 15,58 & 5.21 & $* \star * *$ \\
\hline Auslaqen der Fachgeschafte & 6 & 0.39 & 15,47 & 4,27 & $* * * *$ \\
\hline Gespräche mit Fachhandlern & 2. & 0,35 & 12.04 & 9,86 & $* * * *$ \\
\hline $\begin{array}{l}\text { Gespräche } 1 \mathrm{~m} \text { Freundes- } \\
\text { und Bekanntenkre1s }\end{array}$ & 1 & 0,33 & 10,93 & 1,36 & $\star \star * *$ \\
\hline Ant iquitatenmärkte & 10 & 0.51 & 26.00 & 4.78 & $* * *$ \\
\hline Trödel- und Flohmärkte & 5 & 0,48 & 22.73 & 8,29 & $* * * *$ \\
\hline
\end{tabular}

Tab. 20: Erklärungsbeiträge von Motivationen zur Nutzung von Informationsquellen - Ergebnisüberblick multipler schrittweiser Regressionsanalysen

1 Vgl. Tab. 16 (Anhang) 


\section{A $n h$ a $\mathrm{n} g$ III}

Fragebogen

Jan Honsel - 978-3-631-75024-7

Downloaded from PubFactory at 01/11/2019 09:09:25AM

via free access 
1. Welches war der Grund für Ihren Besuch auf der Antiquitätenmesse in München (Essen / Münster) ?

Antwortmöglichkeiten (Mehrfachnennung möglich) :

Ich wollte mich ganz allgemein über das heutige Angebot informieren

Ich war auf der Suche nach einem ganz speziellen Stück

Mich interessierte der Stand eines bestimmten Händlers

Ich wollte mir eigentlich nur mal wieder schöne Antiquitäten ansehen

Ich hatte mir vorgenommen, etwas $z$ u kaufen

Ich habe tatsächlich eine Antiquität bzw. einen Kunstgegenstand gekauft

Ich bin mit Bekannten bzw. meiner Familie auf der Messe gewesen

2. Mit Antiquitäten kann man die unterschiedlichsten Vorstellungen verbinden. Bitte nennen Sie uns, wenn möglich, drei Begriffe, die Ihnen ganz spontan einfallen, wenn Sie an Antiquitäten denken.

offene Fragestellung

3. Wie stark ist Ihr Interesse an den folgenden Antiquitäten und Kunstgegenständen ?

Antwortmöglichkeiten skaliert ${ }^{1}$ ( sehr / ziemlich / etwas / kaum / gar nicht) für :

Gemälde, Graphik, Silber, Teppiche, Bücher, Schreibmöbel, Truhen/Kommoden, Sitzmöbel, Tische, Schränke /Vitrinen, Kleinmöbel, Schmuck, Porzellan, Uhren, Ostasiatika, Sonstiges

4. Es gibt die unterschiedlichsten Anstöße, die das Interesse für Antiquitäten wecken können. Wie war das bei Ihnen ? Wer hat Ihr persönliches Interesse für Antiquitäten geweckt, bzw. wie sind Sie das erste Mal mit Antiquitäten in Berührung gekommen?

offene Fragestellung

1 Im Originalfragebogen wurden sämtliche skalierten Antwortmöglichkeiten in Form von Skalen vorgezeichnet, so daß die Befragten durch Ankreuzen antworten konnten. 
5. Es gibt verschiedene Gründe, sich Antiquitäten zu kaufen. Wir nennen Ihnen im folgenden eine Reihe solcher Gründe. Bitte geben Sie an, wie wichtig diese Gründe für Sie persönlich bei einem Antiquitätenkauf sind!

Antwortmöglichkeiten skaliert ( sehr wichtig / ziemlich wichtig / etwas wichtig / kaum wichtig / gar nicht wichtig) für die Statements:

Für mich sind Antiquitäten Einrichtungsgegenstände, die ich gebrauchen und nutzen möchte

Ich kaufe Antiquitäten als Dekorationsstücke für meine Wohnung

Ich kaufe Antiquitäten, weil sie zu meiner Person passen und mir eine individuelle Note verleihen

Für mich sind Antiquitäten eine beständige und krisensichere Wertanlage

Ich spekuliere mit dem hohen Wertzuwachs von Antiquitäten

Antiquitäten bedeuten für mich ein Stück Selbstverwirklichung

Ich habe einfach den Wunsch, Antiquitäten $\mathrm{zu}$ besitzen

Ich bin leidenschaftlicher Sammler

Ich kaufe Antiquitäten aus meinem persönlichen Bedürfnis, von ästhetischen Dingen umgeben zu sein

Ich kaufe Antiquitäten als Geschenke, mit denen ich anderen eine Freude bereiten möchte

Ich kaufe Antiquitäten, weil mir die heutigen Möbel nicht zusagen

Antiquitäten bieten steuerliche Vorteile, die ich auch gerne nutzen möchte

Ich kaufe Antiquitäten, weil ich mit Ihnen Wertvorstellungen verbinde, die früher noch von Bedeutung waren, aber in der heutigen Zeit verlorengegangen sind

Ich kaufe Antiquitäten, weil sie für mich ein Hobby, eine Freizeitbeschäftigung sind 
Fortsetzung

Es gibt verschiedene Gründe, sich Antiquitäten zu kaufen. Wir nennen Ihnen im folgenden eine Reihe solcher Gründe. Bitte geben Sie an, wie wichtig diese Gründe für Sie persönlich bei einem Antiquitätenkauf sind!

Antwortmöglichkeiten skaliert ( sehr wichtig / ziemlich wichtig / etwas wichtig / kaum wichtig / gar nicht wichtig) für die Statements:

An einem Antiquitätenkauf reizt mich besonders die Chance, eine Trouvaille bzw. ein "Schnäppchen" zu machen

Den größten Spaß beim Antiquitätenkauf macht mir die Suche nach einem Stück

Ich kaufe Antiquitäten, weil mich die Atmosphäre bzw. die Art und Weise, wie man sie einkauft, anspricht

Die Vorliebe für Antiquitäten hat in unserer Familie Tradition

Ich kaufe Antiquitäten, weil man auf qualitätvolle Stücke mit Recht stolz sein kann

Ich kaufe Antiquitäten, weil ich in einer beruflichen bzw. gesellschaftlichen Stellung bin, in der eine repräsentative Wohnungseinrichtung mit dazu gehört

Antiquitäten bieten mir einen Ausgleich für das Überma $B$ von Technik, Perfektion und Stre $B$ in meiner Umgebung

Antiquitäten zu kaufen empfinde ich als eine wichtige Aufgabe, da ich damit Kultur- bzw. Kunstgegenstände bewahren helfe

Ich kaufe Antiquitäten, da mir die meisten der anderen Ausdrucksformen der modernen Kunst verschlossen bleiben

Mit Antiquitäten verbinde ich Erinnerungen an meine Eltern und GroBeltern

Ich kaufe Antiquitäten, da die heutige Handwerkskunst zu gleichen Leistungen nicht mehr fähig ist

Ich kaufe Antiquitäten, da sie aus schönen und wertvollen Naturstoffen gefertigt sind 
6. Im folgenden haben wir einige Fragen zu Ihrem persönlichen Antiquitätenbesitz.

(a) Denken Sie zunächst ganz allgemein an Ihre Antiquitäten. Es gibt da unterschiedliche Möglichkeiten wie Ihre Antiquitäten zusammengestellt sein können. Welche der folgenden Aussagen trifft auf Sie persönlich zu ?

Antwortmöglichkeiten (Einfachnennung) :

Ich besitze keine Antiquitäten

Ich besitze Antiquitäten nur eines einzigen und zusätzlich nach bestimmten Kriterien eng eingegrenzten Sachgebiets

Ich besitze Antiquitäten eines einzigen aber nicht weiter eingegrenzten Sachgebiets

Ich besitze Antiquitäten mehrerer und jeweils zusätzlich nach bestimmten Kriterien eng eingegrenzter Sachgebiete

Ich besitze Antiquitäten mehrerer aber jeweils nicht weiter eingegrenzter Sachgebiete

Ich besitze Antiquitäten unterschiedlichster Art

(b) Wenn Sie nun einmal an eines Ihrer antiken Möbel denken, wie würden Sie dieses stück beurteilen ? Wenn Sie kein antikes Möbel haben, wozu auch alle Arten von Kleinmöbeln wie Schatullen, Kästchen, Ständer, Tischchen etc. gehören, wählen Sie bitte eine andere Antiquität aus Ihrem Besitz.

Antwortmöglichkeiten skaliert (trifft sehr zu / trifft ziemlich zu / trifft etwas zu / trifft kaum zu / trifft gar nicht zu ) für die beschreibenden Merkmale :

dekorativ, qualitätvoll, gebrauchsfähig, selten, wertbeständig, exklusiv, anziehend, zeitlos, individuell, original, wertvoll, schön, repräsentativ, solide gearbeitet, künstlerisch bedeutend, ausdrucksstark, ausgefallen, stilecht, wiederverkäuflich, mobil, alt, funktional/praktisch

(c) Was für eine Antiquität haben Sie gerade für Ihre Beurteilung herangezogen?

offene Fragestellung

(d) Welchen genauen Platz hat das betrachtete Stück in Ihrer Wohnung ?

offene Fragestellung 
7. Es gibt die unterschiedlichsten Möglichkeiten, wie es zu einem Antiquitätenkauf kommen kann. Bitte sagen Sie uns, in welchem AusmaB die folgenden Aussagen auf Ihr persönliches Verhalten zutreffen.

Antwortmöglichkeiten skaliert (trifft sehr zu / trifft ziemlich $\mathrm{zu} /$ trifft etwas zu / trifft kaum zu / trifft gar nicht zu) für die Statements:

Ich plane einen Antiquitätenkauf ganz gezielt und informiere mich vorher gewissenhaft

Ich entschließe mich meist spontan zum Kauf, wenn ich ein zusagendes stück finde

Eine Antiquität wird nur dann gekauft, wenn auch die anderen Familienmitglieder einverstanden sind Ich entscheide in der Regel allein, wenn eine Antiquität angeschafft werden soll

Auch wenn ich eigentlich nicht die Absicht hatte, etwas zu kaufen, kommt es schon vor, das ich eine Antiquität mit nach Hause bringe

8. Ein Antiquitätenkauf kann von unterschiedlichen Gefühlen und Gedanken begleitet werden. In welchem Ausmaß entsprechen die folgenden Aussagen Ihren persönlichen Erfahrungen?

Antwortmöglichkeiten skaliert (trifft sehr zu / trifft ziemlich zu / trifft etwas zu / trifft kaum zu / trifft gar nicht zu) für die Statements :

Ich habe beim Kauf öfter das Gefühl, daß eine ähnlich gute Gelegenheit so schnell nicht wieder kommt

Wenn man bei einer Antiquität, die einem gefällt, nicht sofort zugreift, wird sie einem meist vor der Nase weggeschnappt

Antiquitäten muB man jetzt kaufen, denn das Angebot wird ständig geringer, und die Preise steigen schnell

Nach dem Kauf von Antiquitäten habe ich schon mal das Gefühl gehabt, doch nicht ganz das richtige Stück gekauft zu haben

Gerade nach einem Kauf fallen mir ähnliche Antiquitäten bei anderen Händlern besonders auf, und ich vergleiche sie mit meiner eigenen 
9. Beim Kauf einer Antiquität kann man seine Auswahl nach verschiedenen Gesichtspunkten treffen. Bitte geben Sie jeweils an, wie wichtig die im folgenden genannten Merkmale für sie persönlich sind.

Antwortmöglichkeiten skaliert (sehr wichtig / ziemlich wichtig / etwas wichtig / kaum wichtig / gar nicht wichtig) für die Merkmale :

Dekorationswert, Qualität, Gebrauchsfähigkeit, Seltenheit, Wertbeständigkeit, Exklusivität, Anziehungskraft, Zeitlosigkeit, Individualität, Originalität, Wert, Schönheit, Repräsentativität, solide Verarbeitung, künstlerische Bedeutung, Ausdrucksstärke, Ausgefallenheit, Stilechtheit, Wiederverkäuflichkeit, Mobilität, Alter, Funktionalität/Praktikabilität

10. Es gibt unterschiedliche Einkaufsquellen für Antiquitäten. Wo haben Sie bisher Ihre Antiquitäten gekauft ?

Antwortmöglichkeiten (Mehrfachnennungen möglich) :

auf Kunst- und Antiquitätenmessen

auf Auktionen durch schriftliches Gebot

auf Auktionen durch persönliches Gebot

von anderen Privatleuten

in einem Kunst- und Antiquitätengeschäft, welches sich auf Kunstgegenstände meines persönlichen

Interesses spezialisiert hat

in einem Kunst- und Antiquitätengeschäft, welches Kunstgegenstände unterschiedlichster Art führt

in einem Fachgeschäft, welches überwiegend andere Dinge wie z.B. Einrichtungsgegenstände und nur nebenbei Kunstgegenstände verkauft

im Trödelladen

auf Antiquitätenmärkten

beim Versandhandel durch Katalogbestellung auf Trödel- und Flohmärkten 
11. Worauf legen Sie persönlich beim Einkauf Ihrer Antiquitäten besonderen Wert ? Bitte beurteilen Sie die folgenden Aussagen, inwieweit sie auf Ihre eigenen Wünsche und Anforderungen an ein Antiquitätengeschäft bzw. Auktionshaus zutreffen.

Antwortmöglichkeiten skaliert (sollte sehr zutreffen / sollte ziemlich zutreffen / sollte etwas zutreffen / sollte kaum zutreffen / sollte gar nicht zutreffen) für die statements :

sichere und verläßliche Beratung bieten

elegant und großzügig wirken

breite Auswahl in einem Spezialgebiet bieten

hohe fachliche Qualifikation der Verkäufer garantieren

beim Preis mit sich handeln lassen

einen bekannten Namen und einen guten Ruf besitzen

ohne viel Zeitaufwand und Mühe erreichbar sein die Chance bieten, auch einmal ein "Schnäppchen" zu machen

Antiquitäten unterschiedlichster Art führen die Möglichkeit geben, selbst herumzustöbern ohne große Umstände ein spezielles Stück besorgen können

den Antiquitätenkauf selbst schon zum Erlebnis werden lassen

ein Stück, das mir nicht mehr gefällt, zurücknehmen

nur stücke von spitzenqualität führen

eine etwas verstaubte und liebevolle Atmosphäre besitzen

die Richtigkeit der gemachten Angaben über die Verkaufsstücke garantieren

zu jeder Antiquität eine Expertise geben

auch Kleinigkeiten, die sich als Geschenke eignen, anbieten

beim Aufbau einer Sammlung'beraten können

12. Wieviel Geld würden Sie höchstens für eine einzelne Antiquität ausgeben ?

Offene Frage mit der Nennung eines DM-Betrages 
13. Es gibt verschiedene Möglichkeiten, sich über Antiquitäten $z u$ informieren. In welchem Ausmaß nutzen Sie die nachfolgend genannten Informationsmöglichkeiten?

Antwortmöglichkeiten skaliert (regelmäßig / häufig / gelegentlich / selten / nie ) für :

Fachbücher, Fachzeitschriften, Tageszeitungen, Auktionskataloge, Auktionsbesuche, Messebesuche, Museumsbesuche, Ausstellungskataloge, Kleinanzeigen, Auslagen der Fachgeschäfte, Gespräche mit Fachhändlern, Gespräche im Freundes-oder Bekanntenkreis, Besuch von AntiquitätenMärkten, Besuch von Trödel- und Flohmärkten

14. Wie informieren Sie sich im allgemeinen iber Antiquitäten? Welche der genannten Aussagen trifft für Sie persönlich zu?

Antwortmöglichkeiten skaliert (trifft sehr zu / trifft ziemlich zu / trifft etwas zu / trifft kaum zu / trifft gar nicht zu, für die Statements :

Ich suche intensiv nach Informationen über Antiquitäten

Ich überlasse es dem Zufall, Neues über Antiquitäten zu erfahren

Ich benutze einen Großteil meiner Freizeit, um mich über Antiquitäten $\mathrm{zu}$ informieren

Ich unternehme gezielt Reisen in andere Städte, um mich über Antiquitäten $\mathrm{zu}$ informieren

Ich unternehme gezielt Reisen ins Ausland, um mich über Antiquitäten $\mathrm{zu}$ informieren

Mich interessieren Informationen über Antiquitäten nur am Rande

15. Welchen Themen schenken Sie im Zusammenhang mit Antiquitäten und dem Antiquitätenmarkt Ihre besondere Aufmerksamkeit ?

Antwortmöglichkeiten skaliert ( siehe Frage 14) für die statements:

Was man im Moment wo kaufen kann / Preise und Preisentwicklungen / Ausstellungs- und Messetermine / kunstwissenschaftliche Abhandlungen / Abbildungen und Beschreibungen schöner Antiquitäten / Monographien über Künstler / steuerrechtliche Fragen / Antiquitäten als Kapitalanlage / was momentan stark gefragt ist / Sammeln mit Gewinn / Dokumentationen über Antiquitäten, die den eigenen möglichst ähnlich sind 
16. Man kann sein Leben in sehr vielfältiger Weise gestalten. Wir nennen Ihnen im folgenden einige Aktivitäten ; bitte geben Sie an, in welchem AusmaB Sie diese persönlich ausüben.

Antwortmöglichkeiten skaliert (regelmäßig / häufig / gelegentlich / selten / nie) für die Aktivitäten :

aktiv Sport treiben / Museums-, Theater- und Konzertbesuche machen / private Auslandsreisen unternehmen / gut und exklusiv Essen gehen / gehobene Literatur lesen / an gesellschaftlichen Ereignissen teilnehmen / dem persönlichen Hobby nachgehen / mit der Familie etwas unternehmen / Tageszeitung lesen / Freunde und Bekannte treffen / die Wohnung neu gestalten

17. Jeder Mensch hat bestimmte Vorstellungen, Wünsche und Ziele. In welchem Ausma $B$ stimmen Sie den folgenden Aussagen zu?

Antwortmöglichkeiten skaliert (trifft sehr zu / trifft ziemlich zu / trifft etwas zu / trifft kaum zu / trifft gar nicht zu) für die statements :

Ich möchte als Person von anderen anerkannt und gemocht werden

Ich möchte mich deutlich vom Durchschnittsmenschen abheben

Ich möchte mehr als bisher aus meinem Leben machen

Ich möchte gern meinen Freundeskreis erweitern

Ich möchte mein eigenes Leben führen, was andere dabei von mir denken, ist mir egal

Ich möchte mein Leben genießen und mich nicht mit den Sorgen von morgen belasten

Ich möchte mehr materielle Sicherheit erlangen

Ich möchte eine unverwechselbare Persönlichkeit darstellen

Ich möchte, daß sich meine Freunde und Bekannte auch bei mir Zuhause wohlfühlen

Ich möchte intensiver meinen Hobbys nachgehen

Ich möchte als eine gepflegte Erscheinung mit sicherem Auftreten gelten

Ich möchte beruflich weiterkommen

Ich möchte mehr von der welt sehen

Ich möchte gern für meine zukunft und die meiner Kinder vorsorgen

Ich möchte mich in meiner Wohnung besser als bisher einrichten 
18. Antiquitäten sind oft Gegenstand von Gesprächen mit Freunden und Bekannten. Welche Beobachtungen haben Sie persönlich bei solchen Gesprächen gemacht? Bitte geben Sie uns an, in welchem Ausma $B$ Sie aus eigenen Erfahrungen den folgenden Aussagen zustimmen.

Antwortmöglichkeiten skaliert (trifft sehr zu / trifft ziemlich zu / trifft etwas zu / trifft kaum zu / trifft gar nicht zu, für die Statements :

Wir führen in unserem Freundes- und Bekanntenkreis oft Gespräche über Antiquitäten

Ich habe Freunde bzw. Bekannte, auf deren Urteil

ich bei Antiquitätenkäufen großen Wert lege

Ich habe bei Freunden bzw. Bekannten das Interesse für Antiquitäten geweckt

Ich glaube, meine Freunde bzw. Bekannten beneiden mich ein wenig um meine Antiquitäten

Ich habe den Ruf, über Antiquitäten gut Bescheid zu wissen und werde oft um meine Meinung gefragt

Ich kenne Leute, die in Gesprächen ganz schön mit ihren Antiquitäten prahlen

19. Welche Eigenschaften verbinden Sie in Ihrer persönlichen Vorstellung ganz allgemein mit Antiquitäten ?

Antwortmöglichkeiten skaliert (siehe Frage 18) für die Eigenschaften :

dekorativ, qualitätvoll, gebrauchsfähig, selten, wertbeständig, exklusiv, anziehend, zeitlos, individuell, original, wertvoll, schön, repräsentativ, solide gearbeitet, künstlerisch bedeutend, ausdrucksstark, ausgefallen, stilecht, wiederverkäuflich, mobil, alt, funktional/praktisch

20. Wir nennen Ihnen nun einige Eigenschaften, mit denen man einen Antiquitätenkäufer beschreiben kann. Welche dieser Eigenschaften treffen nach Ihrer Meinung für Sie persönlich eher $z u$ ? Bitte entscheiden Sie für jedes Eigenschaftspaar, wo Sie Sich selbst tendenziell einordnen.

Antwortmöglichkeiten im Polaritätenprofil für :

Pessimistisch - optimistisch / risikofreudig - risikoscheu / introvertiert - extravertiert / impulsiv planend / entscheidungsfreudig - entscheidungsschwach / rational - emotional / kontaktfreudig -kontaktscheu / geschmackssicher - geschmacklich unsicher / musich nicht musisch / konservativ - progressiv / selbstsicher unsicher / kritisch - unkritisch / fortschrittlich traditionsbewußt / gefühlsarm - gefühlsbetont / angepaßtindividualistisch 
21. Sie finden im folgenden eine Reihe von Aussagen, die man von Antiquitätenkäufern hören kann. Bitte geben Sie an, in welchem Ausmaß Sie persönlich jeweils diesen Aussagen zustimmen.

Antwortmöglichkeiten skaliert (trifft sehr zu / trifft ziemlich zu / trifft etwas zu / trifft kaum zu / trifft gar nicht zu) für die Statements :

Antiquitäten sind eine ebenso sichere Geldanlage wie ein Sparbuch

Antiquitäten sind genauso Spekulationsobjekte wie unsichere Aktien

Antiquitäten dienen vielen Menschen ebenso wie Häuser, Autos oder teurer Schmuck als Statussymbol

Teure Antiquitäten sollte man sich erst leisten, wenn man sonst alles Notwendige hat

Antiquitäten erinnern die Menschen an die gute alte Zeit

Antiquitäten passen $\mathrm{zu}$ jeder Einrichtung

Antiquitäten verleihen ihrem Besitzer eine individuelle Note

Ich werde gern auf meine Antiquitäten angesprochen

Es macht Spaß, auch $a b$ und $z u$ ein Stück wieder mit Gewinn zu verkaufen

Menschen, die sich teure Antiquitäten leisten

können, sind $\mathrm{zu}$ beneiden

Beim Antiquitätenkauf besteht auch heute noch

die Chance, Trouvaillen zu machen

Nur Spitzenstücke finden auch in schlechten

Zeiten einen Käufer

Ohne Fachkenntnisse kommt man beim Antiquitä-

tenkauf nicht aus

Im Moment gibt es eine regelrechte Antiquitätenmode

Antiquitätenkäufer sind ein ganz bestimmter Typ von Menschen

Ein Antiquitätenkauf bleibt immer ein finanzielles Wagnis 
Wenn man Antiquitäten erst einmal besitzt, kann man sich nur sehr schwer wieder davon trennen

Antiquitäten haben einen großen "Schwarzmarkt"

Mit Antiquitäten ist zumeist auch eine Geschichte verbunden, wie man sie erworben hat

Mit Antiquitäten kann man sich wohltuend von einer "normalen" Wohnungseinrichtung abheben

Antiquitäten demonstrieren einen kultivierten Lebensstil

Antiquitäten sind Ausdruck eines Kunstverständnisses ihres Besitzers

An Nachahmungen und Replikaten kann man sich genauso freuen wie an Originalen

Im Antiquitätenmarkt kommt es immer wieder zu spektakulären Preisentwicklungen

Der Preis einer Antiquität wird ausschließlich durch seine Qualität bestimmt

Die Preise für Antiquitäten regulieren sich durch das Zusammenspiel von Angebot und Nachfrage

Es gibt viele Antiquitätenkäufer, aie ohne Sachverstand kaufen, aber mit ihren Antiquitäten angeben

22. Es gibt vielfältige Möglichkeiten, Wohnstile und Wohnatmosphären $z u$ beschreiben. Wir nennen Ihnen hierzu im folgenden eine Reihe von Eigenschaftswörtern. Bitte geben Sie jeweils an, in welchem Ausma $B$ diese Eigenschaften auf Ihre eigene Wohnung zutreffen.

Antwortmöglichkeiten skaliert (trifft sehr zu / trifft ziemlich zu / trifft etwas zu / trifft kaum zu / trifft gar nicht zu) für die Eigenschaften :

nüchtern, elegant, individuell, dezent, unkonventionell, verspielt, gemütlich, praktisch/fuktionell, exklusiv, rustikal, repräsentativ, modern, aufwendig, dekorativ, zeitlos, schön, ausgefallen 
Fragen zur Soziodemographie:

1. Geschlecht

2. Wie alt sind sie?

3. Welcher beruflichen Tätigkeit gehen Sie nach ?

\begin{tabular}{|l|}
\hline Selbständig in Handel, Gewerbe, Industrie \\
\hline Selbständig in einem freien Beruf (z.B.Arzt,Anwalt) \\
\hline Angestellter/e \\
\hline Beamter/e \\
\hline Facharbeiter/in, Arbeiter/in \\
\hline Hausfrau \\
\hline Schüler/in \\
\hline Auszubildender/e \\
\hline Student/in \\
\hline Rentner/in \\
\hline
\end{tabular}

4. Wieviel Personen leben ständig in Ihrem Haushalt, Sie selbst eingeschlossen?

5. Welche abgeschlossene Schulbildung besitzen Sie ?

\begin{tabular}{|l|}
\hline Volks-, Hauptschule ohne abgeschlossene Lehre \\
\hline Volks-, Hauptschule mit abgeschlossener Lehre \\
\hline weiterführende Schule ohne Abitur \\
\hline Abitur, Reifeprüfung \\
\hline Studium \\
\hline
\end{tabular}

6. Wie ist Ihr Familienstand ?

7. Welcher Konfession gehören Sie an?

8. Wieviel Personen haben in Ihrem Haushalt ein eigenes Einkommen ? 
8. Wenn Sie einmal zusammenrechnen: wie hoch ist dann das monatliche Netto-Einkommen Ihres gesamten Haushaltes?

\begin{tabular}{|l|}
\hline unter $1.000 \mathrm{DM}$ \\
\hline 1.000 bis unter $2.000 \mathrm{DM}$ \\
\hline 2.000 bis unter $3.000 \mathrm{DM}$ \\
\hline 3.000 bis unter $4.000 \mathrm{DM}$ \\
\hline 4.000 bis unter $5.000 \mathrm{DM}$ \\
\hline 5.000 bis unter $6.000 \mathrm{DM}$ \\
\hline 6.000 und mehr \\
\hline
\end{tabular}

10. Wie groß ist der Ort oder die Stadt in der Sie leben?

\begin{tabular}{|c|c|c|c|c|}
\hline & & unter & 10. & ০oo \\
\hline 10. 000 & bis & unter & 100. & 000 \\
\hline 100.000 & bis & unter & 500. & 000 \\
\hline 500.000 & bis & unter & 1000 & 000 \\
\hline
\end{tabular}




\section{Literaturverzeichnis}

Ahlert, D. ,

Andreasen, A.R. , Beek, R.W.,

Andritzky, K. ,

Art Address-Verlag (Hrsg.),

Atkinson, J.W.,

Barth, H. ,

Bass, F.M. , Wilkie, W.L.,

Belk, R.W.,

Berekoven, L., Eckert, W. , Ellenrieder, P.,

Beutel, P. et al.,
Grundzüge des Marketing, Düsseldorf 1980

Predictors of Attendence at the Performing Arts, in: JoCR, Vol. 7

(September 1980), S. 112-120

Die Operationalisierbarkeit von Theorien zum Konsumentenverhalten, Berlin 1976

Kunstadressbuch für die Bundesrepublik Deutschland 1977/78, Regional-Ausgabe des : International Directory of Arts/ Internationales Kunst-Adressbuch, Frankfurt 1977

Einführung in die Motivationsforschung, stuttgart 1975

Zum ersten, zum zweiten und zum dritten ... Die Abenteuer eines Kunstsammlers, in: Die Welt (Welt-Report) $\mathrm{Nr} .48$ vom 25.2 .1978$, S. 25

A Comparative Analysis of Attitudinal Predictors of Brand Preference, in: JoMR, Vol. 10 (1973), S. 262-269

Situational Variables and Consumer Behavior, in: JoCR, Vol. 2 (1975), S. 157-164

Marktforschung. Methodische Grundlagen und praktische Anwendung, Wiesbaden 1977

SPSS 7 Statistik-Programm-System für die Sozialwissenschaften, 2. Aufl., Stuttgart, New York 1978 
Bleymüller, J., Gehlert, G., Gülicher, H.,

Böhler, H. ,

Bcngard, W. ,

Bruhn, M.,

Burnkrant, R.E.,

Cofer, C.N., Appley, M.H.,

Cox, D.F. (Hrsg.)

Cunningham, S.M.,

Dahlhoff, H.-D.,
Statistik für Wirtschaftswissenschaftler, München 1979

Beachtete Produktalternativen und ihre relevanten Eigenschaften im Kaufentscheidungsproze $\beta$ von Konsumenten, Beitr. in: Meffert, H., Steffenhagen, H., Freter, H., (Hrsg.), Konsumentenverhalten und Information, Wiesbaden 1979, S. 261-289

Kunst \& Kommerz, Zwischen Passion und Spekulation, Oldenburg-Hamburg 1967

Das soziale Bewußtsein von Konsumenten, Schriftenreihe Unternehmensführung und Marketing, Hrsg. Meffert H., Steffenhagen, H., Bd. 11, Wiesbaden 1978

A Motivational Model of Information Processing Intensity, in: JoCR, Vol. 3 (June 1976), S. 21-30

Motivation: Theory and Research, Sixth Printing, New York, London, Sidney, 1968

Risk Taking and Information Handling in Consumer Behavior, Boston 1967, s. $82-108$

The Major Dimensions of Perceived Risk, in: Cox, D.F. (ed.), Risk Taking and Information Handling in Consumer Behavior, Boston 1967, S. 82-108

Kaufentscheidungsprozesse von Familien Empirische Untersuchung zur Beteiligung von Mann und Frau an der Kaufentscheidung, Frankfurt 1980 


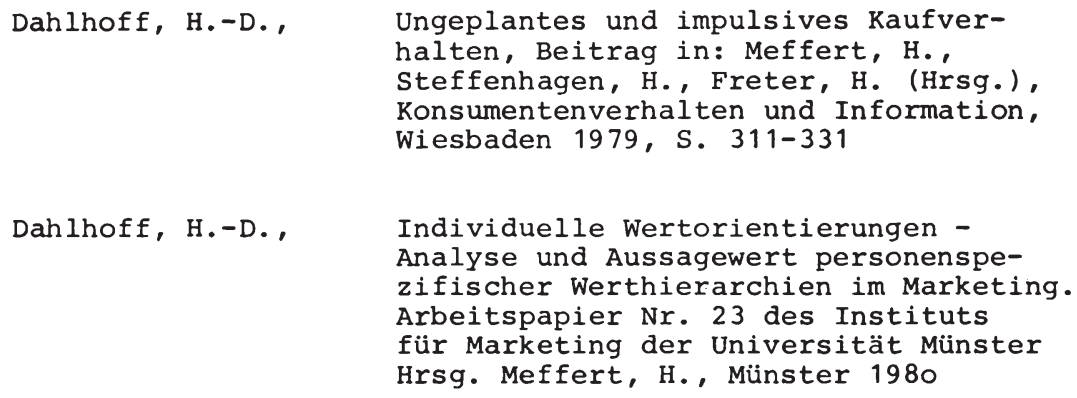
Analyse und Aussagewert personenspezifischer Werthierarchien im Marketing. Arbeitspapier $\mathrm{Nr} .23$ des Instituts für Marketing der Universität Münster Hrsg. Meffert, H., Münster 1980 2nd ed. Stanford 1962, in deutscher Sprache; Irle, M. , Möntemann, V., Theorie der kognitiven Dissonanz, Bern usw. 1978

The Arts and Marketing, in: Mokwa, P. et al., Marketing The Arts, New York 1980, S. 7-13

Congruence Relationships Between Self Images and Product Brands, in: JoMR, Vol. 6 (February 1969), S. $80-84$

Das große Bilderlexikon der Antiquitäten, Gütersloh usw. 1977

A Model of Consumer Motivation and Behavior, in: Research in Consumer Behavior, ed. dieselben, New York, Chicago usw. 1970

Motivation Research Revisited, in: JoAR, Vol. 15, No. 2 (June 1975), S. 23-27

A Theory of Social Comparison Processes, in: HR, Vol. 7 (1954), S. $117-140$ 
Fischer, L.,

Fischer, L.,

Fischer, L.,

Fischer, 0. ,

Freter, H. ,

Frey, D. ,

Gallup, G.H.,

v. Gehren, G. ,

v. Gehren, G. ,

Giachi, A. ,

Grasser, W. (Hrsg.),
Weltrekordpreise nutzen dem Kunstmarkt, in: Die WELT Nr. 192 vom 19. August 1978, S. 19

London bleibt die $\mathrm{Nr} .1$ am Kunstmarkt, in: Die WELT, Nr. 180 vom 5.8.1978, S. 17

Hirsch-Auktion in London: Kunst des Kaufens oder des Verkaufens?, in: Die WELT, Nr. 150 vom 1.6.1978, S.18

Finanzwirtschaft der Unternehmung $I$. Tübingen, Düsseldorf 1977, S. 122-130

Mehrdimensionale Einstellungsmodelle im Marketing. Interpretation, Vergleich und Aussagewert. Arbeitspapier Nr. 12 des Instituts für Marketing der Universität Münster, Meffert, H. (Hrsg.), Münster 1976

Die Theorie der kognitiven Dissonanz, in: derselbe (Hrsg.), Kognitive Theorien der Sozialpsychologie, Bern usw., 1978 , S. 243-294

Human Needs and Satisfactions: A Global Survey, in: POQ Vol. 40 $(1976)$, S. 459-467

Sorgfältige Sammlerpflege, in: HB Nr. 155 vom 14.8.1979, S. 15

Tendenzen zur Internationalisierung, in: WK 51. Jg. (1981), Heft 3 (Februar), S. 205-206

Die Spezialisten geben den Ton an, in: FAZ, Nr. 81 vom 21.4.1978, S. 27

Antiquitäten als Kapitalanlage, 2. Aufl., München 1975 


\begin{abstract}
Green, P.E. ,
Analyzing Multivariate Data, Hinsdale 1978

Gruner + Jahr (Hrsg.), Der Wohnmöbelmarkt in der Bundesrepublik Deutschland von 1960 - 1990 Eine Branchenanalyse, Symposiumsbericht, Hamburg-Saarbrücken 1978
Gruner + Jahr (Hrsg.), Wohnen und Leben - Marketinggerech- te Strukturen der zentralen Lebensbereiche, Hamburg 1978
\end{abstract}

Häußermann, B. ,

Hansen, F.,

Hansen, U.,

Harenberg, H. ,

v. Hassell, Chr.,

v. Hassell, Chr.,

Hawkins, J.,

Heckhausen, H. ,

Heckhausen, H. ,
Ein breitgefächertes Programm, in: WK, 50. Jg. (1980), Heft 7 (April) S. $852-861$

Psychological Theories of Consumer Choice, in: JoCR, Vol. 3 (December 1976), S. 117-142

Absatz- und Beschaffungsmarketing des Einzelhandels. Eine Aktionsanalyse, 1. Teilband, Göttingen 1976

Sammeln mit Gewinn, München 1976

Auktionssaison New York, August 1980, in: Weltkunst, Heft 17, 50. Jg. (1980), S. $2317-2318$

Ein Jahr der Rekorde, in: WK, 51. Jg. (1981), Heft 3 (Februar), S. 207-208

Londons Auktionshäuser auf der Suche nach Millionenobjekten, in: FAZ, $\mathrm{Nr} .251$ vom 28.10 .1980 , S. 18

Motivationsanalyse der Anspruchssetzung, in: Psychologische Forschung, Nr. 25 (1955), S. 126-143

Motiv und Motivation, Beitrag in: Herrmann, T., Hoffstätter, P.R., Huber, H. P., Weinert, F.E. (Hrsg.), Handbuch psychologischer Grundbegriffe, München 1977 , S. 296-313 
Hegewisch, K. ,

Heinemann, M. ,

v. Helmolt, Chr.,

Henry, W.A.,

Herchenröder, Chr. ,

Herchenröder, Chr. ,

Herchenröder, Chr.

Herchenröder, Chr.,

Hill, W. ,

Hillmann, K.H.,
Die Flaute im Mittelfeld - Antiquitätenmesse in München, in: FAZ Nr. 250 vom 28.10 .1981 , S. 28

Einkaufsstättenwahl und Firmentreue des Konsumenten, in: Meffert, $H$. (Hrsg.), Schriftenreihe Unternehmensführung und Marketing, Bd. 6, Wiesbaden 1976

Wo das Alter sehr hoch bewertet wird, in: FAZ Nr. 55 vom 5.3.1980, S. 27

Cultural Values do Correlate with Consumer Behavior, in: JoMR Vol. 13 (May 1976), S. 121-127

Der Kunstmarkt 1981 - besser als sein Ruf, in: WK, 51. Jg. (1981), Heft 20 , S. 2964

Die Kunstmärkte, Sammelgebiete Museumspolitik - Autkionsstrategien Messemärkte - Die großen Sammler Fälschungen - Wert der Expertise, Düsseldorf,Wien 1978

Nach kalter Polarluft eine gewisse Belebung - Freundlichere Tendenzen auf dem Kunstmarkt zum Jahresende, in: Kunst und Antiquitäten ( $K$ \& $A$ ), Heft 6 (November/Dezember) 1981, S. 6-7

Kunstmarkt - Eine bessere Markttransparenz ist anzustreben, in: Deutsche Bank (Hrsg.), Börsenbild und Anlagespiegel 1/82, Frankfurt 1982, S.5-6

Marketing, 3. Aufl., Bern u. Stuttgart 1973

Soziale Bestimmungsgründe des Konsumentenverhaltens, Stuttgart 1971 
Hoepfner, F.G.,

Hoepfner, F.G.,

Hofstätter, P.R. ,

Holbrook, M.B.,

Holloway, R.J.,

Holz, D.,

Howard, J.A.,

Howard, J.A., Sheth, J.N.,

Hull, C.H.,

Nie, N.H.,

Johnson, R.M.,

Jones, E.E.,

Gerard, H.B.,
Beeinflussung des Verbraucherverhaltens, München 1975

Verbraucherverhalten. Informationsprozesse, Einstel lungen, Entschlüsse, Stuttgart usw., 1976

Gruppendynamik, Beitrag in: Herrmann, T., Hofstätter, P.R., Huber, H.P. et al., Handbuch psychologischer Grundbegriffe, München 1977, S. 184-196

Some Preliminary Notes on Research in Consumer Esthetics, in: Advances in Consumer Research, Proceedings of AOCR, Olson, J.C. (ed.), San Francisco 1979, S. 104-108

An Experiment on Consumer Dissonance, in: JoM, Vol. 31 (January 1967), S. $39-43$

Die Optimumbestimmung bei Kauf-LeasingEntscheidungen, Frankfurt, Zürich 1973

Consumer Behavior: Application of Theory, New York usw. 1977

The Theory of Buyer Behavior, New York usw. 1969

SPSS Update, New York usw. 1979

Market Segmentation - A Strategic Management Tool, in: JoMR, Vol. 9 (February 1971), S. 13-18

Foundations of Social Psychology, New York usw. 1967 
Kaplan, L.B.,

Szybillo, C.J.,

Jacoby, J.,

Kassarjian, H.H.,

Kassarjian, H.H., Sheffert, M.J.,
Components of Perceived Risk in

Product Purchase, in: JoAP, Vol. 59 (1974), S. 287-291

Personality and Consumer Behavior: A Review, in: JoMR, Vol. 8 (November 1971), S. 409-418

Personality and Consumer Behavior: One More Time, in: AMA Combined Proceedings 1975, Mazze, M. (ed.),

S. 197-201

Katona, G., Das Verhalten der Verbraucher und Unternehmer, Tübingen 1960

Keysersche Verlags- Der Kunst- und Antiquitäten-Markt
buchhandlung (Hrsg.), 1979/80, München 1978

Kim, J.O.,

Factor Analysis, in: SPSS, Nie, N.H. et al., 2nd ed., New York usw.

S. 468-514

Kim, J.O., Kohout, F.J.,

Multiple Regression Analysis: Subprogramm Regression, Beitr. in: SPSS,Nie, N.H. et al., 2nd ed., New York

S. $320-367$

Klecka, W.R.,

Discriminant Analysis, Beitr. in: Nie, N.H. et al., 2nd ed., New York usw., S. 434-467

Klenger, F.,

Simulation des Käuferverhaltens, Teil Krautter, J., 2, Analyse des Kaufprozesses, Wiesbaden 1972

Kollat, D.T., Customer Impulse Purchasing Behavior, in: JoMR, Vol. 4 (February 1967), S. $21-31$

Kollat, D.T., Is Impulse Purchasing Really a Useful Concept for Marketing Decisions, in: JoM, Vol. 33 (1969), S. 79-83 
Kotler, $\mathrm{Ph} .$,

Kreisel, H. ,

Kroeber-Riel, W. ,

Krohne, H.W. ,

Kruse, L.,

Rogge, K.-E.,

Kunstpreis-Jahrbuch,

Levy, S.J.,

Czepiel, J.A.,

Löhr, H.D. ,

Schütte, R.

Madson, K.B.,

Markin, J.R. (jr.),
Marketing-Management. Analyse, Planung und Kontrolle, Stuttgart 1974

Alte Möbel stehen hoch im Kurs, Beitr.in: Grasser, W., Antiquitäten als Kapitalanalge, 2. Aufl., München 1975, S. 32-35

Konsumentenverhalten, 2. Auf1., München 1980

Persönlichkeitstheorie, in: Herrmann, T. , Hofstätter, P.R., Huber, H.P., Weinert, F.E., Handbuch psychologischer Grundbegriffe, München 1977 , S. 341-355

Motivation, in: Rogge, K.-E., Hrsg., Steckbrief der Psychologie, 2. durchg. Auf1., Heidelberg 1974, S. $104-120$

Der Markt der Kunst international, Bd. XXXVA und XXXVB, Zg. v.: Wellensiek, H., Keyszelitz, R., München 1980

Marketing and Aesthetics, in: AMA Combined Proceedings 1974, Curham, R.C. (ed.) 1975, S. 386-391

Kunstumsatz findet auf einem Sondermarkt statt - Prinzipielles und Aktuelles über Kunstversteigerungen, Beitrag in: Harten, J., de la Motte, M. et al. (Hrsg.), Das Kunstjahrbuch, Bamberg 1979, S. 63-69

Theories of Motivation, 4. Aufl., Kent (Ohio), Munksgaard 1968

Consumer Behavior. A cognitive Orientation, New York 1974 
Maslow, A.M.,

Maslow, A.M.,

Mayntz, R., Holm, K. , Hübner, P. ,

Meffert, H. ,

Meffert, H.,

Meffert, H. ,

Meffert, H.,

Meffert, H. ,

Meffert, H. ,

Meffert, H. ,
A Theory of Human Motivation, in : $P R, 1943$, S. 370-396

Motivation and Personality, New York 1954, als Auszug wiedergegeben in: Levine, F.M. (Hrsg.), Theoretical Readings in Motivation: Perspectives on Human Behavior, Chicago usw. 1975, S. 358-379

Einführung in die Methoden der empirischen Soziologie, 3. Aufl., Opladen 1972

Marketing, Beitrag in: ManagementEnzyklopädie, Bd. 4, München 1971, S. 383-413

Modelle des Käuferverhaltens und ihr Aussagewert für das Marketing, in: ZfgSt, Nr. 127 (1971), S. 326-353

Handelsmarketing und Handelsfunktionen, in: Würfel und Ecken, Heft 2, 1972, S. $4-6$

Instrumente, absatzpolitische, Beitrag in: Tietz, B. (Hrsg.), Handwörterbuch der Absatzwirtschaft, Stuttgart 1974, Sp. 887-896

Die Beurteilung und Nutzung von Informationsquellen beim Kauf von Konsumgütern, in: Meffert, H., Steffenhagen, H., Freter, H. (Hrsg.), Konsumentenverhalten und Information, Wiesbaden 1979 , S. 39-65

Perspektiven des Marketing in den 8oer Jahren, in: DBW 40. Jg. (1980), Heft 1, S. 59-80

Marketing. Einführung in die Absatzpolitik, 5. Aufl., Wiesbaden 1980 
Meffert, H.

Steffenhagen, H.,

Freter, H., (Hrsg.),

Meunier, E.F.,

Miller, $J$. und $M$. (ed.),

Mischel, W.,

Mokwa, M.P., Dawson, W.M., Prieve, E.A.,

Müller-Mehlis, R.,

Müller-Mehlis, R.,

Müller-Mehlis, R.,

Newcomb, T.M.,

Turner, R.H.,

Converse, Ph.E.,
Konsumentenverhalten und Information, Wiesbaden 1979

Die "Bekehrung" Amerikas zur Kunst, Einfuhrung in: Behrmann, S.N., Duveen und die Millionäre. Zur Soziologie des Kunsthandels in Amerika, München 1960, S. 7-11

Miller's Antiques Price Guide, Professional Handbook 1981, Pinguin's Hall usw. 1981

Toward a Cognitive Social Learning Reconceptualization of Personality. in: Psychological Review 80 (1973), S. 252-283

Marketing the Arts, in: Permut, S.E. (Hrsg.), Series in Public and Nonprofit Sector Marketing, No. 1 , New York 1980

Stärkung der mittleren und unteren Ebene, in: WK, 51. Jg. (1981), Heft 3 (Februar), S. 203-205

Nachbericht zur 25. Deutschen Kunstund Antiquitätenmesse in München, in: WK, 5o. Jg. (1980), Heft 23, S. $3541-3543$

Vorschau auf die 26. Deutsche Kunstund Antiquitätenmesse in München, in: WK 51. Jg. (1981), Heft 20 (Oktober), S. $2867-2871$

The Nature of Motivation, in: Research in Consumer Behavior, ed. Kollat, D.T., Blackwell, R.D., Engel, J.F., New York usw. 1970, S. $24-30$ 
Nie, N.H.,

Hull, C.H.,

Jenkins, J.G., et al.,1975

Nielsen, R.P.,

McQueen, Ch.,

Noelle-Neumann, E.,

o.V.,

o.V.,

o.V. ,

o.V.,

o.v.,

o.v. ,

o.V., 1976 1980
Performing Arts Consumer Behavior:

An Exploratory Study, in: AMA

Combined Proceedings 1974, Chicago 1975, S. 392-395

Besitz-Konsum, in: Allensbacher Jahrbuch der Demoskopie, Bd. IV, 1974-

Amerikas Auktionshäuser müssen den Gürtel enger schnallen - Die Flucht in die Sachwerte ebbt ab, in: FAz, $\mathrm{Nr} .56$ vom 8.3.1982, S. 23

Antiquitäten - Eine Stilkunde für Sammler, $H$. Ebeling Int. Publishing Luxembourg (Hrsg.), Luxemburg

Antiquitäten im Warenhaus, Bddw, $\mathrm{Nr} .129$ vom 9.5.1980, S. 5

Antiquitäten. Fast wie Eigentum, in: Der Spiegel, Nr. 46 (November) 1980, S. $254-255$

Das Magnum-Interview, Julius G. Böhler: "Kunsthändler kann man nur sein - aus Leidenschaft", in: Neven DuMont, A. (Hrsg.), Der Kunstmarkt, dumont magnum, Heft 50 (Oktober 63), S. $46-48$

Der Schwerpunkt: Leasing macht Karriere, in: WiWo $\mathrm{Nr} .47$ vom 24.11. 1972 , S. 84-104

Die Geheimnisse des großen Bietens, in: FAZ Nr. 133 vom 27. Juni 1978, S. 19 
o.V.,

o.V.,

O.V.,

o.V.,

o.v.,

o.V.,

o.V.,

o.v. ,

o.V.,

o.V.,

o.V.,

Paschke, M. ,
Eine Legende unter dem Hammer, in: FAZ Nr. 129 vom 22.6.1978, S. 23

Kaufen Sie antizyklisch. Interview mit H. Hanstein, in: Capital, 18. Jg. (1979), Heft 1 (Januar), S. 41

Kaufen und Verkaufen, in: Capital, Heft 1, 18. Jg. (1979), S. 54-56

Künstlerpech. Wie Kunstgegenstände besteuert werden, in: Capital 17. Jg. (1978), Heft 8, S. 140

Kunstgriff. Art Consulting für Unternehmen, in: Capital 19. Jg. (1980), Heft 9, S. 158-161

Reger Konsum honoriert Messearbeit, in: HB Nr. 61 vom 27.3.1979, S. 19

Rückblick, Bestandsaufnahme und Vorschau anläßlich des 25 jährigen Messejubiläums; Presseinformation vom 16.10.1980 zur Eröffnung der Kunstund Antiquitäten-Messe München

Sind Antiquitätengeschäfte antiquiert? in: BddW Nr. 188 vom 16.8.1979, S. 6

Teutonischer Blitz, in: Der Spiegel Nr. 27 (Juli) 1978, S. 133-135

Verbraucher 78: Individualisierungswünsche, in: asw 20. Jg. (1978), Heft 4, S. $36-42$

Zwischen Expansion und Rezession, in: FAZ Nr. 197 vom 27.8.1981, S. 23

Kunstgegenstände im Steuerrecht, Beitrag in: FAZ (Hrsg.), Kunst- und Antiquitäten-Kalender 82 , Frankfurt 1981 , S. 151-153 
Paschke, M. ,

Paschke, M. ,

Polnitz, A.,

Raaij, W.F. von, Wandwossen, K. ,

Raffée, H. ,

v. Rath, K., Simmat, E.W., Schultze, B., Schreib, W., Thwaites, A.J.,

Reitlinger, G.,

Rettelbusch, E.,

Richebächer, K. ,

Rogge, H.-J.,
Zur Marktsituation des Kunst- und Antiquitätenhandels im Frühjahr 1981, in: wK, 51. Jg. (1981), Heft 10 (Mai), S. 1531

Rechtliche Aspekte des Kunsterwerbs und-sammelns, Beitrag in: FAz (Hrsg.), Kunst- und Antiquitäten-Kalender 82 , Frankfurt 1981, S. 148-151

Motivational Research from a Research Viewpoint, in: $\mathrm{POQ}$ Vol. 20 (Winter $1956 / 57)$, S. 663-673

Motivation - Need Theories and Consumer Behavior, in: Faculty Working Paper No. 432, Hrsg. College of Commerce and Business Administration, University of Illinois, Urbana-Champaign 1977

Konsumenteninformation und $\mathrm{Be}-$ schaffungsentscheidung des privaten Haushalts, Stuttgart 1969

Ein provokatorischer Dialog, in: Neven DuMont, A. (Hrsg.), Der Kunstmarkt, dumont magnum Heft 50

(Oktober 1963), S. 72-76

The Economics of Taste, Bd. I-III, London, New York 1961-1970

Stilhandbuch, Ornamentik, Möbel, Innenausbau von den ältesten Zeiten bis zum Biedermeier, 10. erw. Aufl., Stuttgart 1979

Sicherheit durch Sachwerte?, in: BFuP, 33. Jg. (1981), Heft 3, S. $221-230$

Marktforschung. Elemente und Methoden betrieblicher Infornationsgewinnung, iünchen, Wien 1981 
Rokeach, M. ,

Roselius, T.,

Rosenberg, M.J.,

Rosenberg, M.J.,

Rothschild, M.L.,

Rothwell, N.D.,

Rüttinger, B., v. Rosenstiel, L., Molt, W. ,

Ruhfus, E.,

Sachs, L.,

Sanchez, H.T.,

O'Brien, T.V.,

Summers, G.W. ,
Beliefs, Attitudes and Values, San Francisco 1968

Consumer Ranking of Risk Reduction Methods, in: JoM, Vol. 35 (1971), S. 56-61

Cognitive Structure and Attitudinal Affect, in: JoASP, Vol. 53

$(1956)$, S. 367-372

A Structural Theory of Attitude Dynamics, in: POQ, Vol. 24 (1960), S. 319-340

Involvement as a Determinant of Decision Making Styles, in: AMA Combined Proceedings 1975, Mazze, E.M. (ed.), S. 216-220

Motivation Research Revised, in: JoM, Vol. 19 (1955), S. 150-154

Motivation wirtschaftlichen Verhaltens, stuttgart usw. 1974

Kaufentscheidungen von Familien, Ansätze zur Analyse des kollektiven Entscheidungsverhaltens im privaten Haushalt, in: Schriftenreihe Unternehmensführung und Marketing, Meffert, H. (Hrsg.), Bd. 7, Wiesbaden 1976

Angewandte Statistik. Planung und Auswertung. Methoden und Modelle. 4. neu bearb. und erw. Auf1., Berlin, Heidelberg, New York 1974

Self Concept and Consumer Motivation, in: AMA Combined Proceedings 1975, Mazze, E.M. (ed.), S. 225-227 
Schewe, C.D:,

Schloz, G. ,

Schnabel, H. ,

Schroeder, H.M.,

Driver, M.J.,

Streufert, S.,
Selected Social Psychological Models for Analyzing Buyers, in: JoM, Vol. 37 (July 1973), S. 31-39

Kunst und Kommerz - Frivoles Verhältnis, in: WiWo Heft 10 (vom 7. März 1980), S. 50-51

Verhaltenswissenschaftliche Konsumtheorie, Stuttgart, Berlin, Köln, Mainz 1979

Menschliche Informationsverarbeitung. Die Strukturen der Informationsverarbeitung bei Einzelpersonen und Gruppen in komplexen Sozialen Situationen, Weinheim 1975

Schuchard-Ficher, Chr.Multivariate Analysemethoden - Eine Backhaus, K., anwendungsorientierte Einführung, Humme, U. et al., Berlin,Heidelberg, New York 1980

Schütte, R.,

Schulz, R. ,

Selmig, H. (Hrsg.),

Sheth, J.N., Venkatesan, M. ,
Kunst- und Antiquitätenhandel, in: Tietz, B. (Hrsg.), Handwörterbuch der Absatzwirtschaft, stuttgart 1974, Sp. 1165-1169

Kaufentscheidungsprozesse des Konsumenten, Schriftenreihe Unternehmensführung und Marketing. Hrsg. Meffert, H., Bd. 2, Wiesbaden 1972

Keysers Kunst- und Antiquitätenbuch, 8. Aufl., München 1974

Risk Reduction Process in Repetetive Consumer Behavior, in: JoMR, Vol. 5 $(1968)$, No. 3, S. 307-310 
Sheth, J.N.,

Steffenhagen, H. ,

Steinhausen, D. ,

Steinhausen, D., Langer, K.,

Straub, E. ,

Sturm, A.G.,

Tauber, E.M.,

Thomae, H. (Hrsg.),

Thomae, H., Feger, H.,

Thomae, H. ,
Role of Motivation Research in Consumer Psychology, in: Faculty Working Paper No. 153, Hrsg. College of Commerce and Business Administration, University of Illinois, Urbana Champaign 1974

Entscheidungsverhalten unter zeitdruck, in: Die Betriebswirtschaft, 38. Jg. (1978), Heft 4, S. 595-607

Neue Prozedur Cluster im SPSS-8 als lokale Erweiterung, in: inforum 1980, Heft 2, S. 27

Clusteranalyse, Berlin, New York 1977

Das Original in der Kunst - ein Fetisch?, in: FAZ Nr. 100 vom 3o. 4. 1979, S. 21

Schlag auf Schlag, in: Plus Nr. 20 vom 13.5.1981, Düsseldorf, Hamburg, Zürich, S. 22-28

Why do People Shop?, in: JoM, Vol. 36 (October 1972), S. 46-49

Die Motivation menschlichen Handelns, Köln, Berlin 1965

Hauptströmungen der neueren Psychologie, in der Reihe: Graumann, C.F. (Hrsg.), Einführung in die Psychologie, Bd. 7, 2. Aufl., Frankfurt 1972, S. 44-5o, und S. 147-152

Kognition und Motivation - Moderne Aspekte eines alten Problems, Beitrag in: Hartmann, K.D., Koeppler, K., (Hrsg.), Fortschritte der Marktpsychologie, Bd. 1, Frankfurt 1977, S. $9-19$ 
Trommsdorff, $\mathrm{V}$.
Uberla, K.,
Wiegelmann, G.,

Wille, J.G.,

Wiswede, G.,

Wiswede, G.,

Wiswede, G. ,

Wiswede, G.,

Yamane, T.,

Zikmund, W. G., Scott, E.A.,
Die Messung von Produktimages für das Marketing, Grundlagen und Operationalisierung, Köln usw. 1975

Faktorenanalyse, 2. Aufl., Berlin 1971

Anmerkungen zur Antiquitätenmode, unveröffentlichtes Manuskript eines Vortrages in Muinster am 3. Juni 1977 , o.s.

Zürich als internationaler Kunsthandelsplatz, in: SKA (Hrsg.), Bulletin Januar/Februar 1979, S. $38-39$

Motivforschung. Eine Analyse ihrer Erkenntnisgrenzen, Schriftenreihe Marktwirtschaft und Verbrauch, GfK (Hrsg.), Bd. 18, Nürnberg 1962

Rationalität und soziales Wertsystem, in: ZfWirtsch.u.Soz.Wiss. Nr. 4, 1972,92 . Jg. II. Halbband, S. $385-392$

Motivation und Verbraucherverhalten, 2. neubearb. Aufl., München 1973

Motivation zur Information - Determinanten konsumrelevanter Informationssuche, in: JAVF 1975, S. 221-241

Statistik, Frankfurt 1976

A Multivariate Analysis of Perceived Risk, Self-Confidence, and Information Sources, in: AOCR Word, S., Wright, P. (eds.), Advances in Consumer Research, Vol. 2, 1974, S. $406-416$ 


\section{SCHRIFTEN ZUM MARKETING}

Band 1 Friedrich Wehrle: Strategische Marketingplanung in Warenhäusern. Anwendung der Portfolio-Methode. 1981.

Band 2 Jürgen Althans: Die Übertragbarkeit von Werbekonzeptionen auf internationale Märkte. Analyse und Exploration auf der Grundlage einer Befragung bei europaweit tätigen Werbeagenturen. 1982.

Band 3 Günter Kimmeskamp: Die Rollenbeurteilung von Handelsvertretungen. Eine empirische Untersuchung zur Einschätzung des Dienstleistungsangebotes durch Industrie und Handel. 1982.

Band 4 Manfred Bruhn: Konsumentenzufriedenheit und Beschwerden. Erklärungsansätze und Ergebnisse einer empirischen Untersuchung in ausgewählten Konsumbereichen. 1982.

Band 5 Heribert Meffert (Hrsg.): Kundendienst-Management. Entwicklungsstand und Entscheidungsprobleme der Kundendienstpolitik. 1982.

Band 6 Ralf Becker: Die Beurteilung von Handelsvertretern und Reisenden durch Hersteller und Kunden. Eine empirische Untersuchung zum Vergleich der Funktionen und Leistungen. 1982.

Band 7 Gerd Schnetkamp: Einstellungen und Involvement als Bestimmungsfaktoren des sozialen Verhaltens. Eine empirische Analyse am Beispiel der Organspendebereitschaft in der Bundesrepublik Deutschland. 1982.

Band 8 Stephan Bentz: Kennzahlensysteme zur Erfolgskontrolle des Verkaufs und der Marketing-Logistik. Entwicklung und Anwendung in der Konsumgüterindustrie. 1983.

Band 9 Jan Honsel: Das Kaufverhalten im Antiquitätenmarkt. Eine empirische Analyse der Kaufmotive, ihrer Bestimmungsfaktoren und Verhaltenswirkungen. 1984. 
Kimmeskamp, Günter

\section{DIE ROLLENBEURTEILUNG VON} HANDELSVERTRETUNGEN

Eine empirische Untersuchung zur Einschätzung des Dienstleistungsangebotes durch Industrie und Handel

Frankfurt/M., Bern, 1981. XIV, 331 S.

Schriften zum Marketing. Bd. 3

ISBN 3-8204-5700-3

br. sFr. 73.-

Im Spannungsfeld von Industrie und Handel vermitteln Handelsvertretungen nahezu die Hälfte des Inlandsumsatzes der deutschen Industrie. In jüngerer Zeit wird der Aufgabenbereich von Handelsvertretungen unter Marketingaspekten verstärkt diskutiert. Die vorliegende Arbeit strukturiert das Dienstleistungsangebot von Handelsvertretungen und arbeitet wechselseitige Meinungen und insbesondere Anforderungen der Marktpartner an das Dienstleistungsangebot von Handelsvertretungen heraus. Die Aussagen basieren auf den Resultaten einer repräsentativen Befragung bei Industrieunternehmen, Handelsbetrieben und Handelsvertretungen.

Aus dem Inhalt: Verhaltenswissenschaftlicher Bezugsrahmen - Empirische Untersuchung zum Marketing von Handelsvertretungen - Beurteilungen und Anforderungen aus der Sicht von Industrie und Handel - Segmentierung des Hersteller- und Kundenmarktes - Implikationen für das Handelsvertreter-Marketing.

Becker, Ralf

DIE BEURTEILUNG VON HANDELSVERTRETERN UND REISENDEN DURCH HERSTELLER UND KUNDEN

Eine empirische Untersuchung zum Vergleich der Funktionen und Leistungen

Frankfurt/M., Bern, 1982. XVI, 306 S.

Schriften zum Marketing. Bd. 6

ISBN 3-8204-5767-4

br. sFr. 70.-

In den letzten Jahren haben die Verkaufsfunktionen im Marketing wieder eine zunehmende Bedeutung erlangt. Dabei tritt die Frage nach der Vorteilhaftigkeit von Handelsvertretern und Reisenden im Vertrieb erneut in den Vordergrund. Die vorliegende Arbeit unternimmt eine vergleichende Analyse der Leistungen und Funktionen der Absatzformen aus Hersteller- und Kundensicht. Auf der Basis einer Befragung von insgesamt über 1000 leitenden Unternehmensangestellten und Selbständigen aller Branchen und Betriebsformen liegt erstmals für die Bundesrepublik Deutschland eine umfassende Untersuchung zur Beurteilung von Handelsvertretern und Reisenden vor.

Aus dem Inhalt: Handelsvertreter und Reisende als konkurrierende Systeme im Absatzkanal Beurteilung der Vertriebsformen durch Hersteller und Kunden in der wirtschaftswissenschaftlichen Literatur - Einflussgrössen der Beurteilung - Empirische Prüfung von Hypothesen zum Vergleich der Vertriebsformen - Implikationen für ein Handelsvertretermarketing.

\section{Verlag Peter Lang Bern · Frankfurt a.M. New York}

Auslieferung: Verlag Peter Lang AG, Jupiterstr. 15. CH-3000 Bern 15 Telefon $(0041 / 31) 321122$, Telex verlch 32420

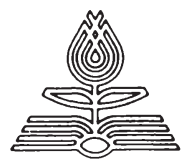


Althans, Jürgen

\section{DIE UBERTRAGBARKEIT VON WERBEKONZEPTIONEN AUF INTERNATIONALE MÄRKTE}

Analyse und Exploration auf der Grundlage einer Befragung bei europaweit tätigen Werbeagenturen

Frankfurt/M., Bern, 1981. XII, 309 S.

Schriften zum Marketing. Bd. 2

ISBN 3-8204-6986-9

br. sFr. 71.-

Die Diskussion, ob Werbekonzeptionen international tätiger Unternehmen international vereinheitlicht werden können (Standardisierung) oder länderspezifisch entwickelt werden müssen (Differenzierung), wird stark kontrovers geführt. Die vorliegende Arbeit strukturiert und analysiert die Bedingungen, unter denen eine Standardisierung internationaler Werbekonzeptionen möglich erscheint. Unter Berücksichtigung der Ergebnisse einer empirischen Untersuchung bei 32 international tätigen Werbeagenturen werden alternative Gestaltungsmöglichkeiten internationaler Werbestrategien abgeleitet.

Aus dem Inhalt: Standardisierung von Werbekonzeptionen internationaler Unternehmen Determinanten der Ubertragbarkeit von Werbekonzeptionen - Ergebnisse einer empirischen Untersuchung bei international tätigen Werbeagenturen.

Schnetkamp, Gerd

EINSTELLUNG UND INVOLVEMENT ALS BESTIMMUNGSFAKTOREN DES SOZIALEN VERHALTENS

Eine empirische Analyse am Beispiel der Organspendebereitschaft in der Bundesrepublik Deutschland

Frankfurt/M., Bern, 1982. XIII, 262 S.

Schriften zum Marketing. Bd. 7

ISBN 3-8204-7191-X

br. sFr. 63.-

In den Forschungsansätzen zur Erklärung menschlichen Verhaltens rückt in jüngster Zeit verstärkt das «soziale Verhalten» in den Mittelpunkt des Interesses. Dabei steht das Bemühen im Vordergrund, wesentliche Einflussgrössen des Verhaltens aufzudecken und zu systematisieren. Die vorliegende Arbeit untersucht den Erklärungs- und Prognosegehalt der verhaltenswissenschaftlichen Konstrukte «Einstellung» und «Involvement» für das soziale Verhalten und versucht eine Integration beider Variablen in einem multivariablen Erklärungskonzept. Die Betrachtungen basieren auf den Resultaten einer repräsentativen Befragung der Bevölkerung der Bundesrepublik Deutschland.

Aus dem Inhalt: Einstellung - Involvement - Social Marketing - «Organspender-Marketing» Verhaltenswissenschaftliche Erklärungsansätze.

\section{Verlag Peter Lang Bern · Frankfurt a.M. New York}

Auslieferung: Verlag Peter Lang AG, Jupiterstr. $15, \mathrm{CH}-3000$ Bern 15

Telefon $(0041 / 31) 32$ 1122. Telex verlch 32420

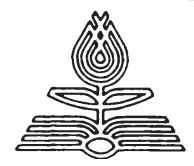


Wehrle, Friedrich

\section{STRATEGISCHE MARKETINGPLANUNG IN WARENHÄUSERN}

Anwendung der Portfolio-Methode

Frankfurt/M., Bern, 1981. XI, 301 S.

Schriften zum Marketing. Bd. 1

ISBN 3-8204-6954-0

br. sFr. 68.-

Die strategische Marketingplanung im Handel muss auch heute noch als vergleichsweise wenig beachtetes Problemfeld angesehen werden. Vielmehr stehen sowohl in Theorie als auch in Praxis Fragestellungen der taktisch-operativen Planung im Mittelpunkt. Die vorliegende Arbeit analysiert die planerischen Probleme des Marketing im Handel aus strategischer Sicht und zeigt am Beispiel der Betriebsform Warenhaus die systematische Entwicklung eines strategischen Marketing-Planungssystems auf, in dessen Mittelpunkt die Anwendung der aktuell stark diskutierten Portfolio-Methode steht.

Aus dem Inhalt: Strategisches Planungssystem im Handel - Diskussion der Anwendung der Portfolio-Methode in Warenhäusern - Ziele und Zielsysteme in Warenhäusern - Entwicklung von Marketing-Strategien in Warenhäusern - Einsatz der Marketinginstrumente in Warenhäusern - Fragen der organisatorischen Umsetzung von Marketingstrategien.

\section{Meffert, Heribert (Hrsg.)}

\section{KUNDENDIENST-MANAGEMENT}

Entwicklungsstand und Entscheidungsprobleme der Kundendienstpolitik

Frankfurt/M., Bern, 1982. VIII, 308 S.

Schriften zum Marketing. Bd. 5

ISBN 3-8204-5764-X

br. sFr. 70.-

Die Entwicklung des Kundendienstes zu einem bedeutenden Instrument der Marketingpolitik ist vor dem Hintergrund gesättigter Märkte, differenzierter Kundenbedürfnisse und verstärktem Konkurrenzdruck zu sehen. Das Angebot an Kundendienstleistungen ist zu einem wesentlichen Kaufentscheidungsfaktor geworden. Die erheblichen Koordinationserfordernisse kundendienstpolitischer Entscheidungen im Rahmen des gesamten Marketingmix legen es nahe, von einem Kundendienst-Management zu sprechen. Der Sammelband umfasst neun Beiträge kompetenter Praktiker und Wissenschaftler zu zentralen Problembereichen der Kundendienstpolitik. Es handelt sich dabei um die Ergebnisse eines Workshops über Kundendienst-Management an der Universität Münster.

\section{Verlag Peter Lang Bern · Frankfurt a.M. $\cdot$ New York}

Auslieferung: Verlag Peter Lang AG, Jupiterstr.15, CH 3000 Bern 15 Telefon $(0041 / 31) 321122$, Telex verlch 32420

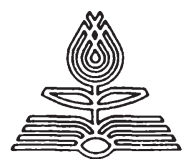

